\title{
Table of Contents
}

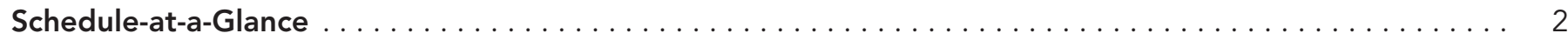

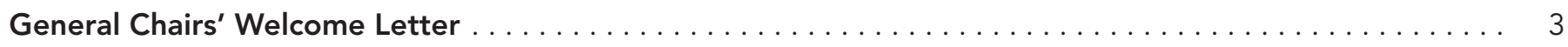

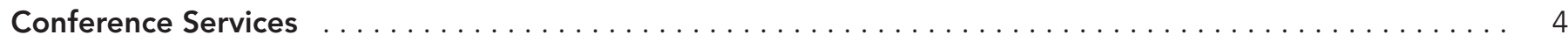

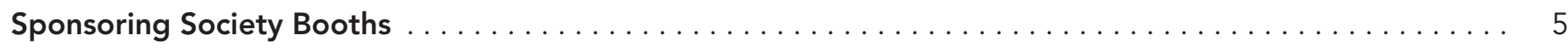

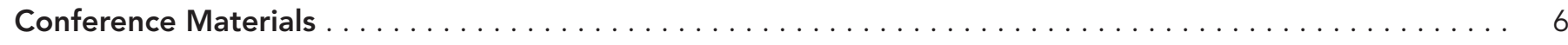

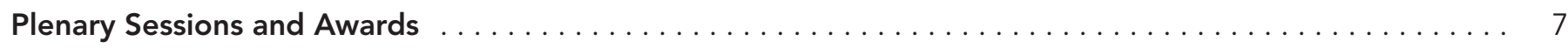

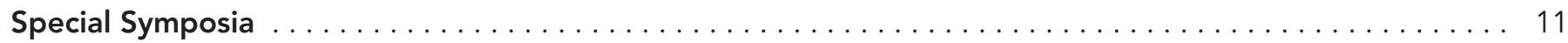

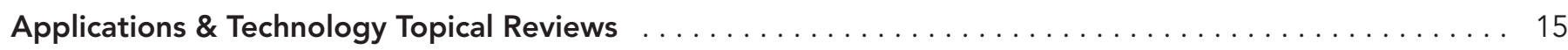

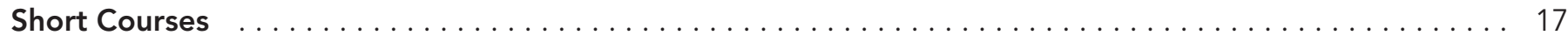

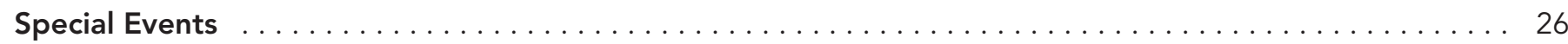

CLEO:EXPO

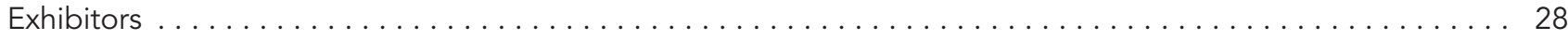

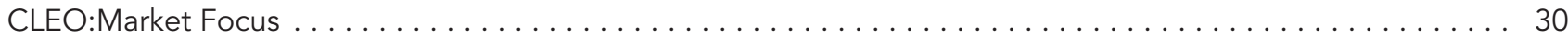

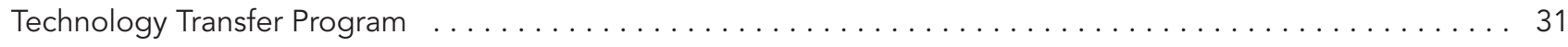

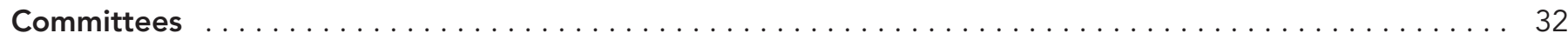

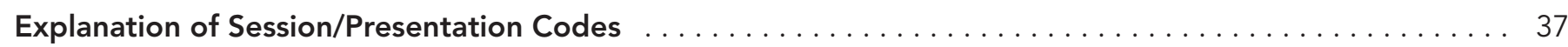

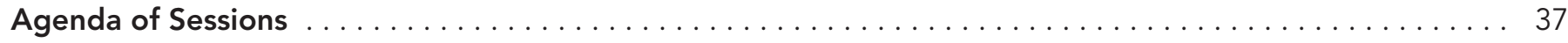

Technical Program

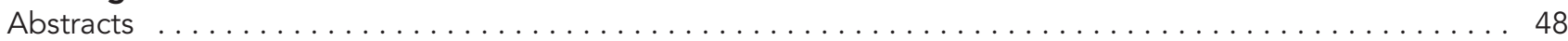

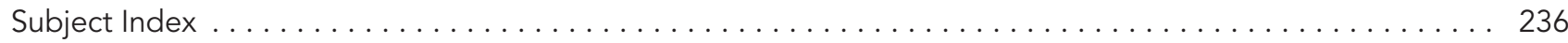

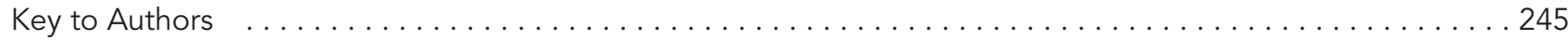

Convention Center Map $\ldots \ldots \ldots \ldots \ldots \ldots \ldots \ldots \ldots \ldots \ldots \ldots \ldots \ldots \ldots \ldots \ldots \ldots \ldots \ldots \ldots \ldots \ldots \ldots$ rear inside cover

CLEO Management thanks the following corporate sponsors for their generous support:

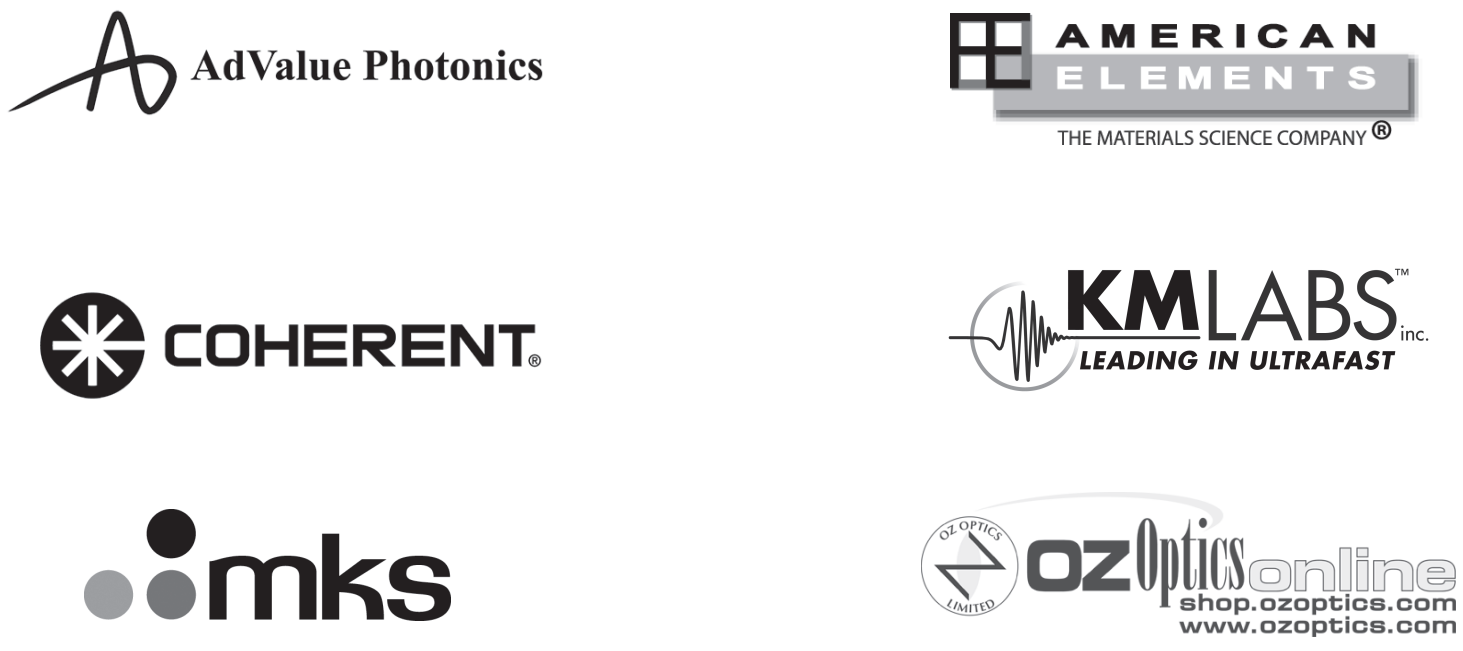

\section{THOR【ABS}

Conference on Lasers and Electro-Optics ${ }^{\circledR}$ 


\section{Schedule-at-a-Glance}

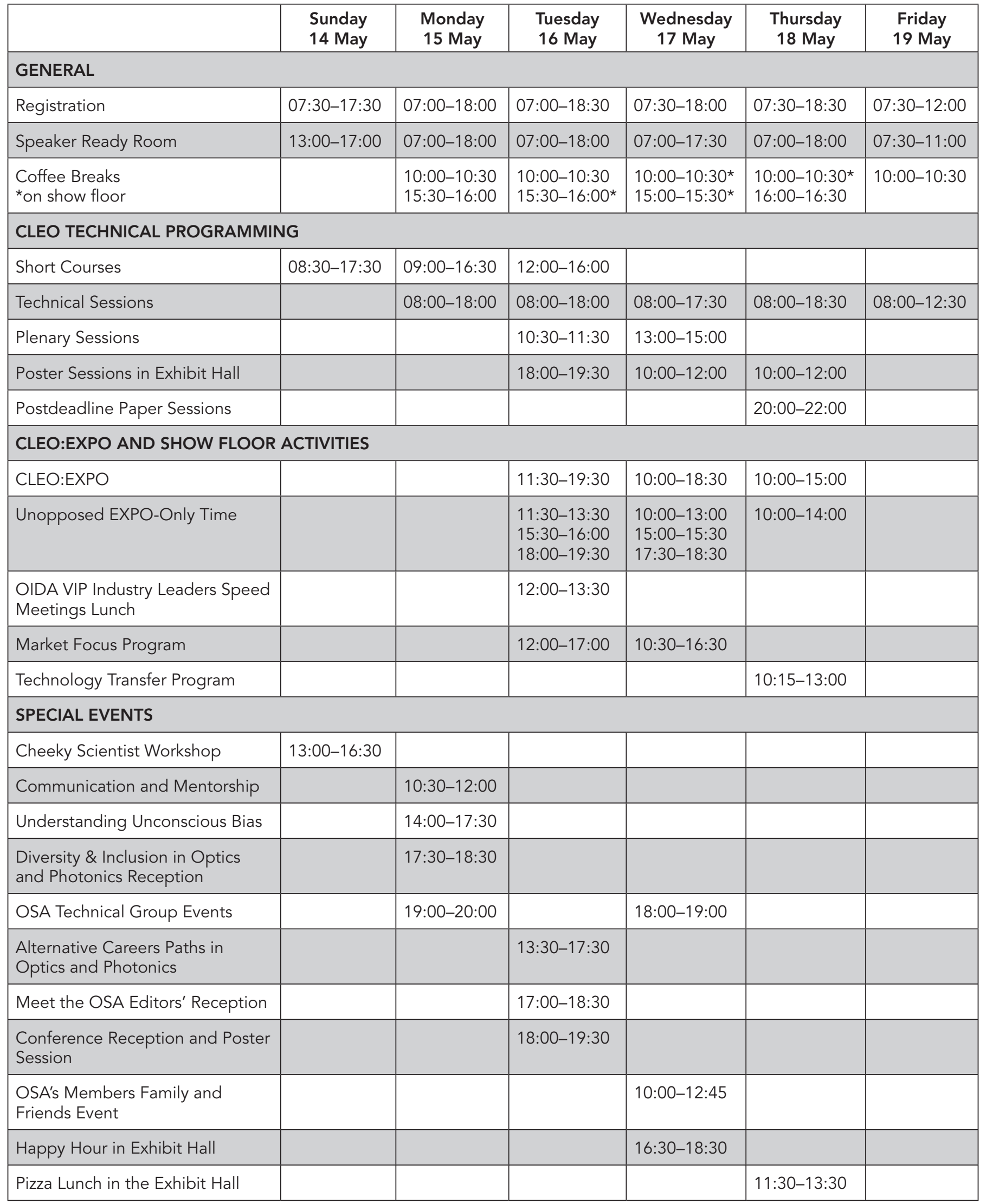




\section{Welcome to CLEO:2017!}

It is our pleasure to welcome you to CLEO 2017 in San Jose, CA. CLEO continues to be the world's premier international forum for scientific and technical optics, uniting the fields of lasers and optoelectronics by bringing together all aspects of laser technology, from basic research to industry applications. Within the scope of a single conference, CLEO provides a forum where attendees can explore new scientific ideas, engineering concepts, and emerging applications in fields such as biophotonics, optical communications, and novel light sources. While the quality of work presented remains assured by CLEO's world-renowned technical program, the conference continues to evolve with new features to enhance your experience.

CLEO offers high quality content in five core event elements:

Fundamental Science: The premier venue for discussion of basic research in optical and laser physics and related fields. Topics include modern spectroscopy, ultrafast and nonlinear lightmatter interactions, quantum optics, low-dimensional, optical materials, quantum information science, nanophotonics, plasmonics, and metamaterials.

Science \& Innovations: World-leading scientific research and innovation in lasers, optical materials, and photonic devices. Topics include laser processing of materials, terahertz sience and technologies, ultrafast optics, biophotonics, nanophotonics, fiber photonics, nonlinear optical and laser technologies, metrology, sensing, and energy-efficient "green" photonics.

Applications \& Technology: Exploration of the transition of fundamental research into emerging applications and products. The scope spans innovative laser and EO components and systems and applications. This topics include biomedical devices for diagnostics and therapeutics, high power laser systems for industry and defense, photonics instrumentation and technologies for metrology, industrial processes, environmental sensing, and energy conservation.

CLEO Expo: The Expo will showcase more than 200 participating companies featuring a wide range of photonics innovations, products and services; it is expected to attract more than 4,000 attendees including researchers, engineers, and leaders from top research institutions and major businesses who represent the fastest growing markets in optics and photonics.

CLEO Market Focus: This program focuses on the latest trends in the photonics marketplace and provides a forum to discuss new products and emerging technologies. All presentations and discussions are focused on the latest in photonics products and services that have been playing an important role in the industry and those that potentially hold a future business opportunity.

This year's CLEO features 4 exceptional plenary speakers. On Tuesday morning Nergis Mavalvala and Atac Imamoglu will be featured. Mavalvala will describe current efforts to improve the sensitivity of gravitational wave detectors and their prospects for future discoveries, while Imamoglu will discuss exciting recent developments on strong light-matter coupled states, or polaritons, in 2D systems. On Wednesday afternoon, we will be joined by Chris Contag and Ursula Keller. Contag will highlight new developments of wearable micro-optical devices for early cancer detection, and Keller will conclude the program with a talk reviewing passive mode locking of solid-state lasers with SESAMs and their many applications.

The CLEO Technical Program committee maintains a rigorous peer review system that emphasizes and maintains high technical quality in all presentations. This rigor is made possible by the combined efforts of over 300 volunteers in 25 technical committees. This year, the conference features an outstanding collection of contributed paper presentations, invited speakers and tutorials. We are excited to offer more than 1000 oral presentations, 194 invited talks by some of the most respected researchers in our international community, and 23 tutorials. This year's poster sessions include an outstanding list of more than 400 posters. Finally we are pleased to offer a comprehensive short course program featuring 18 courses.

We extend our thanks to the Technical Program Co-Chairs; Peter E. Andersen and Michael M. Mielke in Applications \& Technology; and Benjamin J. Eggleton and Irina Novikova in Fundamental Science, Sterling J. Backus and Michal Lipson in Science \& Innovations for coordinating the work of our subcommittees to compile this outstanding CLEO program. We also thank Robert Fisher and Ben Eggleton, Short Course Co-Chairs, and all of the program committee members whose leadership, dedication, and hard work has been critical to maintaining the high quality of the meeting. Additionally, we would like to thank the APS Division of Laser Science, the IEEE Photonics Society, The Optical Society (OSA), and the exhibitors for their support and contributions to the meeting. Finally, we thank the OSA staff for their professional assistance and dedication in organizing this event.

We welcome you to the conference and thank you for your participation.

\section{General Co-Chairs}

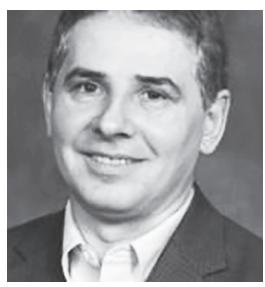

Nicusor Iftimia

Physical Sciences Inc., USA

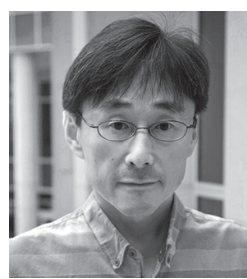

Junichiro Kono Rice University, USA

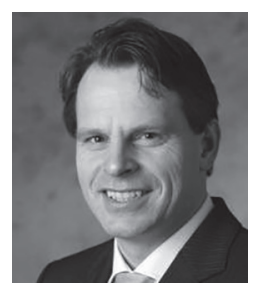

Christian Wetzel

Rensselaer Polytechnic Institute, USA

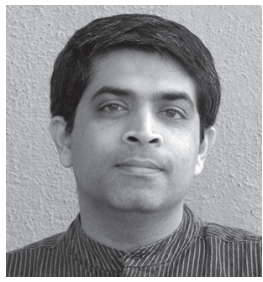

Siddharth Ramachandran University of Boston, USA

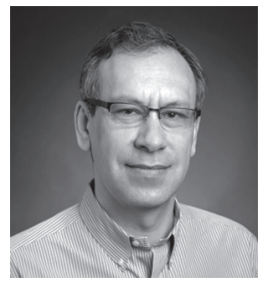

Yurii A. Vlasov University of Illinois at Urbana-Champaign, USA

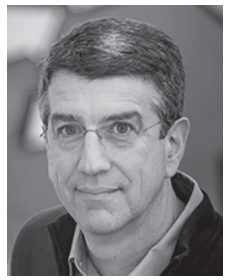

Jonathan D. Zuegel

University of Rochester, USA 


\section{Conference Services}

\section{Business Center}

The San Jose Convention Center does not have a business center on property. The following hotels - located within close proximity to the San Jose Convention Center - provide various levels of Business Center services.

\begin{tabular}{|l|l|}
\hline $\begin{array}{l}\text { San Jose Marriott, } \\
\text { First Floor near } \\
\text { front desk }\end{array}$ & $\begin{array}{l}\text { 24-hour access with guest key } \\
\text { Fax, printer, computers and copiers }\end{array}$ \\
\hline $\begin{array}{l}\text { San Jose Hilton, } \\
\text { Main Lobby }\end{array}$ & $\begin{array}{l}\text { 24-hour access with guest key } \\
\text { Computers and printer } \\
\text { Faxes sent and received at Front Desk } \\
\text { Complimentary wireless-internet in } \\
\text { public areas (lobby and second-floor } \\
\text { concourse) }\end{array}$ \\
\hline
\end{tabular}

\section{CLEO:EXPO}

Exhibit Halls 1, 2 and 3

The CLEO:EXPO is open to all registered attendees. Visit a diverse group of companies representing every facet of the lasers and electro-optics industries. Exhibition information can be found on page 28 .

\begin{tabular}{|l|l|}
\hline Tuesday -16 May & $11: 30-19: 30$ \\
\hline Wednesday, 17 May & $10: 00-18: 30$ \\
\hline Thursday, 18 May & $10: 00-15: 00$ \\
\hline
\end{tabular}

\section{Coat and Baggage Check}

Lower Lobby, Street Level

Coat and baggage check is available to conference attendees for a minimal fee.

\begin{tabular}{|l|l|}
\hline Thursday, 18 May & $07: 30-22: 00$ \\
\hline Friday, 19 May & $07: 30-13: 00$ \\
\hline
\end{tabular}

\section{Conference Information Center}

Concourse Level

Visit the Conference Information Center for help with the conference program, locating sessions, general information and lost and found. Please put your name on all conference materials (Program Book and Short Course Notes) as there is a replacement fee for these materials.

\begin{tabular}{|l|l|}
\hline Sunday, 14 May & $13: 30-17: 30$ \\
\hline Monday, 15 May & $07: 00-17: 00$ \\
\hline Tuesday, 16 May & 08:00-17:00 \\
\hline Wednesday, 17 May & 08:00-17:00 \\
\hline Thursday, 18 May & 08:00-16:00 \\
\hline
\end{tabular}

\section{E-Center Kiosks \\ Concourse Level}

The E-Center provides multiple stations allowing attendees to check email. The E-Center Kiosks will be open during registration hours.

\section{First Aid and Emergency Information}

The First Aid room, which is staffed with emergency medical personnel, is located on the Exhibit Level. This room will be open during all exhibit hours. In the event of an emergency, please contact a security guard or a CLEO staff member. All accidents, injuries or illnesses in the San Jose Convention Center should be reported to the Public Safety Office immediately; call the office at extension 3500 from any white courtesy phone.

\section{Registration \\ Concourse Level}

\begin{tabular}{|l|l|}
\hline Sunday, 14 May & $07: 30-17: 30$ \\
\hline Monday, 15 May & $07: 00-18: 00$ \\
\hline Tuesday, 16 May & $07: 00-18: 30$ \\
\hline Wednesday, 17 May & $07: 30-18: 00$ \\
\hline Thursday, 18 May & $07: 30-18: 30$ \\
\hline Friday, 19 May & $07: 30-12: 00$ \\
\hline
\end{tabular}

\section{Speaker and Presider Ready Room}

\section{Room 211 A-C}

All technical presentation speakers and session presiders are required to check in to the Speaker Ready Room located on the Concourse Level in Room 211 A-C. Speakers are requested to check in 24 hours before their sessions begin.

Session presiders should check in one to two hours prior to their session for instructions on how to use in-room equipment and check for speaker cancellations and changes. Computers will be available to review uploaded slides.

\begin{tabular}{|l|l|}
\hline Sunday, 14 May & $13: 00-17: 00$ \\
\hline Monday, 15 May & $07: 00-18: 00$ \\
\hline Tuesday, 16 May & $07: 00-18: 00$ \\
\hline Wednesday, 17 May & $07: 00-17: 30$ \\
\hline Thursday, 18 May & $07: 00-18: 00$ \\
\hline Friday, 19 May & $07: 30-11: 00$ \\
\hline
\end{tabular}

\section{Wireless Access}

As you'd expect from the capital of Silicon Valley, San Jose offers the nation's best free Wi-Fi experience.

To access the network just connect to the SSID, "Wickedlyfastwifi".

No personal information or password is needed with unlimited Wi-Fi access provided in the San Jose Convention Center.

All conference locations are in the San Jose Convention Center unless otherwise noted. 


\section{Sponsoring Society Booths}

\section{APS Booth \\ Concourse Level}

The American Physical Society (APS) is a non-profit membership organization working to advance and diffuse the knowledge of physics through its outstanding research journals, scientific meetings, and education, outreach, advocacy, and international activities. APS represents over 54,000 members, including physicists in academia, national laboratories, and industry in the United States and throughout the world. Society offices are located in College Park, MD (Headquarters), Ridge, NY, and Washington, D.C. Please stop by our booth near registration to learn more about APS programs, services, and world class journals.

\section{IEEE Photonics Society Booth \& Membership Lounge \\ Concourse Level}

The IEEE Photonics Society is the professional home for a global network of scientists and engineers who represent the laser, optoelectronics and photonics community. The Society provides its members with professional growth opportunities, publishes journals, sponsors conferences and supports local chapter and student activities around the world. Visit the IEEE Photonics Society booth for more information.

IEEE members are welcome to visit the IEEE Member Lounge sponsored by the IEEE Photonics Society, located near Registration. Come to the IEEE members-only lounge to relax, grab a snack and connect to the internet. Not a member? Learn more about how you can join IEEE Photonics by stopping by the lounge or society booth.

IEEE Lounge Schedule:

$\begin{array}{ll}\text { Monday: } & \text { 10:00-16:00 } \\ \text { Tuesday: } & \text { 10:00-16:00 } \\ \text { Wednesday: } & \text { 10:00-16:00 } \\ \text { Thursday: } & 10: 00-14: 00\end{array}$

\section{IEEE Photonics Fund}

The IEEE Photonics Society, in partnership with the IEEE Foundation, is proud to announce the establishment of the IEEE Photonics Fund. This fund will be used to enhance the humanitarian and educational initiatives of the Society by providing members and the photonics community with the ability to contribute directly to mission-driven imperatives, such as the Graduate Student Fellowship Program, Women in Photonics and STEM Outreach.

With the establishment of this fund, you too can play a direct role in this vital work. Visit the IEEE Photonics Society booth or IEEE-Photonics-Fund.org for more information.

\section{The Optical Society Booth \\ Exhibit Hall, \#1221}

Founded in 1916, The Optical Society (OSA) is the leading professional association in optics and photonics, home to accomplished science, engineering, and business leaders from all over the world.

Through world-renowned publications, meetings, and membership programs, OSA provides quality information and inspiring interactions that power achievements in the science of light. More than 20,000 OSA Members, residing in over 100 countries and spanning academic, government and industry, call OSA their professional home.

Stop by to meet OSA staff, and learn more about our publications, conferences and meetings, and membership for individuals and companies.

\section{The Optical Society Member Lounge Concourse Level}

OSA Members are invited to take a brief respite from the conference at the Member Lounge. Whether it's to plan your schedule, meet up with other members or print your boarding pass, the lounge offers comfortable seating, light refreshments, coffee service and a computer/printer.

OSA Member Lounge Schedule:

$\begin{array}{ll}\text { Monday: } & \text { 10:00-16:00 } \\ \text { Tuesday: } & \text { 10:00-16:00 } \\ \text { Wednesday: } & \text { 10:00-16:00 } \\ \text { Thursday: } & \text { 10:00-15:00 }\end{array}$

\section{OSA CAM Lounge}

Room 213

The Centennial Authentic Moments (CAM) videos are an opportunity for OSA members to share their stories in $3 \mathrm{~min}-$ utes or less about what/who inspired them to get into their field and what excites them about their current work. These short vignettes are shown on our website (osa.org/100), on social media and at some of our conferences. Stop by on Monday or Tuesday between 09:00-16:00 for a quick interview. 


\section{Conference Materials}

\section{Access to Technical Digest and Postdeadline Papers}

Technical attendees will have early and continuous access to the CLEO:2017 Technical Digest, including the Postdeadline Papers. The Technical Digest is comprised of the two-page summaries of tutorial, invited and accepted contributed/postdeadline papers. They can be downloaded individually or by downloading daily .zip files. (.zip files are available for 60 days after the conference).

1. Visit the conference website, www.cleoconference.org.

2. Select the Access Digest Papers link on the right side of the web page.

3. Log in using the same email address and password you used to register for the meeting. You will be directed to the conference page where you will see the .zip file links at the top of the page. Please note: if you are logged in successfully, you will see your name in the upper righthand corner.

Access is limited to Full Conference attendees only, not Exhibits Pass Plus or One-Day attendees. If you need assistance with your login information, please use the "forgot password" utility or "Contact Help" link.

The available paper summaries will be submitted to the IEEE Xplore Digital Library (www.ieeexplore.ieee.org), provided that the paper is presented by a co-author during CLEO:2017.

\section{Poster PDFs}

Authors presenting posters have the option to submit the PDF of their poster which will be attached to their papers in OSA Publishing's Digital Library. If submitted, poster PDFs will be available three weeks after the conference.

\section{Short Course Notes}

Notes typically include a copy of the presentation and any additional materials provided by the instructor. Each course has a unique set of notes, which are distributed on-site to registered course attendees only. Notes are not available for purchase separately from the course.

\section{CLEO App}

Manage your conference experience by downloading the CLEO App to your Smartphone or tablet.

\section{Download the app one of three ways}

1. Search for 'CLEO Conference' in the app store.

2. Go to cleoconference.org/app

3. Scan the QR code

\section{Schedule}

Search for conference presentations by day, topic, speaker or program type. Plan your schedule by setting bookmarks on programs of interest. Technical attendees can access technical papers within session descriptions.

\section{Exhibit Hall}

Search for exhibitors in alphabetical order and set a bookmark reminder to stop by their booth. Tap on the map icon within a description, and you'll find their location on the EXPO floor map. View a daily schedule of all activities occurring on the show floor.

\section{Access Technical Digest Papers}

Full technical registrants can navigate directly to the technical papers right from the CLEO mobile app. Locate the session or talk in "Event Schedule" and click on the "Download PDF" link that appears in the description.

IMPORTANT: You will need to log in with your registration email and password to access the technical papers. Access is limited to Full Conference attendees only.

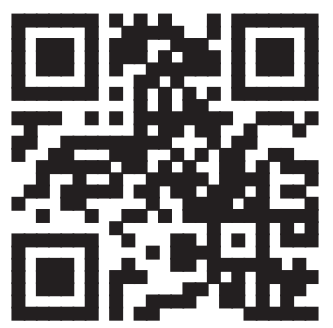

\section{Need assistance?}

Contact our support team, available 24 hours a day Monday through Friday, and from 09:00 to 21:00 EDT on weekends, at +1.888 .889 .3069 option 1. 


\section{Plenary Sessions and Award Ceremony}

\section{Plenary Session I}

Tuesday, 16 May, 10:30-11:30

Grand Ballroom

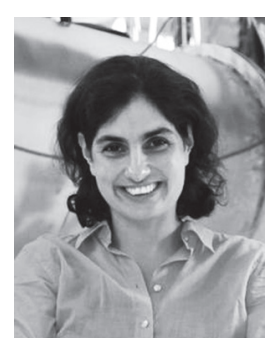

Gravitational Wave Detectors of the Future: Beyond the First LIGO Discoveries

Nergis Mavalvala, Massachusetts Institute of Technology, USA

In February 2016, scientists announced the first ever detection of gravitational waves from colliding black holes, launching a new era of gravitational wave astronomy and unprecedented tests of Einstein's theory of general relativity. Searching for fainter or more distant sources requires ever greater sensitivity for the laser interferometric detectors that made these first discoveries. Mavalvala will describe current efforts to improve the sensitivity of gravitational wave detectors and their prospects for future discoveries.

Biography: Nergis Mavalvala, Marble Professor of Astrophysics at MIT and a 2010 recipient of a MacArthur "genius" award, is a physicist whose research focuses on the detection of gravitational waves. She is a longstanding member of the scientific team that announced the first direct detection of gravitational waves from colliding black holes by the Laser Interferometer Gravitational-wave Observatory. Mavalvala has also conducted pioneering experiments on generation and application of squeezed states of light, and on laser cooling and trapping of macroscopic objects to enable observation of quantum phenomena in human-scale systems. Mavalvala received a B.A. from Wellesley College and a Ph.D. from MIT. She was a postdoctoral fellow and research scientist at the California Institute of Technology before joining the Physics faculty at MIT in 2002.

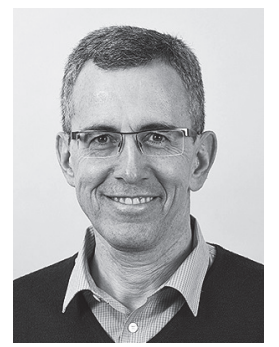

Polaritons in Two-dimensional Electron Systems

Ataç İmamoğlu, ETH Zurich, Switzerland

Cavity-polaritons have emerged as an exciting platform for studying interacting bosons in a driven-dissipative setting. In this talk, I will present cavity spectroscopy of gate-tunable monolayer MoSe2 hosting a degenerate electron gas and exhibiting strongly bound exciton-polaron resonances, as well as nonperturbative coupling to a single microcavity.

Biography: Ataç İmamoğlu graduated with a PhD from Stanford University in 1991. His PhD thesis was on the proposal and first demonstration of electromagnetically induced transparency. He joined the the University of California Santa Barbara from 1993 and 2002. In 2003, he moved to ETH Zurich as a Professor of Physics where his works focuses on quantum optics and condensed matter physics. He is the recepient of an IEEE Quantum Electronics Award and The Optical Society Charles Townes Award.
Plenary Session II \& Awards Ceremony

Wednesday, 17 May, 13:00-15:00

Grand Ballroom

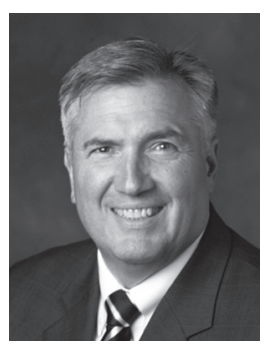

Insertable, Implantable and Wearable Micro-optical Devices for the Early Detection of Cancer

Chris Contag, Stanford University, USA

Optical imaging tools image over a range of scales from macro- to nanoscopic resolution and can provide molecular sensitivity and cellular level resolution. Developments in the field of optical imaging will be useful in informing diagnosis, prognosis and therapy, and for guiding biopsies for multiparameter molecular analyses.

Biography: Christopher Contag is a Professor in the Departments of Pediatrics, Radiology, Bioengineering and Microbiology \& Immunology at Stanford University, and a member of BioX program for interdisciplinary sciences, and the program in Immunology. Dr. Contag is the Associate Chief of Neonatal and Developmental Medicine, director of Stanford's Center for Innovation in In Vivo Imaging (SCl3) and co-director of the Molecular Imaging Program at Stanford (MIPS) and the Child Health Research Institute. Dr. Contag is a pioneer in the field of molecular imaging and is developing and using imaging approaches aimed at revealing molecular processes in living subjects, including humans, and advancing therapeutic strategies through imaging.

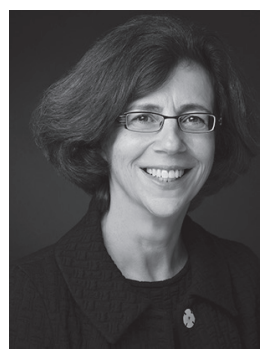

Ultrafast Solid-state Lasers: A Success Story with no End in Sight

Ursula Keller, ETH Zurich, Switzerland

This talk will review and give an outlook on ultrafast solid-state lasers based on SESAMs for passive modelocking. The thin disk laser geometry for efficient heat removal is currently the most successful approach for power scaling of diodepumped ion-doped solid-state and semiconductor lasers. Different gain materials, different performance parameters and different modelocking dynamics require different SESAM parameters. Novel optically pumped semiconductor disk lasers with fully integrated gain and absorber layers (i.e. MIXSEL) give full wavelength flexibility, simple linear cavities and can easily be operated in a dual comb mode. These lasers enable many application ranging from precision micromachining, frequency metrology and nonlinear microscopy.

Biography: Ursula Keller a tenured professor of physics at ETH Zurich since 1993 and currently also a director of the Swiss research program NCCR MUST in ultrafast science since 2010. She received a Ph.D. at Stanford University in 1989 and was a member of technical staff at Bell Labs from 1989 to 1993. She has been a co-founder and board member for Time-Bandwidth Products (acquired by JDSU in 2014) and for GigaTera (acquired by Time-Bandwidth in 2003). Her research interests are exploring and pushing the frontiers in ultrafast science and technology. Awards include the The Optical Society Charles H. Townes Award (2015), LIA Arthur L. Schawlow 
Award (2013), ERC advanced grant (2012), EPS Senior Prize (2011), OSA Fraunhofer/Burley Prize (2008), Leibinger Innovation Prize (2004) and Zeiss Research Award (1998).

\section{IEEE Photonics Society Young Investigator Award}

The IEEE Photonics Society Young Investigator Award honors an individual who has made outstanding technical contributions to photonics (broadly defined) prior to his or her 35th birthday.

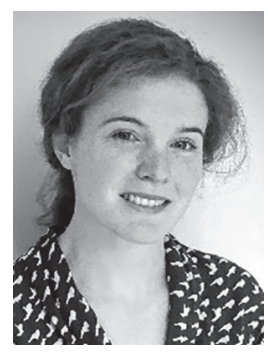

The 2017 award is presented to Hannah Joyce, University of Cambridge, UK, for significant contributions to nanowire optoelectronics and terhertz spectroscopy.

\section{OSA Charles Hard Townes Award}

The Optical Society (OSA) established this award in 1980 to honor Charles Hard Townes, whose pioneering contributions to masers and lasers led to the development of the field of quantum electronics. It is given to an individual or a group of individuals for outstanding experimental or theoretical work, discovery or invention in the field of quantum electronics.

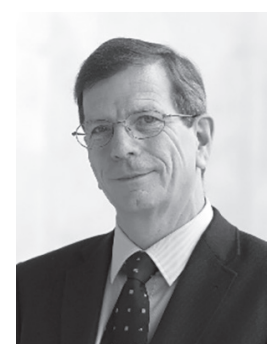

The 2017 recipient is Adolf Giesen, German Aerospace Center, Germany, recognized for pioneering breakthroughs in the field of solid-state lasers by the invention of and fundamental contributions to thin disk lasers.

\section{OSA Nick Holonyak, Jr. Award}

Established in 1997, this award honors Nick Holonyak Jr., who has made distinguished contributions to the field of optics through the development of semiconductor based light emitting diodes and semiconductor lasers. The award is presented to an individual who has made significant contributions to optics based on semiconductor-based optical devices and materials, including basic science and technological applications. This award is endowed by SDL Ventures, LLC, and Donald and Carol Scifres.

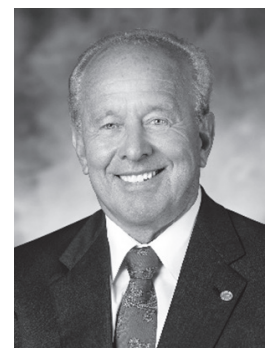

The 2017 recipient is Larry A. Coldren, University of California Santa Barbara, USA, recognized for major contributions to photonic integrated circuits.

\section{OSA R.W. Wood Prize}

Established by OSA in 1975 to honor the many contributions that R.W. Wood made to optics, this award recognizes an outstanding discovery, scientific or technical achievement, or invention in the field of optics. The accomplishment for which the prize is given is measured chiefly by its impact on the field of optics generally, and therefore the contribution is one that opens a new era of research or significantly expands an established one.

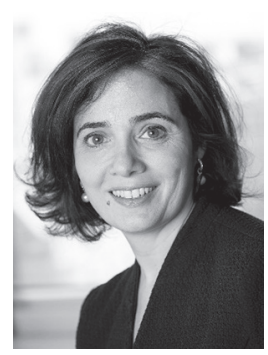

The 2017 recipient is Michal Lipson, Columbia University, USA, recognized for pioneering research contributions in silicon photonics.

\section{IEEE Photonics Society 2017 Fellows}

Robert Boyd, University of Ottawa, Canada For contributions to the fields of nonlinear optics and photonics

Holger Schmidt, University of California Santa Cruz, USA For contributions to optofluidics and integrated photonics

Olav Solgaard, Stanford University, USA

For contributions to optical micro-electro-mechanical devices and systems for sensing, communications and displays

Luc Thevenaz, Ecole Polytechnique Federale de Lausanne, Switzerland

For contributions to Brillouin-based fiber-optic sensors

\section{The Optical Society 2017 Fellows}

Ayman F. Abouraddy, CREOL, University of Central Florida, USA

For pioneering contributions to the development of a new generation of multi-material optical fibers and to the study of optical imaging using entangled photon pairs

Stefan Andersson-Engels, Tyndall National Institute, Ireland For academic, clinical and entrepreneurial contributions in biomedical optics, including techniques for tissue diagnostics and photodynamic therapy

Craig B. Arnold, Princeton University, USA

For significant contribution in the areas of laser materials processing and fabrication, with applications in nanotechnology, optoelectronics, sensing and energy

Wengang (Wayne) Bi, Hebei University of Technology, China For seminal contributions to research and development of photonic materials and device structures, and of their applications to display and solid-state lighting

Gerald S. Buller, Heriot-Watt University, UK For pioneering work in single-photon detection and applications of single-photon technology in three-dimensional imaging and quantum communications

Adrian Carter, Nufern, Australia For pioneering contributions to specialty optical fiber design, fabrication, and commercialization 
Victor L. Gamiz, US Air Force Research Laboratory, USA For 41 years of outstanding research in optics; achievements in the areas of the world's first demonstration of unconventional imaging using diversity of light; spearheading a national level effort to promote research in optical polarization for military applications

Shekhar Guha, US Air Force Research Laboratory, USA For original contributions in the areas of development and characterization of nonlinear optical materials for visible and infrared wavelengths

Robert H. Hadfield, University of Glasgow, UK For pioneering contributions in the development of infrared superconducting single-photon detectors and advanced photon-counting applications

Jeff Hecht, Hecht Associates, USA

For informing and educating professionals as well as the general public in the advances in optics and lasers

Richard B. Holmes, Boeing Company, USA

For contributions in the area of atmospheric and adaptive optics, imaging and remote sensing, and laser beam propagation through the atmosphere

Wei Jin, The Hong Kong Polytechnic University, China For outstanding and sustained contributions to the research, development and application of optical fiber sensor technology, particularly micro- and nano-structured fiber based devices and sensors

Ajoy Kumar Kar, Heriot Watt University, UK For application of nonlinear optics to the development of photonic devices by ultrafast laser inscription. An extensive variety of devices developed include waveguide lasers, supercontinuum generators, Bragg gratings and sensors

Manyalibo J. Matthews, Lawrence Livermore National Laboratory, USA

For outstanding contributions and sustained leadership in the field of high power laser-induced damage science, lasermaterial interactions and processing, and vibrational spectroscopy-based materials characterization

Noureddine Melikechi, University of Massachusetts Lowell, USA

For pioneering scientific, technological and educational contributions to optics, including novel methodologies in laser induced breakdown spectroscopy for space exploration and cancer diagnosis

Rajesh Menon, University of Utah, USA

For pioneering super-resolution optical lithography and innovations in metamaterials, nanophotonics, and micro-optics, with applications in photovoltaics, computational imaging, displays, color sensors, spectroscopy and silicon photonics

Thomas E. Murphy, University of Maryland at College Park, USA

For sustained contributions to nonlinear, electro-optic, and ultrafast processes in nanoscale materials, integrated photonics, devices, and systems

Jeffrey W. Nicholson, OFS Laboratories, USA

For pioneering contributions to fiber modal property measurements and significant contributions to the development of high-power and ultrafast fiber lasers
David E. Spence, Spectra-Physics, USA

For invention and demonstration of the self-modelocked Ti:sapphire laser and for significant and innovative contributions to the design of commercial scientific and industrial laser systems

Xiao Wei Sun, Southern University of Science and Technology, China

For important contributions to the photonic materials and devices for display and lighting applications

Zhiyi Wei, Institute of Physics, Chinese Academy of Sciences, China

For major contributions to ultrahigh intensity femtosecond lasers, carrier-envelope phase stable lasers, ultrafast nonlinear optics and the photonics community

Shinji Yamashita, RCAST, University of Tokyo, Japan For pioneering contributions to fiber lasers, including carbon nanotube and graphene photonics based ultrafast pulse generation and signal processing, and fast wavelength-swept dispersion-tuned lasers for optical coherent tomography

Zhigang Zhang, Peking University, China

For many contributions to ultrafast laser technology such as broadband SESAMs improved grating mirror stretchers model, and high pulse repetition rate fiber lasers

\section{Incubic/Milton Chang Travel Grant}

The OSA Foundation is pleased to award 10 recipients this year's Incubic/Milton Chang Student Travel Grant, endowed by Milton and Rosalind Chang. The list of recipients can viewed at www.osa.org/foundation.

\section{James P. Gordon Memorial Speakership}

Established in 2014 with the support of the Gordon family, The James P. Gordon Memorial Endowment funds a speakership on Quantum Information and Quantum Optics to a CLEO invited speaker. This speakership pays tribute to Dr. Gordon for his numerous high-impact contributions to quantum electronics and photonics, including the demonstration of the maser.

The recipient receives a $\$ 1,500$ honorarium and their presentation will be recorded and archived in OSA's media library. The contents will serve as an educational resource for the next generation of optics and photonics leaders.

Congratulations to

Paul G. Kwiat, University of Illinois, Urbana-Champaign, USA. Kwiat will present his talk, Synchronized Spontaneous Downconversion Supplies Scalable Single-Photon Sources (JM3E.1) at 13:30 on Monday. 


\section{Tingye Li Innovation Prize}

The Tingye Li Innovation Prize, established in 2013, honors the global impact Dr. Li made to the field of Optics and Photonics. This prize is presented to a young professional with an accepted paper that has demonstrated innovative and significant ideas and/or contributions to the field of optics. The recipient of this prize receives a $\$ 3,000$ stipend, a special invitation to the Chairs' Reception, and special recognition at the conference.

Congratulations to

Yoshitomo Okawachi, Columbia University, USA.

Okawachi will present his talk, Silicon Chip-Based Quantum

Random Number Generator (SM1M.1), at 08:00 on Monday.

\section{Maiman Student Paper Competition}

The Maiman Student Paper Competition honors American physicist Theodore Maiman for his demonstration of the first working laser and his other outstanding contributions to optics and photonics. It recognizes student innovation and research excellence in the areas of laser technology and electro-optics. The competition results will be announced during the meeting. The award is endowed by a grant from HRL Laboratories LLC, the IEEE Photonics Society and the APS Division of Laser Science and is administered by the OSA Foundation.

Congratulations to our finalists:

Bowen Li, University of Hong Kong, Hong Kong

Chu Teng, Princeton University, USA

Jinghui Yang, University of California Los Angeles, USA

Zhanshi Yao, Hong Kong University of Science and Technology, Hong Kong

Jingyuan Linda Zhang, Stanford University, USA 


\section{Special Symposia}

\section{Sources of Nonclassical Light and their Scalability \\ Monday, 15 May, 13:30-18:00 \\ Executive Ballroom 210 E}

\author{
Organizers \\ Virginia Lorenz, University of Illinois, USA \\ Joshua Nunn, Oxford University, UK \\ Tracy Northup, University of Innsbruck, Austria \\ Ite A. Yu, National Tsing Hua University, Taiwan
}

Nonclassical states of light possess unique properties that can only be described by quantum theory, and which find applications from quantum information to precision metrology to secure communication. A textbook example is single-photon states, and much effort has been devoted to developing single-photon sources. An ideal single photon source would generate (i) true single photons (ii) rapidly and on demand that are (iii) indistinguishable from both subsequent photons and photons from an identical source. Recent experimental progress has resulted in bright, deterministic and highly indistinguishable sources that approach this ideal. In parallel, there have been significant developments in sources for other types of nonclassical light, including squeezed light and entangled states. These novel sources rely on advances in virtually all branches of optical science, from integrated optics and plasmonics to photonic crystals and nanomaterials. Furthermore, the sources span multiple physical implementations, from nonlinear crystals to single atoms, ions, and molecules and atomic ensembles; from color centers in dielectrics to quantum dots in semiconductors; and, most recently, to optomechanical systems. This symposium will highlight the variety of present-day sources, the underlying technology and science that has made these advances possible, and the outstanding challenges in the field.

\section{Invited Speakers}

Maria Chekhova, Friedrich-Alexander University of ErlangenNürnberg, Germany, Photonic Crystal Fibers for Generating Three-photon States

Thomas Gerrits, NIST Boulder, USA, Utilizing Optical Transition Edge Sensors and Superconducting Nanowire Single Photon Detectors in Quantum Optics

Volkan Inlek, University of Maryland, USA, Entanglement of Quantum Memories by Interfering Distinguishable Photons

Paul Kwiat, University of Illinois at Urbana-Champaign, USA, Synchronized Spontaneous Downconversion Supplies Scalable Single-Photon Sources

Pascale Senellart, CNRS / Paris Sud University, France, Quantum Dot Based Devices for Scaling Up Optical Quantum Technologies

\section{Ultrafast Laser Technology for X-Ray Free Electron Lasers \\ Tuesday, 16 May, 13:30-18:00 \\ Salon I \& II, Marriott}

Organizers

Alan Fry, Stanford University, USA

Ingmar Hartl, DESY, Germany

XUV and X-ray free electron lasers (XFELs) require for their operation highly reliable ultrafast laser systems with unique and challenging capabilities for both the generation and manipulation of the electron beam, and for the delivery of spatially and temporally overlapped laser fields coincident with the X-ray beam on sample for pump-probe experiments. Examples of technical challenges include spatio-temporal pulse shaping, tunability from UV to $\mathrm{THz}$, and sub-fs timing and synchronization to external clocks. With four XFELs currently in operation worldwide and several more under construction, the issues surrounding optical laser systems for XFELs is of significant interest to both the facilities that deliver these capabilities and to the international community of facility users who will rely on this technology during beamtimes. In this symposium we will discuss current and future requirements and opportunities for ultrafast laser systems.

Invited Speakers

Ryan Coffee, SLAC, USA, Timing \& Synchronization of Lasers at XFELs

Miltcho Danailov, Elettra-Sincrotrone Trieste S.C.P.A., Italy, Laser-based Soft X-ray FEL Seeding: Recent Advances and Outlook at FERMI

Christop Hauri, SwissFEL, Switzerland, Intense Laser-based THz Sources for XFEL Experiments

Tino Lang, DESY, Germany, High Rep-rate Pump-probelasers for XFELs

Wilfried Wurth, University of Hamburg, Germany, Ultrafast Laser-Enabled Science at XFELs

Lutz Winkelmann, DESY, Germany, Photocathode Lasers for Free-Electron Lasers 
Military Applications of High Power Lasers

Tuesday, 16 May, 13:30-15:30

Salon V\&VI, Marriott

Organizers

Jony J. Liu, US Army Research Laboratory, USA

Gerarld C. Manke, NSWC-Crane, USA

John Schriempf, US Navy PEO for Integrated Warfare

Systems, USA

Anthony Valenzuela, US Army Research Laboratory, USA

This symposium will review recent developments in the use of high power lasers for military applications, particularly from the point of view of using them as defensive weapons. The recent fielding of a laser weapon by the Navy using laser technology developed for industrial welding and cutting is one example of the benefit which can be gained by the joining of the commercial and military laser development communities. Invited speakers are asked to review the latest developments in their service, with special emphasis on the technical challenges which laser weapon development shares with industrial applications of high power/energy lasers. Contributed papers are solicited which further develop this theme.

\section{Invited Speakers}

Larry Grimes, HEL/JTO, USA, High Energy Laser Joint Technology Office - A Mission Overview

lain McKinnie, Lockheed Martin, USA

Guy Renard, Northrop Grumman, USA, Advances in High Power Laser Systems for Directed Energy

\section{Advances in Metaphotonic Devices}

Wednesday, 17 May, 08:00-10:00; 15:30-17:30

Executive Ballroom $210 \mathrm{G}$

\section{Organizers}

Andrea Alù, The University of Texas at Austin, USA

Xingjie Ni, The Pennsylvania State University, USA

Jie Yao, University of California Berkeley, USA

Metaphotonics - represented by photonic metamaterials and more recently optical metasurfaces - has generated exciting new research paradigms and strengthened our understanding of light-matter interaction as well as material property engineering. Lately, a number of newly emerging sub-fields in metaphotonics go beyond the traditional designs and applications. The community are become more than ever interested in various new aspects, including the effectiveness, new functionality, new physics, and application in multiple disciplines. For example, pure dielectric metamaterials and metasurfaces have started to be explored, their flexibility, tunability, and reconfigurability have attracted wider attention, and they are beginning to be applied to the quantum optics. It greatly advances the scientific knowledge in multiple dimensions, and more importantly, it leads to new devices that are promising to the real-world applications. This symposium emphasizes on the newly emerging sub-fields in the metaphotonics. It seeks for transformative ideas about the future developments for metaphotonic devices, including novel quantum behavior, parity-time symmetry, optical nonreciprocity, nonlinearity and dynamics, and etc. and discusses the potential synergy among these vital directions.
Invited Speakers

Mark Brongersma, Stanford University, USA, Anti-Hermitian Metafilm-based Photodetector for Efficient Subwavelength Photon-sorting

Federico Capasso, Harvard University, USA, Metaoptics Technology in the Visible

Nader Engheta, University of Pennsylvannia, USA, Extreme Platforms for Metaphotonics

Frank Koppens, ICFO -The Institute of Photonic Sciences, Spain, Quantum Plasmonics, Polaritons and Strong LightMatter Interactions with 2d Material Heterostructures

Xiaobo Yin, University of Colorado at Boulder, USA, Scalably Manufactured Metafilms for Effective Day-time Radiative Cooling

\section{Multimodal Imaging in Biophotonics}

Thursday, 18 May, 14:00-18:30

Executive Ballroom 210 A

\section{Organizers}

Stephen Boppart, University of Illinois, USA

Wolfgang Drexler, Medical University of Vienna, Austria

Current clinical medical imaging technologies are expensive and complex with limited sensitivity and specificity. Multimodal optical imaging techniques have the potential to offer low-cost, non-invasive, accurate, rapid alternatives, with the potential to address global medical needs. This symposium focuses on state-of-the-art biophotonics imaging techniques, as well as novel optical sources for instrumentation and applications. Combinations of techniques - also including endoscopic approaches - offer multimodal solutions to diagnostic needs that will exploit the benefits of each modality and will enable optical diagnostics with superior sensitivity, specificity, reliability and clinical utility at reduced cost.

\section{Tutorial Speaker}

Ji-Xin Cheng, Purdue University, USA, Multimodal Spectroscopic Imaging: Instrumentation and Applications

\section{Invited Speakers}

Kishan Dholakia, St. Andrew's University, UK, New Directions in Multimodal Imaging and Light Sheet Microscopy

Brandon Kennedy, University of Western Australia, Australia, Imaging the Micro-Mechanical and Micro-Structural Properties of Tissue using Optical Coherence Tomography

Mengyang Liu, Medical Univeristy Vienna, Austria, Complete Cutaneous Vasculature Imaging and its Clinical Translation using Multimodal Photoacoustic and Optical Coherence Tomography Angiography

Vasilis Ntziachristos, Technische Universität Munchen, Germany, Looking at Tissue with a New Light: Clinical Advances of Multispectral Optoacoustic Tomography

Sylvie Roke, EPFL, Switerland, Multimodal Label-free Low Fluence Nonlinear Imaging of Living Systems with High-Throughput

Melissa Suter, Harvard Medical School, USA, Assessing Airway Smooth Muscle Microstructure and Contractile Force in vivo using Birefringence Microscopy

All conference locations are in the San Jose Convention Center unless otherwise noted. 


\section{Optomechanics: Towards the Second} Quantum Revolution

Thursday, 18 May, 14:00-18:30

Executive Ballroom $210 \mathrm{G}$

\section{Organizers}

Pierre-François Cohadon, Laboratoire Kastler Brossel, Paris, France

Jean-Philippe Poizat, Institut Néel, Grenoble, France

Pierre Verlot, Institut Lumière Matière, Lyon, France

The field of Optomechanics explores the reciprocal interactions between light and mechanical motion. Early proposed for supporting the first Gedanken Experiments at the foundation of Quantum Mechanics, the picture of a movable object interacting with photons has been further formalized and developed in the 1970's for investigating the quantum limits in interferometric measurements, which represents a pivotal contribution to Quantum Measurement Theory.

At present, the field of optomechanics has started stepping into the quantum regime. After 20 years of intense development, the first quantum manifestations have been observed with macroscopic systems, such as quantum groundstate laser cooling and radiation pressure induced quantum backaction. This symposium will review the most recent advances achieved and enabled by quantum optomechanics and will introduce the upcoming challenges towards the Second Quantum Revolution and their consequences in Science and Technology.

\section{Invited Speakers}

Antoine Heidmann, Laboratoire Kastler Brossel, France, Quantum Optomechanics with Micro- and Nano-Mirrors

Tobias Kippenberg, EPFL, Switzerland, Force Metrology Using Quantum Correlations of Light Generated Due to a Room-temperature Mechanical Oscillator

Florian Marquardt, Max Planck Institute for Science of Light, Germany, Topology of Light and Sound

David McClelland, The Australian National University, Australia, Non-Classical Sources of Light and their Applications to Gravitational Wave Detection

Philip Treutlein, University of Basel, Switzerland, Hybrid Atom-Membrane Optomechanics

\section{Optical Microcavities for Ultrasensitive Detection}

Thursday, 18 May, 14:00-18:30

Salon III, Marriott

\section{Organizers}

Yun-Feng Xiao, Peking University, China

Frank Vollmer, Max Planck Institute for the Science of Light, Germany

Warwick P. Bowen, University of Queensland, Australia

Sensing of nanoscale objects with ultrahigh sensitivity is highly desirable for applications in various fields, such as in early-stage diagnosis of diseases, process control in semiconductor manufacturing, environmental monitoring, and homeland security. For instance, the high concentrations of nanoparticles emitted by vehicles and industrial processes contribute significantly to increasing rates of respiratory and cardiac diseases, because nanoscale particles can penetrate the lungs to cause inflammation and even spread to other organs inside the human body.

Over the past few years, the high-Q optical microcavity has shown great potential in ultrasensitive detection because of the strongly enhanced light-matter interaction therein. The microcavity sensing community has been expanding rapidly, with over 100 research groups in the world. The sensing targets range from dispersive to dissipative nanoparticles, including proteins, DNA, nucleic acid and viruses. Significantly, the detection limit of the microcavity sensing has been pushed down to single nanoparticle (molecule) level. This symposium will focus on the nanoparticle detection. It will highlight important recent developments in the field, and aims both to provide a focussed forum to discuss ongoing research and challenges, and to stimulate new interest.

\section{Invited Speakers}

Sile Nic Chormaic, Okinawa Institute of Science and Technology Graduate University, Japan, Cavity Ring-Up Spectroscopy for Sensing in a Whispering Gallery Mode Resonator

Ken Crozier, University of Melbourne, Australia, Trapping Nanoparticles with Plasmonic and Photonic Nanostructures

Xudong Fan, University of Michigan, USA, Monolithically Integrated Ring Resonator Systems On-Chip

Randall H. Goldsmith, University of Wisconsin Madison, USA, Optical Microresonators as Single-Particle Absorption Spectrometers: Fano Resonances, Attometer Sensitivity, and Working Toward Single-Molecule Spectroscopic Identification

Tao Lu, University of Victoria, Canada, Cavity Optomechanics for Sensing Applications 


\section{Thermal Noise in Precision Interferometry}

Friday, 19 May, 08:00-12:30

Executive Ballroom 210 D

\section{Organizers}

Garrett Cole, Crystalline Mirror Solutions LLC, USA

Gregory Harry, American University, USA

Steven Penn, Hobart and William Smith Colleges, USA

Thermal noise in advanced optical systems has emerged as a significant impediment to further progress in precision metrology. This noise process encompasses a number of related effects, with prominent examples being Brownian noise and thermo-optic noise, the latter being the coherent sum of thermoelastic and thermorefractive noise components in optical materials. Thermal noise and its impact in precision optical systems has been investigated in terms of the underlying physics and means for minimization over the previous two decades. Significant progress has been realized in the theoretical understanding of the underlying mechanisms, as well as in modeling the effects at the systems level, and finally in characterizing the performance of various optical materials. We will outline the state-of-the-art in the field and describe its impact on quantum optomechanical systems, cavity QED experiments, narrow-linewidth lasers for optical clocks, and, most prominently, in gravitational wave detector design. The symposium will serve to educate interested parties on the fundamental scientific aspects as well as implications for advanced applications in precision metrology, outlining opportunities for materials scientists, physicists, and optical engineers interested in pursuing basic research efforts as well as the development of components and systems with reduced thermal noise.

\section{Invited Speakers}

Thomas Corbitt, Louisiana State University, USA, Thermal Noise in Microfabricated AIGaAs Structures

David Hume, NIST, USA, Laser Frequency Stabilization for Ion Optical Clocks at NIST

Marty Fejer, Stanford Univ., USA, Thermal Noise in Mirror Coatings for Gravitational Wave Detection

Harald Lück, AEl Hannover, Germany, Thermal Noise Reduction Techniques for High Precision Interferometric Measurements

Stuart Reid, Univ. of Western Scotland, UK, ECR Ion Beam Deposition for the Fabrication of Ultra-low Loss Optical Coatings

Uwe Sterr, Physikalisch Technische Bundesanstalt, Germany, Thermal Noise in Ultrastable Cavity-Referenced Lasers 


\section{Applications \& Technology Topical Reviews}

\author{
Advances in Laser-based Remote Sensing \\ Monday, 15 May, 08:00-12:33 \\ Executive Ballroom $210 \mathrm{~A}$ \\ Organizer \\ Fabio Di Teodoro, Space and Airborne Systems - Raytheon \\ Company, USA
}

Active remote sensing generally refers to the integration of a laser source; optical beam formatting, direction, and collection apparatuses; photodetectors; and data processing infrastructures to perform a broad spectrum of reconnaissance, imaging, and standoff analysis tasks including range finding of still or moving targets, terrain topography, detection of airborne biological/chemical species, wind speed measurements, etc.

This topical review will address cutting-edge research and development of active remote sensors from a system standpoint to include new laser transmitters, detectors, and advanced sensing concepts. Particular emphasis will be placed on long-range sensors intended for deployment in airborne and space platforms. Such systems must address many technological challenges devoted to the maximization of the laser transmitter pulse energy/power; minimization of size, weight, and power consumption (SWaP); increase in ruggedness and support for operation in thermo-mechanical harsh environments; and improvement of long-term reliability.

Topics of interest include but are not limited to direct- or coherent-detection laser radar, 3D imaging, differential absorption lidar and other remote spectroscopic probes, multi-function platforms, and their deployment in the field.

\section{Invited Speakers}

Michael Daly, York University, Canada, The OSIRIS-REx Laser Altimeter

Joseph Marron, Raytheon, USA, Coherent Adaptive Optical System

Nikolaus Schmitt, Airbus Group Innovations, Germany, Research Results, Lessons Learned and Future Perspective of Forward-looking LIDAR for Aircraft

Upendra Singh, NASA, USA, Active Optical Remote Sensor for Carbon dioxide and Water Vapor Measurement from an Air and Space-borne Platform

\section{Scientific and Commercial Progress in Semiconductor Lasers \\ Monday, 15 May, 13:30-18:00 \\ Executive Ballroom $210 \mathrm{~A}$}

\section{Organizer}

Bojan Resan, Lumentum, Switzerland

These sessions will report on the state-of-the-art in various research and applications of semiconductor lasers (SL) including LIDAR requirements and trade-offs for self-driven cars, widely tunable SLs, quantum dot devices, ultrafast VECSELs, low noise ultrafast cavities and signal processing with SLs.
Invited Speakers

Peter J. Delfyett, CREOL, The College of Optics \& Photonics, USA, Low Noise Ultrafast Pulse Generation and Signal Processing using Semiconductor Laser

Ursula Keller, ETH Zurich, Switzerland, Ultrafast Semiconductor Disk Lasers

Christoph Raab, Toptica, Germany, 53 Years Tunable Semiconductor Laser - Past, Present and Future

Edik Rafailov, Aston University, UK, Recent Progress in Quantum dot based Devices: Physics and Applications

Simon Verghese, Waymo - Google, USA, Self-Driving Cars and Lidar

\section{Neurophotonics}

Tuesday, 16 May, 08:00-10:00; 13:30-14:30

Executive Ballroom 210 B

\section{Organizers}

Kishan Dholakia, Univ. of St. Andrews, UK

Chris Xu, Cornell University, USA

Neurophotonics has emerged as very exciting contemporary challenge that aims to merge interface cutting edge photonics technology and neuroscience. Neurophotonics embraces both invasive and non-invasive methods that can be applied right from single cells to whole animal studies. The approaches promise a paradiogm shift in the way we may image, probe and interrogate functional activity in the brain. Grand challenges in this field include recording and manipulating the activity of multiple cells in the brains of live animals using light. Major opportunites exist for using innovations in photonics to address these questions that ultimately may impact our view of the functioning of the brain and the emergence of diseases such as dementia.

This topical section will cover a whole host of methods in this expanding area that may include advanced forms of single photon and multiphoton imaging including light sheet imaging, diffuse correlation spectrocopy, near infrared spectroscopy, novel probes and light sources.

\section{Invited Speakers}

Chris Schaffer, Cornell University, USA, Unexpectedly Stalled: Two-Photon Microscopy Reveals White Blood Cell Adhesion in Capillaries Causes Reduced Brain Blood Flow In Alzheimer's Disease

Junjie Yao, Duke University, USA, Wide-field Fast-scanning Photoacoustic Microscopy of Brain Functions in Action

All conference locations are in the San Jose Convention Center unless otherwise noted. 


\section{Extreme Ultraviolet and Soft x-ray Sources and Applications}

Thursday, 18 May, 14:00-18:30

Executive Ballroom $210 \mathrm{C}$

\section{Organizers}

Carmen S. Menoni, Colorado State University, USA

Alexander Ershov, Cymer, USA

Recent adoption of EUV for microlithography and plans from major chip makers to use EUV scanners in high-volume manufacturing have led to fast progress in EUV source technology and sparked renewed interest to soft X-ray sources from industrial and academic research groups. This session will be focused on latest scientific and technical developments in the field of light generation at $13.5 \mathrm{~nm}$ and in the broader soft $X$-ray range using plasma, high-harmonic generation, freeelectron lasers, synchrotrons and other innovative concepts. It will also include applications of these sources for lithography, metrology, spectroscopy, and other applications as well as integration aspects for the sources, such as collecting and filtering EUV light, debris mitigation, lifetime and reliability issues. This session will provide a forum for researchers and users in the EUV and soft X-ray areas to share their recent results and discuss the wide range of potential applications.

\section{Invited Speakers}

Christopher Barty, Lawrence Livermore National Lab, USA, Nuclear Photonics Enabled by MeV Laser-Compton Sources

Katsumi Midorikawa, RIKEN Center for Advanced Photonics, Japan, Next Generation High-Order Harmonic Sources and Application

Stephane Sebban, Laboratoire d'Optique Appliquée, France, Toward Compact and Ultra-intense Laser Based Soft X-ray Lasers

\section{Supercontinuum and Applications}

Friday, 19 May, 08:00-12:30

Executive Ballroom 210 A

Organizer

Robert Alfano, CUNY City College, USA

The Supercontinuum has become one of the most enabling light source spanning from UV to SWIR used for research in optical and photonic system for a variety of applications in accurate clocks (combs), large capacity optical communication, ultrafast effects, nonlinear optics, imaging, biology and chemistry. These sessions will touch upon the supercontinuum and various supercontinuum applications from various presenters.

\section{Invited Speakers}

Adam Devine, Fianium, USA, Supercontinuum Laser Sources Future Await Wide Applications

Juan Diego Ania Castañón, Instituto De Optica 'Daza De Valdes', Spain, Supercontinuum in Telecom Applications towards Terabits

Robert Fisher, RA Fisher Associates LLC, USA, The Early Days of Self-Phase Modulation and Supercontinuuim Generation

Norihiko Nishizawa, Nagoya University, Japan, Ultrahigh Resolution Optical Coherence Tomography using Supercontinuum and their Wavelength Dependence

Lingyan Shi, CUNY City College, USA, Future Supercontinuum Microscope for Medical and Biological Applications

Laura Sordillo, CUNY City College, USA, Label-free Techniques for the Assessment of Cancer and Other Diseases using the Supercontinuum Light Source at the Four NIR Optical Windows

James R Taylor, Imperial College London, UK, Supercontinuum Sources - Past, Present - Any Future?

Alan Willner, University of Southern California, USA, Structured Light using OAM and Wavelength Domains for Terabit/sec Communications 


\section{Short Course Chairs}

Robert Fisher, R. A. Fisher Associates, USA

Ben Eggleton, Univ. of Sydney, Australia

The CLEO Short Course Program includes a range of topics at a variety of educational levels. Widely recognized experts in industry and academia lead attendees in building skills and/or achieving new insight, and the small-classroom setting provides a tremendous, interactive learning opportunity. Short Courses are an excellent opportunity to learn about new products, cutting edge technology and vital information at the forefront of the laser science and electro-optics fields.

Certificates of Attendance are available for those who register and attend a course. You may request a certificate upon completion of the online course evaluation. If you have any questions about receiving a Certificate of Attendance or completing the course evaluation, please email shortcourses@ cleoconference.org with your name and course name(s).

\section{Sunday, 14 May 2017}

\section{8:30-12:30}

SC149: Foundations of Nonlinear Optics Instructor: Robert Fisher, R. A. Fisher Associates, USA

SC221: Nano Photonics: Physics and Techniques Instructor: Axel Scherer, Caltech, USA

SC361: Coherent Midlnfrared Sources and Applications Instructor: Konstantin Vodopyanov; CREOL, The College of Optics \& Photonics, Univ. Central Florida, USA

\section{8:30-15:00}

SC456: How to Start a Company Instructor: Jes Broeng', Milton Chang2; ${ }^{1}$ DTU, Denmark; 2Incubic, USA

\section{3:30-16:30}

SC403: NanoCavity Quantum Electrodynamics and Applications

Instructor: Jelena Vuckovic, Stanford Univ., USA

SC439: Attosecond Optics

Instructor: Zenghu Chang, Univ. of Central Florida, USA

\section{3:30-17:30}

SC157: Laser Beam Analysis, Propagation, and Shaping Techniques

Instructor: James Leger; Univ. of Minnesota, USA

SC396: Frontiers of Guided Wave Nonlinear Optics Instructor: Ben Eggleton, Univ. of Sydney, Australia
Monday, 15 May 2017

09:00-12:00

SC362: Cavity Optomechanics: Fundamentals and Applications of Controlling and Measuring Nano- and Micro-mechanical Oscillators with Laser Light Instructor: Tobias Kippenberg, École polytechnique fédérale de Lausanne, Switzerland

SC424: Optical Terahertz Science and Technology Instructor: David G. Cooke; Dept. of Physics, McGill University, Canada

\section{$12: 30-16: 30$}

SC301: Quantum Cascade Lasers: Science, Technology, Applications and Markets

Instructor: Federico Capasso, Harvard Univ., USA

SC378: Introduction to Ultrafast Optics

Instructor: Rick Trebino, Georgia Institute of Technology, USA

SC455: Integrated Photonics for Quantum Information Science and Technology Instructor: Dirk Englund; MIT, USA

Tuesday, 16 May 2017

\section{2:00-15:00}

SC352: Introduction to Ultrafast Pulse Shaping-Principles and Applications

Instructor: Marcos Dantus, Michigan State Univ., USA

SC376: Plasmonics

Instructor: Mark Brongersma, Stanford Univ., USA

SC410: Finite Element Modeling Methods for Photonics and Optics

Instructor: Arti Agrawal, City Univ., UK

\section{2:00-16:00}

SC270: High Power Fiber Lasers and Amplifiers Instructor: W. Andrew Clarkson, Optoelectronics Res. Ctr., Univ. of Southampton, UK

SC438: Photonic Metamaterials

Instructor: Nader Engheta, University of Pennsylvania, USA 


\section{Short Course Descriptions}

Courses are listed by date and time. Complete course descriptions are available at www.cleoconference.org/ shortcourses.

\section{SC149 - Foundations of Nonlinear Optics}

Sunday, 14 May, 08:30-12:30

Robert Fisher, R. A. Fisher Associates, USA

This introductory and intermediate level course provides the basic concepts of nonlinear optics. Although some mathematical formulas are provided, the emphasis is on simple explanations. It is recognized that the beginning practitioner in nonlinear optics is overwhelmed by a constellation of complicated nonlinear optical effects, including second-harmonic generation, optical Kerr effect, self-focusing, self-phase modulation, self-steepening, fiberoptic solitons, chirping, stimulated Raman and Brillouin scattering, and photorefractive phenomena. It is our job in this course to demystify this daunting collection of seemingly unrelated effects by developing simple and clear explanations for how each works, and learning how each effect can be used for the modification, manipulation or conversion of light pulses. Examples will address the nonlinear optical effects that occur inside optical fibers and those that occur in liquids, bulk solids, and gases.

\section{Short Course Benefits:}

- Explain and manipulate the Slowly-Varying Envelope Approximation (SVEA)

- Recognize what nonlinear events come into play in different effects

- Appreciate the intimate relationship between nonlinear events which at first appear quite different

- Discuss how a variety of different nonlinear events arise, and how they affect the propagation of light

- Describe how wavematching, phase-matching, and index matching are related

- Summarize how self-phase modulation impresses "chirping" on pulses

- Explain basic two-beam interactions in photorefractive materials

- Develop an appreciation for the extremely broad variety of ways in which materials exhibit nonlinear behavior"

Short Course Level: Beginner

Short Course Audience: Although we start at the very beginning of each topic, we move quite rapidly in order to grasp a deep understanding of each topic. Therefore, both beginners and intermediates will benefit greatly from this course. The material will be of interest to graduate students, to researchers, to members of the legal profession, to experts who are just transferring to this field, to managers, and to anyone else who just wants to learn how nonlinear optics works. This course, offered on Sunday Morning, will also give an excellent nonlinear optics foundation for those feeling the need so they can also take any of the following more specialized nonlinear optics courses at this CLEO conference: SC396: Frontiers of Guided Wave Nonlinear Optics; SC378: Introduction to Ultrafast Optics; SC270: High Power Fiber Lasers and Amplifiers; SC410: Finite Element Modeling Methods for
Photonics and Optics; and SC352: Introduction to ultrafast pulse shaping-principles and applications.

\section{SC221 - Nano Photonics: Physics and Techniques}

Sunday, 14 May, 08:30-12:30

Axel Scherer, Caltech, USA

Students will learn about the applications of printed and integrated optical devices. In particular, optical microcavities and vertical cavity lasers, silicon photonics and plasmonic systems will be introduced and compared. Integrated opto-electronic and opto-fluidic systems for communications and biomedical sensing will be compared.

\section{Short Course Benefits:}

- Compare dielectric (total internal reflection and Braggreflectors) with metallic (surface plasmon) geometries for confining and guiding light

- Identify opportunities for using printed optical systems in silicon (silicon photonics)

- Describe methods for creating quantum-mechanical systems from optical nanostructures

- Design lithographically defined micro- and nanocavities for resonators and lasers

- Define applications of printed optics in biochemical sensing

- Summarize the evolution of printed optical integrated circuits and devices, such as modulators and switches

- Determine the applications of interdisciplinary integration of optics with electronics and fluidics

- Describe optical performance of semiconductor structures when these are made with nanoscale dimensions

Short Course Level: Intermediate

Short Course Audience: This course is designed for participants with interest in miniaturizing optical devices. Methods of microfabricating dielectric and plasmonic devices will be described, along with examples of their applications and description of future opportunities.

\section{SC361 - Coherent Mid Infrared Sources and Applications}

Sunday, 14 May, 08:30-12:30

Konstantin Vodopyanov, CREOL, Univ. Central Florida, USA

This course will make a comprehensive review of different techniques for producing coherent light in this important yet challenging spectral region. It will examine different stateof-the-art approaches from diverse areas of photonics that include: solid-state lasers based on rare-earth and transition metals, fiber lasers, semiconductor lasers (including intra- and intersubband cascade lasers), laser sources based on nonlinear optical frequency downconversion (including difference frequency generators, optical parametric oscillators, generators and amplifiers), Raman sources and others. Since the course is focused mostly on modern-day techniques, such traditional areas as carbon dioxide lasers and free electron lasers will be not be covered. Explaining fundamental principles behind a given technique will precede discussions on each topic. The course will review several emerging technologies such as supercontinuum generation in fibers and waveguides, 
as well as frequency combs generation. Several important mid-IR applications will be also reviewed and include molecular sensing, spectroscopy with frequency combs, and medical and military applications.

\section{Short Course Benefits:}

- Get a clear idea of existing laser sources in the midIR spectral region (2-20 $\mu \mathrm{m})$ and understand their operational principles, as well as advantages and disadvantages.

- Distinguish between different operational regimes, from continuous-wave to few optical cycle pulsed operation.

- Distinguish between broadband and narrow-band sources, as well as between the supercontinuum and the frequency comb regimes.

- Learn about new applications of mid-IR coherent sources, from trace molecular detection and remote sensing to ultrafast spectroscopy and attosecond physics.

- Identify what kind of laser source you need for your particular application.

Short Course Level: Intermediate

Short Course Audience: Students, academics, researchers and engineers in various disciplines who require a broad introduction to the subject and would like to learn more about the state-of-the-art and upcoming trends in mid-infrared coherent source development and applications. Undergraduate training in either engineering or science is assumed.

\section{SC456 - How to Start A Company}

Sunday, 14 May, 08:30-15:00

Jes Broeng' and Milton Chang ${ }^{2}$; ${ }^{1}$ DTU, Denmark, ${ }^{2}$ Incubic, USA

Starting a new business is one of the most rewarding experiences one can imagine. The journey from an idea to a successful company is, however, paved with challenges and puts high demands on technology, business and social skills. The upside, though, is tremendous in personal learning and potentially also in an economic sense.

This short course is aimed to help people thinking about starting their own company and provide practice-oriented tools to help aspiring entrepreneurs who have a scientific or engineering background.

To commercialize a new technology, the short course will help answer the following questions:

- How do I know I have a viable business idea?

- What to look for in co-founders?

- What financing strategy is appropriate for my kind of business?

- How to pitch and work with investors?

- How to bootstrap if all fails?

The course will include a mix of business theory and startup experience based on a number of successful high-tech companies familiar to the scientists and engineers at CLEO. The short course will also include a workshop session, where start-up ideas from the attendees may be introduced and strengthened under the instructor's guidance.
Short Course Benefits:

- Recognize a potential high-tech business opportunity

- Have an understanding of different business models and to identify the right one for your start-up

- Knowledge of how investors think and how you may attract funding for a new venture

- Understand the principles to set up an effective organization

- Establish an eco-system to support your business, including lead customers and R\&D partners.

Short Course Audience: This course is intended for scientists \& engineers with interest in starting up their own high-tech business.

\section{SC403 - NanoCavity Quantum Electrodynamics and Applications}

Sunday, 14 May, 13:30-16:30

Jelena Vuckovic, Stanford Univ., USA

Strong localization of light in nanophotonic structures leads to enhanced light-matter interaction, which can be employed in a variety of applications, ranging from improved (higher speed, lower threshold) optoelectronic devices, to biophotonics, quantum information and low threshold nonlinear optics.

In particular, quantum dots in optical nanocavities are interesting as a test-bed for fundamental studies of such lightmatter interaction (cavity quantum electrodynamics - QED), as well as an integrated platform for information processing. As a result of the strong field localization inside of sub-cubic wavelength volumes, they enable very large emitter-field interaction strengths (vacuum Rabi frequencies in the range of 10 's of $\mathrm{GHz}$ - a few orders of magnitude larger than in atomic cavity QED). In addition to the study of new regimes of cavity QED, this can also be employed to build devices for quantum information processing, such as ultrafast quantum gates, nonclassical light sources, and spin-photon interfaces. Beside quantum information systems, many classical information processing devices greatly benefit from the enhanced light matter interaction in such structures; examples include all-optical switches operating at the single photon level, electro-optic modulators controlled with sub-fJ energy and operating at $\mathrm{GHz}$ speed, and lasers with threshold currents of $100 \mathrm{nA}$. This course will introduce cavity QED (e.g., strong and weak coupling regimes, Purcell effect, etc.), with particular emphasis on semiconductor nanocavities. We will also describe state of the art in solid state cavity QED experiments and applications.

\section{Short Course Benefits:}

- Explain light matter interaction in optical nanostructures

- Discuss state of the art in solid state cavity QED

- Identify benefits of employing nano-cavity QED for certain applications

Short Course Level: Beginner

Short Course Audience: Scientists and engineers interested in cavity QED and nanophotonic devices in general. Some background in electromagnetics, quantum mechanics, and optoelectronics is helpful, but not required.

\section{All conference locations are in the San Jose Convention Center unless otherwise noted.}




\section{SC439 - Attosecond Optics}

Sunday, 14 May, 13:30-16:30

Zenghu Chang, CREOL, Univ. Central Florida, USA

Since the invention of lasers in 1960, various techniques such as mode-locking have been developed to push the pulse duration down first to picoseconds and then to femtoseconds, which is the oscillation period of infrared and visible light. The generation of attosecond pulses requires new methods to produce broadband coherent electromagnetic waves in the UV to x-ray range because of the lack of proper gain media. The discovery of high-order harmonic generation in high intensity laser-atom interaction at the end of 1980s paved the way. In 2001, attosecond light pulses, a train of attosecond bursts or single isolated attosecond pulses, were measured for the first time. It was accomplished by first converting the attosecond photons to photoelectrons in a combination of weak extreme ultraviolet and strong infrared fields, and then retrieve the spectrum phase of the attosecond pulse by reconstructing the photoelectron spectrum. Since then, various sub-optical-cycle gating schemes such as polarization gating and Double Optical Gating have been demonstrated to generation isolated attosecond pulses. By properly compensating the intrinsic chirp, 67 as pulses were characterized in 2012, which is so far the shorted light pulses. The new frontier in attosecond optics research is to significantly increase the photon flux and to extend the spectrum to the "water window." This course covers: (1) High harmonic generation. (2) Carrier-envelope phase of femtosecond driving lasers. (3) Semi-classical model and Strong Field Approximation. (4) Phase-matching in partially ionized media. (5) Sub-cycle gating and attosecond pulse characterization. (6) Attosecond streaking and transient absorption spectroscopy.

\section{Short Course Benefits:}

- Specify parameters of femtosecond driving lasers that are critical to the generation of attosecond pulse trains and single isolated attosecond pulses

- Compare pros and cons of driving lasers based on Ti:Sapphire Chirped Pulse Amplification and Optical Parametric Amplifiers

- Explain the principle and techniques of locking the carrier-envelope offset frequency of femtosecond oscillators and carrier-envelope phase of amplified pulses

- Define short and long trajectories in the attosecond generation process using the Strong Field Approximation in the Lewenstein model

- Estimate the cutoff photon energy and attosecond chirp using the semi-classical model

- Calculate ionization probability of atoms in an intense laser field with the Ammosov-Delone-Krainov (ADK) tunneling rate

- Describe the principle of attosecond streak camera for characterizing attosecond pulses

- Identify the major factors that affects the phase matching of high harmonic generation in partially ionized media.

Short Course Level: Beginner
Short Course Audience: This short course targets senior undergraduate students, graduate students, postdoc fellows, scientists and engineers seeking to enter attosecond optics. The audience should have studied electromagnetism, optics, lasers, quantum mechanics and atomic physics at undergraduate or graduate levels. Prior knowledge of femtosecond lasers is required. Although basic theory is covered, it emphasizes on experimental aspects of attosecond optics, such as locking the carrier-envelope phase of the driving lasers and designing time-of-flight spectrometers for attosecond streak cameras.

\section{SC157 - Laser Beam Analysis, Propagation, and Shaping Techniques}

Sunday, 14 May, 13:30-17:30

James Leger, Univ. of Minnesota, USA

The performance of conventional high power lasers is often compromised by one or more physical effects, limiting the maximum power that can be obtained from a single lasing element. To increase the power from these individual elements, laser beam combining can be employed to convert the outputs from several lower-power modules into a single, high-power beam. This short course establishes general beam combining principles relevant to all laser systems, and emphasizes the limits that are achievable with differ approaches. The practicing engineer and technical manager will be introduced to a wide variety of beam combining methods. Incoherent beam combining attempts to maximize the radiance of an array of incoherent sources. The theoretical limits of this approach will be derived, and a design methodology developed to achieve maximum radiance. Spectral and polarization beam combining techniques employ wavelength and polarization sensitive elements to sum laser power. Several practical issues of this technique will be discussed, and specific systems described. Coherent beam combining is introduced by exploring methods of establishing mutual coherence across laser arrays. The properties and characteristics of these coherent techniques are quantitatively analyzed using simple modal theories. Methods of converting arrays of coherent beams into a single beam are explored, and the sensitivity of these approaches to path length errors investigated. Real-world examples will be used as case studies to illustrate design principles. This offering of the course will make use of recently developed material on coherent beam combining architectures.

\section{Short Course Benefits:}

- Describe the requirements for laser beam combining of all types.

- Estimate the optimum brightness enhancement achievable from incoherent combining.

- Design an ideal incoherent beam combiner.

- Design spectral beam combiners and estimate performance limitations.

- Compare different architectures for establishing mutual coherence across laser arrays.

- Determine the effects of path length errors on beam combining performance.

- Design optical systems to convert coherent arrays of laser beams into a single beam.

Short Course Level: Beginner

All conference locations are in the San Jose Convention Center unless otherwise noted. 
Short Course Audience: The course is designed for students, engineers, scientists and technical managers who are interested in understanding the basics of laser beam combining. No advanced knowledge of laser systems is assumed.

\section{SC396 - Frontiers of Guided Wave Nonlinear Optics}

Sunday, 14 May, 13:30-17:30

Ben Eggleton, Univ. of Sydney, Australia

This course will review recent research and applications in the field of nonlinear guided wave optics with emphasis on both fundamentals and emerging applications. Starting from a strong foundation in the principles of nonlinear optics, we will review recent progress in emerging nonlinear optical platforms with an emphasis on the different materials, including silicon, chalcogenide, III-V semiconductors, lithium niobate, photonic crystal fibres, nanophotonic circuits and others. We will establish key figures of merit for these different material systems and a general framework for nonlinear guided wave optics with emphasis on the applications in emerging areas of science and technology. We will then review recent progress and breakthroughs in the following areas: All-optical processing, Ultra-fast optical communications, Slow light, highly nonlinear and emerging waveguides, Ultrafast measurement and pulse characterization, Frequency combs and optical clock, Optical parametric amplifiers and oscillators, Generation and applications of optical super-continuum, Nonlinear localization effects and solitons, Nonlinear optics for quantum information.

\section{Short Course Benefits:}

This course should enable the participants to:

- Get state of the art knowledge of nonlinear optics in emerging waveguides and materials

- Understand the applications of nonlinear optics in key applications

- Have a foundation of nonlinear waveguide physics for emerging applications and science

Short Course Level: Advanced Beginner

Short Course Audience: This course assumes some basic knowledge/familiarity of nonlinear optics. Individuals lacking such knowledge should consider taking SC149: Foundations of Nonlinear Optics first.

SC362 - Cavity Optomechanics: Fundamentals and Applications of Controlling and Measuring Nanoand Micro-mechanical Oscillators with Laser Light Monday, 15 May, 09:00-12:00

Tobias Kippenberg, École polytechnique fédérale de Lausanne, Switzerland

Radiation pressure denotes the force that optical fields exert and which have wide ranging applications in both fundamental science and applications such as Laser cooling or optical tweezers. Radiation pressure can, however, also have a profound influence on micro- and nanophotonic devices, due to the fact that radiation pressure can couple optical and mechanical modes. This optomechanical coupling gives rise to a host of new phenomena and applications in force, displacement and mass sensing. This course is intended to give an introduction of the Physics and Applications of cavity optomechanics and highlight the rapid developments in this emerging field. Optomechanical coupling can be used to both cool and amplify mechanical motion and thereby allow new light driven photon clocks. Optomechanical refridgeration of mechanical modes gives insights into the quantum limits of mechanical motion. In addition, radiation pressure coupling enables new way of processing light all optically enabling optical mixers, delay lines or storage elements. Moreover, the basic limitations of optomechanical displacement measurements, due to quantum noise and practical laser phase noise limitations, will be reviewed, relevant across a wide range of sensing experiments.

The course will make contact to practical applications of optomechanics in Metrology (force sensors, mass sensors and light driven optical clocks) and review fundamental design principles of optomechanical coupling and the design of high $\mathrm{Q}$ mechanical oscillators. The use of finite element simulations will be covered.

\section{Short Course Benefits:}

- Explain gradient and scattering light forces in microcavities and micromechanical systems

- Design high -Q nano-and micro- mechanical oscillators (finite element modeling, FEM)

- Discuss the fundamental limits of mechanical $\mathrm{Q}$ in NEMS/MEMS

- Describe of the fundamental and practical limits of displacement sensors

- Summarize Applications of optomechanics in mass and force sensing

- Explain the basic optomechanical phenomena (amplification, cooling)

- Discuss the standard quantum limit (SQL)

- Characterize radiation pressure driven oscillations in terms of fundamental oscillator metrics

- Define Phase and frequency noise of oscillators

- Know the influence of phase and amplitude noise of a wide variety of laser systems (fiber lasers, TiSa, diode lasers) in optomechanical systems

Short Course Level: Advanced Beginner

Short Course Audience: This course is intended for physicists and optical and electrical engineers desiring both focused fundamental knowledge of cavity optomechanical coupling (i.e., radiation pressure coupling of light and NEMS/MEMS) but also a view of emerging applications of this new technology. The instruction will be at a level appropriate for graduate students and will assume some basic knowledge of laser. 
SC424 - Optical Terahertz Science and Technology Monday, 15 May, 09:00-12:00

David G. Cooke, McGill University, Canada

The purpose of this short course is to introduce time-domain optical techniques based on femtosecond lasers for generating, manipulating and detecting light in the $0.1-10 \mathrm{THz}$ region, and demonstrate how this interesting part of the spectrum can be used to improve our understanding of materials. I will discuss $\mathrm{THz}$ imaging and sensing applications that are driving the development of this technology and discuss new physics that can be probed with short pulses of $\mathrm{THz}$ light.

\section{Short Course Benefits:}

- Explain methods for the generation and detection of coherent terahertz radiation

- Explain and apply methods for terahertz time - domain spectroscopy

- Understand the physical phenomena taking place on the picosecond - time scale

- Gain hands-on experience with advanced experimental equipment and numerical tools

- Understand and explain the pump - probe techniques for time-resolved spectroscopic measurements in the terahertz range

- Explain and interpret experimental measurements based on the theoretical models

Short Course Level: Advanced Beginner

Short Course Audience: This course is aimed at graduate students interested in the field of time-domain $\mathrm{THz}$ spectroscopy and imaging techniques. Basic knowledge of electromagnetic waves and condensed matter systems is suggested.

\section{SC301 - Quantum Cascade Lasers: Science, Technology, Applications and Markets \\ Monday, 15 May, 12:30-16:30 \\ Federico Capasso, Harvard Univ., USA}

Quantum Cascade Lasers (QCLs) are fundamentally different from diode lasers due to their physical operating principle, which makes it possible to design and tune their wavelength over a wide range by simple tailoring of active region layer thicknesses, and due to their unipolar nature. Yet they use the same technology platform as conventional semiconductor lasers. These features have revolutionized applications (spectroscopy, sensing, etc.) in the mid-infrared region of the spectrum, where molecules have their absorption fingerprints, and in the far-infrared or so called Terahertz spectrum. In these regions until the advent of QCLs there were no semiconductor lasers capable of room temperature operation in pulsed or cw, as well high output power and stable/wide single mode tunability. The unipolar nature of $\mathrm{QCL}$, combined with the capabilities of quantum engineering, leads to unprecedented design flexibility and functionality compared to other lasers. The physics of QCLs, design principles, supported by modeling, will be discussed along with the electronic, optical and thermal properties. State-of-the-art performance in the mid-ir and Terahertz will be reviewed. In particular high power CW room temperature QCLs, broadly tunable $\mathrm{QCL}$, short wavelength MWIR QCLs and recent breakthroughs in THz room temperature operation will be presented. A broad range of applications (IR countermeasures, stand-off detection, chem- bio sensing, trace gas analysis, industrial process control, medical and combustion diagnostics, imaging, etc.) and their ongoing commercial development will be discussed.

\section{Short Course Benefits:}

- Describe underlying $\mathrm{QC}$ Laser physics, operating principles and fundamental differences between standard semiconductor lasers and QC lasers

- Explain quantum design of the key types of QC lasers, which have entered real world applications, and how their electrical and optical properties can be tailored to optimize performance in the mid-infrared and $\mathrm{THz}$ regions.

- Discuss experimental device performance, including physical limits, design constraints and comparison with theory and determine device characteristics (currentvoltage and light-current curves; differential and power efficiency, threshold, gain and losses; spectral behavior, single mode operation; high speed operation)

- Explain the basics of OC laser device technology: fabrication process, materials growth options

- Illustrate the basics of a chemical sensing system; discuss applications of state-of the-art mid-infrared $\mathrm{OC}$ lasers to sensing and present several examples of $\mathrm{QC}$ laser commercialization

- Discuss current and future markets of QC lasers

Short Course Level: Beginner

Short Course Audience: Graduate students; qualified undergraduates (mostly senior level) majoring in EE or physics/ applied physics; researchers in industry, academia and government labs; engineers, sales reps and technical managers.

\section{SC378 - Introduction to Ultrafast Optics}

Monday, 15 May, 12:30-16:30

Rick Trebino, Georgia Institute of Technology, USA

Ultrafast Optics-the science and technology of ultrashort laser pulses -is one of the most exciting and dynamic fields of science. While ultrashort laser pulses seem quite exotic (they're the shortest events ever created!), their applications are many, ranging from the study of ultrafast fundamental events to telecommunications to micro-machining to biomedical imaging - to name a few. Interestingly, these lasers are readily available, and they are easy to understand. But their use requires some sophistication. This course is a basic introduction to the nature of these lasers and the pulses they generate. It will discuss the principles of their generation and amplification and describe their most common distortions in space and time and how to avoid them-or take advantage of them. In addition, it will cover the nonlinear optics of ultrashort pulses for converting pulses to almost any color, as well as the additional interesting and potentially deleterious effects nonlinear optical processes can cause. Finally, it will cover techniques for ultrashort-pulse measurement.

\section{Short Course Benefits:}

- Explain how ultrashort-pulse lasers and amplifiers work.

- Describe and describe ultrashort pulses and their many distortions.

All conference locations are in the San Jose Convention Center unless otherwise noted. 
- Use nonlinear optics to an convert ultrashort laser pulse to virtually any wavelength.

- Take advantage of-or avoid-nonlinear-optical highintensity effects.

- Meaningfully measure ultrashort pulses.

Short Course Level: Beginner

Short Course Audience: Any scientist or engineer interested in the science and technology of the shortest events ever created, especially those new to it.

\section{SC455 - Integrated Photonics for Quantum Information Science and Technology \\ Monday, 15 May, 12:30-16:30 \\ Dirk Englund, MIT, USA}

The rules of quantum mechanics enable applications that are inherently more powerful than their classical counterparts. Quantum key distribution now makes it possible to transmit information with unconditional security; quantum simulation is beginning to address problems that are intractable on classical computers; and quantum metrology techniques push the boundaries of precision measurements.

Many of these quantum technologies rely fundamentally on advanced photonics that place extremely demanding requirements on precision, efficiency, and mode complexity. Over the past decade, new generations of photonic integrated circuits have been developed to begin to address these requirements.

This course will cover basic concepts and recent progress in photonic integrated circuits technology for quantum information processing, with a focus on two primary application areas: quantum communications -- from quantum cryptography to entanglement distribution over quantum networks -- and quantum computing, including analog and digital approaches. Motivated by these applications, the course will discuss nonclassical light sources, photonic interfaces with atomic memories, high-fidelity mode transformation circuits, nonlinear photonic quantum gates, and waveguide-integrated single photon resolving detectors.

\section{Short Course Benefits:}

- Describe a practical quantum key distribution system, estimate performance, and identify central limitations

- Propose methods to extend the reach of quantum secure communications

- Describe the main classes of quantum communications

- Categorize the major areas of quantum computing

- Diagram quantum networks

- Design photonic integrated circuits for quantum key distribution and quantum repeaters

Short Course Level: Advanced Beginner

Short Course Audience: The course is designed for an audience interested in the key ideas and technology of photonic quantum communication and computation. It will probably be most valuable to participants who have some background in quantum information science or integrated optics and who want to better understand where the intersections of these fields and where the challenges and opportunities lie. The course should be useful for graduate students and industrial and academic researchers with an interest in applied photonic quantum technologies.

\section{SC352 - Introduction to Ultrafast Pulse Shaping-- Principles and Applications \\ Tuesday, 16 May, 12:00-15:00 \\ Marcos Dantus, Michigan State Univ., USA}

This course begins by describing pulse shaping with a handson computer simulation that allows one to get a sense of how femtosecond pulses change in response to different phases and amplitudes. The essential physics and a brief background of the development of shapers are provided. The course goes over the experimental implementation requirements and then covers some of the most salient applications of pulse shapers, among them are pulse compression, pulse characterization, creation of two or more pulse replicas, control of nonlinear optical processes such as selective two-photon excitation and selective vibrational mode excitation, material processing, microscopy and others.

\section{Short Course Benefits:}

- Gain a better understanding of femtosecond laser pulses and their applications

- Learn pulse shaper design principles

- Compare among different pulse shaper designs and to determine which one is best suited for a particular application

- Simulate the output pulse from a pulse shaper given a particular phase and amplitude modulation

- Predict the effect caused by introducing a simple phase such as a linear, quadratic or cubic function on a transform-limited pulse

- Learn two different approaches to creating pulse replica that can be independently controlled with attosecond precision in the time domain using the pulse shaper

- Measure the spectral phase of laser pulses using the pulse shaper itself as the measurement tool, and eliminating phase distortions to compress the output pulses

- Summarize the advantages of having an adaptive pulse shaper for controlling the output of ultrafast lasers

Short Course Level: Advanced Beginner

Short Course Audience: This course, updated yearly, is intended for everyone that uses or intends to use femtosecond laser pulses in academic research or industry. Attendees will learn how pulse shaping can greatly enhance femtosecond laser applications. No prior knowledge about pulse shaping is required.

\section{SC376 - Plasmonics}

Tuesday, 16 May, 12:00-15:00

Mark Brongersma, Stanford Univ., USA

Plasmonics is an exciting new field of science and technology that aims to exploit the unique optical properties of metallic nanostructures to enable routing and active manipulation of light at the nanoscale. Nanometallic objects derive these properties from their ability to support collective electron excitations, known as surface plasmons (SPs). Presently we are

All conference locations are in the San Jose Convention Center unless otherwise noted. 
witnessing an explosive growth in both the number and range of plasmonics applications; it is becoming eminently clear that both new fundamental science and device technologies are being enabled by the current plasmonics revolution. The intention of this tutorial is to give the participants a fundamental background and working knowledge of the main physical ideas used in plasmonics, as well as an overview of modern trends in research and applications.

The Short Course will begin with a general overview of the field of plasmonics. This will be followed by an introduction to the basic concepts that enable one to understand and design a range of plasmonic functionalities. This part will be followed by an in-depth discussion of a range of active and passive plasmonic devices that have recently emerged. Particular attention will be given to nanometallic structures in which surface plasmons can be generated, routed, switched, amplified, and detected. It will be shown that the intrinsically small size of plasmonic devices directly results in higher operating speeds and facilitates an improved synergy between optical and electronic components. The field of plasmonics is rapidly growing and has started to provide a whole range of exciting new research and development opportunities that go well beyond chipscale components. A number of such developments will be investigated, including new types of optical sensors, solar cells, quantum plasmonic components, non-linear, and ultrafast devices. At the end of the tutorial, a critical assessment of the entire field is given and some of the truly exciting new opportunities for plasmonics are identified. A comparison of metallic and high-index semiconductor antennas and metamaterials will be made as well.

\section{Short Course Benefits:}

- Obtain a working knowledge of the key physical concepts used in Plasmonics that enable light manipulation at ultra small length- and time-scales

- Understand choices of different metal types, shapes, and sizes to accomplish different plasmonic functionalities.

- Find out about common electromagnetic computational tools to design plasmonic structures and devices

- Get a feel for the current state of the field in terms of fundamental understanding as well as device applications

- Learn about the most recent trends and developments in research and applications

Short Course Level: Beginner

Short Course Audience: Optical engineers and scientists who are interested in learning about the rapidly emerging field of plasmonics and its potential impact.

\section{SC410 - Finite Element Modeling Methods for Photonics and Optics}

Tuesday, 16 May, 12:00-15:00

Arti Agrawal, City University, UK

Numerical modelling and simulation of optical devices and components is a key tool in improving performance by reducing time and monetary costs, design optimization and characterization as well as innovating new ideas. Both passive and active devices are modelled and optimized numerically. In some cases simulation is the only way to explore phenom- ena where technology is not advanced enough for fabrication. The interaction of the optical beam with physical effects such as non-linearity, stress, strain, change in refractive index due to temperature, application of electric fields etc. are now extremely important. Modelling complements experimental work perfectly and almost no research is conducted without it.

The Finite Element (FE) method is one of the most popular and powerful methods for modelling in Photonics. This short course starts with Maxwell's equations and explains the basic principles of numerical modelling and the key assumptions involved. This foundation is used to develop the FE method, including a brief tour of the mathematics. How the method can be applied to various optical devices is discussed in detail. How can physical effects be included with the FE method for modelling is considered. The course ends with an explanation of FE based beam propagation methods and how these can be used to find the evolution of the optical fields.

\section{Short Course Benefits:}

- Identify and explain basic principles of numerical modelling in Photonics

- Discuss and explain Full vector Finite Element Method (FEM) for modal solutions

- Discuss FEM with physical effects (non-linearity, stress/ strain, acousto-optic, electro-optic effect etc.)

- Discuss Finite Element Beam Propagation Methods (FE BPM)

- Discuss and explain how to incorporate Perfectly Matched Layer and Periodic boundary condition

- Summarize how to generate mesh for structures and post-processing of results

- Tips on how to best utilise commercial software

- Discuss the application of the method to practical devices: nano wires, optical fibers, sensors etc.

- Identify the appropriate modeling method for their problem

- How to incorporate PML boundary conditions and write your own code

Short Course Audience: This course is intended for researchers, engineers and students who use simulation in their work in both fundamental and applied aspects of Optics and Photonics, especially for components and devices. The course is useful for members of both academic and industrial institutions. Basic background and familiarity in Optics will be sufficient.

\section{SC270 - High Power Fiber Lasers and Amplifiers Tuesday, 16 May, 12:00-16:00 W. Andrew Clarkson, Univ. of Southampton, UK}

Recent advances in cladding-pumped fiber lasers and amplifiers have been dramatic, leading to unprecedented levels of performance in terms of output power, efficiency, beam quality and wavelength coverage. These achievements have attracted growing interest within the community and have fueled thoughts that fiber-based sources may one day replace conventional "bulk" solid-state lasers in many application areas. The main attractions of cladding-pumped fiber sources 
are derived directly from their geometry, which simultaneously allows very efficient generation of coherent light and almost complete immunity from the effects of heat generation, which are so detrimental to the performance of other types of lasers.

This course aims to provide an introduction to high power fiber lasers and amplifiers, starting from the basic principles of operation and ending with examples of current stateof-the-art devices and some thoughts on future prospects. The course will cover a range of topics, including basic fiber laser and amplifier theory, spectroscopy of the relevant rare earth ions for high power devices, a discussion of the factors influencing laser and amplifier performance, fiber design and fabrication, pump sources and pump launching schemes, fiber resonator design, master-oscillator and power-amplifier configurations, linewidth control and wavelength selection, transverse mode selection, nonlinear loss processes (SBS and SRS) and their impact on performance, and heat generation and its impact on power scalability. The course will also give an overview of techniques (e.g. coherent and spectral beam combining) for further scaling of output power and provide an introduction to hybrid fiber-bulk laser schemes for scaling pulse energy.

\section{Short Course Benefits:}

- Calculate threshold pump power and slope efficiency, and estimate the maximum output power that can be obtained from a given fiber laser oscillator or amplifier configuration.

- Select the optimum pump source for a given rare earth ion transition and fiber design.

- Design the pump light collection and coupling scheme and estimate the pump launch efficiency.

- Specify the fiber parameters (e.g. cladding design, core size, rare earth ion concentration) required for a particular laser or amplifier configuration.

- Design the fiber laser resonator and amplifier and select the operating wavelength.

- Estimate thermally induced damage limit.

- Estimate the power scaling limit.

- Measure fiber laser performance characteristics and relate these to fiber design and resonator parameters.

Short Course Level: Advanced Beginner

Short Course Audience: This course is intended for individuals with a basic knowledge of lasers and optics who wish to learn about the basic principles and capabilities of fiber lasers and amplifiers when operating at high power levels. The course will also cover some of the practical issues of operating these devices and provide an update for those wishing to learn about some of the latest developments in this rapidly advancing field.

\section{SC438 - Photonic Metamaterials}

Tuesday, 16 May, 12:00-16:00

Nader Engheta, University of Pennsylvania, USA

Controlling electromagnetic and optical fields and waves can be achieved via materials. The wave-matter interaction can be engineered using structures made of materials with required parameters and structures with selected shapes, dimensions and sizes. Recent advances in materials science and engineering, condensed matter physics, optical materials, nanoscience and nanotechnology have made it possible to tailor materials with unusual parameters and characteristics. The field of metamaterials, along with its two-dimensional version known as metasurfaces, has seen growing interest and extensive development in recent years. Metamaterials are engineered composite structures made of subwavelength inclusions with suitable materials and proper arrangements. The compositions, arrangements, alignments, densities and distributions of these building blocks in host media provide a variety of degrees of freedom in the design of light-matter interaction with such structures. Manipulation of light at the nano-, micro-, meso- and macroscales using metamaterials and metasurfaces provides rich platforms for tailoring electromagnetic waves with desired functionalities.

In this tutorial, we will begin with the basics of electromagnetic wave interaction with material media and structures. Then the course will get into some of the specifics of the characteristics of metamaterials and metasurfaces including the dispersion properties, scattering mechanisms, effectivemedium phenomena, and unconventional features of waves in such environments. We will then discuss some of the specific topics in photonic metamaterials such as extreme-parameter metamaterials (i.e., epsilon-near-zero (ENZ), mu-near-zero (MNZ), and epsilon-and-mu-near-zero (EMNZ) structures) and their specialized wave-matter interactions, graphene metamaterials as a platform for ideas for one-atom-thick optical device concepts, optical metatronics ("lumped" nanocircuitry) and informatic metastuctures for photonic information processing and computing at the nanoscale, scattering engineering using metamaterials (such as cloaking), guided waves in metamaterials, and nonreciprocal metastructures. Various features and potential applications of these topics will also be presented and discussed. During the course, we will have interactive discussions and question-answer sessions.

\section{Short Course Benefits:}

- Describe the basics of electromagnetic field and wave interaction with metamaterials and metasurfaces

- Explain some of the important properties of photonic metamaterials

- Discuss some of the scenarios in light-matter interaction with "extreme-parameter" metamaterials

- Describe the fundamentals of optical nanocircuits ("optical metatronics"), with potentials for information processing in nanophotonics

- Explain some of the salient features of scattering and guidance of lights in metamaterials and metasurfaces

\section{Short Course Level: Beginner}

Short Course Audience: Graduate students and senior undergraduates with EE, Physics, and Applied Physics interests; Engineers, researchers and technical managers from industry, government labs, and universities; Introductory knowledge of electromagnetics and optics is required. 


\section{Special Events}

\section{Cheeky Scientist Workshops \\ Sunday, 14 May, 13:00-16:30 \\ Winchester Room, Hilton}

Isaiah Hankel works with hundreds of graduate students and postdocs daily assisting them to transition to industry by first showing them how to present themselves as business professionals. These programs will provide you with a strong understanding of what it takes to have a tailored industry resume and how to showcase your transferrable skills.

13:30-14:30, Session 1: The Modern Job Search

15:00-16:30, Session 2: Networking: An Art \& Science

Workshops are complimentary for OSA Members. Learn more about the event at www.osa.org/professionaldevelopment.

Hosted by:
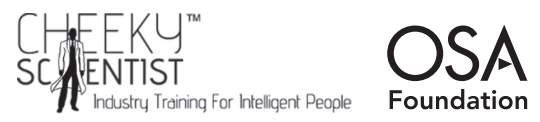

\section{Communication and Mentorship}

Monday, 15 May; 10:30-12:00

Winchester Room, Hilton

Improv for Communication uses techniques drawn from improvisational theater to explore communication skills that can be beneficial for collaboration, mentoring, day-to-day conversations, presentations, or job searches. Workshops focus on storytelling, spontaneity, and the improv principle of "yes, and."

This program is complimentary for OSA Members. Learn more about the event at www.osa.org/professionaldevelopment.

Hosted by:

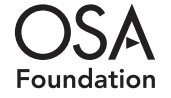

\section{Understanding Unconscious Bias}

Monday, 15 May; 14:00-15:30 \& 16:00-17:30 (repeat session) Winchester Room, Hilton

Research demonstrates that we all have unconscious biases. These biases can result in best and brightest talent made to feel unwelcome, invisible, and not important to the success of the organization. This training will explore concepts and engage participants to better understand implicit bias, increase awareness and understanding the impact on organizational culture and identify ways to promote greater engagement with diversity and inclusion. Choose one session.

This inclusive event has been organized by:

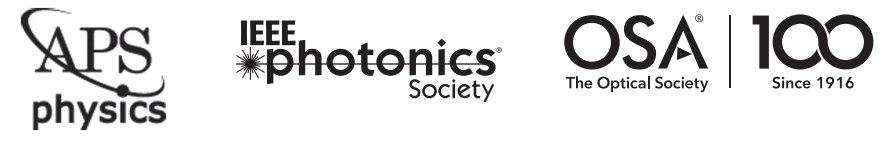

\author{
Diversity \& Inclusion in Optics and \\ Photonics Reception \\ Monday, 15 May, 17:30-18:30 \\ Market Room, Hilton
}

Join us for a reception to connect with the optics and photonics community to discuss diversity in the field. Come to learn, share and engage with colleagues around this important topic.

This inclusive event has been organized by:

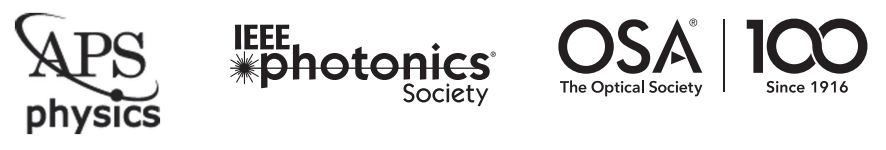

\author{
The National Academies Town Hall Meeting \\ on the Future of Materials Research \\ Monday, 15 May; 18:30-20:00 \\ Salon V \& VI, Marriott
}

The National Academies of Sciences, Engineering and Medicine is seeking community input for a study on the future of materials research (MR). Frontiers of Materials Research: A Decadal Survey will look at defining the frontiers of materials research ranging from traditional materials science and engineering to condensed matter physics. Please join members of the study committee for a town hall to discuss future directions for materials research in the United States in the context of worldwide efforts. In particular, input on the following topics will be of great value: progress, achievements, and principal changes in the R\&D landscape over the past decade; identification of key MR areas that have major scientific gaps or offer promising investment opportunities from 2020-2030; and the challenges that MR may face over the next decade and how those challenges might be addressed.

This study was requested by the Department of Energy and the National Science Foundation. The National Academies will issue a report in 2018 that will offer guidance to federal agencies that support materials research, science policymakers, and researchers in materials research and other adjoining fields. Learn more about the study at http://nas.edu/ materials.

\section{OSA Technical Group Poster Session}

Monday, 15 May, 19:00-20:00

Room 230 B

Join the OSA Technical Groups for a series of focused poster sessions, bringing together students and colleagues for an opportunity to share their latest research findings and exchange ideas. After listening to the poster presentations and connecting with fellow attendees over refreshments, you'll have a chance to cast your vote for the best poster from each of the four participating technical groups. Among the technical groups participating this year will be the Optical Material Studies Technical Group, the Photonic Detection Technical Group, the Ultrafast Optical Phenomena Technical Group and more. Visit www.osa.org/technicalgroups to view a full list of OSA Technical Group events at CLEO.

Hosted by: OSA Technical Groups

All conference locations are in the San Jose Convention Center unless otherwise noted. 


\section{Alternative Careers Paths in Optics and Photonics}

Tuesday, 16 May, 13:00-17:30

Willow Glenn, Marriott

13:30-15:00, March for Science: Scientists + Advocacy Hear from scientists who came together to bring the March for Science to life with a focus on international advocacy of science.

16:00-17:30, Jump-Start a Career in Data Science Have you been looking to utilize your degree in a unique way? Come learn how to jump-start a career in data science by professionals in the field.

This program is complimentary for OSA Members. RSVP at www.OSA.org/professionaldevelopment.

Hosted by: OSA

Foundation

\section{Meet the OSA Editors' Reception}

Tuesday, 16 May, 17:00-18:30

Market Terrace

Join OSA Publishing's Journal Editors for conversation and refreshments. The Editors welcome your questions, concerns, and ideas for any of OSA's Journals. Come along and ask about best practices when submitting a manuscript; elements of a useful manuscript review; criteria editors apply when considering submitted manuscripts; or how to propose a Feature Issue topic for publication in an OSA Journal. All are welcome.

\section{Conference Reception and Poster Session Tuesday, 16 May, 18:00-19:30 \\ Exhibit Halls 1, 2 \& 3}

Enjoy a festive evening with your colleagues, while intermingling with the exhibitors and viewing the first poster session. The reception is open to all attendees and badges must be worn to enter the reception.

\section{Sponsored by: THOR}

\section{Poster Sessions}

Exhibit Halls 1, 2 \& 3

Poster Sessions are an integral part of the Technical program. Each author is provided with a board with six-foot-high by four-foot-wide $(183 \mathrm{~cm} \times 128 \mathrm{~cm})$ of usable space on which to display the summary and results of his or her paper. Authors should remain in the vicinity of their presentation board for the duration of the sessions to answer questions from attendees. Authors may set up one hour prior to their assigned session and must remove their poster one hour following the session. Authors may submit their poster PDF to cstech@osa.org for publication.

\begin{tabular}{|l|l|}
\hline Tuesday, 16 May & $18: 00-19: 30$ \\
\hline Wednesday, 17 May & $10: 00-12: 00$ \\
\hline Thursday, 18 May & $11: 30-13: 00$ \\
\hline
\end{tabular}

\section{OSA Members, Family and Friends Tour - Computer History Museum}

Wednesday, 17 May, 10:15-12:45

Shuttle transportation will depart from the Hilton's Almaden Avenue entrance at 10:15

Located in Mountain View, CA, the Computer History Museum holds a four-decade history as the world's leading institution exploring the history of computing and its ongoing impact on society. The Museum is dedicated to the preservation and celebrating of computer history and is home to the largest international collection of computing artifacts in the world, encompassing computer hardware, software, documentation, photographs, oral histories, and moving images. OSA members and their guests are invited to attend a onehour docent-led tour, which includes exhibits on autonomous vehicles, an IBM demo lab, as well as the first 2,000 years of computing. Advanced registration is required as there are limited spots, please email pwimmer@osa.org to RSVP.

\section{Happy Hour in Exhibit Hall \\ Wednesday, 17 May, 16:30-18:30 \\ Exhibit Hall 1,2 \& 3}

Don't miss the exiciting Happy Hour on the CLEO show floor. This will be a perfect time to relax after a full day of sessions and walking the show floor, while networking with exhibitors and colleagues. The happy hour is open to all attendees.

\section{OSA Nanophotonics Technical Group 20×20 Talks \\ Wednesday, 17 May, 18:00-19:00 \\ Executive Ballroom 210 A}

The OSA Nanophotonics Technical Group is proud to host their first 20x20 Talks session at CLEO! This special session introduces a new platform for presenting members' research innovation in a creative and concise fashion that differs from the usual oral or poster session. Selected participants will showcase their research in a presentation of 20 images, in which each image is displayed for 20 seconds. Presenters will talk along to the images as the slides advance automatically. Immediately following the presentations, attendees are invited to join the Nanophotonics Technical Group for a small reception where they can network with colleagues over refreshments. Visit www.osa.org/technicalgroups to view a full list of OSA Technical Group events at CLEO.

Hosted by: OSA

\section{Pizza Lunch at the CLEO:EXPO}

Thursday, 18 May, 11:30-13:30

Exhibit Halls 1, 2 \& 3

Last chance to visit the exhibit hall! Grab some pizza and network with exhibitors to check out their innovative products and services that can help your organization.

\section{Postdeadline Paper Sessions}

Thursday, 18 May, 20:00-22:00

Locations announced on the Conference Update Sheet

The Technical Program Committee has accepted a limited number of postdeadline papers for oral presentation. The purpose of postdeadline papers is to give participants the opportunity to hear new and significant materials in rapidly advancing areas. 
Exhibit Halls 1, 2 and 3

Make sure to visit the exhibit floor, which features a diverse group of companies, representing every facet of the optics and photonics industries. Learn about new products, find technical and business solutions, and gain the most up-todate perspective of the laser-related business environment. Review the list of exhibitors below to see the wealth of companies you'll meet at CLEO.

There is no charge to attend the exhibit for conference registrants.

\begin{tabular}{|c|c|}
\hline Tuesday, 16 May & $11: 30-19: 30$ \\
Exhibit-Only Times & $11: 30-13: 30$ \\
& $15: 30-16: 00$ \\
& $18: 00-19: 30$ \\
\hline Wednesday, 17 May & $10: 00-18: 30$ \\
Exhibit-Only Times & $10: 00-13: 00$ \\
& $15: 00-15: 30$ \\
& $17: 30-18: 30$ \\
\hline Thursday, 18 May & $10: 00-15: 00$ \\
Exhibit-Only Times & $10: 00-14: 00$ \\
\hline
\end{tabular}

\section{Exhibit Hall Rules}

Children 12 and under must be accompanied by an adult at all times.

Strollers are not allowed on the show floor at any time.

Neither photography nor videotaping is permitted in the Exhibit Hall. Exhibitors need to get permission from Show Management to photograph their own booths. Non-compliance may result in the surrendering of film and removal from the hall.

For further questions, visit Registration on the Concourse Level.
Exhibitors (as of 11 April 2017)

AccuStrata

AdValue Photonics, Inc.

AdvR

AIP Publishing

Allied Laser Solutions

Alpine Research Optics

Altos Photonics, Inc.

American Physical Society (APS)

Amplitude Systemes and Amplitude Technologies

APE - Applied Physics \& Electronics, Inc.

Asphericon

Attocube Systems, Inc.

AUREA Technology

Axiom Optics

Boston Electronics Corporation

Brimrose Corporation of America

Bristol Instruments, Inc.

Calmar Laser, Inc.

CASTECH, Inc.

Changchun New Industries Optoelectronics Tech. Co. Ltd.

Chinese Laser Press

Cobolt, Inc.

Coherent, Inc.

Coherent Solutions

Continuum

CorActive High-Tech, Inc.

Crestec Corporation

Crystalline Mirror Solutions, GmbH

Crystrong Photoelectric Technology Co., Ltd.

CST of America, Inc.

Cybel, LLC

Cycle GmbH

DataRay, Inc.

Dausinger + Giesen GmbH

Daylight Solutions, Inc.

De Gruyter

Discovery Semiconductors, Inc.

Double Helix LLC 
Edmund Optics, Inc.

EKSMA Optics

EKSPLA

Electro-Optics Technology, Inc. Energetiq Technology, Inc.

EOSPACE, Inc.

FASTLITE

Femtochrome Research, Inc.

few-cycle

FiberPro, Inc.

Gentec Electro-Optics, Inc.

Gooch \& Housego, PLC

GT Crystal Systems, LLC.

Hamamatsu Corporation

Hiwin Corporation

HOLOEYE Photonics AG

HTA Photomask

Ibsen Photonics A/S

IEEE Photonics Society

IMRA America, Inc.

InPhenix

Inrad Optics

IOP Publishing Ltd.

IPG Photonics Corp.

IRflex Corporation

Jasper Display Corporation

KMLabs (Kapteyn-Murnane Laboratories)

LaCroix Precision Optics

Laser Focus World

Laser Quantum, Inc.

Lattice Electro Optics, Inc.

Lawrence Livermore National Laboratory

Light Conversion, Ltd.

Lighthouse Photonics

Liquid Instruments

Luvantix ADM Co., Ltd.

Luxmux Technology Corporation

M Squared Lasers Ltd.

Menlo Systems

Mesa Photonics, LLC
Micro Photon Devices

Mindrum Precision, Inc.

MIRTHE Center - Princeton University

MKS Instruments

Montana Instruments

MONTFORT Laser GmbH

National Energetics

Newport Corporation

NKT Photonics

Northrop Grumman Cutting Edge Optronics, Inc.

NPI Lasers

Nuphoton Technologies, Inc.

Ocean Optics, Inc.

OEwaves, Inc.

Ophir

Optics Balzers Optiforms

Optiforms

OptiGrate

Optilab, LLC

Optimax Systems, Inc.

OptoSigma Corporation

Optronics Co., Ltd., The

OSA

Osela, Inc.

Oxford University Press

Oxide Corporation

OZ Optics

PHASICS Corp.

Photodigm, Inc.

Photon Design

Photonics Industries International

Photonix Edge, LLC

Photonics Media/Laurin Publishing

Photop Technologies, Inc.

Physics Today

PI (Physik Instrumente) LP

PicoQuant Photonics North America, Inc.

Princeton Scientific Corporation

PriTel, Inc.

Pure Photonics
Quantel

Quantum Design, Inc.

Quantum Opus

Raicol Crystals Ltd.

Research Electro-Optics, Inc.

RESOLUTION Spectra Systems

RPMC Lasers, Inc.

Sacher Lasertechnik GmbH

Santec USA Corporation

Seiwa Optical America, Inc.

Shasta Crystals

SILIOS Technologies S.A.

Siskiyou Corporation

SmarAct Inc. GmbH

Specialised Imaging

Spectra-Physics

SPIE: The Intl Society for Optics and Photonics

Springer

Srico, Inc.

Stable Laser Systems

STANDA

StellarNet, Inc.

Swamp Optics, LLC

Synopsys, Inc.

Teledyne Judson Technologies

ThermoTek, Inc.

Thorlabs

Toptica Photonics, Inc.

TRUMPF Inc.

University of Central Florida, CREOL

US Fiberoptec Technology, Inc.

Vescent Photonics, Inc.

VST America

Wuhan Industrial Institute for Optoelectronics

Xiamen Freeform Optical Technology Co., Ltd.

Yenista Optics, Inc.

Zaber Technologies

Zurich Instruments 


\section{CLEO:EXPO Technology Playground}

Tuesday - Thursday, Exhibition Hours

Exhibit Hall

This interactive exhibits event allows you to network with top industry leaders, demo their innovative products/services and explore the latest innovations that can help you in your work. Visit all participating companies for a chance to win a $\$ 100$ American Express gift card. Stop by the OSA or IEEE Member booths for an entry form.

\section{Newport Student Lounge}

All student attendees are invited to the Newport Student Lounge, co-sponsored by OSA. The lounge provides an opportunity to relax and spend time networking with other students, while enjoying complimentary, wireless Internet and refreshments.

Sponsored by $\longrightarrow$ Newport. Experience | Solutions

Co-sponsored by

\section{OIDA VIP Industry Leaders Speed Meetings Lunch}

Tuesday, 16 May, 12:00-13:30

Exhibit Hall

Sponsored by

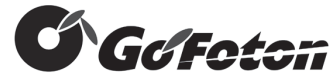

This session brings together Industry Executives to share their business experience - from how they started their careers and lessons learned along the way, to using their degree in an executive position - with Recent Graduates and Students. The program starts with information networking during lunch and then transitions into "speed meetings" - small, brief visits with each executive to discuss careers, industry trends or other career topics. Separate Registration Required

\section{CLEO: Market Focus}

Tuesday, 16 May - Wednesday, 17 May Exhibit Hall Theater

CLEO: Market Focus focuses on the latest trends in the photonics marketplace. The program provides a forum to discuss new products and emerging technologies and markets while also providing networking opportunities. All presentations and discussions will be focused on the latest in photonics products and services that have been playing an important role in the industry and those that potentially hold a future business opportunity. A key feature of this forum will be the survey of market trends and market sector outlook in the selected areas.
Tuesday, 16 May

Session I

12:00-13:30 Precision Applications Using Ultrafast Lasers

Session II

15:30-16:15

Update on Optics and Photonics Markets and Opportunities

Moderator: Tom Hausken, The Optical Society, USA

This presentation will review the outlook for key optics and photonics industry sectors and upcoming market opportunities. It will provide a quantitative look at the present performance and trends, as well as examples of where to find industry growth longer term.

Session III 16:30-17:00

Science Olympiad

Moderator: Mike McKee, CREOL, University of Central Florida, USA

USA Science Olympiad, a competition for middle and high school students, serves as a perfect vehicle to conduct vital outreach in optics and photonics. With over 7000 schools and nearly 2,000,000 students involved across the USA, it is a leader in STEM education. Learn how to get involved in a competition near you, the types of activities that are photonics related, and how best to engage with students to promote photonics as a career choice for K-12 students.

Wednesday, 17 May

Session IV

10:30-12:00

How the Changing Political Landscape will Impact your Company

Moderator: Laura Kolton, The Optical Society, USA

Meet Government Relations experts as they discuss how the national elections in the United States and Europe will impact the optics and photonics industry. The US perspective provides an overview of the priorities of the Trump Administration and U.S. Congress. The EU perspective includes an overview of the upcoming elections in France and Germany and the impact of Brexit on funding and large scale collaboration projects. Don't miss this opportunity to learn how you will be impacted by these changes and how you can get impact policy decisions with your policymakers.

Session V 15:00-16:30

\section{Bright Ideas Competition}

The OSA Foundation (OSAF) have partnered with Quantel Laser to provide organizations the opportunity to compete for a \$30K US laser system and added Gentech Power Meter! Proposals from around the world were submitted and a handful of finalists will compete at CLEO for top honors.

Visit www.cleoconference.org/marketfocus for complete information. 


\section{Plenary Speaker Meet-n-Greets}

Exhibit Hall

Meet CLEO Plenary speakers, ask questions and network with your colleagues.

Ataç İmamoğlu, Tuesday, 16 May, 11:30-12:00

Ursula Keller, Wednesday, 17 May, 15:00-15:30

Please see the update sheet for more information.

\section{Technology Transfer Program}

Thursday, 18 May

Exhibit Hall Theater

The Technology Transfer Program includes a Keynote presentation, a Technology Transfer Tutorial and a Pitch Panel. The Technology Transfer Tutorial provides attendees an opportunity to learn more about the licensing process: funding, entrepreneurship, technology transfer, and intellectual property. The Pitch Panel provides entrepreneurs an opportunity to showcase their technology, explain why it's valuable and discuss the next steps to commercialization.

$\begin{array}{ll}\text { 10:15 - 10:45 } & \text { Keynote Speaker } \\ & \text { Magnus Bengtsson, Coherent, USA } \\ \text { 10:45-11:30 } & \text { Tech Transfer Tutorial } \\ & \text { Moderator: Giacomo Vacca, Kinetic River } \\ & \text { Corp., USA } \\ & \text { Presenters: } \\ & \text { David G Dawes, Lawrence Livermore } \\ & \text { National Lab, USA } \\ & \text { Ruth Houbertz, Multiphoton Optics GmbH, } \\ & \text { Germany } \\ & \text { Pitch Panel w/ Feedback from Panelists } \\ & \text { Moderator: Giacomo Vacca, Kinetic River } \\ & \text { Corp., USA } \\ & \text { Presenters: } \\ & \text { Leslie Kimerling, Double Helix, USA } \\ & \text { Alexander Muhr, Element Six, USA } \\ & \text { Debashis Chanda, e-skin Displays Inc., USA } \\ & \text { Graham L. Randall, Noninvasix Inc., USA } \\ & \text { Pitch Panelists: } \\ & \text { Jason Eichenholz, Open Photonics, USA } \\ & \text { Linda Smith, Ceres, USA } \\ & \text { Mark Tolbert, TOPTICA Photonics Inc., USA }\end{array}$

Media Sponsor: PHOTONICS) MEDIA

Visit www.cleoconference.org/techtransfer for complete information.

\section{CLEO UNconference and Hot Topics}

Thursday, 18 May, 12:00-13:00

Exhibit Hall

Take part in an "unconference" where participants, in an open-floor format, will have the opportunity to discuss the latest Hot Topics and other prevailing challenges facing the science community today.

Please see the update sheet for more information. 


\section{CLEO Committees}

\section{Applications \& Technology}

Nicusor Iftimia, Physical Sciences Inc., USA, General Chair Christian Wetzel, Rensselaer Polytechnic Inst., USA, General Chair

Peter E. Andersen, Danmarks Tekniske Universitet, Denmark, Program Chair

Michael M. Mielke, Lumentum, USA, Program Chair

\section{A\&T 1: Biomedical Applications}

Jin Kang, Johns Hopkins Univ., USA, Subcommittee Chair Ilko K. Ilev, U.S. Food and Drug Admin., USA

Chang-Seok Kim, Pusan National Univ., South Korea

Pilhan Kim, Korea Advanced Inst Sci \& Tech, South Korea

Xuan Liu, New Jersey Inst. of Technology, USA

Laleh Najafizadeh, Rutgers Univ., USA

Jessica C. Ramella-Roman, Florida International Univ., USA

Utkarsh Sharma, Carl Zeiss Meditec AG, USA

Melissa Skala, Vanderbilt Univ., USA

Benjamin J. Vakoc, Harvard Medical School, USA

Martin Villiger, Wellman Center for Photomedicine, USA

Alex Vitkin, Ontario Cancer Institute, Canada

\section{A\&T 2: Industrial Applications}

Andrius Marcinkevicius, TRUMPF Inc., USA, Subcommittee Chair

Peter Fendel, Thorlabs Inc, USA

Oleg Khodykin, KLA-Tencor, USA

Jan Kleinert, ESI, USA

Xiang Peng, Raydiance Inc, USA

Jie Qiao, Rochester Inst. of Technology, USA

Jeffrey Wojtkiewicz, Nufern, USA

\section{A\&T 3: Photonic Instrumentation \& Techniques for Metrology \& Industrial Process \\ Paul Williams, NIST, USA, Subcommittee Chair \\ Giorgio Brida, INRIM, Italy \\ Jana Jagerska, UiT Norges Arktiske Universitet, Norway \\ Dirk Mueller, Coherent Inc, USA \\ Gregory Rieker, Univ. of Colorado at Boulder, USA \\ Andy Sappey, Zolo Technologies, USA \\ Brian Simonds, NIST, USA}

\section{A\&T 4: Laser \& Photonics Applications for Energy \& Environment}

Stephanie Tomasulo, Naval Research Lab., USA, Subcommittee Chair

Mark A. Zondlo, Princeton Univ., USA, Subcommittee Chair Kirsten Alberi, National Renewable Energy Lab, USA

David Bomse, Mesa Photonics, USA

David Canteli, Univ. Politecnica de Madrid, Spain

Jan-Frederik Nekarda, Fraunhofer-Institut für Solare

Energiesysteme, Germany

Joel Silver, Southwest Sciences Inc., USA

\section{Science \& Innovations}

Siddharth Ramachandran, Boston Univ., USA, General Chair Jonathan D. Zuegel, Univ. of Rochester, USA, General Chair

Sterling J. Backus, Kapteyn-Murnane Laboratories, USA, Program Chair

Michal Lipson, Columbia Univ., USA, Program Chair

\author{
S\&I 1: Light-Matter Interactions and Materials \\ Processing \\ Emmanuel Haro-Poniatowski, UAM-Iztapalapa, Mexico, \\ Subcommittee Chair \\ Feng Chen, Shandong Univ., China \\ Maria Dinescu, NILPRP, Romania \\ James Fitz-Gerald, Univ. of Virginia, USA \\ Richard F. Haglund, Vanderbilt Univ., USA \\ Masaki Hashida, Kyoto Univ., Japan \\ Tsing-Hua Her, Univ of North Carolina at Charlotte, USA \\ Alberto Pique, US Naval Research Lab, USA \\ Gagan Saini, Halliburton Energy Services, Inc, USA \\ Javier Solis, Instituto De Optica 'Daza De Valdes', Spain
}

\section{S\&I 2: Advanced Science and Technology for Laser Systems and Facilities}

Klaus Ertel, STFC Rutherford Appleton Lab., UK, Subcommittee Chair

Jake Bromage, Univ. of Rochester, USA

Jay Doster, Northrop Grumman Cutting Edge Optronics, USA

Hiromitsu Kiriyama, National Inst. Quantum \& Radiological Science \& Tech, Japan

Xiaoyan Liang, Shanghai Inst of Optics \& Fine Mechanics, China

Thomas Metzger, TRUMPF Scientific Lasers GmbH + Co. KG, Germany

Dimitrios Nikolaos Papadopoulos, LULI, France

Brendan A. Reagan, Colorado State Univ., USA

Shawn Redmond, MIT Tech Lincoln Lab, USA

Clara Saraceno, Ruhr-Universität Bochum, Germany

David James Spence, Macquarie Univ., Australia

Thomas Spinka, Lawrence Livermore National Lab, USA

\section{S\&I 3: Semiconductor Lasers}

Amr S. Helmy, Univ. of Toronto, Canada, Subcommittee Chair Raffaele Colombelli, Université Paris Sud and CNRS, France

Lan Fu, Australian National Univ., Australia

Nicolas Grandjean, Ecole Polytechnique Federale de Lausanne, Switzerland

Qing Gu, Univ. of Texas at Dallas, USA

Sven Hoefling, Univ. of St Andrews, Germany

Jonathan Klamkin, Univ. of California Santa Barbara, USA

Valentin Loyo-Maldonado, Jenoptik Laser GmbH, Germany

Boon S. Ooi, King Abdullah Univ. of Science \& Technology, Saudi Arabia

Dominic Francis Siriani, MIT Lincoln Lab Periodical Library, USA

Hongping Zhao, Case Western Reserve Univ., USA 


\section{S\&I 4: Nonlinear Optical Technologies}

Jeffrey Moses, Cornell Univ., USA, Subcommittee Chair

Masaki Asobe, Tokai Univ., Japan

Judith M. Dawes, Macquarie Univ., Australia

Majid Ebrahim-Zadeh, ICFO -The Institute of Photonic Sciences, Spain

Leonel P. Gonzalez, Air Force Research Lab, USA

Nicolas Joly, Universität Erlangen-Nürnberg, Germany

Shu-Wei Huang, Univ. of California Los Angeles, USA

Yoshitomo Okawachi, Columbia Univ., USA

Derryck T. Reid, Heriot-Watt Univ., UK

Michelle Y Sander, Boston Univ., USA

Irina T. Sorokina, Norges Teknisk Naturvitenskapelige Univ, Norway

Kartik Srinivasan, NIST, USA

\section{S\&I 5: Terahertz Science and Applications}

Dmitry Turchinovich, Max Planck Inst. for Polymer Research, Germany, Subcommittee Chair

Matthias Clemens Hoffmann, SLAC National Accelerator Lab, USA

Mona Jarrahi, Univ. of California Los Angeles, USA

Peter Uhd Jepsen, Danmarks Tekniske Universitet, Denmark

Hannah Joyce, Univ. of Cambridge, UK

Martin Koch, Philipps Universitat Marburg, Germany

Daniel M. Mittleman, Brown Univ., USA

Rohit Prativadi Prasankumar, Los Alamos National Lab, USA

Masayoshi Tonouchi, Osaka Univ., Japan

Miriam Serena Vitiello, Scuola Normale Superiore di Pisa, Italy

Stephan Winnerl, Forschungszentrum Dresden-Rossendorf, Germany

\section{S\&I 6: Optical Materials, Fabrication and Characterization}

Thomas E. Murphy, Univ. of Maryland at College Park, USA, Subcommittee Chair

Matthew David Escarra, Tulane Univ., USA

Frederic Gardes, Univ. of Surrey, UK

Juejun Hu, MIT, USA

Michael Menard, UQAM, Canada

Robert A. Norwood, Univ. of Arizona, USA

Roberto Paiella, Boston Univ., USA

Jason Pelc, Hewlett Packard Labs, USA

Takehiko Tawara, NTT Basic Research Laboratories, Japan

Frank (Fengqiu) Wang, Nanjing Univ., China

Nanfang Yu, Columbia Univ., USA
S\&I 7: Micro- and Nano-Photonic Devices

Marcelo Davanco, NIST, USA, Subcommittee Chair

Ali Adibi, Georgia Institute of Technology, USA

Paul E. Barclay, Univ. of Calgary, Canada

Kenneth Brian Crozier, Univ. of Melbourne, Australia

Dirk Englund, MIT, USA

Michael Galili, Danmark Tekniske Universitet, Denmark

Zhihong Huang, Hewlett Packard laboratories, USA

Wei Jiang, Rutgers Univ., USA

Christian Koos, Karlsruhe Institute of Technology KIT, Germany

Frank Koppens, ICFO -The Institute of Photonic Sciences, Spain

Qiang Lin, Univ. of Rochester, USA

Jeremy N. Munday, Univ. of Maryland at College Park, USA

Takasumi Tanabe, Keio Univ., Japan

Sharon M. Weiss, Vanderbilt Univ., USA

Chee Wei Wong, Univ. of California Los Angeles, USA

\section{S\&I 8: Ultrafast Optics, Optoelectronics \& Applications}

Christophe Dorrer, Univ. of Rochester, USA, Subcommittee Chair

Jose Azana, INRS-Energie Materiaux et Telecom, Canada

Alan Fry, SLAC National Accelerator Lab, USA

Fumihiko Kannari, Keio Univ., Japan

Cristian Manzoni, IFN-CNR, Italy

Thomas Alexandre Planchon, Delaware State Univ., USA

Bojan Resan, Lumentum, USA

Lawrence Shah, CREOL, Univ. of Central Florida, USA

Thomas Sudmeyer, Université de Neuchâtel, Switzerland

Andreas Vaupel, IPG Photonics Corp, USA

Laszlo Veisz, Umea University, Sweden

Zhiyi Wei, CAS Institute of Physics, China

Tobias Witting, Max Born Institute, Germany

\section{S\&I 9: Components, Integration, Interconnects and Signal Processing}

Weidong Zhou, Univ. of Texas at Arlington, USA, Subcommittee Chair

Adam Michael Crook, Lockheed Martin, USA

Sasan Fathpour, CREOL, Univ. of Central Florida, USA

Mark Aaron Foster, Johns Hopkins Univ., USA

Qiaogiang Gan, State Univ. of New York at Buffalo, USA

Mo Li, Univ. of Minnesota, USA

Marianna Pantouvaki, IMEC Interuniversity Microelectronics Center, Belgium

Richard V. Penty, Univ. of Cambridge, UK

Stefan Francis Preble, Rochester Institute of Technology, USA

Jian Wang, Huazhong Univ. of Science and Technology, China Jianping Yao, Univ. of Ottawa, Canada 
S\&I 10: Biophotonics and Optofluidics

Andreu Llobera, Carl Zeiss Vision GmbH, Germany, Subcommittee Chair

Hatice Altug, Ecole Polytechnique Federale de Lausanne, Switzerland

Kishan Dholakia, Univ. of St Andrews, UK

Ewa M. Goldys, Macquarie Univ., Australia

Aaron Hawkins, Brigham Young Univ., USA

Jessica Perea Houston, New Mexico State Univ., USA

Nien-Tsu Huang, National Taiwan Univ., Taiwan

Ralph Jimenez, Univ. of Colorado at Boulder, USA

Rainer Andreas Leitgeb, Medical Univ. Vienna, Austria

Ute Neugebauer, Center for Sepsis Control and Care Jena, Germany

Kevin K. Tsia, Univ. of Hong Kong, Hong Kong

\section{S\&l 11: Fiber Photonics}

Shinji Yamashita, Univ. of Tokyo, Japan, Subcommittee Chair Kazi S. Abedin, OFS Laboratories, USA

Shahraam Afshar, Univ. of South Australia, Australia

Shaif-UI Alam, Univ. of Southampton, UK

Camille-Sophie Bres, Ecole Polytechnique Federale de Lausanne, Switzerland

Neil Broderick, Univ. of Auckland, New Zealand

Andy Chong, Univ. of Dayton, USA

Liang Dong, Clemson Univ., USA

Peter D. Dragic, Univ of Illinois at Urbana-Champaign, USA

Julien Fatome, Université de Bourgogne, France

Ming-lie Hu, Tianjin Univ., China

Khanh Quoc Kieu, Univ. of Arizona, USA

Sze Yun Set, Univ. of Tokyo, Japan

Kenneth Kin-Yip Wong, Univ. of Hong Kong, Hong Kong

\section{S\&I 12: Lightwave Communications and Optical} Networks

Michael Vasilyev, Univ. of Texas at Arlington, USA, Subcommittee Chair

Anjali Agarwal, Vencore Labs, USA

Nicolas K. Fontaine, Nokia Corporation, USA

David Jason Geisler, MIT Lincoln Lab, USA

Vladimir S. Grigoryan, Ciena Corporation, USA

Yue-Kai Huang, NEC Laboratories America Inc, USA

Francesca Parmigiani, Univ. of Southampton, UK

Yikai Su, Shanghai Jiao Tong Univ., China

Takashi Sugihara, Mitsubishi Electric Corporation, Japan

Stylianos Sygletos, Aston Univ., UK

Ioannis Tomkos, Athens Information Technology Center, Greece

\section{S\&I 13: Active Optical Sensing}

Mark Phillips, Pacific Northwest National Lab, USA,

Subcommittee Chair

Adam J. Fleisher, NIST, USA

Aleksandra Foltynowicz-Matyba, Umeå University, Sweden

Scott Sheridan Howard, Univ. of Notre Dame, USA

R. Jason Jones, Univ. of Arizona, USA

Waruna Kulatilaka, Texas A\&M Univ., USA

Bernhard Lendl, Technische Universität Wien, Austria

Michal Pawel Nikodem, Wroclaw Research Centre EIT+, Poland

Todd H. Stievater, US Naval Research Lab, USA

Damien Weidmann, STFC Rutherford Appleton Lab, UK

Ian M. White, Univ. of Maryland at College Park, USA

Michael Wojcik, Space Dynamics Lab, USA

\section{S\&I 14: Optical Metrology}

lan R. Coddington, NIST, USA, Subcommittee Chair

Katja Beha, Menlo Systems GmbH, Germany

Tara Michele Fortier, NIST, USA

Jérôme Genest, Université Laval, Canada

Jungwon Kim, Korea Advanced Inst. of Science \& Tech, South Korea

Marco Andrea Marangoni, Politecnico di Milano, Italy

Michael Joseph Martin, Sandia National Laboratories, USA

Mark Notcutt, Stable Laser Systems, USA

Stephane Schilt, Université de Neuchâtel, Switzerland

Guanhao Wu, Tsinghua Univ., China

\section{Fundamental Science}

Junichiro Kono, Rice Univ., USA, General Chair

Yurii A. Vlasov, Univ of Illinois at Urbana-Champaign, USA, General Chair

Benjamin J. Eggleton, Univ. of Sydney, Australia, Program Chair

Irina Novikova, College of William \& Mary, USA, Program Chair

FS 1: Quantum Optics of Atoms, Molecules and Solids

Sergey Polyakov, NIST, USA, Subcommittee Chair

Daniel Felinto Pires Barbosa, Universidade Federal de Pernambuco, Brazil

Boris Blinov, Univ. of Washington, USA

Hugues de Riedmatten, ICFO -The Institute of Photonic Sciences, Spain

Ivo Pietro Degiovanni, INRIM, Italy

Edward Flagg, West Virginia Univ., USA

Elizabeth A. Goldschmidt, US Army Research Lab, USA

Virginia O. Lorenz, Univ. of Illinois at Chicago, USA

Andreas Muller, Univ. of South Florida, USA

Tracy Northup, Univ. of Innsbruck, Austria

Joshua Nunn, Univ. of Oxford, UK

Monika Schleier-Smith, Stanford Univ., USA

Olga V. Tikhonova, M. V. Lomonosov Moscow State Univ., Russia

Ite A. Yu, National Tsing Hua Univ., Taiwan 


\section{FS 2: Quantum Science, Engineering and} Technology

Todd B. Pittman, Univ. of Maryland Baltimore County, USA, Subcommittee Chair

Konrad Banaszek, Uniwersytet Warszawski, Poland Joshua Bienfang, NIST, USA

Ryan M Camacho, Sandia National Laboratories, USA

John Charles Howell, Univ. of Rochester, USA

Elanor H. Huntington, Univ. of New South Wales, Australia

Yoon-Ho Kim, Pohang Univ of Science \& Technology, South Korea

Nathan Kim Langford, Technische Universiteit Delft, Netherlands

Olivier Pfister, Univ. of Virginia, USA

Kevin Resch, Univ. of Waterloo, Canada

Shigeki Takeuchi, Kyoto Univ., Japan

Sebastien Tanzilli, Universite de Nice Sophia Antipolis, France

Philip Walther, Universitat Wien, Austria

\section{FS 3: Metamaterials and Complex Media}

Alexey G Yamilov, Missouri Univ of Science \& Technology, USA, Subcommittee Chair

Viktoriia Babicheva, Georgia State Univ., Ukraine

Yaron Bromberg, The Hebrew Univ., Israel

Yidong Chong, Nanyang Technological Univ., Singapore

Nicholas Fang, MIT, USA

Xu Fang, Univ. of Southampton, UK

Sylvain Gigan, Laboratoire Kastler-Brossel, France

Zubin Jacob, Univ. of Alberta, Canada

Andrei V. Lavrinenko, Danmarks Tekniske Universitet, Denmark

Vinod M. Menon, City College of New York, USA

Junsuk Rho, POSTECH, South Korea

Vera Smolyaninova, Towson Univ., USA

Sefaattin Tongay, Arizona State Univ., USA

Xiaobo Yin, Univ. of Colorado at Boulder, USA

\section{FS 4: Optical Excitations and Ultrafast Phenomena in Condensed Matter}

Keshav Moreshwar Dani, Okinawa Inst of Science \&

Technology, Japan, Subcommittee Chair

Hui Deng, Univ. of Michigan, USA

Michael B. Johnston, Univ. of Oxford, UK

Emmanouil Kioupakis, Univ. of Michigan, USA

Chih-Wei Lai, US Army Research Lab, USA

Xiaogin Li, Univ. of Texas at Austin, USA

Kazunari Matsuda, Kyoto Univ., Japan

Janice Musfeldt, Univ. of Tennessee Knoxville, USA

Denis Seletskiy, Univ. of Konstanz, Germany

Mark S. Sherwin, Univ. of California Santa Barbara, USA

Ajay K. Sood, Indian Institute of Science, India

Diyar Talbayev, Tulane Univ., USA

Jerome Tignon, CNRS - Laboratoire Pierre Aigrain, France

Lyubov Titova, Worcester Polytechnic Institute, USA

Xiadong Xu, Univ. of Washington, USA
FS 5: Nonlinear Optics and Novel Phenomena

J. Stewart Aitchison, Univ. of Toronto, Canada, Subcommittee Chair

Matteo Clerici, Univ. of Glasgow, UK

Mercedeh Khajavikhan, CREOL, Univ. of Central Florida, USA

Zhiyuan Li, Chinese Academy of Sciences, China

David J. Moss, Swinburne Univ. of Technology, Australia

Alessia Pasquazi, INRS, Canada

Ulf Peschel, Friedrich-Schiller-Universität Jena, Germany

Mikael C. Rechtsman, The Pennsylvania State Univ., USA

Venugopal Rao Soma, Univ. of Hyderabad, INDIA

Frank W. Wise, Cornell Univ., USA

Jianke Yang, Univ. of Vermont, USA

Kresten Yvind, Danmarks Tekniske Universitet, Denmark

\section{FS 6: Nano-Optics and Plasmonics}

Henri Lezec, NIST, USA, Subcommittee Chair

Amit Agrawal, NIST, USA

Palash Bharadwaj, Rice Univ., USA

Richard Blaikie, Univ. of Otago, New Zealand

Jennifer Dionne, Stanford Univ., USA

Xiaobo Yin, Univ. of Colorado Boulder, USA

Nanfang Yu, Columbia Univ., USA

Rashid Zia, Brown Univ., USA

Wei Zhou, Virginia Tech Univ., USA

\section{FS 7: High-Field Physics and Attoscience}

François Légaré, INRS-Energie Mat \& Tele Site Varennes, Canada, Subcommittee Chair

Michael Chini, Univ. of Central Florida, USA

Oren Cohen, Technion Israel Institute of Technology, Israel

Matthias Fuchs, Univ. of Nebraska Lincoln, USA

Shambhu Ghimire, SLAC/Stanford Univ., USA

Nobuhisa Ishii, Institute for Solid State Physics, Japan

Johan Mauritsson, Lund Univ., Sweden

Mauro Nisoli, Politecnico di Milano, Italy

Markus Roth, Technische Universität Darmstadt, Germany

Artem Rudenko, Kansas State Univ., USA

Olga Smirnova, Max Born Institute, Germany

Xiaoming Wang, Washington State Univ., USA

Amelle Zaïr, Imperial College London, UK

\section{CLEO Steering Committee}

\section{The Optical Society}

Craig Arnold, Princeton Univ., USA

Timothy J. Carrig, Lockheed Martin Coherent Technologies, USA

Ingmar Hartl, DESY, Germany

Yurii Vlasov, IBM TJ Watson Research Center, USA

Jonathan Zuegel, Lab for Laser Energetics, Univ. of Rochester, USA

\section{IEEE/Photonics Society}

Ann Catrina Coleman, Univ. of Texas at Dallas, USA, Chair

Seth Bank, Univ. of Texas at Austin, USA

Kent Choquette, Univ. of Illinois at Urbana-Champaign, USA

Peter Smowton, Cardiff Univ., UK

Weidong Zhou, Univ. of Texas at Arlington, USA 


\section{APS/Division of Laser Science}

Nicholas Bigelow, Univ. of Rochester, USA

Rohit Prasankumar, Los Alamos National Lab, USA

\section{Exhibitor Representative}

Mark Tolbert, Toptica Photonics Inc., USA

\section{Ex-Officio}

Peter Andersen, Danmarks Tekniske Universitet, Denmark Stewart Aichison, Univ. of Toronto, Canada

Sterling J. Backus, Kapteyn-Murnane Labs., USA

Ben Eggleton, Univ. of Sydney, Australia

Amr Helmy, Univ. of Toronto, Canada

Nicusor Iftimia, Physical Sciences Inc., USA

Wilhelm G. Kaenders, Toptica Photonics Inc., Germany

Jin Kang, Johns Hopkins Univ., USA

Junichiro Kono, Rice Univ., USA

Michal Lipson, Columbia Univ., USA

Michael M. Mielke, TRUMPF Inc., USA

Nathan Newbury, NIST, USA

Irina Novikova, College of William \& Mary, USA

Todd Pittman, Univ. of Maryland Baltimore County, USA

Siddharth Ramachandran, Boston Univ., USA

Jessie Rosenberg, IBM TJ Watson Research Center, USA

Jeff Shapiro, MIT, USA

Dirk H. Sutter, TRUMPF Laser GmbH, Germany

Yurii A. Vlasov, Univ. of Illinois at Urbana-Champaign, USA

Christian Wetzel, Rensselaer Polytechnic Inst., USA

Shinji Yamashita, Univ. of Tokyo, Japan

\section{CLEO Budget Committee}

Craig Arnold, Princeton Univ., USA

Joe Haus, Univ. of Dayton, USA

Kent Choquette, Univ. of Illinois at Urbana-Champaign, USA

Chris Jannuzzi, IEEE Photonics Society, USA

Kate Kirby, American Physical Society, USA

Elizabeth A. Rogan, The Optical Society, USA

Peter Delfyett, Jr., Univ. of Central Florida/CREOL, USA

\section{Joint Council on Applications}

Eric Mottay, Amplitude Systemes, France, Chair

Wilhelm G. Kaenders, Toptica Photonics Inc, Germany

Yu Chen, Univ. of Maryland at College Park, USA

Amy Eskilson, Inrad Optics, USA

Peter Fendel, Thorlabs Inc., USA

Klause Klein, Coherent, Inc., USA

Tyler Morgus, Thorlabs Inc., USA

Rick Plympton, Optimax Systems, USA

Mark Tolbert, Toptica Photonics, USA

Chris Wood, Insight Photonic Solutions, USA 


\section{Agenda of Sessions - Sunday, 14 May}

\begin{tabular}{|c|c|}
\hline $\begin{array}{l}07: 30- \\
17: 30\end{array}$ & Registration, Concourse Level \\
\hline $\begin{array}{l}08: 30- \\
12: 30\end{array}$ & $\begin{array}{l}\text { SC149: Foundations of Nonlinear Optics } \\
\text { SC221: Nano Photonics: Physics and Techniques } \\
\text { SC361: Coherent Midlnfrared Sources and Applications }\end{array}$ \\
\hline $\begin{array}{l}08: 30- \\
15: 00\end{array}$ & SC456: How to Start A Company \\
\hline $\begin{array}{l}13: 00- \\
16: 30\end{array}$ & Cheeky Scientist Workshops, Winchester Room/ Hilton \\
\hline $\begin{array}{l}13: 30- \\
16: 30\end{array}$ & $\begin{array}{l}\text { SC439: Attosecond Optics } \\
\text { SC403: NanoCavity Quantum Electrodynamics and Applications }\end{array}$ \\
\hline $\begin{array}{l}13: 30- \\
17: 30\end{array}$ & $\begin{array}{l}\text { SC157: Laser Beam Analysis, Propagation, and Shaping Techniques } \\
\text { SC396: Frontiers of Guided Wave Nonlinear Optics }\end{array}$ \\
\hline
\end{tabular}

\section{Explanation of Session/Presentation Codes}

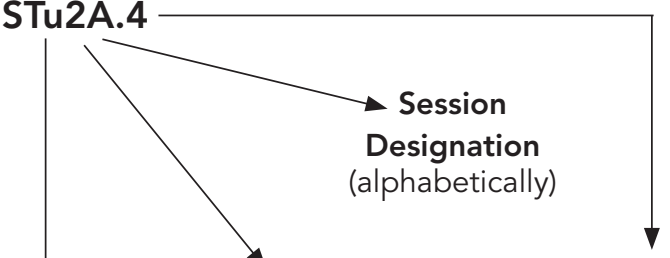

Meeting Name

$\mathrm{A}=$ Applications and Technology

$\mathrm{F}=$ Fundamental Science

$\mathrm{S}=$ Science \& Innovations

$\mathrm{J}=$ Joint

\section{Series Number}

$1=$ First Series of Sessions

$2=$ Second Series of Sessions

$3=$ Third Series of Sessions

$4=$ Fourth Series of Sessions
Number (presentation order within the session)

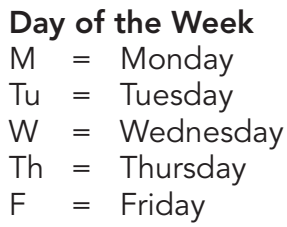

The first letter of the code designates the meeting (For instance, A=Applications \& Technology, $\mathrm{F}=$ Fundamental Science, $\mathrm{S}=$ Science and Innovations, J=Joint). The second element denotes the day of the week (Monday=M, Tuesday=Tu, Wednesday=W, Thursday=Th, Friday=F). The third element indicates the session series in that day (for instance, 2 would denote the second parallel sessions in that day). Each series of sessions begins with the letter $A$ in the fourth element and continues alphabetically through a series of parallel sessions. The number on the end of the code (separated from the session code with a period) signals the position of the talk within the session (first, second, third, etc.). For example, a presentation coded SM2A.4 indicates that this paper is part of Science and Innovations (S) and is being presented on Monday (M) in the second series of sessions (2), and is the first parallel session (A) in that series and the fourth paper (4) presented in that session. 


\section{Agenda of Sessions - Monday, 15 May}

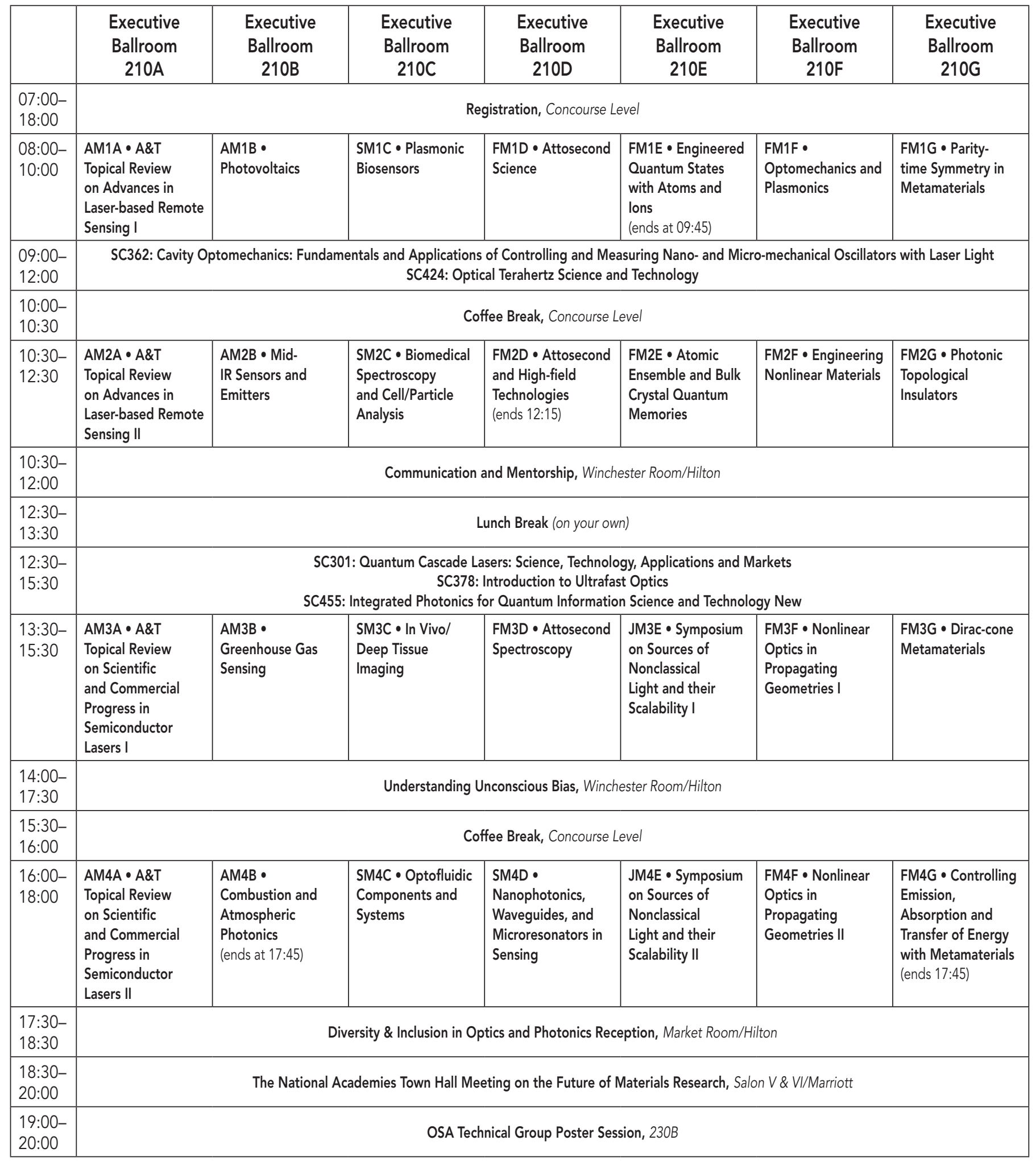




\begin{tabular}{|c|c|c|c|c|c|c|c|}
\hline $\begin{array}{c}\text { Executive } \\
\text { Ballroom } \\
210 \mathrm{H}\end{array}$ & $\begin{array}{l}\text { Meeting } \\
\text { Room } \\
211 \mathrm{~B} / \mathrm{D}\end{array}$ & $\begin{array}{l}\text { Meeting } \\
\text { Room } \\
212 \mathrm{~A} / \mathrm{C}\end{array}$ & $\begin{array}{l}\text { Meeting } \\
\text { Room } \\
212 \mathrm{~B} / \mathrm{D}\end{array}$ & $\begin{array}{l}\text { Marriott, } \\
\text { Salon } \\
\text { I \& II }\end{array}$ & $\begin{array}{l}\text { Marriott, } \\
\text { Salon } \\
\text { III }\end{array}$ & $\begin{array}{l}\text { Marriott, } \\
\text { Salon } \\
\text { IV }\end{array}$ & $\begin{array}{c}\text { Marriott, } \\
\text { Salon } \\
\text { V \& VI }\end{array}$ \\
\hline $\begin{array}{l}\text { FM1H • Plasmonic } \\
\text { and Dielectric } \\
\text { Metasurfaces and } \\
\text { Metamaterials }\end{array}$ & $\begin{array}{l}\text { SM1I • Ultrafast } \\
\text { Modelocked } \\
\text { Oscillators }\end{array}$ & $\begin{array}{l}\mathrm{SM1J} \bullet \mathrm{THz} \\
\text { Photonic } \\
\text { (ends 09:45) }\end{array}$ & $\begin{array}{l}\text { SM1K・Materials } \\
\text { for Quantum } \\
\text { Optics }\end{array}$ & $\begin{array}{l}\text { SM1L・High- } \\
\text { power, High- } \\
\text { energy Fiber } \\
\text { Sources }\end{array}$ & $\begin{array}{l}\text { SM1M • } \\
\text { Integrated } \\
\text { Nonlinear } \\
\text { Photonic } \\
\text { Platforms }\end{array}$ & $\begin{array}{l}\text { SM1N • High-Q } \\
\text { Micro-cavities and } \\
\text { Applications }\end{array}$ & $\begin{array}{l}\text { SM10 • RF } \\
\text { Photonics }\end{array}$ \\
\hline \multicolumn{8}{|c|}{$\begin{array}{l}\text { SC362: Cavity Optomechanics: Fundamentals and Applications of Controlling and Measuring Nano- and Micro-mechanical Oscillators with Laser Light } \\
\text { SC424: Optical Terahertz Science and Technology }\end{array}$} \\
\hline \multicolumn{8}{|c|}{ Coffee Break, Concourse Level } \\
\hline \multicolumn{8}{|c|}{ Communication and Mentorship, Winchester Room/Hilton } \\
\hline \multicolumn{8}{|c|}{ Lunch Break (on your own) } \\
\hline \multicolumn{8}{|c|}{$\begin{array}{l}\text { SC301: Quantum Cascade Lasers: Science, Technology, Applications and Markets } \\
\text { SC378: Introduction to Ultrafast Optics } \\
\text { SC455: Integrated Photonics for Quantum Information Science and Technology New }\end{array}$} \\
\hline $\begin{array}{l}\text { FM3H • Electron } \\
\text { - Plasmon } \\
\text { Interactions }\end{array}$ & $\begin{array}{l}\text { SM3I • Ultrafast } \\
\text { Amplifiers }\end{array}$ & $\begin{array}{l}\mathrm{SM} 3 \mathrm{~J} \cdot \mathrm{THz} \\
\text { Communications }\end{array}$ & $\begin{array}{l}\text { SM3K • Silicon } \\
\text { Photonics I }\end{array}$ & $\begin{array}{l}\text { SM3L } \bullet \text { Mode } \\
\text { Locked Fiber } \\
\text { Lasers I }\end{array}$ & $\begin{array}{l}\mathrm{SM} 3 \mathrm{M} \cdot \text { Progress } \\
\text { in Optical } \\
\text { Frequency } \\
\text { Conversion }\end{array}$ & $\begin{array}{l}\text { SM3N } \\
\text { Plasmonics and } \\
\text { Metamaterials }\end{array}$ & $\begin{array}{l}\text { SM3O } \\
\text { Integrated } \\
\text { Quantum } \\
\text { Photonics }\end{array}$ \\
\hline \multicolumn{8}{|c|}{ Coffee Break, Concourse Level } \\
\hline $\begin{array}{l}\text { FM4H } \bullet \text { Chip-scale } \\
\text { Plasmonic Devices }\end{array}$ & $\begin{array}{l}\text { SM4I • Ultrafast } \\
\text { Pulse Combining } \\
\text { and Manipulation }\end{array}$ & $\begin{array}{l}\mathrm{SM} 4 \mathrm{~J} \cdot \mathrm{THz} \mathrm{QCLS} \\
\text { and Imaging }\end{array}$ & $\begin{array}{l}\text { SM4K • Resonant } \\
\text { Optics }\end{array}$ & $\begin{array}{l}\text { SM4L } \cdot \text { Mode } \\
\text { Locked Fiber } \\
\text { Lasers II }\end{array}$ & $\begin{array}{l}\text { SM4M } \cdot \text { Optical } \\
\text { Parametric } \\
\text { Oscillators }\end{array}$ & $\begin{array}{l}\text { SM4N } \bullet \text { Prevoskite } \\
\text { and Photonic } \\
\text { Crystal Lasers }\end{array}$ & $\begin{array}{l}\text { SM40 • } \\
\text { Heterogeneously } \\
\text { Integrated Si } \\
\text { Photonics } \\
\text { (ends 17:45) }\end{array}$ \\
\hline \multicolumn{8}{|c|}{ Diversity \& Inclusion in Optics and Photonics Reception, Market Room/Hilton } \\
\hline \multicolumn{8}{|c|}{ The National Academies Town Hall Meeting on the Future of Materials Research, Salon V \& VI/Marriott } \\
\hline \multicolumn{8}{|c|}{ OSA Technical Group Poster Session, 230B } \\
\hline
\end{tabular}




\section{Agenda of Sessions - Tuesday, 16 May}

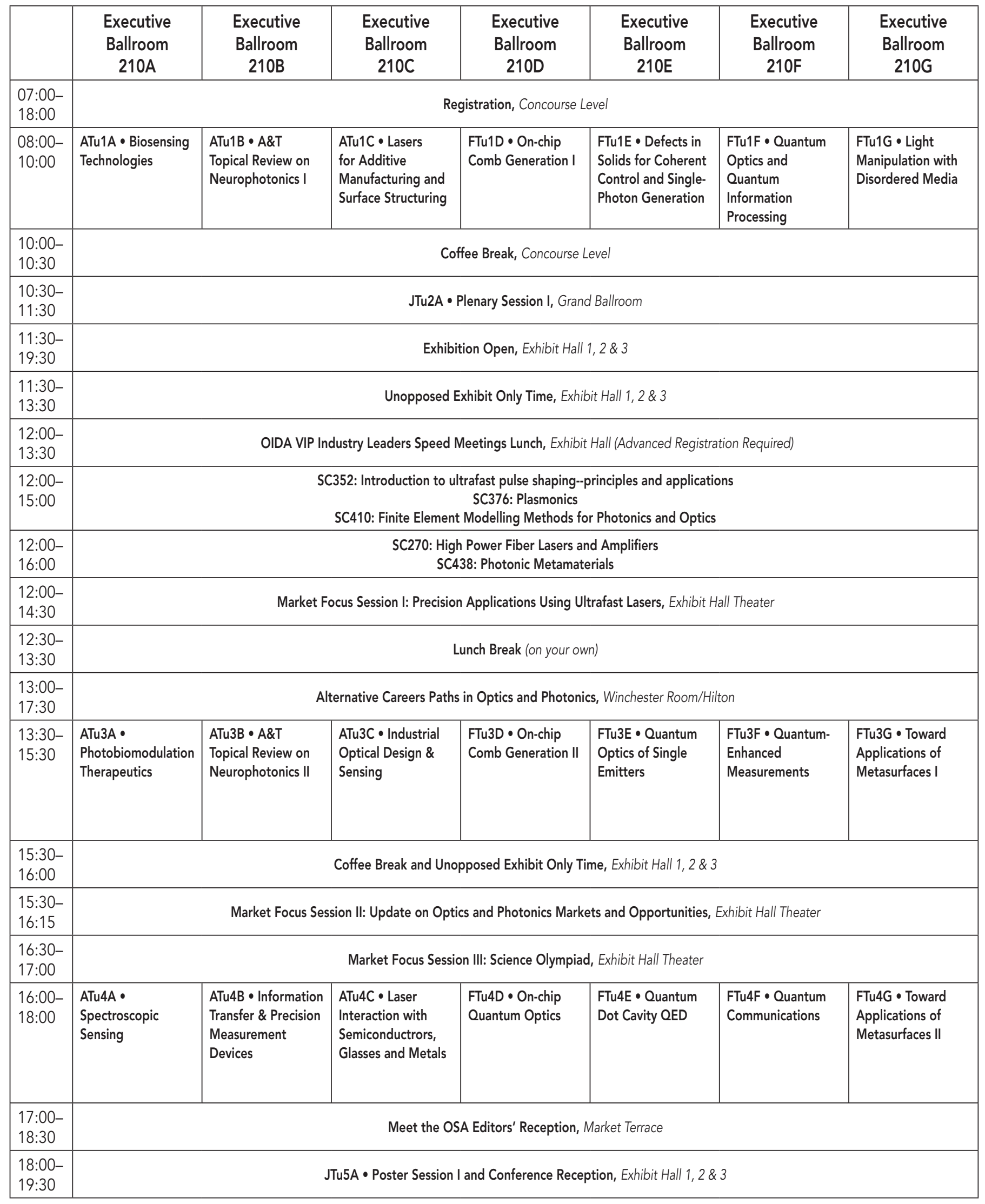




\begin{tabular}{|c|c|c|c|c|c|c|c|}
\hline $\begin{array}{l}\text { Executive } \\
\text { Ballroom } \\
210 \mathrm{H}\end{array}$ & $\begin{array}{l}\text { Meeting } \\
\text { Room } \\
211 \mathrm{~B} / \mathrm{D}\end{array}$ & $\begin{array}{l}\text { Meeting } \\
\text { Room } \\
212 \mathrm{~A} / \mathrm{C}\end{array}$ & $\begin{array}{l}\text { Meeting } \\
\text { Room } \\
212 \mathrm{~B} / \mathrm{D}\end{array}$ & $\begin{array}{l}\text { Marriott, } \\
\text { Salon } \\
\text { | \& || }\end{array}$ & $\begin{array}{l}\text { Marriott, } \\
\text { Salon } \\
\text { III }\end{array}$ & $\begin{array}{l}\text { Marriott, } \\
\text { Salon } \\
\text { IV }\end{array}$ & $\begin{array}{l}\text { Marriott, } \\
\text { Salon } \\
\text { V \& VI }\end{array}$ \\
\hline $\begin{array}{l}\text { FTu1H } \\
\text { Fundamental } \\
\text { Plasmonic \& } \\
\text { Nanophotonic } \\
\text { Effects }\end{array}$ & $\begin{array}{l}\text { STu1I • Ultrafast } \\
\text { Applications }\end{array}$ & $\begin{array}{l}\text { STu1J・THz } \\
\text { Materials Science }\end{array}$ & $\begin{array}{l}\text { STu1K・Mid-IR } \\
\text { Fiber Sensors }\end{array}$ & & $\begin{array}{l}\text { STu1M } \bullet \text { Optical } \\
\text { Interconnect } \\
\text { Systems } \\
\text { (ends at 09:45) }\end{array}$ & $\begin{array}{l}\text { STu1N • } \\
\text { Photodetectors }\end{array}$ & $\begin{array}{l}\text { STu10 • Petawatt } \\
\text { Laser Technology }\end{array}$ \\
\hline \multicolumn{8}{|c|}{ Coffee Break, Concourse Level } \\
\hline \multicolumn{8}{|c|}{ JTu2A • Plenary Session I, Grand Ballroom } \\
\hline \multicolumn{8}{|c|}{ Unopposed Exhibit Only Time, Exhibit Hall 1, 2 \& 3} \\
\hline \multicolumn{8}{|c|}{ OIDA VIP Industry Leaders Speed Meetings Lunch, Exhibit Hall (Advanced Registration Required) } \\
\hline \multicolumn{8}{|c|}{$\begin{array}{l}\text { SC352: Introduction to ultrafast pulse shaping--principles and applications } \\
\text { SC376: Plasmonics } \\
\text { SC410: Finite Element Modelling Methods for Photonics and Optics }\end{array}$} \\
\hline \multicolumn{8}{|c|}{$\begin{array}{l}\text { SC270: High Power Fiber Lasers and Amplifiers } \\
\text { SC438: Photonic Metamaterials }\end{array}$} \\
\hline \multicolumn{8}{|c|}{ Market Focus Session I: Precision Applications Using Ultrafast Lasers, Exhibit Hall Theater } \\
\hline \multicolumn{8}{|c|}{ Coffee Break and Unopposed Exhibit Only Time, Exhibit Hall 1, 2 \& 3} \\
\hline \multicolumn{8}{|c|}{ Market Focus Session II: Update on Optics and Photonics Markets and Opportunities, Exhibit Hall Theater } \\
\hline \multicolumn{8}{|c|}{ Market Focus Session III: Science Olympiad, Exhibit Hall Theater } \\
\hline $\begin{array}{l}\text { FTu4H } \bullet \\
\text { Functional } \\
\text { Plasmonics with } \\
\text { 2D and Novel } \\
\text { Materials }\end{array}$ & $\begin{array}{l}\text { STu4I • Ultrafast } \\
\text { Metrology II }\end{array}$ & $\begin{array}{l}\text { STu4J• } \\
\text { Microcomb } \\
\text { Nonlinear Optical } \\
\text { Technology }\end{array}$ & $\begin{array}{l}\text { STu4K • OAM } \\
\& \text { Higher-Order } \\
\text { Mode Fibers } \\
\text { (ends at 17:45) }\end{array}$ & \begin{tabular}{|l|} 
JTu4L • \\
Symposium on \\
Ultrafast Laser \\
Technology \\
for X-ray Free \\
Electron Lasers II \\
\end{tabular} & $\begin{array}{l}\text { STu4M } \bullet \text { Nonlinear } \\
\text { Impairments } \\
\text { in Optical } \\
\text { Communications } \\
\text { (ends at 17:30) }\end{array}$ & $\begin{array}{l}\text { STu4N } \bullet \\
\text { Optomechanics }\end{array}$ & $\begin{array}{l}\text { STu4O • Spatial } \\
\text { and Temporal } \\
\text { Beam Control }\end{array}$ \\
\hline \multicolumn{8}{|c|}{ Meet the OSA Editors' Reception, Market Terrace } \\
\hline & & JTu5A • Post & ession I and Con & nce Reception, Ex & it Hall $1,2 \& 3$ & & \\
\hline
\end{tabular}




\section{Agenda of Sessions - Wednesday, 17 May}

\begin{tabular}{|c|c|c|c|c|c|c|c|}
\hline & $\begin{array}{l}\text { Executive } \\
\text { Ballroom } \\
210 \mathrm{~A}\end{array}$ & $\begin{array}{l}\text { Executive } \\
\text { Ballroom } \\
210 \mathrm{~B}\end{array}$ & $\begin{array}{l}\text { Executive } \\
\text { Ballroom } \\
210 \mathrm{C}\end{array}$ & $\begin{array}{l}\text { Executive } \\
\text { Ballroom } \\
\text { 210D }\end{array}$ & $\begin{array}{l}\text { Executive } \\
\text { Ballroom } \\
210 \mathrm{E}\end{array}$ & $\begin{array}{l}\text { Executive } \\
\text { Ballroom } \\
210 \mathrm{~F}\end{array}$ & $\begin{array}{l}\text { Executive } \\
\text { Ballroom } \\
210 \mathrm{G}\end{array}$ \\
\hline $\begin{array}{l}07: 30- \\
18: 00\end{array}$ & \multicolumn{7}{|c|}{ Registration, Concourse Level } \\
\hline $\begin{array}{l}08: 00- \\
10: 00\end{array}$ & $\begin{array}{l}\text { AW1A } \bullet \text { Medical } \\
\text { Devices and } \\
\text { Systems }\end{array}$ & $\begin{array}{l}\text { AW1B - Sensing in } \\
\text { Fibers and Free- } \\
\text { space }\end{array}$ & $\begin{array}{l}\text { SW1C } \cdot \text { Nanolasers } \\
\text { and Frequency } \\
\text { Combs }\end{array}$ & $\begin{array}{l}\text { FW1D • Non- } \\
\text { diffracting Beams }\end{array}$ & $\begin{array}{l}\text { FW1E • Rare } \\
\text { Earth Solid State } \\
\text { Quantum Memories }\end{array}$ & $\begin{array}{l}\text { FW1F • Quantum } \\
\text { Entaglement }\end{array}$ & $\begin{array}{l}\text { JW1G - Symposium } \\
\text { on Advances in } \\
\text { Metaphotonic } \\
\text { Devices I }\end{array}$ \\
\hline $\begin{array}{l}10: 00- \\
18: 30\end{array}$ & \multicolumn{7}{|c|}{ Exhibition Open, Exhibit Hall 1, 2 \& 3} \\
\hline $\begin{array}{l}10: 00- \\
12: 00\end{array}$ & \multicolumn{7}{|c|}{ JW2A • Poster Session II, Exhibit Hall 1, 2 \& 3} \\
\hline $\begin{array}{l}10: 00- \\
10: 30\end{array}$ & \multicolumn{7}{|c|}{ Coffee Break, Exhibit Hall 1, 2 \& 3} \\
\hline $\begin{array}{l}10: 00- \\
12: 45\end{array}$ & \multicolumn{7}{|c|}{$\begin{array}{l}\text { OSA Members, Family and Friends Tour - Computer History Museum } \\
\text { Shuttle transportation will depart from the Hilton's Almaden Avenue entrance at 10:15 (Advanced Registration Required) }\end{array}$} \\
\hline $\begin{array}{l}10: 30- \\
12: 00\end{array}$ & \multicolumn{7}{|c|}{ Market Focus Session IV: How the Changing Political Landscape will Impact your Company, Exhibit Hall Theater } \\
\hline $\begin{array}{l}12: 00- \\
13: 00\end{array}$ & \multicolumn{7}{|c|}{ Lunch and Unopposed Exhibit Only Time, Exhibit Hall 1, 2 \& 3 (concessions available) } \\
\hline $\begin{array}{l}13: 00- \\
15: 00\end{array}$ & \multicolumn{7}{|c|}{ JW3A • Plenary Session II and Awards, Grand Ballroom } \\
\hline $\begin{array}{l}15: 00- \\
15: 30\end{array}$ & \multicolumn{7}{|c|}{ Coffee Break and Unopposed Exhibit Only Time, Exhibit Hall 1, 2 \& 3} \\
\hline $\begin{array}{l}15: 00- \\
16: 30\end{array}$ & \multicolumn{7}{|c|}{ Market Focus Session V: Bright Ideas Competition, Exhibit Hall Theater } \\
\hline $\begin{array}{l}15: 30- \\
17: 30\end{array}$ & $\begin{array}{l}\text { AW4A } \bullet \text { Biomedical } \\
\text { Imaging I }\end{array}$ & $\begin{array}{l}\text { AW4B } \bullet \text { Lasers and } \\
\text { Applications } \\
\text { (ends at 17:15) }\end{array}$ & $\begin{array}{l}\text { SW4C } \\
\text { Semiconductor } \\
\text { Lasers on Silicon }\end{array}$ & $\begin{array}{l}\text { FW4D • Nonlinear } \\
\text { Optical Sources }\end{array}$ & $\begin{array}{l}\text { FW4E • } \\
\text { Quantum State } \\
\text { Generation and } \\
\text { Characterization }\end{array}$ & $\begin{array}{l}\text { FW4F • Quantum } \\
\text { States and } \\
\text { Sensing with } \\
\text { Optomechanical } \\
\text { Systems } \\
\text { (starts at 16:00) }\end{array}$ & $\begin{array}{l}\text { JW4G • Symposium } \\
\text { on Advances in } \\
\text { Metaphotonic } \\
\text { Devices II }\end{array}$ \\
\hline $\begin{array}{l}16: 30- \\
18: 30\end{array}$ & \multicolumn{7}{|c|}{ Happy Hour in Exhibit Halll, Exhibit Hall 1, 2 \& 3} \\
\hline $\begin{array}{l}18: 00- \\
19: 00\end{array}$ & \multicolumn{7}{|c|}{ OSA Nanophotonics Technical Group 20x20 Talks, Executive Ballroom 210A } \\
\hline
\end{tabular}




\begin{tabular}{|c|c|c|c|c|c|c|c|}
\hline $\begin{array}{l}\text { Executive } \\
\text { Ballroom } \\
210 \mathrm{H}\end{array}$ & $\begin{array}{l}\text { Meeting } \\
\text { Room } \\
211 \mathrm{~B} / \mathrm{D}\end{array}$ & $\begin{array}{l}\text { Meeting } \\
\text { Room } \\
212 \mathrm{~A} / \mathrm{C}\end{array}$ & $\begin{array}{l}\text { Meeting } \\
\text { Room } \\
212 \mathrm{~B} / \mathrm{D}\end{array}$ & $\begin{array}{l}\text { Marriott, } \\
\text { Salon } \\
\text { | \& || }\end{array}$ & $\begin{array}{l}\text { Marriott, } \\
\text { Salon } \\
\text { III }\end{array}$ & $\begin{array}{l}\text { Marriott, } \\
\text { Salon } \\
\text { IV }\end{array}$ & $\begin{array}{c}\text { Marriott, } \\
\text { Salon } \\
\text { V \& VI }\end{array}$ \\
\hline $\begin{array}{l}\text { FW1H • Extreme } \\
\text { Electromagnetic } \\
\text { Radiation-THZ to } \\
\text { EUV: Generation, } \\
\text { Detection \& } \\
\text { Applications }\end{array}$ & $\begin{array}{l}\text { SW1/• } \\
\text { Space-Division } \\
\text { Multiplexed } \\
\text { Optical } \\
\text { Communications } \\
\text { (ends at 09:45) }\end{array}$ & $\begin{array}{l}\text { SW1J • Precision } \\
\text { References and } \\
\text { Optical Synthesis }\end{array}$ & $\begin{array}{l}\text { SW1K } \bullet \text { Flexible } \\
\text { and Soft } \\
\text { Optoelectronics }\end{array}$ & $\begin{array}{l}\text { SW1L • Sensing } \\
\text { in Dynamic } \\
\text { and Extreme } \\
\text { Environments, } \\
\text { Plasmas, and } \\
\text { Explosions }\end{array}$ & $\begin{array}{l}\text { SW1M } \bullet \text { Nonlinear } \\
\text { Optics for } \\
\text { Spectroscopy and } \\
\text { Sensing }\end{array}$ & $\begin{array}{l}\text { SW1N・ Silicon } \\
\text { Photonic Devices } \\
\text { and Structures }\end{array}$ & \begin{tabular}{|l|} 
SW10 \\
Optical Comb \\
\& Integrated \\
Systems
\end{tabular} \\
\hline \multicolumn{8}{|c|}{ Exhibition Open, Exhibit Hall 1, 2 \& 3} \\
\hline \multicolumn{8}{|c|}{ JW2A • Poster Session II, Exhibit Hall 1, 2 \& 3} \\
\hline \multicolumn{8}{|c|}{$\begin{array}{l}\text { OSA Members, Family and Friends Tour - Computer History Museum } \\
\text { Shuttle transportation will depart from the Hilton's Almaden Avenue entrance at 10:15 (Advanced Registration Required) }\end{array}$} \\
\hline \multicolumn{8}{|c|}{ Market Focus Session IV: How the Changing Political Landscape will Impact your Company, Exhibit Hall Theater } \\
\hline \multicolumn{8}{|c|}{ Lunch and Unopposed Exhibit Only Time, Exhibit Hall 1,2 \& 3 (concessions available) } \\
\hline \multicolumn{8}{|c|}{ JW3A • Plenary Session II and Awards, Grand Ballroom } \\
\hline \multicolumn{8}{|c|}{ Coffee Break and Unopposed Exhibit Only Time, Exhibit Hall 1, 2 \& 3} \\
\hline \multicolumn{8}{|c|}{ Happy Hour in Exhibit Halll, Exhibit Hall 1,2 \& 3} \\
\hline \multicolumn{8}{|c|}{ OSA Nanophotonics Technical Group 20x20 Talks, Executive Ballroom 210A } \\
\hline
\end{tabular}




\section{Agenda of Sessions - Thursday, 18 May}

\begin{tabular}{|c|c|c|c|c|c|c|c|}
\hline & $\begin{array}{l}\text { Executive } \\
\text { Ballroom } \\
210 \mathrm{~A}\end{array}$ & $\begin{array}{l}\text { Executive } \\
\text { Ballroom } \\
210 B\end{array}$ & $\begin{array}{l}\text { Executive } \\
\text { Ballroom } \\
210 \mathrm{C}\end{array}$ & $\begin{array}{l}\text { Executive } \\
\text { Ballroom } \\
\text { 210D }\end{array}$ & $\begin{array}{l}\text { Executive } \\
\text { Ballroom } \\
210 E\end{array}$ & $\begin{array}{l}\text { Executive } \\
\text { Ballroom } \\
210 F\end{array}$ & $\begin{array}{l}\text { Executive } \\
\text { Ballroom } \\
210 G\end{array}$ \\
\hline $\begin{array}{l}07: 30- \\
18: 30\end{array}$ & \multicolumn{7}{|c|}{ Registration, Concourse Level } \\
\hline $\begin{array}{l}08: 00- \\
10: 00\end{array}$ & $\begin{array}{l}\text { ATh1A } \bullet \text { Biomedical } \\
\text { Imaging II }\end{array}$ & $\begin{array}{l}\text { ATh1B • } \\
\text { Active Remote } \\
\text { Environmental } \\
\text { Sensing }\end{array}$ & STh1C・III-V Lasers & $\begin{array}{l}\text { FTh1D } \bullet \text { Topological } \\
\text { Photonics }\end{array}$ & $\begin{array}{l}\text { JTh1E • Quantum } \\
\text { Photonics I }\end{array}$ & $\begin{array}{l}\text { FTh1F • Ultrafast } \\
\text { Exciton Dynamics } \\
\text { in Van Der Waals } \\
\text { Materials }\end{array}$ & $\begin{array}{l}\text { FTh1G • Nonlinear } \\
\text { and Hyperbolic } \\
\text { Metamaterials }\end{array}$ \\
\hline $\begin{array}{l}10: 00- \\
15: 00\end{array}$ & \multicolumn{7}{|c|}{ Exhibition Open, Exhibit Hall 1, 2 \& 3} \\
\hline $\begin{array}{l}10: 00- \\
11: 30\end{array}$ & \multicolumn{7}{|c|}{ Coffee Break (10:00-10:30) and Unopposed Exhibit Only Time, Exhibit Hall 1, 2 \& 3} \\
\hline $\begin{array}{l}10: 00- \\
12: 00\end{array}$ & \multicolumn{7}{|c|}{ JTh2A • Poster Session III, Exhibit Hall 1, 2 \& 3} \\
\hline $\begin{array}{l}10: 15- \\
13: 00\end{array}$ & \multicolumn{7}{|c|}{ Technology Transfer Program, Exhibit Hall Theater } \\
\hline $\begin{array}{l}11: 30- \\
13: 30\end{array}$ & \multicolumn{7}{|c|}{ Pizza Lunch, Exhibit Hall 1, 2 \& 3} \\
\hline $\begin{array}{l}14: 00- \\
16: 00\end{array}$ & $\begin{array}{l}\text { JTh3A } \cdot \text { Symposium } \\
\text { on Multimodal } \\
\text { Imaging in } \\
\text { Biophotonics I }\end{array}$ & $\begin{array}{l}\text { ATh3B • Optical } \\
\text { Devices \& } \\
\text { Components }\end{array}$ & $\begin{array}{l}\text { ATh3C • A\&T } \\
\text { Topical Review on } \\
\text { Extreme Ultraviolet } \\
\text { and Soft X-ray } \\
\text { Sources and } \\
\text { Application I }\end{array}$ & $\begin{array}{l}\text { FTh3D • PT } \\
\text { Symmetry and } \\
\text { Beyond }\end{array}$ & $\begin{array}{l}\text { JTh3E • Quantum } \\
\text { Photonics II }\end{array}$ & $\begin{array}{l}\text { FTh3F • Ultrafast } \\
\text { Lattice and } \\
\text { Molecular Dynamics }\end{array}$ & $\begin{array}{l}\text { JTh3G • Symposium } \\
\text { on Optomechanics: } \\
\text { Towards the } \\
\text { Second Quantum } \\
\text { Revolution I }\end{array}$ \\
\hline $\begin{array}{l}16: 00- \\
16: 30\end{array}$ & \multicolumn{7}{|c|}{ Coffee Break, Concourse Level } \\
\hline $\begin{array}{l}16: 30- \\
18: 30\end{array}$ & $\begin{array}{l}\text { JTh4A • Symposium } \\
\text { on Multimodal } \\
\text { Imaging in } \\
\text { Biophotonics II }\end{array}$ & $\begin{array}{l}\text { ATh4B • Process } \\
\text { Evaluation \& } \\
\text { Microscopy }\end{array}$ & $\begin{array}{l}\text { ATh4C • A\&T } \\
\text { Topical Review on } \\
\text { Extreme Ultraviolet } \\
\text { and Soft X-ray } \\
\text { Sources and } \\
\text { Application II }\end{array}$ & $\begin{array}{l}\text { FTh4D • Solitons } \\
\text { and Temporal Wave } \\
\text { Guiding }\end{array}$ & $\begin{array}{l}\text { FTh4E • Single- } \\
\text { Photon Sources } \\
\text { and Quantum } \\
\text { Communications }\end{array}$ & $\begin{array}{l}\text { FTh4F • Imaging } \\
\text { Electron Dynamics } \\
\text { on the Nano-, } \\
\text { Femto- Scale }\end{array}$ & $\begin{array}{l}\text { JTh4G • Symposium } \\
\text { on Optomechanics: } \\
\text { Towards the } \\
\text { Second Quantum } \\
\text { Revolution II }\end{array}$ \\
\hline $\begin{array}{l}18: 30- \\
20: 00\end{array}$ & \multicolumn{7}{|c|}{ Dinner Break (on your own) } \\
\hline $\begin{array}{l}20: 00- \\
22: 00\end{array}$ & \multicolumn{7}{|c|}{ Postdeadline Paper Sessions } \\
\hline
\end{tabular}




\begin{tabular}{|c|c|c|c|c|c|c|c|}
\hline $\begin{array}{c}\text { Executive } \\
\text { Ballroom } \\
210 \mathrm{H}\end{array}$ & $\begin{array}{l}\text { Meeting } \\
\text { Room } \\
211 \mathrm{~B} / \mathrm{D}\end{array}$ & $\begin{array}{l}\text { Meeting } \\
\text { Room } \\
212 \mathrm{~A} / \mathrm{C}\end{array}$ & $\begin{array}{l}\text { Meeting } \\
\text { Room } \\
212 \text { B/D }\end{array}$ & $\begin{array}{l}\text { Marriott, } \\
\text { Salon } \\
\text { | \& II }\end{array}$ & $\begin{array}{l}\text { Marriott, } \\
\text { Salon } \\
\text { III }\end{array}$ & $\begin{array}{l}\text { Marriott, } \\
\text { Salon } \\
\text { IV }\end{array}$ & $\begin{array}{l}\text { Marriott, } \\
\text { Salon } \\
\text { V \& VI }\end{array}$ \\
\hline \multicolumn{8}{|c|}{ Registration, Concourse Level } \\
\hline $\begin{array}{l}\text { FTh1H } \bullet \\
\text { Nanoscale } \\
\text { Optomechanics }\end{array}$ & $\begin{array}{l}\text { STh1I • 2D } \\
\text { Materials and } \\
\text { Devices II }\end{array}$ & $\begin{array}{l}\text { STh1J・ Laser } \\
\text { Ablation } \\
\text { Fundamentals and } \\
\text { Applications }\end{array}$ & $\begin{array}{l}\text { STh1K • Nonlinear } \\
\text { Fiber Photonics I }\end{array}$ & $\begin{array}{l}\text { STh1L • High } \\
\text { Average Power } \\
\text { Lasers }\end{array}$ & $\begin{array}{l}\text { STh1M • Phased } \\
\text { Arrays Related } \\
\text { Device }\end{array}$ & $\begin{array}{l}\text { STh1N • Optical } \\
\text { Computing \& } \\
\text { Communications } \\
\text { using Photonic } \\
\text { Nanostructures }\end{array}$ & $\begin{array}{l}\text { STh10 • Direct } \\
\text { Detection } \\
\text { Multicarrier } \\
\text { Optical } \\
\text { Communications }\end{array}$ \\
\hline \multicolumn{8}{|c|}{ Exhibition Open, Exhibit Hall 1, 2 \& 3} \\
\hline \multicolumn{8}{|c|}{ Coffee Break (10:00-10:30) and Unopposed Exhibit Only Time, Exhibit Hall 1, 2 \& 3} \\
\hline \multicolumn{8}{|c|}{ Pizza Lunch, Exhibit Hall 1, 2 \& 3} \\
\hline $\begin{array}{l}\mathrm{FTh} 3 \mathrm{H} \bullet \text { Photonics } \\
\text { Crystals for Light } \\
\text { Manipulation and } \\
\text { Concentration }\end{array}$ & $\begin{array}{l}\text { STh3I • Quantum } \\
\text { Confined Materials } \\
\text { \& Devices }\end{array}$ & $\begin{array}{l}\text { STh3J • Ultrafast } \\
\text { Laser-Material } \\
\text { Interactions }\end{array}$ & $\begin{array}{l}\text { STh3K • Nonlinear } \\
\text { Fiber Photonics II }\end{array}$ & $\begin{array}{l}\text { STh3L • Dual } \\
\text { Frequency Comb } \\
\text { Techniques }\end{array}$ & $\begin{array}{l}\text { JTh3M • } \\
\text { Symposium } \\
\text { on Optical } \\
\text { Microcavities for } \\
\text { Ultrasensitive } \\
\text { Detection I }\end{array}$ & $\begin{array}{l}\text { STh3N • Light } \\
\text { Emitters and } \\
\text { Lasers }\end{array}$ & $\begin{array}{l}\text { STh30 • Free- } \\
\text { Space Optical } \\
\text { Communications }\end{array}$ \\
\hline \multicolumn{8}{|c|}{ Coffee Break, Concourse Level } \\
\hline \multicolumn{8}{|c|}{ Dinner Break (on your own) } \\
\hline \multicolumn{8}{|c|}{ Postdeadline Paper Sessions } \\
\hline
\end{tabular}




\section{Agenda of Sessions - Friday, 19 May}

\begin{tabular}{|c|c|c|c|c|c|c|}
\hline & $\begin{array}{l}\text { Executive } \\
\text { Ballroom } \\
210 \mathrm{~A}\end{array}$ & $\begin{array}{l}\text { Executive } \\
\text { Ballroom } \\
210 \mathrm{~B}\end{array}$ & $\begin{array}{l}\text { Executive } \\
\text { Ballroom } \\
210 \mathrm{C}\end{array}$ & $\begin{array}{l}\text { Executive } \\
\text { Ballroom } \\
\text { 210D }\end{array}$ & $\begin{array}{l}\text { Executive } \\
\text { Ballroom } \\
210 E\end{array}$ & $\begin{array}{l}\text { Executive } \\
\text { Ballroom } \\
210 F\end{array}$ \\
\hline $\begin{array}{l}07: 30- \\
12: 00\end{array}$ & \multicolumn{6}{|c|}{ Registration, Concourse Level } \\
\hline $\begin{array}{l}08: 00- \\
10: 00\end{array}$ & $\begin{array}{l}\text { AF1A } \bullet \text { A\&T } \\
\text { Topical Review on } \\
\text { Supercontinuum and } \\
\text { Applications I }\end{array}$ & $\begin{array}{l}\text { AF1B - Application \& } \\
\text { Advances of Frequency } \\
\text { Combs }\end{array}$ & $\begin{array}{l}\text { SF1C } \bullet \text { Frequency } \\
\text { Comb Technology }\end{array}$ & $\begin{array}{l}\text { JF1D • Symposium } \\
\text { on Thermal Noise } \\
\text { in Precision } \\
\text { Interferometry I }\end{array}$ & $\begin{array}{l}\text { FF1E - Single-Photon } \\
\text { Detectors }\end{array}$ & $\begin{array}{l}\text { FF1F • Optical \& } \\
\text { THZ Spectroscopy of } \\
\text { Quantum Matter }\end{array}$ \\
\hline $\begin{array}{l}10: 00- \\
10: 30\end{array}$ & \multicolumn{6}{|c|}{ Coffee Break, Concourse Level } \\
\hline $\begin{array}{l}10: 30- \\
12: 30\end{array}$ & $\begin{array}{l}\text { AF1A } \bullet A \& T \\
\text { Topical Review on } \\
\text { Supercontinuum and } \\
\text { Applications II }\end{array}$ & $\begin{array}{l}\text { AF2B • Applied } \\
\text { Spectroscopy }\end{array}$ & $\begin{array}{l}\text { SF2C } \cdot \text { Frequency } \\
\text { Comb Applications }\end{array}$ & $\begin{array}{l}\text { JF2D • Symposium } \\
\text { on Thermal Noise } \\
\text { in Precision } \\
\text { Interferometry II }\end{array}$ & $\begin{array}{l}\text { FF2E • Quantum } \\
\text { Technologies }\end{array}$ & $\begin{array}{l}\text { FF2F • Valley } \\
\text { Coherence and } \\
\text { Polariton Dynamics in } \\
\text { 2D Materials }\end{array}$ \\
\hline
\end{tabular}




\begin{tabular}{|c|c|c|c|c|c|c|}
\hline $\begin{array}{l}\text { Executive } \\
\text { Ballroom } \\
210 \mathrm{G}\end{array}$ & $\begin{array}{l}\text { Executive } \\
\text { Ballroom } \\
210 \mathrm{H}\end{array}$ & $\begin{array}{l}\text { Meeting } \\
\text { Room } \\
211 \mathrm{~B} / \mathrm{D}\end{array}$ & $\begin{array}{l}\text { Meeting } \\
\text { Room } \\
212 \mathrm{~A} / \mathrm{C}\end{array}$ & $\begin{array}{l}\text { Meeting } \\
\text { Room } \\
212 \mathrm{~B} / \mathrm{D}\end{array}$ & $\begin{array}{l}\text { Marriott, } \\
\text { Salon } \\
\text { | \& I| }\end{array}$ & $\begin{array}{l}\text { Marriott, } \\
\text { Salon } \\
\text { III }\end{array}$ \\
\hline $\begin{array}{l}\text { FF1G } • \text { Nanoparticle } \\
\text { Mediated } \\
\text { Emission and Field } \\
\text { Enhancement }\end{array}$ & $\begin{array}{l}\text { SF1H • Waveguides } \\
\text { and Ring Resonators }\end{array}$ & $\begin{array}{l}\text { SF1I } \bullet \text { Integrated } \\
\text { Photonic Devices }\end{array}$ & $\begin{array}{l}\text { SF1J • Micro- and } \\
\text { Nanophotonic } \\
\text { Devices }\end{array}$ & $\begin{array}{l}\text { SF1K • Laser Facilities } \\
\text { and Applications }\end{array}$ & $\begin{array}{l}\text { SF1L • Data Center } \\
\text { Communications } \\
\text { (ends at 09:30) }\end{array}$ & $\begin{array}{l}\mathrm{SF} 1 \mathrm{M} \cdot \text { Aerosol and } \\
\text { Gas Sensing }\end{array}$ \\
\hline \multicolumn{7}{|c|}{ Coffee Break, Concourse Level } \\
\hline $\begin{array}{l}\text { FF2G • Nanoscale } \\
\text { Control of Quantum } \\
\text { Emission }\end{array}$ & & $\begin{array}{l}\text { SF2I - Detectors and } \\
\text { Other Novel Devices }\end{array}$ & $\begin{array}{l}\text { SF2J・Surface } \\
\text { Emitting Lasers }\end{array}$ & $\begin{array}{l}\text { SF2K • Infrared Laser } \\
\text { Sources }\end{array}$ & $\begin{array}{l}\text { SF2L • Advanced } \\
\text { Fiber Devices and } \\
\text { Concepts } \\
\text { (ends at 12:15) }\end{array}$ & $\begin{array}{l}\mathrm{SF} 2 \mathrm{M} \cdot \text { Combustion } \\
\text { Diagnostics and } \\
\text { Imaging }\end{array}$ \\
\hline
\end{tabular}


Executive Ballroom 210A

Executive Ballroom 210B

\section{CLEO: Applications \& Technology}

08:00-10:00

AM1A • A\&T Topical Review on Advances in Laser-based Remote Sensing I

Presider: To be Determined

\section{AM1A.1 08:00 Invited}

Coherent Adaptive Optical System, Joseph Marron'; 'Raytheon, USA. An adaptive optical system in which coherent images are processed to determine wavefront error and the error is corrected by applying the inverse to a deformable mirror is discussed.

08:00-10:00

AM1B • Photovoltaics

Naval Research Lab, USA

AM1B. $1 \bullet 08: 00$

\section{AM1A.2 • 08:30 Invited}

Research Results, Lessons Learned and Future Perspective of Forward-looking LIDAR for Aircraft, Nikolaus P. Schmitt ${ }^{1} ;{ }^{1}$ Airbus Group Innovations, Germany. Forward-looking LIDAR measurements on-board aircraft have been realized. Results show potential for real-time anticipation of atmospheric disturbances and automated counter-maneuvering in the future. However, a multi-functiona design approach is required. Results, lessons and perspectives will be discussed.
Presider: Stephanie Tomasulo;

Extremely Cost-effective and Efficient Solar Vapor Generation Using Thermally Isolated Black Paper, Zhejun Liu ${ }^{1,2}$, Haomin Song ${ }^{1}$ Dengxin Ji', Chenyu Li', Alec Cheney', Youhai Liu', Nan Zhang' ', Xie Zeng', Borui Chen', Jun $\mathrm{Gao}^{2}$, Xiang Liư ${ }^{3}$, Diana Aga ${ }^{4}$, Suhua Jiang ${ }^{2}$ Zongfu Yu', Qiaogiang Gan'; 'Dept. of Electrical Engineering, The State Univ. of New York at Buffalo, USA; ${ }^{2}$ Material Science Dept. Fudan Univ., China; ${ }^{3}$ Dept. of Environmenta Science and Engineering, Fudan Univ., China; ${ }^{4}$ The State Univ. of New York at Buffalo, Dept. of Chemistry, USA; ${ }^{5}$ Dept. of Electrical and Computer Engineering, Univ. of Wisconsin, USA. We report an efficient strategy using extremely low-cost materials. Due to the excellent thermal insulation, a record thermal efficiency of $\sim 88 \%$ was obtained under one sun without concentration, corresponding to the evaporation rate of $1.28 \mathrm{~kg} /\left(\mathrm{m}^{2} \bullet \mathrm{h}\right)$.

AM1B.2 • 08:15

Broadband Polarization-Insensitive $\mathrm{Ab}$ sorption In Solar Spectrum Enhanced By Magnetic Polaritons, $\mathrm{Xu} \mathrm{Han}{ }^{2}$, Kebo $\mathrm{He}^{1}$ Ziqi Liü, Zhubing He ${ }^{3}$, Zhaoyu Zhang'; 'The Chinese Univ, of Hong Kong, Shenzhen, China: ${ }^{2}$ Peking Univ. Shenzhen Graduate School, China; ${ }^{3}$ South Univ. of Science and Technology of China, China. A broadband polarization-insensitive solar absorber with simple structure based on metamaterials is proposed. The excitation of SPPs, MPs and CMPs enables a wide plateau exceeding absorbance of $90 \%$, which is favorable for solar harvesting

AM1B.3 • 08:30

Highly Reproducible- Organometallic Halide Perovskite Microdevices Based on Top-Down Lithography, Nan Zhang ${ }^{1}$ Wenzhao Sun', Zhiyuan Gu', Wang Kaiyang ${ }^{1}$ Shuai Wang ${ }^{1}$, Wenshan $\mathrm{Cai}^{2}$, Shumin Xiao ${ }^{1}$ Qinghai Song1: 'State Key Lab on Tunable laser Technology, Ministry of Industry and Information Technology Key Lab of MicroNano Optoelectronic Information System, Shenzhen Graduate School, Harbin Inst. of Technology, China; ${ }^{2}$ School of Electrical and Computer Engineering, Georgia Inst. of Technology, USA. Herein we fabricate highly reproducible-organometallic-halideperovskite based devices, various device shapes that are hard to directly synthesize, unique properties and an improved photodetector have been successfully achieved. The advances will shed light on the practical applications.
Executive Ballroom

$210 \mathrm{C}$

\section{CLEO: Science \&} Innovations

08:00-10:00

SM1C • Plasmonic Biosensors

Presider: Nien-Tsu Huang;

National Taiwan Univ., Taiwan

\section{SM1C.1 08:00 Invited}

High-Speed Super-Resolution Microscopy for Biological Imaging, Anna Bezryadina ${ }^{1}$ Junxiang Zhao ${ }^{1}$, Joseph Ponsetto ${ }^{1}$, Yang $\mathrm{Xia}^{2}$, Xiang Zhang ${ }^{2}$, Zhaowei Liu'; ' ${ }^{1}$ Electri$\mathrm{cal}$ and Computer Engineering, Univ. of California, San Diego, USA; ${ }^{2}$ Physics, Univ. of California, Berkeley, USA. We used new super resolution imaging method, localized plasmonic structure illumination microscopy (LPSIM), to observe biological specimens movement. This approach allows biologically friendly video rate imaging with wide field of view and $50 \mathrm{~nm}$ resolution.

SM1C. $2 \bullet 08: 30$

Quantitative Label-free Imaging of Live-cell Adhesion Using Photonic Crystal Enhanced Microscopy (PCEM), Yue Zhuo' ${ }^{1}$, Ji Sun Choi' Thibault Marin², Hojeong Yu', Brendan Harley', Brian T. Cunningham"; ' Univ of Illinois at Urbana-Champaign, USA ; ${ }^{2}$ Research Park, UIUC, USA. To quantify live-cell adhesion, a photonic crystal biosensor surface with an extracellular matrix coating is monitored within a PCEM instrument to dynamically image changes in attached cell mass-density during live-cell attachment, spreading, and drug response.
Executive Ballroom 210D

\section{CLEO: QELS- Fundamental Science}

08:00-10:00

FM1D • Attosecond Science

Presider: Francois Legare; INRS-

Energie Mat \& Tele Site Varennes, Canada

\section{FM1D.1 08:00 Tutorial}

Versatile Sources of High-order Harmonics for Multiple Applications, Anne L'Huillier', 'Lund Univ., Sweden. This tutorial will introduce the physics of high-order harmonic generation and attosecond pulses and give an orientation of the performances and main applications of current HHG sources.

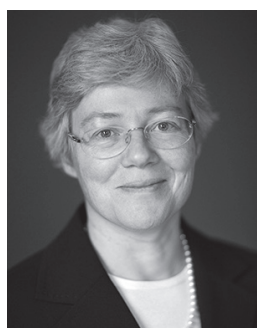

Anne L'Huillier defended her thesis in 1986 in Paris and was permanent researcher at the CEA Saclay, France until 1995. She moved to Lund University, Sweden, becoming full professor in 1997. Her research is centered around high-order harmonic generation in gases and its applications, in particular in attosecond science. 
Executive Ballroom $210 \mathrm{E}$
Executive Ballroom

210F
Executive Ballroom

210G
Executive Ballroom

$210 \mathrm{H}$

\section{CLEO: QELS-Fundamental Science}

\section{8:00-09:45}

FM1E • Engineered Quantum States with Atoms and lons Presider: Ilja Gerhardt; Max Planck Inst. for Solid State Research, Germany

FM1E.1・08:00

Macroscopically Visible Quantum Interference Due to Strong Interactions in Colliding BECs, Rachel E. Wooten ${ }^{1}$, Mackillo Kira ${ }^{1}$ 'Center for Ultrafast Optical Science, Univ. of Michigan, USA. We use a non-perturbative many-body theory to explore macroscopic quantum interference in strongly interacting, colliding Bose-Einstein condensates.

\section{FM1E.2 • 08:15 Invited}

Quantum Many-body Physics with Multimode Cavity QED, Benjamin Lev'; 'Stanford Univ., USA. We report our observation of a novel form of nonequilibrium phase transition, the condensation of supermodedensity-wave-polaritons, by placing a BEC of atoms inside a multimode optical cavity.

\section{8:00-10:00 \\ FM1F • Optomechanics and}

Plasmonics

Presider: Mercedeh Khajavikhan; Univ. of Central Florida, CREOL, USA

FM1F.1 • 08:00

Guided Entropy-Wave Scattering, William H. Renninger ${ }^{1}$, Ryan Behunin ${ }^{1}$, Peter T. Rakich'; "Yale Univ., USA. Driven optical scattering from guided entropy-waves is demonstrated in superfluid helium-4 in a hollow-core fiber. A single strongly-coupled second-sound resonance is observed, which varies with temperature in proportion to the known second-sound speed, as predicted.
FM1F.2 • 08:15

Enabling Strong Coupling in Nanoscale Silicon Optomechanical Waveguides, Raphaë Van Laer', Amir H. Safavi-Naeini'; ' 'Stanford Univ., USA. We simulate giant sidebandresolved interaction between telecom photons and megahertz phonons traveling along a silicon double-slot waveguide. The calculations predict a Rabi flop length below $100 \mu \mathrm{m}$ and a Brillouin gain coefficient above $10^{9} \mathrm{~W}^{-1} \mathrm{~m}^{-1}$.
08:00-10:00

FM1G • Parity-time Symmetry in Metamaterials

Presider: Liang Feng, The State Univ. of New York at Buffalo, USA

\section{FM1G.1 08:00 Tutorial}

Parity-time Symmetry in Metamaterials and Metasurfaces, Andrea Alu'; ${ }^{1}$ Univ. of Texas at Austin, USA. In this tutorial we discuss metamaterials and metasurfaces with balanced loss and gain, yielding unusual electromagnetic responses for a variety of applications, from imaging to scattering manipulation and sensing.

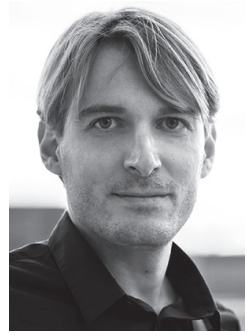

Andrea Alù is the Temple Foundation Endowed Professor \#3 at the University of Texas at Austin. His research interests span over nano-optics, photonics, electromagnetics and acoustics. He is an OSA, APS, IEEE, SPIE Fellow and recipient of several awards, including the NSF Waterman award and the OSA Lomb medal.
08:00-10:00

$\mathrm{FM} 1 \mathrm{H} \bullet$ Plasmonic and

Dielectric Metasurfaces and Metamaterials

Presider: Yuanmu Yang; Sandia National Lab, USA

FM1H.1 • 08:00

Printed Large-area Flat Optical Compo nent: Metasurfaces for Cylindrical Vector Beam Generation, Cheng Zhang ${ }^{1}$, Qiaochu Li', Lei Jin', Xi Chen', L. Jay Guo'; ' 'Univ. of Michigan, USA. We develop a cost-effective and highly reproducible method for manufacturing large-area dielectric metasurfaces. A high-performance silicon metasurface fo generating cylindrical polarized vector beam is demonstrated as an example.
FM1H.2 • 08:15

Achromatic Metalens over $60 \mathrm{~nm}$ Bandwidth in the Visible, Zhujun Shi" Mohammadreza Khorasaninejad', Wei-Ting Chen', Alexander Y. Zhu', Vyshakh Sanjeev ${ }^{1,2}$, Aun Zaidi', Federico Capasso'; 'harvard Univ. USA: ${ }^{2}$ Univ. of Waterloo, Canada. We demonstrate an achromatic metalens with a constan focal length over $60 \mathrm{~nm}$ bandwidth $(\lambda=490$ $\mathrm{nm}$ to $550 \mathrm{~nm}$ ). We also design metalenses with reverse chromatic dispersion, opposite of a Fresnel lens.
FM1F. $3 \bullet 08.30$

Noise analysis under nonlinear optica spring effect in cavity optomechanical sensors, Lingzhi Li ${ }^{1}$, Jaime Flor Flores ${ }^{1}$, Chee Wei Wong'; ${ }^{1}$ Mesoscopic Optics and Quantum Electronic, USA. Cavity optomechanical sensors with high resolution can be affected by nonlinear optical spring effect. Theoretica analysis and experiment have been done to illustrate this effect and thermal Langevin force under nonlinearity has been studied.
FM1H.3 • 08:30

Broadband Achromatic Metasurface Lens es, Sajan Shrestha' ${ }^{1}$ Adam Overvig', Nanfang $\mathrm{Yu}^{1} ;{ }^{1}$ Columbia Univ., USA. We experimentally demonstrated broadband achromatic metasurface lenses that show $\pm 3 \%$ focal distance error over a wavelength range of $\sim 400 \mathrm{~nm}$ in the near-infrared. Converging and diverging metasurface lenses with different numerica apertures have been realized. 


\section{CLEO: Science \& Innovations}

\section{8:00-10:00}

SM1I • Ultrafast Modelocked Oscillators

Presider: Thomas Sudmeyer;

Université de Neuchâtel,

Switzerland

\section{SM1I.1 08:00 Invited}

High-power Modelocked Thin-disk Oscillators: Latest Progress and Future Perspectives, Clara J. Saraceno'; ${ }^{1}$ Ruhr Universitat Bochum, Germany. High-power ultrafast thin-disk oscillators have made tremendous progress in the last decade, creating a path towards compact high-power systems from the XUV to the $\mathrm{THz}$ domain. We will review most recent progress and future trends.
SM11.2 • 08:30

$10-\mathrm{GHz}$ Straight-Cavity SESAM-Modelocked Yb:CALGO Laser Operating in the Normal Dispersion Regime, Aline Sophie Mayer ${ }^{1}$, Christopher R. Phillips ${ }^{1}$, Ursula Keller'; ' ${ }^{1}$ ETH Zurich, Switzerland. We demonstrate a 10-GHz SESAM-modelocked $\mathrm{Yb}: C A L G O$ laser delivering $240 \mathrm{fs}$ at $0.64 \mathrm{~W}$ from a straight cavity containing a fanoutapodized-PPLN crystal, which provides soliton modelocking via cascaded second-order nonlinearities and a defocusing-lens effect suppressing Q-switching-damage.

\section{8:00-09:45}

SM1J • THz Photonic

Presider: Iwao Kawayama; Osaka

Univ., Japan

SM1J.1 • 08:00

Modulation of Terahertz Polarization on Picosecond Timescales using PolymerEncapsulated Semiconductor Nanowires, Sarwat A. Baig ${ }^{1}$, Jessica L. Boland², Djamshid Damry ${ }^{2}$, Hoe Tan ${ }^{3}$, Chennupati Jagadish ${ }^{3}$, Michael B. Johnston ${ }^{2}$, Hannah Joyce 1: ' Univ. of Cambridge, UK; ${ }^{2}$ Univ. of Oxford, UK ${ }^{3}$ Australian National Univ., Australia. We exploit the photoconductivity of semiconductor nanowires to achieve ultrafast broadbandwidth modulation of $\mathrm{THz}$ pulses. $\mathrm{A}$ modulation depth of $-8 \mathrm{~dB}$ was exhibited by a polarizer consisting of 14 layers of nanowires encapsulated in polymer.

\section{SM1J.2 $\bullet$ 08:15}

Terahertz Power Enhancement by Improving Metal Adhesion Layer of Plasmonic Photoconductive Sources, Deniz Turan' Sofia Carolina Corzo García², Enrique Castro Camus ${ }^{2}$, Mona Jarrahi': ' $U$ Univ. of California Los Angeles, USA; ${ }^{2}$ Centro de Investigaciones en Óptica, Mexico. Impact of metal adhesion layer on performance of plasmonic terahertz sources is investigated. Up to $50 \%$ terahertz power enhancement is achieved when using $\mathrm{Cr}$ adhesion, compared to $\mathrm{Ti}$ adhesion used in existing plasmonic terahertz sources.

SM1J.3 • 08:30

Superior Terahertz Generation using Plasmon-Enhanced Sub-bandgap Photo conductive Antenna, Afshin Jooshesh ${ }^{1}$ Thomas E. Darcie', Reuven Gordon'; ' $U$ Univ. of Victoria, Canada. We demonstrate eight times larger $\mathrm{THz}$ field emission with twice the bandwidth for a plasmon-enhanced lowtemperature grown $\mathrm{GaAs}$ photoconductive antenna in comparison with a commercial InGaAs device at $1570 \mathrm{~nm}$ excitation.

\section{8:00-10:00 \\ SM1K • Materials for Quantum Optics \\ Presider: Takehiko Tawara; NTT \\ Basic Research Labs, Japan}

\section{SM1K.1 08:00 Invited}

New Color Centers in Diamond for Long Distance Quantum Communication, $\mathrm{Na}$ thalie de Leon'; 'Princeton Univ., USA. We have developed new methods to stabilize $\mathrm{SiV}^{0}$ in diamond, and observe $T_{1}>1$ minute at $4 \mathrm{~K}$, and $>90 \%$ of its emission into its zero phonon line, making it a promising single atom quantum memory.

\section{SM1K.2・08:30}

Anomalous Spectral Characteristics of Ultrathin sub-nm Colloidal CdSe Nanoplatelets, Sumanta Bose 1,2, Savas Delikanli3,4 Aydan Yeltik ${ }^{4}$, Manoj Sharma ${ }^{4}$, Onur Erdem $^{4}$, Cuong Dang ${ }^{3,2}$, Weijun Fan ${ }^{1,2}$, Dao H. Zhang1,3 Hilmi V. Demir3,4. 'OPTIMUS, Nanyang Technological Univ., Singapore; ${ }^{2}$ School of Electrical and Electronic Engineering, Nanyang Technological Univ., Singapore: ${ }^{3}$ LUMINOUS! Centre of Excellence for Semiconductor Lighting \& Displays and TPI - The Photonics Inst., Nanyang Technological Univ., Singapore: ${ }^{4}$ UNAM , Inst. of Materials Science and Nanotechnology, Bilkent Univ., Turkey. We demonstrate high quantum yield broad photoluminescence emission of ultrathin sub-nanometer $\mathrm{CdSe}$ nanoplatelets (two-monolayer). They also exhibit polarization-characterized lateral size dependent anomalous heavy hole and light/ split-off hole absorption intensities.
08:00-10:00

SM1L $\bullet$ High-power, Highenergy Fiber Sources

Presider: Darren Hudson;

Macquarie Univ., USA

\section{SM1L.1 • 08:00 Invited}

High Power Narrow Linewidth Microstructured Fiber Amplifiers, Benjamin Pulford', Cody Mart², lyad Dajani', Thoma Ehrenreich ${ }^{4}$, Roger Holten ${ }^{3}$, Craig Robin ${ }^{5}$ Air Force Research Lab, USA; ${ }^{2}$ Univ. of Arizona, USA; ${ }^{3}$ Leidos, USA; ${ }^{4} \mathrm{KBR}$ Ryle, USA ${ }^{5}$ Lockheed Martin, USA. An acoustic and gain tailored photonic crystal fiber (PCF), and hybrid microstructured fiber, were used to demonstrate near diffraction limited output powers of $1160 \mathrm{~W}$ and $820 \mathrm{~W}$, respectively, with spectral linewidths $\leq 1 \mathrm{GHz}$.

SM1L.2・08:30

Multi-mJ Ultrashort Pulse Coherent Pulse Stacking Amplification in a Yb-doped $85 \mu \mathrm{m}$ CCC Fiber Based System, Hanzhang Pei ${ }^{1}$ John Ruppe', Siyun Chen', Morteza Sheikhsofla ${ }^{1}$, John Nees ${ }^{1}$, Almantas Galvanauskas ${ }^{1}$ 'Univ. of Michigan, USA. Multi-mJ 81ns effectively-long burst of chirped pulses is amplified through fiber amplification system based on $85 \mu \mathrm{m}$ Yb-doped Chirally-CoupledCore fiber and coherently stacked into a single pulse. $5.4 \mathrm{~mJ}$ energy extraction at $1 \mathrm{kHz}$ repetition rate is demonstrated. 


\section{CLEO: Science \& Innovations}

08:00-10:00

$\mathrm{SM} 1 \mathrm{M} \bullet$ Integrated Nonlinear

Photonic Platforms

Presider: Kartik Srinivasan; NIST, USA

SM1M.1 • 08:00

Silicon Chip-Based Quantum Random Number Generator, Yoshitomo Okawachi ${ }^{1}$ Mengije Yu ${ }^{1,2}$, Kevin Luke ${ }^{2}$, Daniel O. Carvalho ${ }^{2,3}$, Michal Lipson', Alexander L. Gaeta'; ${ }^{1}$ Columbia Univ., USA; ${ }^{2}$ Cornell Univ., USA; ${ }^{3}$ São Paulo State Univ. (UNESP), Brazil. We demonstrate an all-optical quantum random number generator using a degenerate optical parametric oscillator in a silicon-nitride microresonator. We achieve a 2-MHz generation rate and verify the randomness using the NIST Statistical Test Suite.

\section{SM1M.2 • 08:15}

A Nonlinear Enhanced Microresonator Gyroscope, Jonathan M. Silver', Leonardo Del Bino ${ }^{1}$, Pascal Del'Haye ${ }^{1}{ }^{1}{ }^{1}$ National Physical Lab, UK. We present the experimental demonstration of a nonlinear enhanced gyroscope using counterpropagating light in a microresonator. This could enable the realization of integrated optical Sagnac sensors with enhanced sensitivity via the Kerr nonlinearity.

SM1M.3 • 08:30

1.25-Gb/s All-Optical NAND/AND Logic Gates in a Hydrogenated Amorphous Silicon Waveguide, Kangmei Li ${ }^{1}$, Amy Foster ${ }^{1}$; 1 Johns Hopkins Univ., USA. We demonstrate 1.25-Gb/s all-optical NAND/AND logic gates in a hydrogenated amorphous silicon waveguide via four-wave mixing Bragg scattering with only $85-\mathrm{mW}$ peak pump powers in the waveguide.

\section{8:00-10:00 \\ SM1N • High-Q Micro-cavities and Applications \\ Presider: Steven Rogers; Univ. of Rochester, USA}

\section{SM1N.1・08:00}

Experimental Demonstration of an OmniResonant Optical Micro-Cavity, Soroush Shabahang ${ }^{1}$, Hasan E. Kondakci ${ }^{1}$, Massimo Villinger', Joshua Perlstein², Ayman F. Abouraddy'; 'CREOL, Univ. of Central Florida, USA; ${ }^{2}$ Materials Science and Engineering Dept., Univ. of Central Florida, USA. By simultaneously phase-matching an angularly multiplexed 60-nm-wide spectrum, we render a Fabry-Perot microcavity having $0.7-\mathrm{nm}$ wide resonances 'omni-resonant', such that it continuously resonates across multiple bare-cavity FSRs.

\section{SM1N.2 • 08:15}

Silicon Microring with Ferrofluid Cladding, Abdelkrim El Amili', Mário C. Souza², Felipe Vallini ${ }^{1}$, Newton C. Frateschi ${ }^{2}$, Yeshaiahu Fainman ${ }^{2} ;{ }^{1}$ Univ. of California at San Diego, USA; ${ }^{2}$ Univ. of Campinas, Brazil. We experimentally investigate a ferrofluid-clad silicon microring resonator-based magnetic field sensor. The device presents relatively high loaded quality factors $(\sim 6,000)$ and resonance shifts of $185 \mathrm{pm}$ in response to 110 Oe strong magnetic field.

\section{SM1N.3・08:30}

Towards Ultra-High $\mathrm{Q}$ Microresonators in High-Index Contrast AlGaAs-On-Insulator, Minhao Pu', Ayman N. Kamel', Erik Stassen ${ }^{1}$, Yi Zheng', Luisa Ottaviano', Elizaveta Semenova' ${ }^{1}$ Kresten Yvind'; ' 'Danmarks Tekniske Universitet, Denmark. We demonstrate an AlGaAs-on-insulator microresonator with intrinsic $Q$ as high as 690,000. We optimized the fabrication and investigated the impact of waveguide dimension on the $Q$ in such a high-index contrast platform.
08:00-10:00

SM10 • RF Photonics

Presider: Jian Wang; Huazhong

Univ of Science and Technology,

China

SM10.1 08:00

Tri-color Optical Transmitter with Embedding 28-GHz Millimeter-wave Carrier for 5 G Mobile over Fiber, Huai-Yung Wang ${ }^{1}$, Yu-Chieh Chi', You-Wei Chen ${ }^{1}$, Gong-Ru . Lin'; ${ }^{1}$ Graduate Inst. of Photonics and Optoelectronics, and Dept. of Electrical Engineering, National Taiwan Univ., Taiwan. Data throughput enhanced tri-color optical transmitter with single-mode modulation for 28-GHz photonic millimeter-wave over fiber link in 50-km-long SMF is demonstrated to achieve QAM-OFDM baseband data rates of $18-\mathrm{Gb} / \mathrm{s}$ optical and $12-\mathrm{Gb} / \mathrm{s} 3-\mathrm{m}$ wireless communications.

\section{SM10.2 • 08:15}

High Efficiency 36-50 GHz Millimeter-wave Down-Conversion Utilizing a Wideband Tunable Optoelectronic Oscillator Based on Stimulated Brillouin Scattering, Huanfa Peng ${ }^{1}$, Yongchi $\mathrm{Xu}^{1}$, Xiaofeng Peng ${ }^{1}$, Yuanxiang Chen ${ }^{1}$, Cheng Zhang ${ }^{1}$, Lixin Zhu' ${ }^{1}$, Weiwei $\mathrm{Hu}^{1}$, Zhangyuan Chen'; 'Peking Univ., China. A novel high-efficiency $36-50 \mathrm{GHz}$ millimeterwave down-conversion system utilizing an optoelectronic oscillator based on stimulated brillouin scattering is demonstrated. $35-49 \mathrm{GHz}$ signals are down-converted to $1 \mathrm{GHz}$ IF with $-35.72 \mathrm{~dB}$ conversion-efficiency and without optical or electronic amplifiers.

SM10.3 • 08:30

Gigahertz tuning of on-chip RF photonic delay line, Yang Liu ${ }^{1,2}$, Amol Choudhary ${ }^{1,2}$, David Marpaung ${ }^{1,2}$, Benjamin J. Eggleton ${ }^{1,2}$; 'The Univ. of Sydney, Australia; ${ }^{2}$ Centre for Ultrahigh bandwidth Devices for Optical Systems (CUDOS), Australia. We demonstrate a technique that enables three-fold enhancement of time delay and seamless delay switching for RF signals at gigahertz speed from optical ring resonators on an integrated Si3N4 chip, solely by optical power control. 
Executive Ballroom $210 \mathrm{~A}$

Executive Ballroom 210B

\section{CLEO: Applications \& Technology}

AM1A • A\&T Topical Review on Advances in Laser-based Remote Sensing I-Continued

AM1B • PhotovoltaicsContinued
AM1B. $4 \cdot 08: 45$

Electrosprayed $\mathrm{TiO}_{2}$ Nanoporous Hemisphere Arrays for Enhanced Efficiency of Perovskite Solar Cells, Shaoyang Ma', Tao $\mathrm{Ye}^{2}$, Lei Wei ${ }^{1} ;{ }^{1}$ Nanyang Technological Univ. Singapore; ${ }^{2}$ National Univ. of Singapore, Singapore. We demonstrate the enhanced performances of perovskite solar cells based on electrosprayed $\mathrm{TiO}_{2}$ nanoporous hemisphere (NHS) arrays. The optimized PCE is $19.3 \%$ with a $J_{S C}$ of $23.8 \mathrm{~mA} / \mathrm{cm}^{2}, V_{\text {OC }}$ of $1.14 \mathrm{~V}$ and FF of 0.71 .

\section{AM1A.3 • 09:00}

Standoff Detection of Isotopes in a $\mathrm{NH}_{3}$ Chemical Plume, Mark C. Phillips', Brian Brumfield'; 'Pacific Northwest National Lab, USA. We perform standoff detection of ${ }^{14} \mathrm{NH}_{3}$ and ${ }^{15} \mathrm{NH}_{3}$ at a $10 \mathrm{~Hz}$ rate in a chemical plume with varying concentration using an external cavity quantum cascade laser swept over the range $930-1065 \mathrm{~cm}^{-1}$

\section{AM1A.4 0 09:15}

Multi-Wavelength Laser Transmitter for the Two-Step Laser Time-of-Flight Mass Spectrometer, Anthony W. Yu' ${ }^{1}$, Steven X. Li ${ }^{1}$ Molly E. Fahey', Andrej Grubisic', Benjamin Farcy ${ }^{1}$, Kyle Uckert ${ }^{1}$, Xiang Li ${ }^{1}$, Stephanie A. Getty'; ${ }^{1}$ NASA Goddard Space Flight Center, USA. We are developing a multi-wavelength laser for the two-step laser time-of-flight mass-spectrometer (L2MS). The L2MS is designed to detect hydrocarbons in organicallydoped analog minerals, including cryogenic Ocean World-relevant ices and mixtures for future astrobiology missions.

\section{AM1B $5 \bullet 09: 00$ Tutorial}

Photovoltaics as a Branch of Optoelectronics:Solar Cells, Heat Engines Electroluminescent Refrigerators, Eli Yablonovitch', T. Patrick Xiao': 'Univ. of California Berkeley, USA. The photovoltaic cell and the LED are really the reciprocal of one another. The slogan: "A great solar cell has to be a great LED" has led to all the new solar cell efficiency records. Very efficient light emitting diodes (LED's), surprisingly, do actually become cold as they operate, since LED light carries away entropy. This refrigeration requires superb LED efficiency, which is enabled by $2 \mathrm{~d}$ photonic crystal patterning for luminescence extraction.

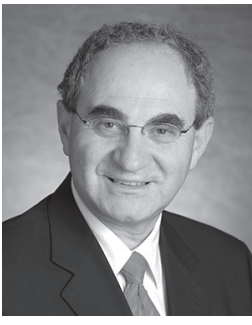

Eli Yablonovitch is Director of the NSF Center for Energy Efficient Electronics Science (E3S), a multi-University Center headquartered at Berkeley, which aims to fundamentally reduce the energy consumption of digital electronics. He is also a Co-Founder of Luxtera Inc the pioneer in Silicon Photonics, and the largest supplier of $100 \mathrm{~Gb} / \mathrm{s}$ optical interconnects.

\section{Executive Ballroom} $210 \mathrm{C}$

\section{CLEO: Science \& Innovations}

SM1C $\bullet$ Plasmonic BiosensorsContinued

SM1C.3 • 08:45

Ultrasound Detection with Surface Plasmon Resonance on Fiber End-facet, Xin Zhou ${ }^{1}$ De Cai', Xiaolong He ${ }^{1,2}$, Sung-Liang Chen ${ }^{1}$ Xueding Wang ${ }^{3}$, Tian Yang ${ }^{1}{ }^{1}$ State Key Lab of Advanced Optical Communication Systems and Networks, Key Lab for Thin Film and Microfabrication of the Ministry of Education, Shanghai Jiao Tong Univ., China; ${ }^{2} \mathrm{Xu}$ Yuan Biotechnology Company, China: ${ }^{3}$ Dept. of Biomedical Engineering, Univ. of Michigan, USA. A surface plasmon resonance cavity on an optical fiber end-facet is designed and demonstrated for ultrasound detection. A noise equivalent pressure of $25 \mathrm{KPa}$ over 20 $\mathrm{MHz}$, almost omni-directional response and stable performance are reported.

\section{SM1C.4 09:00}

Monolayer WS Enhanced High Sensitivity Plasmonic Biosensor based on Phase Modulation, Qingling Ouyang ${ }^{1,2}$, Nishtha Panwar $^{1}$, Shuwen Zeng ${ }^{1,2}$, Xingli Wang ${ }^{1,2}$ Li Jiang ${ }^{3,2}$, Xuan-Quyen Dinh ${ }^{2}$, Beng Kang Tay 1,2, Philippe Coquet ${ }^{2,4}$, Ken-tye Yong ${ }^{1,2}$ School of Electrical and Electronic Engineering, Nanyang Technological Univ. Singapore; ${ }^{2}$ CINTRA CNRS/NTU/THALES Singapore; ${ }^{3}$ State Key Lab of Modern Optical Instrumentation, Centre for Optical and Electromagnetics Research, Zhejiang Univ., China; ${ }^{4}$ IEMN, CNRS UMR 8520, France. A monolayer $\mathrm{WS}_{2}$ enhanced surface plasmon resonance biosensor is proposed. We theoretically and experimentally demonstrate that the sensitivity could reach to $3.5 \times 10^{4} \mathrm{deg} /$ RIU, which was $300 \%$ higher than that of the conventional configuration.

\section{SM1C.5・09:15}

On-chip detection of immune-cell secretion using a circular nanoplasmonic interferometer array , Xie Zeng ', Yifeng Qian' ', Yongkang $\mathrm{Gao}^{3}$, Hang $\mathrm{Li}^{1}$, Sushil Kumar', Qiaogiang Gan $^{2}$, Xuanhong Cheng', Filbert Bartoli ${ }^{1}$ Lehigh Univ., USA; ${ }^{2}$ The State Univ. of New York at Buffalo, USA: ${ }^{3} \mathrm{Al}$ catel-Lucent Bell Labs, USA. The MMP-9 protein secreted by THP1 cells was detected using an on-chip circular nanoplasmonic interferometer array. Sensitive dynamic analysis of MMP-9 in the supernatant was demonstrated, suggesting the device a potential for cell function analysis.
Executive Ballroom 210D

CLEO: QELSFundamental Science

FM1D • Attosecond ScienceContinued
FM1D.2 • 09:00

Observing the Ultrafast Buildup of a Fano Resonance in the Time Domain, Andreas Kaldun 1,2, Alexander Blättermann1, Veit Stooß ${ }^{1}$, Stefan Donsa ${ }^{3}$, Hui Wei ${ }^{4}$, Renate Pazourek ${ }^{3}$, Stefan Nagele ${ }^{3}$, Christian Ott ${ }^{1}$, Chii-Dong Lin ${ }^{4}$, Joachim Burgdörfer ${ }^{3}$, Thomas Pfeifer ${ }^{1,5}$; ${ }^{1}$ Max-Planck-Institut für Kernphysik, Germany; ${ }^{2}$ PULSE Inst. for Ultrafast Energy Science, SLAC National Accelerator Lab, USA; ${ }^{3}$ Inst. for Theoretical Physics, Vienna Univ. of Technology, Austria; ${ }^{4}$ Dept. of Physics, Kansas State Univ., USA; ${ }^{5}$ Center for Quantum Dynamics, Universität Heidelberg, Germany. First experimental observation of the time-dependent build-up of an asymmetric Fano resonance achieved by using a tunable temporal gate to interupt the autoionization process of a correlated twoelectron excited state in $\mathrm{He}$ with a strong laser field.

\section{FM1D.3・09:15}

Investigating Impulsive Strong Field Perturbation of Molecular Rydberg States with XUV Transient Absorption, Chen-Ting Liao Nathan Harkema', Arvinder Sandhu': 'Univ. of Arizona, USA. We probe the laser induced modification of XUV initiated molecular polarization using attosecond transient absorption. Spectral evolution is used to investigate the vibrationally resolved dynamics. We also simulate the spectrogram under impulsive laser perturbation. 
Executive Ballroom 210E
Executive Ballroom 210F
Executive Ballroom

210G
Executive Ballroom

$210 \mathrm{H}$

\section{CLEO: QELS-Fundamental Science}

\section{FM1E • Engineered Quantum} States with Atoms and lonsContinued

\section{FM1E.3 • 08:45}

Time-delayed Einstein-Podolsky-Rosen Entanglement between Single Photon and Collective Atomic Excitation, Michal Dabrowski ${ }^{1}$, Michal Parniak1, Wojciech Wasilewski': 'Faculty of Physics, Univ. of Warsaw, Poland. We create 12-dimensional entangled state exhibiting quantum correlations between photon and atomic ensemble in positions and momenta. This state allows us to demonstrate the original EPR paradox with an unprecedented delay time of $6 \mu \mathrm{s}$ between the entanglement generation and detection.

FM1E.4 09:00

Photon-Photon to Atom-Photon Entanglement Transfer, Stephan Kucera', Jan Arenskötter ${ }^{1}$, Pascal Eich ${ }^{1}$, Matthias Kreis ${ }^{1}$ Philipp Müller', Jürgen Eschner'; ${ }^{1}$ Saarland Univ., Germany. Using a high-brightness narrowband source of ${ }^{40} \mathrm{Ca}^{+}$-resonant entangled photon pairs, we transferred the photon-photon polarization entanglement to atom-photon entanglement by heralded absorption of one photon of the pair.
FM1E.5 • 09:15

Coherent Quantum Fourier Transform Using 3-Qubit Conditional Gates and Ultrasensitive Magnetometry with RFDriven Trapped lons, Christof Wunderlich ${ }^{1}$; 'Universität Siegen, Germany. Using longrange magnetic gradient induced coupling between three effective spins, a coherent QFT is efficiently realized with trapped $\mathrm{Yb}^{+}$ ions. With a single $\mathrm{Yb}^{+}$ion, RF magnetic fields are measured close to the quantum limit.

\section{FM1F - Optomechanics and Plasmonics-Continued}

\section{FM1F.4 • 08:45}

In-Fiber Fabry-Perot Microresonator with 100 Million O-Factor, Ewelina Obrzud ${ }^{1,2}$ Steve Lecomte 1 , Tobias Herr'; ' ${ }^{1}$ CSEM, Switzerland; ${ }^{2}$ Observatoire de Geneve, Universite de Geneve, Switzerland. A single-mode, 100 million Q-factor, Kerr-nonlinear Fabry-Perot microresonator with $10 \mathrm{GHz}$ free-spectral range is fabricated. The resonator's group elocity dispersion, coupling ratio and nonlinearity can be engineered via choice of fibre and dielectric Bragg-reflection coating.

FM1F.5 • 09:00

Losses and Intensity Clamping During Filamentation of Mid-IR Pulses in Ambient Air, Valentina Shumakova' ${ }^{1}$, Audrius Pugzlys ${ }^{1}$ Skirmantas Alisauskas ${ }^{1}$, Andrius Baltuska ${ }^{1}$ Alexander Voronin², Alexander Mitrofanov², Dmitriy Sidorov-Biryukov², Aleksei Zheltikov² Daniil Kartashov'. 'Vienna Univ. of Technology, Austria; ${ }^{2}$ Moscow State Univ., Russia; 3 Jena Univ., Germany. Different regimes of filamentation of multi-millijoule mid-infrared pulses in ambient air can be achieved by varying focusing and chirp of driving pulses. Dynamic absorption losses are identified as a possible mechanism of observed plasma-less filamentation.
FM1G • Parity-time Symmetry in Metamaterials-Continued

M1G.2・09:00

Eigenvalue dynamics in the presence of non-uniform gain and loss, Alexande Cerjan', Shanhui Fan'; ${ }^{1}$ Stanford Univ., USA We introduce a general set of conditions for observing reverse pump dependence in lasers and other counterintuitive phenomena, which demonstrate that any irreducible system with patterned gain and loss can exhibit such exotic behaviors.

FM1F.6 • 09:15

Impact of Landau Damping on Field Enhancement in Plasmonic Dimers, Jacob Khurgin ${ }^{2}$, Wei-Yi Tsai ${ }^{3}$, Din P. Tsai ${ }^{3}$, Yujie Ding ${ }^{4}$, Gregory Sun ${ }^{1} ;{ }^{1}$ Engineering, Univ. of Massachusetts Boston, USA; ${ }^{2}$ Johns Hopkins Univ., USA: ${ }^{3}$ Physics, National Taiwan Univ. Taiwan; ${ }^{4} E C E$, Lehigh Univ., USA. We show that Landau damping presents the most practically-relevant limit to the achievable plasmonic enhancement inside the narrow gaps of plasmonic dimers and other similarlyshaped plasmonic nanoantennas.

\section{FM1G.3・09:15}

Virtual electromagnetic absorption and energy storage by a Hermitian system via complex frequency excitation, Alexand Krasnok', Denis Baranov², Andrea Alu'. 'The Univ. of Texas at Austin, USA; ${ }^{2}$ Dept. of Physics, Chalmers Univ. of Technology, Sweden. Hermitian systems support virtual absorbing modes located in the complex frequency plane. Here, we access these modes and use them for virtual absorption and storage of light by specifically shaping the incident excitation.
$\mathrm{FM} 1 \mathrm{H} \bullet$ Plasmonic and

Dielectric Metasurfaces and

Metamaterials-Continued

FM1H.4 0 08:45

High-Efficiency Amplitude-Phase Modulation Holograms Based on Dielectric Metasurfaces, Adam C. Overvig', Sajan Shrestha' Chanxi Zheng', Nanfang Yu'; ' Columbia Univ., USA. We report a high-efficiency dielectric metasurface with continuous and arbitrary control of both amplitude and phase. We experimentally demonstrated the advantages of complete wavefront control by comparing amplitude-phase modulation metasurface holograms to phase-only metasurface holograms.
FM1H.5 09:00

Variable Emissivity Coatings Based on Plasmonic Metasurfaces Integrated with Phase-Transition Materials, Chongzhao $\mathrm{Wu}^{1}$, Zhaoyi $\mathrm{Li}^{1}$, Derek Schwanz ${ }^{2}$, Zhen Zhang ${ }^{2}$, Shriram Ramanathan ${ }^{2}$, Nanfang Yu' 'Columbia Univ., USA; ${ }^{2}$ Purdue Univ., USA Modulation of emissivity in the mid-infrared is experimentally realized using thin-film phase-transition material $\mathrm{SmNiO}_{3}$. Design of variable emissivity coatings based on metasurfaces integrated with $\mathrm{SmNiO}_{3}$ are also reported.

\section{FM1H.6・09:15}

Accurate Permittivity Extraction of Hyperbolic Metamaterials using Attenuated Tota Internal Reflection Ellipsometry, Cheng Zhang ${ }^{2,1}$, Nina Hong ${ }^{3}$, Chengang $\mathrm{Ji}^{2}$, Wenq Zhu $^{1}$, Amit Agrawal', L. Jay Guo², Lezec Henri' ${ }^{1}$, Tom T. Tiwald ${ }^{3}$, Stefan Schoeche ${ }^{3}$ James N. Hilfiker ${ }^{3} .{ }^{1}$ NIST USA. ${ }^{2}$ Univ of Michigan, USA; ${ }^{3}$ J. A. Woollam Co., USA. We demonstrate a new measurement procedure using attenuated total internal reflection ellipsometry for accurately extracting the permittivity of hyperbolic metamaterials. 


\section{CLEO: Science \& Innovations}

\section{SM1I • Ultrafast Modelocked Oscillators-Continued}

\section{SM11.3 • 08:45}

128-fs Pulses from a Kerr-Lens Modelocked Yb:LuO Thin-Disk Laser, Clement Paradis ${ }^{1}$ Norbert Modsching ${ }^{1}$, Valentin J. Wittwer ${ }^{1}$ Bastian Deppe ${ }^{2}$, Christian Kränkel ${ }^{2}$, Thomas Sudmeyer': ${ }^{1}$ Laboratoire Temps-Fréquence, Institut de Physique, Université de Neuchâtel, Switzerland; 'Inst. of Laser-Physics, Univ. of Hamburg, Germany. We discuss different configurations of KLM thin-disk lasers based on $\mathrm{Yb}: \mathrm{LuO}$. We achieve shorter pulses than previously demonstrated for this gain material in the thin-disk configuration and up to $11.0 \mathrm{~W}$ in 184-fs pulses.

\section{SM1I.4 09:00}

Femtosecond Yb:YAG Laser Mode-Locked using Intracavity SHG, Veselin S. Aleksandrov $^{1,2}$, Luben S. Petrov', Nickolai Belashenkov ${ }^{3}$, Ivan C. Buchvarov 1,3. 'Sofia Univ. St. Kliment Ohridski, Bulgaria; ${ }^{2}$ IBPhotonics Ltd. Bulgaria; ${ }^{3}$ TTMO Univ., Russia. $X^{(2)}$-lens modelocking of an Yb:YAG laser is demonstrated using intracavity SHG in LBO crystal. Stable operation is achieved with pulse duration of $560 \mathrm{fs}$, output power of $0.8 \mathrm{~W}$ at repetition rate of $110 \mathrm{MHz}$

\section{SM11.5 09:15}

$5.9 \mathrm{GHz}$ Q-Switched Mode-locked Midinfrared Ho:YAG Waveguide Laser, Fiona Thorburn', Adam Lancaster', Sean A. McDaniel2, Gary Cook ${ }^{3}$, Ajoy K. Kar'; ' 'Institue of Photonics and Quantum Sciences, Heriot Watt Univ., UK; ${ }^{2}$ Leidos Inc., USA; ${ }^{3}$ Air Force Research Lab, USA. A Q-Switched Modelocked Holmium doped YAG waveguide laser is reported. With a Graphene saturable output coupler, Q-switched mode-locking operation was realized in the $2 \mu \mathrm{m}$ spectra region, with a mode-locked repetition rate of $5.9 \mathrm{GHz}$.

\section{SM1J • THz Photonic- Continued}

\section{SM1J.4 • 08:45}

Highly Efficient Photoconductive Terahertz Generation through Photon Trapping, Nezih Yardimci', Semih Cakmakyapan ${ }^{1}$, Soroosh Hemmati', Mona Jarrahi'; ' Univ. of California - Los Angeles, USA. We present a highly efficient photoconductive terahertz generation scheme based on photon trapping. We utilize this scheme to demonstrate record-high pulsed terahertz radiation powers as high as $4 \mathrm{~mW}$ over 0.1-5 THz frequency.

\section{SM1J.5 09:00 Invited}

Injection-seeded THz Parametric Generator/Amplifier, Kodo Kawase ${ }^{1}$, Kosuke Murate'; ${ }^{1}$ Nagoya Univ., Japan. We report a $\mathrm{THz}$-wave amplifier using nonlinear optical effects in $\mathrm{MgO}: \mathrm{LiNbO}_{3}$ crystals. The amplifier operates at room temperature and has a gain of $55 \mathrm{~dB}$ based on the fundamental principle of injection-seeded $\mathrm{THz}$ parametric generator/detector.

\section{SM1K • Materials for Quantum Optics-Continued}

\section{SM1K.3 • 08:45}

Frequency-Domain Measurement of Spontaneous Emission Lifetime in Rare-EarthDoped Gain Media, Emir S. Magden'1, Patrick T. Callahan', Nanxi Li ${ }^{1}{ }^{1}$, Katia Shtyrkova ${ }^{1}$ Alfonso Ruocco', Neetesh K. Singh', Ming Xin', Diedrik Vermeulen', Jonathan Bradley ${ }^{1,3}$, Gerald Leake ${ }^{4}$, Douglas Coolbaugh ${ }^{4}$, Leslie Kolodziejski', Franz Kaertner ${ }^{1,5}$, Erich P. Ippen', Michael Watts'; ${ }^{1}$ MIT, USA; ${ }^{2}$ John A. Paulson School of Engineering and Applied Science, Harvard Univ., USA; ${ }^{3}$ Dept. of Engineering Physics, McMaster Univ. Canada; ${ }^{4}$ College of Nanoscale Science and Engineering, Univ. at Albany, USA; ${ }^{5}$ Center for Free-Electron Laser Science and Deutsches Elektronen-Synchrotron, Germany. The spontaneous emission lifetime in $\mathrm{Al}_{2} \mathrm{O}_{3}: \mathrm{Tm}^{3+}$ waveguides is measured to be $568 \pm 48 \mu \mathrm{s}$, using a frequency-domain method. The method is studied and verified in $\mathrm{Er}^{3+}$-doped silica fiber yielding a measured lifetime of $9.73 \pm 0.08 \mathrm{~ms}$.

\section{SM1K.4 09:00 Invited}

Quantum Nano-photonic Devices Based on Rare-earth lons, Andrei Faraon'; 'Applied Physics, Caltech, USA. I present our progress towards developing optical quantum memories based on photonic crystal cavities fabricated in rare-earth-doped crystals. These devices exhibit large spontaneous emission rate enhancement, enabling optical control of single rare-earth ion quantum bits.
SM1L • High-power, Highenergy Fiber SourcesContinued

SM1L.3 • 08:45

High Power Tm-doped All-Fiber Amplifier at $2130 \mathrm{~nm}$, Brian Anderson' Angel Flores' Jacob Grosek', lyad Dajani'; 'Air Force Research Lab, USA. A diode pumped (793nm), long-wavelength thulium-doped all-fiber amplifier was demonstrated. Laser gain competition was used to suppress parasitic lasing and an output power of $80 \mathrm{~W}$ was generated at $2130 \mathrm{~nm}$ with $50 \%$ optical efficiency.
SM1L.4 09:00

A Kilo-Watt all-fiber distributed-pumping oscillator, Jianqiu $\mathrm{CaO}^{1}, \mathrm{Yu} \mathrm{Yu}^{1}$, Hanyuan Ying ${ }^{1}$, Zhiyong Pan', Zefeng Wang ${ }^{1}$, Jinbao Chen'; 'National Univ of Defense Technology, China. An all-fiber distributed-pumped oscillator with 1.14-kW output power is demonstrated firstly to the best of our knowledge. No pump-light stripper is needed in this oscillator.

SM1L.5 • 09:15

Single-mode $60 \mu \mathrm{m}$-core multiple-claddingresonance photonic bandgap fiber lase with $\sim 1 \mathrm{~kW}$ output power, Guancheng $\mathrm{Gu}^{1}$, Fanting Kong ${ }^{1}$, Thomas Hawkins ${ }^{1}$ Maxwell Jones ${ }^{2}$, Joshua Parsons ${ }^{1}$, Monica T. Kalichevsky-Dong', Benjamin Pulford ${ }^{3}$, lyad Dajani ${ }^{3}$, Stephen Palese ${ }^{4}$, Eric Cheung ${ }^{5}$, Liang Dong1: ' ${ }^{1}$ lemson Univ., USA: ${ }^{2}$ Nufern, USA; ${ }^{3}$ Air Force Research Lab, USA; ${ }^{4}$ Raytheon Space \& Airborne Systems, USA; ${ }^{5}$ Northrop Grumman Aerospace Systems, USA. We report the effectiveness of multiple-claddingresonance photonic bandgap fiber for suppressing mode instability by demonstrating pump-limited single-mode output power of $\sim 1 \mathrm{~kW}$ in a $60 \mu \mathrm{m}$-core fiber, a record for any fiber lasers at this core diameter. 


$\begin{array}{lcc}\text { Marriott } & \text { Marriott } & \text { Marriott } \\ \text { Salon III } & \text { Salon IV } & \text { Salon V \& VI }\end{array}$

CLEO: Science \& Innovations

$\mathrm{SM} 1 \mathrm{M} \bullet$ Integrated Nonlinear Photonic Platforms-Continued

SM1M.4 • 08:45

All-optically induced quasi phase matching in $\mathrm{SiN}$ waveguides for second harmonic generation enhancement, Davide Grassani', Adrien Billat ${ }^{1}$, Martin Pfeiffer ${ }^{1}$, Svyatoslav Kharitonov' ${ }^{1}$, Tobias J. Kippenberg' CamilleSophie Bres'; ' ${ }^{E} E P F L$, Switzerland. We report more than $30 \mathrm{~dB}$ second harmonic generation enhancement in SiN waveguide by all-optical writing of a persistent $X^{(2)}$ grating. Phase matching peaks are observed for different writing wavelengths.

SM1M.5 09:00 Invited

Nonlinear Optical Frequency Conversion in Aluminum Nitride Photonic Circuits, Hong Tang'; ' ${ }^{1}$ Electrical Engineering, Yale Univ., USA. I will discuss the development of high efficiency optical frequency converters based on AIN nonlinear photonic circuits, including visible-to-IR conversion leveraging AlN's x(2) nonlinearity and microwave driven noiseless in-band frequency conversion at single photon levels.
SM1N • High-Q Micro-cavities and Applications-Continued

SM1N.4 • 08:45

Transversely Coupled Fabry-Perot Resonators in SOI, Md. Ghulam Saber', Zhenping Xing ${ }^{1}$, Eslam El-Fiky ${ }^{1}$, David Patel ${ }^{1}$, Luhua $\mathrm{Xu}^{1}$, Nicolás Abadía', David V. Plant'; ' ${ }^{1}$ McGill Univ., Canada. We experimentally demonstrate transversely coupled Fabry-Perot resonators using sidewall Bragg gratings and loop mirrors as reflectors in siliconon-insulator platform. The resonators have channel spacing of $\sim 50 \mathrm{GHz}$, extinction ratio of $\sim 12 \mathrm{~dB}$ and $\mathrm{Q}$-factor of $\sim 15350$.

\section{SM10 • RF Photonics-} Continued

SM10.4 • 08:45

Silicon Optical-Phased-Array Prototypes Using Electro-Optical Phase Shifters, Che Zhao ${ }^{1}$, Haiyang Zhang ${ }^{1}$, Zhong Zheng ${ }^{1}$, Chao Peng ${ }^{1}$, Weiwei Hu'; ${ }^{1}$ Peking Univ., China. High-speed silicon optical-phased-array (OPA) prototypes are achieved using electrooptical phase shifters with quick response $(4.2$ ns) and small footprint $(500 \mu \mathrm{m} / 2 \pi) \cdot 11.1^{\circ}$ $(1 \mathrm{D})$ and $7.4^{\circ} \times 3.7^{\circ}(2 \mathrm{D})$ beam steering is observed.
SM1N.5・09:00

Post processing resonance trimming of a silicon micro-ring resonator using Flash memory technology, Meir Y. Grajower ${ }^{1}$, Noa Mazurski ${ }^{1}$, Joseph Shappir ${ }^{1}$, Uriel Levy ${ }^{1} ;{ }^{1} \mathrm{He}-$ brew Univ. of Jerusalem, Israel. A new post processing approach for resonance trimming of a micro-ring resonator is presented. The approach is based on charge trapping in thin layer of silicon nitride as done in Flash memories device.

\section{SM1N.6 • 09:15}

On-chip beam positioning sensor via frequency locked cascaded ring resonators, Alex Naiman', Liron Stern', Uriel Levy'; 'The Hebrew Univ. of Jerusalem, Israel. We present a novel technique for on-chip beam positioning, based on tracking the thermo-optic driven spectral resonance shifts between two cascaded microring resonators vs. the position of an NSOM tip which illuminates the structure.
SM10.5 09:00

Compressed Sensing of Sparse RF Signals Based on Silicon Photonic Microcavities, Hongcheng Sun', Bryan T. Bosworth', Brian C. Grubel', Michael Kossey', Mark A. Foster'1, Amy Foster'; 'The Johns Hopkins Univ., USA. We demonstrate accurate reconstructions of sparse radio frequency signals using silicon photonic microcavities to generate the pseudorandom pattern. A compression ratio of $8 \%$ is achieved for 24 patterns.

\section{SM10.6 09:15}

Silicon microring weight banks for multivariate RF photonics, Alex Tait', Thomas Ferreira de Lima', Ellen Zhou', Allie X. Wu', Matt Chang', Mitchell A. Nahmias', Bhavin J. Shastri', Paul R. Prucnal'. 'Princeton Univ., USA. Microring weight banks enable novel analog processing approaches in silicon photonics. Incorporating statistical techniques, they can implement wideband dimensionality reduction. We demonstrate principal component analysis of three $1 \mathrm{GHz}$ signals in a microring weight bank. 
Executive Ballroom

$210 \mathrm{~A}$

Executive Ballroom

210B

\section{CLEO: Applications \\ \& Technology}

AM1A • A\&T Topical Review on Advances in Laser-based Remote Sensing I-Continued

AM1B • PhotovoltaicsContinued
Executive Ballroom $210 \mathrm{C}$

\section{CLEO: Science \& Innovations}

SM1C • Plasmonic BiosensorsContinued

SM1C.6 • 09:30

Computational Sensing in Plasmonics: Design of Low-cost and Mobile Plasmonic Readers Using Machine Learning, Zachary S. Ballard ${ }^{1,2}$, Daniel Shir ${ }^{1,2}$, Aashish Bhardwaj ${ }^{3}$, Sarah Bazargan ${ }^{3}$, Shyama Sathianathan ${ }^{3}$ Aydogan Ozcan 1,3; ' ${ }^{1}$ Electrical Engineering, Univ. of California Los Angeles, USA; ${ }^{2}$ California NanoSystems Inst., Univ. of California Los Angeles, USA: ${ }^{3}$ Bioengineering, Univ. of California Los Angeles, USA. We introduce computational sensing framework to select the optimal set of illumination bands for given plasmonic sensor design and fabrication method. This framework enables optimized designs for cost-effective, sensitive and mobile plasmonic readers.

\section{SM1C.7 • 09:45}

Mid-Infrared Nanoplasmonics for Labelfree Real-time Biosensing of Proteins and Lipid Membranes, Dordaneh Etezadi ${ }^{1}$ Odeta Limaj', Nathan J. Wittenberg², Danie Rodrigo ${ }^{1}$, Daehan $\mathrm{Yoo}^{2}$, Sang-Hyun $\mathrm{Oh}^{2}$, Hatice Altug '; 'École Polytechnique Fédérale de Lausanne, Switzerland; ' Univ. of Minnesota USA. We present our surface-engineered Mid-infrared plasmonic biosensor for realtime, label-free chemical-specific monitoring of biomimetic lipid membrane kinetics. Ou experiments and simulations demonstrate high field enhancements with probing depth of tens of nanometers suitable for bioassays.
Executive Ballroom 210D

\section{CLEO: QELS- Fundamental Science}

FM1D • Attosecond ScienceContinued

FM1D.4 • 09:30

Attosecond kinetics of photoexcited Germanium, Peter Kraus ${ }^{1}$ Chris Kaplan ${ }^{1}$, Michae Zurch', Hung-Tzu Chang ${ }^{1}$, Marieke F. Jager ${ }^{1}$ Scott Cushing', Lauren J. Borja', Daniel M. Neumark', Stephen R. Leone'; 'Chemistry, UC Berkeley, USA. Attosecond transient reflectivity is developed to observe the photoexcitation dynamics in germanium. Attosecond time-resolved measurements of the dielectric function reveal a few-femtosecond collective electronic response time, which renormalizes the Coulomb interaction between the excited carriers.

\section{FM1D.5 • 09:45}

Relativistic-intensity 1.3 Optical Cycle Lase Pulses at $1 \mathrm{khz}$ from a Stretched Hollowfiber Compressor, Frederik Boehle ${ }^{1}$, Andreas Blumenstein ${ }^{4}$, Aline Vernier ${ }^{1}$, Maïmouna Bocoum¹, Magali Lozano', Jean-Philippe Rousseau', Aurélie Jullien', Domynikas Gustas $^{1}$, Diego Guénot ${ }^{1}$, Jérôme Faure ${ }^{1}$, Mate Kovacs ${ }^{2}$, Martin Kretschmar ${ }^{3}$, Peter Simon ${ }^{4}$ Uwe Morgner ${ }^{3,5}$, Tamas Nagy, ${ }^{6,3}$, Rodrigo B. Lopez-Martens'; ; 'Laboratoire d'Optique Appliquée, France; ${ }^{2}$ ELI-Hu NonProfit Ltd Hungary; ${ }^{3}$ Institut für Quantenoptik, Leibniz Universität Hannover, Germany; ${ }^{4}$ Laser Laboratorium Göttingen e.V., Germany; ${ }^{5}$ Laser Zentrum Hannover e.V., Germany ${ }^{6}$ Max Born Inst. for Nonlinear Optics and Short Pulse Spectroscopy, Germany. We generate high temporal contrast 1.3 optical cycle laser pulses with TW peak power at $1 \mathrm{kHz}$ from a stretched hollow-fiber compressor. These pulses are then used to drive relativistic-intensity laser-plasma interactions downstream.

09:00-12:00 SC362: Cavity Optomechanics: Fundamentals and Applications of Controlling and Measuring Nano- and Micro-mechanical Oscillators with Laser Light SC424: Optical Terahertz Science and Technology 

Executive Ballroom
$210 \mathrm{E}$
Executive Ballroom 210F
Executive Ballroom

210G
Executive Ballroom

$210 \mathrm{H}$

\section{CLEO: QELS-Fundamental Science}

FM1E • Engineered Quantum States with Atoms and lonsContinued

\section{FM1E.6 • 09:30}

Integrated Optical Addressing of Ion Qubits with Waveguide-Based Focusing Gratings, Karan Mehta', Colin Bruzewicz², Robert McConnell2, Rajeev Ram', Jeremy Sage $^{2}$, John Chiaverini ${ }^{2} ;{ }^{1} M I T$, USA; ${ }^{2}$ Lincoln Labs, USA. Waveguide optics integrated within planar ion traps can enable scalable addressing of atomic ions with diffractionlimited, focused beams. We discuss our experimental work on integrated optics based on such devices for large-scale trapped-ion quantum information processing.

\section{FM1F • Optomechanics and} Plasmonics-Continued

FM1F.7 • 09:30

Splashing Transients of 2D Plasmons Launched by Swift Electrons, Xiao Lin ${ }^{1,4}$, Ido Kaminer ${ }^{2}$, Xihang Shi', Fei Gao', Zhaoju Yang', Zhen Gao', Hrvoje Buljan" John Joannopoulos ${ }^{2}$, Marin Soljacic ${ }^{2}$, Hongsheng Chen ${ }^{4}$, Baile Zhang ${ }^{1}$; ${ }^{1}$ Nanyang Technological Univ., Singapore; ${ }^{2}$ Physics, MIT, USA; ${ }^{3}$ Physics, Univ. of Zagreb, Croatia; ${ }^{4}$ ISEE, Zhejiang Univ., China. We predict a jet-like rise of excessive charge concentration that delays $2 \mathrm{D}$ plasmon generation in electron-energyloss spectroscopy; revealing an analogy to the Rayleigh jet in a splashing phenomenon prior to the launching of ripples.

\section{FM1F.8 • 09:45}

Ultrafast Transient Nonlinear Dynamics of Two-Layer Graphene Sheets, Jennifer M Reed', Manuel R. Ferdinandus ${ }^{2,1}$, Kathleen Brockdorf ${ }^{1,3}$, Shin Mou', Augustine Urbas ${ }^{1}$ ${ }^{1}$ US Air Force Research Lab, USA: ${ }^{2}$ Air Force Inst. of Technology, USA; ${ }^{3}$ Wright State Univ., USA. We measure the ultrafast nonlinear dynamics of two-layer graphene. One instantaneous electronic and three non-instantaneous free carrier responses are observed. Measurements indicate a large saturable absorption effect due to Pauli blocking.
FM1G • Parity-time Symmetry in Metamaterials-Continued

\section{FM1G.4 • 09:30}

Extending Edge Modes with non-Hermitian Forcing, Hanan H. Herzig Sheinfux', Eran Lustig', Yaakov Lumer², Yonatan Plotnik', Mordechai Segev'; 'Technion Israel Inst. of Technology, Israel; ' 2 Univ. of Pennsylvania, USA. We show that introducing asymmetric coupling can force all the modes of a waveguide lattice to localize, except for one topologically protected "edge-state" which becomes extended. This mode has real eigenvalues and retains topological properties.

FM1G.5 • 09:45

Fast Tunable Terahertz Absorber Based on a MEMS-driven Metamaterial, Mingkai Liu' Mohamad Susli², Dilusha Silva², Gino Putrino², Hemendra Kala ${ }^{2}$, Shuting Fan ${ }^{3}$, Michael Cole ${ }^{1}$ Lorenzo Faraone ${ }^{2}$, Vincent Wallace ${ }^{3}$, Willie J. Padilla ${ }^{4}$, David A. Powell1', Mariusz Martyniuk ${ }^{2}$ Ilya Shadrivov'; ${ }^{1}$ Nonlinear Physics Centre, Australian National Univ., Australia; ${ }^{2}$ Schoo of Electrical, Electronic and Computer Engineering, Univ. of Western Australia, Australia; ${ }^{3}$ School of Physics, Univ. of Western Australia, Australia; ${ }^{4}$ Dept. of Electrical and Computer Engineering, Duke Univ., USA. We design, fabricate and experimentally study ultra-thin tunable terahertz absorbers based on MEMS driven metamaterials. We demonstrate gian tuning of resonant absorption, with practical modulation speeds that can be useful for terahertz detection and imaging applications.
FM1 $\bullet$ Plasmonic and

Dielectric Metasurfaces and Metamaterials-Continued

FM1H.7 • 09:30

On-Resonance Chiral Metamaterial for Chiroptical Sensing at the Molecular Level, Hamed Shams Mousavi', Sajanlal R. Panikkanvalappil' ', Ali A. Eftekhar', Mostafa El-Sayed', Ali. Adibi'; ' ${ }^{1}$ Georgia Inst. of Technology, USA. We present a novel three-dimensiona chiral metamaterial design for chiroptica spectroscopy. Utilizing the chiral light-matte interaction between the designed metamaterial and molecule that is chiral in the same wavelength range, we demonstrate chiroptical sensing at the molecular level.

\section{FM1H.8 • 09:45}

Plasmonic Metamaterial Device for Optomechanical Amplification and Dampening Hai Zhu', Fei Yi'2, Ertugrul Cubukcu 3,4; ${ }^{1}$ Dept. of Materials Science and Engineering, Univ. of Pennsylvania, USA: ${ }^{2}$ School of Optical and Electronic Information, Huazhong Univ. of Science and Technology, China; ${ }^{3}$ Dept. of Nanoengineering, Univ. of California, San Diego, USA; ${ }^{4}$ Dept. of Electrical and Computer Engineering, Univ. of California San Diego, USA. We designed, fabricated and characterized an optomechanical device based on plasmonic metamaterial absorber which demonstrated optical manipulation of mechanical resonances. This device is able to achieve coherent mechanical oscillation and compatible with broadband light source.

09:00-12:00 SC362: Cavity Optomechanics: Fundamentals and Applications of Controlling and Measuring Nano- and Micro-mechanical Oscillators with Laser Light SC424: Optical Terahertz Science and Technology 


\section{Meeting Room $211 \mathrm{~B} / \mathrm{D}$}

Meeting Room
$212 \mathrm{~A} / \mathrm{C}$
Meeting Room

212 B/D
Marriott

Salon I \& II

\section{CLEO: Science \& Innovations}

\section{SM1I • Ultrafast Modelocked Oscillators-Continued}

\section{SM11.6 09:30}

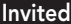

Generation of $220 \mathrm{fs}, 20 \mathrm{~W}$ pulses at $2 \mu \mathrm{m}$ from Kerr-lens mode-locked Ho:YAG thindisk oscillator, Jinwei Zhang', Ka Fai Mak' Sebastian Gröbmeyer ${ }^{2}$, Dominik Bauer ${ }^{3}$, Dirk Sutter $^{3}$, Vladimir Pervak ${ }^{2}$, Ferenc Krausz ${ }^{1,2}$ Oleg Pronin ${ }^{1} ;{ }^{1}$ Max-Planck-Inst. of Quantum Optics, Germany: ${ }^{2}$ Ludwig-Maximilians-Univ. Munich, Germany; ${ }^{3}$ TRUMPF Laser GmbH and Co. KG, Germany. We report the first mode-locked Ho:YAG thin-disk oscillato delivering 220-fs pulses at 20-W average power and 2090-nm central wavelength. The output parameters constitute the highest average power of any mode-locked oscillator around $2 \mu \mathrm{m}$.

\section{SM1J • THz Photonic- Continued}

SM1J.6 • 09:30

Complete Wavefront Control of SingleCycle THz Pulses via Optical Pulse Envelope Manipulation, Bradley Smith', John Whita$\mathrm{ker}^{1} ;{ }^{1}$ Univ. of Michigan, USA. The generation of $\mathrm{THz}$ pulses by tilted and curved optical pulse-fronts is modeled from first-principles -- agreeing with initial experimental results. We also propose a novel method to rapidly vary the tilt of optical pulse-fronts.

\section{SM1K • Materials for Quantum Optics-Continued}

SM1K.5 • 09:30

Improving Photoluminescence Collection from Nitrogen Vacancy Ensembles in Diamond via Surface Texturing, Samuel M. Parks ${ }^{1}$, Richard Grote ${ }^{1}$, David Hopper ${ }^{1,2}$, Lee Bassett'; 'Dept. of Electrical and Systems Engineering, Univ. of Pennsylvania, USA; ${ }^{2}$ Dept. of Physics, Univ. of Pennsylvania, USA We demonstrate a $60 \%$ increase in collected photoluminescence from nitrogen-vacancy ensembles in single-crystal diamond by surface texturing. Enhancements of up to a factor of 23 are predicted for optimized texturing and collection.
SM1K.6 09:45

Quantum Photonic Wavelength Conversion and Modulation using Low Loss Aluminum Nitride, Gong Zhang ' ${ }^{1}$, Jianguo Huang ${ }^{1}$, Wee $\mathrm{Ser}^{1}$, Weibo $\mathrm{Gao}^{2}$, Yidong Chong ${ }^{2}$, Jiangbin Gong ${ }^{3}$, Leong Chuan Kwek ${ }^{4}$, Ai Qun Liu'; ${ }^{1} S c h o o l ~ o f$ Electrical and Electronic Engineering, Nanyang Technological Univ., Singapore; ${ }^{2}$ School of Physical and Mathematical Sciences, Nanyang Technological Univ., Singapore; ${ }^{3}$ Dept. of Physics and Centre for Computational Science and Engineering, National Univ. of Singapore, Singapore; ${ }^{4}$ Centre for Quantum Technologies, National Univ. of Singapore, Singapore. An AIN photonic chip for quantum wavelength convention and modulation is designed and fabricated. The waveguide has low loss down to $1.2 \mathrm{~dB}$ / $\mathrm{cm}$. Fast switching using Pockels effect and second harmonic generation is achieved.
SM1L $\bullet$ High-power, Highenergy Fiber SourcesContinued

SM1L.6 • 09:30

Transverse-Mode Instability Mitigation using Photonic-Lantern Adaptive Spatial Mode Control, Juan Montoya', Chris Aleshire', Christopher Hwang', Dale Martz' Niyom Lue ${ }^{1}$, Andrew Benedick', Tso-Yee Fan', Dan Ripin'; 'Massachusetts Inst of Tech Lincoln Lab, USA. We report on mitigating transverse mode instability using a photoniclantern all-fiber-based adaptive spatial mode control (ASMC) system. We demonstrate control of polarization, phase, and amplitude to combat optical disturbances.

\section{SM1L.7 • 09:45}

Tunable, All-Fiber, Continuous Wave Oscillator in the E-band Operating on the ${ }^{4} \mathrm{~F}_{3 / 2}$ to ${ }^{4} I_{13 / 2}$ transition in Neodymium, Leily $S$. Kiani', Jay Dawson ${ }^{1}$, Paul H. Pax', Graham S. Allen', Victor V. Khitrov' ${ }^{1}$, Derrek R. Drachenberg', Michael J. Messerly', Nick Schenkel ${ }^{1}$, Matthew J. Cook', Robert P. Crist'; ' 'Lawrence Livermore National Lab, USA. We demonstrate $80 \mathrm{~nm}$ of tuning in the $1400 \mathrm{~nm}$ range with $116 \mathrm{~mW}$ maximum output power in an $\mathrm{Nd}^{3+}$ doped fiber oscillator with distributed spectral filtering.

09:00-12:00

SC362: Cavity Optomechanics: Fundamentals and Applications of

Controlling and Measuring Nano- and

Micro-mechanical Oscillators with Laser Light

SC424: Optical Terahertz Science and Technology

10:00-10:30 Coffee Break, Concourse Level 


$\begin{array}{llc}\text { Marriott } & \text { Marriott } & \text { Marriott } \\ \text { Salon III } & \text { Salon IV } & \text { Salon V \& VI }\end{array}$

CLEO: Science \& Innovations

SM1M • Integrated Nonlinear Photonic Platforms-Continued

SM1M.6 0 09:30

Enhanced Effective Second-order Nonlinearities in Si-rich $\mathrm{SiN}_{\mathrm{x}}$ Thin Films, Hung-Hsi Lin', Rajat Sharma', Mu-Han Yang', Matthew W. Puckett' ${ }^{1}$, Christian D. Wurm', Felipe Vallini', Yeshaiahu Fainman': 'Electrical and Computer Engineering, Univ. of California, San Diego, USA. We develop Si-rich $\mathrm{SiN}_{\mathrm{x}}$ thin films exhibiting large effective secondorder nonlinearity $\left(x^{(2)}\right)$ as high as $22.7 \mathrm{pm} / \mathrm{V}$ by combining the nonlinear contribution from pre-existing $X^{(2)}$ in $\mathrm{SiN}_{\mathrm{x}}$ and from the electric-field induced second-harmonic (EFISH) effect.

SM1M.7 • 09:45

Silicon photonic crystal cavity enhanced second-harmonic generation from monolayer $\mathrm{WSe}_{2}$, Taylor K. Fryett ${ }^{1}$, Kyle Seyler ${ }^{1}$ Jiajiu Zheng ${ }^{1}$, Xiadong Xu' ${ }^{1}$, Arka Majumdar ${ }^{1}$ ${ }^{1}$ Univ. of Washington, USA. We demonstrate a silicon photonic crystal cavity enhanced second-harmonic generation (SHG) in tungsten diselenide. The observed SHG is enhanced by a factor of $\sim 200$ compared to a bare monolayer on silicon.
SM1N • High-Q Micro-cavities and Applications-Continued

SM1N.7 • 09:30

Subwavelength Grating Racetrack Resonator Based Ultrasensitive Refractive Index Sensor, Lijun Huang ${ }^{1,2}$, Hai Yan', Xiaochuan $\mathrm{Xu}^{3}$, Swapnajit Chakravarty ${ }^{3}$, Naimei Tang ${ }^{3}$, Huiping Tiann', Ray T. Chen ${ }^{1,3}$; ${ }^{1}$ Dept. of Electrical and Computer Engineering, The Univ. of Texas at Austin, USA; ${ }^{2}$ State Key Lab of Information Photonics and Optical Communications, School of Information and Communication Engineering, Beijing Univ. of Posts and Telecommunications, China; ${ }^{3}$ Omega Optics Inc, USA. An ultrasensitive transverse magnetic mode subwavelength grating racetrack resonator with a sensitivity of $429.7 \mathrm{~nm} /$ RIU and a detection of limit of $3.71 \times 10^{-4}$ RIU is demonstrated experimentally.

SM1N.8 09:45

The Switchable EIT-like and Fano Resonances in Microring-Bragg Grating Based Coupling Resonant System, Zecen Zhang ${ }^{1}$, Geok Ing Ng${ }^{1}$, Ting Hu${ }^{1}$, Haodong Qiu', Xin Guo', Mohamed S. Rouifed', Chongyang Liu', Hong Wang'; 'School of Electrical and Electronic Engineering, Nanyang Technological Univ., Singapore. A microring-Bragg grating based coupling resonant system is experimentally demonstrated to generate switchable EIT-like and Fano transmissions for the first time. The resonance state is dependent on the relation between coupling coefficient and round-trip-intensity-attenuation.

\section{SM10 • RF Photonics-} Continued

SM10.7 • 09:30

Highly selective and reconfigurable Si3N4 RF photonic notch filter with negligible RF losses, Yang Liu',2, David Marpaung ${ }^{1,2}$, Amol Choudhary ${ }^{1,2}$, Benjamin J. Eggleton ${ }^{1,2}$;

TThe Univ. of Sydney, Australia; ${ }^{2}$ Centre for Ultrahigh bandwidth Devices for Optical System (CUDOS), Australia. We present an integrated RF photonic notch filter on a Si3N4 chip, with tunable bandwidth (150-300 $\mathrm{MHz})$, high rejection $(>50 \mathrm{~dB})$, tuning range of $1-12 \mathrm{GHz}$ and negligible RF insertion loss, using resonators' unique phase responses.

\section{SM10.8 • 09:45}

Microwave frequency-doubling based on a coupling-modulated silicon ring resonator, Yiming Zhong', Linjie Zhou', Yanyang Zhou', Yujie Xia², Minjuan Wang', Jianping Chen'. 'Shanghai Jiaotong Univ., China: 'Univ. of California, Santa Barbara, USA. We demonstrate microwave signal generation with frequency doubling using a couplingmodulated silicon ring resonator at critical coupling. The average electrical harmonic suppression ratio is around $20 \mathrm{~dB}(29 \mathrm{~dB})$ for $5 \mathrm{dBm}(10 \mathrm{dBm})$ input microwave power.

\begin{tabular}{c}
\hline $\begin{array}{c}\text { 09:00-12:00 } \\
\text { SC362: Cavity Optomechanics: Fundamentals and Applications of } \\
\text { Controlling and Measuring Nano- and } \\
\text { Micro-mechanical Oscillators with Laser Light } \\
\text { SC424: Optical Terahertz Science and Technology }\end{array}$ \\
\hline 10:00-10:30 Coffee Break, Concourse Level \\
\hline 10:30-12:00 Communication and Mentorship, Winchester Room/ Hilton
\end{tabular}


Executive Ballroom 210A

Executive Ballroom 210B

\section{CLEO: Applications \& Technology}

$10: 30-12: 30$

AM2A • A\&T Topical Review on Advances in Laser-based Remote Sensing II Presider: Fabio Di Teodoro; Raytheon, USA

AM2A.1 - 10:30

nvited

Active Optical Remote Sensor for Carbon dioxide and Water Vapor Measurement from an Air and Space-borne Platform, Upendra Singh ${ }^{1,2}$, Tamer Refaat ${ }^{1,2}$, Mulugeta Petros ${ }^{1,2}$, Syed Ismail1,2; 'NASA Langley Research Center, USA; ${ }^{2}$ Analytical Services and Materials Inc, USA. Challenges towards development and demonstration of an airborne 2- $\mu \mathrm{m}$ triple-pulse integrated path differential absorption (IPDA) lidar for simultaneous measurements of carbon dioxide and water vapor column measurements from air and space-borne platform will be presented.

\footnotetext{
AM2A.2 - 11:00 Invited
}

Differential Absorption Lidar Monitoring of Atmospheric Atomic Mercury in China using a Novel Mobile System, Guangyu Zhao', Ming Lian ${ }^{1}$, Zheng Duan', Yiyun Li ${ }^{1}$ Shiming Zhu', Sune R. Svanberg, ${ }^{1,2}$; ${ }^{1}$ South China Normal Univ., Sweden; ${ }^{2}$ Lund Univ., Sweden. Mercury is a severe pollutant in China. A novel mobile laser spectroscopy Lab was constructed and used in differential absorption lidar mapping in major Chinese cities, a heavily polluted mining area and an archeological site.
10:30-12:30

AM2B • Mid-IR Sensors and

Emitters

Presider: To be Determined

\section{AM2B 1 • 10:30 Invited}

New Sources and Sensors for Mid- to Far-IR Optical Sensing, Lan $Y u^{2}$, Daehwan Jung $^{3}$, Sukrith Dev ${ }^{1}$, Narae Yoon ${ }^{1}$, Leland J. Nordin', Anthony J. Hoffman', Minjoo Lee², Dan Wasserman'; 'Univ. of Texas at Austin, USA: ${ }^{2}$ Electrical and Computer Engineering, Univ. of Illinois Urbana Champaign, USA ${ }^{3}$ Electrical and Computer Engineering, Univ. of California Santa Barbara, USA; ${ }^{4}$ Electrical Engineering, Univ. of Notre Dame, USA. The mid- to far-IR wavelength ranges offer unique opportunities to engineer a wide range of light matter interactions. We will present new optical and optoelectronic devices and materials leveraging these interactions for next generation optical systems.

AM2B.2 • 11:00

Narrow-Linewidth Oxide-Confined Heterogeneously Integrated Si/III-V Semiconductor Laser, Wang Huolei', Dongwan Kim', Mark Harfouche ${ }^{3}$, Naresh Satyan², George Rakuljic², Amnon Yariv'1,3; 'Dept. of Applied Physics and Materials Science, California Inst. of Technology, USA; ${ }^{2}$ Telaris Inc., USA; ${ }^{3}$ Dept. of Electrical Engineering, California Inst. of Technology, USA. We demonstrate a narrowlinewidth heterogeneously integrated silicon/ III-V laser based on the oxide-confinement method. The laser achieves an output power of $4 \mathrm{~mW}$ and a linewidth of $28 \mathrm{kHz}$ with a threshold current of $60 \mathrm{~mA}$ and a side mode suppression ratio of $50 \mathrm{~dB}$ at $1574 \mathrm{~nm}$.
Executive Ballroom $210 \mathrm{C}$

\section{CLEO: Science \& Innovations}

10:30-12:30

$\mathrm{SM} 2 \mathrm{C} \cdot$ Biomedical

Spectroscopy and Cell/Particle Analysis

Presider: Andreu Llobera; Centre Nacional de Microelectronica, Spain

\section{SM2C.1・10:30 Tutorial}

Applications of Laser Spectroscopy to Meet Challenges in Medicine, Katarina Svanberg1,2; ' 'Lund Laser Centre, Sweden ${ }^{2}$ Center for Optical and Electromagnetic Research, China. Laser based spectroscopic techniques can be used in the detection and therapy of human diseases. Examples from oncology, ortopedics and pediatrics as well as from the field of food quality control will be given.

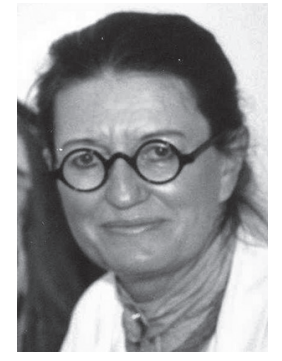

Katarina Svanberg is an M.D. and a Ph.D and holds a professorship in Oncology at Lund University, Sweden as well as at South China Normal University in Guangzhou, China. Her main research interest concerns light interaction in tissue in biomedical optics and photonics for applications in the clinic.
Executive Ballroom 210D

\section{CLEO: QELS - Fundamental Science}

10:30-12:15

FM2D - Attosecond and High-

field Technologies

Presider: Nirit Dudovich;

Weizmann Inst. of Science, Israel

FM2D.1 10:30 Invited

53 Attosecond X-ray Pulses Glancing Through the Water Window, Xiaoming Ren ${ }^{1}$, Jie Li' ${ }^{1}$ Yanchun Yin ${ }^{1}$ Kun Zhao ${ }^{2}$, Andrew Chew', Yan Cheng', Eric Cunningham ${ }^{1}$, Yang Wang ${ }^{1}, Y_{i} W^{1}{ }^{1}$, Michael Chini ${ }^{3}$, zenghu chang1,3. 'Univ. of Central Florida, CREOL, USA; ${ }^{2}$ CAS Inst. of Physics, China; ${ }^{3}$ Dept. of Physics, Univ. of Central Florida, USA. 53 attosecond $X$-ray pulses with photon energies up to $300 \mathrm{eV}$ are generated using polarization gated CEP-stable two-cycle pulses around $1.8 \mu \mathrm{m}$ and characterized using photoelectron streaking technique combined with the PROOF retrieval method.

\section{FM2D.2 • 11:00}

Time-Resolved X-ray Absorption Spectroscopy with a Water-Window High-Harmonic Source, Yoann Pertot' ${ }^{1}$, Jean-Pierre Wolf ${ }^{2}$ Hans Jakob Woerner'; ; ${ }^{1}$ ETH Zurich, Switzerland; ${ }^{2}$ Universite de Geneve, Switzerland. Femtosecond X-ray absorption spectroscopy is demonstrated at the carbon K-edge (290 $\mathrm{eV}$ ) and sulfur L-edges (180-240 eV) and applied to study the photodissociation of $\mathrm{CF}_{4}+$ and $\mathrm{SF}_{6}{ }^{+}$by element-specific core-to-valence transitions. 
Executive Ballroom 210E
Executive Ballroom

210F
Executive Ballroom

210G
Executive Ballroom

$210 \mathrm{H}$

\section{CLEO: QELS-Fundamental Science}

10:30-12:30

FM2E - Atomic Ensemble and Bulk Crystal Quantum Memories Presider: Benjamin Lev; Stanford Univ., USA

FM2E.1 • 10:30

Spatially Multimode Holographic Quantum Memory for Single and Multiple Photons Generation, Michal Dabrowski', Radoslaw Chrapkiewicz ${ }^{1,2}$, Wojciech Wasilewski ${ }^{1}$ 'Faculty of Physics, Univ. of Warsaw, Poland ${ }^{2}$ Stanford Univ., USA. We experimentally demonstrate $\mu$ s-lifetime holographic quantum memory capable of intrinsic generation storage and retrieval up to 60 photons, based on Raman interaction in warm rubidium-87 vapors. Such a multimode memory could practically enhance rates of single and multiple photons generation.

\section{FM2E.2 • 10:45}

QLad: A Noise-Free Quantum Memory for Broadband Light at Room Temperature, Joshua Nunn ${ }^{1}$, Krzysztof T. Kaczmarek ${ }^{1}$ Patrick M. Ledingham ${ }^{1}$, Benjamin Brecht ${ }^{1}$ Amir Feizpour1, Guillaume S. Thekkadath², Sarah E. Thomas', Joseph H. Munns', Dylan J. Saunders', Ian A. Walmsley'; ' 'Univ. of Oxford UK; ${ }^{2}$ National Research Council, Canada. We implement a low-noise, broadband quantum memory for light via off-resonant two-photon absorption in warm atomic vapour. We store heralded single photons and verify that the retrieved fields are anti-bunched.

\section{FM2E.3 • 11:00}

Storage of Ultra-Broadband Pulses in Hot Atomic Barium Vapor, BIN FANG ${ }^{1}$, Shuai Dong', Seth Meiselman², Offir Cohen², Virginia O. Lorenz'; ${ }^{1}$ Physics, Univ. of Illinois at Urbana-Champaign, USA; ${ }^{2}$ Physics, Univ. of Delaware, USA. We demonstrate the potential for an ultra-broadband quantum memory in hot atomic barium vapor using an off-resonance Raman interaction. It may enable storage of $\mathrm{THz}$-bandwidth photons for high-speed quantum information processing in the telecom range.
10:30-12:30

FM2F • Engineering Nonlinear

Materials

Presider: Kresten Yvind;

Danmarks Tekniske Universitet,

Denmark

FM2F.1 - 10:30 Invited

Engineered Nonlinearities in Transparent Conducting Oxides, Marcello Ferrera ${ }^{1}$ Matteo Clerici ${ }^{3}$, Nathaniel Kinsey², Clayton DeVault ${ }^{4}$, Jonngbum Kim ${ }^{4}$, Enrico Carnemolla ${ }^{1}$, Lucia Caspani ${ }^{5}$, Amr Shaltout ${ }^{4}$ Daniele Faccio ${ }^{1}$, Vladimir M. Shalaev ${ }^{4}$, Alexandra Boltasseva ${ }^{4}$; ${ }^{1}$ Heriot-Watt Univ., UK ${ }^{2}$ Virginia Commonwealth Univ., USA; ${ }^{3}$ Univ. of Glasgow, UK; ${ }^{4}$ Purdue Univ., USA; ${ }^{5}$ Univ. of Strathclyde, UK. Towards the fabrication of all-dielectric nanophotonic devices with tunable capabilities, we combined interband and intraband nonlinearities in aluminum-doped zinc oxide thin films thus enlarging the material bandwidth and gaining ultra-fast contro over the transmitted spectrum.

\section{FM2F.2 • 11:00}

Time-Resolved Nonlinear Refraction of Indium Tin Oxide at Epsilon Near Zero, Sepehr Benis ${ }^{1}$, Peng Zhao', Himansu Pattanaik1, David Hagan ${ }^{1}$, Eric Van Stryland ${ }^{1}$ ${ }^{1}$ CREOL, The College of Optics and Photonics, Univ. of Central Florida, USA. Using Beam-Deflection measurements we directly measure the temporal dynamics of nonlinear refraction and transmittance of an ITO thin film in the $\varepsilon$-near-zero regime, and find nonlinear index 5000 times that of glass.
10:30-12:30

FM2G • Photonic Topological Insulators

Presider: Mikael Rechtsman;

Pennsylvania State Univ., USA

FM2G.1 • 10:30 Invited

Photonic Topological Insulators in Two and Three Dimensions, Alexander B. Khanikaev'; ' ${ }^{1}$ City College of New York, USA. Magneto-electric coupling is shown to give rise to topological surface states in $2 \mathrm{D}$ and 3D systems with electromagnetic duality. The ability to control magneto-electric coupling locally enables reflectionless routing of electromagnetic states along arbitrarily shaped pathways.

FM2G.2 • 11:00

Artificial Gauge Fields and Topological Phenomena Through All-Dielectric Birefringence, Jonathan Nemirovsky', Yonatan Plotnik', Miguel Bandres ${ }^{1}$, Radoslaw Kolkowski' , Oded Zewi ${ }^{1}$, Mordechai Segev' ${ }^{1} T e c h n i o n$ Israel Inst. of Technology, Israel. We design tailored artificial optical gauge fields with ordinary dielectric birefringent materials. Using this method, we realize a wide range of phenomena: Quantum Hall effect, Haldane Topological-Insulators, Rashba effect and more.
10:30-12:30

$\mathrm{FM} 2 \mathrm{H} \bullet$ Plasmonic and

Nanophotonic Sensors, Switches, \& Photodetectors Presider: Nathalie de Leon; Princeton Univ., USA

FM2H.1 $10: 30$

Label-free Detection of Nucleic Acid Composition within DNA Strands using Surface-enhanced Raman Spectroscopy, Lindsay Freeman ${ }^{1}$, Lin Pang ${ }^{1}$, Yeshaiahu Fainman': 'Univ. of California, San Diego, USA We demonstrate label-free detection of the composition of a single strand of DNA using surface-enhanced Raman spectroscopy in which DNA is linearized to plasmonic surfaces and hundreds of Raman spectra are acquired and statistically analyzed.

\section{$\mathrm{FM} 2 \mathrm{H} .2 \cdot 10: 45$}

Active Metasurface Sensors for High Sensitivity Detection of the Concentration and Mid-Infrared Spectral Fingerprints of Biomolecules, Zhaoyi Li ${ }^{1}$ Yibo Zhu' ${ }^{1}$ James C. Hone ${ }^{1}$, Qiao Lin ${ }^{1}$, Nanfang Yu'; ${ }^{1}$ Columbia Univeristy, USA. We report biosensors based on tunable graphene plasmonic metasurfaces that enable high sensitivity detection of both the concentration and mid-infrared spectral fingerprints of human anti-body immunoglobulin (lgG).

\section{FM2H.3 • 11:00}

Optical Fractal Dimensional Analysis for Biosensing, Alexei I. Smolyaninov ${ }^{1}$, Yeshaiahu Fainman'; 'Univ. of California San Diego, USA. A novel optical biosensing scheme based on the interaction between the abbe limit and the optical power spectrum of a nanoscale fractal pattern in the Fourier plane is presented. 


\section{CLEO: Science \& Innovations}

10:30-12:30

SM2I • Ultrafast Pulse

\section{Generation}

Presider: Jose Azana; INRS-

Energie Materiaux et Telecom,

\section{Canada}

SM2I.1 • 10:30

Directly stabilized solitons in silicon-nitride microresonators, Chengying Bao ${ }^{1}$, Andrew Weiner ${ }^{1}$, Yi Xuan ${ }^{1}$, Daniel Leaird', Minghao Qi'; ' 'Purdue Univ., USA. We investigate soliton generation dynamics with the influence of thermal effects. Either soliton annihilation or survival can occur in different trials with the same tuning method, and a spontaneous route to soliton formation is observed.

\section{SM2I.2 • 10:45}

Optical Linewidth and RF Phase Noise Reduction of a Chip-scale CPM Laser Using COEO Multi-tone Injection Locking, Ricardo Bustos Ramirez', Michael Plascak', Kristina Bagnell1, Ashish Bhardwaj2, James Ferrara², Gloria Hoefler ${ }^{2}$, Ming C. Wu ${ }^{3}$, Peter J. Delfyett'; ' $C R E O L$, The College of Optics \& Photonics, USA; ${ }^{2}$ Infinera Corporation, USA ${ }^{3}$ Electrical Engineering and Computer Science, Univ. of California at Berkeley, USA. A novel method of regenerative mode-locking using multi-tone optical injection locking of a chip-scale CPM laser is demonstrated. This technique achieves simultaneous reduction in RF phase noise (8x) and narrows the longitudinal mode by $4,000 x$.

\section{SM2I.3 • 11:00}

High-Dynamic-Range Relative Arrival Time Measurement for Accurate and Precise Parametric Waveform Synthesis, Giulio Maria Rossi ${ }^{1,2}$, Roland E. Mainz ${ }^{1,2}$, Giovanni Cirmi ${ }^{1,2}$, Yudong Yang ${ }^{1,2}$, Oliver Muecke ${ }^{1,2}$, Franz Kaertner ${ }^{1,2}$; 'DESY' - CFEL, Germany; ${ }^{2}$ Physics, Center for Ultrafast Imaging, Hamburg Univ., Germany. We introduce an all-inline scheme for relative timing measurement of ultrabroadband pulses, featuring FPGA-based detection/feedback. High common-mode rejection and long-term stability were validated by out-of-loop measurement, paving the way for ultrabroadband optical parametric waveform synthesis.
10:30-12:30

SM2J • THz Quantum Optics and Metamaterials

Presider: Dmitry Turchinovich; Max Planck Inst. for Polymer Research, Germany

\section{SM2J.1 • 10:30 Tutorial}

Time-domain Quantum Electrodynamics, Alfred Leitenstorfer ${ }^{1} ;{ }^{1}$ Univ. of Konstanz, Ger many. Ultrabroadband electro-optic sampling with few-femtosecond laser pulses allows direct detection of electric-field vacuum fluctuations. Subcycle analysis of multi-terahertz squeezed light is introduced as a first application of this new type of quantum metrology.

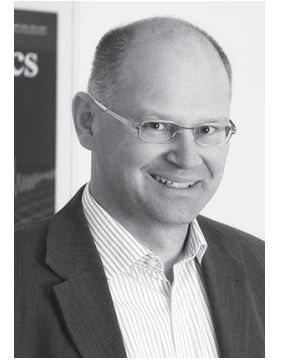

Alfred Leitenstorfer is Professor of Experimental Physics and heads the Center for Applied Photonics at University of Konstanz, Germany. His research covers fundamenta dynamics and quantum phenomena of light and matter as well as femtosecond lasers and terahertz technology.
10:30-12:30

SM2K • Micro and Nanoscale

Fabrication

Presider: Michael Menard;

UQAM, Canada

SM2K.1 • 10:30

Low-Loss Ge-on-GaAs Platform for Mid-Infrared Photonics, Hsien-Yu Liao', Seungyong Jung ${ }^{1}$ Swapnajit Chakravarty ${ }^{2}$, Ray T. Chen ${ }^{1,2}$ Mikhail A. Belkin'; 'The Univ. of Texas at Austin, USA; ${ }^{2}$ Omega Optics Inc, USA. Low-loss photonic platform that can span the entire mid-infrared spectral region is reported based on the Ge-on-GaAs wafers. Optical loss of approximately $4.2 \mathrm{~dB} / \mathrm{cm}$ is measured in ridge-waveguides at $10 \mu \mathrm{m}$ wavelength.

\section{SM2K.2 • 10:45}

Stable Lasing from Perovskite $\mathrm{CsPb}_{2} \mathrm{Br}_{5} \mathrm{Mi}$ croplate, Juan $\mathrm{Du}^{1}$, Zhiping $\mathrm{Hu}^{2}$, Zhengzheng Liu', Xiaosheng Tang', Yuxin Leng'; ' 'Shanghai Inst of Optics and Fine Mech, China; ${ }^{2}$ Chongqing Univ., China. We reported the low-threshold and stable lasing performance from a new type of all-inorganic perovskite $\mathrm{CsPb}_{2} \mathrm{Br}_{5}$ microplate with superior crystallization, enhanced stability, and tunable optical properties under both one- and two-photon excitation.

\section{SM2K.3 • 11:00 Invited}

3D Laser Printing for Photonics: Recent Progress, Martin Wegener'; ${ }^{1}$ Karlsruhe Inst. of Technology, Germany. We review our recent progress on $3 \mathrm{D}$ laser nanoprinting. Examples are masters for freeform surfaces made by dip-in shell writing to make contacts on solar cells invisible and multi-material bio-scaffolds.
10:30-12:15

SM2L • Multiwavelength and Comb Fiber Sources

Presider: Kazi Abedin; OFS Labs, USA

\section{SM2L.1 - 10:30 Invited}

Ultra-low Noise Robust Er Fiber-based Optical Frequency Combs with a Graphene Modulator, Naoya Kuse ${ }^{2}$, Noriaki Ohmae ${ }^{3,4}$ Chien-Chung Lee ${ }^{5}$, Thomas R. Schibli5, $\mathrm{Hi}_{-}$ detoshi Katori3,4, Martin E. Fermann': ${ }^{1}$ IMRA America Inc, USA; ${ }^{2}$ IMRA America, BRL, USA ${ }^{3}$ RIKEN, Japan; ${ }^{4}$ The Univ. of Tokyo, Japan; ${ }^{5}$ Univ. of Colorado, USA. An ultra-low noise al polarization-maintaining Er fiber comb based on nonlinear amplifying mirror was demonstrated. Instability of the frequency transfer was evaluated at optical clock wavelengths.
SM2L. $2 \bullet 11: 00$

Optical frequency synthesizer based on a fully stabilized $750-\mathrm{MHz} \mathrm{Yb}$ fiber lase frequency comb, Bo Xu ${ }^{1,2}$, Hideaki Yasui ${ }^{1,2}$ Thomas R. Schibli ${ }^{3}$, Kaoru Minoshima ${ }^{1,2}$. 'The Univ. of Electro-Communications, Japan; ${ }^{2}$ ERATO MINOSHIMA Intelligen Optical Synthesizer Project, Japan Science and Technology Agency, Japan; ${ }^{3}$ Physics, Univ. of Colorado at Boulder, USA. We developed a microwave-referenced optical single frequency generator based on a fully stabilized, self-referenced 750-MHz Yb fiber laser frequency comb. The device could continuously generate the optical frequencies and the tuning speed reaches $60 \mathrm{GHz} / \mathrm{s}$. 


$\begin{array}{llc}\text { Marriott } & \text { Marriott } & \text { Marriott } \\ \text { Salon III } & \text { Salon IV } & \text { Salon V \& VI }\end{array}$

\section{CLEO: Science \& Innovations}

10:30-12:30

$\mathrm{SM} 2 \mathrm{M} \cdot$ Nonlinear Dynamics and Harmonic Generation Presider: Shu-Wei Huang; UCLA Engineering IV, USA

\section{SM2M.1 10:30 Invited}

Topology Optimization in Nonlinear Nanophotonics: from Frequency Conversion to Exceptional Points, Alejandro Rodriguez'; 'Princeton Univ., USA. We exploit topology optimization to design complex nanophotonic structures (new kinds of micropillars, photonic-cyrstal slabs, and waveguides), with applications to efficient nonlinear frequency conversion and dualpolarization, dual-wavelength, and highly degenerate Dirac cones, with implications to zero-index metamaterials and exceptional points.
10:30-12:30

SM2N • Whispering Gallery

Mode Micro-cavities

Presider: Xiaoge Zeng; Hewlett

Packard Labs, USA

SM2N.1 • 10:30

Adiabatic frequency conversion in an ultrahigh-O silica microcavity using the Kerr effect, Wataru Yoshiki' Y Yoshihiro Honda', Misako Kobayashi', Tomohiro Tetsumoto', Takasumi Tanabe'; ${ }^{1}$ Keio Univ., Japan. We experimentally demonstrate adiabatic frequency conversion in an ultra-high-Q silica toroid microcavity using the Kerr effect. We show that the amount of conversion, conversion time width, and number of conversions can be freely controlled.

SM2N.2 $10: 45$

Demonstration of all-optical tunable buffering using coupled ultra-high- $\mathrm{Q}$ silica toroid microcavities, Wataru Yoshiki', Yoshihiro Honda', Tomohiro Tetsumoto', Kentaro Furusawa ${ }^{2}$, Norihiko Sekine ${ }^{2}$, Takasumi Tanabe ${ }^{1}$. ${ }^{1}$ Keio Univ., Japan; ${ }^{2}$ Advanced ICT Research Inst., National Inst. of Information and Communications Technology, Japan. We describe the first experimental demonstration of alloptical tunable buffering with coupled silica toroid microcavities. We prove that a $10-n s$ optical pulse can be buffered for 20 ns thanks to the microcavities' ultra-high $Q$ factor.

SM2N.3 $111: 00$

SM2M.2 • 11:00

Waveform Dynamics in Air-slot Photonic Crystal Optomechanical Oscillators, Jiagui $\mathrm{Wu}^{1}{ }^{1,2}$, Shu-Wei Huang ${ }^{2}$, Yongjun Huang ${ }^{3}$, Hao Zhou ${ }^{2}$, Mingbin Yu ${ }^{4}$, Guogiang Lo ${ }^{4}$, Dim-Lee Kwong ${ }^{4}$, Shukai Duan', Cheewei Wong'; ${ }^{1}$ Southwest Univ., College of Electronic and Information Engineering, China: ${ }^{2}$ Univ. of California, Los Angeles, Fang Lu Mesoscopic Optics and Quantum Electronics Lab, USA; ${ }^{3}$ Univ. of Electronic Science and Technology of China, School of Communication and Information Engineering, China; ${ }^{4} A^{\star} S T A R$, Inst. of Microelectronics, Singapore. We study experimentally and theoretically the use of photonic crystal nano-cavity to generate a broad range of waveforms, mediating by Drude electron-hole plasma in silicon, and coming from the dynamical states at varying operating conditions.
10:30-12:30

SM2O • Modulators

Presider: Qiaogiang Gan; State

Univ. of New York at Buffalo, USA

SM2O.1 • 10:30

An Integrated Racetrack Colliding-Pulse Mode-Locked Laser with Pulse-Picking Modulator, Ashish Bhardwaj ${ }^{1}$, James Ferrara', Ricardo Bustos Ramirez ${ }^{2}$, Michael Plascak², Gloria Hoefler', Vikrant Lal ${ }^{1}$, Fred Kish ${ }^{1}$, Peter J. Delfyett ${ }^{2}$, Ming C. Wu ${ }^{3}$. ${ }^{1}$ Infinera Corporation, USA; ${ }^{2}$ CREOL, College of Optics and Photonics, Univ. of Central Florida, USA; ${ }^{3}$ Dept. of Electrical Engineering and Computer Science, Univ. of California at Berkeley, USA. We present a novel racetrack colliding-pulse mode-locked laser with external pulse-picking and optical amplification monolithically integrated on InP. Optical pulses with FWHM of 2.36 ps are observed under hybrid mode-locking at $10 \mathrm{GHz}$ repetition rate.

SM2O.2 $10: 45$

Over 10-Gbit/s Pulsed RZ-OOK Wavelength and Format Switching in TwoPhoton-Absorption-Free SiC Waveguide, Bo-Ji Huang ${ }^{1}$, Chung-Lun Wu', Chih-Hsien Cheng' ${ }^{1}$ Yung-Hsiang Lin' ${ }^{1}$ Huai-Yung Wang ${ }^{1}$, Cheng-Ting Tsai', Yu-Chieh Chi', Gong-Ru Lin'; ${ }^{1}$ National Taiwan Univ., Taiwan. Alloptical wavelength and format conversion of pulsed return-to-zero on-off-keying data in two-photon-absorption-free carbon-rich silicon carbide ring waveguide with enhanced nonlinear Kerr switching effect at 12 Gbits/s is demonstrated with an extinction ratio of $20 \mathrm{~dB}$.

\section{SM20.3 - 11:00 Invited}

A C-band Push-pull Dual-ring Silicon Photonic Modulator for $20 \mathrm{~km}$ SSMF transmission without $C D$ compensation, Rui $\mathrm{Li}^{1}$, David Patel', Eslam El-Fiky', Alireza Samani', Zhenping Xing', Luhua Xu', David V. Plant'; ${ }^{1}$ McGill Univ., Canada. We experimentally present a C-band push-pull dual-ring silicon photonic modulator operating at $60 \mathrm{~Gb} / \mathrm{s}$ in B2B configuration. Without CD compensation, $23 \mathrm{~Gb} / \mathrm{s}$ transmission over $20 \mathrm{~km}$ of SSMF with measured BER below $3.8 \times 10^{-3}$ is demonstrated. 
Executive Ballroom

$210 \mathrm{~A}$

Executive Ballroom

210B

\section{CLEO: Applications \\ \& Technology}

AM2A • A\&T Topical Review on Advances in Laser-based Remote Sensing II-Continued

AM2A.3 • 11:30

Cavity attenuated phase shift Faraday rotation spectroscopy, Charles L. Patrick', Jonas Westberg', Gerard Wysocki'; 'Princeton, USA. A cavity attenuated phase shift Faraday rotation spectrometer has been developed for oxygen detection near $762 \mathrm{~nm}$. The system incorporates a high-finesse cavity for sensitivity enhancement and achieves minimum detectible polarization rotation of $2 \times 10^{-9} \mathrm{rad} / \sqrt{\mathrm{Hz}}$.

AM2B.3 • 11:15

\section{AM2B.4 • 11:30} hanced Infrared Response Using Indium-Tin Oxide Nanorods, Lung-Hsing $\mathrm{Hsu}^{1}$, Yuh-Jen Cheng ${ }^{2}$, Peichen $\mathrm{Yu}^{1}$, Hao-chung Kuo ${ }^{1}$, Chien Chung Lin'; ${ }^{1}$ National Chiao-Tung Univ. Taiwan; ${ }^{2}$ Academia Sinica, Taiwan. Enhanced infrared photoresponse is observed in $\ln N$ pillars/ITO rods photodetectors fabricated by LP-MOCVD and oblique-angle electron beam evaporation. The enhanced IR portion photocurrent as high as $19 \%$ can be measured via AM1.5G solar simulated spectra.

Interband Cascade LEDs with Split Active Stages, William W. Bewley ${ }^{1}$, Chul Soo Kim¹, Mijin Kim², Chadwick L. Canedy ${ }^{1}$, Michae V. Warren ${ }^{3}$, Charles. Merritt ${ }^{1}$, Stephanie Tomasulo', Igor Vurgaftman', Jerry R. Meyer' Naval Research Lab, USA; ${ }^{2}$ Sotera Defense Solutions, USA; ${ }^{3}$ ASEE Fellow Residing at Naval Research Lab, USA. Midwave infrared ICLEDs with split active stages positioned at antinodes of the optical electric field are shown to generate up to $1.86 \mathrm{~mW}$ of output power and $0.3 \%$ wallplug efficiency in $\mathrm{CW}$

\section{AM2B.5 • 11:45}

AM2A.4 11:45 Invited

The OSIRIS-REx Laser Altimeter, Michae Daly ${ }^{1}$, Oliver Barnouin ${ }^{2}$, Catherine Johnson ${ }^{3}$ Cameron Dickinson ${ }^{4}$, Timothy Haltigin ${ }^{5}$ Dante Lauretta6; ${ }^{1}$ York Univ., Canada; ${ }^{2} A p$ plied Physics Lab, Johns Hopkins Univ. , USA ${ }^{3}$ Univ of British Columbia, Canada: ${ }^{4} \mathrm{MDA}$ Canada; ${ }^{5}$ Canadian Space Agency, Canada; ${ }^{6}$ Univ. of Arizona, USA. The OSIRIS-REx Laser Altimeter (OLA) is a scanning laser altimete onboard the NASA mission to the near-Earth asteroid 101955 Bennu. We will describe the operation and unique capabilities of the instrument for an asteroid mission.

\section{AM2B • Mid-IR Sensors and} Emitters-Continued
InN Nanopillar Photodetector with En operation at $25^{\circ} \mathrm{C}$.

Executive Ballroom $210 \mathrm{C}$

\section{CLEO: Science \&} Innovations

SM2C $•$ Biomedical Spectroscopy and Cell/Particle Analysis-Continued

SM2C.2・11:30

Ptychography for Nonlinear Optical Microscopy: Retrieving Phase without Interferometry, Jarno N. van der Kolk'1, Lora Ramunno': 'Univ. of Ottawa, Canada. The nonlinear susceptibility phase is obtained by adapting ptychography to nonlinear optical microscopy. We develop a reconstructive technique to retrieve phase from far-field diffractive images, obtaining enhanced resolution despite distorted input beams.

SM2C. $3 \cdot 11: 45$

Automated Detection and Enumeration of Waterborne Pathogens Using Mobile Phone Microscopy and Machine Learning Hatice Ceylan Koydemir', Steve Feng ${ }^{1}$, Kyle Liang ${ }^{1}$, Derek Tseng' ${ }^{1}$, Rohan Nadkarni' ${ }^{1}$, Paru Benien', Aydogan Ozcan'; ' UCLA, USA. We present a field portable and cost-effective smartphone based microscopy platform for rapid and sensitive detection and automated counting of waterborne pathogens, i.e. Giardia lamblia cysts, in large volume water samples using machine learning.

SM2C. $4 \cdot 12: 00$

AM2B.6 $\bullet 12: 00$

FPGA Locking to Acetylene (C2H2) Hyperfine Structure, Fatemeh Yazdandoust Herve Tatenguem Fankem ${ }^{1}$, Tobias Milde ${ }^{1}$ Marc Strohwald ${ }^{1}$, Alvaro Jimenez ${ }^{1}$, Christian Assmann ${ }^{1}$, Niklas Staacke', Joachim Sacher ${ }^{1}$. 'Sacher Lasertechnik GmbH, Germany. A $1530 \mathrm{~nm}$ high power tunable diode laser system with FPGA locking to acetylene hyperfine structure is presented. The system is locked to the hyperfine structure of the R5 state of acetylene with excellent long term stability.
Executive Ballroom 210D

CLEO: QELS Fundamental Science

FM2D - Attosecond and Highfield Technologies-Continued

FM2D.3 • 11:15

Two-cycle, 2.5 TW pulse generation at 1.8 $\mu \mathrm{m}$ via Frequency domain Optical Parametric Amplification, Vincent Gruson 1,2, Guilmo Ernotte ${ }^{1}$, Philippe Lassonde ${ }^{1}$, Lou DiMauro² Paul B. Corkum ${ }^{3}$, Heide Ibrahim¹, Bruno Schmidt ${ }^{1,4}$, Francois Legare ${ }^{1} ;{ }^{1}$ INRS-EMT Canada; ${ }^{2}$ Dept. of Physics, The Ohio State Univ., USA; ${ }^{3}$ Univ. of Ottawa, Canada; ${ }^{4} \mathrm{Few}$ Cycles, Canada. A non-collinear Frequency domain Optical Parametric Amplifier is used to produce a $1.8 \mu \mathrm{m}, 30 \mathrm{~mJ}, 13 \mathrm{fs}$ laser source, leading to $2.5 \mathrm{TW}$ peak power. This laser opens the way for high brightness soft $\mathrm{X}$-ray attosecond pulses.

\section{FM2D.4 • 11:30}

Intense attosecond soft $x$-ray pulse by a high-energy three-channel waveform synthesizer, Bing Xue ${ }^{1}$, Eiji J. Takahashi ${ }^{1}$, Yux $\mathrm{Fu}^{1}$, Katsumi Midorikawa'; ${ }^{1} \mathrm{RIKEN}$, Japan We report on the generation of high-flux continuum harmonics using a three-channe waveform synthesizer. The soft-x-ray supercontinuum supporting $<300$ as isolated pulses at $48 \mathrm{eV}$ is generated. The continuum soft-x-ray energy is evaluated to $>100 \mathrm{~nJ}$.

\section{FM2D.5 • 11:45}

Faraday Rotation Probe of Laser-Plasma Bubble Structures in Petawatt-Driven Wakes, Yen-Yu Chang ${ }^{1}$, Joseph Shaw ${ }^{1}$, James R. Welch ${ }^{1}$, Kathleen Weichman ${ }^{1}$, Andrea Hannasch', Max LaBerge', Watson Henderson' Rafael Zgadzaj', Aaron Bernstein ${ }^{1}$, Craig Wagner $^{1}$, Joe Gordon', Michael Martinez ${ }^{1}$ Michael Spinks' ${ }^{1}$, Toma Toncian', Gilliss Dyer ${ }^{1}$ Erhard W. Gaul' ${ }^{1}$, Michael Donovan', Todd Ditmire ${ }^{1}$, Michael Downer ${ }^{1} ;{ }^{1}$ Univ. of Texas at Austin, USA. We exploited the Faraday effect to imprint polarization shifts on a transverse probe that captured the structure of a $\mathrm{GeV}$ laser-plasma accelerator. Our measurements suggested a plasma bubble diameter was around $50.8 \pm 10.1$ um.

FM2D.6 • 12:00

Mid-IR, $\mathrm{CO}_{2}$-Laser driven, Self-Modulated Wakes, James R. Welch'; ' Univ. of Texas a Austin, USA. We report a study of self-modulated laser plasma wakefields, at densities down to $\sim 5 \times 10^{17} \mathrm{~cm}^{-3}$, driven by the mid IR (lambda $=10.6 \mathrm{um}$ ), 4ps long, 2-Joule laser pulses from the BNL/ATF $\mathrm{CO}_{2}$ laser. 

Executive Ballroom
$210 \mathrm{E}$
Executive Ballroom 210F
Executive Ballroom

210G
Executive Ballroom

$210 \mathrm{H}$

\section{CLEO: QELS-Fundamental Science}

FM2E - Atomic Ensemble and Bulk Crystal Quantum Memories-Continued

\section{FM2E.4 • 11:15}

Highly Efficient and Long-lived Optical Quantum Memory with Cold Atoms, Young-Wook Cho', G. T. Campbell', J. L. Everett' ${ }^{1}$ J. Bernu', D. Higginbottom ${ }^{1}$ M. T. Cao', J. Geng', N. P. Robins' ${ }^{2}$, P. K. Lam', B. C. Buchler'; ' Centre for Quantum Information, Dept. of Quantum Science, The Australian National Univ., Australia; ${ }^{2}$ Dept. of Quantum Science, The Australian National Univ., Australia. We report a highly efficient coherent optical memory via the gradient echo memory (GEM) technique in cold atoms. The efficiency is as high as $87 \%$ with the e-1 coherence time of $1 \mathrm{~ms}$. The ability of quantum storage is verified using heterodyne tomography of small coherent states.

\section{FM2E.5 • 11:30 Invited}

A Quantum Light-Matter Beamsplitter in Diamond, Duncan England' ${ }^{1}$, Kent Fisher ${ }^{2}$ Jean-Philippe MacLean², Khabat Heshami', Philip Bustard', Kevin Resch², Ben Sussman 1,3, 'National Research Council, Canada; ${ }^{2}$ Inst. for Quantum Computing, Univ. of Waterloo Canada; ${ }^{3}$ Dept. of Physics, Univ. of Ottawa, Canada. A quantum memory can be viewed as a light-matter beam-splitter, mapping a photon to a superposition of the output optical mode and stored mode. We use this mechanism to demonstrate non-classical one-photon and two-photon interference.

\author{
FM2F • Engineering Nonlinear \\ Materials-Continued
}

FM2G • Photonic Topological Insulators-Continued

FM2F.3 • 11:15

Electrically Tunable Optical Nonlinearity of Graphene-covered SiN waveguides, Koen Alexander ${ }^{1,2}$, Muhammad Mohsin ${ }^{3}$ Utsav D. Dave ${ }^{1,2}$, Leili Abdollahi shiramin ${ }^{1,2}$ Stéphane Clemmen ${ }^{1,2}$, Daniel Neumaier ${ }^{3}$, Bart Kuyken ${ }^{1,2}$, Dries Van Thourhout 1,2; 1 Photonics Research Group, INTEC, Ghent Univ. Belgium; ${ }^{2}$ Center for Nano-and Biophotonics (NB-Photonics), Ghent Univ., Belgium ${ }^{3}$ Advanced Microelectronic Center Aachen, AMO GmbH, Germany. Electrical tunability of the optical nonlinearity of graphene is demonstrated on a SiN platform using four-wave mixing. The nonlinearity $y$ of the graphenecovered waveguide more than doubles when tuning $E_{F}$ to the vicinity of $-h v / 2$.

\section{FM2F.4 • 11:30}

Enhanced second-harmonic generation from two-dimensional $\mathrm{MoSe}_{2}$ by waveguide integration, Haitao Chen', Vincent Corboliou², Alexander S. Solntsev', Duk-Yong Choi $^{1}$, Maria a. Vincentiti,4, Domenico Ceglia ${ }^{3}$, Costantino Angelis ${ }^{4}$, Yuerui Lu', Dragomi N.Neshev1: ${ }^{1}$ Australian National Univ., Australia; ${ }^{2}$ Université PAris Sud, France; ${ }^{3}$ National Research Council, USA; ${ }^{4}$ Univ. of Brescia, Italy. We demonstrate enhanced second-harmonic generation from a monolayer $\mathrm{MoSe}_{2}$ through Si waveguide integration. This is achieved by exciting the monolayer through the guided mode, which dramatically increases the interaction length and allows for phase matching.

FM2F.5 • 11:45

Comparison of surface and bulk contributions to SHG in meta-atoms made of centrosymmetric materials, Daniel Timbrell ${ }^{1}$ Jian Wei You', Yuri Kivshar², Nicolae C. Panoiu'; 'Univ. College London, UK; ${ }^{2}$ Australian National Univ., Australia. We analyze the contributions of surface and bulk effects to second-harmonic generation from crosses made of centrosymmetric dielectric and metallic materials and demonstrate that bulk and surface effects in dielectric structures can be comparable.
FM2G.3 • 11:15

Molding the Spin Flow of Light in Valley Photonic Crystals, Xiaodong Chen ${ }^{1}$, Jianwen Dong ${ }^{1,2}$, Hanyu Zhu'2, Yuan Wang ${ }^{2}$, Xiang Zhang ${ }^{2} ;{ }^{1}$ Sun Yat-sen Univ., China; ${ }^{2}$ Univ. of California, USA. We show the manipulation of spin flow of light in valley photonic crystals. Due to the coupled valley spin physics, both photonic valley Hall effect and unidirectional spin flow are realized in bulk crystal.

\section{FM2G.4 • 11:30}

Temporal Defects in Photonic Topological Insulators, Christina I. Jörg ${ }^{1}$, Fabian Letscher ${ }^{1,2}$, Michael Fleischhauer ${ }^{1}$, Georg von Freymann 1,3; 'Physics Dept. and Research Center OPTIMAS, Univ. of Kaiserslautern, Germany; ${ }^{2}$ Graduate School Materials Sci ence in Mainz, Germany; ${ }^{3}$ Fraunhofer-Inst. for Physical Measurement Techniques (IPM), Germany. We experimentally study timedependent defects in a waveguide model of a topological insulator. Backscattering is not observed, but in contrast to static defects, edge modes propagate through the defects.

\section{FM2G.5 • 11:45}

Realization of Photonic Anomalous Floquet Topological Insulators, Lukas Maczewsky ${ }^{1}$ Julia Zeuner ${ }^{1}$, Stefan Nolte', Alexander Szameit ${ }^{2,1}$; ${ }^{1}$ Friedrich-Schiller-Universität Jena, Germany; ${ }^{2}$ Inst. of Physics, Universität Rostock, Germany. We realize the first observation of a photonic anomalous Floquet insulator in the waveguide regime. In contrast to the common understanding, the system exihibts topological edge modes despite vanishing Chern number of all bands.
$\mathrm{FM} 2 \mathrm{H} \bullet$ Plasmonic and Nanophotonic Sensors, Switches, \& PhotodetectorsContinued

\section{FM2H.4 • 11:15}

High-Contrast, All-Optical Switching of Infrared Light using a Cadmium Oxide Perfect Absorber, Yuanmu Yang', Kyle Kelley², Edward Sachet ${ }^{2}$, Salvatore Campione ${ }^{1}$, Ting S. Luk', Jon-Paul Maria ${ }^{2}$, Igal Brener'; ' ${ }^{1}$ Sandia National Labs, USA; ${ }^{2}$ North Carolina State Univ., USA. We experimentally demonstrate high-contrast, ultrafast switching of infrared light at $2.1 \mu \mathrm{m}$ via intraband pumping of a high quality factor perfect absorber made from a highly doped cadmium oxide thin film.

\section{FM2H.5 • 11:30}

Sub-Picosecond All-Optical Switching of Tamm Plasmons in Photonic Crystals, Boris Afinogenov', Vladimir Bessonov', Andrey Fedyanin ${ }^{1}$; ${ }^{1}$ Lomonosov Moscow State Univ., Russia. Photoinduced change in metal permittivity leads to a spectral shift of the Tamm plasmon resonance excited in a photonic crystal/metal sample. Proper selection of a probe wavelength allows observing a 200 fs-long reflectance modulation.
FM2E.6 6 12:00

Single Photon Generation Using Raman Transitions in Sapphire, Daniel A. Inafuku' Kai Shinbrough ${ }^{2}$, BIN FANG ${ }^{1}$, Virginia $O$. Lorenz'; ' Univ. of Illinois, USA; ${ }^{2}$ Oberlin College, USA. We implement the DuanLukin-Cirac-Zoller protocol to produce single photons using Raman scattering in sapphire. We present data of the Stokes/anti-Stokes cross-correlation with a value of $32 \pm 5$.
FM2F.6 • 12:00

Cross-Interaction of Quadratic and Cubic Nonlinearities in Four-Wave Mixing in GaAs at $10 \mu \mathrm{m}$, Daniel A. Matteo', Jeremy Pigeon', Sergei Tochitsky', Chandrashekhar Joshi'; ' ${ }^{1}$ Dept. of Electrical Engineering, Univ. of California at Los Angeles, USA. We have measured the efficiency of collinear four-wave mixing of $\mathrm{CO}_{2}$ laser beat-waves in $\mathrm{GaAs}$ for different crystal orientations. We deduce significant contribution of cascaded difference frequency generation to the effective nonlinear refractive index.
FM2G.6 • 12:00

Prediction and Realization of a Photonic Topological Phase Transition, Jonathan Guglielmon ${ }^{1}$, Sheng Huang ${ }^{2}$, Kevin P. Chen ${ }^{2}$ Mikael C. Rechtsman'; 'The Pennsylvania State Univ., USA; ${ }^{2}$ Univ. of Pittsburgh, USA. We predict a topological phase transition for paraxial light propagating through an array of evanescently-coupled helical waveguides. As a result of the transition, we observe a topological edge mode reverse its transverse propagation direction.

\section{FM2H.6 • 11:45}

GaAs/AlGaAs Core-shell Ensemble Nanowire Photodetectors, Fajun Li ${ }^{1,2}$, Liying Tan ${ }^{1}$, Ziyuan $\mathrm{Li}^{2}$, Jing $\mathrm{Ma}^{1}$, Lan $\mathrm{Fu}^{2}$, Hark Hoe $\operatorname{Tan}^{2}$, Chennupati Jagadish ${ }^{2} ;{ }^{1}$ Harbin Inst. of Technology, China; ${ }^{2}$ The Australian National Univ., Australia. We report the growth of GaAs/AlGaAs core-shell nanowire ensembles grown on $\mathrm{p}$ - and $\mathrm{n}$-doped GaAs substrate respectively and the fabrication of these nanowire photodetectors. The I-V characteristics and spectral response of both detectors were investigated by comparison.
FM2H.7 • 12:00

Actively-Tunable Plasmonic Metasurfaces Using a Phase-Change Material, Andrew Boyce $^{1,2}$, Jon Stewart ${ }^{1,2}$, Virginia Wheeler ${ }^{4}$ Maiken Mikkelsen,3; ${ }^{1}$ Electrical and Computer Engineering, Duke Univ., USA; ${ }^{2}$ Center for Metamaterials and Integrated Plasmonics, Duke Univ., USA; ${ }^{3}$ Dept. of Physics, Duke Univ., USA; ${ }^{4}$ U.S. Naval Research Lab, USA We demonstrate active tuning of the absorption resonance of a plasmonic metasurface by integrating $\mathrm{VO}_{2}$, a phase change material. Thermal switching of the resonance yields shifts close to the metasurface's linewidth. 


\section{SM2I • Ultrafast Pulse Generation-Continued}

\section{SM2I.4 • 11:15}

Energy-Scalable, 150-fs Fiber Source Seeded by a Gain-Switched Diode, Walter P. Fu' ${ }^{1}$ Logan Wright ${ }^{1}$, Frank W. Wise ${ }^{1} ;{ }^{1}$ Cornell Univ., USA. We generate 150 -fs pulses from a fiber system based on a gain-switched diode. A combination of nonlinear spectral-temporal filtering and amplification by parabolic preshaping compresses 14-ps pulses by nearly 100 -fold with transform-limited pulse quality.

\section{SM2I.5 • 11:30}

Ultrafast laser mode-locked using Nonlinear Polarization Evolution in Polarization Maintaining fibers, Jan Szczepanek', Tomasz M. Kardas ${ }^{2}$, Michal Nejbauer ${ }^{1}$, Czeslaw Radzewicz', Yuriy Stepanenko ${ }^{2}$; ${ }^{1}$ Faculty of Physics, Univ. of Warsaw, Poland; ${ }^{2}$ Inst. of Physical Chemistry Polish Academy of Sciences, Poland. We demonstrate an ytterbium laser oscillator mode-locked by means of Nonlinear Polarization Evolution realized in Polarization Maintaining (PM) fibers. The all-PM-fiber laser generates 150 fs pulses at $20.54 \mathrm{MHz}$ repetition rate.

\section{SM2J • THz Quantum Optics and Metamaterials-Continued}

\section{SM2K • Micro and Nanoscale Fabrication-Continued}

\section{SM2L • Multiwavelength and Comb Fiber Sources- Continued}

SM2L.3 • 11:15

Octave-spanning dual-comb spectroscopy with a free-running bidirectional modelocked femtosecond fiber laser, $\mathrm{Yi}-\mathrm{H} \sin \mathrm{N}$ Ou', Joshua Olson', Soroush Mehravar', Robert A. Norwood', Nasser Peyghambarian ${ }^{1}$ Khanh Q. Kieu'; 'College of Optical Science, Univ. of Arizona, USA. We demonstrate an octave-spanning laser source for dual-comb spectroscopy (DCS). Two optical frequency combs (OFCs) are generated from a single laser cavity and frequency broadened to cover over an octave for broadband single shot DCS.

SM2J.2 • 11:30

Characterization of Switchable Terahertz Metasurfaces, Nicholas Karl ${ }^{1}$, Martin S Heimbeck ${ }^{2}$, Henry Everitt ${ }^{2}$, Houtong Chen ${ }^{3}$, Antionette Taylor ${ }^{3}$, Alex Benz ${ }^{4}$ John Reno ${ }^{4}$ Igal Brener ${ }^{4}$, Rajind Mendis', Daniel M Mittleman'; ' 'Brown Univ., USA; ${ }^{2}$ U.S. Army AMRDEC, Redstone Arsenal, USA; ${ }^{3}$ CINT Los Alamos National Lab, USA: ${ }^{4} \mathrm{CINT}$ Sandia National Labs, USA. We perform experimental characterization of switchable $\mathrm{THz}$ metasurfaces using variable-angle broadband $\mathrm{THz}$ ellipsometry. We extract frequency dependent surface susceptibility tensors and model the applied DC bias as tuning the conductivity of the active layer.

\section{SM2I.6 • 11:45}

Generation of $65-\mathrm{fs}$ pulses at $2 \mu \mathrm{m}$ in an all-fiber laser, Biao Sun ${ }^{1}$, Jiagi Luo ${ }^{1,2}$, Junhua $\mathrm{Ji}^{1}$, Xia Yu'; ' Precision measurement group, SIMTech, A-STAR, Singapore; ${ }^{2}$ School of Electrical and Electronic Engineering, Nanyang Technological Univ., Singapore. We demonstrate for the first time a sub-100 fs all-fiber laser at $2 \mu \mathrm{m}$. The output pulse can be as short as $65 \mathrm{fs}$ with a spectral bandwidth of $\sim 160 \mathrm{~nm}$ via nonlinear compression.

\section{SM2I.7 • 12:00}

Mode-locking via Dissipative Faraday Instability in a Fiber Laser, Nikita Tarasov ${ }^{1,2}$ Auro Perego', Dmitry V. Churkin ${ }^{2,3}$, Kestutis Staliunas ${ }^{4,5}$, Sergei Turitsyn ${ }^{1,3} ;{ }^{1}$ Aston Univ., UK; ${ }^{2}$ Inst. of Computational Technologies, Russia: ${ }^{3}$ Novosibirsk State Univ., Russia ${ }^{4}$ Departament de Física i Enginyeria Nuclear, Universitat Politècnica de Catalunya, Spain; ${ }^{5}$ Institució Catalana de Recerca i Estudis Avançats, Spain. In this work we experimentally demonstrate the dissipative Faraday instability mode-locking in a Raman fiber laser. The instability was induced by spatially periodic zig-zag modulation of spectrally dependent losses, which resulted in temporal pattern formation and high order harmonic mode-locking.

\section{SM2J.3 • 11:45}

Metamaterial-integrated Non-electric Focal Plane Array for Real-time Terahertz Imaging, Yongzheng Wen ${ }^{1,3}$, Delin Jia ${ }^{1}$, Wei $\mathrm{Ma}^{1}$, Yun Feng ${ }^{2}$, Ming Liu², Liquan Dong ${ }^{2}$ Yuejin Zhao ${ }^{2}$, Xiaomei Yu'; ${ }^{1}$ Inst. of Microelectronics, Peking Univ., China; ${ }^{2}$ School of Optoelectronics, Beijing Inst. of Technology, China; ${ }^{3}$ School of Materials Science and Engineering, Tsinghua Univ., China. We report a non-electric focal plane array with metamaterials integrated into bi-materials cantilever pixels for real-time terahertz imaging. The fabricated device experimentally captured THz images of different objects with fast response and high sensitivity.

\section{SM2J.4 $\bullet 12: 00$}

Artificial Dielectric Polarizing Beam Splitter for the THz Region, Rajind Mendis ${ }^{1}$, Masaya Nagai ${ }^{2}$, Wei Zhang ${ }^{1}$, Daniel M. Mittleman ${ }^{1}$; ${ }^{1}$ Brown Univ., USA; ${ }^{2}$ Osaka Univ., Japan. We experimentally demonstrate a polarizing beam splitter (PBS) for the $\mathrm{THz}$ frequency region, based on artificial dielectrics. The PBS exhibits power efficiencies as high as $95 \%$ and extinction ratios as high as $10^{4}: 1$.

\section{SM2K.4 • 11:30}

Dispersion Characterization of Microresonators for Broadband Kerr Frequency Comb Generation, Junqiu Liu', Martin Pfeiffer ${ }^{1}$, Victor Brasch', Hairun Guo', Michael Zervas", Michael Geiselmann", Tobias J. Kippenberg'; ' ${ }^{1} E P F L$, Switzerland. Measuring higher order dispersion to determine the position of dispersive waves is important for broadband Kerr frequency comb generation and requires broad measurement bandwidth. Here we present a method of $1355-1630 \mathrm{~nm}$ bandwidth using frequency-comb-assisted cascaded diode laser spectroscopy.

\section{SM2K.5 • 11:45}

Single Crystal Small Core Semiconductor Optical Fibers for All-Fiber Optoelec tronics, Xiaoyu Ji', Shih-Ying Yu', Shiming Lei ${ }^{1}$, Hiu Yan Cheng ${ }^{1}$, Subhasis Chaudhuri ${ }^{1}$ Wenjun Liu², Suzanne Mohney, John Badding', Venkatraman Gopalan'; 'The Pennsylvania State Univ., USA; ${ }^{2}$ Argonne National Lab, USA. Recent development of fabricating small core single-crystal silicon and germanium optical fibers using a visible laser crystallization technique is reviewed. These fibers have potential applications in fiber-based nonlinear optical devices and optoelectronic applications.

\section{SM2K.6 • 12:00}

A Chip-based Silicon Nitride Platform for Mid-Infrared Nonlinear Photonics, Clemens Herkommer ${ }^{2,1}$, Hairun Guo ${ }^{2}$, Adrien Billat ${ }^{3}$ Davide Grassani ${ }^{3}$, Martin Pfeiffer ${ }^{2}$, Michael Zervas $^{2}$, Camille-Sophie Bres ${ }^{3}$, Tobias J. Kippenberg2. 'Walter-Schottky Institut Technical Univ. Munich, Germany; ${ }^{2}$ Lab for Photonics and Quantum Measurements, École Polytechnique Fédérale de Lausanne, Switzerland; ${ }^{3}$ hhotonics Systems Lab, École Polytechnique Fédérale de Lausanne, Switzerland. We developed a chip-based silicon nitride platform with thick waveguides $(\geq 2 \mu \mathrm{m})$ that overcomes the usual fabrication limitation. We demonstrate both microresonato frequency comb generation at $2.5 \mu \mathrm{m}$ and supercontinuum generation extending beyond $4.0 \mu \mathrm{m}$ in this platform.
SM2L.4 $\bullet 11: 30$

Multi-Line Regeneration of Noise Limited Frequency Combs by Brillouin Amplification via a Self-Seeded Dispersed Pump Mark D. Pelusi' , Amol Choudhary', Takashi Inoue $^{2}$, Benjamin Eggleton', David Marpaung ${ }^{1}$ Shu Namiki2; ' CUDOS, School of Physics, Univ. of Sydney, Australia; ${ }^{2}$ National Inst. of Advanced Industrial Science and Technology (AIST), Japan. We demonstrate noise suppression for 40 frequency comb-lines of narrow $10 \mathrm{GHz}$ pitch using narrowband Brillouin amplification pumped by dispersionmanaged spectral-lines seeded from the comb itself. Improved carrier performance for 96Gb/s-DP-64QAM signals is shown.
SM2L.5 • 11:45

Subpicosecond Coherent Nyquist Pulse Generation for 1-Tbaud Transmission Using a $\mathrm{C}_{2} \mathrm{H}_{2}$ Frequency-Stabilized CW Laser and a $40 \mathrm{GHz}$ Optical Comb Generator, Daiki Suzuki', Keisuke Kasai ${ }^{1}$, Toshihiko Hirooka ${ }^{1}$ Masataka Nakazawa'; 'Tohoku Univ., Japan. A $40-\mathrm{GHz}$ coherent Nyquist pulse was generated from a $\mathrm{C}_{2} \mathrm{H}_{2}$ frequency-stabilized $\mathrm{CW}$ fiber laser and an optical comb generator. A 1.5-THz flat-top comb was shaped into 680 -fs Nyquist pulses, which are applicable to 1.28-Tbaud transmission.

SM2L.6 • 12:00

Multi-wavelength Coherent Brillouin Random Fiber Laser with High Optical Signalto-Noise Ratio, Liang Zhang', Yanping Xu', Song Gao', Bhavaye Saxena', Liang Chen', Xiaoyi Bao'; 'Dept. of Physics, Univ. of Ottawa, Canada. A coherent multi-wavelength Brillouin random fiber laser with randomly distributed Rayleigh scattering feedback was demonstrated. Up to six orders Stokes random lasing radiations were generated with an unprecedented high optical signalto-noise ratio of $\sim 47 \mathrm{~dB}$. 


$\begin{array}{llc}\text { Marriott } & \text { Marriott } & \text { Marriott } \\ \text { Salon III } & \text { Salon IV } & \text { Salon V \& VI }\end{array}$

\section{CLEO: Science \& Innovations}

\section{$\mathrm{SM} 2 \mathrm{M} \bullet$ Nonlinear Dynamics and Harmonic Generation- Continued}

\section{SM2M.3 • 11:15}

Polarization Chaos in Nonlinear Optical Fibers Induced by a Reflective Delayed Loop, jacopo morosi ${ }^{1,2}$, Akram Akrout ${ }^{2}$, Antonio Picozzi ${ }^{2}$, Marin Gilles², Massimiliano Guasoni ${ }^{3}$, Julien Fatome2; ${ }^{1}$ Dipartimento di Elettronica, Informazione e Bioingegneria (DEIB), Politecnico di Milano, Italy; ${ }^{2}$ Laboratoire Interdisciplinaire Carnot de Bourgogne (ICB), Université Bourgogne Franche-Comté, France; ${ }^{3}$ Optoelectronics Research Centre, Univ. of Southampton, UK. We demonstrate that the nonlinear interaction in an optical fiber between an incident beam and its backward delayed replica leads to a chaotic dynamics of its output polarization state enabling a powerful scrambling process.

\section{SM2M.4 $111: 30$}

Linearizing Nonlinear Optics, Bruno E. Schmidt', Philippe Lassonde², Guilmot Ernotte ${ }^{2}$, Matteo Clerici ${ }^{3}$, Roberto Morandotti ${ }^{2}$, Heide Ibrahim² ${ }^{2}$ Francois Legare ${ }^{2}{ }^{1}$ few-cycle Inc., Canada; ${ }^{2}$ INRS-EMT, Canada; ${ }^{3}$ Univ. of Glasgow, UK. We demonstrate how Fourier Nonlinear Optics elegantly merges the simplicity of linear optics with the power of conventional nonlinear optics to achieve the decoupling of frequencies, amplitudes and phases in nonlinear processes.
SM2M.5 • 11:45

Bandwidth Control of Near Infrared Frequency Combs in High-Order Sideband Generation, Darren C. Valovcin ${ }^{1,2}$, Hunter Banks ${ }^{1,2}$, Shawn Mack ${ }^{5}$, Art Gossard ${ }^{3}$, Loren $\mathrm{Pf}^{4}$, Mark S. Sherwin ${ }^{1,2}$; 1 UCSB Physcis, USA; ${ }^{2}$ Inst. for Terahertz Science and Technology, USA; ${ }^{3}$ Materials, Univ. of California, Santa Barbara, USA; ${ }^{4}$ Electrical Engineering, Princeton, USA: ${ }^{5}$ U.S. Naval Research Lab, USA. Optical excitation of excitons in semiconductor quantum wells driven by intense, monochromatic terahertz fields results in several-hundred meV-wide frequency combs. Appropriate scaling of the driving field and frequency result in predictable comb bandwidths.

\section{SM2M.6 • 12:00}

Efficient multi-stage frequency mixing in multiple QPM device for optical carrier processing, Masaki Asobe', Kazuki Nakamura', Koji Enbutsu², Takeshi Umeki2; ' Tokai Univ., Japan; ${ }^{2}$ NTT Device Technology Labs, NTT Corporation, Japan. The multi-stage frequency mixing opens up the possibility of optical carrier processing. We propose efficient carrier phase recovery of multi-level phase modulated signal using new configuration. We also demonstrated data transmission using multiple carriers.

\section{SM2N • Whispering Gallery Mode Micro-cavities- Continued}

\section{SM2N.4 • 11:15}

Tunable Split-Disk Whispering Gallery Mode Resonators, Tobias M. Siegle ${ }^{1}$ Michael Remmel', Sarah Kraemmer', Heinz Kalt ${ }^{1}$; Insitute of Applied Physics, Karlsruhe Inst. of Technology, Germany. Polymeric dyedoped disk resonators, split in two halves, are structured on a flexible elastomer substrate by direct laser writing. A controlled substrate deformation enables precise resonance tuning verified by reversible shifts of the lasing modes.

\section{SM2N.5 - 11:30 Invited}

Whispering Gallery Micro-cavities for Functional Devices, Lan Yang'; 'Washington Univ. in St Louis, USA. I will discuss fundamental physics, such as parity-time symmetry and exceptional point (EP), in whispering-gallerymode (WGM) resonators, which can be used to achieve a new generation of optical systems enabling unconventional control of light flow.

\section{SM2N.6 $12: 00$}

Isolators and Circulators Based on Kerr Nonreciprocity in Microresonators, Leonardo Del Bino ${ }^{1,2}$, Jonathan Silver ${ }^{1}$, Xin Zhao ${ }^{1,3}$, Sarah L. Stebbings', Pascal Del'Haye'; ${ }^{1}$ National Physical Lab (NPL), UK; ${ }^{2}$ Inst. of Photonics and Quantum Sciences, HeriotWatt Univ., UK; ${ }^{3}$ School of Electronic and Information Engeneering, Beihang Univ., China. We demonstrate nonreciprocal light propagation in microresonators based on Kerr-effect-mediated symmetry breaking between counterpropagating light. In proof-of-principle experiments, we realize isolators and circulators with more than 20 $\mathrm{dB}$ isolation.

\section{SM2O • Modulators-} Continued
SM2O.4 $111: 30$

Monolithically Integrated CMOS Nanophotonic Segmented Mach Zehnder Transmitter, Andri Mahendra, ${ }^{1,2}$, Douglas Gill'3, Chi Xiong ${ }^{3}$, Jason Orcutt ${ }^{3}$, Benjamin Lee $^{3}$, Tam Huynh $^{3}$, Jonathan Proesel ${ }^{3}$, Nicolas Dupuis ${ }^{3}$, Philip Leong', Benjamin Eggleton², William Green'; ' 'School of Electrical and Information Engineering at The Univ. of Sydney, Australia; ${ }^{2}$ Centre for Ultrahigh Bandwidth Devices for Optical Systems (CUDOS) at School of Physics at The Univ. of Sydney, Australia; ${ }^{3}$ IBM Thomas J. Watson Research Center, USA. We present a monolithic segmented Mach-Zehnder transmitter, fully integrated in a $90 \mathrm{~nm}$ CMOS process. The transmitter exhibits a link sensitivity of $-14.1 \mathrm{dBm}$ optical modulation amplitude (OMA) at bit error rate $(\mathrm{BER})=10^{-12}$ at $12.5 \mathrm{Gbps}$.

SM2O.5 • 11:45

Ultra-Broadband Mach-Zehnder Hybrid Electro-Optic Polymer/Sol-Gel Silica Waveguide Modulators, Yasufumi Enami ${ }^{1,2}$, Atsushi Seki ${ }^{3}$, Shin Masuda ${ }^{3}$, Jingdong Luo ${ }^{4}$, Alex Jen"; ${ }^{1}$ Kochi Univ. of Technology, Japan; ${ }^{2}$ College of Optical Sciences, Univ. of Arizona, USA; ${ }^{3}$ Advantest Lab, Ltd, Japan; ${ }^{4}$ Materials Sciences and Engineering, Univ. of Washington, USA. We demonstrate ultrabroadband Mach-Zehnder (MZ) electro-optic polymer/sol-gel silica waveguide modulators. A bandwidth of an electrical transmission $S_{21}$ is measured at a modulation frequency of up to $50 \mathrm{GHz}$, which extrapolated the $6 \mathrm{~dB}$ bandwidth of $130 \mathrm{GHz}$.

\section{SM2O.6 • 12:00}

Integrated lithium niobate microresonators with in-plane microelectrodes for electro-optic tuning, Min Wang', Yingxin $\mathrm{Xu}^{2}$, Zhiwei Fang ${ }^{1}$, Jintian Lin', Wei Fang², Ya Cheng'; 'Shanghai Inst of Optics and Fine Mech, China; ${ }^{2}$ Zhejiang Univ., China. We report on on-chip electro-optic tuning of high-Q lithium niobate microresonator with integrated in-plane microelectrodes fabricated by femtosecond laser. Due to the compact spatial arrangement, high electrooptical tuning coefficient of $3.41 \mathrm{pm} / \mathrm{V}$ was demonstrated. 
Executive Ballroom $210 \mathrm{~A}$

Executive Ballroom 210B

\section{CLEO: Applications \\ \& Technology}

AM2A • A\&T Topical Review on Advances in Laser-based Remote Sensing II-Continued

\section{AM2A.5 • 12:15}

Early-stage Plasma Spectra Improvement Using a Field-portable Double-pulse Laser System, Shuo Li', Lei Liu², Aidong Yan', Sheng Huang ${ }^{1}, X_{i}$ Huang ${ }^{2}$, Yongfeng $\mathrm{Lu}^{2}$, Kevin Chen'; 'Univ. of Pittsburgh, USA ${ }^{2}$ Electrical and Computer Engineering, Univ. of Nebraska-Lincoln, USA. A field-portable double-pulse laser system was developed to study early-stage plasma in laser-induced breakdown spectroscopy. Emission spectral quality was significantly improved in terms of enhanced peak emission and reduced background emission.

\section{AM2B • Mid-IR Sensors and} Emitters-Continued

\section{AM2B.7 • 12:15}

Broadband Terahertz-Light Emission by Current-Injection Distributed-Feedback Dual-Gate Graphene-Channel Field-Effect Transistor, Deepika Yadav', Youssef To-

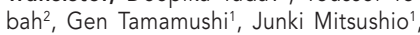
Takayuki Watanabe', Alexander Dubinov ${ }^{3}$, Maxim Ryzhii ${ }^{4}$, Victor Ryzhii ${ }^{1,5}$, Taiichi Otsuji ${ }^{1}$ Research Inst. of Electrical Communication Tohoku Univ., Japan; ${ }^{2} E C E$, Univ. of Texas, USA; ${ }^{3}$ Inst. for Physics of Microstructures, RAS, Russia; ${ }^{4}$ Univ. of Aizu, Japan; ${ }^{5}$ Inst. of Ultra-High-Frequency Semiconductor Electronics, Russia. Observed spontaneous $\mathrm{THz}$ emission $(1-7.6 \mathrm{THz})$ at $100 \mathrm{~K}$ by current injection in distributed-feedback dual-gate graphene transistor. We saw nonlinea threshold-like behavior w.r.t the currentinjection level. Precise DFB cavity design is expected to transcend spontaneous emission to stimulated emission.

\section{Executive Ballroom}

$210 \mathrm{C}$

\section{CLEO: Science \& Innovations}

$\mathrm{SM} 2 \mathrm{C} \cdot$ Biomedical Spectroscopy and Cell/Particle Analysis-Continued

\section{SM2C.5 ・ 12:15}

Single Particle Fluorescence Analysis on Demand on Electro-Optofluidic Chip with Gated Particle Delivery, Md Mahmudur Rahman', Mark Harrington ${ }^{1}$, Matthew A. Stott ${ }^{2}$ Aaron Hawkins ${ }^{2}$, Holger Schmidt'; ' ${ }^{1}$ Univ. of California, Santa Cruz, USA; ${ }^{2}$ Electrical and Computer Engineering, Brigham Young Univ. USA. Electronic feedback enables introduction of single microbeads and DNA molecules into a liquid-core waveguide through a micro/nanopore. Subsequent fluorescence detection from a controlled number of individual particles is demonstrated, enabling single particle analysis on demand.
Executive Ballroom 210D

CLEO: QELSFundamental Science

FM2D - Attosecond and Highfield Technologies-Continued

12:30-13:30 Lunch Break (on your own)

12:30-15:30 SC301: Quantum Cascade Lasers: Science, Technology, Applications and Markets SC378: Introduction to Ultrafast Optics

SC455: Integrated Photonics for Quantum Information Science and Technology New
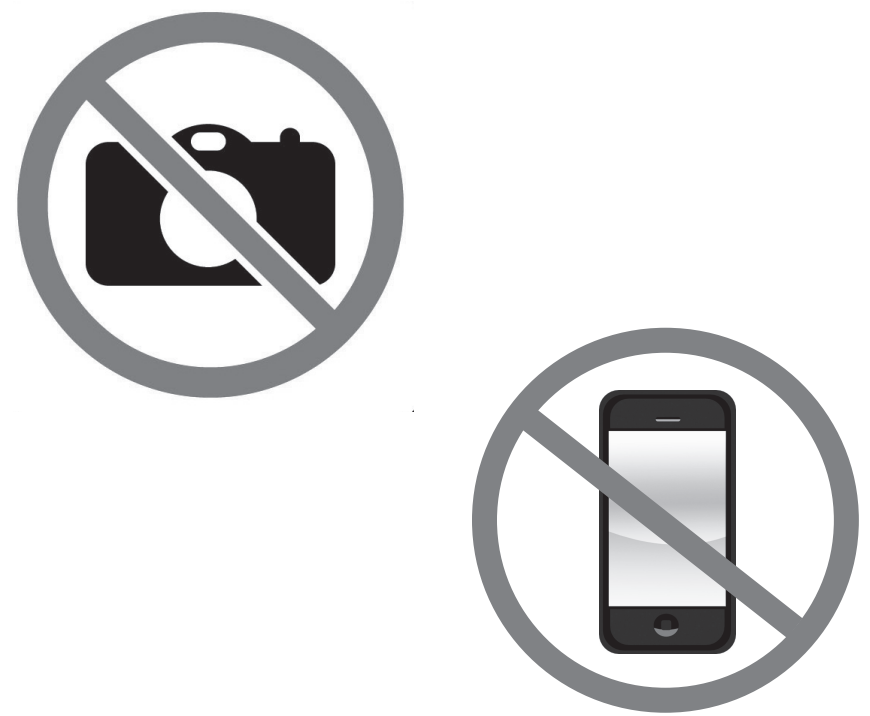

Executive Ballroom
$210 \mathrm{E}$
Executive Ballroom

210F
Executive Ballroom

210G
Executive Ballroom

$210 \mathrm{H}$

\section{CLEO: QELS-Fundamental Science}

FM2E • Atomic Ensemble and Bulk Crystal Quantum Memories-Continued

\section{FM2E.7 • 12:15}

Designing Quantum Repeaters for Continuous Variable Quantum Communication, William Munro ${ }^{1,2}$, fabian Furrer ${ }^{1} ;{ }^{1} N T T$

Basic Research Labs, Japan; ${ }^{2}$ National Inst. for Informatics, Japan. In this presentation we discuss the design of continuous variable quantum repeaters that can distribute entangled and pure two-mode squeezed states over arbitrarily long distances with a success probability that scales polynomially with distance.

\author{
FM2F • Engineering Nonlinear \\ Materials-Continued
}

FM2F.7 • 12:15

Dynamical Birefringence: High-order Sideband Generation as a Probe of Berry Curvature, Hunter B. Banks ${ }^{1,2}$, Darren C. Valovcin ${ }^{1,2}$ Qile Wu ${ }^{3}$, Shawn Mack ${ }^{4}$, Art Gossard ${ }^{5}$, Loren Pfeiffer $^{6}$, Renbao Liu ${ }^{3}$, Mark S. Sherwin ${ }^{1,2}$; ${ }^{1}$ Physics Dept., UCSB, USA; ${ }^{2}$ Inst. for Terahertz Science and Technology, UCSB, USA; ${ }^{3}$ Chinese Univ. of Hong Kong, China; ${ }^{4} \mathrm{Naval}$ Research Lab, USA; ${ }^{5}$ Materials Dept., UCSB, USA; ${ }^{6}$ Electrical Engineering Dept., Princeton Univ., USA. Continuous optical excitation of electron-hole pairs in quantum wells driven by intense, monochromatic terahertz fields leads to high-order sideband generation, up to $90^{\text {th }}$ order. Manipulations of polarization reveal sensitivity to Berry curvature.
FM2G • Photonic Topological Insulators-Continued

FM2G.7 • 12:15

Dispersion Topological Darkness, Haomin Song', Nan Zhang ${ }^{1}$, Jiyuan Duan', Zhejun $\mathrm{Liu}^{3}$, Jun $\mathrm{Gao}^{3}$, Matthew H. Singer ${ }^{1}$, Dengxin Ji', Alec R. Cheney', Xie Zeng', Borui Chen', Suhua Jiang ${ }^{3}$, Qiaoqiang Gan'; ${ }^{1}$ Dept. of Electrical Engineering, The State Univ. of New York at Buffalo, USA: ${ }^{2}$ Technical Center for Industrial Product and Raw Material Inspection and Testing, China; ${ }^{3}$ Material Science Dept., Fudan Univ., China. We present a complete description of "topological darkness" in a three-dimensional space regarding optical constants (i.e., $n$ and $k$ ) of effective media, wavelengths and incident angles, which is essential for enhanced light-matter interaction in thin-films.
$\mathrm{FM} 2 \mathrm{H} \bullet$ Plasmonic and Nanophotonic Sensors, Switches, \& PhotodetectorsContinued

FM2H.8 • 12:15

Plasmonic Nanoantenna based Ultrafast and Broadband Graphene Photodetectors, Semih Cakmakyapan ${ }^{1}$, Ping Keng Lu' ${ }^{1}$, Mona Jarrahi'; ' 'Univ. of California Los Angeles, USA. We present broadband photodetection from $800 \mathrm{~nm}$ to $20 \mu \mathrm{m}$ with operation speeds exceeding $50 \mathrm{GHz}$ and responsivity levels as high as $0.6 \mathrm{~A} / \mathrm{W}$ at $0.8 \mu \mathrm{m}$ and $11.5 \mathrm{~A} / \mathrm{W}$ at 20 $\mu \mathrm{m}$ by using plasmonic nanoantennas as photodetector contact electrodes on graphene.

\section{CLEO Mobile App}

Use the conference app to plan your schedule; view program updates; receive special events reminders and access Technical Papers (separate log-in required).

- Go to www.cleoconference.org/app.

- Select the Apple App Store or Google Play link.

- Download the app.

- Log in to use app features such as contacting fellow conference attendeesusing your registration I.D. and email address. 
Meeting Room

$211 \mathrm{~B} / \mathrm{D}$
Meeting Room

$212 \mathrm{~A} / \mathrm{C}$
Meeting Room

$212 \mathrm{~B} / \mathrm{D}$
Marriott

Salon I \& II

\section{CLEO: Science \& Innovations}

\section{SM2I • Ultrafast Pulse}

Generation-Continued

SM2I.8 • 12:15

Frequency-halved Orthogonally Polarized

Vector Soliton States from a Single Fiber

Laser Source, Ahmet E. Akosman'1, Michelle

Y. Sander, ${ }^{1,2}$; ${ }^{1}$ Electrical and Computer

Engineering, Boston Univ., USA: ${ }^{2}$ Materials

Science and Engineering, Boston Univ.

USA. An ultrafast pulse train composed of

co-generated, consecutive, equal intensity

and orthogonally polarized pulses is experi-

mentally shown for the first time in a vector

soliton mode-locked fiber laser.
SM2J • THz Quantum Optics and Metamaterials-Continued

SM2J.5 • 12:15

Electrically Modulated Nonlinear Terahertz Metamaterials, George R. Keiser ${ }^{1}$, Nicholas Karl$^{1}$, Qiang Liu², Caleb Tulloss' ${ }^{1}$, Houtong $\mathrm{Chen}^{3}$, Antionette Taylor ${ }^{3}$, Igal Brener ${ }^{2}$, Alex Benz ${ }^{2}$, John Reno ${ }^{2}$, Daniel M. Mittleman ${ }^{1}$ ${ }^{1}$ Brown Univ., USA; ${ }^{2}$ Sandia National Lab, USA; ${ }^{3}$ Los Alamos National Lab, USA. We present an electrically modulated nonlinear erahertz metamaterial. The device consists of an array of split-ring resonators fabricated on n-type $\mathrm{GaAs}$. Applying a 15V bias to the $\mathrm{MM}$ reduces the nonlinear terahertz modulation by $\sim 60 \%$.

\section{SM2K • Micro and Nanoscale Fabrication-Continued}

SM2K.7 • 12:15

Telecommunications Band Photoluminescence from Hydrogenated Amorphous Silicon Ring Resonators, Michael G. Wood ${ }^{1}$ Ryan J. Patton', Ronald M. Reano'; 'Ohio State Univ., USA. We report enhanced photoluminescence from $1300 \mathrm{~nm}$ to $1600 \mathrm{~nm}$ wavelength in ring resonators composed of hydrogenated amorphous silicon thin films. Enhancement of up to $5 \mathrm{~dB}$ occurs at the resonant modes of the rings.
SM2L • Multiwavelength and Comb Fiber SourcesContinued

12:30-13:30 Lunch Break (on your own)

12:30-15:30 SC301: Quantum Cascade Lasers: Science, Technology, Applications and Markets SC378: Introduction to Ultrafast Optics

SC455: Integrated Photonics for Quantum Information Science and Technology New 


$\begin{array}{llc}\text { Marriott } & \text { Marriott } & \text { Marriott } \\ \text { Salon III } & \text { Salon IV } & \text { Salon V \& VI }\end{array}$

\section{CLEO: Science \& Innovations}

$\mathrm{SM} 2 \mathrm{M} \bullet$ Nonlinear Dynamics and Harmonic GenerationContinued

SM2M.7 • 12:15

A Two-Photon Shack-Hartmann Wavefront Sensor for The Near-Infrared Wavelength, Fei Xia', David Sinefeld', Bo Li', Chris Xu ${ }^{1}$ Cornell Univ., USA. We present a novel wavefront sensing scheme based on twophoton absorption in a conventional silico camera for measuring aberrations of pulsed laser beams in the near-infrared wavelengths up to $2.0 \mu \mathrm{m}$
SM2N • Whispering Gallery Mode Micro-cavitiesContinued

\section{SM2N.7 • 12:15}

Self-Referenced Temperature Sensing with a Lithium Niobate Microdisk Resonator, Rui Luo', Haowei Jiang 2,1, Hanxiao Liang', Qiang Lin ${ }^{1 ;}{ }^{1}$ Univ. of Rochester, USA; ${ }^{2}$ Shanghai Jiao Tong Univ., China. We report self-referenced temperature sensing with a high-O Z-cut lithium niobate microdisk resonator, based on thermo-optic birefringence. We achieved a temperature sensitivity of $0.834 \mathrm{GHz} / \mathrm{K}$ and temperature sensing resolution of $0.8 \mathrm{mK}$
SM2O - ModulatorsContinued

SM2O.7 • 12:15

A Dual-drive PAM-4 Si Mach-Zehnder Modulator for $50 \mathrm{~Gb} / \mathrm{s}$ Data Transmission at $1550 \mathrm{~nm}$ Wavelength, ChihKuo Tseng ${ }^{1}$, Jhih-Heng Yeh', Po-Wei Chen', Wei-Lun Chung ${ }^{1}$, Tzu-Yu Yeh', Kai-Ming Feng ${ }^{1}$, Meng-Chyi Wu', Ming-Chang Lee'; ' $N T H U$, Taiwan. A low-voltage dual-drive push-pull Si Mach-Zehnder modulator is demonstrated to implement an optical $25 \mathrm{Gbaud}(50 \mathrm{~Gb} / \mathrm{s}$ ) PAM-4 transmission.

12:30-13:30 Lunch Break (on your own)

12:30-15:30 SC301: Quantum Cascade Lasers: Science, Technology, Applications and Markets SC378: Introduction to Ultrafast Optics

SC455: Integrated Photonics for Quantum Information Science and Technology New 
Executive Ballroom 210A

Executive Ballroom

210B

\section{CLEO: Applications \\ \& Technology}

13:30-15:30

AM3A • A\&T Topical Review Progress in Semiconductor Lasers I

Presider: Bojan Resan; Lumentum, Univ. of Applied Sciences, Switzerland

\section{AM3A.1 • 13:30 Invited}

Self-driving Cars and Lidar, Simon Verghese'; 'Waymo, USA. Before graduating from X as Waymo, Google's self-driving car project had been using custom lidars for several years. In their latest revision, the lidars are designed to meet the challenging requirements we discovered in autonomously driving 2 million highly-telemetered miles on public roads. Our goal is to approach price points required for advanced drive assistance systems (ADAS) while meeting the performance needed for safe self-driving This talk will review some history of the project and describe a few use-cases for lidars on Waymo cars. Out of that will emerge key differences between lidars for self-driving and traditional applications (e.g. mapping) which may provide opportunities for semiconductor lasers.

13:30-15:30

AM3B • Greenhouse Gas

Sensing

Univ., USA

\section{AM3B.1 1 13:30 Invited} on Scientific and Commercial

Presider: Mark Zondlo; Princeton

Laser-Based Sensors for Address ing Climate Change, Michael B. Frish"; ${ }^{1}$ Physical Sciences Inc., USA Identifying, measuring, and reducing mankind's contribution to climate change is an urgent international endeavor. This paper describes our work dedicated towards developing and applying laser sensors to support efforts to reduce greenhouse gas emissions.

AM3A.2 • 14:00 Invited

53 Years Tunable Semiconductor Lase - Past, Present and Future, Christoph Raab1, Rudolf Neuhaus ${ }^{1}$, Stephan Falke ${ }^{1}$ Christian Nölleke' ${ }^{1}$, Jürgen Stuhler ${ }^{1}$, Wilhelm Kaenders'; 'TOPTICA Photonics AG Germany. We present an overview from firs external cavity diode lasers to current and future designs. Based on applications the requirements and realizations of tunable diode lasers are shown with respect to their importance to "Quantum Technology" initiatives.
AM3B.2 • 14:00

Fiber-Pigtailed Silicon Photonic Sensors for Methane Leak Detection, Chu Teng ${ }^{1}$, Ch Xiong ${ }^{2}$, Eric Zhang ${ }^{2}$, Yves Martin², Marwan Khater $^{2}$, Jason Orcutt ${ }^{2}$, William Green ${ }^{2}$, Gerard Wysocki'; 'Princeton Univ., USA; ${ }^{2}$ IBM T. J. Watson Research Center, USA. We present comprehensive characterization of silicon photonic sensors for methane leak detection. Sensitivity of 40 ppmv after 1 second integration is reported. Fourier domain characterization of on-chip etalon drifts is used for further sensor improvement.
Executive Ballroom $210 \mathrm{C}$

\section{CLEO: Science \&} Innovations

\section{3:30-15:30 \\ SM3C ・ In Vivo/Deep Tissue Imaging \\ Presider: To be Determined}

\section{SM3C.1 • 13:30 Invited}

The Importance of Knowing You are Sick: Biophotonics For The 'Other' Brain, Mark R. Hutchinson 1,2; ${ }^{1}$ School of Medicine, The Univ. of Adelaide, Australia: ${ }^{2}$ Australian Research Council Centre of Excellence for Nanoscale BioPhotonics, Australia. The next frontier in neuroscience is the exploration of the "other brain" or the other $90 \%$ of cells of the central nervous system, termed glia. Currently there are no tools available to explore the real-time function of these underappreciated cells. This presentation will explore new opportunities for biophotonics in this space.
SM3C 2 14.00 Invited

Biophotonics - A Powerful Tool for Noninvasive and Labelfree Cell- and Tissue Screening, Juergen Popp ${ }^{1,2}$; 'Leibniz Inst. of Photonic Technology, Germany; ${ }^{2}$ Inst. of Physical Chemistry, Friedrich-Schille Univ., Germany. In this presentation, we will highlight our recent advances in translating biophotonic approaches with special focus on linear and non-linear Raman spectroscopy towards routine clinical applications with focus on infectious diseases and cancer.
Executive Ballroom 210D

\section{CLEO: QELS- Fundamental Science}

13:30-15:30

FM3D • Attosecond

Spectroscopy

Presider: Michael Chini; Univ. of Central Florida, USA

\section{FM3D.1 • 13.30 Invited}

Multidimensional Attosecond Spectroscopy, Nirit Dudovich'; 'Weizmann Inst. of Science, Israel. Photoemission is a fundamental light-matter interaction in nature. We demonstrate how the liberated electron can perform a self-probing measurement of the interaction. We reconstruct, with attosecond precision, the photoemission dynamics in a strong-field environment.
FM3D.2 • 14:00

Attosecond-precision Coherent Control of Electron Recombination in the Polarization Plane, Ofer Kfir ${ }^{2,1}$, Sergey Zayko², Christina Nolte $^{3}$, Stefan Mathias ${ }^{3}$, Oren Cohen ${ }^{1}$, Claus Ropers"; ${ }^{2}$ Solid State Inst. and Physics Dept., Technion Israel Inst. of Technology, Israel; ${ }^{2}$ IV. Physical Inst., Georg-August Univ. of Göttingen, Germany; ${ }^{3}$. Physical Inst., Georg-August Univ. of Göttingen, Germany. We demonstrate attosecond-precision control over the timing, phase, and orientation of electron recollisions in high harmonic generation. Employing elliptically-polarized bi-chromatic driving fields, we exert farreaching control over the enhancement and suppression of different harmonic sets. 
Executive Ballroom

210E

\section{Joint}

13:30-15:30

JM3E • Symposium on Sources of Nonclassical Light and their Scalability I

Presider: Virginia Lorenz; Univ. of Illinois at Chicago, USA

\section{JM3E.1 • 13:30 Invited}

Synchronized Spontaneous Downconversion Supplies Scalable Single-Photon Sources, Paul G. Kwiat', Fumihiro Kaneda ${ }^{1}$ Fedor Bergmann', Michelle Victora'; 'Univ of Illinois at Urbana-Champaign, USA. Very efficient sources of pure single photons can be realized by combining optimized, heralded nondeterministic photon sources with spatial or temporal multiplexing. Low-loss synchronization elements can further enhance scalability, enabling various multi-photon quantum information applications.

\section{Executive Ballroom 210F}

Executive Ballroom

210G
Executive Ballroom $210 \mathrm{H}$

\section{CLEO: QELS-Fundamental Science}

\section{3:30-15:30}

FM3F • Nonlinear Optics in Propagating Geometries I

Presider: Tobias Kippenberg; Ecole Polytechnique Federale de Lausanne, Switzerland

\section{3:30-15:30 \\ FM3G • Dirac-cone}

Metamaterials

Presider: Andrea Alu; Univ. of

Texas at Austin, USA

FM3F.1 • 13:30

Direct Observation of Multimode Solitons in Few-Mode Optical Fiber, Zimu Zhu', Logan Wright', Demetrios Christodoulides ${ }^{2}$, Frank W. Wise'; 'School of Applied and Engineering Physics, Cornell Univ. , USA ${ }^{2}$ The College of Optics and Photonics, Univ. of Central Florida, USA. We experimentally observe Raman shifted multimode solitons in few-mode graded-index fiber. They display spatiotemporal properties that depend on the specific launch conditions. Multimode solitons exhibit energy-volume relations distinct from both single-mode and spatiotemporal solitons.

\section{FM3G.1 • 13:30} optical range.
FM3F.2 • 13:45

Modulational Instability in Normally Dispersive Tapered Multimode Fibers, Helena E. Lopez Aviles', Mohammad Amin Eftekhar' Z. Sanjabi Eznaveh', Rodrigo A. Correa', Demetrios Christodoulides'; ' ${ }^{1} \mathrm{CREOL}$, College of Optics and Photonics, Univ. of Central Florida, USA. We show that modulational instability can occur in normally dispersive adiabatically tapered parabolic multimode fibers. Experimental results corroborate this intriguing phenomenon at $1.064 \mu \mathrm{m}$, indicating that the sideband gain is dictated by the tapering rate.

JM3E.2 • 14:00 Invited

Entanglement of Quantum Memories by Interfering Distinguishable Photons, Volkan Inlek', Grahame Vittorini', David Hucul', Clayton Crocker', Chris Monroe'; 'Univ. of Maryland at College Park, USA. Time-resolved photon detection can be used to generate entanglement between non-identical qubits that emit distinguishable photons, which may be necessary for future modular quantum systems. We experimentally realize this process using remotely trapped ytterbium ions.

\section{FM3F.3 • 14:00}

\section{FM3G.3 • 14:00 Invited}

Drive Acceleration, Yumiao Pei', Yi Hu', Cibo

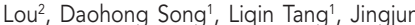
$\mathrm{Xu}^{1}$, Zhigang Chen',3; 'Nankai Univ., China; ${ }^{2}$ Ningbo Univ., China; ${ }^{3}$ San Francisco State Univ., USA. We study nonlinear interaction of two light beams with opposite effective "mass" in an optical waveguide array under a self-defocusing nonlinearity, and demonstrate the optical diametric drive acceleration in a spatial configuration.
Experiment Realization of Synthetic Weyl Points In Optical Regime, Hui Liu', Qiang Wang1; 'Nanjing Univ., China. We demonstrate that generalized Weyl points can exist in a parameter space and we report the first observation of such nodal points in one-dimensional photonic crystals in the

FM3G.2 • 13:45

Giant optical cross section induced by conical dispersion in photonic crystals, Ming Zhou', Ling Lu', Lei Shi', Jian Zi ', Zongfu Yu'; ${ }^{1}$ Univ. of Wisconsin - Madison, USA; ${ }^{2}$ Chinese Academy of Sciences, China; ${ }^{3}$ Fudan Univ, China. We show that the optical cross section of a single two-level system embedded in photonic crystals can be enhanced by 4 orders of magnitude near the vicinity of Dirac and Weyl points.

Experimental observation of optical Weyl points and Fermi arcs, Jiho Noh', Sheng Huang ${ }^{3}$, Daniel Leykam², Yidong Chong ${ }^{2}$, Kevin P. Chen ${ }^{3}$, Mikael C. Rechtsman'; 'Pennsylvania State Univ., USA; ${ }^{2}$ Physics, Nanyang Technological Univ., Singapore; ${ }^{3}$ Electrical Engineering, Univ. of Pittsburgh, USA. We present the experimental observation of type-Il optical Weyl points and corresponding Fermi arcs in a three-dimensional photonic structure. We employ a system composed of an array of staggered helical waveguides fabricated using the direct laser writing technique.
13:30-15:30

$\mathrm{FM} 3 \mathrm{H} \bullet$ Electron - Plasmon Interactions

Presider: Jacob Khurgin; Johns Hopkins Univ., USA
FM3H.1 • 13:30

Probing the Femtosecond Response of Plasmonic Nanoparticles with Strong-field Photoemission, William Putnam ${ }^{1,2}$, Phillip D. Keathley ${ }^{1}$, Richard G. Hobbs ${ }^{1,3}$, Karl K. Berggren ${ }^{1}$, Franz Kaertner ${ }^{1,2}$; ${ }^{1}$ Dept. of Electrical Engineering and Computer Science and Research Lab of Electronics, MIT, USA; ${ }^{2}$ Dept. of Physics and Center for Ultrafast Imaging Univ. of Hamburg, Germany; ${ }^{3}$ Centre for Research on Adaptive Nanostructures and Nanodevices (CRANN), Advanced Materials and Bio-Engineering Research Centre (AM$B E R)$, and School of Chemistry, Trinity College Dublin, Ireland. We illuminate resonant and off-resonant plasmonic nanoparticles with few-cycle laser pulses and measure strongfield photoemission. Recording interfero metric autocorrelations with the strong-field photocurrent, we study the response of the nanoparticle near-fields to ultrafast excitation.

\section{FM3H.2 • 13:45}

Plasmonics at the Space-Time Limit, Martin Aeschlimann"; ${ }^{1}$ Dept. of Physics and Research Center OPTIMAS, Univ. of Kaiserslautern, Germany. The ultrafast dynamics of nanoplasmonic vortex evolution using time-resolved photoemission electron microscopy with several attosecond time steps have been recorded. The study demonstrates three key stages in the vortex lifetime resulting from spin-orbit conversion. 


\section{CLEO: Science \& Innovations}

\section{3:30-15:30}

SM3I - Ultrafast Amplifiers

Presider: Alan Fry; SLAC National Accelerator Lab, USA

\section{SM3I.1 - 13:30 Invited}

Scaling Mid-Infrared Ultrafast Parametric Sources to High Peak and Average Power, Igor Jovanovic'; 'Univ. of Michigan, USA. Scaling mid-infrared parametric sources to high power motivates the use of highperformance nonlinear materials transparent beyond $\sim 2 \mu \mathrm{m}$. Recent progress in development of parametric amplifiers based on $\mathrm{ZnGeP}_{2}$, associated long-wavelength pumps, and diagnostics is discussed.

\section{3:30-15:30}

$\mathrm{SM} 3 \mathrm{~J} \cdot \mathrm{THz}$ Communications

Presider: David Cooke; McGill

Univ., Canada

\section{SM3J.1 • 13:30 Invited}

Advances in THz Wireless Communications, Tadao Nagatsuma'; ' ${ }^{\circ}$ ssaka Univ., Japan. This talk reviews latest advances in $\mathrm{THz}$ communications based on photonics as well as electronics, and discusses future technological challenges towards an unprecedented data rate of 1 Tbit/s.

\section{3:30-15:30 \\ SM3K • Silicon Photonics I}

Presider: Nanfang Yu; Columbia Univ., USA

SM3K.1 • 13:30

Hybrid Silicon / Lithium Niobate Waveguide Micro-chips Stable to $300 \mathrm{C}$, Peter Weigel', Shayan Mookherjea'; 'Univ. of California San Diego, USA. Bonded siliconon-insulator and lithium niobate-on-insulator dies are shown to be thermally stable up to $300 \mathrm{C}$ over a $67.2 \mathrm{~mm}^{\wedge} 2$ bonded area. Optical propagation through a waveguide on this bonded hybrid silicon-lithium niobate platform is performed.

\section{SM3K.2 • 13:45}

Surface textured silicon single-photon avalanche diode, Kai Zang ${ }^{1}$, Xun Ding ${ }^{2}$, Xiao Jiang ${ }^{2}, Y_{i j i e ~ H u o}{ }^{1}$, Matthew Morea', Xiaochi Chen ${ }^{1}$, Ching-Ying Lu', Muyu Xue ${ }^{1}$, Yusi Chen', Colleen Shang ${ }^{1}$, Theodore Kamins ${ }^{1}$, Qiang Zhang' ${ }^{2}$ Jian-Wei Pan², James Harris' ${ }^{1}$ Stanford Univ., USA; ${ }^{2}$ Univ. of Science and Technology of China, China. We present a surface textured Si SPAD with improved detection efficiency and without sacrificing dark count rate or jitter distribution. Texturing reduces reflection, allows weak light trapping and is CMOS and lithography compatible.

\section{SM3I.2 $\bullet 14: 00$}

High-Power Optical Parametric ChirpedPulse Amplifier Operating at $2.2 \mu \mathrm{m}$ Nicolas Bigler ${ }^{1}$, Christopher R. Phillips ${ }^{1}$, Justinas Pupeikis', Lukas Gallmann', Hideki Ishizuki' ${ }^{2}$, Takunori Taira ${ }^{2}$, Ursula Keller'; ; Dept. of Physics, Inst. of Quantum Electronics, ETH Zürich, Switzerland; ' Inst. for Molecular Science, Laser Research Center for Molecular Science, Japan. We present a high-power mid-infrared OPCPA system generating ultrabroadband pulses centered at $2.2 \mu \mathrm{m}$ with a bandwidth supporting pulse compression to $15 \mathrm{fs}$. We achieve a record average power of $9.1 \mathrm{~W}$ at $100 \mathrm{kHz}$.

Wireless THz Communications Using Opto-
SM3K.3 $14: 00$

CMOS-Compatible ALD Zinc Oxide Coating for On-Chip Second-Order Nonlinear Optical Functionalities, Artur Hermans ${ }^{1}$, Michiel Van Daele ${ }^{1}$, Clemens Kieninger ${ }^{2}$, Jolien Dendooven', Stéphane Clemmen', Christophe Detavernier ${ }^{1}$, Christian Koos ${ }^{2}$ Roel Baets'; ' 'Universiteit Gent, Belgium; ${ }^{2}$ Karlsruhe Inst. of Technology, Germany. We report on the atomic layer deposition of $\mathrm{ZnO}$ for interfacing with existing $\mathrm{Si}_{3} \mathrm{~N}_{4}$ photonics which lacks $2^{\text {nd }}$-order nonlinear functionalities. We measure a $X^{(2)}$ of $15 \mathrm{pm} / \mathrm{V}$ in line with a bulk $\mathrm{ZnO}$ crystal.
SM3J.2 $\bullet 14: 00$ electronic Techniques for Signal Generation and Coherent Reception, Tobias Harter ${ }^{1,2}$ Marco Weber ${ }^{1}$, Sascha Muehlbrandt ${ }^{1,2}$, Stefan Wolf', Juned Kemal', Florian Boes ${ }^{3}$, Simon Nellen ${ }^{4}$, Thorsten Goebel ${ }^{4}$, Joachim Giesekus ${ }^{4}$, Thomas Zwick ${ }^{3}$, Sebastian Randel', Wolfgang Freude ${ }^{1}$, Christian Koos ${ }^{1,2}$; IInst. of Photonics and Quantum Electronics, Karlsruhe Inst. of Technology (KIT), Germany; 2Inst. of Microstructure Technology, KIT, Germany; ${ }^{3}$ Inst. of Radio Frequency Engineering, KIT, Germany; ${ }^{4}$ Fraunhofer Inst. for Telecommunications, Heinrich Hertz Inst., Germany. We show coherent wireless transmission at carrier frequencies of $0.25 \mathrm{THz}$ and $0.35 \mathrm{THz}$ relying exclusively on optoelectronic concepts for RF signal generation and coherent reception. In a proof-of-concept experiment, we demonstrate transmission of a BPSK signal at a symbol rate of $1 \mathrm{GBd}$.
13:30-15:30

SM3L • Mode Locked Fiber Lasers I

Presider: Shinji Yamashita; RCAST, The Univ. of Tokyo, Japan

\section{SM3L.1 • 13:30 Invited}

50-nJ and 40-fs Pulses from a Mamyshev Ocillator, Zhanwei Liu', Zachary Ziegler ${ }^{1}$, Frank W. Wise ${ }^{1}$; ${ }^{1}$ Cornell Univ., USA. We demonstrate a fiber pulse generator based on reshaping and reamplification, which generates pulses of 50-nJ energy and 40-fs duration. The pulse peak power is two-orders of magnitude higher than the previous best result.

\section{SM3L.2 $\bullet 14: 00$}

Er-fiber laser enabled femtosecond source tunable from 1.3 to $1.7 \mu \mathrm{m}$ for nonlinear optical microscopy, Hsiang-Yu Chung 1,2, Wei Liu', , Qian Cao, ${ }^{1,2}$, Guoqing Chang ${ }^{1,3}$; ${ }^{\prime} \mathrm{Cen}$ ter for Free-Electron Laser Science, DESY, Germany; ${ }^{2}$ Physics Dept., Univ. of Hamburg, Germany; ${ }^{3}$ The Hamburg Centre for Ultrafast Imaging, Germany. We demonstrate an Er-fiber laser based source that produces 100-200 fs pulses widely tunable from 1300 to $1700 \mathrm{~nm}$, constituting an ideal driving source for three-photon excitation nonlinear optical microscopy. 


$\begin{array}{llc}\text { Marriott } & \text { Marriott } & \text { Marriott } \\ \text { Salon III } & \text { Salon IV } & \text { Salon V \& VI }\end{array}$

\section{CLEO: Science \& Innovations}

13:30-15:30

$\mathrm{SM} 3 \mathrm{M} \bullet$ Progress in Optical

Frequency Conversion

Presider: Masaki Asobe; Tokai

Univ., Japan

SM3M.1 - 13:30

Broadband and Wideband Parametric

Gain via Intermodal Four-Wave Mixing in Optical Fiber, Jeffrey Demas', Gautam Prabhakar1, Tao He ${ }^{1,2}$, Siddharth Ramachandran': 'Boston Univ., USA; ${ }^{2}$ School of Optoelectronics, Beijing Inst. of Technology, China. We demonstrate a novel intermodal four-wave mixing process by dividing a pump at $1047 \mathrm{~nm}$ between the LP and LP fiber modes, leading to simultaneously broadband ( $28 \mathrm{~nm}$ at $1550 \mathrm{~nm})$ and wideband ( 1 octave) spontaneous parametric gain.

SM3M.2 • 13:45

Visible Raman Generation from Ambient Air in a Nodeless Hollow-Core Fiber, Shoufei Gao', Yingying Wang', Pu Wang'; ${ }^{1}$ Beijing Univ. of Technology, China. A nodeless hollow-core fiber, exposed to ambient air, enables multiline Raman generation from nitrogen, oxygen, water and carbon oxide by a $532 \mathrm{~nm}$ picosecond laser for the first time.

SM3M.3 • 14:00

Picosecond Pulse Generation at $1177 \mathrm{~nm}$ by SRS in PbWO, Pumped by a Multi-mJ, Multi-W Sub-ns Laser System, Bozhidar Oreshkov', Ruijun Lan², Luben S. Petrov', Hui Yuan ${ }^{3}$, Wei Xiong ${ }^{3}$, Ivan C. Buchvarov ${ }^{1,4}$ Valentin Petrov'; 'Faculty of Physics, Sofia Univ. St. Kliment Ohridski, Bulgaria; ${ }^{2}$ School of Opto-Electronic Information Science and Technology, Yantai Univ., China; ${ }^{3}$ Key Lab of Transparent and Opto-Functional Inorganic Materials, Shanghai Inst. of Ceramics, China; ${ }^{4}$ ITMO Univ., Russia; ${ }^{5}$ Max Born Inst. for Nonlinear Optics and Short Pulse Spectroscopy, Germany. We report on high energy $(\sim 1 \mathrm{~mJ})$, high average power ( 0.5 W), sub-500-ps stimulated Raman scattering in a $\mathrm{PbWO}_{4}$ crystal, pumped by a sub-nanosecond Nd:YAG based master-oscillator power-amplifier (MOPA) laser system operating at $500 \mathrm{~Hz}$.
$13: 30-15: 30$

$\mathrm{SM} 3 \mathrm{~N} \cdot$ Plasmonics and

Metamaterials

Presider: Wenqi Zhu; NIST, USA

SM3N.1 • 13:30

Design and Fabrication of 2 um Metasurface-based Orbital Angular Momentum (OAM) Mode Generator Employing Reflective Optical Antenna Array, Yifan Zhao', Jing Du', Zhengsen Ruan', Li Shen ${ }^{1}$, Shuhui Li', Jian Wang ${ }^{1} ;{ }^{1}$ Huazhong Univ of Science and Technology, China. A chip-scale reflective metasurface is designed and fabricated for generating 2 um orbital angular momentum (OAM) mode with topological charge of +1 or -1 . We also study the operation performance of fabricated OAM mode generator in experiment.

\section{SM3N.2 • 13:45}

Extraordinary Optical Transmission of Ultra-Thin Freestanding Plasmonic Membranes, Longju Liu', Hsin-Yu Wu ${ }^{2}$, Meng Lu, ${ }^{1,3} ;{ }^{1}$ Dept. of Electrical and Computer Engineering, lowa State Univ., USA; ${ }^{2}$ Graduate Inst. of Nanomedicine and Medical Engineering, Taipei Medical Univ., Taiwan; ${ }^{3}$ Dept. of Mechanical Engineering, lowa State Univ., USA. We demonstrate a 30 $\mathrm{nm}$-thick freestanding plasmonic membrane that supports mid-infrared surface plasmon resonances. The membrane is perforated using the imprint-and-transfer approach. The device was used to measure the absorption of a thin polymer film.

\section{SM3N.3 • 14:00}

Bloch long-range surface plasmon polaritons in metallic stripe waveguides, Norman Fong ${ }^{2,4}$, Matteo Menotti ${ }^{1}$, Ewa Lisicka-Skrzek ${ }^{2,4}$, Howard Northfield2,4, Anthony Olivierii, ${ }^{2,}$, Niall Tait ${ }^{3}$, Marco Liscidini ${ }^{1}$, Pierre Berini2,4: 'Dept. of Physics, Univ. of Pavia, Italy; ${ }^{2}$ School of Electrical Engineering and Computer Science, Univ. of Ottawa, Canada; ${ }^{3}$ Dept. of Electronics, Carleton Univ. Canada; ${ }^{4}$ Centre for Research in Photonics, Univ. of Ottawa, Canada. We propose and demonstrate a thin Au stripe on a truncated 1D dielectric photonic crystal covered with Cytop as a waveguide for Bloch long-range surface plasmon polaritons.
13:30-15:30

SM3O • Integrated Quantum Photonics

Presider: Qiaoqiang Gan; State

Univ. of New York at Buffalo, USA

SM30.1 • 13:30 Invited

Photonic Gauge Potential and Synthetic Dimension with Integrated Photonics Platforms, Shanhui Fan'; 'Stanford Univ., USA. Abstract not available.

SM30.2 • 14:00

On-chip Coherent Conversion of Photonic Quantum Entanglement Between Different Degrees of Freedom, Lantian Feng', Ming Zhang ${ }^{2}$, Zhi-Yuan Zhou', Ming Li', Xiao Xiong ${ }^{1}$, Le Yu', Bao-Sen Shi', Guo-Ping Guo', Dao-Xin Dai², Xi-Feng Ren'1, GuangCan Guo'; 'Univ Sci \& Tech China, China; ${ }^{2} Z$ hejiang Univercsity, China. We introduce the transverse waveguide-mode degree of freedom to quantum photonic integrated circuits, and demonstrate the coherent conversion of a photonic quantum state between path, polarization and transverse waveguidemode degrees of freedom on a single chip. 
Executive Ballroom

$210 \mathrm{~A}$

Executive Ballroom

210B

\section{CLEO: Applications \\ \& Technology}

AM3A • A\&T Topical Review on Scientific and Commercial Progress in Semiconductor Lasers I-Continued

\section{AM3B • Greenhouse Gas Sensing-Continued}

\section{Executive Ballroom} $210 \mathrm{C}$

\section{CLEO: Science \& Innovations}

\section{$\mathrm{SM} 3 \mathrm{C} \cdot$ In Vivo/Deep Tissue Imaging-Continued}

Executive Ballroom 210D

CLEO: QELS Fundamental Science

\author{
FM3D • Attosecond \\ Spectroscopy-Continued
}

AM3B.3 • 14:15

Open-Path $\mathrm{C}_{2} \mathrm{H}_{6}$ Sensor for Fast, LowPower, Measurement of Natural Gas Emissions, Levi Golston', Dana Caulton ${ }^{1}$ James McSpiritt ${ }^{1}$, Bernhard Buchholz ${ }^{1}$, Da Pan', Mark A. Zondlo'; ' Princeton Univ., USA An open-path, ICL-based ethane sensor is developed with low power consumption (24 W) and fast response $(1 \mathrm{~Hz})$. Use on a mobile Lab allows for discriminating between thermogenic (fossil fuels) and biogenic (wetlands, agriculture) methane.

\section{AM3A.3 • 14:30 Invited}

Recent Progress in Quantum Dot Based Devices: Physics and Applications, Edik U. Rafailov'; ${ }^{1}$ Aston Inst. of Photonic Technologies, Aston Univ., UK. The unique properties offered by quantum-dot semiconductor structures allowed for the development of compact CW and ultrashort pulse lasers. In this paper we review recent progress in fabrication of quantum-dot based devices and their applications.
AM3B.4 • 14:30

Cryptophane-Cladded Interferometric Waveguide Sensor for Aqueous Methane Detection, Jana Jágerská', Firehun T. Dullo1, Susan M. Lindecrantz', Jacqueline M. Boergers ${ }^{1,2}$, Jørn H. Hansen ${ }^{1}$, Laura M. Lechuga ${ }^{3}$, Olav G. Helles $\varnothing^{1} ;{ }^{1}$ UiT Norges Arktiske Universitet, Norway; ${ }^{2}$ TU Dortmund, Germany: ${ }^{3}$ Catalan Inst. of Nanoscience and Nanotechnology (ICN2), CSIC, Spain. A nanophotonic sensor for sensitive detection of methane in water solution is presented. Cryptophane-A doped waveguide cladding provides for methane pre-concentration directly on a chip, resulting in a detection limit of 60 ppm (86 nM).

AM3B.5 • 14:45

High spectral resolution of overlapping molecular transitions of $\mathrm{CH}_{4}$ and $\mathrm{N}_{2} \mathrm{O}$ in the mid-infrared region, May Hlaing ${ }^{1,2}$ Amir M. Khan ${ }^{1,2}$, Caio S. Azevedo ${ }^{1,2}$, Seth A. Fair ${ }^{1,2}$; ${ }^{1}$ Delaware State Univ., USA; ${ }^{2}$ Physics, Optical Science Center for Applied Research, Delaware State Univ., USA. We show novel detection techniques and quantitative metrics of instrument performance of a mid-IR quantum cascade laser-based system using higher harmonic ( $2 f$ and $4 f$ ) detection to resolve overlapping line transitions of methane, nitrous-oxide and water-vapor.
SM3C.3 $14: 30$

Depth-Resolved Characterization of the In Vivo Tympanic Membrane using NanoSensitive Optical Coherence Tomography, Roshan Dsouza', Jenny Won ${ }^{1,3}$, Guillermo L. Monroy ${ }^{1,3}$, Ryan Porter ${ }^{4,5}$, Michael A. Novak ${ }^{4,5}$, Malcom C. Hill ${ }^{6}$, Stephen A. Boppart ${ }^{1,2}$; ${ }^{1}$ Beckman Inst. for Advanced Science and Technology, Univ. of Illinois at Urbana-Champaign, USA; ${ }^{2}$ Dept. of Electrical and Computer Engineering, Univ. of Illinois at Urbana-Champaign, USA; ${ }^{3}$ Dept. of Bioengineering, Univ. of Illinois at UrbanaChampaign, USA; ${ }^{4}$ Dept. of Otolaryngology, Carle Foundation Hospital, USA; ${ }^{5}$ College of Medicine, Univ. of Illinois at UrbanaChampaign, USA; ${ }^{6}$ Dept. of Pediatrics, Univ. of Illinois at Urbana-Champaign, USA. We report nano-sensitive optical coherence tomography (nsOCT) structural changes of the in vivo human tympanic membrane (TM) under normal and infected conditions. nsOCT enables diagnostics which may impact the clinical treatment of middle-ear infection.

\section{SM3C. $4 \cdot 14: 45$}

High Confinement and Low Loss $\mathrm{Si}_{3} \mathrm{~N}_{4}$ Waveguides for Miniaturizing Optical Coherence Tomography, Xingchen $\mathrm{Ji}^{1,2}$ Xinwen Yao', Mohammad A. Tadayon ${ }^{1}$ Aseema Mohanty ${ }^{1,2}$, Christine P. Hendon', Michal Lipson'; ' Columbia Univ., USA; ${ }^{2}$ Cornell Univ., USA. We show high confinement thermally tunable, low loss $(0.27 \pm 0.04 \mathrm{~dB}$ / $\mathrm{cm}) \mathrm{Si}_{3} \mathrm{~N}_{4}$ waveguides that are $42 \mathrm{~cm}$ long. We show that this platform can enable the miniaturization of traditionally bulky active OCT components.
FM3D.3 • 14:15

Waveform control of high-harmonic generation in solids, Yongsing You', Mengxi $W^{3}$, Yanchun Yin ${ }^{2}$, Andrew Chew ${ }^{2}$, Xiaoming Ren $^{2}$, Shima Gholam-Mirzaei ${ }^{2}$, Dana Browne ${ }^{3}$, Michael Chini ${ }^{2}$, Zenghu Chang ${ }^{2}$, Kenneth Schafer ${ }^{3}$, Mette Gaarde ${ }^{3}$, Shambhu Ghimire ${ }^{1}$ 'SLAC National Lab, USA; '2Univ. of Central Florida, USA; ${ }^{3}$ Louisiana State Univ., USA. We report a strong carrier-envelope-phase dependence of high-harmonics in bulk solids subjected to strong few-cycle laser fields. We discover that harmonics are delayed with respect to each other at the sub-cycle level, yielding an atto-chirp.
FM3D.4 • 14:30

Harmonic Generation in Solids from a Fiber Laser, Kevin F. Lee ${ }^{1}$, Xiaoyan Ding ${ }^{2}$ T J. Hammond², Martin E. Fermann1, Giulio Vampa ${ }^{2}$, Paul B. Corkum²; 'IMRA America, Inc., USA; ${ }^{2}$ Joint Attosecond Science Lab, Univ. of Ottawa and National Research Council Canada, Canada. We generate up to the seventh harmonic in silicon and zinc oxide from a femtosecond Tm fiber laser a $93 \mathrm{MHz}$, rather than a multi-stage Ti:sapphire system as in earlier experiments.
FM3D.5 • 14:45

Solid-State Three-Step Model for HighHarmonic Generation from Periodic Crystals, Takuya Ikemachi ${ }^{1}$, Yasushi Shinohara ${ }^{2}$ Takeshi Sato 2,3, Junji Yumoto ${ }^{1,4}$, Makoto Kuwata-Gonokami ${ }^{1}$, Kenichi L. Ishikawa ${ }^{2,3}$ 'Dept. of Physics, The Univ. of Tokyo, Japan; 2Photon Science Center, The Univ. of Tokyo, Japan; ${ }^{3}$ Dept. of Nuclear Engineering and Management, The Univ. of Tokyo, Japan ${ }^{4}$ Inst. for Photon Science and Technology, The Univ. of Tokyo, Japan. Based on direct spatial-grid numerical simulations, we present a simple three-step model capable of explaining multiple cutoff positions, time-frequency structure, and other experimentally observed unique features of high-harmonic generation from solids. 
Executive Ballroom 210E

Joint

JM3E • Symposium on Sources of Nonclassical Light and their Scalability I-Continued
JM3E.3 • 14:30

Two-Photon Interference from Multiple Solid-State Quantum Emitters, Jehyung Kim¹, Christopher Richardson², Richard Leavitt ${ }^{2}$, Edo Waks'; ' Univ. of Maryland, USA; ${ }^{2}$ Lab for Physical Sciences, Univ. of Maryland USA. Indistinguishable single photons are key elements of quantum information and communication. We demonstrate multiple identical quantum emitters on-a-chip that show two-photon interference. The advantages of photonic crystal platform for scalable quantum photonic devices are highlighted. Executive Ballroom
$210 \mathrm{~F}$
Executive Ballroom

210G
Executive Ballroom

$210 \mathrm{H}$

\section{CLEO: QELS-Fundamental Science}

FM3F • Nonlinear Optics in
Propagating Geometries IContinued

FM3F.4 • 14:15

Multi-octave supercontinuum driven by soliton explosion in dispersion-designed antiresonant hollow-core fibers, Michael Zuerch $^{2,1}$, Rudrakant Sollapur², Daniil Kartash$\mathrm{ov}^{2}$, Andreas Hoffmann², Teodora Grigorova², Gregor Sauer ${ }^{2}$, Alexander Hartung ${ }^{3}$, Anka Schwuchow $^{3}$, Jörg Bierlich ${ }^{3}$, Jens Kobelke ${ }^{3}$ Markus Schmidt ${ }^{3,4}$, Christian Spielmann ${ }^{2,5}$ ${ }^{1}$ Dept. of Chemistry, UC Berkeley, USA; ${ }^{2}$ Inst. of Optics and Quantum Electronics, Friedrich Schiller Univ., Germany; ${ }^{3}$ Leibniz Inst. of Photonic Technology e.V., Germany; ${ }^{4}$ Otto Schott Inst. of Material Research, Friedrich Schiller Univ., Germany: ${ }^{5} \mathrm{Helmholtz}$ Inst. Jena, Germany. Broadband supercontinuum generation mediated by non-adiabatic mode dispersion profiles resulting in new type of soliton dynamics in dispersion-designed antiresonant hollow-core fibers is reported. A new concept of soliton explosion is demonstrated in experiment and simulation.

FM3F.5 • 14:30

Akhmediev Breathers in Nonlinear Partially Coherent Environments, Fan $\mathrm{Wu}^{1}$ Mohammad Amin Eftekhar ${ }^{1}$, Zhigang Chen², Demetrios Christodoulides'; ${ }^{1} \mathrm{CREOL}$, College of Optics and Photonics, Univ. of Central Florida, USA; ${ }^{2}$ Dept. of Physics and Astronomy, San Francisco State Univ., USA. We show that Akhmediev breathers can appear even in partially coherent nonlinear systems. We find that the degree of coherence can significantly affect the onset of these waves and can critically alter their ensuing dynamics.

\author{
FM3G - Dirac-cone \\ Metamaterials-Continued
}

FM3H • Electron - Plasmon Interactions-Continued

\section{FM3G.4 • 14:30}

Reflection and refraction in artificial photonic gauge fields, Moshe-lshay Cohen ${ }^{1}$ Yaakov Lumer' ${ }^{2}$, Hanan H. Herzig Sheinfux ${ }^{1}$ Yonatan Plotnik', Jonathan Nemirovsky? Mordechai Segev'; 'Technion Israel Inst. of Technology, Israel; ${ }^{2}$ Dept. of Electrical and Systems Engineering, Univ. of Pennsylvania, USA. We study the interface between two artificial gauge fields in a 2D photonic lattice, and find the analogues of Snell's law and Fresnel coefficients of such interfaces.

\section{JM3E.4 • 14:45}

Scalable Quantum Photonics with Single Color Centers in Silicon Carbide, Marina Radulaski ${ }^{1}$, Matthias Widmann², Matthias Niethammer ${ }^{2}$, Jingyuan L. Zhang ${ }^{1}$, Sang-Yun Lee $^{2}$, Torsten Rendler ${ }^{2}$, Konstantinos Lagoudakis ${ }^{1}$, Nguyen Son ${ }^{3}$, Erik Janzen ${ }^{3}$, Takeshi Ohshima ${ }^{4}$, Jorg Wrachtrup ${ }^{2}$, Jelena Vuckovic ${ }^{1}$. 'Stanford Univ., USA; ${ }^{2}$ Univ. of Stuttgart, Germany; ${ }^{3}$ Linkoping Univ., Sweden; ${ }^{4}$ National Inst.s for Quantum and Radiological Science and Technology, Japan. We develop a scalable array of $4 \mathrm{H}-\mathrm{SiC}$ nanopillars incorporating single silicon vacancy centers, readily available to serve as efficient single photon sources or quantum bits interfaced with freespace or lensed-fiber optics.

\section{FM3F.6 • 14:45}

Experimental Demonstration of the Necklace Beam Formation in Engineered Nonlinear Media, Jingbo Sun ${ }^{1}$, Salih Silahli', Wiktor Walasik', Eric Johnson², Alexandra M. Nikiforov', Natalia M. Litchinitser'; ' ${ }^{1}$ State Univ. of New York at Buffalo, USA; ${ }^{2}$ Electrical and Computer Engineering, Clemson Univ., USA. We experimentally investigate the formation, dynamics and stability of complex necklace beams using structured light with different orbital angular momentum propagating in nonlinear nano-colloidal suspensions with negative polarizabilities.
FM3H.3 • 14:15

Efficient hard X-ray source enabled by metallic nano-gratings, Gilles D. Rosolen ${ }^{1,3}$ Liang Jie Wong ${ }^{2}$, Ido Kaminer ${ }^{1}$, Nicholas Rivera', Bjorn Maes ${ }^{3}$, Marin Soljacic'; ${ }^{1}$ MIT, USA; ${ }^{2}$ Singapore Inst. of Manufacturing Technology, Singapore; ${ }^{3}$ UMONS, Belgium. We propose practical nano-grating configurations for highly directional and monochromatic hard X-ray generation from modestly relativistic electrons. By optimizing the nanograting to support high field enhancement we show efficient radiation generation at multiple harmonics.

FM3H.4 • 14:30

Numerical Simulation of Electron Energy Loss Spectroscopy of Aluminum Nanodisk Surface Plasmons, Yujia Yang ${ }^{1}$, Richard G. Hobbs', Vitor R. Manfrinato', Sarah A. Goodman', Karl K. Berggren'; 'MIT, USA ${ }^{2}$ Brookhaven National Lab, USA. We perform simulations to model electron energy loss spectroscopy of aluminum nanodisk surface plasmons. Nanodisk geometry and e-beam position determine excitation and energy of plasmonic modes. Multipolar modes are explained with a circulating waveguide model.
FM3G.5 • 14:45

Photonic Weyl Point in a 2D Resonator Array with a Synthetic Frequency Dimension, Qian Lin', Meng Xiao', Luqi Yuan ${ }^{1}$, Shanhui Fan'; 'Stanford Univ., USA. We propose a realization of Weyl point in two-dimensiona arrays of resonators undergoing dynamic modulation. Our system provides an on-chip platform to explore Weyl points under different symmetries and demonstrate topological surface state in synthetic space.

\section{FM3H.5 $\bullet 14: 45$}

Nano-chirality detection with vortex plasmon modes, Jordan Hachtel $1^{1,2}$, Roderick Davidson',1, Matthew Chisholm1', Richard F. Haglund $^{2}$, Sokrates Pantelides ${ }^{2,1}$, Sang-Yeon $\mathrm{Cho}^{3}$, Benjamin Lawrie ${ }^{1}$; Oak Ridge National Lab, USA; ${ }^{2}$ Vanderbilt Univ., USA; ${ }^{3}$ New Mexico State Univ., USA. Cathodoluminescence spectroscopy in a scanning transmission electron microscope is used to probe the spatio-spectral response of nanospiral ap erture plasmonic vortex generators. Further we demonstrate that plasmonic vortex modes can probe the chirality of nanoscale materials. 


\section{CLEO: Science \& Innovations}

\section{SM3I • Ultrafast Amplifiers- Continued}

\section{SM3I.3 • 14:15}

Generation of a $200-\mathrm{mJ}$ class infrared femtosecond laser by dual-chirped optical parametric amplification, Yuxi FU', Eiji J. Takahashi', Bing Xue ${ }^{1}$, Katsumi Midorikawa'; ${ }^{\prime} R I K E N$, Japan. Total output energy of $1.4 \mu \mathrm{m}$ (signal) and $1.9 \mu \mathrm{m}$ (idler) reaches $210 \mathrm{~mJ}$ by a dual-chirped optical parametric amplifier (DC-OPA). The obtained IR spectra support $41 \mathrm{fs}$ and $40 \mathrm{fs}$ transform limited durations, respectively.

\section{SM3I.4 $\bullet 14: 30$}

Sub-8 fs, $210 \mu \mathrm{J}$ Pulses at $100 \mathrm{kHz}$ from a Noncollinear Optical Parametric Amplifier, Federico J. Furch ${ }^{1}$, Achut Giree ${ }^{1,2}$, Felix Schell', Tobias Witting ${ }^{1}$, Gunnar Arisholm³, Claus Peter Schulz', Marc J. J. Vrakking ${ }^{1} ;{ }^{1}$ Max Born Inst., Germany; ${ }^{2}$ Amplitude Technologies, France; ${ }^{3}$ Norwegian Defence Research Establishment, Norway. A noncollinear optical parametric amplifier delivering more than $23 \mathrm{~W}$ at $100 \mathrm{kHz}$ at a central wavelength of $850 \mathrm{~nm}$ is presented. After compression pulses of $7.2 \mathrm{fs}$ with more than $210 \mu \mathrm{J}$ are obtained.

\section{SM3I.5 • 14:45}

Direct diode pumpedTi:Sapphire ultrafast regenerative amplifier system, Sterling J. Backus ${ }^{1,2}$, mathew kirchner ${ }^{1}$, Charles Durfee ${ }^{3}$ Henry Kapteyn ${ }^{1,4}$, Margaret Murnane ${ }^{4,1}$ ${ }^{1}$ KMLabs, USA; ${ }^{2} E C E$, Colorado State Univ. USA; ${ }^{3}$ Colorado School of Mines, USA; ${ }^{4}$ Univ. of Colorado, USA. We report on a cryogenically cooled Ti:sapphire ultrafast regenerative amplifier laser system producing multi-uJ energies with repetition rates continuously tunable from $50 \mathrm{kHz}$ up to $250 \mathrm{kHz}$ pumped with $450 \mathrm{~nm}$ fiber coupled laser diodes.

\section{SM3J • THz Communications- Continued}

SM3J.3 • 14:15

Active $\mathrm{THz}$ Waveguides Enabled by Liquid Metal Actuation, Kimberly S. Reichel ${ }^{1}$ Nicolas Lozada-Smith', Rajind Mendis' ${ }^{1}$ I Ishan Joshipura ${ }^{2}$, Michael Dickey², Daniel M. Mittleman': 'School of Engineering, Brown Univ. USA; ${ }^{2}$ Dept. of Chemical \& Biomolecular Engineering, North Carolina State Univ., USA. We utilize electronically reconfigurable liquid metals to dynamically modify the coupling between two $\mathrm{THz}$ waveguides, to realize an active tunable filter with channel add-drop functionality.

\section{SM3K • Silicon Photonics - Continued}

\section{SM3K.4 • 14:15}

Laser Annealing of Low Temperature Deposited Silicon Waveguides, Yohann Franz Antoine F. Runge ${ }^{1}$, Swe Z. Oo ${ }^{1}$, Noel Healy ${ }^{1}$, Gregorio Martinez-Jimenez', Ali Z. Khokhar' Antulio Tarazona ${ }^{1}$, Harold M. Chong ${ }^{1}$, Sakellaris Mailis', Anna C. Peacock'; 'Univ. of Southampton, UK. We report the fabrication of low temperature deposited polysilicon waveguides using a laser annealing process. Micro-Raman and XRD measurements revea the quasi-single crystal-like quality of the material, which exhibits low optical losses of $5.13 \mathrm{~dB} / \mathrm{cm}$.

\section{SM3L • Mode Locked Fiber Lasers I-Continued}

\section{SM3L.3 • 14:15}

Dirac Semimetal Thin-film Mode-locked Fiber Laser, Yafei Meng' Chunhui Zhu'1, Wenbin Gao', Yao Li', Xiang Yuan², Faxian Xiu², Yongbing $X^{1}{ }^{1}$, Yi Shi ${ }^{1}$, Frank (Fengqiu) Wang ${ }^{1}$ ${ }^{1}$ Nanjing Univ., China; ${ }^{2}$ Fudan Univ., China. We report that $\mathrm{MBE}$-grown three-dimensiona (3D) topological Dirac semimetal $\mathrm{Cd}_{3} \mathrm{As}_{2}$ thinfilm exhibits remarkable saturable absorption effects at 1, 1.5 and $2 \mu \mathrm{m}$. A mode-locked Tm fiber laser is demonstrated using such a SESAM-like material.
SM3J. $4 \cdot 14: 30$

Dispersion Compensation in Terahertz Communication Links Using Metallized 3D Printed Hollow Core Waveguide Bragg Gratings, Tian $\mathrm{Ma}^{1}$, Kathirvel Nallapan ${ }^{1}$ Hichem Guerboukha', Maksim Skorobogatiy'; 'Dept. of Engineering Physics, École Polytechnique de Montréal, Canada. A nove terahertz $(\mathrm{THz})$ waveguide Bragg grating is proposed for dispersion compensation. The results confirm single mode guidance of the fundamental mode, as well as large negative group velocity dispersion in the vicinity of $0.14 \mathrm{THz}$

\section{SM3J.5 • 14:45}

Patch array antenna coupling of $\mathrm{THz}$ source and detector, Lorenzo Bosco', Giacomo Scalari', Mattias Beck', Jerome Faist'; ${ }^{1}$ ETH Zurich, Switzerland. We study the performance of a Terahertz (THz) source-detector system coupled through equal patch-array antennas, using a single mode Quantum Cascade Laser and a Quantum Well Infrared Photodetector. The antenna allows surface emission and detection of light and use Benzocyclobutene as support.

\section{SM3K.5 1 14:30 Invited}

Effects of Dielectric Cladding on Si Nanophotonics, Yeshaiahu Fainman': 'Univ. of California San Diego, USA. We discuss effects of dielectric claddings on nanoscale engineered optical nonlinearities for $\mathrm{Si}$ nanophotonics applications to modulation and wave mixing of optical fields.
SM3L $4 \cdot 14: 30$

Novel Robust 2- $\mu \mathrm{m}$ All-PM Thulium/ Holmium Based Femtosecond Fiber Laser Oscillator, Heinar Hoogland ${ }^{1,2}$, Wolfgang Hänsel', Ronald Holzwarth'; ${ }^{1}$ Menlo Systems $\mathrm{GmbH}$, Germany; ${ }^{2}$ Dept. of Physics, Univ. of Erlangen-Nuremberg, Germany. We report on an all-PM mode locked 2-um central wavelength figure-9 fiber laser oscillator based on Thulium/Holmium codoped gain fiber supporting femtosecond pulses at $\mathrm{MHz}$-repetition rate.
SM3L.5 • 14:45

Broadband High Energy Breathing Laser at $1.6 \mu \mathrm{m}$, Jiqiang $\mathrm{Kang}^{1}$, Xiaoming $\mathrm{Wei}^{1}$ Kenneth Wong'; 'The Univ. of HongKong, China. We demonstrate a broadband high energy breathing fiber laser at $1.6 \mu \mathrm{m}$ with a robust configuration. It delivers $52.4-\mathrm{nm}$ flat optical spectrum and about 3.9-nJ pulse energy by 220-mW pump power. 


$\begin{array}{llc}\text { Marriott } & \text { Marriott } & \text { Marriott } \\ \text { Salon III } & \text { Salon IV } & \text { Salon V \& VI }\end{array}$

\section{CLEO: Science \& Innovations}

\section{SM3M • Progress in Optical Frequency Conversion- Continued}

SM3M.4 • 14:15

A Compact, Efficient Deep UV Optically Pumped VECSEL, Mikhail A. Yakshin ${ }^{1}$, Mahmoud Fallahi'; ' ${ }^{2}$ SESI, USA; ${ }^{2}$ College of Optical Sciences, The Univ. of Arizona, USA. We describe a compact and efficient UV laser that is under development. Over $190 \mathrm{~mW}$ power at $234 \mathrm{~nm}$ wavelength is obtained by harmonic conversion of the intra-cavity doubled output of an optically pumped $936 \mathrm{~nm}$ wavelength InGaAs VECSEL.
SM3M.5 • 14:30

263-nm Deep Ultraviolet Femtosecond Laser Pulses Generation in $\mathrm{K}_{3} \mathrm{~B}_{6} \mathrm{O}_{10} \mathrm{Cl}$ Crystal, Ninghua Zhang ${ }^{1}$, Hao Teng ${ }^{2}$, Peng $\mathrm{He}^{1}$, Hangdong Huang ${ }^{1}$, Jiangfeng Zhu', Wenlong Tian', Hongping $\mathrm{Wu}^{3}$, Shilie $\mathrm{Pan}^{3}$, shaobo fang ${ }^{2}$, Zhiyi Wei ${ }^{2}$; ${ }^{1}$ Xidian Univ., China; ${ }^{2}$ Inst. of Physics, Chinese Academy of Science, China; ${ }^{3}$ Xinjiang Technical Inst. of Physics \& Chemistry, Chinese Academy of Science, China. We reported the third harmonic generation using a $\mathrm{K}_{3} \mathrm{~B}_{6} \mathrm{O}_{10} \mathrm{Cl}$ crystal for the first time. Output power of $5.9 \mathrm{~mW}$ was obtained at the wavelength of $263-\mathrm{nm}$, corresponding to conversion efficiency of $4.5 \%$ to second harmonic.

SM3M.6 $14: 45$

Highly-efficient Broadband Second Harmonic Generation in Compact Fiber-integrated Thin-film $\mathrm{LiNbO}_{3} \mathrm{Nano}$-waveguides, Lu-Tong Cai ${ }^{1,2}$, Hui Hu${ }^{2}$, Andrey Gorbach ${ }^{3}$, Yi-Wen Wang', Wei Ding'; 'CAS Inst. of Physics, China; ${ }^{2}$ School of Physics, Shandong Univ., China; ${ }^{3}$ Dept. of Physics, Univ. of Bath, UK. We report a highly efficient (eta $\sim 450$ $\% W^{\wedge}(-1) c m^{\wedge}(-2)$ ) and broadband (phase matching bandwidth Delta_lambda 100nm) second harmonic generation in a 50 micron long fiber-integrated LNOI waveguide. We demonstrate phase matching tunability by small adjustments of microfiber diameter.

\section{SM3N • Plasmonics and Metamaterials-Continued}

\section{SM3N.4 $14: 15$}

Multispectral Metasurface Absorbers for Optoelectronic Devices, Jon W. Stewart ${ }^{2}$, Gleb M. Akselrod², David R. Smith"2, , Maiken Mikkelsen 2,1; 'Dept. of Physics, Duke Univ., USA; ${ }^{2}$ Dept. of Electrical and Computer Engineering, Duke Univ., USA. We demonstrate multispectral metasurfaces over wafer-scale areas exhibiting greater than 85 percent absorption, 100 nm linewidths from 580-1125 $\mathrm{nm}$ by patterning plasmonic resonators in micron-scale pixels using a fusion of bottomup and top-down fabrication techniques.

\section{SM3O • Integrated Quantum Photonics-Continued}

SM30.3 • 14:15

An Integrated Photonic Chip for Continuous-variable Quantum Key Distribution, Gong Zhang ${ }^{1}$, Jian $\mathrm{Wu}^{2}$, Song $\mathrm{Yu}^{2}$, Leong Chuan Kwek ${ }^{3}$, Jiangbin Gong ${ }^{4}$, Weibo Gao ${ }^{5}$, Yidong Chong ${ }^{5}$, Wee Ser ${ }^{1}$, Ai Qun Liu ${ }^{1}$ ${ }^{1}$ School of Electrical and Electronic Engineering, Nanyang Technological Univ., Singapore; ${ }^{2}$ State Key Lab of Information Photonics and Optical Communications, Beijing Univ. of Posts and Telecommunications, China; ${ }^{3} \mathrm{Cen}$ tre for Quantum Technologies, National Univ. of Singapore, Singapore: ${ }^{4}$ Dept. of Physics and Centre for Computational Science and Engineering, National Univ. of Singapore, Singapore; ${ }^{5}$ School of Physical and Mathematical Sciences, Nanyang Technological Univ., Singapore. An integrated photonic chip for continuous-variable quantum key distribution is designed and fabricated. The modulation speed reaches $0.4 \mathrm{GHz}$. High extinction ratio up to $40 \mathrm{dBm}$ for polarization multiplexing components is achieved.

SM3N.5 • 14:30

Graphene metasurface devices for spatial light modulation and imaging, Beibei Zeng ${ }^{1}$, Akhilesh Singh"', Abul Azad', Aditya Mohite', Houtong Chen'; 'Los Alamos National Lab, USA. We demonstrate an ultrathin solidstate modulator employing metasurface/ graphene/semiconductor heterostructures, where the optical properties can be efficiently tuned through applying low voltage biases in each pixel, serving as infrared spatial light modulators for imaging and sensing.

\section{SM3N.6 $14: 45$}

Metasurfaces Based on Nano-Patterned Phase-Change Memory Materials, Shane A. Colburn', Alan Zhan' ${ }^{1}$ Sanchit Deshmukh², Jason Myers ${ }^{3}$, Jesse Frantz ${ }^{3}$, Eric Pop ${ }^{2}$, Arka Majumdar'; ' 'Univ. of Washington, USA; ${ }^{2}$ Stanford Univ., USA; ${ }^{3}$ U.S. Naval Research Lab, USA. We fabricate nano-patterned films of GeSbTe to make optical metasurfaces of isolated scatterers with the goal of arbitrary electrical reconfigurability, and induce phase changes by heating with a femtosecond pulsed laser.
Implementation and Verification of Boson Sampling with Integrated Photonics, Fabio Sciarrino'; 'Univ degli Studi di Roma La Sapienza, Italy. Boson sampling is a computational task hard for classical computers, but efficiently solvable via bosonic interference in a specialized quantum computer. We report several experiments of boson sampling implemented with integrated quantum photonics.

\section{SM30.4 14:30 Invited}


Executive Ballroom

$210 \mathrm{~A}$

Executive Ballroom

210B

\section{CLEO: Applications \\ \& Technology}

AM3A • A\&T Topical Review on Scientific and Commercial Progress in Semiconductor Lasers I-Continued

AM3A.4 • 15:00

Mid-infrared Quantum Cascade Lasers Transfer-printed on Silicon-on-Sapphire, Seungyong Jung', Jeremy Kirch', Jae Hyun Kim', Luke J. Mawst², Mikhail A. Belkin' Dan Botez'; ' 'Univ. of Texas at Austin, USA ${ }^{2}$ Univ. of Wisconsin-Madison, USA. We experimentally demonstrate transfer-printing of mid-infrared quantum cascade lasers onto a silicon-on-sapphire platform and laser light coupling to a silicon waveguide via an adiabatically tapered mode converter Transferred devices operate in pulsed mode at room-temperature.

AM3A.5 • 15:15

Coupled-Cavity Lasers for a Low-Power Integrated Coherent Optical Receiver Shamsul Arafin', Gordon Morrison², Milan Mashanovitch 2', Leif Johansson², Larry A. Coldren'; 'Univ. of California Santa Barbara, USA; ${ }^{2}$ Freedom Photonics LLC, USA Compact, tunable, low-power consumption coupled-cavity lasers are designed and experimentally demonstrated. Single-mode operation with an SMSR $>24 \mathrm{~dB}$ and $>11 \mathrm{~nm}$ tuning range are achieved, being suitable as on-chip local oscillators in low-power integrated optical coherent receivers.

\section{AM3B • Greenhouse Gas Sensing-Continued}

\section{AM3B.6 • 15:00}

Plasmonic Nanoantenna Array with SingleChip Integrated Metal-Organic Framework for Infrared Absorption $\mathrm{CO}_{2}$ Sensing, Alan X. Wang'; 'Oregon State Univ., USA. Surface-enhanced infrared absorption is a spectroscopic technique but unsuitable for gas spectroscopy due to the need for long absorption path lengths. We demonstrate a device using metal-organic framework integrated with plasmonic nanoantennas for $\mathrm{CO}_{2}$ sensing.

AM3B.7 • 15:15

Development of $\mathrm{IR}^{2}$-Hi5 multipass MIR isotope analyzer for plant photosynthesis and respiration study, Zhenyou Wang ${ }^{1}$ Yan Zhuang', Andrei Deev', Sheng Wu'; ${ }^{1}$ Arrow Grand Technologies, USA. A fast, low-sample-volume mid-infrared carbon isotope ratio analyzer was developed and characterized. It's employed to demonstrate real-time monitoring the $\mathrm{CO}_{2}$ concentration and carbon isotope fractionation in plant photosynthesis and respiration processes.
Executive Ballroom $210 \mathrm{C}$

\section{CLEO: Science \&} Innovations

\author{
SM3C • In Vivo/Deep Tissue \\ Imaging-Continued
}

SM3C. 5 ・ 15:00

Deep Tissue Coherent Imaging Using Speckle Intensity Correlations Over Object Position, Qiaoen Luo', Kevin J. Webb' 'Purdue Univ., USA. Spatial speckle intensity correlation measurements in relation to object position are used to image moving objects embedded inside heavily scattering centimeter-thick chicken breast tissue.

SM3C.6・15:15

Doubling the Sensitivity of Multiphoton Frequency-Domain Fluorescence Lifetime Images, Yide Zhang', Genevieve Vigil', Aamir Khan', Scott S. Howard'; 'Electrical Engineering, Univ. of Notre Dame, USA. We demonstrate super-sensitive multiphoton frequency-domain fluorescence lifetime images using the recently developed DC\&1 $\omega$ method by showing a two-fold improvement in imaging sensitivity compared to the conventional $1 \omega$ phase fluorometry.
FM3D.7 • 15:15

Harmonic Sideband Generation in Monolayer Transition Metal Dichalcogenides, Peter G. Hawkins 1,2 , Johannes Steiner ${ }^{1}$ Ulrich Huttner ${ }^{1,2}$, Fabian Langer ${ }^{3}$, Christoph Schmid ${ }^{3}$, Stefan Schlauderer ${ }^{3}$, Stephan W. Koch' Rupert Huber ${ }^{3}$ Mackillo Kira2. ${ }^{1}$ Univ. of Marburg, Germany; ${ }^{2}$ Univ. of Michigan, USA ${ }^{3}$ Univ. of Regensburg, Germany. Harmonic sidebands are compared in monolayer vs. bulk WSe $e_{2}$. We find strong Coulomb enhancement of sidebands in monolayer WSe when resonant vs. non-resonant 1s-exciton excitations are compared, this is virtually absent in bulk. 

Executive Ballroom
$210 \mathrm{E}$

Joint

JM3E • Symposium on Sources of Nonclassical Light and their Scalability I-Continued

\section{JM3E.5 • 15:00}

Tunable Quantum Emission from Atomic Defects in Hexagonal Boron Nitride, Gabriele Grosso', Hyowon Moon ${ }^{1}$, Benjamin Lienhard' ${ }^{1}$ Sajid Ali², Marco Furchi', Michael Walsh', Dmitri K. Efetov', Pablo JarilloHerrero', Mike J. Ford², Igor Aharonovich², Dirk Englund'; 'MIT, USA; ${ }^{2}$ Univ. of Technology Sydney, Australia. We demonstrate that strain control of hexagonal boron nitride allows spectral tuning of single photon emitters over $6 \mathrm{meV}$. We propose a material processing that sharply improves the single-photon purity with $\mathrm{g}^{2}(0)=0.077$, and brightness with emission rate exceeding $10^{7}$ counts/s at saturation.

\section{JM3E.6 • 15:15}

Nanoscale Strain-Engineering and Optics of Quantum Emitters in a Two-Dimensional Semiconductor, Santosh Kumar ${ }^{1}$ Artur Branny', Mauro Brotons-Gisbert², Rima Al-Khuzheyri', Raphaël Proux' ${ }^{1}$, Guillem Ballesteros-Garcia ${ }^{1}$, Juan F. Sanchez-Royo ${ }^{2}$ Brian D. Gerardot'; ; Heriot-Watt Univ., UK ${ }^{2}$ ICMUV, Instituto de Ciencia de Materiales, Universidad de Valencia, Spain. We present deterministic fabrication of a two-dimensional lattice of quantum emitters in an atomically thin semiconductor. Resonant laser spectroscopy of these emitters reveals localized exciton states that exhibit stable, bright and high-purity single photon emission.

\section{Executive Ballroom $210 \mathrm{~F}$}

Executive Ballroom
210G
Executive Ballroom

$210 \mathrm{H}$

\section{CLEO: QELS-Fundamental Science}

FM3F • Nonlinear Optics in Propagating Geometries IContinued

FM3F.7 • 15:00

Loss of Polarization in Collapsing Beams of Elliptical Polarization, Gauri Patwardhan ${ }^{1,4}$ Xiaohui Gao ${ }^{4}$, Avik Dutt ${ }^{2,3}$, Jared Ginsberg ${ }^{4}$ Alexander L. Gaeta ${ }^{4} ;{ }^{1}$ School of Applied and Engineering Physics, Cornell Univ., USA; ${ }^{2} S$ chool of Electrical and Computer Engineering, Cornell Univ., USA: ${ }^{3}$ Dept. of Electrical Engineering, Columbia Univ., USA; ${ }^{4}$ Dept. of Applied Physics and Applied Mathematics, Columbia Univ., USA. We predict that elliptically polarized beams undergoing multiple collapsing-defocusing cycles experience a loss of polarization and demonstrate this experimentally by measuring a large increase in fluctuations of nonlinear ellipse rotations for pulses undergoing filamention.

FM3F.8 • 15:15

Three-Dimensional Spatiotemporal Pulse Train Solitons, Oren Lahav' ${ }^{1}$, Ofer Kfir ${ }^{1}$, Pave Sidorenko ${ }^{1}$, Maor Mutzafi ${ }^{1}$, Avner Fleischer ${ }^{1}$ Oren Cohen'; 'Technion Israel Inst. of Technology, Israel. We experimentally demonstrate three-dimensional spatiotemporal solitons. A spatially-bright temporally-dark pulse-train beam is trapped spatially, mainly by a slowly responding photorefractive selffocusing nonlinearity while each pulse is trapped by the fast Kerr nonlinearity.

\author{
FM3G - Dirac-cone \\ Metamaterials-Continued
}

\section{FM3G.6 • 15:00}

Transition metamaterials for local-field en hancement, Yang Li' ${ }^{1}$ Philip Camayd-Muñoz ${ }^{1}$ Daryl Vulis ${ }^{1}$, Peter Saeta ${ }^{1}$, Yu Peng ${ }^{1}$, Orad Reshef ${ }^{1}{ }^{2}$, Olivia L. Mello ${ }^{1}$, Haoning Tang ${ }^{1}$ Marko Lončar', Eric Mazur ${ }^{1} ;{ }^{1}$ Harvard Univ. USA; ${ }^{2}$ Univ. of Ottawa, Canada. We achieve strong local-field enhancement within a nove inhomogeneous metamaterial whose graded refractive index is realized using Dirac-cone metamaterials. This transition metamaterial provides a powerful tool for sensing, nonlinear optics, and quantum optics.

\section{FM3G.7 • 15:15}

Optical Phase Retrieval Using Conical Refraction Imaging in Structured Media, Zun Huang ${ }^{1,2}$, Evgenii E. Narimanov ${ }^{1,2}$; ${ }^{1}$ Electrical and Computer Engineering, Purdue Univ. USA; ${ }^{2}$ Birck Nanotechnology Center, Purdue Univ., USA. We present a new optical phase retrieval method using the conical refraction imaging in structured media, which enables a single simultaneous measurement of multiple images and allows a stable recovery of the optical phase.

\section{$\mathrm{FM} 3 \mathrm{H} \cdot$ Electron - Plasmon Interactions-Continued}

\section{FM3H.6 • 15:00}

Smith-Purcell radiation from low-energy electrons, Aviram Massuda1,2, Charles Roques-Carmes ${ }^{1,2}$, Yujia Yang ${ }^{1}$, Steven E. $\mathrm{Kooi}^{3}$, Yi Yang ${ }^{1,2}$, Chitraang Murdia ${ }^{2}$, Karl K. Berggren 1 , Ido Kaminer², Marin Soljacic ${ }^{2}$ 'Electrical'Engineering and Computer science, MIT, USA: ${ }^{2}$ Dept of Physics, MIT, USA; ${ }^{3}$ Inst. for Soldier Nanotechnologies, USA. Focused electron beams can induce electromagnetic radiation from periodic surfaces. We have used low-energy electrons $(1.5-6 \mathrm{kV})$ to induce visible light emission from nanoscale gratings ( $50 \mathrm{~nm}$ and $60 \mathrm{~nm}$ ). Our results coincide well with numerical simulations.

FM3H.7 • 15:15

Investigation of the light generation from crystalline Ag-cubes based metal-insulatormetal tunnel junctions, Haoliang Qian ${ }^{1}$ Su-Wen Hsu', Kargal Gurunatha', Jie Zhao' Conor T. Riley', Dylan Lu', Andrea Tao', Zhaowei Liu'; 'Univ. of California, San Diego, USA Efficient light generation from inelastic tunneling using self-assembled edge-to-edge single crystalline silver nanocubes has been demonstrated with efficiency up to $\sim 2 \times 10^{-3}$ which brings on-chip ultrafast optical sources one step closer to reality.

14:00-17:30 Understanding Unconscious Bias, Winchester Room/Hilton

15:30-16:00 Coffee Break, Concourse Level 


\section{Meeting Room $211 \mathrm{~B} / \mathrm{D}$}

Meeting Room
$212 \mathrm{~A} / \mathrm{C}$
Meeting Room
$212 \mathrm{~B} / \mathrm{D}$
Marriott

Salon I \& II

\section{CLEO: Science \& Innovations}

\section{SM3I • Ultrafast Amplifiers- Continued}

\section{SM3I.6 $\bullet 15: 00$}

Original Ti:Sa $10 \mathrm{kHz}$ Front-End Design Delivering $17 \mathrm{fs}$, 170 mrad CEP Stabilized Pulses up to 7 W., Anna Golinelli, ${ }^{2}$, Chen Xiaowei ${ }^{2}$, Emilien Gontier ${ }^{2}$, Benoit Bussiere ${ }^{2}$, Olivier Tcherbakoff', Pascal D'Oliveira ${ }^{1}$ Pierre Mary Paul3 ${ }^{3,2}$, Jean-Francois Hergott ${ }^{1}$. 'Commissariat a l'Energie Atomique, France; ${ }^{2}$ Amplitude Technologies, France; ${ }^{3} \mathrm{Con}$ tinuum Inc, Amplitude Laser Group, USA We present an original compact, $10 \mathrm{kHz} \mathrm{Ti}: \mathrm{Sa}$ regenerative amplifier design that minimizes existing thermal effects allowing to produce $17 \mathrm{fs}$ pulse duration with a $170 \mathrm{mrad}$ shot to shot residual CEP noise.

SM3I.7 • 15:15

Spectral phase instabilities during amplification in Ti:Sapphire, Roland Nagymihály ${ }^{1,2}$ Peter Jojart ${ }^{1,2}$, Adam Borzsonyi ${ }^{1,2}$, Huabao Cao', Karoly Osvay'; ' ${ }^{1}$ LLI-HU Non-Profit Ltd. Hungary; ${ }^{2}$ Univ. of Szeged, Dept. of Optics and Quantum Electronics, Hungary. Stability of the spectral phase, including the carrierenvelope phase in water- and cryogenically cooled multipass Ti:Sapphire amplifiers was measured with spectral interferometry. Low phase noise operation of polarization encoded Ti:Sapphire amplification is also under investigation.

\section{SM3J • THz Communications- Continued}

SM3J.6 $\bullet 15: 00$

A Demultiplexer for Terahertz Wireless Links, Jianjun $\mathrm{Ma}^{1}$, Nicholas Karl ${ }^{1}$, Sara Bretin², Guillaume Ducournau², Daniel Mittleman'; 'School of Engineering, Brown Univ. USA; ${ }^{2}$ Institut d'Electronique de Microélectronique et de Nanotechnologie (IEMN), France. A leaky-wave antenna based terahertz ( $\mathrm{THz}$ ) demutiplexer is presented in this paper. It can realize demultiplexing of real-time $\mathrm{THz}$ data streams at different carrier frequencies under identical or different data rates.

SM3J.7 • 15:15

Fiber-Coupled, Photoconductive Heterodyne Receiver Operating at Frequencies up to $1 \mathrm{THz}$, Simon Nellen', Björn Globisch"1, Robert Kohlhaas', Dennis Stanze' Thorsten Göbel', James O' Gorman², Liam Barry ${ }^{3}$, Martin Schell'; ${ }^{1}$ Fraunhofer Heinrich Hertz Inst., Germany; ${ }^{2} X y l o p h o n e ~ O p t i c s$, Ireland; ${ }^{3}$ Dublin City Univ., Ireland. An optoelectronic InGaAs-based terahertz $(\mathrm{THz})$ receiver for heterodyne detection up to 1 $\mathrm{THz}$ is demonstrated for the first time operating at $1.5 \mu \mathrm{m}$. This wavelength allows for a fully fiber-coupled heterodyne system, which simplifies applications spectroscopy and $\mathrm{THz}$ communications.

\section{SM3K • Silicon Photonics L- Continued}

SM3K.6 $15: 00$

Sidewall Roughness in $\mathrm{Si}_{3} \mathrm{~N}_{4}$ Waveguides Directly Measured by Atomic Force Microscopy, Samantha P. Roberts ${ }^{1}$, Xingchen $\mathrm{Ji}^{1,3}$, Jaime Cardenas ${ }^{1,2}$, Alex Bryant ${ }^{4}$, Michal Lipson'; ' ${ }^{1}$ Columbia Univ., USA; ${ }^{2}$ The Inst. of Optics, Univ. of Rochester, USA: ${ }^{3}$ School of Electrical and Computer Engineering, Cornell Univ., USA; ${ }^{4}$ School of Materials Science and Engineering, Georgia Inst. of Technology, USA. We have developed a robust method to measure side-wall-roughness of sub-micron feature waveguides using atomic-force-microscopy. We measure the side-wall-roughness of silicon-nitride waveguides patterned by DUV photolithography and compare results of two different etch chemistries.

\section{SM3K.7 • 15:15}

Telecom Band Plasmonic Enhanced Internal Photoemission Photodetector Based On Deposited Amorphous Silicon, Nir Kaplan ${ }^{1}$ Meir Y. Grajower ${ }^{1}$, Noa Mazurski ${ }^{1}$, Joseph Shappir', Uriel Levy'; 'The Hebrew Univ. Of Jerusalem, Israel. We present a first demonstration of plasmonic enhanced internal photoemission Schottky photodetector implemented by low temperature deposited amorphous silicon for the telecom regime. The detector show responsivity of $53 \mu \mathrm{A} / \mathrm{V}$.

\section{SM3L・ Mode Locked Fiber Lasers I-Continued}

SM3L.6 • 15:00

Fixed-Point Tuning of a Frequency Comb from a Passively Mode-Locked Soliton $\mathrm{Fi}$ ber Laser, Ken Kashiwagi ${ }^{1,2}$, Hajime Inaba ${ }^{1,2}$; ${ }^{\prime}$ National Inst. of Advanced Industrial Science and Technology (AIST), Japan; ${ }^{2}$ JST, ERATO MINOSHIMA Intelligent Optical Synthesizer (IOS), Japan. We report wavelength-dependent pump-induced responses of repetition and carrier-envelope-offset frequencies of a soliton fiber laser comb. Lasing wavelength adjustment can tune a fixed point and design controlling orthogonality between a pumplaser current and another actuator.
SM3L.7 • 15:15

Diode-pumped CNT Mode-locked $\mathrm{Ho}^{3+}$ doped Fluoride Fiber Laser at 1.2 um, Junfeng Wang ${ }^{1}$, Xiushan Zhu' ${ }^{1}$ Yunxiu Ma' ${ }^{1}$, Jie Zong ${ }^{2}$, Kort Wiersma ${ }^{2}$, Arturo Chavez-Pirson ${ }^{2}$ Robert A. Norwood ${ }^{1}$, Shijie $\mathrm{Fu}^{3}$, Wei Shi ${ }^{3}$ Nasser Peyghambarian ${ }^{1} ;{ }^{1}$ College of Optical Sciences, Univ. of Arizona, USA : ${ }^{2}$ NP Photonics, USA $:{ }^{3}$ College of Precision Instrument and Optoelectronics Engineering, Tianjin Univ. China. Mode-locked operation of a $1150 \mathrm{~nm}$ diode-pumped holmium-doped fluoride fiber laser at 1.2 um based on carbon nanotube saturable absorber is reported. Mode-locked laser at $1192 \mathrm{~nm}$ with a repetition rate of

18.47 $\mathrm{MHz}$ was obtained. 


$\begin{array}{llc}\text { Marriott } & \text { Marriott } & \text { Marriott } \\ \text { Salon III } & \text { Salon IV } & \text { Salon V \& VI }\end{array}$

\section{CLEO: Science \& Innovations}

\section{SM3M • Progress in Optical Frequency Conversion- Continued}

\section{SM3M.7 • 15:00}

Intracavity Difference-Frequency Mixing of OPO Signal and Idler Pulses in $\mathrm{BaGa}_{4} \mathrm{Se}_{7}$ Andrey A. Boyko ${ }^{1,2}$, Nadezhda Y. Kostyukova $^{1,2}$, Valeriy Badikov ${ }^{3}$, Dmitrii Badikov ${ }^{3}$, Vladimir Panyutin ${ }^{1}$, Galina Shevyrdyaeva ${ }^{3}$, Valdas Pasiskevicius ${ }^{4}$, Andrius Zukauskas ${ }^{4}$, Georgi M. Marchev', Dmitry Kolker ${ }^{5}$, Valentin Petrov': 'Max Born Inst., Germany; ${ }^{2}$ Special Technologies, Ltd., Russia; ${ }^{3}$ Kuban State Univ., Russia; ${ }^{4}$ Royal Inst. of Technology, Sweden; ${ }^{5}$ Novosibirsk State Univ., Russia. An overall quantum conversion efficiency of $7.8 \%$ is achieved by intracavity mixing the signal and idler of a $1.064-\mu \mathrm{m}$ pumped $\mathrm{Rb}$ :PPKTP OPO in $\mathrm{BaGa}_{4} \mathrm{Se}_{7}$, generating $>0.7 \mathrm{~mJ}$ pulse energy at $\sim 7 \mu \mathrm{m}$ and $100 \mathrm{~Hz}$.

SM3M.8 • 15:15

Burst-Mode Pumping for Single-Pulse Parametric Amplification in the Long Wave IR, Ignas Astrauskas', Edgar Kaksis ${ }^{1}$ Tobias Flöry', Giedrius Andriukaitis' ${ }^{1}$, Pave Malevich', Tadas Balciunas ', Audrius Pugzlys ${ }^{1}$ Andrius Baltuska'; ${ }^{1}$ Photonics Inst., Vienna Univ. of Technology, Austria. We propose and demonstrate a scheme for LWIR pulse amplification based on spatial and spectral demultiplexing of a pulse burst from a 1 um laser amplifier. This method enables multi-color multi-beam pumping of an OPA without complications intrinsic to coherent pulse combining techniques.

\section{$\mathrm{SM} 3 \mathrm{~N} \cdot$ Plasmonics and Metamaterials-Continued}

SM3N.7 • 15:00

Thermal Homeostasis Device Using PhaseChange Materials, Shao-Hua Wu', Mingkun Chen ${ }^{1}$, Luqi Wang ${ }^{1}$, Michael Barako², Vladan Jankovic ${ }^{2}$, Philip Hon², Luke Sweatlock ${ }^{2}$, Michelle Povinelli'; 'Univ. of Southern California, USA; ${ }^{2} N G$ Next Northrop Grumman Corporation, USA. We design a thermal homeostasis device that passively regulates temperature $20 \mathrm{x}$ better than regular semiconductor materials. The thermal emission changes by a factor of 10 as the material temperature crosses a phase transition.

\section{SM3O • Integrated Quantum Photonics-Continued}

SM30.5 • 15:00

On-Chip Auto-Correlator Using TwoPhoton-Absorption Photodiode Array and Counter-Propagating Slow Light, Keisuke Kondo', Toshihiko Baba'; 'Dept. of Electrical and Computer Engineering, Yokohama National Univ., Japan. We demonstrate an ultra-compact solid-state auto-correlator without delay scanning. We observe counterpropagating slow light pulses in a Si photonic crystal waveguide with a two-photon-absorption photodiode array. Sensitive detection is available for picosecond pules.
SM3N.8 • 15:15

Surface-Plasmon Opto-Magnetic Field Enhancement for Magnetization Reversal of On-Chip Nanomagnets, Aveek Dutta', Deesha Shah¹, Bradley Beauchamp', Kuntal Roy', Vladimir M. Shalaev', Ernesto E. Marinero', Alexandra Boltasseva ${ }^{1}$; ${ }^{1}$ Purdue Univ., USA. We study, computationally, TiN plasmonic resonator coupled to a nanomagnet for magnetization switching. We find that compared to an isolated nanomagnet under similar illumination conditions, localized surface plasmon resonances in the coupled system generate larger magnetic fields in the nanomagnet.

\section{SM30.6 $15: 15$}

Electrically Pumped, Waveguide-Coupled Si Light Emitting Diodes, Sonia M. Buckley', Martin Stevens ${ }^{1}$, Sae Woo Nam¹, Richard Mirin', Jeffrey Shainline ${ }^{1}$; ${ }^{1}$ NIST, USA. We describe fabrication and testing of LEDs based on emissive defect centers in Si and discuss our progress toward low-temperature on-chip integrated sources and detectors.

14:00-17:30 Understanding Unconscious Bias, Winchester Room/Hilton

15:30-16:00 Coffee Break, Concourse Level 
Executive Ballroom 210A
Executive Ballroom

210B
Executive Ballroom

$210 \mathrm{C}$
Executive Ballroom 210D

\section{CLEO: Applications \\ \& Technology}

16:00-18:00

AM4A • A\&T Topical Review on Scientific and Commercial Progress in Semiconductor Lasers II

Presider: Bojan Resan; Lumentum, Univ. of Applied Sciences, Switzerland

\section{AM4A.1 • 16:00 Invited}

Low Noise Ultrafast Pulse Generation and Signal Processing Using Semiconductor Lasers, Peter J. Delfyett ${ }^{1}$, A Ardey ${ }^{1}$, S Bhooplapur ${ }^{1}$, E Sarailou'; ${ }^{1}$ CREOL, The College of Optics \& Photonics, USA. This talk covers novel techniques and applications of low noise ultrafast optical pulses and stabilized optical frequency combs. Applications are focused on photonic ultrawide-band signal processing, such as waveform generation and measurement, and matched filtering.
16:00-17:45

AM4B - Combustion and

Atmospheric Photonics

Presider: To be Determined

\section{AM4B.1 - 16:00 Invited}

In-Vivo Monitoring of Energy Chemistry and Energy Production with High Spatia Resolution, aidong yan ${ }^{1}$, Paul Ohodnicki ${ }^{2}$ Michael Buric ${ }^{2}$, Shiwoo Lee ${ }^{2}$, Ming-Jun Li ${ }^{3}$ Kevin P. Chen': 'Univ. of Pittsburgh, USA ${ }^{2}$ National Energy Technology Lab, USA; ${ }^{3}$ Corning Inc., USA. This talk discusses developments of distributed fiber sensors for real-time and simultaneous monitoring of fuel consumption and resulting temperature/ strain variation with sub-cm spatial resolution in reactors such as solid oxide fuel cells during their operations.

\section{CLEO: Science \& Innovations}

16:00-18:00

SM4C $\bullet$ Optofluidic

Components and Systems

Presider: Aaron Hawkins; Brigham Young Univ., USA

SM4C.1 1 16:00 Invited

Optofluidic Chips for Raman Spectroscopy and Optical Trapping, Heidi Ottevaere ${ }^{1}$ Qing Liu', Diane De Coster ${ }^{1}$, Jürgen Van Erps ${ }^{1}$, Michael Vervaeke ${ }^{1}$, Hugo Thienpont ${ }^{1}$, ${ }^{1}$ Vrije Universiteit Brussel, Belgium. We present the modeling, design and fabrication of microfluidic devices incorporating Raman spectroscopy, from which one enables confocal Raman measurements on-chip, as well as optical trapping. In a proof-of-concept demonstration, we measure the Raman spectra of various solutions and investigate the trapping capabilities of the replicated chips.

\begin{abstract}
AM4A.2 • 16:30 Invited
Ultrafast Semiconductor Disk Lasers, Ursula Keller'; 'Physics Dept., ETH Zurich, Switzerland. The performance of ultrafas semiconductor disk lasers rapidly advanced over the last decades. There is a strong interest from industry for inexpensive, compact and reliable ultrafast laser sources in the picosecond and femtosecond domain. The aim of this review is to describe the application potential and to give an overview of the current status of modelocked semiconducto disk lasers. Particular focus is placed on the ongoing efforts to achieve shorter pulses with higher peak powers.
\end{abstract}

AM4B.2 • 16:30

Withdrawn.

\author{
SM4C.2 • 16:30 \\ Vertically Embedded Multimode-Interfer- \\ ence Waveguide-Based Optical Stretchers \\ for Mechanical Characterization of Cells, \\ Zhanshi Yao ${ }^{1}$, Andrew W. Poon ${ }^{1}{ }^{1}$ Hong Kong \\ Univ. of Sci. and Tech., Hong Kong. We dem- \\ onstrate an on-chip optical cell stretcher us- \\ ing optical lattices generated from SU8-filled \\ vertically embedded multimode-interference \\ waveguides in a silicon substrate. We extract \\ the shear modulus of $\sim 2 \mu \mathrm{N} / \mathrm{m}$ from swollen \\ rabbit red blood cells.
}

\section{6:00-18:00 \\ SM4D - Nanophotonics, Waveguides, and} Microresonators in Sensing Presider: Aleksandra Foltynowicz; Umea Univ., Sweden

\section{SM4D.1 • 16:00}

Transform-limited dual-comb spectroscopy using free-running waveguide lasers, Nicolas Bourbeau Hebert ${ }^{1}$, Jean-Danie Deschênes', Hugo Bergeron', George Chen², Champak Khurmi², David Lancaster², Jérôme Genest1: 'Université Laval, Canada; ${ }^{2}$ Univ. of South Australia, Australia. We present a standalone dual-comb platform based on two mutually stable waveguide lasers integrated in the same glass chip. Residual fluctuations are compensated using an algorithm seeded only by interferograms, which yields transform-limited spectra.

\section{SM4D 2 • 16:15 Invited}

Waveguide Cavities for Absorption Detection of Chemicals, Hans-Peter Loock 'Queen's Univ. - Chemistry, Canada. Fibe optic cavities based on either on fiber loops or identical FBGs are used to detect the optical loss due to trace chemicals based on the cavity ring-down time or the photoacoustic effect, respectively. 
Executive Ballroom

210E

\section{Joint}

16:00-18:00

JM4E • Symposium on Sources

of Nonclassical Light and their

Scalability II

Presider: Joshua Nunn; Univ. of

Oxford, UK
Executive Ballroom

210F
Executive Ballroom

210G
Executive Ballroom

$210 \mathrm{H}$

\section{JM4E.1 16:00 Invited}

Quantum Dot Based Devices for Scaling Up Optical Quantum Technologies, Pascale Senellart'; ${ }^{1}$ Center for Nanoscience and Nanotechnology, CNRS, France. We discuss our recent progresses on developing efficient quantum devices for scaling up optical quantum technologies. We insert semiconductor quantum dots in microcavities and use these artificial atoms to fabricate near-optimal single photon sources and single photon filters.

\section{JM4E.2 • 16:30}

Heterogeneous III-V / $\mathrm{Si}_{3} \mathrm{~N}_{4}$ integration for scalable quantum photonic circuits, Marcelo I. Davanco', Jin Liu'1,2, Luca Sapienza ${ }^{3}$, Chen-Zhao Zhang ${ }^{4}$, Jose Vinicius De Miranda Cardoso ${ }^{1,5}$, Varun Verma ${ }^{6}$, Richard Mirin ${ }^{6}$, Sae Woo Nam ${ }^{6}$, Liu Liu ${ }^{4}$, Kartik Srinivasan ${ }^{1} ;{ }^{1}$ NIST, USA; ${ }^{2}$ Physics, Sun Yat-Sen Univ., China; ${ }^{3}$ Physics \& Astronomy, Univ. of Southampton, UK: ${ }^{4}$ South China Normal Univ., China; ${ }^{5}$ Federal Univ. of Campina Grande, Brazil; ${ }^{6}$ National Inst. of Standards and Technology, USA. We develop a scalable heterogeneous integration platform for quantum photonic circuits based on $\mathrm{Si}_{3} \mathrm{~N}_{4}$ waveguides and on-chip, self-assembled InAs quantum dot-based single-photon sources. Hybrid waveguides, photonic crystals, and microring resonators are demonstrated.

\section{CLEO: QELS-Fundamental Science}

16:00-18:00

FM4F • Nonlinear Optics in Propagating Geometries II Presider: J. Stewart Aitchison; Univ. of Toronto, Canada

FM4F.1 • 16:00

Rogue waves in red blood cell suspensions, Yuxuan Ren ${ }^{1}$, Josh Lamstein ${ }^{1}$, Trevor S. Kelly' Chensong Zhang' ${ }^{1}$, Yong Sun ${ }^{1}$, Claudio Conti ${ }^{3}$, Demetrios Christodoulides ${ }^{4}$, Zhigang Chen"1,2; 'San Francisco State Univ., USA ${ }^{2}$ Nankai Univ., China; ${ }^{3}$ Univ. Sapienza, Italy; ${ }^{4} \mathrm{CREOL} / \mathrm{College}$ of Optics, Univ. of Central Florida, USA. We observe rogue-wave-like events in red-blood-cell suspensions driven by light scattering and Brownian motion. In contradistinction with results from polystyrene bead suspensions, at high powers, the optical nonlinearity leads to altogether different probability distributions.

FM4F.2 • 16:15

Deep penetration of light through suspensions of red blood cells, Josh Lamstein ${ }^{1}$ Rekha Gautam1, Tobias Hansson ${ }^{3}$, Anna Bezryadina ${ }^{1,4}$, Benjamin Wetzel ${ }^{3,5}$, Roberto Morandotti ${ }^{3}$, Zhigang Chen ${ }^{1,2 .}{ }^{1}$ San Francisco State Univ., USA; ${ }^{2}$ Nankai Univ., China; ${ }^{3}$ INRS Université du Québec, Canada; ${ }^{4}$ Univ. of California, San Diego, USA; ${ }^{5}$ Univ. of Sussex, UK. We demonstrate nonlinear self-trapping of a light beam in human red blood cell suspensions with varying liquid buffer concentrations, along with a numerical model that features an effective nonlocal nonlinearity to explain our experimental observations.

\section{FM4F.3 • 16:30}

Second-harmonic focusing by nonlinear turbid medium via feedback-based wavefront shaping, Yanqi Qiao ${ }^{1,2}$, Xianfeng Chen ${ }^{1,2}, Y a-$ jun Peng ${ }^{1,2}$, Yuanlin Zheng ${ }^{1,2} ;{ }^{1}$ Dept. of Physics and Astronomy, Shanghai JiaoTong Univ. China; ${ }^{2}$ Collaborative Innovation Center of IFSA (CICIFSA), Shanghai Jiao Tong Univ. China. Here, purposeful focusing of secondharmonic waves, which are generated and scattered from nonlinear turbid media via feedback-based wavefront shaping, is presented, indicating more controllable degrees of freedom for future focusing and imaging through turbid media.
16:00-17:45

FM4G • Controlling Emission, Absorption and Transfer of Energy with Metamaterials Presider: Vinod Menon; City Univ. of New York, USA

FM4G.1 • 16:00

On-chip Integrated Cherenkov Radiation Emitter, Fang Liu', Long Xiao', Yu Ye ${ }^{1}$ Mengxuan Wang ${ }^{1}$, Kaiyu Cui ${ }^{1}$, Xue Feng ${ }^{1}$ Wei Zhang', Yidong Huang'; 'Tsinghua Univ., China. We demonstrate an on-chip integrated Cherenkov radiation (CR) emitter in which the no-threshold CR could be realized and a broadband CR is observed with electron energy of only $0.25-1.4 \mathrm{keV}$.

\section{FM4G.2 • 16:15}

Zero-Differential Thermal Emission Using Thermochromic Samarium Nickelate, Patrick J. Roney ${ }^{3}$, Alireza Shahsafi ${ }^{3}$, Zhen Zhang ${ }^{1}$, You Zhou ${ }^{2}$, Yuzhe Xiao ${ }^{3}$, Chenghao $W^{4}{ }^{4}$, Raymond Wambold ${ }^{3}$, Jad Salman ${ }^{3}$, Shriram Ramanathan ${ }^{1}$, Mikhail Kats ${ }^{3,4}$; ${ }^{1}$ School of Materials Engineering, Purdue Univ., USA ${ }^{2} S c h o o l$ of Engineering and Applied Sciences, Harvard Univ., USA; ${ }^{3}$ Electrical and Computer Engineering, Univ. of Wisconsin - Madison, USA; ${ }^{4}$ Materials Science and Engineering Univ of Wisconsin - Madison USA. We demonstrate a thermal emitter whose radiated power remains nearly constant over a temperature range of $\Delta \mathrm{T} \sim 30^{\circ} \mathrm{C}$ implemented using thermochromic samarium nickelate. This zero-differential thermal emitter can be used for infrared camouflage and obfuscation.

\section{FM4G.3 • 16:30}

Tailoring Thermal Emission with Epsilon Near-Zero Media Augmented with Dielectric Rods, Inigo Liberal ${ }^{1}$, Nader Engheta ${ }^{1}$ Univ. of Pennsylvania, USA. We theoretically nvestigate the thermal emission capabilities of epsilon-near-zero (ENZ) bodies containing dielectric rods. Effective enlargement of the wavelength at the ENZ frequency empowers directive and reconfigurable emission patterns, as well as geometry-invariant spectral features.
16:00-18:00

FM4H • Chip-scale Plasmonic

Devices

Presider: Euclides Almeida;

Weizmann Institute of Science, Israel
FM4H.1 • 16:00

Chip-size Plasmonic Spectropolarimeters Fei Ding ${ }^{1}$, Anders Pors ${ }^{1}$, Yiting Chen ${ }^{1}$, Vladimir Zenin', Sergey Bozhevolnyi'1. 'Centre for Nano Optics, Univ. of Southern Denmark, Denmark. Chip-size plasmonic spectropolarimeters for simultaneous polarization state and wavelength determination have been demonstrated. The fabricated spectropolarimeter operating in the wavelength range of 750 - $950 \mathrm{~nm}$ exhibits expected polarization selectivity and high angular dispersion $(0.0133 \% \mathrm{~nm})$

\section{FM4H.2 • 16:15 Invited}

Dynamic plasmonic colour display, Laura Na Liu', Xiaoyang Duan', Simon Kamin'; 'Max Planck Inst. for Intelligent Systems, Germany. We demonstrate a dynamic plasmonic colour display technique based on catalytic magnesium metasurfaces. Controlled hydrogenation and dehydrogenation of the constituent magnesium nanoparticles, which serve as dynamic pixels, allow for plasmonic colour tuning, erasing, and restoring. 


\section{CLEO: Science \& Innovations}

16:00-18:00

SM4I • Ultrafast Pulse

Combination and Manipulation

Presider: Igor Jovanovic; Univ. of Michigan, USA

\section{SM4I.1 16:00 Invited}

Coherent Pulse Stacking Amplification Extending Chirped Pulse Amplification by Orders of Magnitude, Almantas Galvanauskas'; ' 'Univ. of Michigan, USA. A new technique of time-domain pulse combining - coherent pulse stacking amplification - is enabling nonlinearity-free energy extraction at the stored energy limit from rare-earth doped fiber based ultrashort pulse amplification systems.
16:00-18:00
SM4J - THz QCLs and Imaging

Presider: Kimberly Reichel; Brown Univ, USA

\section{SM4J.1 • 16:00 Invited}

Terahertz Quantum Cascade Laser Frequency Combs, David P. Burghoff'; 'MIT, USA. Optical frequency combs are light sources that consist of many evenly-spaced lines. I will discuss recent developments in frequency combs based on terahertz quantum cascade lasers, which emit broadband comb light in a compact package.
SM4I.2 • 16:30

Femtosecond Beam Combination Using Diffractive Optic Pairs, Russell wilcox', Dar Dahlen', Tyler Sano'; ' 'LBNL, USA. A new, scalable method of ultrashort pulse, coherent beam combination is modeled numerically and demonstrated experimentally. 110fs, $1040 \mathrm{~nm}$ pulses in a $1 \times 4$ array are combined using two diffractive optics, preserving pulse duration and spectral width.

\section{6:00-18:00 \\ SM4K • Resonant Optics}

Presider: Roberto Paiella; Boston Univ., USA

\section{SM4K.1 16:00 Invited}

Merging Micro- and Nano-Optics, Harald W. Giessen', S Thiele', S Ristok', A Herkommer ${ }^{2} ;{ }^{1} 4$ th Physics Inst. and Research Center SCOPE, Universitiat Stuttgart, Germany; ${ }^{2}$ Inst. for Technical Optics and Research Center SCoPE, Universitiat Stuttgart, Ghana. We demonstrate miniaturized micro- and nanooptics. Our approach uses femtosecond 3D direct laser writing. Aberration-corrected performance for large angles of incidence is achieved, and our approach solves the common problem of off-axis coma in metasurfaces.
16:00-18:00

SM4L • Mode Locked Fiber Lasers II

Presider: Andy Chong; Univ. of Dayton, USA

\section{SM4L.1 • 16:00}

Mode-locked Er-doped Fiber Laser by Pump Modulation beyond Emission Lifetime Limit, Shoko Yokokawa', Yu Wang' ', Sze Y. Set', shinji Yamashita'; ' ${ }^{1}$ the Univ. of Tokyo, Japan. Mode-locked EDF laser using active mode-locking via pump modulation (AMPM) is demonstrated. The repetition rate is 99.250 $\mathrm{kHz}$, which is beyond the emission lifetime limit of Er ion.
SM4J.2 $\bullet 16: 30$

Simultaneous Phase-Locking of Quantum Cascade Lasers Using Multi-Frequency $\mathrm{THz}$ Source System Composed of MZM-Based Flat Comb Generator, Isao Morohashi ${ }^{1}$ Yoshihisa Irimajiri', Motoaki Kumagai ${ }^{1}$, Akira Kawakami', Takahide Sakamoto', Norihiko Sekine ${ }^{1}$, Akifumi Kasamatsu', Iwao Hosako ${ }^{1} N I C T$, Japan. By using frequency-stabilized multi-frequency terahertz $(\mathrm{THz})$ source system composed of a Mach-Zehnder-modulatorbased flat comb generator, simultaneous phase-locking of two quantum cascade lasers was successfully demonstrated.
SM4K.2 • 16:30

Towards Planar Dielectric Metasurfaces, Jonathan Bar-David', Noa Mazurski', Uriel Levy ${ }^{1}{ }^{1}$ Hebrew Univ. of Jerusalem, Israel. We use LOCOS technique to fabricate quasiplanar Silicon nanoantennas which are building blocks for future planar metasurfaces. Antenna arrays are characterized, and their measured optical transmission is shown to match simulations.
SM4L.3 • 16:30

Self-optimization and oscillation state mapping of polarization additive pulse mode-locked fiber laser, Manuel Ryser ${ }^{1}$ Christoph Bacher ${ }^{1}$, Philippe Raisin', Danie Paardekooper ${ }^{1}$, Thomas Feurer ${ }^{1}$, Valerio Romano'; ' Universitat Bern, Switzerland. With motorized polarization controllers and online monitoring we map the oscillation states of additive pulse mode-locked fiber lasers. These fingerprint-like maps allow to optimize a given fiber laser configuration and to implement self-optimization schemes. 


$\begin{array}{llc}\text { Marriott } & \text { Marriott } & \text { Marriott } \\ \text { Salon III } & \text { Salon IV } & \text { Salon V \& VI }\end{array}$

\section{CLEO: Science \& Innovations}

16:00-18:00

$\mathrm{SM} 4 \mathrm{M} \bullet$ Optical Parametric Oscillators

Presider: Shekhar Guha; US Air

Force Research Lab, USA

\section{SM4M.1 • 16:00}

Room-Temperature, Rapidly-Tunable, Green-Pumped Continuous-Wave Optical Parametric Oscillator Based on FanOut-Grating MgO:sPPLT, Kavita Devi ${ }^{1}$, Majid Ebrahim-Zadeh"1,2; ${ }^{1}$ CCFO -The Inst. of Photonic Sciences, Spain; ${ }^{2}$ Institucio Catalana de Recerca i Estudis Avancats (ICREA), Spain. We report the first realization of a stable, room-temperature, green-pumped cw OPO using fan-out-grating MgO:sPPLT, providing rapid, continuous and wide-tuning across $734-1929 \mathrm{~nm}, 2.2 \mathrm{~W}$ of output-power, frequency-stability of $518 \mathrm{MHz}(2 \mathrm{mins}$.) and linewidth of $6.9 \mathrm{MHz}$, in good-beam-quality.

\section{SM4M.2 $16: 15$}

Octave-wide Gallium Phosphide OPO Centered at $3 \mu \mathrm{m}$ and Pumped by an Erfiber Laser, Qitian Ru', Zachary E. Loparo², Xiaosheng Zhang ${ }^{3}$, Sean Crystal ${ }^{1}$, Subith Vasu $^{2}$, P. G. Schunemann ${ }^{4}$, Konstantin L. Vodopyanov'; 'CREOL, College of Optics and Photonics, Univ. of Central Florida, USA; ${ }^{2} \mathrm{Mechanical}$ and Aerospace Engineering, Univ. of Central Florida, USA: ${ }^{3}$ State Key Lab, Dept. of Precision Instrum, Tsinghua Univ., China; ${ }^{4}$ BAE Systems, USA. We achieved 2.35-4.75 $\mu$ m continuous spectrum from an Er-fiber pumped subharmonic OPO based on orientation-patterned $\mathrm{GaP}$ that is suitable for ultra-broad bandwidth comb generation. Less than 67-fs pulse duration and 29-mW output power were measured.

\section{SM4M.3 • 16:30}

Instantaneous Spectral Span of 2.85 - 8.40 $\mu \mathrm{m}$ Achieved in a Cr:ZnS Laser Pumped Subharmonic GaAs OPO, Qitian Ru1, Kai Zhong $^{2}$, Nathaniel P. Lee ${ }^{1}$, Zachary E. Loparo $^{3}$, P. G. Schunemann ${ }^{4}$, Sergey Vasilyev ${ }^{5}$, Sergey B. Mirov ${ }^{5}$, Konstantin L. Vodopyanov'; 'CREOL, College of Optics and Photonics, Univ. of Central Florida, USA; ${ }^{2}$ College of Precision Instrument and Optoelectronics Engineering, Tianjin Univ., China; ${ }^{3}$ Mechanical and Aerospace Engineering, Univ. of Central Florida, USA; ${ }^{4}$ BAE Systems, USA; ${ }^{5}$ Dept. of Physics, Univ. of Alabama at Birmingham, USA. We report a broadband mid-IR output reaching $>1.5$ octaves at $-30 \mathrm{~dB}$ level from a subharmonic orientation-patterned $\mathrm{GaAs}$ OPO pumped by an ultrafast (62-fs) Kerr-lens mode-locked $\mathrm{Cr}: \mathrm{ZnS}$ laser at $2.35 \mu \mathrm{m}$ with 800-mW average power.
16:00-18:00

SM4N • Prevoskite and

Photonic Crystal Lasers

Presider: Qing Gu; Univ. of

California San Diego, USA

\section{SM4N.1 • 16:00 Invited}

Halide Perovskite Lasers, Tze Chien Sum"; ${ }^{\dagger}$ Nanyang Technological Univ., Singapore. Solution-processed halide perovskites possess exceptional photovoltaic properties. Amazingly, these materials are also outstanding optical gain media. In this talk, I will review the milestones, state-of-the-art and prospective outlook of this new family of lasers.

SM4N.2 $16: 30$

Organic-inorganic Lead Halide Perovskite $\mathrm{CH}_{3} \mathrm{NH}_{3} \mathrm{PbBr}_{3}$ Nanolaser Array based on Silicon Grating, Wang Kaiyang ${ }^{1}$, Zhiyuan Gu', Shuai Liu', Wenzhao Sun', Nan Zhang ${ }^{1}$, Qinghai Song ${ }^{1}$; ${ }^{1}$ Harbin Inst. of Technology, Shenzhen Graduate School, China. We report perovskite $\mathrm{CH}_{3} \mathrm{NH}_{3} \mathrm{PbBr}_{3}$ nanolaser array with high density and uniform lasing wavelength on silicon. By transferring a perovskite microwire onto silicon grating, spatially periodic laser array is achieved without modifying the cavity and gain.

\section{6:00-17:45 \\ SM4O • Heterogeneously} Integrated Si Photonics

Presider: Qiaogiang Gan; The

State Univ. of New York at Buffalo, USA

\section{SM40.1 • 16:00}

Hybrid Integration of UTC-PDs on Silicon Photonics, Brandon Isaac' ${ }^{1}$, Yuan Liu' ${ }^{1}$, Bowen Song ${ }^{1}$, Xiaojun $\mathrm{Xia}^{2}$, Andreas Beling ${ }^{2}$, Jonathan Klamkin'; ' $U C S B$, USA; ${ }^{2}$ Univ. of Virgina, USA. A method of hybrid integration using grating couplers is demonstrated providing a way to utilized high speed InP photodetectors in silicon photonics. Responsivity measurements before and after bonding are reported to quantify coupling efficiency.

SM4O.2 • 16:15

A lithium niobate- $\mathrm{Si}_{3} \mathrm{~N}_{4}$ platform on silicon by heterogeneous wafer bonding, Lin Chang ${ }^{1}$, Martin Pfeiffer ${ }^{2}$, Nicolas Volet ${ }^{1}$, Michael Zervas², Jon Peters', Costanza Manganelli ${ }^{1}$, Eric Stanton', Yifei Li ${ }^{1}$, Tobias J. Kippenberg ${ }^{2}$, John Bowers ${ }^{1}$; ${ }^{1}$ Univ. of California Santa Barbara, USA; ${ }^{2}$ École Polytechnique Fédérale de Lausanne, Switzerland. A lithium niobate- $\mathrm{Si}_{3} \mathrm{~N}_{4}$ platform on silicon is demonstrated. It combines second- and third-order nonlinearities and has low-loss waveguides with mode converters. This is a key step for integrating nonlinear materials in silicon photonics.

SM40.3 - 16:30 Invited

Monolithic $8 \times 40 \mathrm{~Gb} / \mathrm{s}$ Tunable WDM Transmitter Based on Generic III-V Technology, Weiming Yao ${ }^{1}$, Meint K. Smit ${ }^{1}$, Michael J. Wale'; 'Univ. of Technology Eindhoven, Netherlands. We demonstrate an 8-channel tunable WDM transmitter capable of $320 \mathrm{~Gb} / \mathrm{s}$ operation. It exhibits high integration density on $36 \mathrm{~mm}^{2}$ chip area and was fabricated in an experimental generic integration platform. 
Executive Ballroom

210A

Executive Ballroom

210B

Executive Ballroom $210 \mathrm{C}$
Executive Ballroom 210D

\section{CLEO: Applications \\ \& Technology}

AM4A • A\&T Topical Review on Scientific and Commercial Progress in Semiconductor Lasers II-Continued AM4B - Combustion and
Atmospheric Photonics-
Continued

AM4B. $3 \cdot 16: 45$

Evaluation of Air Turbulence Impact Based on Wavefront Reconstruction, Wenbo Gao ${ }^{1}$ Milorad Cvijetic'. ' Univ. of Arizona, USA. We have established and experimentally verified a convenient relation between the wavefront error variance and the phase structure function, which can be readily used to estimate the atmospheric coherence diameter and the refraction-index structure constant.

AM4A.3 • 17:00

Tunable 3D Hybrid Integrated Silicon Photonic External Cavity Laser, Bowen Song ${ }^{1}$ yuan liu', Sasa Ristic ${ }^{2}$ Jonathan Klamkin ${ }^{1}$ ${ }^{\prime} E C E$, Univ. of California Santa Barbara, USA; ${ }^{2}$ McGill Inst. for Advanced Materials, McGill Univ., Canada. A 3D integrated hybrid silicon laser was demonstrated with tuning range of $30 \mathrm{~nm}$, a side-mode suppression ratio of 34 $\mathrm{dB}$, optical output power of $2 \mathrm{~mW}$, and peak relative intensity noise of $-135 \mathrm{~dB} / \mathrm{Hz}$.
AM4B. $4 \cdot 17: 00$

Iterative holographic reconstruction based on the grating illumination with improved resolution by interpolation, Shaodong Feng'; ' ${ }^{1}$ Shanghai Jiao Tong Univ., China. We proposed an iterative reconstruction method with interpolation based on the grating il lumination with improved resolution. UASF target as the sample in Numerical simulation and experiment were conducted to prove the feasibility of this method.

\section{CLEO: Science \& Innovations}

\section{SM4C • Optofluidic Components and Systems- Continued}

AM4B.5 $\bullet 17: 15$

Visibility Enhancement of Hazy Images dehazing methods are proven very effective Switzerland. We demonstrate the utility of femtosecond semiconductor disk lasers for multi-photon microscopy with several in vivo imaging experiments. These compact and affordable short pulse lasers are promising new sources for widespread bio-imaging.

AM4A.5 • 17:30

III-nitride nanowire array based $1.3 \mu \mathrm{m}$ monolithic photonic integrated circuit on (001) silicon substrate, Arnab Hazari ${ }^{1}$ Junseok $\mathrm{Heo}^{2}$, Pallab Bhattacharya'; ' ${ }^{1}$ Univ. of Michigan, USA; ${ }^{2}$ Dept. of Electrical and Computer Engineering, Ajou Univ., Korea (the Republic of). III-nitride nanowire arrays are employed to demonstrate the first monolithically integrated active photonic circuit on (001)silicon, composed of a diode laser emitting at $1.3 \mu \mathrm{m}$, a dielectric waveguide, and a photodiode with high responsivity at $1.3 \mu \mathrm{m}$. Using Polarimetric Dehazing Method Based on Stokes Parameters, Jian Liang ${ }^{1}$, Wenfei Zhang', Liyong Ren', Haijuan Ju' Zhaofeng Bai', Enshi Qu'; ' ${ }^{1}$ i'an Inst Optics \& Precision Mech, CAS, China. Polarimetric in enhancing the contrast and visibility of images captured in hazy weather. In this paper, we analyze the capability of visibility enhancement in experiments.

Multimodal Multiplexing of Single-Virus Detection Using Multi-Mode Interference Waveguides, Damla Ozcelik', Matthew A Stott' ${ }^{2}$, Joshua W. Parks ${ }^{1}$, Aadhar Jain ${ }^{1}$, Aaron Hawkins ${ }^{2}$, Holger Schmidt'; ' UC Santa Cruz, USA: ${ }^{2}$ Brigham Young Univ., USA. Multimode interference waveguides are used to implement simultaneous spectral and spatia multiplex fluorescence analysis in liquid-core waveguide channels. A six-times multiplex identification of single influenza viruses is demonstrated with two excitation colors and three channels.

SM4C.6 6 17:30

AM4B.6 • 17:30

First-Photon Ghost Imaging at Low Light Level, Xialin Liu', Jianhong Shi', Guihua Zeng1: ' Center of Quantum Information Sensing and Processing, State Key Lab of Advanced Optical Communication Systems and Networks, Shanghai Jiao Tong Univ. China. We propose a photon-limited imaging technique, first-photon ghost imaging, which reconstructs image by counting the pulses before the first photon arrives. It can achieve visibility enhancement with $<1$ photon detection per pixel.

Lab-on-a-chip Detection of Illicit Pigment in Food using Photonic Crystal Biosilica Alan X. Wang': ' ${ }^{1}$ Oregon State Univ., USA We developed a facile route for detecting illicit pigment in real food sample using photonic crystal biosilica, which serves as a new lab-on-a-chip platform combining surface-enhanced Raman scattering sensing and thin layer chromatography.

\section{SM4C. $3 \cdot 16: 45$}

Standing-wave Raman Tweezers for Optical TrappingaAnd Sensitive Characterization of Nano-sized Structures, Mu-ying $\mathrm{Wu}^{1}$, Guang Yang', Guihua Chen', Yong-Qing $\mathrm{Li}^{1}, 2 ;{ }^{1}$ Dongguan Univ. of Technology, China; ${ }^{2}$ East Carolina Univ., USA. A standing-wave optical trap integrated with confocal Raman spectroscopy enables stable trapping and characterization of individual single-walled carbon nanotubes, graphene flakes, biological cells, SERS-active metal nanoparticles and other nanoparticles based on their increased Raman fingerprints.

SM4C.4 4 17:00

Single Gold Nanoparticle Trapping using an Optofluidic Chip, Yuzhi Shi ${ }^{1}, 2$, Sha Xiong ${ }^{1}$ Lip Ket Chin', Jiuhui Wu ${ }^{2}$, Tianning Chen ${ }^{2}$, Ai Qun Liu'; 'Nanyang Technological Univ., Singapore; ${ }^{2} X i ' a n$ Jiaotong Univ., China. We present an optofluidic chip for the trapping and separation of single gold nanoparticles (60-100 nm). Size and refractive index of a gold nanoparticle are determined by the trapping position using Drude and Mie theories.

SM4C.5 • 17:15

SM4D • Nanophotonics, Waveguides, and Microresonators in SensingContinued

\section{SM4D.3 $16: 45$}

Absorption Spectroscopy of Doped Conjugated Polymer Single-Particles with Toroidal Optical Microresonators, Erik H. Horak ${ }^{1}$, Kassandra A. Knapper ${ }^{1}$, Morgan Rea' Feng Pan', Kevin D. Heylman', Randall H. Goldsmith"; 'Univ. of Wisconsin - Madison, USA. Applying toroidal optical microresonators as absorption spectrometers to examine the heterogeneous electronic structure of the doped conjugated polymer PEDOT:PSS from a single particle basis.

SM4D.4 $\bullet 17: 00$

Microresonator soliton dual-comb spectroscopy, Qifan Yang', Myoung-Gyun Suh'1, Ki Y. Yang ${ }^{1}$, Xu Yi ${ }^{1}$, Kerry Vahala ${ }^{1}{ }^{1}$ California Inst. of Technology, USA. Dual-comb spectroscopy is demonstrated using microresonator soliton frequency combs by measuring the absorption spectrum of $\mathrm{H}^{13} \mathrm{CN}$ in the nearinfrared. The results show the potential fo a chip-based, high-precision spectroscopic system.

\section{SM4D.5 • 17:15}

Near-Infrared Waveguide-Enhanced Raman Spectroscopy of Trace Gases, Todd $\mathrm{H}$. Stievater ${ }^{1}$, Kee Koo ${ }^{1}$, Nathan Tyndall' ${ }^{1}$, Dmitry Kozak', Scott Holmstrom², R. Andrew McGill' Marcel W. Pruessner ${ }^{1}$, William Rabinovich' Jacob Khurgin3. 'US Naval Research Lab, USA; ${ }^{2}$ Univ. of Tulsa, USA; ${ }^{3}$ Johns Hopkin Univ., USA. Functionalized silicon nitride waveguides are used to detect Raman scattering from trace concentrations of toxic chemical species. Parts-per-billion detection imits are measured using single-mode rib and nanoslot waveguides pumped at $785 \mathrm{~nm}$.

\section{SM4D.6 $17: 30$}

Detection of Surface-enhanced Raman Signals from a Single Nano plasmonic Antenna Integrated on a Single Mode Waveguide, Ali A. Raza' V. Dope ${ }^{3}$, Stéphane Clemmen', Roel Baets ${ }^{1}$ INTEC, Ghent Univ., Belgium; ${ }^{2}$ MIT, USA ${ }^{3}$ IMEC, Belgium. We present the first demonstration of on-chip Raman spectroscopy using Frederic Peyskens², Pieter Wuytens ${ }^{1}$, Paul a single nanoplasmonic antenna integrated on a single mode nanophotonics waveguide. To achieve this goal, shot noise associated with waveguide background is investigated. 

Executive Ballroom
$210 \mathrm{E}$

Joint

JM4E • Symposium on Sources of Nonclassical Light and their Scalability II-Continued

\section{JM4E.3 • 16:45}

Controlling the temporal behavior of photon emission from a quantum dot molecule, Brennan C. Pursley ${ }^{1}$, Sam Carter ${ }^{1}$, Mijin $\mathrm{Kim}^{2}$, Chul Soo Kim', Sophia E. Economou ${ }^{3}$, Michael Yakes ${ }^{1}$, Allan S. Bracker ${ }^{1}$, Daniel Gammon'; ${ }^{1}$ Naval Research Lab, USA; ${ }^{2}$ Sotera Defense Solutions, USA; ${ }^{3}$ Physics, Virginia Tech, USA. We demonstrate that properties of photons emitted from a doubly charged quantum dot molecule can be modified using spin-flip Raman emission. The temporal and spectral bandwidth of the emission matches that of the pulsed laser.

\section{JM4E.4 • 17:00 Invited}

Utilizing Optical Transition Edge Sensors and Superconducting Nanowire Single Photon Detectors in Quantum Optics, Thomas Gerrits ${ }^{1}$, Georg Harder ${ }^{2}$, Timothy Bartley ${ }^{2}$ Christine Silberhorn², Omar Magana-Loaiza ${ }^{1}$ Krister Shalm', Adriana Lita', Varun Verma', Sae Woo Nam'; 'NIST, USA; '2Univ. of Paderborn, Germany. We present the current state-of-the art of single-photon detection in quantum optics using high-efficiency superconducting single photon detectors, the implementation of high-efficiency sources and the measurement of large photon number squeezing in waveguides. Executive Ballroom
$210 \mathrm{~F}$
Executive Ballroom 210G
Executive Ballroom $210 \mathrm{H}$

\section{CLEO: QELS-Fundamental Science}

FM4F • Nonlinear Optics in Propagating Geometries IIContinued

FM4F.4 • 16:45

Femtosecond Localized Electric Field Measurement in Gases via Second Harmonic Generation, Arthur Dogariu', Benjamin Goldberg ${ }^{1}$, Sean O'Byrne ${ }^{2}$, Richard Miles ${ }^{1}$. ${ }^{1}$ Princeton Univ., USA; ${ }^{2}$ The Univ. of New South Wales, Australia. We demonstrate a non-intrusive method of measuring electric fields in arbitrary gases using second harmonic generation. This simple method allows for sub-mm spatial and femtosecond temporal resolution with high sensitivity.

FM4F.5 • 17:00

Soliton-mediated orientation and birefrigence in gold nanorod suspensions, Yuxuan Ren ${ }^{1}$ Trevor S. Kelly ${ }^{1}$ Chensong Zhang ${ }^{1}$ Huizhong $\mathrm{Xu}^{1}$, Zhigang Chen ${ }^{1,2}$; 'Dept. of Physics and Astronomy, San Francisco State Univ, USA. ${ }^{2}$ Nankai Univ, China. We demonstrate soliton-mediated orientational ordering in gold nanorod suspensions. Polarization transmission spectrum shows orientation-enhanced birefringence along the soliton channel, indicating a disorderto-order transition of nanorods due to the torque exerted by the soliton beam.

FM4F.6 • 17:15

Programmable, Time-Dependent Optical Heating in Water using a Nano-patterned Silicon Membrane, Ahmed M. Morsy', Roshni Biswas ${ }^{1}$, Michelle Povinelli'; ${ }^{1}$ Ming Hsieh Dept. of Electrical Engineering, Univ. of Southern California, USA. We use photonic-crystal pattern to create an absorptive resonance near $970 \mathrm{~nm}$. We carry out experiments and simulations to show that all-dielectric, programmable microheaters can be achieved using this design and operate in water.

\section{FM4G - Controlling Emission, Absorption and Transfer of Energy with Metamaterials- Continued}

\section{FM4G.4 • 16:45}

Broadband enhancement of thermal emission, Gaurang Bhatt', Raphael St-Gelais ${ }^{2}$ Avik Dutt' ${ }^{1}$, Felippe A. Barbosa ${ }^{3}$, Michal Lipson': 'Columbia Univ., New York, USA USA; ${ }^{2}$ McGill Univ., Canada; ${ }^{3}$ Universidade Estadual de Campinas, Brazil. We present wide-band enhancement of thermal emission from a semitransparent weak thermal emitter optically coupled to an external cavity. We demonstrate $\sim 2.7 x$ enhancement of far-field total thermal emission from a suspended silicon carbide membrane.

\section{FM4G.5 • 17:00}

Long Range Energy Transfer Across an Epsilon-Near-Zero Metamaterial, Rahu Deshmukh',2, S. A. Biehs ${ }^{3}$, Emaad Khwaja ${ }^{4}$ Girish Agarwal ${ }^{5}$, Vinod M. Menon ${ }^{1,2} ;{ }^{1}$ City College of New York, USA; ${ }^{2}$ Graduate Center of the City Univ. of New York, USA; ${ }^{3}$ Inst. of Phsyics, Univ. of Oldenberg, Germany; ${ }^{4}$ Hunter College, USA; ${ }^{5}$ Texas A \& M Univ. USA. We experimentally demonstrate long range ( 160 nm) energy transfer in a donoracceptor pair across a metamaterial designed such that the epsilon-near-zero regime coincided with the donor emission.

\section{FM4G.6 • 17:15}

Integrated zero-index waveguides, Orad Reshef ${ }^{1,2}$, Philip Camayd-Muñoz ${ }^{2}$, Daryl Vulis ${ }^{2}$ Yang Li ${ }^{2}$, Eric Mazur'; ' ${ }^{1}$ Dept. of Physics, Univ. of Ottawa, Canada; ${ }^{2}$ School of Engineering and Applied Sciences, Harvard Univ., USA We present small-footprint $(\approx \lambda / 2$-wide $)$ silicon-based waveguides with an effective index of zero at a wavelength near $\lambda=1630$ $\mathrm{nm}$. We characterize the refractive index using on-chip interferometry and measure the propagation loss to be $1.3 \mathrm{~dB} / \mu \mathrm{m}$.

\section{FM4H • Chip-scale Plasmonic Devices-Continued}

FM4H.3 • 16:45

Full RGB Liquid Crystal-Tunable Plasmonic Color and TFT Integration, Daniel Franklin' Shin-Tson Wu', Debashis Chanda'; ' Univ. of Central Florida, USA. We demonstrate a full RGB liquid crystal-tunable reflective surface where the color of the aluminum nanostructure is changed as a function of applied voltage. We then integrate the system with a TFT to depict images.

\section{FM4H.4 • 17:00}

Vertical Metallic Grating Couplers Enabling Direct Access to Plasmonic Devices, Masafumi Ayata', Yuriy Fedoryshyn", Claudia Hoessbacher', Juerg Leuthold'; ${ }^{1}$ Inst. of Electromagnetic Fields (IEF), Switzerland. We demonstrate direct conversion of light to and from plasmonic devices via a vertically aligned multicore fiber. New metallic grating couplers are introduced. The tota fiber-to-fiber losses are $28 \mathrm{~dB}$.

\section{FM4H.5 • 17:15}

Highly Efficient Excitation of Surface Plasmons Using a Si Gable Tip, Arnab Dewanjee1, Muhammad Alam², J. Stewart. Aitchison', Mo. Mojahedi'; ' $U$ niv. of Toronto Canada; ${ }^{2}$ California Inst. of Technology, USA We experimentally demonstrate an integrated silicon gabled tip to excite an SPP mode at $1550 \mathrm{~nm}$ wavelength at an $\mathrm{Au} / \mathrm{SiO}_{2}$ interface with $25.5 \%$ input to SPP output efficiency which can reach as high as $52 \%$.

\section{JM4E.5 • 17:30 Invited}

Photonic Crystal Fibers for Generating Three-photon States, Maria Chekhova ${ }^{1}$ Andrea Cavanna', Michael Taheri', Cameron Okoth ${ }^{1}$, Xin Jiang ${ }^{1}$, Nicolas Joly', Philip S. Russell'; ' ${ }^{1}$ Max-Planck-Inst Physik des Lichts, Germany. Direct decay of pump photons into triplets is an interesting but not-yet-realized nonlinear effect. We are exploring two approaches using photonic crystal fibers: gas-filled hollow-core PCF and the recently designed hybrid solid-core PCF.
FM4F.7 • 17:30

Two dimensional acoustic horizon and ergosphere in a nonlocal photon superfluid, David Vocke', Calum Maitland ${ }^{1}$, Angus Prain ${ }^{1}$, Francesco Marino ${ }^{2}$, Daniele Faccio'; ${ }^{1}$ Heriot Watt Univ., UK; ${ }^{2}$ Dipartimento di Fisica, Universita di Firenze, Sezione di Firenze INFN Italy. We present experimental evidence of a two-dimensional black hole horizon and ergosphere for the first time in an analogue system using a nonlocal photon fluid based on a thermal nonlinearity.
FM4G.7 • 17:30

Dynamically Tunable, Vanadium Dioxide Huygens Source Metasurfaces, Adam Ollanik', Yaping Ji', David Bar-Or ${ }^{1}$, Nathan Kurtz', ${ }^{1,}$ Matthew D. Escarra'; 'Tulane Univ., USA; ${ }^{2}$ Univ. of Southern Mississippi, USA. We design and simulate dynamically tunable metasurfaces comprised of vanadium dioxide Huygens source nanoantennas. Simulations demonstrate metasurfaces capable of transmittance, reflectance, and absorbance modulation of $>85 \%$, with experimental realization in progress.
FM4H.6 $\bullet 17: 30$

Magneto-optical Isolator for Nanoplas monic Waveguides, Vahid Foroughi $\mathrm{Ne}$ zhad', Georgios Veronis'; 'Louisiana State Univ., USA. We introduce an extremely compact magneto-optical isolator consisting of a cavity placed in the proximity of metal-dielectric-metal plasmonic waveguide. The transmission spectra of the structure depend on the direction of the incident waveguide mode. 


\section{CLEO: Science \& Innovations}

\section{SM4I • Ultrafast Pulse \\ Combination and Manipulation-Continued}

\section{SM4I.3 • 16:45}

A Pulse-Pattern-Based Phase-Locking Method for Multi-cavity Coherent Pulse Stacking, Yawei Yang ${ }^{1}$, John Byrd ${ }^{1}$, Jay Dawson², Lawrence Doolittle', Qiang Du', Almantas Galvanauskas ${ }^{3}$, Gang Huang ${ }^{1}$, Wim Leemans', John Ruppe ${ }^{3}$, Russell Wilcox', Yilun Xu'; ' ${ }^{1}$ Lawrence Berkeley National Lab, USA; ${ }^{2}$ Lawerence Livermore National Lab, USA; ${ }^{3}$ Univ. of Michigan, USA. A novel phaselocking method, which locks cavity phase based on the pulse patterns detected from each cavity, has stabilized the output pulse intensity in a four-cavity Coherent Pulse Stacking experiment.

\section{SM4I.4 $17: 00$}

Fractional Temporal Self-Imaging for Mitigation of Nonlinear Propagation Impairments of Ultrashort Pulses, Seghilani Mohamed', Reza Maram', Jose Azana'; 'Énergie Matériaux Télécommunications, Institut National de la Recherche Scientifique, Canada. We propose a new approach to mitigate nonlinear propagation-impairments of pulses through pulse-division using fractional temporal self-imaging, overcoming limitations of previous methods for application at high repetition-rates. Successful demonstration on $\mathrm{GHz}$-rate picosecond-pulses is shown.

\section{SM4I.5 • 17:15}

High Repetition rate fs Pulse Burst Generation using the Vernier effect, Tobias Flöry' Giedrius Andriukaitis ${ }^{1}$, Martynas Barkauskas $^{2}$, Edgar Kaksis', Ignas Astrauskas ${ }^{1}$, Audrius Pugzlys ${ }^{1}$, Andrius Baltuska', Romas Danielius², Almantas Galvanauskas ${ }^{3}$, Tadas Balciunas'; 'Technische Universität Wien, Austria; ${ }^{2}$ Light Conversion Ltd., Lithuania; ${ }^{3}$ Center for Ultrafast Optical Science, Univ. of Michigan, USA. We demonstrate pulse burst generation method based on the Vernier effect using a femtosecond oscillator and regenerative amplifier cavity that have slightly different round trip times. This can be used for coherent pulse stacking, rapid material miroprocessing and rapid scan spectroscopy.

\section{SM4I.6 $\bullet 17: 30$}

Generation of Programmable Envelope in High-Speed Optical Pulse Train by Fractional-Rate Intensity Modulation, Qijie $\mathrm{Xie}^{1}$, Chester Shu'; ${ }^{1}$ Chinese Univ. of Hong Kong, China. We experimentally demonstrate periodic envelope programming of $\sim 80$ $\mathrm{GHz}$ optical pulse trains by using $\sim 10 \mathrm{GHz}$ multilevel electrical patterns. The method synthesizes $78.08 \mathrm{GHz}$ optical binary patterns and pulse trains with triangular and parabolic envelopes.

\section{SM4J $\bullet T H z$ QCLs and Imaging-Continued}

SM4J.3 • 16:45

Chip-scale Turing frequency comb for coherent high-power $\mathrm{THz}$ radiation, Jinghui Yang', Shu-Wei Huang ${ }^{1}$, Shang-Hua Yang ${ }^{1}$, Mingbin Yu ${ }^{2}$, Dim-Lee Kwong ${ }^{2}$, Tanya Zelevinsky ${ }^{3}$, Mona Jarrahi' ${ }^{1}$ Chee Wei Wong ${ }^{1}$ ${ }^{1}$ Univ. of California, Los Angeles, USA; ${ }^{2}$ Inst. of Microelectronics, Singapore; ${ }^{3}$ Dept. of Physics, Columbia Univ., USA. We report chip-scale Turing frequency comb with narrow linewidth of $9 \mathrm{kHz}$ and long term stability of $160 \mathrm{kHz}$ on THz carriers. The Turing comb is transferred onto a plasmonic photomixer achieving $600 \mathrm{~mW}$ terahertz radiation with high $1.1 \%$ optical-to-terahertz power conversion at room temperature.

\section{SM4J.4 • 17:00}

Terahertz quantum cascade dipole-antenna vertically emitting continuous wave laser Luca Masini ${ }^{1}$, Alessandro Pitanti', Lorenzo Baldacci², Miriam S. Vitiello1, Riccardo Degl'innocenti ${ }^{3}$, Harvey E. Beere ${ }^{3}$, David A Ritchie $^{3}$, Alessandro Tredicucci ${ }^{4}$; ${ }^{1} N E S T$, Istituto Nanoscienze-CNR and Scuola Normale Superiore, Italy; ${ }^{2}$ Inst. of Life Sciences, Scuola Superiore Sant'Anna, Italy; ${ }^{3}$ Cavendish Lab, Univ. of Cambridge, UK; ${ }^{4} \mathrm{NEST}$, CNR - Istituto Nanoscienze and Dipartimento di Fisica, Università degli studi di Pisa," Italy. In this work we show how a continuous wave, low threshold and well collimated terahertz laser vertical source can be obtained by coupling two sub-wavelength whispering galleries quantum cascade optical resonators with a metallic bridge

SM4J.5 ・ 17:15

A Source-free Single-chip Terahertz Spec troscope through Sub-wavelength Sensing of Antenna Near-fields, Xue Wu' Kaushik Sengupta'; 'Princeton Univ., USA. In this work, near-field electromagnetic sensing is exploited to enable compact broadband $\mathrm{THz}$ spectroscope in silicon-based integrated technology. Integrating passives with active devices in a silicon chip creates a new class of miniaturized $\mathrm{THz}$ systems.

\section{SM4J.6 6 17:30}

Imaging Polarization in GaN Surfaces by Laser Terahertz Emission Microscopy, Yuji Sakai ${ }^{1}$, Iwao Kawayama', Hidetosh Nakanishi' ${ }^{2}$, Masayoshi Tonouchi'; ' Osaka Univ., Japan; ${ }^{2}$ SCREEN Holdings, Japan. Polarizations in $\mathrm{GaN}$ surfaces are visualized using terahertz emission microscopy. A nonradiative-inversion domain that is hardly distinguishable with photoluminescence imaging was clearly observed with this method.

\section{SM4K • Resonant Optics- Continued}

\section{SM4K.3 • 16:45}

Optical Properties of Ultrathin Plasmonic TiN Films, Deesha Shah', Harsha Reddy ${ }^{1}$ Nathaniel Kinsey ${ }^{2}$, Vladimir M. Shalaev ${ }^{1}$, Alexandra Boltasseva'; 'Purdue Univ., USA ${ }^{2}$ Virginia Commonwealth Univ., USA. Epitaxial, ultrathin $(<10 \mathrm{~nm})$ plasmonic TiN films are characterized using spectroscopic ellipsometry and Hall measurements. Thin films with thicknesses down to $2 \mathrm{~nm}$ remain highly metallic with a carrier concentration on the order of $10^{22} \mathrm{~cm}^{-3}$.

\section{SM4K.4 17:00 Invited}

Light Management in Resonant Structures, Zongfu Yu'; ' Univ. of Wisconsin-Madison, USA. Light management based on resonant structures offer extraordinary optical concentration and novel functionalities for optoelectronic devices. We will discuss the limit of optical concentration and the nonHermitian interactions that lead to opticalcoupled electrical-isolated photodetectors for multi-modal light detection.

\section{SM4K.5 • 17:30}

Optimized Multilayer Interference for Color-tuning in Colloidal Quantum Dot Solar Cells, Botong H. Qiu', Ebuka S. Arinze', Nathan Palmquist', Yan Cheng ${ }^{1}$, Yida Lin ${ }^{1}$, Gabrielle Nyirjesy², Gary Qian', Susanna M. Thon'; ' ${ }^{1}$ Electrical and Computer Engineering, Johns hopkins Univ., USA; ${ }^{2}$ Material Science and Engineering, Johns Hopkins Univ., USA. We develop a method that combines thin film interference modeling with population-based optimization algorithms to demonstrate infrared-responsive color-tuned colloidal quantum dot solar cells with 10 to $15 \mathrm{~mA}$ $\mathrm{cm}^{2}$ photocurrents and semi-transparent cells with $\sim 30 \%$ transparencies.
SM4L • Mode Locked Fiber Lasers II-Continued

\section{SM4L.4 • 16:45}

Pump-to-Signal Modulation Transfer in a Tm-doped Fiber for Active Mode-locking Yu Wang', Sze Y. Set', Shinji Yamashita'; ' 'Univ. of Tokyo, Japan. Recently, we proposed a new class of actively mode-locking technique using pump modulation and realized a mode-locked Tm-doped fiber laser. Here, we investigate a pump-to-signal modulation transfer function in a Tm-doped fiber numerically and experimentally.
SM4L.5 • 17:00

Dual Repetition-Rate Femtosecond Pulses Directly from a Tm-doped Fiber Laser, Ruoyu Liao', Youjian Song', Lu Chai', Ming-lie $\mathrm{Hu}^{1}$; ${ }^{1}$ Tianjin Univ., China. A passively modelocked Tm-doped fiber laser directly output two sets of pulse trains with $2.7 \mathrm{kHz}$ offset repetition-rate, having potential for dualcomb spectroscopic applications.
SM4L.6 • 17:15

Fast Wavelength-Switchable Figure-Nine Er Fiber Laser Using a Galvanometer-Driven Intracavity Filter, Toshiro Fujita², Yasuyuk Ozeki'; ${ }^{1}$ Electrical Engineering and Information Systems, The Univ. of Tokyo, Japan ${ }^{2}$ Electrical and Electronic Engineering, The Univ. of Tokyo, Japan. We demonstrate fast wavelength switching of a polarizationmaintaining Er fiber laser within $<10 \mathrm{~ms}$ Within the wavelength range of $30 \mathrm{~nm}$, the pulse duration was $<1.1 \mathrm{ps}$ and the timebandwidth product was $<0.34$.

SM4L.7 • 17:30

Effects of Non-reciprocal Phase Bias in Figure-8/9 Fiber Lasers, Tomoyasu Honda' Sze Y. Set', shinji Yamashita'; 'Univ. of Tokyo, Japan. We investigate the dependence of phase bias on the pulse qualities in figure- 8 and figure-9 fiber lasers. Numerical simulation results show that phase bias can facilitate NALM operation and reduce pulse duration. 


$\begin{array}{llc}\text { Marriott } & \text { Marriott } & \text { Marriott } \\ \text { Salon III } & \text { Salon IV } & \text { Salon V \& VI }\end{array}$

\section{CLEO: Science \& Innovations}

\section{SM4M • Optical Parametric Oscillators-Continued}

\section{SM4M.4 $16: 45$}

High-repetition-rate Picosecond Deepinfrared Optical Parametric Oscillator Based on $\mathrm{CdSiP}_{2}$, Chaitanya Kumar Suddapalli ${ }^{1,2}$, Josep C. Casals', Shahrzad Parsa', K. T. Zawilski ${ }^{3}$ P. G. Schunemann ${ }^{3}$, Majid Ebrahim-Zadeh"1,4; ${ }^{1}$ CFO -The Inst. of Photonic Sciences, Spain; ${ }^{2}$ Radiantis, Spain; ${ }^{3} B A E$ Systems Incorporated, USA; ${ }^{4}$ nstitucio Catalana de Recerca i Estudis Avancats (ICREA), Spain. We report the first high-repetition-rate picosecond OPO based on $\mathrm{CdSiP}_{2}$, tunable across 6205-6695 nm in deep-IR, providing $105 \mathrm{~mW}$ at $6205 \mathrm{~nm}$ at $79.5 \mathrm{MHz}$, with $2.3 \%$ rms passive power stability over $12 \mathrm{~h}$ in high-beam-quality.

\section{SM4M.5 • 17:00 Invited}

Optical Parametric Sources for Atmospheric Sensing, Antoine Godard', Julie Armougom', Erwan Cadiou', Guillaume Walter $^{1}$, Jean-Baptiste Dherbecourt ${ }^{1}$, Guillaume Gorju', Jean-Michel Melkonian', Myriam Raybaut', Michel Lefebvre'; 'ONERA - The French Aerospace Lab, France. We present our activities on the development of tunable optical parametric sources for gas sensing. In particular, we introduced the nested cavity OPO. We have also developed rapidly tunable OPOs based on aperiodic quasi-phase matching.

\section{SM4N • Prevoskite and Photonic Crystal Lasers- Continued}

\section{SM4N.3 • 16:45}

Continuous-wave Optically Pumped Lasing of Hybrid Perovskite VCSEL at Green Wavelength, Mohammed A. Sharizal', Zhixiong Liu', Abdullah Al-Atawi ${ }^{1}$, Tien Khee $\mathrm{Ng}^{1}$, tao wu ${ }^{1}$, Boon S. Ooi ${ }^{1}$; ${ }^{1}$ KAUST, Saudi Arabia. We demonstrate the lasing of a perovskite vertical-cavity surface-emitting laser at green wavelengths, which operates under continuous-wave optical pumping at room-temperature by embedding hybrid perovskite between dielectric mirrors deposited at low-temperature.

\section{SM4N.4 17:00 Invited}

Narrowband Thermal Emitters Bsed on Photonic Crystals, Takashi Asano1, Takuya Inoue ${ }^{1}$, Susumu Noda'; ${ }^{1}$ Kyoto Univ., Japan. Single-peak narrowband thermal emitters with high energy utilization efficiencies are realized by controlling both photonic and electronic states. Ultrafast $(\sim \mathrm{MHz})$ direct intensity modulation is also demonstrated.

\section{SM4O • Heterogeneously Integrated Si Photonics- Continued}

SM40.4 $\bullet 17: 00$

Polarization Diversity Quantum Dot Semiconductor Optical Amplifier Module for T-band Communication, Hiroyuki Tsuda ${ }^{1}$, Takafumi Chiba², Tadashi Hajikano ${ }^{3}$, Katsumi Yoshizawa ${ }^{4}$, Yasunori Tomomatsu ${ }^{5}$, Hiroshi Takahashi ${ }^{6}$, Takayuki Kawashima ${ }^{2}$, Shojiro Kawakami², Yudai Okuno', Koki Sugiyama'; ${ }^{1}$ Keio Univ., Japan; ${ }^{2}$ Photonic Lattice, Inc., Japan; ${ }^{3}$ OPTOQUEST CO., LTD., Japan; ${ }^{4}$ Pioneer Micro Technology Corp, Japan ${ }^{5}$ Koshin Kogaku Co., Ltd., Japan; ${ }^{6}$ Sophia Univ., Japan. The polarization independent SOA module for T-band communication was fabricated using the quantum dot gain chip and the polarization diversity circuits with photonic crystal waveplates. The polarization dependent gain was successfully reduced to $0.5 \mathrm{~dB}$.

SM40.5 • 17:15

Self-Amplified Filter Fabricated in a SOI Photonics Foundry, Paulo F. Jarschel de Siqueira', Mário C. Souza', Rafael B. Merlo', Newton C. Frateschi"; " "Gleb Wataghin" Physics Inst., Univ. of Campinas, Brazil. We demonstrate a self-amplified filter based on Silicon Ring Resonators with Er-doped cladding. It is capable of filtering and routing resonant wavelengths, with simultaneous signal amplification. An equivalent internal gain of $4.7 \mathrm{~dB} / \mathrm{mm}$ was observed.

\section{SM4M.6 • 17:30}

Simulton Formation in Mid-Infrared Femtosecond Optical Parametric Oscillators, Marc Jankowski', Alireza Marandi', Christopher R. Phillips' ${ }^{2}$, Ryan Hamerly' ${ }^{1}$, Kirk Ingold ${ }^{3}$, Robert L. Byer', Martin M. Fejer'; 'Stanford Univ., USA; ${ }^{2}$ ETH Zurich, Switzerland; ${ }^{3}$ West Point Academy, USA. We report on observations of simultons, bright-dark soliton pairs, in femtosecond optical parametric oscillators. Simulton formation generates stable sub-50-fs half-harmonic pulses with sech^2 envelopes, slope efficiencies $>400 \%$, and conversion efficiencies $>50 \%$.
SM4N.5 • 17:30

Green Vertical-Cavity Surface-Emitting Laser from Perovskite $\left(\mathrm{CH}\left(\mathrm{NH}_{2}\right)_{2} \mathrm{PbBr}_{3}\right)$ Thin Films, Songtao Chen', Joonhee Lee', Arto Nurmikko'; ${ }^{1}$ Engineering, Brown Univ., USA. We demonstrate green lasing from solution processed perovskite thin films, sandwiched within planar $\mathrm{SiO}_{2} / \mathrm{HfO}_{2}$ dielectric DBRs to form a high-Q surface emitting vertical cavity. Spectrally and spatially coherent lasing with a low threshold is achieved.
SM40.6 • 17:30

Flat-top Frequency Comb Generation with Silicon Microring Modulator and Filter, Xinru Wu', Hon Ki Tsang'; 'The Chinese Univ. of Hong Kong, Hong Kong. An optical frequency comb with five lines having $<0.86$ $\mathrm{dB}$ intensity deviation and $10 \mathrm{GHz}$ spacing is experimentally obtained using a silicon microring modulator and a microring resonator filter with only $3.6 \mathrm{~V}_{\mathrm{pp}}$ driving voltage. 
Executive Ballroom

$210 \mathrm{~A}$
Executive Ballroom

210B
Executive Ballroom

$210 \mathrm{C}$
Executive Ballroom

210D

\section{CLEO: Applications \\ \& Technology}

AM4A • A\&T Topical Review on Scientific and Commercial Progress in Semiconductor Lasers II-Continued

AM4A.6 • 17:45

Toward fully monolithic $1550-\mathrm{nm}$ lasers on silicon by direct hetero-epitaxy growth on patterned substrates, Ludovico Megalini',

Brian Cabinian', Hongwei Zhao', Douglas Oakley', John Bowers', Jonathan Klamkin'; 'UCSB, USA. We demonstrate diode rectify-' ing behavior of $1550-\mathrm{nm}$ laser structures on exact-oriented (001) Si substrates after coalescence of densely-packed, smooth, high crystalline quality, and millimeter-long InP nanowires grown by MOCVD using aspect-ratio-trapping and selective-areagrowth technique.

\section{CLEO: Science \& Innovations}

SM4C • Optofluidic Components and SystemsContinued

SM4C.7 • 17:45

Enhancing the Response Time of Electrowetting Lenses Using Voltage Shaping, Omkar D. Supekar ${ }^{1}$, Mo Zohrabi², Joseph Brown', Juliet T. Gopinath², Victor M. Bright ${ }^{1}$ ${ }^{1}$ Dept. of Mechanical Engineering, Univ. of Colorado Boulder, USA; '2Dept. of Electrical, Computer, and Energy Engineering, Univ. of Colorado Boulder, USA. We have demonstrated tunability of the response time of electrowetting lenses from underdamped to overdamped through input voltage shaping. This strategy shows great promise to further optimize the response time of electrowetting lenses.
SM4D • Nanophotonics, Waveguides, and Microresonators in SensingContinued

SM4D.7 • 17:45

Chip-Based Tunable Direct Comb Spectroscopy, Mengjie Yu ${ }^{1,2}$, Yoshitomo Okawachi ${ }^{1}$ Austin G. Griffith², Michal Lipson', Alexander L. Gaeta'; ${ }^{1}$ Columbia Univ., USA; ${ }^{2}$ Cornell Univ., USA. We demonstrate mode-hop-free tuning of a modelocked frequency comb over $60 \mathrm{GHz}$ in a silicon microresonator. A gas-phase spectroscopy of acetylene is performed with a high-spectral-resolution (<80 $\mathrm{MHz}$ ) over a bandwidth of $40 \mathrm{THz}$

17:30-18:30 Diversity \& Inclusion in Optics and Photonics Reception, Market Room/Hilton

18:30-20:00 The National Academies Town Hall Meeting on the Future of Materials Research, Salon V \& VI/Marriott

19:00-20:00 OSA Technical Group Poster Session, 230B 
Executive Ballroom $210 \mathrm{E}$

Joint

JM4E • Symposium on Sources of Nonclassical Light and their Scalability II-Continued
Executive Ballroom 210F
Executive Ballroom

210G
Executive Ballroom $210 \mathrm{H}$

\section{CLEO: QELS-Fundamental Science}

FM4F • Nonlinear Optics in Propagating Geometries IIContinued

FM4F.8 • 17:45

Alkali Vapors in Mid-Infrared - Towards Gain, Yoel Sebbag', Uriel Levy'; 'Dept. of Applied Physics, The Hebrew Univ. of Jerusalem, Israel. We measured direct influence of a mid-infrared laser at $5.23 \mu \mathrm{m}$ on the $5 \mathrm{D}_{5 / 2}-6 \mathrm{P}_{3 / 2}$ transition of rubidium ( ${ }^{85} \mathrm{Rb}$ ) pumped by two photons at $780 \mathrm{~nm}$ and $776 \mathrm{~nm}$. The hyperfine structure of the $6 \mathrm{P}_{3 / 2}$ level is clearly identified.
FM4G • Controlling Emission, Absorption and Transfer of Energy with MetamaterialsContinued
FM4H • Chip-scale Plasmonic Devices-Continued
FM4H.7 • 17:45

Ultra-compact and High-performance Silicon Photonic TE-pass Polarizer Based on a Si Stripe Waveguide Coated with Multilayer Hyperbolic Metamaterial Cladding, Lei Chen ${ }^{1}$, Yumin Liu', Zhongyuan Yu', Li Yu' 'Beijing Univ of Posts \& Telecom, China. A standard silicon stripe waveguide coated with $220 \mathrm{~nm}$-thick multilayer hyperbolic metamaterial cladding makes itself become an ultra-compact TE-pass polarizer, which is characterized by ultrahigh extinction ratio of $41 \mathrm{~dB}$ and low insertion loss of $1.35 \mathrm{~dB}$.

17:30-18:30 Diversity \& Inclusion in Optics and Photonics Reception, Market Room/Hilton

18:30-20:00 The National Academies Town Hall Meeting on the Future of Materials Research, Salon V \& VI/Marriott

19:00-20:00 OSA Technical Group Poster Session, 230B 
Meeting Room
$211 \mathrm{~B} / \mathrm{D}$
Meeting Room

$212 \mathrm{~A} / \mathrm{C}$
Meeting Room

$212 \mathrm{~B} / \mathrm{D}$
Marriott

Salon I \& II

\section{CLEO: Science \& Innovations}

\section{SM4I • Ultrafast Pulse}

Combination and

Manipulation-Continued

SM4I.7 • 17:45

Frequency Domain Invisibility Enables Phase-Preserving Broadband Cloaking, Luis Romero Cortes', Seghilani Mohamed', Reza Maram', Jose. Azana'; ' ${ }^{1}$ NRS-EMT, Canada. We propose and experimentally demonstrate the first invisibility cloaking principle capable of preserving both the amplitude and the phase of a broadband illumination wave. A multiple frequency resonance is successfully cloaked over a $500-\mathrm{GHz}$ bandwidth.

\author{
$\mathrm{SM} 4 \mathrm{~J} \cdot \mathrm{THz} \mathrm{OCLs}$ and \\ Imaging-Continued
}

SM4J.7 • 17:45

GaN Terahertz Photodetectors for the Reststrahlen Gap of Intersubband Optoelectronics, Habibe Durmaz ${ }^{1,2}$, Denis Nothern', Gordie Brummer', Theodore D. Moustakas ${ }^{1}$, Roberto Paiella': ${ }^{1}$ Boston Univ., USA; ${ }^{2}$ Recep Tayyip Erdogan Univ. Turkey. Terahertz intersubband photodetectors are developed based on GaN/AlGaN quantum wells grown on a semi-polar $\mathrm{GaN}$ substrate, covering the frequency range that is fundamentally inaccessible to existing III-V semiconductor devices due to Reststrahlen absorption.

\section{SM4K • Resonant Optics- Continued}

SM4K.6 • 17:45

Engineered Pores of Hydrophilic Nanoporous Materials Using Wet-drying and Freeze-drying, Dengxin Ji', Haomin Song ${ }^{1}$, Borui Chen', Feng Yang ${ }^{2}$, Alec R. Cheney ${ }^{1}$ Feng Zhang ${ }^{2}$, Nan Zhang ${ }^{1}$, Xie Zeng ${ }^{1}$, John D. Atkinson ${ }^{3}$, Chi Zhou'2, Alexander N. Cartwright ${ }^{1}$, Qiaoqiang Gan ${ }^{1}$; ${ }^{1}$ Dept. of Electrical Engineering, The State Univ. of New York at Buffalo, USA; ${ }^{2}$ Dept. of Industrial and Systems Engineering, The State Univ. of New York at Buffalo, USA; ${ }^{3}$ Dept. of Civil, Structural and Environmental Engineering, The State Univ. of New York at Buffalo, USA. We manipulate the pore size of nanoporous polymeric photonic crystals using phase change between water and ice, demonstrating accurate postmanipulation of reflection resonances and thereby providing an approach to address grand challenges in nanomanufacturing and materials engineering.

\section{SM4L • Mode Locked Fiber} Lasers II-Continued

\section{SM4L.8 • 17:45}

Dispersion compensation of a compact NPE mode-locked Yb-doped all fiber laser oscillator by using tapered-fiber, Peilong Yang ${ }^{1,3}$, Zhongqi Hu ${ }^{1,4}$, Hao Teng ${ }^{5}$, Shaobo Fang ${ }^{6}$, Zhiguo $\mathrm{Lv}^{2}$, Zhiyi $\mathrm{Wei}^{7} ;{ }^{1}$ School of Physics and Optoelectronic Engineering, Xidian Univ., China; ${ }^{2} \mathrm{Xi}^{\prime}$ an Inst. of Optics and Precision Mechanics, Chinese Academy of Sciences, China: ${ }^{3}$ nst. of Physics, Chinese Academy of Sciences, China. A mode-locked Yb-doped all fiber oscillator using tapered-fiber dispersion compensation was demonstrated. The spectrum was broadened to $20 \mathrm{~nm}$ and compressed to 112 $\mathrm{fs}$ using diameter of $1 \mathrm{~mm}$ with length of 10 $\mathrm{cm}$ tapered fiber.

17:30-18:30 Diversity \& Inclusion in Optics and Photonics Reception, Market Room/Hilton

18:30-20:00 The National Academies Town Hall Meeting on the Future of Materials Research, Salon V \& VI/Marriott 


$\begin{array}{llc}\text { Marriott } & \text { Marriott } & \text { Marriott } \\ \text { Salon III } & \text { Salon IV } & \text { Salon V \& VI }\end{array}$

\section{CLEO: Science \& Innovations}

SM4M • Optical Parametric Oscillators-Continued

SM4M.7 • 17:45

Electro-Optic Controlled, Highly Spectrum

Narrowed Multiline Intracavity Optical Parametric Oscillators, Hung-Pin Chung Wei-Kun Chang ${ }^{1,2}$, Yen-You Chou', Reinhard Geiss $^{3}$, Shang-Da Yang ${ }^{4}$, Thomas Pertsch ${ }^{3}$ Yen-Hung Chen'; 'National Central Univ. Taiwan; ${ }^{2}$ Brain Research Center, Nationa Tsinghua Univ., Taiwan; ${ }^{3}$ nst. of Applied Physics, Abbe Center of Photonics, FriedrichSchiller-Universität Jena, Germany; ${ }^{4}$ nst. of Photonics Technologies, Dept. of Electrical Engineering, National Tsinghua Univ., Taiwan. An electro-optically spectrum narrowed, mu tiline optical parametric oscillator was buil based on a novel aperiodically poled lithium niobate device. The power spectral density of the EO controlled system is enhanced by a factor of $\sim 7.8$.
SM4N • Prevoskite and Photonic Crystal LasersContinued

\section{SM4N.6・17:45}

Photonic Crystal Surface-Emitting Lasers on Bulk Silicon Substrate, Shih-Chia Liu $^{1}$, Deyin Zhao', Hongjun Yang ${ }^{1}$, Carl Reuterskiöld-Hedlund², Mattias Hammar², Zhenqiang $\mathrm{Ma}^{3}{ }^{3}$ Weidong Zhou ${ }^{1}$. ${ }^{1}$ Univ of Texas at Arlington, USA; ${ }^{2} \mathrm{KTH}$-Royal Inst. of Technology, Sweden; ${ }^{3}$ Univ. of Wisconsin, USA. We report here heterogeneous photonic crystal (PC) bandedge surface emitting lasers on bulk silicon (Si) substrates. Thermal resistance was investigated to evaluate the heat dissipation and lasing characteristics afforded by this integration and potential for high efficiency application.
SM4O • Heterogeneously Integrated Si PhotonicsContinued

17:30-18:30 Diversity \& Inclusion in Optics and Photonics Reception, Market Room/Hilton

18:30-20:00 The National Academies Town Hall Meeting on the Future of Materials Research, Salon V \& VI/Marriott

19:00-20:00 OSA Technical Group Poster Session, 230B 


\section{Executive Ballroom $210 \mathrm{~A}$}

\section{8:00-10:00}

ATu1A $\bullet$ Biosensing

\section{Technologies}

Presider: Xuan Liu; New Jersey

Inst. of Technology, USA

ATu1A.1 $\bullet$ 08:00

Digital DNA Detection based on Compact Optofluidic Laser with Ultra-Low Sample

Consumption, Wonsuk Lee', Qiushu Chen² Xudong Fan', Dong Ki Yoon'; 'KAIST, USA; 2Univ. of Michigan, USA. Optofluidic laser that has a single layer of DNA molecules on the ring resonator surface is proposed. A target DNA can be detected in truly digital manner only with a single pulse of laser excitation.

ATu1A.2 • 08:15

Integrated Sensor based on a-Si:H Photodiodes and Diffused Glass Waveguides for Biomedical Applications, Giampiero de Cesare ${ }^{1}$, Rita Asquini ${ }^{1}$, Alessio Buzzin ${ }^{1}$ Antonio d'Alessandro', Augusto Nascetti ${ }^{1}$, Domenico Caputo'; 'DIET - Univ. of Rome "La Sapienza", Italy. We present the design and fabrication of an evanescent waveguide sensor based on a-Si:H photodiodes for biomedical applications. The complete device presents a noise level lower than $2 f A$ and a responsivity of $300 \mathrm{~mA} / \mathrm{W}$ at $532 \mathrm{~nm}$.

\section{ATu1A.3 • 08:30}

3D Refractive Index Mapping of Single Cells, Patricia Y. Liu',2, Chao-mao Hsieh', L. K. Chin', Yamin Wang ${ }^{2}$, Wee Ser', Tarik Bourouina², Jingbo Zhang'; 'Nanyang Technological Univ., Singapore; ${ }^{2}$ Université Paris-Est, France. We present the study of quantitative $3 \mathrm{D}$ refractive index mapping of single cells and intracellular lipid droplets with an Optica Diffractive Tomography system. Intracellular organelles, such as nucleus, mitochondria, lipid droplets are studied.

\section{Executive Ballroom 210B}

Executive Ballroom $210 \mathrm{C}$

\section{CLEO: Applications \& Technology}

08:00-10:00

ATu1B • A\&T Topical Review on Neurophotonics I

Presider: Kishan Dholakia; Univ. of St Andrews, UK

\section{ATu1B.1 • 08:00 Invited}

Unexpectedly Stalled: Two-Photon Microscopy Reveals White Blood Cell Adhesion in Capillaries Causes Reduced Brain Blood Flow In Alzheimer's Disease, Chris B. Schaffer': ${ }^{1}$ Biomedical Engineering, Cornell Univ. USA. About $2 \%$ of brain capillaries were oc cluded by leukocytes adhered to the vessel wall in mouse models of Alzheimer's. When this adhesion was blocked, brain blood flow immediately increased by $~ 30 \%$ and spatial memory performance improved.
08:00-10:00

ATu1C • Lasers for Additive

Manufacturing and Surface

Structuring

Presider: Jie Qiao; Rochester Inst. of Technology, USA

ATu1C.1 • 08:00

Thin Disk Lasers for Research and Industria Applications, Adolf Giesen, German Aerospace Center, Germany. The status of thin disk laser development will be discussed in detail as well as some important applications in research and industry.

\section{ATu1B.2 $08: 30$ Invited}

Transcutical three-photon fluorescence imaging of Drosophila brain at subcellular resolution with adaptive optics, Xiaodong $\mathrm{TaO}^{2}$, Hui-Hao Lin'1, Tuwin Lam², Ramiro Rodriguez ${ }^{2}$, Jing W. Wang ${ }^{1}$, Joel Kubby ${ }^{2}$. ${ }^{1}$ Univ. of California, San Diego, USA; ${ }^{2}$ Univ. of California, Santa Cruz, USA. We demonstrate non-invasive structural and functional imaging of neurons labeled with genetically encoded red fluorescent proteins in the living Drosophila brain at cellular and subcellular resolution using three-photon microscopy and wavefront correction.
Executive Ballroom 210D

\section{CLEO: QELS- Fundamental Science}

\section{8:00-10:00 \\ FTu1D • On-chip Comb}

Generation I

Presider: Marcello Ferrera; Heriot-

Watt Univ., UK

\section{FTu1D.1 • 08:00 Tutorial}

Chip-Based Optical Frequency Combs, Alexander L. Gaeta'; ' ${ }^{1}$ Columbia Univ., USA. Microresonator-based optical frequency combs could enable the realization of time and frequency metrology instruments in highly compact and robust platforms. will provide an overview of the underlying principles and applications of such combs.

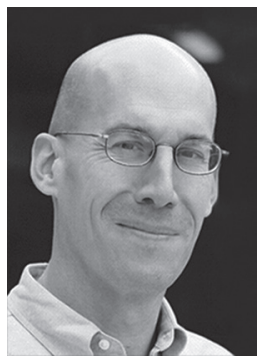

Alex Gaeta received his $\mathrm{PhD}$ from the University of Rochester. From 1992 to 2015 he was on the faculty at Cornell University. In 2015 he joined the faculty at Columbia University where he is the David M. Rickey Professor of Applied Physics. He co-founded PicoLuz, Inc. along with Michal Lipson and Alex Cable and is the founding Editor-in-Chief of Optica. He is a Fellow of The Optical Society and of the America Physical Society.
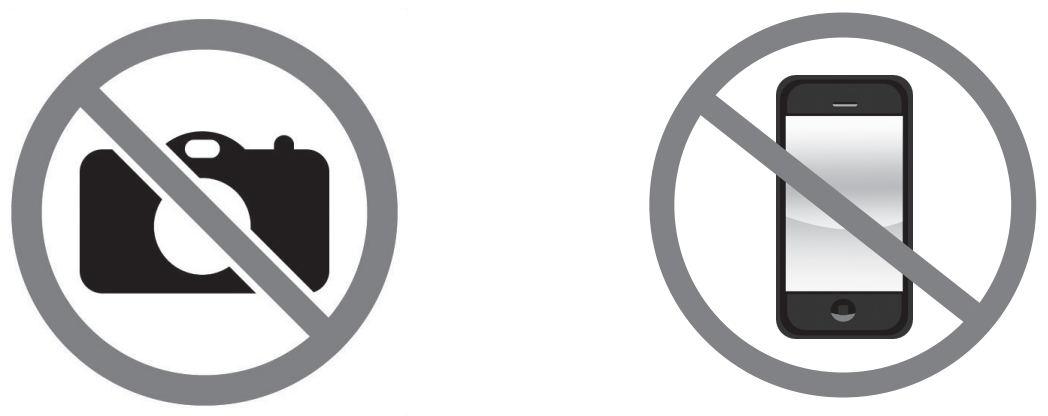
Executive Ballroom 210E
Executive Ballroom

210F
Executive Ballroom

210G
Executive Ballroom

$210 \mathrm{H}$

\section{CLEO: QELS-Fundamental Science}

08:00-10:00

FTu1E - Defects in Solids for Coherent Control and SinglePhoton Generation

Presider: Joshua Nunn; Univ. of Oxford, UK

FTu1E.1 $\bullet$ 08:00

Complete Coherent Control of SiliconVacancies in Diamond Nanopillars Containing Single Defect Centers, Jingyuan Linda Zhang', Konstantinos Lagoudakis', Yan-kai Tzeng ${ }^{1}$, Constantin Dory ${ }^{1}$, Marina Radulaski ${ }^{1}$, Yousif Kelaita' ${ }^{1}$ Kevin Fischer ${ }^{1}$, Zhi-Xun Shen ${ }^{1}$ Nicholas Melosh', Steven Chu', Jelena Vuckovic' ${ }^{1}$ 'Stanford Univ., USA. We fabricate diamond nanopillar arrays containing single SiV- centers with high yield and spectral stability, and perform ultrafast, all-optical complete coherent control over the state of individual SiV- centers, as demonstrated by Rabi oscillation, Ramsey interference, and SU(2) control.

FTu1E.2 • 08:15

Enhanced Quantum Sensing with NitrogenVacancy Centers in Nanodiamonds Using All-Optical Charge Control, David Hopper ${ }^{\prime}$ Richard Grote ${ }^{1}$, Lee Bassett'; ' ${ }^{1}$ Univ. of Pennsylvania, USA. We demonstrate an all-optical protocol for ensemble charge readout and spin-to-charge conversion of nitrogenvacancy centers in room-temperature nanodiamonds. This technique provides drastic improvements for electrochemical sensing and an order-of-magnitude speedup for spin-based quantum sensors.

\section{FTu1E.3 • 08:30}

The neutral silicon split-vacancy defect in diamond, a promising color center for quantum communication, Brendon C. Rose Ding Huang', Alexei Tyryshkin'1, Sorawis Sangtawesin', Daniel J. Twitchen ${ }^{6}$, Matthew L. Markham ${ }^{6}$, Andrew M. Edmonds ${ }^{6}$, Adam Gali $^{2}$, Alastair Stacey ${ }^{5}$, Wuyi Wang ${ }^{4}$, Ulrika D'Haenens-Johansson ${ }^{4}$, Alexander Zaitsev ${ }^{3}$ Stephen A. Lyon', Nathalie de Leon'; 'Princeton Univ., USA; ${ }^{2}$ Wigner Research Center Hungary; ${ }^{3}$ CUNY College of Staten Island USA; ${ }^{4}$ Gemological Inst. of America, USA; ${ }^{5}$ Univ. of Melbourne, Australia; ${ }^{6}$ Element Six, UK. We investigate the neutral charge state of the interstitial silicon split-vacancy defect in diamond for use in quantum communication applications using pulsed electron spin resonance at X-Band magnetic fields ( 3500 $\mathrm{G})$ and confocal microscopy.
08:00-10:00

FTu1F • Quantum Optics

and Quantum Information

Processing

Presider: Michael Brodsky; US

Army Research Laboratory, USA

FTu1F.1 • 08:00

Frequency-Domain Boson Sampling, Chaitali Joshi ${ }^{1}, 2$, Alessandro Farsi ${ }^{2}$, Alexander Gaeta ${ }^{2}{ }^{1}$ Cornell Univ. , USA : ${ }^{2}$ Applied Physics and Applied Mathematics, Columbia Univ. USA. We present a scheme to efficiently perform boson sampling using frequency modes which yields exponential reduction in losses and significantly reduced experimental complexity compared to conventional spatial-mode implementations.

FTu1F.2 • 08:15

Gaussian Boson Sampling, Craig S. Hamilton², Regina Kruse', Linda Sansoni', Sonja Barkhofen ${ }^{1}$, Christine Silberhorn ${ }^{1}$, Igor Jex ${ }^{2}$ ${ }^{1}$ Univ. of Paderborn, Germany; ${ }^{2}$ FNSPE, Czech Technical Univ. in Prague, Czech Republic. We present the protocol for Gaussian Boson Sampling with single-mode squeezed states. We eliminate heralding and show that our proposal with the Hafnian matrix function can retain the higher photon number contributions at the input.

\section{FTu1F.3 08:30 Invited}

Multiparticle distinguishability: three photons are different in four ways, Adrian J. Menssen ${ }^{1}$, Alex Jones ${ }^{1,2}$, Malte Tichy ${ }^{3}$, Benjamin Metcalf1', Stefanie Barz', Steven Kolthammer', Ian A. Walmsley'; 'Univ. of Oxford, UK: ${ }^{2}$ Imperial College, UK. ${ }^{3}$ Univ of Aarhus, Denmark. Quantum interference of two independent photons is fully described by the particles' distinguishability. We demonstrate that for three photons, the scattering depends on their three mutual distinguishabilities and a fourth parameter we named triad phase.
08:00-10:00

FTu1G • Light Manipulation with Disordered Media

Presider: Alexandra Boltasseva;

Purdue Univ., USA

FTu1G.1 • 08:00

Anderson Localization of Light in Spectrally-Tailored Disordered Potentials,

Alex Dikopoltsev1, Hanan Herzig Sheinfux

1, Mordechai Segev'; 'Technion, Israel. We demonstrate, against current knowledge, that Anderson localization can occur fo wavepackets outside the spectral exten of the disordered potential, mediated by second order transitions.
FTu1G.2 • 08:15

Phase transitions in the diffusion of light, Roxana Rezvani Naraghi3,2, Aristide Dogariu'; 'Univ. of Central Florida, CREOL, USA; ${ }^{2}$ Physics, Univ. of Central Florida, USA ${ }^{3}$ CREOL, Univ. of Central Florida, USA. We demonstrate a new phenomenon occurs in the propagation of light through random media. Due to different mechanisms of interaction, recurrent scattering of on-shel propagating fields is impeded by strongly localized evanescent couplings.

\section{FTu1G.3 • 08:30 Invited}

Wavefront Shaping in Complex Media: From the Compensation to the Harnessing of Disorder, Sebastien Popoff1,2; ${ }^{1}$ Langevin Inst., ESPCl, France; ${ }^{2} \mathrm{CNRS}$, France. In the past ten years, many techniques were developed to control light propagation in complex media using spatial light modulators. The applications evolved from compensating for disorder to taking advantage of the randomness.
08:00-10:00

FTu1H • Fundamental Plasmonic \& Nanophotonic Effects

Presider: Benjamin Lawrie; Oak

Ridge National Lab, USA

FTu1H.1・08:00

Plasmon Drag in Nanostructured Metal and Effects of Spin Angular Momentum of Plasmons, Maxim Durach ${ }^{1}$ Natalia Noginova2; ${ }^{2}$ Georgia Southern Univ., USA; ${ }^{2}$ Norfolk State Univ., USA. Here we review recent advances in plasmon drag studies, and describe the plasmogalvanic effects associated with absorption of spin angula momentum (SAM) of plasmons.

\section{FTu1H.2 $08: 15$ Invited}

The Role of Coherence in Plasmonic Interferometry, Domenico Pacifici'; ' ${ }^{1}$ Brown Univ, USA. Here we discuss methods that employ surface plasmons to measure and strongly modulate the degree of optica spatial coherence of light, paving the way for multifunctional optical elements beyond conventional refractive- and diffractive-based photonics metasurfaces. 


\section{CLEO: Science \& Innovations}

08:00-10:00

STu1l • Ultrafast Applications

Presider: Fumihiko Kannari; Keio Univ., Japan

\section{STu1I.1 • 08:00 Invited}

Extreme Ultraviolet Vector Beams Driven by Multicycle Infrared Laser Pulses, Carlos Hernandez-Garcia', Alex Turpin ${ }^{2}$, Julio San Roman' ${ }^{1}$ Antonio Picon', Rokas Drevinskas ${ }^{3}$, Aura Cerkauskaite ${ }^{3}$, Peter Kazansky ${ }^{3}$, Charles Durfee ${ }^{4}$, Iñigo J. Sola'; 'Universidad de Salamanca, Spain; ${ }^{2}$ Universitat Autonoma de Barcelona, Spain; ${ }^{3}$ Univ. of Southampton, UK; ${ }^{4}$ Colorado School of Mines, USA. We experimentally produce extreme-ultraviolet vector beams -from radially to azimuthally polarized- through high-order harmonic generation. Our simulations predict the generation of unique spatio-temporal structures in the form of attosecond vector beams.

\section{8:00-10:00}

STu1J - THz Materials Science

Presider: Rohit Prasankumar; Los Alamos National Lab, USA

STu1J.1 $\bullet$ 08:00

Off-resonant magnetization dynamics in $\mathrm{Co}, \mathrm{Fe}$ and $\mathrm{Ni}$ thin films driven by an intense single-cycle $\mathrm{THz}$ field, Mostafa Shalaby', C. Vicario', Flavio Giorgianni', Andreas Donges ${ }^{2}$, Karel Carva ${ }^{3}$, Peter Oppeneer $^{4}$, Ulrich Nowak ${ }^{2}$, Christoph P. Hauri ${ }^{1,5}$; ${ }^{1}$ Paul scherrer institut, Switzerland; ${ }^{2}$ Dept. of Physics, Univ. of Konstanz, Germany; ${ }^{3}$ Charles Univ., Czech Republic; ${ }^{4}$ Uppsala Univ., Sweden; ${ }^{5}$ Ecole Polytechnique Federale de Laus anne, Switzerland. We present time-resolved measurements exploring the $\mathrm{THz}$-induced magnetization dynamics as function of the driving field strength in the ferromagnetic thin film samples $\mathrm{Co}, \mathrm{Fe}$ and $\mathrm{Ni}$. The experimental results are excellently reproduced by ab-initio calculations.

\section{STu1J.2 $08: 15$ Invited}

Dynamics, Control, and Metastability in Correlated Oxides, Richard D. Averitt'; ' 'Univ. of California San Diego, USA. I will present results of photoinduced insulator-to-metaltransition dynamics in $\mathrm{La}_{0.7} \mathrm{Ca}_{0.3} \mathrm{MnO}_{3}$ films that have been strain-engineered to quench the thermal IMT. Photoexcitation initiates a nonthermal transition to a "hidden" metallic phase that is metastable yet robust.
STu11.2 $08: 30$

Time-resolved Femtosecond Photoemission Spectroscopy using a $60-\mathrm{MHz} \mathrm{En}-$ hancement Cavity XUV Source, Arthur K. Mills', Sergey Zhdanovich', Fabio Boschini' MengXing $\mathrm{Na}^{1}$, Michael Schneider', Pinder Dosanjh', Doug Wong', Giorgio Levy ${ }^{1}$, Andrea Damascelli', David J. Jones'; ' $D$ Dept. of Physics and Stewart Blusson Quantum Matter Inst., Univ. of British Columbia, Canada. We perform time-resolved photoemission on the topological insulator $\mathrm{Bi}_{2} \mathrm{Se}_{3}$ at $60 \mathrm{MHz}$ repetition rate, with a $25 \mathrm{eV}$ probe and a $1.2 \mathrm{eV}$ pump, with demonstrated time and energy resolution of $<400 \mathrm{fs}$ and $<25 \mathrm{meV}$, respectively.
08:00-10:00

STu1K • Mid-IR Fiber Sensors

Presider: Khanh Kieu; Univ. of

Arizona, USA

\section{STu1K.1 • 08:00 Invited}

Ultrafast Fiber Lasers in the Mid-IR Water Vapor Window, Darren D. Hudson', Sergei Antipov1, Stuart D. Jackson', Alexander Fuerbach': 'Macquarie Univ., Australia. We demonstrate record performance in a mid-IR ultrafast fiber laser by using holmium instead of erbium as the active gain medium. The $2.9 \mu \mathrm{m}$ laser emits $180 \mathrm{fs}$ pulses with $37 \mathrm{~kW}$ peak power.
STu1K.2 • 08:30

Raman Generation in $2.9-3.5 \mu \mathrm{m}$ Spectral Range in Revolver Hollow-Core Silica Fiber Filled by $\mathrm{H}_{2} / \mathrm{D}_{2}$ Mixture, Alexey Gladyshev1', Alexey F. Kosolapov', Maxim M. Khudyakov ${ }^{1,2}$, Yury P. Yatsenko', Andrey K. Senatorov ${ }^{1}$, Anton N. Kolyadin ${ }^{1}$, Alexander A. Krylov', Victor G. Plotnichenko', Mikhail E. Likhachev', Igor A. Bufetov', Evgeny M. Dianov'; 'Fiber Optics Research Center of the Russian Academy of Sciences, Russia; ${ }^{2}$ Moscow Inst. of Physics and Technology (State Univ.), Russia. Mid-infrared Raman generation is demonstrated in gas-filled hollow-core silica fiber pumped by high-power $1.56 \mu \mathrm{m}$ Er-doped fiber laser. Quantum conversion efficiency up to $8 \%$ and peak output power up to $0.9 \mathrm{~kW}$ are achieved. 
Marriott

Salon III
Marriott

Salon IV
Marriott

Salon V \& VI

\section{CLEO: Science \& Innovations}

08:00-09:45

STu1M • Optical Interconnect Systems

Presider: Michael Vasilyev; Univ.

of Texas at Arlington, USA

\section{STu1M.1 • 08:00}

\section{Invited}

Silicon Photonic Systems-on-Chip, Michael Hochberg'; ' 'Elenion Technologies LLC, USA. We continue to see silicon photonics chip complexity doubling every 12-18 months, providing an excellent platform for developing photonic systems-on-chip (SoC). The complexity scaling is enabling more functionality for high-bandwidth applications and enabling new application domains, while bringing the overall system costs down.
08:00-10:00

STu1N • Photodetectors

Presider: Shiqiang Li; Univ. of Melbourne, Australia

\section{STu1N.1 $\bullet$ 08:00}

Flexible waveguide-integrated photodetectors, Hongtao Lin ${ }^{1}$, Lan Li ${ }^{1}$, Yizhong Huang ${ }^{1}$, Junying $\mathrm{Li}^{1}$, Spencer Novak ${ }^{2}$, Kathleen Richardson'2, Juejun Hu'; ' Materials Science and Engineering, MIT, USA; ${ }^{2} C R E O L$, Univ. of Central Florida, USA. We demonstrated a flexible waveguide-integrated metal-semiconductor-metal photodetector with $0.5 \mathrm{~A} / \mathrm{W}$ responsivity near $1550 \mathrm{~nm}$ wavelength. The device can withstand a small bending radius of $0.7 \mathrm{~mm}$ without optical performance degradation.
STu1N.2・08:15

High Speed Photoconductive Plasmonic Germanium Detector, Yannick Salamin', Ping $\mathrm{Ma}^{1}$, Alexandros Emboras', Yuriy Fedoryshyn ${ }^{1}$, Bojun Cheng ${ }^{1}$, Christian Hafner ${ }^{1}$, Juerg Leuthold'; 'ETH Zurich, Switzerland. We demonstrate a new concept of a photoconductive plasmonic photodetector that features high speed at nanometer scale. The concept is based on the electro-absorption effect in an plasmonic slot-waveguide with amorphous $\mathrm{Ge}$ as active material.
STu1M.2 • 08:30

A 3x3 Switch Exploiting an Optical Vortex Beam Emitter based on a Silicon ThreeGrating Microring, Mirco Scaffardi ${ }^{1}$, Muhammad N. Malik' ${ }^{1,2}$, Emma Lazzeri ${ }^{2}$, Charalambos Klitis $^{3}$, Laura Meriggi ${ }^{3}$, Ning Zhang ${ }^{3}$, Marc Sorel $^{3}$, Antonella Bogoni2; ${ }^{2}$ CNIT, Italy; ${ }^{2}$ Scuola Superiore Sant'Anna, Italy; ${ }^{3}$ Univ. of Glasgow, UK. A silicon three-grating microring is proposed and characterized as a device enabling $3 \times 3$ optical switching based on orbital angular momentum and wavelength. Bit error rate measurements show penalties $<1 \mathrm{~dB}$ for OOK traffic up to $20 \mathrm{Gbaud}$.
08:00-10:00

STu10 • Petawatt Laser

Technology

Presider: Jake Bromage; Univ. of Rochester, USA

\section{STu10.1 0 08:00 Invited}

High Energy, High Repetition Rate Nd:Glass Laser Technology, Erhard W. Gaul'; ; ${ }^{1}$ Univ. of Texas at Austin, USA. Nd:Glass lasers enable high energy, ultra-high intensity pulses with 10PW peak power. Lasers technology with active cooling provides orders of magnitudes improved over previous shots rates will be discussed.

\section{STu1N.3 • 08:30 Invited}

Subwavelength Angle Sensing Photodetector, Soongyu Yi', Ming Zhou', Zongfu $\mathrm{Yu}^{1}$, Pengyu Fan ${ }^{2}$, Dianmin Lin ${ }^{2}$, Shanhui Fan', Mark Brongersma2; 'Univ. of Wisconsin - Madison, USA; ${ }^{2}$ Stanford Univ., USA. By mimicking internally coupled ears directional hearing capability of small animals, we demonstrate subwavelength angle sensing using coupled optical resonators in extremely small distance.
STu10.2 • 08:30

Temporal dual-pulse pumped Ti:Sapphire Amplilier, Zebiao Gan', Lianghong Yu', Xiaoyan Liang', Yanqi Liu', Wenqi Li', Cheng Wang ${ }^{1}$, Zhen Guo', Zutao Fan ${ }^{1}$, Xiaolong Yuan', Lu Xu', Zhengzheng Liu', Shuai Li', Yi $X u^{1}$, Jun Lu', Haihe Lu', Dingjun Yin ${ }^{1}$, Yuxin Leng', Ruxin Li', Zhizhan $\mathrm{Xu}^{1}$; 'Shanghai Inst of Optics \& Fine Mechanics, China. We report that $202.8 \mathrm{~J}$ output energy from a $150-\mathrm{mm}$ diameter Ti:sapphire amplifier was achieved with $320 \mathrm{~J}$ pump energy. A temporal dualpulse pumped scheme was used to suppress the transverse parasitic lasing. 
Executive Ballroom $210 \mathrm{~A}$

Executive Ballroom 210B

Executive Ballroom $210 \mathrm{C}$

\section{CLEO: Applications \\ \& Technology}

ATu1A $\bullet$ Biosensing Technologies-Continued

ATu1B • A\&T Topical Review on

ATu1A.4 0 08:45

Thermal Expansion Feedback for Wavefront Shaping, Omer Tzang ${ }^{1}$, Eyal Niv' ${ }^{1}$, Rafael Piestun'; ${ }^{1}$ Univ. of Colorado at Boulder, USA. We present a technique for focusing inside scattering media that combines optical-coherence-tomography (OCT) and wave-front-shaping (WFS). We use OCT as a non-invasive feedback for WFS optimization of a separate penetrating laser, based on light-induced thermal-expansions.

ATu1A.5 • 09:00

Continuous characterization of viscoelasticity-modulated biopolymer hydrogels, Jose Guzman-Sepulveda ${ }^{2}$, Jinan Deng ${ }^{1}$ Jiyu Fang ${ }^{1}$, Aristide Dogariu'; ' Dept. of Materials Science and Engineering, Univ. of Central Florida, USA; ${ }^{2}$ CREOL, The College of Optics and Photonics, Univ. of Central Florida, USA. We present a spatiotemporal coherence-gated dynamic light scattering technique that permits continuously assess non-equilibrium, long-term dynamical processes. We demonstrate it experimentally by characterizing the evolution of mechanical properties in viscoelasticity-modulated biopolymer hydrogels.

ATu1A.6 0 09:15

Gallium Nitride Based Tactile Sensors, Jingyang Sui ${ }^{1}$, Pei-Cheng Ku' ${ }^{1}$; ${ }^{1}$ Dept. EECS, Univ. of Michigan, USA. An optical tactile sensor is proposed using GaN LEDs and $\mathrm{Si}$ imagers. Performance comparable to human fingertips including high spatial resolution, fast response and multidirectional discrimination was shown. visualization of microvasculature and fluores-
ATu1A.7 • 09:30

Diagnostics of Femoral Head Status in Humans usingHigh-Resolution Lase Spectroscopy -In Vitro Studies, Katarina Svanberg 2,1 , Huiying Lin², Wansha Li², Hao Zhang $^{2}$, Peng Chen ${ }^{3}$, Delong Chen ${ }^{4}$, Wei $\mathrm{He}^{3}$, Sune R. Svanberg ${ }^{2,1}$; ${ }^{1}$ Lund Lase Centre, Sweden: ${ }^{2}$ Center for Optical and Electromagnetic Research, South China Normal Univ., China; ${ }^{3}$ Orthopedics Dept. First Affiliated Hospital, China: ${ }^{4}$ First Clinical Medical School of Chinese Medicine, China. Bone decay processes, due to lacking blood supply, was studied by diode laser absorption spectroscopy detecting gas-filled pores in hip replacement operation specimen from 19 orthopedical patients. Minimally invasive diagnostics seems feasible. Neurophotonics I-Continued
ATu1B.3 • 09:00

In Vivo Deep Tissue Visualization by Needle-type Side-view Confocal Endomicroscopy, Jinhyo Ahn', Eunji Kong ${ }^{1}$, Kibaek Choe', Eunjoo Song' ${ }^{1}$, Yoonha Hwang ${ }^{1}$, Inwon Park', Pilhan Kim"; ${ }^{1}$ Korea Advanced Inst of Science \& Tech, Korea (the Republic of). In vivo longitudinal and repetitive cellular-leve cent cells in deep tissue such as skin dermis solid tumor and brain of single mouse in minimally invasive manner was demonstrated by using a needle-type side-view confocal endomicroscopy.

ATu1B.4 • 09:15

End-Fire Silicon Optical Phased Arrays for Infrared Neural Stimulation Applications Michael Kossey', Shannon Alt ${ }^{1}$, Charbel Rizk' Amy Foster'; 'Johns Hopkins Univ., USA. We propose infrared neural stimulation as an application for end-fire integrated optical beam steering devices. We show some initial results and discuss its implications for the future development of the technique.

\section{ATu1C $\bullet$ Lasers for Additive Manufacturing and Surface Structuring-Continued}

\section{ATu1C.2 • 08:45}

Energy coupling efficiency and melt pool dynamics associated with the laser melting of metal powder layers, Manyalibo J. Matthews ${ }^{1}$, Johannes Trapp', Gabe Guss Alexander Rubenchik' ; 'Lawrence Livermore National Lab, USA. Micro-calorimetry and high speed imaging are used to characterize energy coupling mechanisms in laser powder bed fusion additive manufacturing. Materialdependent keyhole formation onset and melt pool dynamics are investigated as a function of laser parameters.

\section{ATu1C. $3 \bullet 09: 00$}

Laser beam ellipticity and microstructura control in metal additive manufacturing Sheldon S. Wu' ${ }^{1}$, Tien T. Roehling ${ }^{2,1}$, Saad A. Khairallah', Alexander Rubenchik', John D. Roehling', Stefan S. Soezeri², Michael F. Crumb', Gabe Guss' ${ }^{1}$, Manyalibo Matthews ${ }^{1}$ 'Lawrence Livermore National Lab, USA ${ }^{2}$ Dept. of Mechanical Engineering, Univ. of the Pacific, USA. Spatial beam profile tailor ing is a potential means for microstructura control during laser additive manufacturing. Microstructures produced by circular and elliptical laser profiles in 316 L stainless stee single-tracks and applications to more general beam shapes are presented.

\section{ATu1C.4 09:15}

High throughput laser-scribing processes for industrial production of flexible CIGS thin-film solar modules, Andreas Burn ${ }^{4}$ Christian Heger ${ }^{4}$, Stephan Buecheler ${ }^{1}$, Lukas Greuter $^{1}$, Patrick Reinhard², Roger Ziltener ${ }^{2}$, Lukas Krainer ${ }^{3}$, Gabriel Spuehler ${ }^{3}$, Valerio Romano ${ }^{4} ;{ }^{1}$ Lab for Thin Films \& Photovoltaics, Empa, Swiss Federal Labs for Materials Science and Technology, Switzerland ${ }^{2}$ Flisom AG, Switzerland; ${ }^{3}$ onefive $\mathrm{GmbH}$ Switzerland: ${ }^{4}$ Applied Laser-, Photonics- and Surface Technologies - ALPS, Bern Univ. of Applied Sciences, Switzerland. Robust high-throughput laser scribing processes for monolithic interconnection of $\mathrm{Cu}(\ln \mathrm{Ga})$ $\mathrm{Se}_{2}$ absorber based thin-film solar cells were developed, validated and assessed for industrial roll-to-roll production of photovoltaic modules. Here we present results of the FP7-project APPOLO.

ATu1B.5 • 09:30 Invited

Wide-field Fast-scanning Photoacoustic Microscopy of Brain Functions in Action, Junjie Yao', Jun Zou', Lihong Wang ${ }^{3}$; 'Duke Univ., USA; ${ }^{2}$ Texas A\&M Univ., USA; ${ }^{3}$ Washington Univ. , USA. We have developed fast functional photoacoustic microscopy for 3D high-resolution high-speed imaging of the mouse brain. In particular, a novel singlewavelength pulse-width-based method can image blood oxygenation with capillary-leve resolution at $100 \mathrm{kHz}$ frame rate.

\section{ATu1C.5 • 09:30}

Multifunctional Properties of High-speed Highly Uniform Femtosecond Lase Patterning on Stainless steel, Laroslav Gnilitskyi', Alberto Rota' ${ }^{1}$ Radim Ctvrtlik2 ${ }^{2}$, Ana Paula Serro ${ }^{3,4}$, Enrico Gualtieri', Leonardo Orazi'; ' $U N I M O R E$, Italy; ${ }^{2}$ Palacky Univ. and Inst. of Physics, Czech Republic; ${ }^{3}$ Instituto Superior Tecnico, Portugal; ${ }^{4} \mathrm{CIIEM}$, Portugal. Highly-regular laser-induced periodic surface structures essentially change surface properties of treated surfaces. Here, effect of HR-LIPSS on optical, tribological, mechanical and wetting properties of stainless steel were systematically investigated.
FTu1D.2 • 09:00

Dynamics of soliton crystals in optical microresonators, Maxim Karpov' ${ }^{1}$, Hairun Guo ${ }^{1}$, Martin Pfeiffer ${ }^{1}$, Erwan Lucas ${ }^{1}$, Michae Geiselmann', Miles Anderson', Tobias J. Kip-

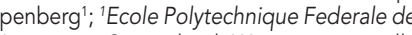
Lausanne, Switzerland. We experimentally demonstrate and study the formation and switching dynamics of collectively-ordered ensembles of strongly interacting dissipative Kerr solitons (soliton crystals) in optical microresonators.

FTu1D.3 • 09:15

Low Threshold Frequency Comb Generation in AlGaAs-on-Insulator Microresonato in the Normal Dispersion Regime, Ayman N. Kamel', Minhao Pu' ${ }^{1}$ Kresten Yvind ${ }^{1}$; 1 Danmarks Tekniske Universitet, Denmark. We present milli-Watt threshold frequency comb generation in AlGaAs-on-insulator integrated microresonators exhibiting normal GVD by employing the effects of mode interaction.

\section{FTu1D.4 09:30 Invited}

Aluminum-nitride-waveguide supercontinuum and harmonic generation across 500 to 4000 nm, Daniel Hickstein ${ }^{1}$, Hojoon Jung², David R. Carlson', Alex Lind', Ian R. Coddington ${ }^{4}$, Kartik Srinivasan ${ }^{3}$, Gabriel Ycas ${ }^{1}$, Daniel Cole', Abijith Kowligy', Nate Newbury ${ }^{4}$, Hong Tang ${ }^{2}$, Scott Diddams ${ }^{1}$ Scott Papp'; ${ }^{1}$ Time and Frequency Division, NIST, USA; ' ${ }^{2}$ Dept. of Electrical Engineering Yale Univ., USA; ${ }^{3}$ Center for Nanoscale Science and Technology, NIST, USA; ${ }^{4}$ Applied Physics Division, NIST, USA. Using aluminumnitride photonic-chip waveguides, we generate optical frequency comb supercontinuum spanning 500 to $4000 \mathrm{~nm}$. We detect and stabilize the offset frequency of the compact laser comb oscillator directly using the waveguide output. 

Executive Ballroom
$210 \mathrm{E}$
Executive Ballroom

$210 \mathrm{~F}$
Executive Ballroom

210G
Executive Ballroom

$210 \mathrm{H}$

\section{CLEO: QELS-Fundamental Science}

\section{FTu1E - Defects in Solids for Coherent Control and Single- Photon Generation-Continued}

\section{FTu1E.4 • 08:45}

Photonic Crystal Cavities in Bulk Diamond for Efficient Spin-Photon Interfaces, Sara L. Mouradian', Noel Wan', Michael Walsh', Eric Bersin', Tim Schröder ${ }^{1,2}$, Dirk Englund ${ }^{1}$; ${ }^{1}$ MIT, USA; ${ }^{2}$ Niels Bohr Inst., Denmark. We report a new fabrication process of planar photonic crystal nanocavities from bulk diamond. Experimental devices have quality factors $Q>1 \times 10^{\wedge} 4$ with resonances matched to the negatively charged nitrogen vacancy zero phonon line, allowing for an enhanced spin-photon interface.

\section{FTu1E.5 • 09:00}

Efficient Dielectric Reflectors for Solidstate Emitters in Bulk Diamond, Noel Wan', Sara L. Mouradian', Benjamin Lienhard', Donggyu Kim', Michael Walsh', Tim Schröder ${ }^{1}$, Brendan Shields ${ }^{2}$, Dirk Englund ${ }^{1}$; ${ }^{1}$ MIT, USA; ${ }^{2}$ Univ. of Basel, Switzerland. We report on a dielectric reflector fabricated directly on the surface of bulk diamond. We measure saturated count rates of up to 2.9 million cps from a single nitrogen-vacancy center in diamond.

\section{FTu1F • Quantum Optics and Quantum Information Processing-Continued}

FTu1G • Light Manipulation with Disordered MediaContinued
FTu1F.4 • 09:00

Physical meaning of the radial index of Laguerre-Gauss beams, William Plick², Mario Krenn'; ${ }^{1}$ QOOQI, Austria; ${ }^{2}$ Dept. of Physics, Univ. of Dayton, USA. Laguerre-Gauss modes are fundamental optical fields, characterized by two numbers: the azimuthal index indicating the orbital angular momentum, and the radial index. We give for the first time the meaning of the radial number.
FTu1E.6・09:15

Protecting The Spin Coherence of Silicon Vacancy Color Centers from Thermal Noise using Diamond MEMS, Young-lk Sohn'1, Srujan Meesala' ${ }^{1}$ Benjamin Pingault ${ }^{2}$, Haig Atikian', Jeffrey Holzgrafe ${ }^{1,2}$, Mustafa Gündogan², Camille Stavrakas², Alp Sipahigil', Michae Burek', Mian Zhang ${ }^{1}$, Jose Pacheco ${ }^{3}$, John Abraham $^{3}$, Edward Bielejec ${ }^{3}$, Mikhail Lukin ${ }^{1}$ Mete Atatüre ${ }^{2}$, Marko Lončar ${ }^{1} ;{ }^{1}$ Harvard Univ. USA: ${ }^{2}$ Cambridge Univ., UK; ${ }^{3}$ Sandia National Lab, USA. Spin coherence time, $T_{2}^{*}$, of silicon vacancy color centers in diamond is limited by thermal phonons. This process in engineered using a electro-micro-mechanical-system (MEMS) device. Suppressing the thermal process, we demonstrate the improvement of spin $T_{2}$

\section{FTu1E.7 • 09:30 Invited}

Single Photons from Single Molecules: Hong-Ou-Mandel Experiments and Beyond, Ilja Gerhardt1,2, Mohammad Rezai ${ }^{2}$ Jörg Wrachtrup ${ }^{1,2 ;}{ }^{1}$ Max Planck Inst for Solid State Research, Germany; ${ }^{2}$ Physics, Univ. of Stuttgart, Germany. Single molecules under cryogenic conditions are excellent single photon sources. They are bright $\left(10^{6}\right.$ clicks per second), narrow-band (tens of $\mathrm{MHz}$ ) and have an excellent Hong-Ou-Mandel indistighuishability (>90\%). We show experiments implementing an atom-compatible all-optical quantum gate.
FTu1F.5 • 09:15

Learning nitrogen-vacancy electron spin dynamics on a silicon quantum photonic simulator, Jianwei Wang ${ }^{1}$, Stefano Paesani ${ }^{1}$ Raffaele Santagati ${ }^{1}$, Sebastian Knauer ${ }^{1}$ Antonio A. Gentile', Nathan Wiebe'2, Maurangelo Petruzzella ${ }^{3}$, Anthony Laing ${ }^{1}$, John Rarity', Jeremy L. O'Brien ${ }^{1}$, Mark Thompson ${ }^{1}$. ${ }^{1}$ Quantum Engineering Technology Labs, H. H. Wills Physics Lab, Univ. of Bristol, UK ${ }^{2}$ Quantum Architectures and Computation Group, Microsoft Research, USA; ${ }^{3}$ Dept of Applied Physics, Eindhoven Univ. of Technology, Netherlands. We present the experimental demonstration of quantum Hamiltonian learning. Using an integrated silicon-photonics quantum simulator with the classical machine learning technique, we successfully learn the Hamiltonian dynamics of a diamond nitrogen-vacancy center's electron ground-state spin.

\section{FTu1F.6 • 09:30}

Experimental Tests of Indefinite Causa Orders, Lee Rozema', Giulia Rubino', Adrien Feix ${ }^{1}$, Mateus Araújo ${ }^{1}$, Časlav Brukner', Philip Walther'; ' 'Univ. of Vienna, Austria. It has been predicted that quantum processes without a defined causal order exist. Here we present an overview of our experimental research program creating and characterizing such processes, discussing also their potentia applications.

\section{FTu1G.4 • 09:00}

Inverse Design of Eigenchannels in Scattering Media, Milan Koirala' ${ }^{1}$ Raktim Sarma ${ }^{2}$ Hui $\mathrm{Cao}^{2}$, Alexey G. Yamilov'; 'Missouri Univ of Science \& Technology, USA; ${ }^{2}$ Applied Physics, Yale Univ., USA. We develop a comprehensive theoretical model for the maximum transmission eigenchannel and obtain a closed-form analytical expression relating its spatial profile to the shape of a disordered waveguide.
FTu1H • Fundamental Plasmonic \& Nanophotonic EffectsContinued

\section{FTu1H.3 • 08:45}

Brewster Plasmons - The Second Plasmonic Degree of Freedom, Gilad Rosenblatt ${ }^{1}$, Boris Simkhovich², Guy Bartal'1,2, Meir Orenstein ${ }^{1}$; 'Dept. of Electrical Engineering, Technion Israel Inst. of Technology, Israel: ${ }^{2}$ The Russell Berrie Nanotechnology Inst., Technion - Israel Inst. of Technology, Israel. We experimentally observe and theoretically prove the existence of a second class of plasmonic oscillations, distinct from standard surface plasmons, we call Brewster plasmons. We demonstrate far-field coupling to both Brewster plasmons and surface plasmons.

\section{FTu1H.4 09:00}

Beyond Toroidal Multipoles, Shiqiang $\mathrm{Li}^{1}$, Kenneth B. Crozier ${ }^{1,2}$; ${ }^{1}$ Electrical and Electronic Engineering, Univ. of Melbourne Australia; ${ }^{2}$ Physics, Univ. of Melbourne, Australia. We propose to separate out the toroidal multipoles in the polar coordinates that is simpler than previous methods done in the Cartesian coordinates. We uncover highe order current configurations other than the toroidal electric multipole.
FTu1G.5 • 09:15

Periodic Behavior in Aperiodic Multilayers Yonatan Sharabi' ', Hanan H. Herzig Sheinfux ${ }^{1}$ Mordechai Segev', Gadi Eisenstein'; 'physics, Technion, Israel. We present a family of one-dimensional quasiperiodic crystal which simultaneously display both the fractal bandstructure typical to quasiperiodic structures and properties normally exclusive to periodic structures, including Bloch-like modes.
FTu1H.5 09:15

Quantum Optics Picture of Surface Enhanced Raman Scattering in Lossy Plasmonic Systems, Mohsen Kamandar ${ }^{1}$, Stephen Hughes': ' ${ }^{1}$ Queen's Univ. at Kingston, Canada. Using an open-system quantum optics approach of a vibrating molecule coupled to a photonic reservoir, we present a new analytical solution to SERS in arbitrary environments. We exemplify the technique using several nanoplasmonic resonator systems.

\section{FTu1G.6・09:30}

Smith-Purcell radiation in the presence of short-range disorder, Ido Kaminer' ${ }^{1}$, Steven E. Kooi ${ }^{1}$, Roy Shiloh², Bo Zhen ${ }^{1}$, Yichen Shen ${ }^{1}$ Josue Lopez', Roei Remez², Scott Skirlo', Yi Yang', John Joannopoulos', Marin Soljacic ${ }^{1}$; ${ }^{1} \mathrm{MIT}$, USA; ${ }^{2}$ Tel Aviv Univ., Israel. The emission of light from electrons passing near a grating includes resonant plasmonic features and Smith-Purcell collective excitations. We observe both and distinguish between them, finding surprising robustness to disorder in the Smith-Purcell radiation.
FTu1H.6 • 09:30

Spin-Hall effect and circular birefringence of a uniaxial crystal plate, Graciana Puentes ${ }^{1}$, Konstantin Bliokh², C. T. Samlan ${ }^{3}$ Chandravati Prajapati ${ }^{3}$, Nirmal Viswanathan ${ }^{3}$, Franco Nori'; ; ${ }^{2}$ Univ. of Buenos Aires, Argentina; ${ }^{2}$ RIKEN, Japan; ${ }^{3}$ Univ. of Hyderabad, India. We demonstrate theoretically and experimentally the fine lateral circular birefringence of uniaxial crystal plates, an example of the spin-Hall effect of light. We report experimental observations of this effect using polarimetric and quantum-weakmeasurement techniques. 


\section{CLEO: Science \& Innovations}

\section{STu1I • Ultrafast Applications- Continued}

STu11.3 • 08:45

Development and Application of Ultrashort Mid-infrared Pulses for Pump-Probe Experiments at the LCLS, Scott Wandel', Grant Welch', Joseph Robinson', Alan Fry', Giacomo Coslovich': 'SLAC, USA. We report on recent developments using intense and narrowband Mid-infrared (MIR) ultrashort optical pulses at the LCLS. We describe the laser system and experimental methods that enable cutting-edge MIR/X-ray pump-probe experiments.

\section{STu1J • THz Materials Science- Continued}

STu1J.3 • 08:45

Terahertz Surface Plasmons in GratingCoupled Graphene, Khwanchai Tantiwanichapan', Xuanye Wang', Habibe Durmaz', Yuyu Li', Anna Swan', Roberto Paiella', 'Boston Univ., USA. Pronounced plasmonic absorption features at terahertz frequencies are measured in large-area graphene sheets coupled to periodic arrays of metallic nanoparticles, and limitations on their tunability caused by carrier density inhomogeneities are investigated.
STu11.4 • 09:00

Multiphoton Imaging with Blue-DiodePumped SESAM-Modelocked Ti:Sapphire Oscillator, Bojan Resan ${ }^{1,2}$, Andreas Rohrbach$\mathrm{er}^{1}$, Vesna Villamaina', Marina Cunquero ${ }^{3}$, Jacob Licea-Rodriguez ${ }^{3}$, Omar E. Olarte ${ }^{3}$, Pablo Loza-Alvarez ${ }^{3}$; ${ }^{1}$ Lumentum, Switzerland ${ }^{2} \mathrm{Sch}$ ool of Engineering, Univ. of Applied Sciences Northwestern Switzerland, Switzerland ${ }^{3}$ ICFO-Institut de Ciencies Fotoniques, The Barcelona Inst. of Science and Technology, Spain. Multicolor two-photon fluorescence imaging is performed using blue-diodepumped SESAM-modelocked Ti:Sapphire oscillator generating $5 \mathrm{~nJ}$ pulse energy, $82 \mathrm{fs}$ pulse duration, at $780 \mathrm{~nm}$ central wavelength, with $92 \mathrm{MHz}$ pulse repetition rate.

\section{STu1I.5 • 09:15}

Ultra-High Speed Microscopy of Complex (Amplitude and Phase) Samples Using Single Camera Snapshot, Pavel Sidorenko' Oren Cohen', Oren Lahav'; 'Technion Israe Inst. of Technology, Israel. We propose and demonstrate numerically a simple method for ultra-high speed microscopy of complex (amplitude and phase) samples. Our method exploits redundancy in (single-shot) ptychography for reconstruction of multiple frames from a single camera snapshot.
STu1 J.4 • 09:00

Broadband Single-Nanowire Photoconductive Terahertz Detectors, Kun Peng ${ }^{1}$, Patrick Parkinson'2, Qian Gao', Jessica Boland ${ }^{3}$ Ziyuan $\mathrm{Li}^{1}$, Fan Wang ${ }^{4}$, Yesaya Wenas ${ }^{1}$, Christopher Davies ${ }^{3}$, Lan Fu', Michael B. Johnston ${ }^{3}$, Hark Hoe Tan', Chennupati Jagadish'; 'Australian National Univ., Australia, ${ }^{2}$ Univ. of Manchester, UK; ${ }^{3}$ Univ. of Oxford, UK: ${ }^{4}$ Macquarie Univ., Australia. Broadband photoconductive terahertz detectors based on undoped InP single nanowires were demonstrated. By further design and growth of an axial $n^{+}-i-n^{+}$structure to reduce the contact resistance, highly-sensitive $n^{+}-i-n^{+}$InP singlenanowire terahertz detectors were achieved.

\section{STu1J.5 • 09:15 Invited}

Ultrafast Photophysics of Single Crystal Metal Halide Perovskites Measured by Transient Multi-THz Spectroscopy, David G. Cooke'; 'McGill Univ., Canada. Organometallic halide perovskites are a promising class of materials for optoelectronic devices, including photovoltaics. Recent multi-THz spectroscopy measurements of single crystal $\mathrm{CH}_{3} \mathrm{NH}_{3} \mathrm{Pbl}_{3}$ has revealed free charge generation dynamics, intrinsic mobilities and exciton binding energies.

\section{STu1K • Mid-IR Fiber Sensors- Continued}

STu1K.3 • 08:45

Mid-IR Spectrum Tailoring in ErbiumDoped Fluoride Fiber Amplifiers, Vincent Fortin ${ }^{1}$, Simon Duval', Jean-Christophe Gauthier $^{1}$, Louis-Rafaël Robichaud', Pascal Paradis $^{1}$, Michel Olivier ${ }^{1,2}$, Michel Piché ${ }^{1}$ Martin Bernier ${ }^{1}$, Réal Vallée'; 'Universite Laval, Canada; ${ }^{2}$ Cégep Garneau, Canada. We present an innovative laser system based on an erbium doped fluoride fiber amplifier for generating either high-power mid-IR supercontinuum or continuously tunable femtosecond pulses in the 3-4 $\mu \mathrm{m}$ spectral band.
STu1K.4 09:00 Invited

4.4-5.2 $\mu \mathrm{m}$ Wavelength Tunable, Coherent MIR Frequency Comb Generation Based on Yb-doped Fiber Laser, Lei Jin ${ }^{1}$ Masahito Yamanaka' Volker Sonnenschein ${ }^{1}$ Hideki Tomita', Tetsuo Iguchi', Atsushi Sato ${ }^{2}$ Akira Ideno2, Toshinari Oh-hara², Norihiko Nishizawa'; ' ${ }^{1}$ agoya Univ., Japan; ${ }^{2}$ Sekisui Medical Co. Ltd. Japan. Offset free, 4.4-5.2 $\mathrm{mm}$ wavelength tunable, mid-infrared optical frequency comb was generated through DFG pumped by $\mathrm{Yb}$-doped fiber laser system. Coherence verification was performed by beat-note measurement using quantum cascade laser.
STu1I.6 • 09:30

$1 \mathrm{GS} / \mathrm{s}$ time-stretch imaging at $532 \mathrm{~nm}$ through fiber optics, Cihang Kong ${ }^{1}$, Xiaoming Wei ${ }^{1}$, Kevin Tsia' ${ }^{1}$ Kenneth Kin-Yip Wong'; 'eee, The Univ. of Hong Kong, Hong Kong. We demonstrate a 6- $\mathrm{MHz}$ green-light timestretch imaging at a low data-stream of 1 $\mathrm{GS} / \mathrm{s}$. The highly-chirped pulse at $532 \mathrm{~nm}$ is generated through double-pass high-power frequency doubling, which provides a dispersion of $\sim 7 \mathrm{~ns} / \mathrm{nm}$.
STu1K.5 • 09:30

Short wavelength mode-locked thuliumdoped fiber laser based on nonlinear polarization rotation, Can $\mathrm{Li}^{1}$, Xiaoming $\mathrm{Wei}^{1}$, Sisi Tan' ${ }^{1}$, Nan Chen', Jiqiang Kang ${ }^{1}$, Kenneth Kin-Yip Wong'; 'The Univ. of Hong Kong, China. We demonstrate a short wavelength harmonically mode-locked thulium-doped fiber laser based on nonlinear polarization rotation. Stable soliton pulsing with record short wavelength of $1787 \mathrm{~nm}$ and FWHM of $6.5 \mathrm{~nm}$ is achieved. 


$\begin{array}{lcc}\text { Marriott } & \text { Marriott } & \text { Marriott } \\ \text { Salon III } & \text { Salon IV } & \text { Salon V \& VI }\end{array}$

CLEO: Science \& Innovations

\section{STu1M • Optical Interconnect Systems-Continued}

Phase Drift in Depletion-Mode Silicon Photonics Modulators, Jiachuan Lin", Hassan Sepehrian', Wei Shi', Leslie Rusch'; 'Université Laval, Canada. We experimentally examine a modulation-induced phase drift in depletion-mode silicon phase modulator and Mach-Zehnder modulator. The impact on BPSK signal is studied under varying modulator operating conditions.
STu1M.3 • 08:45

\section{STu1N • Photodetectors- \\ Continued}

STu1M.4 • 09:00

Directly-modulated IM/DD OFDM Transmission over $100-\mathrm{km}$ SSMF using SSB Filtering with Two Silicon Micro-ring Resonators, Mohamed Essghair Chaibi', Karim Hassan², Laurent Bramerie', Christophe Peucheret'; 'FOTON Lab, Univ. of Rennes 1, ENSSAT, France; 'Univ. Grenoble-Alpes, CEA, LETI, France. Optical single sideband signals generation using two silicon microring resonators is demonstrated for $5.3-\mathrm{GHz}$ wideband OFDM modulation. Transmission at $14.69 \mathrm{~Gb} / \mathrm{s}$ over $100-\mathrm{km}$ SSMF is made possible thanks to the SSB filtering provided by the MRRs.

\section{STu1M.5 • 09:15}

4-PAM Dispersion-Uncompensated Transmission with Micro-Ring Resonator Enhanced 1.55- $\mathrm{mm} \mathrm{DML,} \mathrm{Francesco} \mathrm{Da}$ Ros', Valentina Cristofori', Oskars Ozolins², Mohamed Chaibi ${ }^{3}$, Xiaodan Pang ${ }^{2}$, Gunnar Jacobsen², Sergei Popovi, Michael Galili', Leif K. Oxenløwe ${ }^{1}$, Christophe Peucheret ${ }^{3}$; ${ }^{1}$ Technical Univ. of Denmark, Denmark; ${ }^{2}$ NETLAB, Acreo Swedish ICT, Sweden; ${ }^{3}$ FOTON Lab, Univ. of Rennes, France; ${ }^{4}$ School of ICT, Royal Inst. of Technology, Sweden. Realtime transmission of 14-GBd 4-PAM signal is demonstrated by combining a commercial 1.55- $\mu \mathrm{m}$ DML with a silicon MRR. BER below the HD-FEC threshold is measured after 26$\mathrm{km}$ SSMF transmission without offline digital signal processing

STu1M.6 09:30

High-Speed IQ Modulator Based on Injection-Locked VCSEL Array, Xian Xiao ${ }^{2,1}$, Nicolas K. Fontaine ${ }^{2}$, Haoshuo Chen², Bin Huang $^{2}$, David T. Neilson², Kwangwoong $\mathrm{Kim}^{2}$, Jeffrey H. Sinsky², Roland R. Ryf², Gregory Raybon², Peter Winzer ${ }^{2}$, Aidan Daly ${ }^{3}$, Christian Neumeyr ${ }^{3}$, Markus Ortsiefer ${ }^{3}$, S. J. Ben Yoo'; ' 'Univ. of California, Davis, USA; ${ }^{2}$ Bell Labs/Nokia, USA; ${ }^{3}$ VERTILAS GmbH, Germany. We demonstrate an $1 \mathrm{Q}$ modulator by employing two monolithic injectionlocked VCSELs which are driven to produce pure amplitude modulation. QPSK signals are produced at $10 \mathrm{GBaud}$ with a peak-to-peak modulation voltage of $600 \mathrm{mV}$.
STu1N.4 09:00

CMOS-compatible Mid-Infrared Silicon Detector, Romy Fain ${ }^{1,2}$, Steven Miller ${ }^{1,2}$, Mengjie $\mathrm{Yu}^{5}$, Austin G. Griffith ${ }^{3}$, Jaime Cardenas ${ }^{4}$, Michal Lipson'1,2; 'Electrical and Computer Engineering, Cornell Univ., USA; ${ }^{2}$ Electrical Engineering, Columbia Univ., USA; ${ }^{3}$ Applied Physics, Cornell Univ., USA; ${ }^{4}$ Inst. of Optics, Univ. of Rochester, USA; ${ }^{5}$ Applied Physics and Mathematics, Columbia Univ., USA. We demonstrate a CMOS-compatible mid-infrared detector at wavelengths ranging from 3.36 $\mu \mathrm{m}$ to $3.74 \mu \mathrm{m}$ by exciting mid bandgap states in a sulfur-doped silicon waveguide with responsivities up to $2.2 \mathrm{~mA} / \mathrm{W}$.

STu1N.5 • 09:15

Self-Aligned Local Electrolyte Gating of 2D Materials for Mid-Infrared Photodetection, Cheng Peng', Dmitri K. Efetov', Sebastien Nanot ${ }^{2}$, Ren-Jye Shiue', Gabriele Grosso', Yafang Yang ${ }^{1}$, Marek Hempel', Pablo JarilloHerrero' ${ }^{1}$ Jing Kong ${ }^{1}$, Frank Koppens ${ }^{2,3}$, Dirk Englund'; ${ }^{1} \mathrm{MIT}$, USA; ${ }^{2}$ ICFO - Institut de Ciencies Fotoniques, Spain; ${ }^{3}$ ICREA-Institucio Catalana de Recerca i Estudis Avancats, Spain. We present a new gating concept based on a self-aligned electrolyte technique that can enable spatially modulating charges with nanometer resolution. We use this technique to demonstrate a graphene mid-infrared thermopile photodetector with novel geometry.

STu1N.6 0 09:30

Investigation of Si-based $\mathrm{Ge}_{0.89} \mathrm{Sn}_{0.11}$ Photoconductors with $3.0 \mu \mathrm{m}$ photoresponse, Thach Pham ${ }^{1,2}$, Huong Tran', Wei $\mathrm{Du}^{3}$, Joe Margetis ${ }^{4}$, Yiyin Zhou', Perry Grant ${ }^{1,2}$, Gregory Sun $^{5}$, Richard Soref ${ }^{5}$, John Tolle ${ }^{4}$, Baohua Li ${ }^{2}$, Mansour Mortazavi ${ }^{3}$, Shui-Qing Yu'; ' ${ }^{1}$ Univ. of Arkansas, USA; ${ }^{2}$ Arktonics LLC, USA; ${ }^{3}$ Chemistry and Physics, Univ. of Arkansas at Pine Bluff, USA; ${ }^{4}$ ASM, USA; ${ }^{5}$ Engineering, Univ. of Massachusetts Boston, USA. The $\mathrm{Ge}_{0.89} \mathrm{Sn}_{011}$ photoconductors with interdigitated electrodes have been investigated. High responsivity of $28.5 \mathrm{~A} / \mathrm{W}$ and spectral response cutoff at $3.0 \mu \mathrm{m}$ were achieved. The $D^{*}$ of $4.5 \times 10^{10} \mathrm{cmHz}^{1 / 2} \mathrm{~W}^{-1}$ is close to that of extended-InGaAs detector.
STu10 • Petawatt Laser Technology-Continued

STu10.3 • 08:45

Spectral shaping of an OPCPA preamplifier for a multi-PW laser at $20 \mathrm{fs}, \mathrm{H}$. W. Lee', Yeong Gyu Kim², Je Yoon Yoo', Jin Woo Yoon ${ }^{3,1}$, Jae Hee Sung ${ }^{3,1}$, Seong Ku Lee $^{3,1}$, Chang Won Lee ${ }^{3,1}$, Jung Moon Yang ${ }^{1}$ Chang Hee Nam ${ }^{1,2} ;{ }^{1}$ Center for Relativistic Laser Science, Inst. for Basic Science, Korea (the Republic of); ${ }^{2}$ Dept. of Physics and Photon Science, Gwangju Inst. Science and Technology, Korea (the Republic of); ${ }^{3} \mathrm{Ad}$ vanced Photonics Research Inst., Gwangju Inst. Science and Technology, Korea (the Republic off. We developed an OPCPA preamplifier for the 4-PW laser at CoReLS. The output spectrum was shaped by controlling the pump laser temporal profile. Spectrally shaped laser pulse was generated with an energy of $240 \mathrm{~mJ}$.

STu10.4 09:00

Experimental Demonstration for $808 \mathrm{~nm}$ centered OPCPA Based on High Deuteration DKDP Crystals and the Potential Utilization in SGII-5PW System, Xiao Liang', Meizhi Sun ${ }^{1}$, Jun Kang ${ }^{1}$, Jian Zhou' ', Xinglong $X_{i e}{ }^{1}$, Jianqiang Zhu', Zunqi Lin'; 'Shanghai Inst. of Optics and Fine Mechanics, China. Broadband exceeding 30nm amplification has been realized experimentally based on 95\% deuteration DKDP crystal with 532nm pump pulses, which meets numerical analysis well. The results indicate potential utilization for OPCPA systems of compressed 30fs pulses.

STu10.5 0 09:15

High Repetition Rate Thin Disk Ti:Sa Amplifiers for Sub-PW class Laser Systems, Vladimir V. Chvykov', Roland Nagymihaly', Huabao Cao', Mikhail Kalashnikov', Karoly Osvay'; ' ${ }^{1}$ ELI-HU Non-Profit Ltd., Hungary. Resuts of the proof-of-principal experiments with two types thin disc water cooled Ti:Sa amplifiers will be presented. Scaling simulations based on experimental results demonstrate feasibility of hundreds $\mathrm{Hz}$ sub-PW Ti:Sa laser systems.

STu10.6 09:30

Picosecond Contrast of Recompressed Ti:Sapphire Laser Pulses, Mikhail P. Kalashnikov', Nikita Khodakovskiy'; ' ${ }^{1}$ Max Born Inst., Germany. The degradation of picosecond contrast in Ti:Sapphire lasers was investigated for different stretcher-compressor combinations. During amplification the coherent ragged post-pedestal, the feature of Ti:Sapphire medium, generates a coherent pre-pedestal limiting the temporal contrast. 
Executive Ballroom

$210 \mathrm{~A}$

Technologies-Continued

ATu1A.8 • 09:45

Robust stiffness quantification using quantitative optical coherence elastography, Xuan Liu', Farzana Zaki', Yahui Wang ${ }^{1}$; ${ }^{1}$ New Jersey Inst. of Technology, USA. We demonstrated the capability of quantitative optical coherence elastography (qOCE) for robust measurement of material stiffness under different boundary conditions using the reaction force and displacement field established in the sample.
Executive Ballroom 210B

Executive Ballroom

$210 \mathrm{C}$

\section{CLEO: Applications \\ \& Technology}

ATu1B • A\&T Topical Review on Neurophotonics I-Continued
ATu1C $\bullet$ Lasers for Additive Manufacturing and Surface Structuring-Continued

\section{ATu1C.6 • 09:45}

Tuning the Wettability of Steel by Femtosecond Laser Structuring, Daniel Puerto Camilo Florian Baron', Evangelos Skoulas ${ }^{2}$ Emmanuel Stratakis², Javier Solis', Jan Siegel': 'Instituto de Optica, Spanish National Research Council, Spain; 'Inst. of Electronic Structure and Laser, Foundation for Research and Technology, Greece. We present a strategy to control the wetting properties of stee using high-repetition rate femtosecond laserwritten parallel lines and grids with variable spacing. This approach also allows generating lateral anisotropy of the wetting angle.
Executive Ballroom 210D

CLEO: QELS Fundamental Science

FTu1D • On-chip Comb Generation I-Continued

10:00-10:30 Coffee Break, Concourse Level

10:30-11:30 JTu2A • Plenary Session I, Grand Ballroom

11:30-19:30 Exhibition Open, Exhibit Hall 1, 2 \& 3

11:30-13:30 Unopposed Exhibit Only Time, Exhibit Hall 1, 2 \& 3

12:00-13:30 OIDA VIP Industry Leaders Speed Meetings Lunch, Exhibit Hall (Advanced Registration Required)

12:00-15:00 SC352: Introduction to ultrafast pulse shaping--principles and applications

SC376: Plasmonics

SC410: Finite Element Modelling Methods for Photonics and Optics

12:00-16:00 SC270: High Power Fiber Lasers and Amplifiers

SC438: Photonic Metamaterials

12:30-13:30 Lunch Break (on your own)

12:00-13:30 Market Focus Session I: Precision Applications using Ultrafast Lasers, Exhibit Hall Theater

13:00-17:30 Alternative Careers Paths in Optics and Photonics, Willow Glenn/Marriott 
Executive Ballroom
$210 \mathrm{E}$

Executive Ballroom

210F
Executive Ballroom

210G
Executive Ballroom

$210 \mathrm{H}$

\section{CLEO: QELS-Fundamental Science}

FTu1E - Defects in Solids for Coherent Control and SinglePhoton Generation-Continued
FTu1F • Quantum Optics and Quantum Information Processing-Continued

FTu1F.7 • 09:45

Experimental Implementation of QuantumCoherent Mixtures of Causal Relations, Jean-Philippe MacLeann, ${ }^{3,1}$ Katja Ried ${ }^{1,2}$, Robert W. Spekkens' ${ }^{2}$ Kevin Resch ${ }^{3,1}$; ${ }^{1}$ Dept. of Physics \& Astronomy, Univ. of Waterloo, Canada; ${ }^{2}$ Perimeter Inst. for Theoretical Physics, Canada; ${ }^{3}$ nst. for Quantum Computing, Univ. of Waterloo, Canada. We realize a nonclassical mixture of causal relations in a quantum optics experiment using a partial swap and derive a set of criteria for witnessing the coherence based on a quantum version of Berkson's paradox.
FTu1G • Light Manipulation with Disordered MediaContinued

\section{FTu1G.7 • 09:45}

Metasurfaces With Random Nanoantennas for Ultra-broadband Surface Enhanced Nonlinear Optics, Nan Zhang ${ }^{1}$, Ziheng $\mathrm{Ji}^{2}$, Alec R. Cheney ${ }^{1}$, Haomin Song ${ }^{1}$, Dengxin $\mathrm{Ji}^{1}$, Xie Zeng ${ }^{1}$, Borui Chen ${ }^{1}$, Alexander N. Cartwright ${ }^{1}$, Kebin Shi², Qiaogiang Gan ${ }^{1}$; ${ }^{1}$ Dept. of Electrical Engineering, The State Univ. of New York at Buffalo, USA; ${ }^{2}$ Physics Dept., Peking Univ., China. We demonstrate a strong enhancement of second harmonic generation based on a three-layered super absorbing metasurface consisting of a dielectric spacer layer sandwiched by an array of random metallic nanoparticles and a metal ground plate.
FTu1H • Fundamental Plasmonic \& Nanophotonic EffectsContinued

FTu1H.7 • 09:45

Visible Frequency Plasmon Resonator exhibiting Quality Factors exceeding 750, Shawn Divitt ${ }^{2,1}$, Wenqi Zhu'2,1, Jared Strait ${ }^{2}$, Henri J. Lezec', Amit K. Agrawal2,1 ${ }^{1}$ Maryland Nanocenter, Univ. of Maryland, USA; ${ }^{2}$ Center for Nanoscale Science and Technology, National Inst. of Standards and Technology, USA. We propose a plasmon resonator consisting of a cylindrical hole in a metal film that supports whispering gallery type surface plasmon polariton modes exhibiting record-high quality factors $(>750)$ in the visible frequency range.

10:00-10:30 Coffee Break, Concourse Level

10:30-11:30 JTu2A • Plenary Session I, Grand Ballroom

11:30-19:30 Exhibition Open, Exhibit Hall 1, 2 \& 3

11:30-13:30 Unopposed Exhibit Only Time, Exhibit Hall 1, 2 \& 3

12:00-13:30 OIDA VIP Industry Leaders Speed Meetings Lunch, Exhibit Hall (Advanced Registration Required)

12:00-15:00 SC352: Introduction to ultrafast pulse shaping--principles and applications

SC376: Plasmonics

SC410: Finite Element Modelling Methods for Photonics and Optics

12:00-16:00 SC270: High Power Fiber Lasers and Amplifiers

SC438: Photonic Metamaterials

12:30-13:30 Lunch Break (on your own)

12:00-13:30 Market Focus Session I: Precision Applications using Ultrafast Lasers, Exhibit Hall Theater

13:00-17:30 Alternative Careers Paths in Optics and Photonics, Willow Glenn/Marriott 


\section{Meeting Room \\ $211 \mathrm{~B} / \mathrm{D}$}

\section{Meeting Room \\ $212 \mathrm{~A} / \mathrm{C}$}

Meeting Room

$212 \mathrm{~B} / \mathrm{D}$
Marriott

Salon I \& II

\section{CLEO: Science \& Innovations}

STu1I • Ultrafast ApplicationsContinued

STu11.7 • 09:45

Multiplexed detection for higher performance quantitative phase time-stretch microscopy, Bryan T. Bosworth', Mark A. Foster'; 'Johns Hopkins Univ., USA. We present a simple fiber-based technique for multiplexing interferometric signals as a means to improve the SNR and electronic bandwidth of quantitative phase time-stretch microscopy, enabling better sampling rates and signal quality.
STu1J • THz Materials ScienceContinued

STu1J.6 • 09:45

Selective Modulation of Terahertz Radiation using Photo-excited 2D Hybrid Lead Halide Perovskite, Ashish Chanana', Ajay Nahata'; 'Univ. of Utah, USA. We demonstrate $100 \%$ modulation of selective Terahertz resonances using series of two-dimensional hybrid lead halide perovskites. The device operation in perovskite/silicon devices was achieved using a simple halogen lamp and a set of color filters.

\section{STu1K • Mid-IR Fiber Sensors-} Continued

\section{STu1K.6・09:45}

Mode-locking Regime Switching by Wavelength Tuning in a Tm-fiber Laser, Ruoyu Liao $^{1}$, Youjian Song ${ }^{1}$, Lu Chai ${ }^{1}$, Ming-lie Hu'; ${ }^{1}$ Tianjin Univ., China. We demonstrate a passively mode-locked thulium-doped fiber laser which can switch among soliton, stretched pulse and dissipative soliton regimes by simply tuning central wavelength.

10:00-10:30 Coffee Break, Concourse Level

10:30-11:30 JTu2A • Plenary Session I, Grand Ballroom

11:30-19:30 Exhibition Open, Exhibit Hall 1, 2 \& 3

11:30-13:30 Unopposed Exhibit Only Time, Exhibit Hall 1, 2 \& 3

12:00-13:30 OIDA VIP Industry Leaders Speed Meetings Lunch, Exhibit Hall (Advanced Registration Required)

12:00-15:00 SC352: Introduction to ultrafast pulse shaping--principles and applications

SC376: Plasmonics

SC410: Finite Element Modelling Methods for Photonics and Optics

12:00-16:00 SC270: High Power Fiber Lasers and Amplifiers

SC438: Photonic Metamaterials

12:30-13:30 Lunch Break (on your own)

12:00-13:30 Market Focus Session I: Precision Applications using Ultrafast Lasers, Exhibit Hall Theater

13:00-17:30 Alternative Careers Paths in Optics and Photonics, Willow Glenn/Marriott 


$\begin{array}{llc}\text { Marriott } & \text { Marriott } & \text { Marriott } \\ \text { Salon III } & \text { Salon IV } & \text { Salon V \& VI }\end{array}$

\section{CLEO: Science \& Innovations}

STu1M • Optical Interconnect Systems-Continued
STu1N $\bullet$ PhotodetectorsContinued

STu1N.7 • 09:45

Surface Micromachined MEMS-Tunable PIN-Photodiodes around 1550-nm, Julijan Cesar', Sujoy Paul', Mohammad T. Haidar', Brian Corbett ${ }^{2}$, Arkadi Chipouline ${ }^{1}$, Franko Küppers'; 'Technische Universität Darmstadt, Germany; ${ }^{2}$ Tyndall National Inst., Ireland. We report continuously widely tunable InP-based pin-photodiodes by integrating a surface micromachined MEMS-based Fabry-Pérot filter with FWHM/FSR of $0.3 \mathrm{~nm} / 189 \mathrm{~nm}$. Detection of $36 \mathrm{~nm}$ is obtained for WDM- or sensing-applications around $1550 \mathrm{~nm}$.
STu10 • Petawatt Laser Technology-Continued

STu10.7 • 09:45

Dependence of Compressed Pulse Contrast on Grating Surface Roughness, Yunxin Tang1', David Egan², Chris Hooker ${ }^{1}$, Chris Gregory', Oleg Chekhlov', Cristina Hernandez-Gomez', John Collier', P P. Rajeev'; ${ }^{1}$ Central Laser Facility, STFC Rutherford Appleton Lab, UK; ${ }^{2}$ The Orion Laser Facility, AWE, UK. We report a novel method to evaluate the impact of stretcher gratings on the compressed pulse contrast by quantitative characterization of the grating surface, demonstrating a correlation between the contrast pedestal and grating natural property.

10:00-10:30 Coffee Break, Concourse Level

10:30-11:30 JTu2A • Plenary Session I, Grand Ballroom

11:30-19:30 Exhibition Open, Exhibit Hall 1, 2 \& 3

11:30-13:30 Unopposed Exhibit Only Time, Exhibit Hall 1, 2 \& 3

12:00-13:30 OIDA VIP Industry Leaders Speed Meetings Lunch, Exhibit Hall (Advanced Registration Required)

\begin{tabular}{c}
\hline $\begin{array}{c}\text { 12:00-15:00 SC352: Introduction to ultrafast pulse shaping--principles and applications } \\
\text { SC376: Plasmonics }\end{array}$ \\
SC410: Finite Element Modelling Methods for Photonics and Optics \\
\hline 12:00-16:00 $\begin{array}{r}\text { SC270: High Power Fiber Lasers and Amplifiers } \\
\text { SC438: Photonic Metamaterials }\end{array}$ \\
\hline 12:30-13:30 Lunch Break (on your own) \\
\hline 12:00-13:30 Market Focus Session I: Precision Applications using Ultrafast Lasers, Exhibit Hall Theater \\
\hline Alternative Careers Paths in Optics and Photonics, Willow Glenn/Marriott \\
\hline
\end{tabular}


Executive Ballroom $210 \mathrm{~A}$
Executive Ballroom 210B
Executive Ballroom $210 \mathrm{C}$

Executive Ballroom 210D

\section{CLEO: Applications \& Technology}

13:30-15:30

ATu3A - Photobiomodulation Therapeutics

Presider: Ilko llev; U.S. Food and Drug Admin., USA

\section{ATu3A.1 • 13:30 Invited}

Emerging Photobiomodulation Therapeutics, Juanita Anders': ' 'USUHS, USA. The current status of photobiomodulation therapy will be presented. The importance of device parameters, wavelength selection related to the target tissue, photon dose related to cellular mechanisms, and opportunities for

\section{3:30-14:30 \\ ATu3B - A\&T Topical Review on Neurophotonics II}

Presider: Chris Xu; Cornell Univ., USA

\section{ATu3B.1 • 13:30}

Predicting Behavior from Cortical Activity Recorded through Widefield Transcranial Imaging, Li Zhu', Christian R. Lee', David J. Margolis', Laleh Najafizadeh'; 'Rutgers Univ., USA. We present a method based on visibility graph capable of predicting whisking activities with an accuracy of $93.57 \%$ from calcium signals of excitatory neurons that are recorded through widefield imaging in GCaMP6f reporter mice.

\section{3:30-15:30 \\ ATu3C $\bullet$ Industrial Optical \\ Design \& Sensing}

Presider: Jan Kleinert; ESI, USA

\section{ATu3C.1 1 13:30 Invited}

Polygon Scanners Deliver Speed and Accuracy, Ronny De Loor ${ }^{1}$; ${ }^{1}$ Next Scan Technology, Belgium. Polygon scanner systems were introduced to the laser material processing market in 2013. Process developers worked out new strategies to exploit the high scan speed offered by the polygon scanner system technology. An updated product status and overview of emerging applications will be presented.
ATu3B.2 • 13:45

Assessment of Lexiscan for Blood Brain Barrier disruption to facilitate Fluorescence brain imaging, Rebecca W. Pak', Hanh Le ${ }^{2}$ Heather Valentine ${ }^{3}$, Daniel Thorek ${ }^{3}$, Arman Rahmim ${ }^{3}$, Dean Wong ${ }^{3}$, Jin U. Kang'; ${ }^{1}$ Biomedical Engineering, Johns Hopkins Univ. USA; ${ }^{2}$ Electrical and Computer Engineering, Johns Hopkins Univ., USA; ${ }^{3}$ Radiology and Radiological Sciences, Johns Hopkins Univ. USA. Mouse brain fluorescence was imaged after the tail vein injections of indocyanine green (ICG) dye and Lexiscan. The throughskull images showed dye in the vasculatures and permeating through the blood brain barrier into the tissue.

\section{ATu3B.3 • 14:00 Invited}

ATu3A.2 $14: 00$ Invited Biology: Can Engineering Lead to a Cancer Cure?, Vadim Backman',2. ${ }^{1}$ Northwestern Univ., USA; ${ }^{2}$ Cancer and Physical Sciences, Robert H. Lurie Comprehensive Cancer Center, USA. The development of new optical nanoimaging technologies and the physics-based modeling of gene expression can help decipher the gene expression code, elucidate early events in carcinogenesis, and lead to new physico-chemical approaches to anti-cancer therapy. length-tunable, femtosecond pulse at $1600-2520 \mathrm{~nm}$ and its second-harmonic for multiphoton imaging, Bo Li ${ }^{1}$, Mengran Wang', Chris Xu'; ${ }^{1}$ Cornell Univ., China. We demonstrate 1600-2520 nm wavelengthtunable, high energy soliton generation using a large-mode-area fiber pumped by a compact fiber source. Using second harmonic generation, we show their applications for in vivo multi-photon mouse brain imaging.
Generation of high-pulse energy, wave-

CLEO: OELSFundamental Science

\section{3:30-15:30 \\ FTu3D • On-chip Comb}

Generation II

Presider: Roberto Morandotti; INRS-Energie Mat \& Tele Site

Varennes, Canada

\section{FTu3D.1 • 13:30}

Broadband visible comb generation in AINon-sapphire microresonators, Xianwen Liu' Changzheng Sun ${ }^{1}$, Bing Xiong', Lai Wang ${ }^{1}$ Jian Wang ${ }^{1}$, Yanjun $\mathrm{Han}^{1}$, Zhibiao $\mathrm{HaO}^{1}$ Hongtao Li' ${ }^{1}$, Yi Luo', Jianchang Yan², Tongbo Wei ${ }^{2}$, Yun Zhang ${ }^{2}$, Junxi Wang ${ }^{2}{ }^{1}{ }^{T}$ singhua Univ., China; ${ }^{2}$ Inst. of Semiconductors, Chinese Academy of Sciences, China. On-chip visible comb generation covering from 720 to $840 \mathrm{~nm}$ is demonstrated in AlN-on-sapphire microring resonators. Broadband frequency conversion of near-infrared Kerr comb into visible region is achieved in slightly phasemismatched condition.

ATu3C.2 $14: 00$

Laser Active Optical Systems (LAOS) for industrial applications, Vladimir V. Chvykov ${ }^{1,2}$ ${ }^{1}$ ELI-HU Non-Profit Ltd., Hungary; ${ }^{2} \mathrm{CUOS}$ Univ. of Michigan, USA. LAOS are discussed as a new generation of laser systems for industrial processing. The proof of principa experiment results with LAOS Ti:Sapphire (TI:Sa) amplifiers have demonstrated the realistic abilities to achieve required parameters.
FTu3D.2 • 13:45

Raman-assisted broadband Kerr frequency comb generation in AIN-on-sapphire microresonators, Xianwen Liu' ' Changzheng Sun Bing Xiong ${ }^{1}$, Lai Wang ${ }^{1}$, Jian Wang ${ }^{1}$, Yanjun Han $^{1}$, Zhibiao Hao', Hongtao Li', Yi Luo' Jianchang YAN ${ }^{2}$, Tongbo Wei ${ }^{2}$, Yun Zhang ${ }^{2}$ Junxi Wang2: 'Tsinghua Univ., China; ${ }^{2}$ Inst. of Semiconductors, Chinese Academy of Sciences, China. Coexistence of stimulated Raman scattering and four-wave mixing is observed in high quality factor AlN-on-sapphire microring resonators, which facilitates broadband Kerr frequency comb generation covering from 1.2 to $2.2 \mu \mathrm{m}$ along with strong Raman lines.

\section{FTu3D.3 • 14:00 Invited}

Bright square pulse generation by pump modulation in a normal GVD microresonator, Hao Liu' ', Shu-Wei Huang ${ }^{1}$, Jinghui Yang ${ }^{1}$ Mingbin Yu², Dim-Lee Kwong ${ }^{2}$, Cheewe Wong ${ }^{1} ;{ }^{1}$ UCLA, USA; ${ }^{2}$ The Inst. of Microelectronics, Singapore. We report a novel way to generate frequency comb in normal GVD microresonator. By using single FSR intensitymodulated pump, we successfully observe a mode locked frequency comb and bright square pulse for the first time. 


\section{CLEO: QELS-Fundamental Science}

\section{3:30-15:30 \\ FTu3E - Quantum Optics of Single Emitters}

Presider: Virginia Lorenz; Univ. of Illinois at Chicago, USA

\section{FTu3E.1 • 13:30}

On-demand source of entangled photonpairs using the biexciton-exciton radiative cascade, Roni Winik ${ }^{1,2}$, Dan cogan ${ }^{1}$, Yaroslav Don 1', Ido schwartz', Liron Gantz', Emma Schmidgall ${ }^{1}$, Nitzan Livneh ${ }^{3}$, Ronen Rapaport $^{3}$, Eyal Buks ${ }^{2}$, David Gershoni'; 'The Physics Dept. and the Solid State Inst. Technion - Israel Inst. of Technology, Israel; ${ }^{2}$ Andrew and Erna Viterbi Dept. of Electrical Engineering, Technion - Israel Inst. of Technology, Israel; ${ }^{3}$ Applied Physics Dept., engineering, The Hebrew Univ., Israel. We show that pairs of photons resulting from the radiative cascade of the confined biexciton are maximally entangled. The measured entanglement depends on the resolution by which the time between the pair emissions is determined.

\section{FTu3E.2 • 13:45}

Engineering Spins in Quantum Dot Molecules for Scalable Quantum Photonics, Xiangyu $\mathrm{Ma}^{1}$, Garnett Bryant ${ }^{2}$, Matthew Doty'; ${ }^{1}$ Univ. of Delaware, USA; ${ }^{2}$ National Inst. of Science and Technolgy, USA. We analyze the physics underlying emergent spin properties in self-assembled InAs Quantum Dot Molecules. We describe opportunities to engineer these structures for scalable and in situ tunable quantum photonic devices. The Benin School of computer sciences and
13:30-15:30

FTu3F • Quantum-Enhanced

\section{Measurements}

Presider: James Franson; Univ. of Maryland Baltimore County, USA

\section{FTu3F.1 • 13:30 Invited}

Quantum Illumination: From Enhanced Target Detection to Gbps Quantum Key Distribution, Jeffrey H. Shapiro'; ${ }^{1}$ MIT, USA. We review theory and experiments for quantum illumination-entanglement- based protocols for enhanced target detection and secure classical communication-and show that its unentangled descendant, floodlight quantum key distribution, affords Gbps secret-key rates over metropolitan-area distances.
13:30-15:30

FTu3F.2 • 14:00

Phonon limit to simultaneous near-unity efficiency and indistinguishability in semiconductor single photon sources, Jake lles-Smith ${ }^{1}$, Ahsan Nazir ${ }^{2}$, Dara McCutcheon ${ }^{3}$, Jesper Mørk'; 'DTU, Denmark; ${ }^{2}$ The Univ. of Manchester, UK; ${ }^{3}$ Univ. of Bristol, UK. We investigate the role of phonons on the emission properties of solid-state single photon sources. We demonstrate a fundamental trade-off between indistinguishability and efficiency of sources based on both cavity and waveguide architectures.
Transverse localization of light for singlemode and secure information transport, Marco Leonetti ${ }^{1,2}$, Salman Karbasi ${ }^{3}$, Arash $\mathrm{Mafi}^{4}$, Behnam Abaie ${ }^{4}$, Eugenio DelRe ${ }^{5}$, Giancarlo Ruocco ${ }^{5} ;{ }^{1}$ Italian Inst. of technology, Italy; ${ }^{2}$ Nanotec, CNR, Italy; ${ }^{3}$ Dept. of Electrical and Computer Engineering "Univ. of California, USA; ${ }^{4}$ Dept. of Physics and Astronomy and Center for High Technology Materials, Univ. of New Mexico, , USA 5Physics, Univ. or Rome "Sapienza", Italy. We demonstrate how reconfigurable localized optical patterns allow to encode up to 6 bits of secure information in disorder-induced high transmission channels loaded with single photons.
FTu3G - Toward Applications of Metasurfaces I

Presider: Xiaobo Yin; Univ. of

Colorado at Boulder, USA

\section{FTu3G.1 • 13:30}

On-Chip Demonstration of a Transparent Perfect Mirror, Ali Kazemi Jahromi ${ }^{1}$ Soroush Shabahang ${ }^{1}$, Hasan E. Kondakci ${ }^{1}$ Petri Melanen², Seppo Orsilla², Ayman F. Abouraddy'; ' Univ. of Central Florida, CREOL, USA; ${ }^{2}$ Modulight, Inc., Finland. We experimentally demonstrate an active cavity exhibiting $100 \%$ spectrally flat reflection with no vestiges of structural resonances while still transmitting light across the gain bandwidth. This non-Hermitian structure is realized in an indium-phosphide platform.

\section{FTu3G.2 • 13:45}

Metasurface-Enabled On-Chip Quantum Entanglement, Nir Shitrit ${ }^{1}$, Pankaj K. Jha ${ }^{1}$, Jeongmin Kim ${ }^{1}$, Xuexin Ren ${ }^{1}$, Yuan Wang ${ }^{1}$, Xiang Zhang 1,2; ${ }^{1}$ Univ. of California Berkeley, USA; ${ }^{2}$ Lawrence Berkeley National Lab, USA We report on on-chip quantum entanglement between two microscopically separated qubits by engineering their long-range interactions via a metasurface. The metasurface route to quantum state engineering opens a new paradigm for on-chip quantum technology.

FTu3G.3 • 14:00

13:30-15:30

FTu3H - Active Plasmonics and Nanophotonics

Presider: Wei Zhou; Virginia Tech, USA

\section{FTu3H.1 • 13:30}

Massive Parallel Positioning of Nanodiamonds on Nanophotonic Structures, Justus C. Ndukaife ${ }^{1}$, Benjamin Isaacoff ${ }^{2}$, Mikhail Y Shalaginov', Simeon Bogdanov', Agbai G. Nnanna', Julie S. Biteen², Mordechai Segev ${ }^{3}$, Vladimir M. Shalaev' ${ }^{1}$ Alexandra Boltasseva ${ }^{1}$ ${ }^{1}$ Purdue Univ., USA; ${ }^{2}$ Univ. of Michigan, USA ${ }^{3}$ Physics, Technion Israel Inst. of Technology, Israel. Precise and scalable positioning of nanoscale emitters, such as nanodiamonds with color centers, on solid substrates is essential for realizing integrated quantum devices and sensor arrays. We present a novel approach to meet this need.

\section{FTu3H.2 • 13:45}

A Multi-frequency Finite-difference Frequency-domain Algorithm for Active Nanophotonic Device Simulations, Yu Shi' Wonseok Shin'2, Shanhui Fan'1, 'Stanford Univ., USA; ${ }^{2}$ Mathematics, Massachussetts Inst. of Technology, USA. We introduce a multi-frequency finite-difference frequencydomain algorithm for active nanophotonic devices simulations from first principles. This algorithm overcomes large time-scale differences between optical and modulation frequencies and efficiently simulates performances of modulated devices.

FTu3H.3 • 14:00

Broadband transparent all-dielectric metasurfaces, Sergey S. Kruk' ${ }^{1}$, Lei Wang ${ }^{1}$, Hanzhi Tang ${ }^{1,2}$, Ben Hopkins ${ }^{1}$, Andrey Miroshnichen$\mathrm{ko}^{1}$, Tao Li ${ }^{2}$, Ivan Kravchenko ${ }^{3}$, Dragomi Neshev', Yuri Kivshar'; ' $A$ ustralian National Univ., Australia; ${ }^{2}$ Nanjing Univ., China; ${ }^{3}$ Oak Ridge National Lab, USA. We employ the generalized Huygens principle to design and fabricate highly transparent dielectric metasurfaces for complex wavefront manipulation with $99 \%$ polarization conversion and $99 \%$ diffraction efficiencies and broadband operation at telecom wavelengths.
Modeling Nonlinear Resonators Comprising Graphene: A Coupled Mode Theory Approach, Thomas A. Christopoulos' ${ }^{1}$, Odysseas Tsilipakos², Nikolaos Grivas' ${ }^{1}$, Georgios Sinatkas ${ }^{1}$, Emmanouil E. Kriezis'; ; Dept. of Electrical and Computer Engineering, Aristotle Univ. of Thessaloniki, Greece; ${ }^{2}$ Inst. of Electronic Structure and Laser, Foundation of Research and Technology - Hellas, Greece. We develop a perturbation theory framework for modeling nonlinear resonators comprising dispersive sheet materials. It is applied to model optical bistability with graphene based nonlinear resonant structures in the $\mathrm{THz}$ and near-infrared regimes. 


\section{Meeting Room $211 \mathrm{~B} / \mathrm{D}$}

Meeting Room

$212 \mathrm{~A} / \mathrm{C}$
Meeting Room $212 \mathrm{~B} / \mathrm{D}$

\section{CLEO: Science \& Innovations}

13:30-15:30

STu3I • Ultrafast Metrology I Presider: Christophe Dorrer; Univ. of Rochester, USA

\section{STu3I.1 • 13:30 Tutorial}

Beyond the Fringe: Interferometry for Ultrafast Optics, lan A. Walmsley'; ${ }^{1}$ Univ. of Oxford, UK. Interferometry has proven to be a spectacularly successful technique in ultrafast optics, enabling the complete characterization of light pulses from atto- to nano-seconds. I will describe the principles of spectral interferometry in this application.

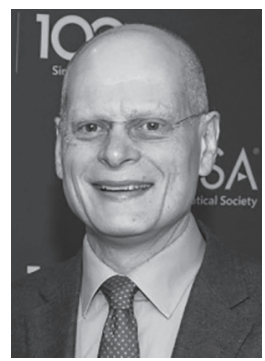

Ian Walmsley is the Hooke Professor of Experimental Physics and the Pro-Vice-Chancellor for Research and Innovation at the University of Oxford, UK. His research in optical science and technology ranges from ultrafast optics to quantum information science. Currently he is the Director of the Networked Quantum Information Technology Hub, the largest collaboration in the UK National Quantum Technologies Programme.

\section{3:30-15:30 \\ STu3J • High-field THz}

Generation

Presider: Matthias Hoffmann; SLAC National Accelerator Lab, USA

\section{STu3J.1 • 13:30}

Broadband terahertz generation with a stair-step echelon, Koustuban Ravi ${ }^{1,2}$ Benjamin Ofori-Okai ${ }^{1}$, Prasahnth Sivarajah' Wenqian Huang ${ }^{1}$, Franz Kaertner ${ }^{2,1}$, Keith Nelson'; ' ${ }^{M}$ IT, USA; ${ }^{2}$ Ultrafast Optics and X Rays, Center for free electron lasers, Germany. A method to overcome limitations of conventional broadband terahertz generation techniques is presented. A stair-step echelon allows for the creation of superior tiltedpulse-fronts to yield larger frequencies and bandwidths, energy conversion efficiencies exceeding $5 \%$.

STu3J.2 • 13:45

Towards high power and low noise midinfrared DFG ultrafast source, Qian $\mathrm{CaO}^{1,2}$ Franz Kaertner', 2 , Guoging Chang'; 'DESY' Germany; ${ }^{2}$ Physics, Univ. of Hamburg, Germany. We theoretically demonstrate that high power low noise mid-IR pulses can be obtained by using SPM-enabled pulses as the signal. Compared with Raman soliton pulse SPM-enabled pulse exhibits high energy and low timing jitter.

\section{STu3J.3 $14: 00$}

Aperiodically poled structures for high ef ficiency broadband terahertz generation Koustuban Ravi ${ }^{1,2}$, Alireza Yahaghi ${ }^{2}$, Arya Fallahi ${ }^{2}$, Franz Kaertner2,1; ${ }^{1}$ MIT, USA; ${ }^{2}$ Ultrafast Optics and $X$ Rays, Center for free electron lasers, Germany. We introduce a combination of aperiodically poled structures and chirped mirrors for broadband terahertz generation. Unprecedented spectral and tempora shaping possibilities with energy conversion efficiencies $>5 \%$ and terahertz output energies of $\sim 10 \mathrm{~mJ}$ are predicted.
13:30-15:30

STu3K • Structured and Gasfilled Fibers

Presider: Benjamin Pulford; Air

Force Research Lab., USA

\section{STu3K.1 • 13:30 Tutorial}

Nanowire-based Hybrid Optical Fibers: A Platform for Nonlinear Light Generation Nanoscale Plasmonics and Single Nanoobject Detection, Markus Schmidt'1,2; 'Leibniz Inst. of Photonic Technology, Germany; ${ }^{2}$ Otto Schott Inst. of Material Research, Germany. Nanowires inside hybrid optical fibers provide new functionalities for various fields such as plasmonics, nonlinear optics and biophotonics. Here I review our results on plasmonic nanotips, nanoparticle tracking and coherent mid-IR light generation.

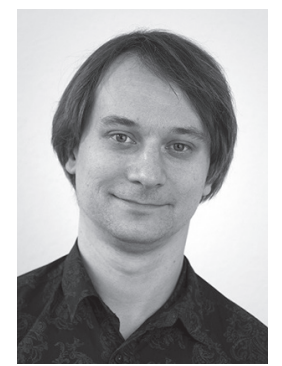

Markus A. Schmidt is professor at the University of Jena and leads a Research Group at the Leibniz Institute of Photonic Technology. He was team leader at the Max Planck Institute for the Science of Light and spent a research stay Imperial College London. He obtained his PhD in Hamburg.
Marriott

Salon I \& II

\section{Joint}

13:30-15:30

JTu3L • Symposium on Ultrafast Laser Technology for X-ray Free

\section{Electron Lasers}

Presider: Ingmar Hartl; DESY,

Germany

\section{JTu3L.1 • 13:30 Invited}

Ultrafast Laser-Enabled Science at XFELs, Wilfried Wurth',2: 'Dept. Physik and Center for Free-Electron Laser Science, Universität Hamburg, Germany; ${ }^{2}$ DESY Photon Science, Germany. Ultrafast science with x-ray free-electron lasers requires in many cases ultrashort laser pulses ranging from $\mathrm{THz}$ to UV. Science examples ranging from physics and chemistry to life science will be given.
JTu3L.2 • 14:00 Invited

High Rep-Rate Pump-Probe-Lasers for XFELs, Tino Lang'; ' 'Deutsches ElektronenSynchrotron DESY, Germany. Abstract not available. 
Marriott

Salon III

Marriott

Salon IV

\section{CLEO: Science \& Innovations}

13:30-15:30

STu3M • Coherent Transmission

Systems

Presider: Vladimir Grigoryan;

Ciena Corporation, USA

STu3M.1 • 13:30

Modulation Format Independent and Low Complexity CPE Algorithm for Elastic Optical Networks, Yang Tao', Xue Chen', Huan Chen'; 'State Key Lab of Information Photonics and Optical Communications, Beijing Univ. of Posts and Telecommunications, China. We propose a modulation format independent blind carrier phase estimation (MFI-CPE) algorithm for arbitrary mQAM coherent systems. Comprehensive numerical simulations and experimental results demonstrate its effectiveness and comparable performance to traditional BPS

\section{STu3M.2 • 13:45}

A High-sensitivity Coherent Receiver without Frequency Recovery Enabled by Doubly Differential QPSK, Tingting Zhang ${ }^{1}$, Christian Sanchez', Stylianos Sygletos ${ }^{1}$, Lida Sadeghioon", Mary E. McCarthy', Andrew D. Ellis'; 'Aston Univ., UK. A high-sensitivity coherent receiver with reduced complexity and high frequency offset tolerance was numerically demonstrated using doubly differential QPSK (DDQPSK). The sensitivity for the 134-Gb/s coherent system transmission over $80-\mathrm{km}$ SMF was below $-27.5 \mathrm{dBm}$.

STu3M.3 $14: 00$

Quasi-Single-Mode Raman amplification in hybrid FMF/SMF span for CO-OFDM transmission, Liang $\mathrm{Xu}^{1}$, Jingchi Cheng ${ }^{1}$, Ming Tang', Zhenhua Feng', Qiong Wu', Huibin Zhou', Xi Chen', Ruoxu Wang', Songnian $\mathrm{Fu}^{1}$, Deming Liu'; ${ }^{1}$ Huazhong Univ of Science and Technology, China. We propose Raman amplified quasi-single-mode transmission with hybrid few-mode-fiber (FMF) and singlemode-fiber (SMF) span using CO-OFDM signals. The OFDM signal is robust to multi-path interference (MPI) and DD-LMS algorithm can be eliminated.
13:30-15:30

STu3N • Electro-Optic \&

Acousto-Optic Devices

Presider: Gregory Steinbrecher; MIT, USA

STu3N.1 1 13:30

Micrometer Scale Lithium Niobate Electrooptic Modulators, Mian Zhang2 ${ }^{2}$ Cheng Wang ${ }^{2}$, Brian Stern ${ }^{3,1}$, Michal Lipson ${ }^{3}$, Marko Lončar ${ }^{2} ;{ }^{1}$ Cornell Univ., USA; ${ }^{2}$ John A. Paulson School of Engineering and Applied Sciences, Harvard Univ., USA: ${ }^{3}$ Electrical Engineering, Columbia Univ., USA. We demonstrate electro-optic modulation on a monolithic lithium niobate nanophotonic platform up to $40 \mathrm{~Gb} / \mathrm{s}$. We show electro-optic efficiency as high as $7 \mathrm{pm} / \mathrm{V}$ in microresonators and half-wave voltage length product as low as 2 $\mathrm{Vcm}$ in micro-Mach-Zehnder interferometers.

\section{STu3N.2 • 13:45}

Record-High In-Device Electro-Optic Coefficient of $359 \mathrm{pm} / \mathrm{V}$ in a Silicon-Organic Hybrid (SOH) Modulator, Clemens Kieninger ${ }^{1,2}$, Yasar Kutuvantavida ${ }^{1,2}$, Heiner Zwickel ${ }^{1}$, Stefan Wolf', Matthias Lauermann ${ }^{3}$, Delwin Elder $^{4}$, Larry Dalton ${ }^{4}$, Wolfgang Freude ${ }^{1}$, Sebastian Randel', Christian Koos ${ }^{1,2}$; ${ }^{1}$ Inst. of Photonics and Quantum Electronics, Karlsruhe Inst. of Technology, Germany; ${ }^{2}$ Inst. of Microstructure Technology, Karlsruhe Inst. of Technology, Germany; ${ }^{3}$ Infinera Corporation, USA; ${ }^{4}$ Dept. of Chemistry, Univ. of Washington, USA. We demonstrate a record-high electro-optic coefficient of $\mathrm{r} 33=359 \mathrm{pm} / \mathrm{V}$ in a silicon-organic hybrid (SOH) modulator using the electro-optic chromophore JRD1. The $\pi$-voltage-length product amounts to $U \pi \mathrm{L}=320 \mathrm{~V} \mu \mathrm{m}$, enabling error-free $25 \mathrm{Gbit} / \mathrm{s}$ signaling at drive voltages of $180 \mathrm{mVpp}$.

\section{STu3N.3 $14: 00$}

Inter-modal Brillouin Scattering in an Integrated Waveguide, Eric Kittlaus ${ }^{1}$, Nils T. Otterstrom', Peter T. Rakich'; 'Yale Univ., USA. We report strong stimulated Brillouin scattering between light guided in separate spatial modes of an integrated waveguide for the first time. Through this process, we demonstrate $2.3 \mathrm{~dB}$ of single-sideband optical amplification in a silicon waveguide.
Marriott

Salon V \& VI

\section{Joint}

13:30-15:30

JTu30 • Symposium on Military Applications of High Powered Lasers I

Presider: J. Thomas Schriempf; US NAVY/PEOIWS 2, USA

\section{JTu30.1 • 13:30 Invited}

High Energy Laser Joint Technology Office - A Mission Overview, Larry Grimes' ${ }^{1}$; 'HEL/JTO, USA. The High Energy Laser Joint Technology Office (HEL-JTO) was established in 2000 for the purpose of developing and executing a comprehensive investment strategy for HEL science and technology that would underpin weapons development. The JTO is currently sponsoring 70 programs across industry, academia, and government agencies with a budget of approximately $\$ 50$ million. The competitively awarded programs are chosen to advance the current state of the art in HEL technology, stimulate the industrial base and fill technology gaps, thus providing a broad capability that can be harvested in acquisition programs of record by the military services.

\section{JTu30.2 • 14:00 Invited}

Title to be Determined, lain McKinnie ${ }^{1 .}$ 'Lockheed Martin, USA. Abstract not available 
Executive Ballroom $210 \mathrm{~A}$
Executive Ballroom 210B
Executive Ballroom $210 \mathrm{C}$
Executive Ballroom 210D

\section{CLEO: Applications \& Technology}

ATu3A - Photobiomodulation Therapeutics-Continued
ATu3B • A\&T Topical Review on Neurophotonics II-Continued

\section{ATu3C • Industrial Optical Design \& Sensing-Continued}

ATu3C.3 • 14:15

Laser-induced Fluorescence for Detection of Alloying Elements During Laser Welding of Austenitic Stainless Steel, Brian Simonds', Jeffrey W. Sowards', Paul A. Williams': ' National Inst. of Standards and Tech, USA. We demonstrate a sensitivity increase of $10^{4}$ over optical emission spectroscopy by applying laser-induced fluorescence to detect evaporating alloying elements during laser welding. As proof-of-principle, we target silicon in stainless steel in the near-UV.
ATu3A.3 • 14:30

Label-Free Sensing of Intrinsic Biomarkers Related to Medical Device Performance Employing a Noninvasive Fingerprint Infrared Spectroscopy Method, Moinuddin Hassan', Ilko K. Ilev'; 'U S Food and Drug Adminstration, USA. An advanced sensing methodology based on a noninvasive labelfree fingerprint infrared spectroscopy approach for detecting and identifying intrinsic biomarkers related to the safety and efficacy of optical diagnostics and therapeutics technologies and devices is presented.
ATu3A.4 • 14:45

Eliciting Host Immunity Selectively against Cancer Cells Treated with Silica-Phthalocyanine-Based Near Infrared Photoimmunotherapy, Hisataka Kobayashi'; ${ }^{1} \mathrm{Na}$ tional Inst. of Health, USA. Near infrared photoimmunotherapy (NIR-PIT) is a new type of molecularly-targeted cancer phototherapy based on antibody-photosensitizer conjugates. By crashing cancer or immunosuppressor cells, NIR-PIT efficiently activates host immunity against NIR-PIT treated cancer cells growing in patients.

\section{ATu3C. $4 \cdot 14: 30$}

Interferometric time-stretch microscopy for three-dimensional microstructure recognition, Shan Jiao', Hongwei Chen', Yuxi Wang', Qiang Guo', Sigang Yang', Minghua Chen', Shizhong Xie'; 'Tsinghua Univ. China. The key principle and architecture of interferometric time-stretch microscopy are presented in this paper. And the application for three-dimensional microstructure recognition is also demonstrated, achieving a frame rate of $50 \mathrm{MHz}$.
CLEO: QELSFundamental Science

\section{FTu3D • On-chip Comb} Generation II-Continued
FTu3D . 4 - 14:30

Coherent on-chip spectral-engineered mid-IR frequency comb generation in Si waveguides, Nima Nader ${ }^{1}$, Daniel L. Maser ${ }^{1,3}$, Flavio C. Cruz ${ }^{1}$, Connor Fredrick ${ }^{1,3}$ Gabriel Y' cas $^{1}$, Daron Westly², Richard Mirin' Jeffrey Shainline ${ }^{1}$, Scott Diddams ${ }^{1,3} ;{ }^{1}$ Nationa Inst of Standards and Tech, USA. ${ }^{2}$ NIST, USA ${ }^{3}$ Dept. of Physics, Univ. of Colorado Boulder USA. Engineered spectral broadening of mid-IR frequency combs is presented in silicon waveguides. Supercontinuum generated light is shown to be coherent for dua comb spectroscopy by heterodyne detection against a second independent comb.
ATu3C.5 • 14:45

An Optical Remote Sensor for Fingerprint Identification using Speckle Pattern, Ariel Schwarz ${ }^{1}$, Amir Shemer ${ }^{1}$, Nisan Ozana', Ran Califa $^{3}$, Javier García², Zeev Zalevsky'; ${ }^{1}$ Fac ulty of Engineering, Bar Ilan Univ., Israel, ${ }^{2}$ Departamento de Óptica, Universitat de València, Spain; ${ }^{3}$ Continuse Biometrics, Israel. The implementation of a simple, inexpensive optical device for remote fingerprint identification is presented. The sensor is based on temporal tracking of back-reflected secondary speckle patterns generated while illuminating a finger with a laser.
FTu3D.5 • 14:45

Large Effective $X^{(2)}$ Nonlinearity via Coherent Photon Conversion on a $\mathrm{Si}_{3} \mathrm{~N}_{4}$ Chip, Alessandro Farsi ${ }^{1}$, Sven Ramelow ${ }^{2}$, Stéphane Clemmen ${ }^{3}$, Xingchen $\mathrm{Ji}^{4}$, Michal Lipson ${ }^{4}$, Alexander L. Gaeta': ${ }^{1}$ APAM, Columbia Univ., USA; ${ }^{2}$ Inst. for Physics, Humboldt Univ. Germany; ${ }^{3}$ Dep. of Inf. Tech, Ghent Univ., Belgium; ${ }^{4} E E$, Columbia Univ., USA. We generate large effective $\gamma^{(2)}$ nonlinearities in a purely $\chi^{(3)}$-nonlinear $\mathrm{Si}_{3} \mathrm{~N}_{4}$ microring-resonator using the coherent photon conversion scheme and measure a normalized effective second harmonic generation efficiency above $77 \% / \mathrm{mW}$. 

Executive Ballroom
$210 \mathrm{E}$
Executive Ballroom
$210 \mathrm{~F}$
Executive Ballroom

210G
Executive Ballroom

$210 \mathrm{H}$

\section{CLEO: QELS-Fundamental Science}

\section{FTu3E - Quantum Optics of Single Emitters-Continued}

FTu3E.4 • 14:15

Spectroscopy of Single Quantum Emitters in Hexagonal Boron Nitride Using Linear and Non-Linear Excitation, Andreas W. Schell', Hideaki Takashima', Toan T. Tran², Igor Aharonovich2', Shigeki Takeuchi'; 'Kyoto Univ., Japan; '2Univ. of Technology Sydney, Australia. Excitation of single photon emitters via a two-photon process can be employed for high resolution imaging and has applications in quantum optics. Here, we present one- and two-photon excitation of single defects in hexagonal boron.

FTu3E.5 • 14:30

Photo-induced modification of singlephoton emitters in hexagonal boron nitride, Zav Shotan', Harishankar Jayakumar' Christopher R. Considine', Helmut Fedder ${ }^{2}$ Jorg Wrachtrup ${ }^{2}$, Audrius Alkauskas ${ }^{3}$, Marcus W. Doherty4, Vinod M. Menon', Carlos A. Meriles'; 'Physics, CUNY-City College of New York, USA; ${ }^{2}$ rd Physics Inst., Univ. of Stuttgart, Germany; ${ }^{3}$ Center for Physical Sciences and Technology, Lithuania; ${ }^{4}$ Laser Physics Centre, Research School of Physics and Engineering, Australia. We report on the room-temperature single photon emission dynamics originating from defect states in hBN. Photo induced modification of the emission characteristics of thee defects under blue and green illumination is shown.

\section{FTu3E.6 • 14:45}

Robust Multicolor Single Photon Emission from Point Defects in Hexagonal Boron Nitride, Toan T. Tran', Christopher Elbadawi', Daniel Totonjian', Charlene J. Lobo', Gabriele Grosso ${ }^{2}$, Hyowon Moon 2 , Dirk Englund ${ }^{2}$, Igor Aharonovich', Mike J. Ford', Milos Toth'; 'School of Mathematical and Physical Sciences, Univ. of Technology, Sydney, Australia; ${ }^{2}$ Dept. of Electrical Engineering and Computer Science, MIT, USA. We demonstrates engineering of quantum emitters in hBN multi-layers using either electron beam irradiation or annealing. The defects exhibit a broad range of multicolor room-temperature single photon emissions across the visible and the near-infrared ranges.

\section{FTu3F • Quantum-Enhanced Measurements-Continued}

FTu3F.3 • 14:15

Single-Shot Orbital-Angular-Momentum Spectrum Measurement Using ImageInversion, Girish Kulkarni', Rishabh Sahu', Omar Magana-Loaiza², Robert Boyd ${ }^{2,3}$ Anand K. Jha'; 'Dept. of Physics, Indian Inst. of Technology, Kanpur, India; ${ }^{2}$ Univ. of Rochester, USA; ${ }^{3}$ Univ. of Ottawa, Canada. We propose a single-shot scheme to measure the orbital-angular-momentum (OAM) spectrum of a partially coherent field. We first test the scheme for a known field, and then use it to measure the OAM spectrum of the signalidler field from parametric down-conversion over a range of more than 150 modes.

FTu3F.4 • 14:30

Optimum Mixed-State Discrimination for Noisy Entanglement-Enhanced Sensing, Quntao Zhuang', Zheshen Zhang', Jeffrey H. Shapiro'; 'MIT, USA. We propose a structured receiver for optimum mixed-state discrimination in quantum illumination target detection, paving the way for entanglement-enhanced minimum-error-probability sensing in an entanglement-breaking environment.

\section{FTu3F.5 • 14:45}

M-state frequency shift keying discrimination below the standard quantum limit, Ivan A. Burenkov', Sergey V. Polyakov'; ' 'Joint Quantum Inst., NIST and UMD, USA; ${ }^{2} \mathrm{Na}$ tional Inst. of Standards and Technology, USA. We introduce a new quantum receiver based on frequency-shift keying encoding. We show that with the appropriate discrimination strategy the accuracy of this method significantly exceeds that of phase-shift keying-based quantum receivers.

\section{FTu3G - Toward Applications of Metasurfaces I-Continued}

FTu3G.4 • 14:15

Large Area Electrically Tunable Metasurface Lenses, Alan She ${ }^{1}$, Shuyan Zhang ${ }^{1}$, Samuel Shian', David Clarke', Federico Capasso'; 'Harvard Univ., USA. Our tunable optics technology enables dynamic tuning with voltage-resolved precision. We have demonstrated electrically controlled focal length tuning of over $100 \%$ with a metasurface lens 2 centimeters in diameter.

FTu3G.5 • 14:30

All-Dielectric Metasurface for PolarizationInsensitive Color Printing, Shang Sun 2,1, Zhenxing Zhou ${ }^{2,1}$, Zonghui Duan ${ }^{2,1}$, Shumin Xiao 2,1, Qinghai Song ${ }^{2,1}$; ' ${ }^{1}$ HIT Shenzhen, China; ${ }^{2}$ Ministry of Industry and Information Technology Key Lab of Micro-Nano Optoelectronic Information System, China. All-dielectric, low-loss, and full-color structural color printing has been realized by $\mathrm{TiO}_{2}$-based all-dielectric metasurface. The highest recorded reflectance reached $64 \%$ and the full width at half maximum (FWHM) of reflection peak was around $30 \mathrm{~nm}$.

FTu3G.6 • 14:45

All-Silica Multifunctional Beam Information Detector without Destroying Original Wave Fronts, Qitong $\mathrm{Li}^{2,1}$, Fengliang Dong ${ }^{3}$, Bo Wang ${ }^{2}$, Weiguo $\mathrm{Chu}^{3}$, Qihuang Gong ${ }^{2,4}$ Mark Brongersma', Yan Li2,4.; 'Stanford Univ. USA; ${ }^{2}$ Peking Univ., China; ${ }^{3}$ National Center for Nanoscience and Technology, China; ${ }^{4}$ Shanxi Univ., China. We demonstrated an all-silica beam information detector based on Pancharatnam-Berry phase which can effectively detect the signal while the original wavefronts are strictly protected. A chiroptical spectrometer and a 12-channel angular momentum detector were proposed.
FTu3H - Active Plasmonics and Nanophotonics-Continued

FTu3H.4 • 14:15

Voltage tunable dual wavelength light source via optomechanically controlled CdS nanoplates, Fei $\mathrm{Yi}^{3}$, Mingliang Ren ${ }^{2}$ Hai Zhu ${ }^{2}$, Wenjing Liư ${ }^{2}$, Ritesh Agarwal ${ }^{2}$ Ertugrul Cubukcu'; 'Dept. of Electrical and Computer Engineering, Univ. of California, San Diego, USA; ${ }^{2}$ Dept. of Materials Science and Engineering, Univ. of Pennsylvania, USA

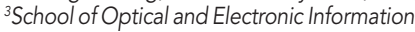
Huazhong Univ. of Science and Technology, China. We experimentally demonstrate optomechanically controlled second harmonic generation and two photon excited photoluminescence in microcavity coupled $\mathrm{CdS}$ nanoplates. The device can be used as a voltage reconfigurable single or dual wavelength light source.

\section{FTu3H.5 • 14:30}

Higher-Order Surface Plasmon Contributions to Plasmonic Interferometry, Dongfang Li', Jing Feng ${ }^{1}$, Domenico Pacifici ${ }^{1}$ 'Brown Univ., USA. We experimentally unveiled up to the sixth-order surface plasmon contributions to hole-groove plasmonic interferometry using discrete Fourier transform. This method is further extended to double-slit plasmonic structures to deconvolve competing interference effects from asymmetric interfaces.

\section{FTu3H.6 • 14:45 Invited}

Light Vapor Interactions on a Chip, Uriel Levy'; ' 'Hebrew Univ. of Jerusalem, Israel. In this talk we discuss our recent results related to chip scale enhanced light-vapor interactions. We also discuss the potential of ou platform for applications such as metrology, communications and photonic information technology. 


\section{Meeting Room $211 \mathrm{~B} / \mathrm{D}$}

Meeting Room

$212 \mathrm{~A} / \mathrm{C}$
Meeting Room

212 B/D

\section{CLEO: Science \& Innovations}

STu3I • Ultrafast Metrology IContinued

\author{
STu3J • High-field THz \\ Generation-Continued
}

STu3K - Structured and Gasfilled Fibers-Continued
Marriott

Salon I \& II

\section{Joint}

JTu3L • Symposium on Ultrafast Laser Technology for X-ray Free Electron Lasers I-Continued

\begin{abstract}
STu3J.4 $\bullet$ 14:15
Generation of Narrowband, High-intensity, Carrier-envelope Phase-stable Pulses Tunable Between 4 and $18 \mathrm{THz}$, Biaolong Liu', Hubertus Bromberger', Andrea Cartella', Thomas Gebert', Michael Först', Andrea Cavalleri ${ }^{1}, 2{ }^{1}{ }^{1} \mathrm{MPI}$ for Structure and Dynamics of Matter, Germany; ${ }^{2}$ Oxford Univ., UK. We demonstrate the generation of narrowband $(<1 \mathrm{THz}$ ) high-energy $(\sim 2 \mu \mathrm{J})$ carrier-envelope phase-stable pulses, tunable between 4 and $18 \mathrm{THz}$ as achieved by difference-frequency mixing between chirped near-infrared pulses in organic DSTMS
\end{abstract}

STu31.2 • 14:30

Near single-cycle pulse characterization with time-domain ptychography, Tobias Witting 2,1. 'Max-Born-Inst., Germany; ${ }^{2}$ Physics, Imperial College London, UK. We show the application of time-domain ptychography for the self-referenced characterization of near single-cycle laser pulses. Using spatia multiplexing spatio-temporal pulse characterization is demonstrated.

\section{STu31.3 • 14:45}

Reconstruction of an isolated burst of (non-repetitive) pulses from a single FROG trace, Gil Ilan Haham', Pavel Sidorenko', Oren Cohen'; 'Technion, Israel. We propose and demonstrate numerically an approach for reconstructing multiple pulses from a single multiplexed FROG trace, opening the door for complete characterization of laser pulses in isolated pulse-bursts with $\mathrm{MHz}-\mathrm{THz}$ pulse repetition rate.

\section{STu3J.5 • 14:30}

Coherent Field Transients below $15 \mathrm{THz}$ from Phase-Matched Difference Frequency Generation in $4 \mathrm{H}-\mathrm{SiC}$, Marco Patrick Fischer', Johannes Bühler', Takayuki Kurihara', Gabriel Fitzky', Alfred Leitenstorfer', Daniele Brida'; 'Dept. of Physics and Center for Applied Photonics, Univ. of Konstanz, Germany. We experimentally demonstrate tunable, phase-matched difference frequency generation fully covering the spectral regime below $15 \mathrm{THz}$ using $4 \mathrm{H}-\mathrm{SiC}$ as nonlinear crystal. The material is also exploited as a broadband detector for electro-optic sampling.

\section{STu3J.6 $14: 45$}

Broadband and narrowband terahertz source at extreme field strength, $\mathrm{C}$. Vicario ${ }^{1}$, Mostafa Shalaby ${ }^{1}$, Flavio Giorgianni ${ }^{1}$ Andrey Ovchinnikov ${ }^{3}$, Oleg Chefonov ${ }^{3}$, Christoph P. Hauri'1,2; 'Paul Scherrer Institut, Switzerland; ' ${ }^{2}$ Ecole Polytechnique Federale de Lausanne, Italy; ${ }^{3}$ Joint Institut for High Temperatures of Ras, Russia. We present a THz source providing multi-octave as well as narrowband spectra at electric field up to tens $\mathrm{MV} / \mathrm{cm}$. The emission is tunable in frequency over $0.5-8 \mathrm{THz}$ and in relative bandwidth over $2 \%-300 \%$.

\section{STu3K.2 • 14:30}

Liquid-Core Nodeless Anti-Resonant Fiber for Biochemical Sensing, Xiaolu Liu', Yingying Wang', Wei Ding', Shoufei Gao', Ling $\mathrm{Cao}^{1}$, Xian Feng', Pu Wang'; 'Beijing Univ. of Technology, China; ${ }^{2}$ Inst. of Physics, Chinese Academy of Sciences, China. A low refractive index liquid-core fiber is formed under antiresonant guidance mechanism with broad transmission band and single modeness. A Raman spectroscopy experiment shows the versatility of this fiber platform for biochemical sensing

\section{STu3K.3 • 14:45}

Efficient fiber gas Raman amplifier based on hydrogen-filled hollow-core fiber, Zefeng Wang', Bo Gu', Yubin Chen', Xiaoming $\mathrm{Xi}^{\prime}$, Jianqiu Cao', Jinbao Chen'; 'National Univ of Defense Technology, China. A highly efficiency 1908 nm gas Raman amplifier based on hydrogen-filled hollow-core fiber is reported for the first time. Seeded by a tunable laser, the maximum power conversion efficiency of $41 \%$ is obtained.

\section{JTu3L.3 • 14:30 Invited}

Intense Laser-based THz Sources for XFEL Experiments, Christoph P. Hauri'; ' SwissFEL, Paul Scherrer Inst., Switzerland. We present recent progress in $\mathrm{THz}$ pulse generation, shaping technology and $\mathrm{THz}$ diagnostics in the low-frequency range $(0.1-10 \mathrm{THz}$ for strong-field experiments at the Free Electron Laser.

\section{CLEO Mobile App}

Use the conference app to plan your schedule; view program updates; receive special events reminders and access Technical Papers (separate log-in required).

- Go to www.cleoconference.org/app.

- Select the Apple App Store or Google Play link.

- Download the app.

- Log in to use app features such as contacting fellow conference attendeesusing your registration I.D. and email address. 
Marriott

Salon III

Marriott

Salon IV

\section{CLEO: Science \& Innovations}

STu3M • Coherent Transmission Systems-Continued

STu3M.4 • 14:15

Long-haul Transmission of $4 \times 100 \mathrm{~Gb} / \mathrm{s}$ DP-QPSK Signals over $2800 \mathrm{~km}$ with Span Lengths Greater than 250 km, Chao Li' , Jian Zhao ${ }^{1}$, Lin Zhang ${ }^{1}$, Qi Mo ${ }^{3}$, Zhiqun Yang ${ }^{1}$, Wei Wang', Guifang Li ${ }^{2,1}$; ${ }^{1}$ Tianjin Univ., China; ${ }^{2}$ Univ. of Central Florida, USA; ${ }^{3}$ Fiberhome \& Fujikura Optics Co,.Ltd, China. We experimentally demonstrate $4 \times 100-G b / s$ PM-QPSK transmission with a $50-\mathrm{GHz}$ channel spacing and a span length greater than $250 \mathrm{~km}$ over a record reach of $2800 \mathrm{~km}$. The transmission system uses a low-loss large-effective-area fiber and EDFA/DRA amplification.

STu3M.5 • 14:30 Invited Progress of Digital Coherent Optical Communication Systems, Maurice O'Sullivan'; ${ }^{1}$ Ciena incorporated, Canada. We describe the progress of single mode digital optical coherent transmission towards increasing practical network capacity.

\section{STu3N • Electro-Optic \& Acousto-Optic Devices- Continued}

STu3N.4 • 14:15

Integrated Two-Dimensional Free-Space Acousto-Optics on Suspended Membranes, Huan Li', Qiyu Liu', Mo Li'; 'Dept. of Electrical and Computer Engineering, Univ. of Minnesota, USA. We propose and have experimentally demonstrated integrated two-dimensional free-space acousto-optics on suspended membranes. Both the Stokes and anti-Stokes sidebands due to Brillouin frequency shift have been observed in a prototype aluminum nitride device.

\section{STu3N.5 • 14:30}

Integrated Graphene Electro-Optic Phase Modulator, Ipshita Datta', Christopher Phare $^{2,1}$, Avik Dutt2,1, Aseema Mohanty2,1, Michal Lipson ${ }^{1} ;{ }^{1}$ Columbia Univ., USA; ${ }^{2}$ Electrical and Computer Engineering, Cornell Univ., USA. We report the first experimental demonstration of a graphene electro-refractive modulator with $\mathrm{V}_{\pi} \mathrm{L}$ of $0.14 \mathrm{Vcm}$, and minimal absorption modulation based on graphene capacitor integrated on $\mathrm{Si}_{3} \mathrm{~N}_{4}$ with embedded high-K and high breakdown dielectric

STu3N.6 $14: 45$

High-speed Active Devices Integrated in Hybrid Silicon on Silicon Nitride Platform, Amir H. Hosseinnia', Majid Sodagar ${ }^{2}$, Hesam Moradinejad', Tianren Fan', Ali A. Eftekhar', Ali Adibi'; ' ${ }^{1}$ Georgia Inst. of Technology, USA; ${ }^{2}$ Skorpios Technologies, USA. We present a hybrid resonant linear frequency comb generator and a modulator for pulse formation and fast electro-optic modulation. Integrated in a hybrid silicon-on-silicon nitride platform, such devices utilize the high-speed plasma dispersion in Si and high-O resonance in SiN.
Marriott

Salon V \& VI

\section{Joint}

JTu30 • Symposium on Military Applications of High Powered Lasers I-Continued

\section{JTu30.3 • 14:30 Invited}

Advances in High Power Laser Systems for Directed Energy, Guy Renard'; 'Northrop Grumman Corp, USA. Laser Weapon (Directed Energy) Systems are experiencing increased interest and nearing deployment. The latest technology research to address these applications is reviewed. Progress with ongoing research activities and remaining challenges will be discussed. 


\section{Executive Ballroom} 210A

\section{Executive Ballroom 210B}

\section{Executive Ballroom $210 \mathrm{C}$}

\section{CLEO: Applications \\ \& Technology}

\section{ATu3A - Photobiomodulation Therapeutics-Continued}

ATu3A.5 • 15:00

Wound Healing Study and Ablation Rate Measurements with the Novel Picosecond Infrared Laser (PIRL), Stephanie Maier ${ }^{2}$, Nils-Owe Hansen², Sebastian Kruber², Tobias Gosau', Dennis Eggert ${ }^{2}$, Alexandra Gliese ${ }^{3}$, Hannes Petersen ${ }^{3}$, Hartmut Schlüter ${ }^{4}$, R. J. Dwayne Miller ${ }^{2} ;{ }^{1}$ Anatomy and Experimental Morphology, Univ. Medical Center HamburgEppendorf, Germany; ${ }^{2}$ Atomically Resolved Dynamics, Max Planck Inst. for the Structure and Dynamics of Matter, Germany; ${ }^{3}$ Otorhinolaryngology, Head and Neck Surgery and Oncology, Univ. Medical Center HamburgEppendorf, Germany; ${ }^{4}$ Clinical Chemistry, Univ. Medical Center Hamburg-Eppendorf, Germany. We present the first wound healing study in rat skin, showing minimal scar formation with the Picosecond-Infrared-Laser (PIRL) compared to a conventional scalpel and electrosurgical device. In addition, we show first ablation rate measurements.

\section{ATu3A.6 • 15:15}

Multi-dimensional Imaging in the Terahertz Regime for Theranostic Applications, Holger Breitenborn', Rafik Naccache², Anna Mazhorova', Matteo Clerici ${ }^{3}$, Riccardo Piccoli', Larousse K. Khorashad ${ }^{4}$, Alexander O. Govorov ${ }^{4}$, Luca Razzari ${ }^{1}$, Fiorenzo Vetrone ${ }^{1,5}$ Roberto Morandotti'; ' Energy Materials Telecommunications, INRS, Canada; ${ }^{2}$ Dept. of Chemistry and Biochemistry, Concordia Univ., Canada; ${ }^{3}$ School of Engineering Univ. of Glasgow, UK; ${ }^{4}$ Dept. of Physics and Astronomy, Ohio Univ., USA; ${ }^{5}$ Centre for SelfAssembled Chemical Structures, McGill Univ. Canada. We demonstrate a novel terahertz radiation-based joint thermal-hyperspectral imaging method for theranostic applications. Hyperspectral imaging of a drug formulation was realized in the stratum granulosum of skin, in the presence of plasmonically heated gold nanoparticles.

\section{ATu3C $\bullet$ Industrial Optical Design \& Sensing-Continued}

ATu3C.6 1 15:00

Photo-Acoustic Sensor for Detection of Oil Contamination in Compressed Air Systems, Mikael Lassen ${ }^{1}$, David BaslevHarder 1 , Anders Brusch 1. Dita Heikens ${ }^{2}$ Stefan Persijn ${ }^{2}$, Jan C. Petersen'; ' Danish Fundamental Metrology, Denmark; ${ }^{2}$ VSL - The Dutch Metrology Inst., Thijsseweg 11, Netherlands. We demonstrate an in-situ sensor to detect oil contamination in compressed air complying with the ISO-8573 standard. The sensor is based on the photo-acoustic effect and will be beneficial for a large category of industries.

ATu3C.7 • 15:15

Design and Fabrication Toward a Shorter Lightweight Night Vision Goggle Objective Assembly with a Nanolayered Polymer Gradient Refractive Index Lens, Howard Fein ${ }^{1}$ Michael Ponting'; 'Peak Nano Optics, USA. A night vision goggle objective is described leveraging a spherical gradient refractive index lens fabricated from polymeric nanolayered materials. The objective achieves a reduction in optical elements to reduce system length $15 \%$ and weight $28 \%$.
Executive Ballroom 210D

\section{CLEO: QELS} Fundamental Science

\section{FTu3D • On-chip Comb Generation II-Continued}

\section{FTu3D.6 1 15:00}

Efficient Broadband Optical Parametric Amplification in Non-Uniform Bulk Crystals, Andrey Markov', Anna Mazhorova' Holger Breitenborn ${ }^{1}$, Andrew Bruhacs ${ }^{1}$ Matteo Clerici ${ }^{2}$, Daniele Modotto ${ }^{3}$, Ottavia Jedrkiewicz ${ }^{4}$, Paolo Di Trapani ${ }^{4}$, Arkady Major ${ }^{5}$, Francois Vidal' ${ }^{1}$, Roberto Morandotti ${ }^{1}$,

'Energy, Materials, Telecommunications, Institut National de la Recherche Scientifique (INRS), Canada; ${ }^{2}$ School of Engineering, Univ. of Glasgow, UK: ${ }^{3}$ Dipartimento di Ingegneria dell'Informazione, Università di Brescia, Italy; ${ }^{4}$ Dipartimento di Scienza e Alta Tecnologia Univ. of Insubria and CNISM Udr Como, Italy; ${ }^{5}$ Univ. of Manitoba, Canada. We demonstrate $\sim 50 \%$ efficient adiabatic optical parametric amplification in bulk crystals by introducing temperature-gradient phase-matching. We provide details on the choice of temperature profile that maximizes conversion efficiency and increases bandwidth to over $300 \mathrm{~nm}$.

\section{FTu3D.7 • 15:15}

$30 \mathrm{GHz}$ Frequency Comb Spanning 160 $\mathrm{THz}$ in the Near-Infrared, Andrew J. Metcalf ${ }^{1,2}$, Connor Fredrick ${ }^{1,2}$, Ryan Terrien ${ }^{1}$, Scott Papp', Scott Diddams 1,2; 'Time and Frequency Division, National Inst. of Standards and Technology, USA: ${ }^{2}$ Physics, Univ. of Colorado - Boulder, USA. We generate a $30 \mathrm{GHz}$ electro-optic frequency comb at 1064 $\mathrm{nm}$ and extend its spectral bandwidth to 160 $\mathrm{THz}$ through nonlinear spectral broadening. Further, we frequency double the broadened spectrum producing a visible comb which spans over $100 \mathrm{THz}$

15:30-16:00 Coffee Break and Unopposed Exhibit Only Time, Exhibit Hall 1, 2 \& 3

15:30-16:15 Market Focus Session II: Update on Optics and Photonics Markets and Opportunities, Exhibit Hall Theater 

Executive Ballroom
$210 \mathrm{E}$
Executive Ballroom
$210 \mathrm{~F}$
Executive Ballroom

210G
Executive Ballroom

$210 \mathrm{H}$

\section{CLEO: QELS-Fundamental Science}

FTu3E - Quantum Optics of Single Emitters-Continued

\section{FTu3E.7 • 15:00}

High Fidelity Source of a Single Atom in its 2D Quantum Ground State, Pimonpan Sompet $^{1}$, Yin H. Fung ${ }^{1}$, Eyal Schwartz', Matthew D. Hunter ${ }^{1}$, Jindaratsamee Phrompao', Mikkel F. Andersen'; ${ }^{1}$ The Dodd-Walls Centre for Photonic and Quantum Technologies, Dept. of Physics, Univ. of Otago, New Zealand. We combine the near-deterministic preparation of a single atom in optical tweezers with magnetically-insensitive Raman sideband cooling, to prepare an atom in its motional ground state with 2D fidelity of $\sim 0.7$ for the entire procedure.

\section{FTu3F • Quantum-Enhanced} Measurements-Continued

\section{FTu3F.6 • 15:00}

A nonlinear interferometer with intrinsic stability, Joseph M. Lukens ${ }^{1}$, Nicholas A. Peters ${ }^{1,2}$, Raphael C. Pooser ${ }^{1,3} ;{ }^{1}$ Quantum Information Science Group, Oak Ridge National Lab, USA; ${ }^{2}$ The Bredesen Center for Interdisciplinary Research and Graduate Education, The Univ. of Tennessee, USA ${ }^{3}$ Dept. of Physics, The Univ. of Tennessee, USA. We realize a passively stable nonlinear interferometer based on a single parametric amplifier, attaining $99.9 \%$ visibility by combining RF modulation and spatial filtering Our configuration offers new capabilities for robust interferometric sensors.
FTu3G • Toward Applications of Metasurfaces I-Continued

\section{FTu3G.7 • 15:00 Invited}

Lasing and Anti-Lasing in a Single Cavity , Xiang Zhang'; ' 'Univ. of California Berkeley, USA. Using Parity-time symmetry, we experimentally realize lasing and anti-lasing at the same equency in a single cavity. Because of the time-reversal property, the demonstrated lasing and anti-lasing resonances share common resonant features such as identical frequency dependence, coherent in-phase response and line spectral resolution. Lasing and anti-lasing in a single device offers a new route for light modulation with high contrast approaching the ultimate limit.

\section{FTu3E.8 • 15:15}

Deterministic single-atom array preparation using dynamic holographic optical tweezers, Hyosub Kim1, Woojun Lee1 Jaewook Ahn'; ' ${ }^{\prime}$ AIST, Korea (the Republic of). We report a new method to load $\mathrm{N}=20$ single-atoms near-deterministically $(90 \%$ for 3 -by- 3 square and $80 \%$ for $\mathrm{N}=19$ ring lattice) in 2D lattices, using dynamic holographic optical tweezers implemented with a 2D liquid-crystal spatial-light modulator.
FTu3F.7 • 15:15

Single-photon fiber bundle cameras (SFICAMs) for quantum enhanced superresolution microscopy, Yonatan Israel', Ron Tenne ${ }^{1}$, Dan Oron', Yaron Silberberg'; 'Weizmann Inst. of Science, Israel. We present a method that utilizes quantum correlation measurements for multi-emitter sub-diffraction localization in a time-dependent scene. This is demonstrated using a newly developed imaging configuration based on fiber bundle coupled single-photon avalanche detectors.
FTu3H • Active Plasmonics and Nanophotonics-Continued
FTu3H.7 • 15:15

Coupled Metallic Nanolaser Arrays, William Hayenga ${ }^{1}$, Midya Parto ${ }^{1}$, Hossein Hodaei ${ }^{1}$ Patrick LiKamWa', Demetrios Christodou lides ${ }^{1}$, Mercedeh Khajavikhan'; 'Univ. of Central Florida, CREOL, USA. Heptamer arrays of coupled metallic nanolasers are demonstrated. The lasers operate in a single transverse and longitudinal mode, have low thresholds, and are capable of generating high output powers.

15:30-16:00 Coffee Break and Unopposed Exhibit Only Time, Exhibit Hall 1, 2 \& 3

15:30-16:15 Market Focus Session II: Update on Optics and Photonics Markets and Opportunities, Exhibit Hall Theater 


\section{Meeting Room $211 \mathrm{~B} / \mathrm{D}$}

Meeting Room
$212 \mathrm{~A} / \mathrm{C}$
Meeting Room

$212 \mathrm{~B} / \mathrm{D}$

\section{CLEO: Science \& Innovations}

STu3I • Ultrafast Metrology IContinued

\section{STu3I.4 1 15:00}

Improved principal components generalized projections algorithm for frequency resolved optical gating, Daniel J. Kane ${ }^{1}$; 'Mesa Photonics, LLC, USA. I present an improvement to the principal components generalized projections (PCGP) algorithm used for Frequency Resolved Optical Gating (FROG) inversion that still maintains the speed and simplicity of the PCGP algorithm.

STu31.5 $15: 15$

Deep UV pulse shaping at $207 \mathrm{~nm}$ via Frequency domain Nonlinear Optics (FNO), Bruno E. Schmidt 3,1, Philippe Lassonde', Guilmot Ernotte', Matteo Clerici', Roberto Morandotti', Heide Ibrahim', Francois Legare'; 'INRS-Energie Mat \& Tele Site Varennes, Canada; ${ }^{2}$ Univ. of Glasgow, UK; ${ }^{3}$ few-cycle Inc., Canada. FNO enables deep UV pulse shaping through direct phase transfer of conventionally shaped NIR pulses at $830 \mathrm{~nm}$ to their $4^{\text {th }}$ harmonic. We demonstrate first time pulse characterization with a transient grating FROG at $207 \mathrm{~nm}$.

\author{
STu3J • High-field THz \\ Generation-Continued
}

STu3J.7 • 15:00

High-Power Terahertz Generation from Two-Color Laser Filamentation in Various Types of Gases, Yung Jun Yoo', Donghoon Kuk', Zheqiang Zhong ${ }^{1,2}$, Ki-Yong Kim ${ }^{1} ;{ }^{1}$ Univ. of Maryland, USA: ${ }^{2}$ College of Electronics and Information Engineering, Sichuan Univ., China. We report strong $\mathrm{THz}$ field generation from two-color laser filamentation in various types of gases. We observe enhanced $\mathrm{THz}$ generation at low pressures for certain gas species with laser-to- $\mathrm{THz}$ conversion ef ficiency of $0.07 \%$

\section{STu3J.8 • 15:15}

Terahertz-induced optical birefringence in liquid water, Liwei Song 1,2, Peter Zalden 1,3 Xiaojun $\mathrm{Wu}^{1,2}$, Haoyu Huang ${ }^{2,4}$, Oliver Muecke ${ }^{1,2}$, Christian Bressler ${ }^{1,3}$, Franz Kaertner ${ }^{1,2} ;{ }^{1}$ The Hamburg Centre for Ultrafast Imaging CUI, Univ. of Hamburg, Germany; ${ }^{2}$ Center for Free-Electron Laser Science CFEL, Deutsches Elektronen-Synchrotron DESY, Germany; ${ }^{3}$ European XFEL GmbH, Germany; ${ }^{4}$ Dept. of Physics, Univ. of Hamburg, Germany. We have observed optical birefringence in neat water induced by single-cycle intense terahertz pulses. The refractive index changes are explained by the electronic polarizability modulated by molecular orientation driven by the strong terahertz electric field.

\section{STu3K • Structured and Gas- filled Fibers-Continued}

\section{STu3K.4 1 15:00}

Efficient Dispersive Waves Generation From Argon-Filled Anti-Resonant Nodeless Fiber, Fanchao Meng', Shoufei $\mathrm{Gao}^{2}$, Yingying Wang ${ }^{2}$, Pu Wang ${ }^{2}$, Junku Liu ${ }^{3}$, Sijia Wang ${ }^{3}$, Bowen Liu', Yanfeng Li ${ }^{1}$, Chingyue Wang', Ming-lie Hu'; 'Tianjin Univ., China; ${ }^{2}$ Beijing Univ. of Technology, China; ${ }^{3}$ China Academy of Space Technology, China. We report dispersive waves generation in an argon-filled anti-resonant nodeless fiber pumped by ultrashort pulses centered at different wavelengths. The conversion efficiency can be as high as $16 \%$ with a bandwidth below $10 \mathrm{~nm}$.

\section{STu3K.5 • 15:15}

Experimental generation of deep-ultraviolet second-harmonics in an air-silica photonic crystal fiber, Jinhui Yuan ${ }^{1}$, Zhe Kang ${ }^{1}$ Feng $\mathrm{Li}^{2}$, Xianting Zhang ${ }^{1}$, Xinzhu Sang ${ }^{1}$ Binbin Yan', Chao Mei' , Xian Zhou ${ }^{2}$, Kangping Zhong ${ }^{2}$, Kuiru Wang', Chongxiu Yu' ${ }^{1}$, Chao $\mathrm{Lu}^{2}$, Hwa Yaw Tam², P. K. A. Wai'; 'Beijing Univ. of Posts and Telecomm, China; ${ }^{2}$ Dept. of Electronic and Information Engineering, The Hong Kong Polytechnic Univ., Hong Kong. We experimentally generate secondharmonics within deep-ultraviolet wavelength range of 334.5 to $306 \mathrm{~nm}$. We combine four-wave mixing and surface nonlinearity polarization by coupling femtosecond pump pulses at $\sim 800 \mathrm{~nm}$ into an air-silica photonic crystal fiber.

\section{Marriott \\ Salon I \& II}

\section{Joint}

JTu3L • Symposium on Ultrafast Laser Technology for X-ray Free Electron Lasers I-Continued

JTu3L.4 • 15:00

Integration of the ANGUS 200 TW LaserSystem into the Accelerator Infrastructure at DESY, Andreas R. Maier ${ }^{1}$, Spencer W. Jolly ${ }^{1,2}$, Vincent Leroux ${ }^{1,2}$, Matthias Schnepp ${ }^{1}$ ${ }^{1}$ Univ. of Hamburg, Germany; ${ }^{2}$ ELI Beamlines, Czech Republic. Aiming for clystron-like operation, we integrated the ANGUS 200 TW laser system into the accelerator infrastructure at DESY, to reliably drive the LUX laser-plasma acceleration experiment. We report on commissioning results and lessons learned.

JTu3L.5 • 15:15

Temporal characterization on FLASH FEL Pulses, Rosen Ivanov', Stefan Duesterer ${ }^{1}$ Guenter Brenner', Jia Liu2; ${ }^{1}$ FS-FLASH-D, Deutsches Elektronen Synchrotron - DESY Germany; ${ }^{2}$ European XFEL, Germany. A terahertz ( $\mathrm{THz}$ )-field driven streak camera has been commissioned at FLASH providing XUV pulse duration and arrival time information with around $10 \mathrm{fs}$ resolution for each single XUV free-electron laser (FEL) pulse.

15:30-16:00 Coffee Break and Unopposed Exhibit Only Time, Exhibit Hall 1, 2 \& 3

15:30-16:15 Market Focus Session II: Update on Optics and Photonics Markets and Opportunities, Exhibit Hall Theater 
Marriott

Marriott

Salon III

Salon IV

\section{CLEO: Science \& Innovations}

STu3M • Coherent Transmission Systems-Continued

STu3M.6 $\cdot 15: 00$

An Improvement in Delay Mismatch Tolerance for 128 Gbaud 160AM Spectral Slicing Transmission System Employing Multicarrier Technique, Tu Nguyen', Son T. $\mathrm{Le}^{2}$, Marc Wuilpart ${ }^{1}$, Patrice Megret ${ }^{1}{ }^{1}$ Univ. of Mons, Belgium; ${ }^{2}$ Nokia Bell Labs, Germany. We demonstrate for the first time the remarkable delay mismatch tolerance of the filter bank multicarrier over orthogonal frequency division multiplexing for the transmission of a 128 Gbaud 160AM supersignal with single coherence receiver.

STu3M.7 • 15:15

Extended Kalman Filter for Carrier Frequency Offset and Carrier Phase Noise, Li Lingian', Yiqiao Feng ${ }^{1}$, Wenbo Zhang ${ }^{1,2}$, Nan Cui ${ }^{1}$, Hengying $X^{1}{ }^{1}$, Xianfeng Tang ${ }^{1}$ Lixia Xi', Xiaoguang Zhang'; ' State Key Lab of Information Photonics and Optical Communications, Beijing Univ of Posts \& Telecom, China; ${ }^{2}$ School of Sciences, Beijing Univ of Posts \& Telecom, China. A joint recovery scheme for carrier frequency offset (CFO) and carrier phase noise (CPN) utilizing extended Kalman filter is proposed, which has good performance of high CFO estimation accuracy, large CPN tolerance and low complexity.

\section{STu3N • Electro-Optic \& Acousto-Optic Devices- Continued}

\section{STu3N.7 • 15:00}

$\mathrm{SiN}$-on-LiNbO ${ }_{3}$ Integrated Optical Modulation at Visible Wavelengths, Karan Mehta', Gavin West ${ }^{1}$, Rajeev Ram ${ }^{1}$; ${ }^{2}$ MIT, USA. Waveguides formed with $\mathrm{SiN}$ ridges on thin-film $\mathrm{LiNbO}_{3}$ enable a platform for compact integrated electro-optic devices in the visible and infrared. We demonstrate Mach-Zehnder modulators at $\lambda=674 \mathrm{~nm}$ with $\mathrm{V}_{7} \mathrm{~L}$ products $<0.5 \mathrm{Vcm}$, as well as ring resonators with $\mathrm{Os}$ up to 340,000 at $\lambda=1590 \mathrm{~nm}$.

STu3N.8 • 15:15

Silicon Waveguide Modulator with In-Line Phase Change Material, Kevin J. Miller ${ }^{1}$, Kent A. Hallman'1, Richard F. Haglund', Sharon M. Weiss'; 'Vanderbilt Univ., USA. We demonstrate a silicon waveguide modulator with an in-line vanadium dioxide segment as a platform for optical switching. A modulation depth greater than $10 \mathrm{~dB}$ is achieved with a device footprint of only $0.28 \mu \mathrm{m}^{2}$.
Marriott

Salon V \& VI

\section{Joint}

JTu30 • Symposium on Military Applications of High Powered Lasers I-Continued

JTu30.4 • 15:00

Few cycle pulse Damage morphology of an ultra-broad band chirped mirror, Enam Chowdhury',2, Kyle Kafka1, Noah Talisa', Drake Austin ${ }^{1}$, Gabriel Tempea ${ }^{2}$, Catalin Neacsu2.' 'Physics, Ohio State Univ., USA; ${ }^{2}$ Spectra-Physics, Austria. Few cycle pulse damage of ultra-broad band chirped mirror was studied with $5 \mathrm{fs}$ pulses showing dramatic damage morphology, which has been analyzed with cross-sectional scanning electron microscopy, showing delamination behavior of the top few layers.

JTu30.5 • 15:15

Damage Performance of lon Beam Sputtered $\mathrm{Sc}_{2} \mathrm{O}_{3}$ and $\mathrm{HfO}_{2}$ Single Layers Tested in Air and Ultra-high Vacuum, Carmen S. Menoni ${ }^{1}$, Peter Langston ${ }^{1}$, Dinesh Patel ${ }^{1}$, Brendan A. Reagan', Federico J. Furch', Alden Curtis ${ }^{1}$, Jorge Rocca': ${ }^{1}$ Colorado State Univ., USA. Results of a study of laser induced damage of ion beam sputtered single layers and capped single layers of $\mathrm{Sc} 2 \mathrm{O} 3, \mathrm{HfO} 2$ and $\mathrm{Ta} 2 \mathrm{O} 5$ in a controlled atmosphere and in an ultra-high vacuum (UHV) show the choice of material is paramount to achieving the best performance.

15:30-16:00 Coffee Break and Unopposed Exhibit Only Time, Exhibit Hall 1, 2 \& 3

15:30-16:15 Market Focus Session II: Update on Optics and Photonics Markets and Opportunities, Exhibit Hall Theater 
Executive Ballroom $210 \mathrm{~A}$

Executive Ballroom 210B

Executive Ballroom $210 \mathrm{C}$

\section{CLEO: Applications \& Technology}

16:00-18:00

ATu4A - Spectroscopic Sensing

Presider: Ilko llev; U.S. Food and Drug Admin, USA

ATu4A.1 1 16:00

Noninvasive Glucose Measurements in Skin using Mid-IR Quantum Cascade Laser Spectroscopy, Alexandra Werth1', Grant Schultheis ${ }^{1}$, Anqi Dong ${ }^{1,2}$, Sabbir Liakat ${ }^{1,3}$ Claire Gmachl'; 'Princeton Univ., USA; ${ }^{2}$ Google Inc., USA; ${ }^{3}$ Princeton Identity, USA A mobile sensor using a quantum cascade laser and integrating sphere has been implemented to detect glucose noninvasively in human skin. Principal component analysis of the backscattered spectra closely matched the known glucose absorption spectrum.

16:00-18:00 Metals TRUMPF Inc., USA

\section{ATu4C.1 16:00 Invited}

ATu4A.2 • 16:15

In vivo Raman Spectroscopic Sensing of Biophysical Changes in Skin Cancer, Xu Feng ${ }^{1}$, Austin Moy ${ }^{1}$, Hieu Nguyen ${ }^{1}$, Jason Zhang', Matthew Fox ${ }^{2}$, Jason Reichenberg ${ }^{2}$, Mia Markey', James Tunnell'; 'Dept. of Biomedical Engineering, Univ. of Texas at Austin, USA; ${ }^{2}$ Dept. of Medicine, Dell Medical School, Univ. of Texas at Austin, USA. We developed a biophysical Raman model of human skin and validated it using in vivo clinical screening data. Key biophysical changes were used for fast and accurate diagnosis of melanoma and nonmelanoma skin cancer.

ATu4A.3 • 16:30

Label-free Mid-Infrared Photothermal Spectroscopy and Imaging of Neurological Tissue, Atcha Totachawattana ${ }^{1,2}$, Michael S. Regan $^{4}$, Nathalie Y. Agar ${ }^{4,5}$, Shyamsunder Erramilli, ${ }^{3,7}$, Michelle Y. Sander ${ }^{1,6},{ }^{1}$ Electrical and Computer Engineering, Boston Univ., USA ${ }^{2}$ Photonics Center, Boston Univ., USA; ${ }^{3}$ Dept. of Physics, Boston Univ., USA; ${ }^{4}$ Neurosurgery, Brigham and Women's Hospital, USA ${ }^{5}$ Surgery and Radiology, Harvard Medical School, USA; ${ }^{\circ}$ Division of Materials Science and Engineering, Boston Univ., USA; ${ }^{7}$ Dept. of Biomedical Engineering, Boston Univ. USA. We present mid-infrared photothermal spectroscopy for label-free characterization of various healthy and diseased brain tissue types in a mouse model. The photothermal spectroscopy and imaging results allow to quantitatively distinguish between different tissue types.
16:00-18:00

ATu4B • Information Transfer \&

Precision Measurement Devices

Presider: Paul Williams; NIST, USA

ATu4B.1 • 16:00

Photonic-Assisted Ultra-wideband Arbitrary Waveform Generation with Extended Time Aperture for Multipath Channe Sounding and Compensation, Bohao Liu' Pragjnesh Reddy ${ }^{1}$, Andrew Weiner ${ }^{1}$; ${ }^{1}$ Electrical and Computer Engineering, Purdue Univ. USA. Photonic-assisted radio-frequency arbitrary waveform generation via pulse shaping and frequency-to-time mapping is demonstrated with a time-bandwidth product of 250 ( $34 \mathrm{~ns} \times 7.3 \mathrm{GHz}$ ). It is applied to multipath wireless channel sounding and subsequent precompensation.
ATu4B.2 • 16:15

Fully Integrated Photonic Microwave Tracking Generator on Heterogeneous Si/III-V Platform, Rui-Lin Chao ${ }^{2,1}$, Linjun Liang ${ }^{2,3}$, Jin-Wei Shi ${ }^{2,1}$, Jared Hulme ${ }^{2}$, M. J. Kennedy ${ }^{2}$, Tin Komljenovic ${ }^{2}$, Doug Baney ${ }^{4}$ Bogdan Szafraniec ${ }^{4}$, John Bowers ${ }^{2}$; 1 Dept. of EE, National Central Univ., Taiwan; ${ }^{2}$ Electrical and Computer Engineering Dept., Univ. of California Santa Barbara, USA: ${ }^{3}$ Inst. of Lightwave Technology , Beijing Jiaotong Univ., China; ${ }^{4}$ Keysight Technologies, USA. Fully-integrated photonic microwave tracking generators are demonstrated for the firs time. By sweeping wavelength separations among two-locked DFBs and one tunable laser, a two-tone photo-generated RF signal with tunable differences in frequency can be generated.
ATu4C • Laser Interaction with Semiconductrors, Glasses and

Presider: Andrius Marcinkevicius;

Relevance of Modeling Laser-Materia Interactions in the Industrial Context Wolfgang Schulz', 2, ${ }^{1}$ Fraunhofer ILT Aachen, Germany; ${ }^{2}$ RWTH Aachen Univ., Germany. mplementing a virtual production system is challenging due to machine specific interactions, uncertainties and unknowns. The theory of design oriented thinking adapted for manufacturing favours fast iteration in digitised design cycles instead of optimising the model quality in one step. A virtua production system is seen to become a set of "digital shadows" emulating relevant properties of the underlying techno-physical sys tems. Different model reduction techniques are demonstrated resulting in "digital shadows" able to generate dense data by million of runs within acceptable calculation time.

\section{ATu4C. $2 \cdot 16: 30$}

ATu4B.3 • 16:30

Precision Multiple-access RF Dissemination by Hybrid Frequency Modulation Technique, Yajie Cui', Tianwei Jiang', Song $\mathrm{Yu}^{1}$, Chenxia Liu', Ruihuan Wu', Wanyi Gu${ }^{1}$ Bin Luo', Guohua Wu', ${ }^{1}$ Beijing Univ. of Posts and Telecommunications, China. A precise multiple-access radio frequency dissemination scheme with anti-dispersion function by hybrid frequency modulation technique is proposed. The residual phase jitter at arbitrary node is less than 0.034-rad over 50.2-km fiber link.
Executive Ballroom 210D

\section{CLEO: QELS-} Fundamental Science

\section{6:00-18:00 \\ FTu4D • On-chip Quantum \\ Optics \\ Presider: Marco Liscidini; \\ Universita degli Studi di Pavia, Italy}

\section{FTu4D.1 • 16:00}

Integrated Quantum Spectroscopy on a Nonlinear Chip, Alexander S. Solntsev ${ }^{1,2}$ Pawan Kumar², Thomas Pertsch², Frank Setzpfandt ${ }^{2}$, Andrey A. Sukhorukov'; ${ }^{1}$ Australian National Univ., Australia; ${ }^{2}$ Univ. of Jena Germany. We demonstrate experimentally on-chip-integrated quantum spectroscopy by generating biphotons in a $\mathrm{LiNbO}_{3}$ waveguide through spontaneous parametric down-conversion, and using signal photon detection in the NIR to study the dynamics of idler photons in the MIR.
Comparison of nano particle implantation with picosecond lasers by concerning different wavelengths from Aluminum and Copper on Silicon wafer substrate Mohammad Hossein Azhdast ${ }^{1}$, Martin Kossatz $^{2}$, Hans Joachim Eichler ${ }^{3}$, Klaus Lang ${ }^{4}$ Veronika Glaw'; ${ }^{1}$ PacTech \& TU-Berlin, Germany; ${ }^{2}$ PacTech GmBH, Germany; ${ }^{3}$ Technical Univ. of Berlin, Germany; ${ }^{4}$ IZM Fraunhofer, Germany. Copper and Aluminum have similar reactions in Infra-red lasers for nano particle printing and different behavior in frequency doubled lasers. Absorption of two different metals shows various roughness of thin film on silicon wafer substrates.
FTu4D.2 • 16:15

Sum-Frequency Generation and PhotonPair Creation in AlGaAs Nano-Scale Resonators, Giuseppe Marino ${ }^{1,2}$, Alexander S Solntsev ${ }^{2}$, Lei Xu ${ }^{2}$, Valerio Gili ${ }^{4}$, Luca Carletti ${ }^{5}$ Alexander N. Poddubny ${ }^{3}$, Daria Smirnova ${ }^{2}$ Hitao Chen ${ }^{2}$, Guoquan Zhang ${ }^{6}$, Anatoly Zayats ${ }^{1}$, Costantino Angelis ${ }^{5}$, Giuseppe Leo ${ }^{4}$ Yuri Kivshar', Andrey Sukhorukov², Dragomir N. Neshev2; ' ${ }^{1}$ Dept. Of Physics, King's College London, UK; ${ }^{2}$ Nonlinear Physics Centre, Re search School Of Physics and Engineering, Australian National Univ., Australia; ${ }^{3}$ ITMO Univ. , Russia: ${ }^{4}$ Université Paris Diderot Paris 7, France; ${ }^{5}$ Dipartimento di Ingegneria dell'Informazione, Univ. of Brescia, Italy ${ }^{6}$ Nankai Univ., China. We demonstrate experimentally sum-frequency generation in AlGaAs nano-resonators, establishing a quantum-classical correspondence with spontaneous parametric down-conversion. We predict that $\mathrm{AlGaAs}$ nano-resonators can be utilized as high-rate sources of photon pairs with non-classical correlations.

\section{FTu4D.3 • 16:30}

Four-Wave Mixing Photon Pair Generation Statistics for a Nonlinear Microcavity with Chaotic and Pulsed Excitation, Piot Roztocki ${ }^{1}$, Michael Kues ${ }^{1,6}$, Christian Reimer ${ }^{1}$ Brent Little2, Sai Chu ${ }^{3}$, David J. Moss ${ }^{1,4}$ Roberto Morandotti ${ }^{1,5},{ }^{1}$ INRS-EMT, Canada; ${ }^{2} X i{ }^{\prime}$ an Inst. of Optics and Precision Mechanics of CAS, China; ${ }^{3}$ City Univ. of Hong Kong China; ${ }^{4}$ Swinburne Univ. of Technology, Australia; ${ }^{5}$ National Research Univ. of Information Technologies, Russia; ${ }^{6}$ Univ. of Glasgow, UK. We characterize four-wave mixing photon pair generation statistics in the chaotic pulsed regime associated with a self-locked nonlinear microcavity excitation scheme, and contrast these against a pulsed actively mode-locked excitation. 
Executive Ballroom $210 \mathrm{E}$
Executive Ballroom 210F
Executive Ballroom

210G
Executive Ballroom

$210 \mathrm{H}$

\section{CLEO: QELS-Fundamental Science}

\section{6:00-18:00 \\ FTu4E • Quantum Dot Cavity QED}

Presider: Edward Flagg; West

Virginia Univ., USA

\section{FTu4E.1 1 16:00}

Tuning the Photon Statistics of a Strongly Coupled Nanophotonic System, Constantin Dory ${ }^{1}$, Kevin Fischer ${ }^{1}$, Kai Müller ${ }^{1}$, Konstantinos Lagoudakis ${ }^{1}$, Tomas Sarmiento ${ }^{1}$, Armand Rundquist', Linda J. Zhang'1, Yousif Kelaita', Neil Sapra', Jelena Vuckovic'; ' ${ }^{1}$ Stanford Univ. Germany. We demonstrate the generation of single- and two-photons at a time from a quantum dot-photonic crystal resonator system. Controlling the detuning between emitter and cavity allows us to drive a nonlinear ladder of hybridized light-matter states.

FTu4E.2 • 16:15

Effects of Homodyne Interference on Jaynes-Cummings Emission for Single Photon Generation, Kevin Fischer ${ }^{1}$, Yousif Kelaita ${ }^{1}$, Neil Sapra ${ }^{1}$, Constantin Dory ${ }^{1}$, Konstantinos Lagoudakis', Kai Müller², Jelena Vuckovic'; 'Stanford Univ., USA; ${ }^{2}$ Dept. of Physik, Walter Schottky Institut, Germany. We investigate the effects of homodyne interference on light transmitted through a dissipative Jaynes-Cummings system and use quantum optical simulations to show how interference can dramatically improve the generation of high-quality single photons.

\section{6:00-18:00 \\ FTu4F • Quantum \\ Communications}

Presider: Claude Fabre; Universite

Pierre et Marie Curie, Paris,

France

\section{FTu4F.1・16:00}

Secret Sharing of a Quantum State, He Lu $^{1,2}$, Zhen Zhang ${ }^{3}$, Luo-Kan Chen ${ }^{1,2}$, Zheng Da Li ${ }^{1,2}$, Chang Liu ${ }^{1,2}$, Li Li ${ }^{1,2}$, Nai-Le Liu ${ }^{1,2}$ Xiongfeng $\mathrm{Ma}^{3}$, Yu-Ao Chen ${ }^{1,2}$, Jian-We Pan ${ }^{1,2}$; ' National Lab for Physical Sciences at Microscale and Dept. of Modern Physics, Univ. of Science and Technology of China China; ${ }^{2}$ CAS Center for Excellence and Synergetic Innovation Center in Quantum Information and Quantum Physics, Univ. of Science and Technology of China, China; ${ }^{3}$ Center for Quantum Information, Inst. for Interdisciplinary Information Sciences, Tsinghua Univ. China. We experimentally demonstrate $(3,3)$ threshold secret sharing scheme by employing employing a six-photon entangled state. The shared quantum secrecy can be efficiently reconstructed with a state fidelity as high as $93 \%$. Moreover, we show that any one or two parties cannot recover the secrecy.

FTu4F.2 • 16:15

Large-Alphabet Encoding Schemes for Floodlight Quantum Key Distribution, Quntao Zhuang ${ }^{1}$, Zheshen Zhang ${ }^{1}$, Jeffrey H. Shapiro'; 'MIT, USA. Floodlight quantum key distribution (FL-OKD) uses binary phaseshift keying (BPSK) of multiple optical modes to achieve Gbps secret-key rates (SKRs) at metropolitan-area distances. We show that FL-QKD's SKR can be doubled by using 32-ary PSK.

\section{6:00-18:00 \\ FTu4G • Toward Applications of Metasurfaces II}

Presider: Alexey Yamilov; Missouri Univ of Science \& Technology, USA

\section{FTu4G.1 • 16:00}

Ultrafast all-optical tuning of magnetic modes in GaAs metasurfaces, Maxim R. Shcherbakov ${ }^{1}$, Sheng Liu², Varvara Zubyuk' Aleksandr Vaskin ${ }^{3}$, Polina Vabishchevich ${ }^{1}$ Gordon Keeler², Thomas Pertsch ${ }^{3}$, Tatyana Dolgova ${ }^{1}$, Isabelle Staude ${ }^{3}$, Igal Brener ${ }^{2}$ Andrey Fedyanin 1 : 1 M. V. Lomonosov Moscow State Univ., Russia; ${ }^{2}$ Center for Integrated Nanotechnologies, Sandia National Labs, USA; ${ }^{3}$ Inst. of Applied Physics, Abbe Center of Photonics, Friedrich-Schiller Univ. Jena, Germany. We experimentally realize an ultrafast tunable metasurface consisting of subwavelength gallium arsenide nanoparticles supporting Mie-type resonances, which are all-optically tuned by $30 \mathrm{~nm}$ in spectral domain under pump fluences as low as $<400 \mu \mathrm{J} / \mathrm{cm}^{2}$.

\section{FTu4G.2・16:15}

Shared-aperture multitasking Pancharatnam-Berry phase dielectric nanoantenn array, Elhanan Maguid', Igor Yulevich ${ }^{1}$ Michael Yannai ${ }^{1}$, Vladimir Kleiner ${ }^{1}$, Mark Brongersma², Erez Hasman'; 'Technion, Israel; ' ${ }^{2}$ Geballe Lab for Advanced Materials, Stanford Univ., USA. We present the alliance of the interleaved phased antenna array and spin-enabled optics of dielectric nanoantennas incorporated within a two-dimensional nanophotonic system, enabling the detection of the fundamental properties of light.
16:00-18:00

FTu4H • Functional Plasmonics with 2D and Novel Materials Presider: Li-Jing Cheng; Oregon State Univ., USA

\section{FTu4H.1 • 16:00}

Plasmonic Resonances in Nanostructured MXene: Highly Broadband Absorber Krishnakali Chaudhuri ${ }^{1}$ Mohamed Alhabeb² Zhuoxian Wang', Vladimir M. Shalaev' ${ }^{1}$ Yury Gogotsi ${ }^{2}$, Alexandra Boltasseva'; ${ }^{1}$ Purdue Univ., USA; ${ }^{2}$ Drexel Univ., USA. Localized surface plasmon type resonances have been demonstrated in nanostructured films of recently discovered $2 \mathrm{D} \mathrm{Ti}_{3} \mathrm{C}_{2}$. A planar design of highly broadband plasmonic absorber is implemented as an application of this new plasmonic material.
FTu4H.2 • 16:15

All-angle Negative Refraction of Highly Squeezed Polaritons in Graphene-boron nitride Heterostructures, Xiao Lin 1,3 $Y_{i}$ Yang ${ }^{2}$, Nicholas Rivera ${ }^{2}$, Josue Lopez ${ }^{2}$, Yichen Shen ${ }^{2}$, Ido Kaminer ${ }^{2}$, Hongsheng Chen ${ }^{3}$, Baile Zhang ${ }^{1}$, John Joannopoulos ${ }^{2}$, Marin Soljacic ${ }^{2}$. 'Nanyang Technological Univ., Singapore; ${ }^{2}$ Physics, MIT, USA; ${ }^{3}$ ISEE, Zhejiang Univ. China. Graphene-boron nitride (BN) heterostructures provide a versatile platform to flexibly tune the sign of the group velocity of the hybrid plasmon-phonon-polaritons, enabling all-angle negative refraction between graphene plasmons, BN's phonon polaritons and their hybrid polaritons.
FTu4E.3 • 16:30

Strong Photon-Photon Interactions Mediated By a Single Quantum Dot Spin, Shuo Sun ${ }^{1}$, Zhouchen Luo', Glenn S. Solomon ${ }^{2}$ Edo Waks'; ${ }^{1}$ Univ. of Maryland, College Park, USA; ${ }^{2}$ NIST, USA. We show that the presence of a single photon deterministically controls the transmission of another photon. Thei strong interactions are mediated by a single quantum dot spin that is strongly coupled to a nanophotonic cavity.
FTu4F.3 • 16:30

Towards an Implementation of Superdense Teleportation in Space, Joseph C. Chap man ${ }^{1}$, Trent Graham ${ }^{1,2}$, Francesco Marsili ${ }^{3}$ Matthew Shaw ${ }^{3}$, Christopher Zeitler' ${ }^{1}$, Paul G. Kwiat '; ' Univ of Illinois at Urbana-Champaign, USA; ${ }^{2}$ Univ. of Wisconsin - Madison, USA; ${ }^{3} \mathrm{Je}$ Propulsion Lab, California Inst. of Technology, USA. In our effort to implement superdense teleportation (SDT) from space to earth, we have incorporated Doppler compensation methods and we installed 4 superconducting nanowire detectors so our system can operate efficiently with high loss, showing that we retain the ability to efficiently perform SDT.

\section{FTu4G.3 • 16:30 Invited}

Oxides and Nitrides for Nanophotonics and Energy Applications, Aveek Dutta ${ }^{1}$ Clayton DeVault ${ }^{1}$, Krishnakali Chaudhuri ${ }^{1}$ Soham Shah', Deesha Shah', Harsha Reddy Eragamreddy, Urcan Guler ${ }^{1}$, Alberto $\mathrm{Na}$ Idoni ${ }^{1}$, Vladimir M. Shalaev', Alexandra Boltasseva'; ${ }^{1}$ Purdue Univ., USA. We study transition metal nitrides and transparen conducting oxides as alternate plasmonic materials in the visible and near-infrared for applications such as on-chip interconnects, metasurfaces, local heating, photocatalysis and ultrafast modulation.

\section{FTu4H.3 • 16:30 Invited}

Novel Classical and Quantum Photonic Devices by Manipulating Light-matte Interactions in One and Two-Dimensional Systems, Ritesh Agarwal'; ' Univ. of Pennsylvania, USA. With an emphasis on nanowires and monolayer $\mathrm{MoS}_{2}$, we will discuss how confinement of symmetry breaking electric and plasmonic fields interacting with lowdimensional materials produces unexpected response such as emission from $\mathrm{Si}$ and induced chirality. 


\section{Meeting Room $211 \mathrm{~B} / \mathrm{D}$}

Meeting Room
$212 \mathrm{~A} / \mathrm{C}$
Meeting Room $212 \mathrm{~B} / \mathrm{D}$

\section{CLEO: Science \& Innovations}

16:00-18:00

STu4I • Ultrafast Metrology II

Presider: Tobias Witting; Max-

Born-Inst., Germany

\section{STu4I.1 • 16:00}

Single-Shot Optical Recording with SubPicosecond Resolution Implementing a Differentiated Semiconductor Nonlinearity, Ryan D. Muir ${ }^{1}$, John E. Heebner' 'Lawrence Livermore National Lab, USA We demonstrate a novel optical recording method implementing carrier-based nonlinear optical phase response in semiconductors. The integrating carrier response is mitigated with an all-optical derivative technique. A 50 ps record with sub-ps resolution was demonstrated.

STu4I.2 • 16:15

A Simple, Picojoule Sensitive Ultraviolet Autocorrelator Based on Two-Photon Conductivity in Sapphire, Kenneth Leedle ${ }^{1}$, Karel Urbanek', Robert L. Byer'; 'Stanford Univ., USA. We present a simple autocorrelator for picojoule 226-278 nm pulses from femtosecond-picosecond laser oscillators based on two-photon conductivity in sapphire. The sub-20 W peak power sensitivity is over $10 \mathrm{X}$ better than previous UV autocorrelators.

STu4I.3 • 16:30

Noiseless Spectral Amplification of Optical Frequency Combs, Luis Romero Cortes ${ }^{1}$ Reza Maram1, Hugues Guillet de Chatellus', Jose Azana'; 'INRS-EMT, Canada. We propose a technique capable of increasing the power of the lines of a frequency comb without introducing noise, and demonstrate it by resolving the lines of a comb, originally buried below the background noise.
16:00-18:00

STu4J • Microcomb Nonlinear

Optical Technology

Presider: Yoshitomo Okawachi;

Columbia Univ., USA

STu4J.1 • 16:00 Invited

A Carrier-offset-stabilized Dual Kerr Microresonator Frequency $\mathrm{Cmb}$, Scott Papp'; ${ }^{1}$ NIST, USA. We present a photonic-chip Kerr microresonator frequency comb with carrier envelope-offset frequency stabilization. The system leverages a dual reduction strategy from optical to microwave frequencies using $1 \mathrm{THz}$ and $22 \mathrm{GHz}$ repetition rate Kerr microcombs.

STu4J.2 • 16:30

Accessing octave-spanning soliton microcomb states in a thermally stable way, Qing Li $^{1,2}$, Travis C. Briles ${ }^{3}$, Daron Westly ${ }^{1}$, Tara Drake $^{3}$, Jordan R. Stone ${ }^{3}$, Bogdan $\|_{i c^{1}}$, Scott Diddams ${ }^{3}$, Scott Papp ${ }^{3}$, Kartik Srinivasan' ${ }^{1}$ CNST, NIST, USA; ${ }^{2}$ Maryland NanoCenter, Univ. of Maryland, USA; ${ }^{3}$ Time and Frequency Division, NIST, USA. We report the demonstration of octave-spanning soliton microcomb states in high- $\mathrm{Q} \mathrm{Si}_{3} \mathrm{~N}_{4}$ microresonators. These states are shown to be thermally stable and therefore can be accessed with slow frequency tuning of the pump laser.
16:00-17:45

STu4K • OAM \& Higher-Order

Mode Fibers

Presider: Peter Dragic; Univ of Illinois at Urbana-Champaign, USA

\section{STu4K.1 • 16:00}

Polarization-maintaining fiber for guiding light in large-effective-area higher-ordermodes, Raja Ahmad', Jeffrey W. Nicholson ${ }^{1}$ Kazi S. Abedin', Paul S. Westbrook', Clifford Headley', Patrick W. Wisk', Eric M. Monberg' ${ }^{1}$ Man F. Yan', David J. DiGiovanni'; ' OFS Labs, USA. We present a polarization-maintaining (PM) fiber for higher-order optical modes (HOMs) with effective area 1200-2800 $\mu \mathrm{m}^{2}$. The $L P_{08}\left(L P_{01}\right)$ mode exhibits a birefringence of $1.8 \times 10^{-4}\left(2.4 \times 10^{-4}\right)$, and propagates with $13(23) \mathrm{dB}$ polarization-extinction ratio over $1(1.3) \mathrm{m}$.

STu4K.2 • 16:15

$200 \mathrm{~nm}$ tunable acousto-optic fiber grating for OAM mode generation in the visible spectral range, Du-Ri Song ${ }^{1}$, Tao He $\mathrm{He}^{1,2}$, Lu Yan', Siddharth Ramachandran'; 'Boston Univ. , USA; ${ }^{2}$ Beijing Inst. of Technology China. We demonstrate low loss $(0.7 \mathrm{~dB})$ efficient (>90\%) tunable generation of OAM modes over a record $200 \mathrm{~nm}$ in the visible spectral range using acousto-optic fiber gratings, of utility in numerous applications requiring spectrally diverse OAM light.

STu4K.3 • 16:30

Generation of Higher-Order Orbital Angular Momentum in Polarization-Maintaining Fiber, Brendan M. Heffernan ${ }^{1}$, Robert Niederriter', Mark Siemens ${ }^{2}$, Juliet T. Gopinath ${ }^{3,1}$; 'Physics, Univ. of Colorado, Boulder, USA; ${ }^{2}$ Physics and Astronomy, Univ. of Denver, USA; ${ }^{3}$ Electrical, Computer and Energy Engineering, Univ. of Colorado, Boulder, USA. Light with orbital angular momentum of $+/-2 \mathrm{hbar}$ per photon is produced in commercially available polarization maintaining fiber with modal purity $>96 \%$. Twist measurements demonstrate that the orbital angular momentum can be continuously tuned between $+/-2 \mathrm{hbar}$
Marriott

Salon I \& II

\section{Joint}

16:00-18:00

JTu4L • Symposium on Ultrafast Laser Technology for X-ray Free Electron Lasers II

Presider: Alan Fry; SLAC National Accelerator Lab, USA

\section{JTu4L.1 • 16:00 Invited}

Photocathode Lasers for Free-Electron Lasers, Lutz Winkelmann'; 'Deutsches Elektronen-Synchrotron DESY, Germany. Freeelectron laser require precisely triggered bright electron bunches. These bunches are generated by a short laser pulse irradiating a charged photocathode. An overview on current and future laser technology for this application will be given.

\section{JTu4L.2 • 16:30 Invited}

Timing \& Synchronization of Lasers at XFELs, Ryan Coffee'. ' ${ }^{1}$ SLAC, USA. I will show preliminary results for an interference enabled cross-correlation that promises both improved signal levels and high-speed onboard processing that is expected for few-fs synchronization at high repetition rate $x$-ray free-electron lasers. 


$\begin{array}{llc}\text { Marriott } & \text { Marriott } & \text { Marriott } \\ \text { Salon III } & \text { Salon IV } & \text { Salon V \& VI }\end{array}$

\section{CLEO: Science \& Innovations}

16:00-17:30

STu4M • Nonlinear Impairments in Optical Communications

Presider: Takashi Sugihara;

Mitsubishi Electric Corporation, Japan

\section{STu4M.1 1 16:00 Invited}

Nonlinear Propagation in Fibers for Space Division Multiplexing, Cristian Antonelli ${ }^{\top}$ Antonio Mecozzi ${ }^{1}$, Ori Golani ${ }^{2}$, Mark Shtaif 'Università degli Studi dell'Aquila, Italy; ${ }^{2} \mathrm{Te}$ Aviv Univ., Israel. We review the modeling of nonlinear propagation in fibers for Space Division Multiplexing (SDM), and discuss the impact of mode coupling and modal dispersion on the nonlinear interference between the WDM channels of an SDM systems.
16:00-18:00

STu4N • Optomechanics

Presider: Paul Barclay; Univ. of Calgary, Canada

\section{STu4N.1 1 16:00 Invited}

Optomechanical Crystals at Millikelvin Temperatures, Oskar J. Painter ${ }^{1} ;{ }^{1}$ California Inst. of Technology, USA. We will present pulsed optical measurements of silicon optomechanical crystals at millikelvin temperatures. These measurements highlight the exciting new opportunities for application of microwave phononic devices in the quantum realm, but also some of the challenges.
STu4M.2 • 16:30

Impacts of Signal Nonlinearity on 106 \& 112 Gb/s PAM4 Transmission, Jinwoo Cho', Rohit Mittal', Deepthi Chakilam1, Mahan Movassaghi', Hai-Feng Liu'; ${ }^{1}$ Intel Corporation, USA. We experimentally demonstrate 106 and 112 Gbps PAM4 transmission and quantify the link penalty induced by a nonlinear MZM (Mach-Zehnder Modulator). Less than $0.5 \mathrm{~dB}$ penalty requires higher than $90 \%$ linearity.
STu4N.2・16:30

Optomechanics in Bulk Crystalline Phonon Resonators, William H. Renninger ${ }^{1}$, Prashanta Kharel', Ryan Behunin', Peter T. Rakich'; 'Yale Univ., USA. Shaping photonphonon coupling in bulk crystalline solids, we demonstrate a new paradigm for coherent optomechanical interaction with access to high frequency $(>10 \mathrm{GHz})$ ultra-high quality factor $\left(40 \times 10^{6}\right)$ phonon modes. Both experiment and theory are presented.
16:00-18:00

STu4O - Spatial and Temporal Beam Control

Presider: Jay Doster; Northrop Grumman, USA

STu40.1 • 16:00

High-Accuracy, Model-Based Near-Field Beam Shaping, Christophe Dorrer' ${ }^{1}$, Jeremy Hassett'; ' 'Univ. of Rochester, USA. Modelbased optimization via direct binary search is performed for binary pixelated beam shapers used in high-energy lasers, yielding significant reduction of beam modulation and higher resilience to fabrication errors.
STu40.2 • 16:15

High-speed Polarisation Shaping of Arbitrary Vector Beams Using a Digital Micro-mirror Device, Kevin J. Mitchell ${ }^{3}$, Sergey Turtaev ${ }^{1,2}$, Miles J. Padgett ${ }^{3}$, Tomas Cizmar', David B. Phillips'; 'School of Science and Engineering, Univ. of Dundee, UK ${ }^{2}$ School of Life Sciences, Univ. of Dundee, UK: ${ }^{3}$ School of Physics and Astronomy, Univ. of Glasgow, UK. We present the use of a digital micro-mirror device to generate and rapidly switch between vector beams with spatially controllable intensity, phase and polarisation. We demonstrate this functionality by creating radially polarised, azimuthally polarised and Poincaré beams at a frame rate of $4 \mathrm{kHz}$.

\section{STu40.3 • 16:30}

Optical Vortex with Comb-like Laser Spectra in $\mathrm{Yb}: \mathrm{YAG} / \mathrm{YVO}_{4}$ Microchip Raman Laser, Jun Dong ${ }^{1}$, Xiaolei Wang ${ }^{1}$, Xiaojie

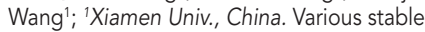
optical vortices with comb-like laser spectra have been generated in $\mathrm{Yb}: Y A G / M V O$ microchip Raman laser. Wide span comb-like laser spectra of $1.98 \mathrm{THz}$ with 30 laser lines around $1.07 \mu \mathrm{m}$ has been obtained. 
Executive Ballroom $210 \mathrm{~A}$

Executive Ballroom 210B

Executive Ballroom $210 \mathrm{C}$

\section{CLEO: Applications \& Technology}

ATu4A • Spectroscopic Sensing-Continued

\section{ATu4B • Information Transfer \& Precision Measurement Devices-Continued}

ATu4B.4 4 16:45

ATu4A.4 • 16:45

Localized Surface Plasmon Resonance Platform for Multi-point and Real-time Biosensing, Hana T. Lin ${ }^{1}$, Chi-Chen Lin ${ }^{1}$, NienTsu Huang ${ }^{1,2} ;{ }^{1}$ Graduate Inst. of Biomedical Electronics and Bioinformatics, Nationa Taiwan Univ., Taiwan; ${ }^{2}$ Dept. of Electrical Engineeering, National Taiwan Univ., Taiwan. We develop a LSPR platform composed of a nanoplasmonic sensor, a spectrometer, and a motorized stage to achieve multi-point and real-time biosensing. The assay can be done in 1 hour with $60 \mu \mathrm{L}$ sample requirement.

\section{ATu4A.5 • 17:00}

Mobile Microscope for Quantitative Fluorescence Sensing Through Highly Autofluorescent and Scattering Media, Zoltán Göröcs ${ }^{1,3}$, Yair Rivenson 1,3, Hatice Ceylan Koydemir $^{1,3}$, Derek Tseng ${ }^{1,3}$, Tamara Troy ${ }^{2}$ Vasiliki Demas ${ }^{2}$, Aydogan Ozcan 1,3: ${ }^{1}$ Electrical Engineering Dept., Univ. of California, Los Angeles, USA; ${ }^{2}$ Verily Life Sciences, LLC, USA; ${ }^{3}$ Bioengineering Dept., Univ. of California Los Angeles, USA. We created a mobile fluorescence microscope weighing $<40$ grams and demonstrated a computational sensing method for quantitative measurement of fluorescent dyes through highly autofluorescent, scattering and absorbing tissue phantoms.

ATu4A.6 $17: 15$

CMOS-based Florescence Biosensor with Integrated Nanoplasmonic Filters, Lingyu Hong ${ }^{1}$, Kaushik Sengupta ${ }^{1}$; ${ }^{1}$ Princeton Univ. USA. In this work, we present the first integrated biosensor chip, fabricated in standard CMOS technology with integrated copperbased nanoplasmonic filters that allows elimination of all external optical instruments and encompasses the sensing platform, sensors, scanner in a mm-sized CMOS chip
Demonstration of a Silicon Photonic Transceiver for Polarization-Based Discrete Variable Quantum Key Distribution, Hong Cai ${ }^{1}$, Christopher Long ${ }^{1}$, Christopher DeRose ${ }^{1}$ Nicholas Boynton ${ }^{1,2}$, Juni Urayama ${ }^{1}$, Andrew Pomerene ${ }^{1}$, Andrew Starbuck ${ }^{1}$, Douglas Trotter ${ }^{1}$, Paul Davids' ${ }^{1}$, Anthony Lentine'; ' ${ }^{1}$ Sandia National Labs, USA; ${ }^{2}$ Dept. of Electrical \& Computer Engineering, Univ. of New Mexico, USA. We demonstrate a silicon photonic transceiver circuit to implement polarization encoding/decoding for DV-QKD. The circuit is capable of encoding BB84 states with $>30$ $\mathrm{dB}$ PER and decoding with $>20 \mathrm{~dB}$ ER.

\section{ATu4B.5 $17: 00$}

Airborne Demonstration of a Quantum Key Distribution Receiver Payload, Christopher J. Pugh ${ }^{1,2}$, Sarah Kaiser ${ }^{3}$, Jean-Philippe Bourgoin ${ }^{1,2}$, Jeongwan Jin ${ }^{1,2}$, Nigar Sultana ${ }^{1,4}$, Sascha Agne ${ }^{1,2}$, Elena Anisimova ${ }^{1,2}$, Vadim Makarov ${ }^{1,2}$, Eric $\mathrm{Choi}^{5}$, Brendon Higgins ${ }^{1,2}$ Thomas Jennewein ${ }^{1,2} \cdot{ }^{1}$ Inst. for Quantum Computing, Canada; ${ }^{2}$ Physics and Astronomy, Univ. of Waterloo, Canada; ${ }^{3}$ Physics and Astronomy, Macquarie Univ., Australia, ${ }^{4}$ Electrical and Computer Engineering, Univ. of Waterloo, Canada; ${ }^{5}$ Magellan Aerospace, Canada. We demonstrate the viability of components of a quantum receiver satellite payload by successfully performing quantum key distribution in an uplink configuration to an airplane. Each component has a clea path to flight for future satellite integration.

\section{ATu4B.6・17:15}

Mode Shape Engineering of Silicon Nitride Nano-strings for Quantum Optomechanics Ryan D. Schilling ${ }^{1}$, Amir Ghadimi', Sergey Fedorov ${ }^{1}$, Hendrik Schütz ${ }^{1}$, Vivishek Sudhir ${ }^{1}$ Tobias J. Kippenberg ${ }^{1}{ }^{1}{ }^{\text {Ecole Polytechnique }}$ Federale de Lausanne, Switzerland. We detail the design and performance of silicon nitride nanostrings optimized for room-temperature quantum optomechanics. Specifically, our devices feature a low effective mass and high mechanical $Q$, for a localized highorder mode.

Executive Ballroom 210D

\section{CLEO: QELS-} Fundamental Science

\section{ATu4C • Laser Interaction with Semiconductrors, Glasses and Metals-Continued}

\section{ATu4C.3 $16: 45$}

Laser glass cutting by spatio-temporal control of energy deposition using bursts of femtosecond pulses, Konstantin Mishchik', John Lopez², O Dematteo Caulier ${ }^{2}$ Guillaume Duchateau², Bruno Chassagne ${ }^{3}$ Rainer Kling ${ }^{3}$, Clemens Hoenninger ${ }^{1}$, Eric Mottay'; ${ }^{1}$ Amplitude Systemes, France; ${ }^{2}$ Univ. of Bordeaux, France; ${ }^{3}$ Alphanov, France. We demonstrate the beneficial effect of bursts of femtosecond laser pulses for volume processing of transparent material. Nonlinear effects can be significantly reduced, and the temperature increase is sufficient to induce high mechanical stress and in-volume crack.

\section{ATu4C.4 • 17:00 Invited}

Industrialization of a Laser Produced Plasma EUV Light Source for Lithography, Igor Fomenkov ${ }^{1}$, Alex Schafgans ${ }^{1}$, Slava Rokitski', Michael Kats', Jayson Stewart ${ }^{1}$ Andrew LaForge ${ }^{1}$, Alex Ershov ${ }^{1}$, Michae Purvis', Yezheng Tao', Mike Vargas ${ }^{1}$, Jonathan Grava', Palash Das', Lukasz Urbanski', Rob Rafac', Joshua Lukens', Chirag Rajyaguru' Georgiy Vaschenko', Matthew Abraham ${ }^{1}$ David Brandt', Daniel Brown ${ }^{1} ;{ }^{1}$ Cymer, an ASML company, USA. ASML is committed to develop high power EUV source technology for use in EUV lithography for high-volumemanufacturing (HVM) of semiconductors. A stable dose controlled Laser-Produced Plasma (LPP) EUV source has been successfully developed and introduced using a $\mathrm{CO}_{2}$ laser and small tin (Sn) droplets.

\section{FTu4D • On-chip Quantum Optics-Continued}

\section{FTu4D.4 • 16:45}

Spectral Compression of Single Photons Coherent Pulse, Yuanhua Li', Tong Xiang', Yiyou $\mathrm{Nie}^{2}$, Minghuang Sang ${ }^{2}$, Xianfeng Chen'; 'Shanghai Jiao Tong Univ., China; ${ }^{2}$ Jiangxi Normal Univ., China. We experimentally demonstrate that the spectrum of single photons coherent pulse is compressed by a factor of 58 in a periodically poled lithium niobate waveguide chip. The frequency and bandwidth of single photons are simultaneously converted.

FTu4D.5 • 17:00

Highly efficient frequency conversion with bandwidth compression of quantum light, Markus Allgaier ${ }^{1}$ Vahid Ansari ${ }^{1}$, Linda Sansoni ${ }^{1}$, Christof Eigner' ${ }^{1}$, Viktor Quiring', Raimund Ricken', Georg Harder', Benjamin Brecht ${ }^{1,2}$, Christine Silberhorn ${ }^{1}$; ${ }^{2}$ Univ. of Paderborn, Germany; ${ }^{2}$ Clarendon Lab, Univ. of Oxford, UK. We demonstrate an engineered sum-frequency-conversion process in lithium niobate that provides a bandwidth compression factor of 7.47 at a high efficiency of 61.5 $\%$, thus outperforming spectral filtering. The process preserves non-classical photonnumber statistics.

(17:15

Smith-Purcell radiation in periodic nanostructures: quantum effects and applicability, Shai Tsesses', Guy Bartal', Ido Kaminer². 'Technion-Israeli Inst. of technology, Israel; ${ }^{2}$ MIT - MIT, USA. We show that, using nano metric periodic structures, non-relativistic particles can generate light via Smith-Purcell radiation, ranging from Terahertz to visible; in this regime, quantum corrections become nevitable and lead to new effects. 


\section{CLEO: QELS-Fundamental Science}

\section{FTu4E • Quantum Dot Cavity QED-Continued}

\section{FTu4E.4 • 16:45}

Efficient deterministic giant photon phase shift from a single charged quantum dot, Petros Androvitsaneas ${ }^{1}$, Andrew Young ${ }^{1}$, Joseph Lennon ${ }^{1}$, Christian Schneider ${ }^{2}$ Sebastian Maier ${ }^{2}$, Janna Hinchliff', George Atkinson', Edmund Harbord', Martin Kamp ${ }^{2}$ Sven Hoefling ${ }^{2,3}$, John Rarity ${ }^{1}$, Ruth Oulton'; Univ. of Bristol, UK; ${ }^{2}$ Universität Würz burg, Germany; ${ }^{3}$ Univ. of St Andrews, UK We demonstrate a deterministic shift in phase of an input single photon by a negatively charged quantum dot in a low $\mathrm{Q}$-factor, high output efficiency micropillar cavity, with values up to $2 \pi / 3$.

\section{FTu4E 5 • 17:00}

Bright and Coherent On-Chip Single Photons from a Very High Purcell Factor Photonic Crystal Cavity, Alistair Brash ${ }^{1}$ Feng Liu', John O'Hara', Luis Martins', Rikk J. Coles' ${ }^{1}$, Catherine L. Phillips' ${ }^{1}$, Ben Royall' Christopher Bentham', Igor E. Itskevich², Luke Wilson', Maurice S. Skolnick', Mark Fox'; 'Dept. of Physics and Astronomy, Univ. of Sheffield, UK; ${ }^{2}$ School of Engineering and Computer Science, Univ. of Hull, UK. Using a novel two-pulse resonance fluorescence technique we demonstrate a Purcell factor of $\sim 35$ in a cavity quantum dot system. Highly coherent single photons are efficiently emitted into a waveguide, forming a near-ideal source for integrated quantum circuits.

\author{
FTu4F • Quantum \\ Communications-Continued
}

\section{FTu4G • Toward Applications of Metasurfaces II-Continued}

FTu4H • Functional Plasmonics with 2D and Novel MaterialsContinued
FTu4F.4 • 16:45

Superdense codes transmitted over optical fiber links decoded deterministically using time-polarization hyperentanglement Brian P. Williams', Travis S. Humble'; ${ }^{1} \mathrm{O} a k$ Ridge National Lab, USA. We demonstrate superdense coding over optical fiber using time-polarization hyperentanglement, linear optics, and common single-photon detec tors. We achieve the highest single-qubit channel capacity to date encoding on a single-degree of freedom with linear optic decoding.

\section{FTu4F.5 17:00 Invited}

Battling with Quantum Hackers, HoiKwong Lo': 'Univ. of Toronto, Canada. Quantum hacking threatens the security of practical quantum key distribution (QKD) systems. Fortunately, measurement-deviceindependent(MDI) QKD automatically foils all attacks on detectors. I will survey MDI-QKD together with other recent progress on QKD security research.
FTu4G.4 • 17:00

Tunable Metasurfaces using Alkali Vapors, Jonathan Bar-David', Liron Stern', Uriel Levy ${ }^{1}$. ${ }^{1}$ Hebrew Univ of Jerusalem, Israel. We use the circular dichroism of Alkali vapors to tune the optical transmission of metasurfaces. The output of the metasurface system is controlled by applying magnetic fields, changing input polarization or shifting the optical frequency.
FTu4E.6 • 17:15

Photoluminescence imaging based nanopositioning of single quantum dots for high-performance single-photon generation, Jin Liu',3, Yu-ming $\mathrm{He}^{2}$, Luca Sapienza ${ }^{4}$ Kumarasiri Konthasinghe ${ }^{5}$, Stephan Gerhardt², Jose Vinicius De Miranda Cardoso', Jin Dong Song ${ }^{6}$, Antonio Badolato ${ }^{7}$, Christian Schneider ${ }^{2}$, Sven Höfling ${ }^{2}$, Marcelo I. Davanco ${ }^{1}$, Kartik Srinivasan ${ }^{1} ;{ }^{1}$ Center for Nanoscale Science and Technology, National Inst. of Standards and Technology, USA ${ }^{2}$ Technische Physik and Wilhelm Conrad Röntgen Research Center for Complex Material Systems, Physikalisches Institut, Universität Würzburg, Germany; ${ }^{3}$ Dept. of Physics, Sun-Yat Sen Univ., China; ${ }^{4}$ Dept. of Physics and Astronomy, Univ. of Southampton, UK ${ }^{5}$ Dept. of Physics, Univ. of South Florida, USA ${ }^{6}$ Center for Opto-Electronic Convergence Systems, Korea Inst. of Science and Technology, Korea (the Republic of); ${ }^{7}$ Dept. of Physics and Astronomy, Univ. of Rochester, USA. We present a wide-field, high-throughput optical technique for locating solid-state quanutm emitters with $<10 \mathrm{~nm}$ accuracy, and apply it in the creation of micropillar sources with near-optimal single-photon emission.
FTu4G.5 • 17:15

Metasurface Terahertz Laser With Electronically-Controlled Polarization, Daguan Chen', Luyao Xu1, ${ }^{1,2}$ Christopher Curwen ${ }^{1,2}$ Mohammad Memarian', John Reno ${ }^{3}$, Tat suo Itoh', Benjamin Williams ${ }^{1,2}$; ${ }^{1}$ Dept. of Electrical Engineering, Univ. of California, Los Angeles, USA; ${ }^{2}$ California NanoSystems Inst., Univ. of California, Los Angeles, USA ${ }^{3}$ Sandia National Labs, Center of Integrated Nanotechnologies, USA. We report a terahertz metasurface quantum-cascade VECSEL laser without moving parts that can electronically switch between near-orthogonal linearly polarized output. It exhibits excellent beam pattern, single-mode operation, and power up to $93 \mathrm{~mW}$ at $77 \mathrm{~K}$

FTu4H.4 • 17:00

Random Perfect Absorption in 2D Atomic Layers on All-Dielectric Substrates Mediated by Anderson Localization, Judson Ryckman ${ }^{1} ;{ }^{1}$ Holcombe Dept. of Electrical and Computer Engineering, Clemson Univ., USA I present an approach for achieving perfect absorption in 2D atomic layers utilizing randomized dielectric layers. The emergence of high Q optical modes featuring $>99.9 \%$ absorption in single layer graphene is shown.

FTu4H.5 • 17:15

Plasmonic Antenna Resonance Pinning and Suppression of Near-Field Coupling from Epsilon-Near-Zero Substrate, Clayton DeVault ${ }^{1}$, Vladimir Zenin ${ }^{2}$, Anders Pors ${ }^{2}$ Jonngbum Kim ${ }^{1}$, Krishnakali Chaudhuri ${ }^{1}$ Sergey Bozhevolnyi', Vladimir M. Shalaev' Alexandra Boltasseva': 'Purdue Univ. USA ${ }^{2}$ Southern Denmark Univ., Denmark. The resonance wavelength of single gold nanorods patterned on an epsilon-near-zero substrate is observed to be independent of antenna length. Additionally, the near-field coupling between dimer antennas is suppressed at the epsilon-near-zero wavelength. 


\section{Meeting Room $211 \mathrm{~B} / \mathrm{D}$}

Meeting Room $212 \mathrm{~A} / \mathrm{C}$
Meeting Room

$212 \mathrm{~B} / \mathrm{D}$

\section{CLEO: Science \& Innovations}

STu4I • Ultrafast Metrology IIContinued

\section{STu4I.4 • 16:45}

How Short should your Nonlinear Crystal be for Pulse Diagnostic?, Ning Hsu', JeanClaude M. Diels ${ }^{1}$; 'Univ. of New Mexico, USA. Ultrashort pulse diagnostic require a nonlinear crystal satisfying the conflicting requirements of fidelity in reconstruction and efficiency. It is shown that correct autocorrelations can be obtained with longer crystals than generally assumed.

\section{STu4I.5 • 17:00}

Single-Shot Measurement of TemporallyDependent Polarization State of Femtosecond Pulses by Angle-Multiplexed Spectral-Spatial Interferometry, Ming-wei Lin', Igor Jovanovic; ${ }^{1}$ Inst. of Nuclear Engineering and Science, National Tsing Hua Univ., Taiwan; ${ }^{2}$ Dept. of Nuclear Engineering and Radiological Sciences, Univ. of Michigan, USA. Various temporally-dependent polarization states of ultrashort laser pulses have been reconstructed in a single shot measurement by angle-multiplexed spatial-spectral interferometry.

\author{
STu4J • Microcomb Nonlinear \\ Optical Technology-Continued
}

STu4J.3 • 16:45

Soliton Kerr Frequency Combs with Octave Bandwidth in Integrated $\mathrm{Si}_{3} \mathrm{~N}_{4}$ Microresonators, Martin Pfeiffer' ${ }^{1}$ Junqiu Liu', Clemens Herkommer', Hairun Guo', Erwan Lucas ${ }^{1}$ Maxim Karpov ${ }^{1}$, Michael Zervas ${ }^{1}$, Michae Geiselmann', Tobias J. Kippenberg'; ' ${ }^{1}$ cole Polytechnique Federale de Lausanne, Switzerland. Coherent Kerr soliton frequency combs with $200 \mathrm{THz}$ spectral bandwidth are generated in high-Q silicon nitride microresonators pumped at $1.3 \mu \mathrm{m}$ wavelength. We discuss design and wafer-scale fabrication of such microresonators enabling on-chip self-referencing at low powers.

STu4J.4 $\bullet$ 17:00

Initiating Kerr-Soliton Frequency Combs Apart from Thermal Bistability and Mode Perturbation Effects, Jordan R. Stone ${ }^{1}$ Travis Briles', Tara Drake', Daryl Spencer ${ }^{1}, X^{1}$ ' $\mathrm{Yi}^{2}$, Ki Y. Yang ${ }^{2}$, Kerry Vahala ${ }^{2}$, Scott Diddams ${ }^{1}$ Scott Papp'; ' NIST, USA; ${ }^{2}$ Caltech, USA We implement an optical single-sideband suppressed-carrier modulator with a Kerr microcomb pump laser. Rapid frequency scanning enables exploration of Kerr-comb physics, and practically reduces its sensitivity to challenging resonator thermal bistability and mode-structure imperfection effects.

\section{STu4K • OAM \& Higher-Order Mode Fibers-Continued}

Marriott

Salon I \& II

\section{Joint}

JTu4L • Symposium on Ultrafast Laser Technology for X-ray Free Electron Lasers II-Continued

\section{STu4K.4 • 16:45}

Erbium-Doped Fiber Amplifier for OAM Modes Using an Annular-Core Photonic Lantern, Ning Wang ${ }^{1}$, Z. Sanjabi Eznaveh ${ }^{1}$ Juan Carlos Alvarado Zacarias', Jose E. Antonio-Lopez ${ }^{1}$, Sergio Leon-Saval2 ${ }^{2}$, Pierre Sillard ${ }^{4}$, Cedric Gonnet ${ }^{4}$, Axel Schülzgen ${ }^{1}$ Guifang Li ${ }^{1,3}$, Rodrigo A. Correa'; 'Univ. of Central Florida, USA: ${ }^{2}$ The Univ. of Sydney, Australia; ${ }^{3}$ Tianjin Univ., China; ${ }^{4}$ Prysmian Group, France. We experimentally demonstrated an erbium-doped fiber amplifier for OAM modes using an annular-core photonic lantern. The small signal gain for OAM modes with $|\mathrm{L}|=1$ and 2 are obtained to be $22.1 \mathrm{~dB}$ and $16.7 \mathrm{~dB}$.

\section{STu4K.5 • 17:00}

High energy, radially polarized picosecond laser pulses from a Yb-doped fiber MOPA Di Lin ${ }^{1}$, Neda Baktash ${ }^{1}$, Shaif-ul Alam ${ }^{1}$, David Richardson'; ' 'Univ. of Southampton, UK. We report the generation of radially polarized laser pulse from a gain-switched diode-seeded Yb-doped fiber MOPA system delivering $110 \mathrm{ps}$ pulses at a repettion rate of $1.367 \mathrm{MHz}$ with up to $25.8 \mu \mathrm{J}$ pulse energy.
STu4l.6 • 17:15

High Resolution Single-shot Time Stretch Spectroscopy with Wavelength Demultiplexer at One Billion Frames per Second, Takeshi Makino', Hideaki Furukawa', Mohammad Asghari², Paul Trinh², Bahram Jalali ${ }^{3}$, Xiaomin Wang ${ }^{4}$, Tetsuya Kobayashi ${ }^{4}$, Wa Man ${ }^{5}$, Kwong Tsang ${ }^{5}$, Naoya Wada'; ${ }^{1}$ Nationa Inst Information \& Comm Tech, Japan; ${ }^{2}$ Time Photonics Inc., USA; ${ }^{3}$ Univ. of California, Los Angeles, USA; ${ }^{4}$ Optoquest, Japan; ${ }^{5}$ Amonics Ltd., Hong Kong. To improve spectral resolution or capturable pulse-repetition-rate, we introduce a wavelength demultiplexing technique into 1-GHz pulse-by-pulse singleshot spectrum measurement based on time stretch dispersive Fourier Transform. Three times higher resolution of conventional one is achieved.
STu4J.5 • 17:15

Broadband Frequency Comb Generation in the Near-Visible using Higher-Order Modes in Silicon Nitride Microresonators, Prathamesh Donvalkar ${ }^{2,1}$, Felippe A. Barbosa $^{3}$, Xingchen $\mathrm{Ji}^{3,4}$, Yoshitomo Okawachi ${ }^{2}$, Rees Mcnally ${ }^{5}$, Alessandro Farsi ${ }^{2}$, Alexande Klenner $^{2}$, Michal Lipson ${ }^{3}$, Alexander L. Gaeta2; 'Applied and Engineering Physics, Cornell Univ., USA; ${ }^{2}$ Applied Physics and Applied Mathematics, Columbia Univ. , USA ${ }^{3}$ Electrical Engineering, Columbia Univ., USA; ${ }^{4}$ School of Electrical and Computer Engineering, Cornell Univ. , Namibia; ${ }^{5}$ Dept. of Physics, Columbia Univ., USA. We demonstrate frequency comb generation in the visible optical spectrum via excitation of higher-order modes in silicon nitride microresonators. Anomalous group-velocity dispersion from the higher-order mode allows for broadband comb generation spanning $45 \mathrm{THz}$.

\section{STu4K.6 • 17:15}

Passively Q-switched erbium fiber laser using few-mode fiber long-period grating and carbon nanotube for cylindrical vector beam generation, Tianxing Wang ${ }^{1}$, Yunhe Zhao 1,2, Changle Wang ${ }^{2}$, Zuyao Liu' Chengbo Mou', Yunqi Liu', Mohammed AlAraimi2,3, Aleksey Rozhin 2,4, Raz Arif5, Lin Zhang', Tingyun Wang'; 'Shanghai Univ., China; ${ }^{2}$ Aston Inst. of Photonic Technolo-

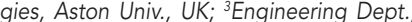
Al Musanna College of Technology, Oman; ${ }^{4}$ Nanoscience Research Group, Aston Univ. UK; ${ }^{5}$ Physics Dept., Univ. of Sulaimani, Iraq. We have demonstrated an all-fiber erbium laser incorporating a few-mode fiber longperiod grating passively Q-switched by carbon nanotube for cylindrical vector beam generation. The laser can output both radially and azimuthally polarized beams.
JTu4L.3 • 17:00

Large-Scale Turnkey Timing Distribution System for Attosecond Photon Science Facilities, Kemal Shafak ${ }^{1,2}$, Haynes Pak Hay Cheng ${ }^{2}$, Johann Derksen ${ }^{1,2}$, Damian N. Schimpf', Andrej Berg', Andrej Berlin², Erwin $\mathrm{Cano}^{2}$, Anan Dai ${ }^{2}$, Dariush Forouher ${ }^{2}$ Aram Kalaydzhyan ${ }^{1}$, Joachim Meier ${ }^{1,3}$, Wahid Nasimzada², Mathias Neuhaus², Philipp Schiepel ${ }^{2}$, Eduard Seibel ${ }^{2}$, Thomas Tilp ${ }^{1}$ Franz Kaertner 1,3: ${ }^{1}$ Center for Free-Electron Laser Science, Germany; ${ }^{2} \mathrm{Cycle} \mathrm{GmbH}$ Germany; ${ }^{3}$ The Hamburg Center for Ultrafast Imaging, Hamburg Univ., Germany. We report a turnkey timing distribution system able to serve multiple remote optical and microwave sources. The system exhibits only $0.3-f s$ timing jitter at the outputs of stabilized fiber links.

\section{JTu4L.4 • 17:15 Invited}

Laser-based Soft X-ray FEL Seeding: Recent Advances and Outlook at FERMI, Miltcho B. Danailov', Paolo Cinquegrana', Alexander Demidovich', Gabor Kurdi', Ivaylo Nikolov', Paolo Sigalotti'; 'Elettra-Sincrotrone Trieste S.C.P.A., Italy. The talk describes the status, recent progress and near future plans in the Free Electron Laser seeding by ultrashort UV pulses implemented at FERMI as a root to generate XUV pulses with very exciting properties 


$\begin{array}{llc}\text { Marriott } & \text { Marriott } & \text { Marriott } \\ \text { Salon III } & \text { Salon IV } & \text { Salon V \& VI }\end{array}$

\section{CLEO: Science \& Innovations}

STu4M • Nonlinear Impairments in Optical CommunicationsContinued

\section{STu4M.3 • 16:45}

Nonlinear Fourier Based Spectral Filtering, Morteza Kamalian Kopae ${ }^{1}$, Jaroslaw E. Prilepsky', Stanislav A. Derevyanko², Son T. LE $^{3}$, Sergei Turitsyn ${ }^{1} ;{ }^{1}$ Aston Inst. of Photonic Technologies, Aston Univ., UK; ${ }^{2}$ Electrical and Computer Engineering, Ben-Gurion Univ. of the Negev, Israel; ${ }^{3}$ Nokia Bell Labs, Germany. The new concept of filtering the nonlinear spectrum of signal in order to reduce the noise-induced signal degradation is introduced and, as the result, the performance improvement at high powers is demonstrated.

STu4M.4 • 17:00 Invited

Nonlinearity-tolerant modulation formats at 3.5 bits/symbol, Keisuke Kojima' Tsuyoshi Yoshida², Toshiaki Koike-Akino', David S. Millar ${ }^{1}$, Keisuke Matsuda², Kieran Parsons ${ }^{1} ;{ }^{1}$ Mitsubishi Electric Research Labs, USA; Information Technology R\&D Center, Mitsubishi Electric Corp., Japan. We propose two nonlinearity-tolerant high dimensional modulation format for 3.5 bits/symbol, which can also be an alternative for PS-QPSK (3 bits) symbol) or DP-QPSK (4 bits/symbol).

\section{STu4N • Optomechanics- Continued}

\section{STu4N.3 $16: 45$}

Optimal Coupling in Cavity Optomechanical Systems, Marcel W. Pruessner ${ }^{1}$, Todd H. Stievater', Doewon Park', Christopher L. Panuski ${ }^{2,1}$, William S. Rabinovich'; ' $U$ S Naval Research Lab, USA: ${ }^{2}$ U.S. Naval Academy, USA. We analyze a cavity optomechanical system suitable for foundry-level fabrication. Contrary to many structures, in which only the cavity design is optimized, we find an optimal coupling strength resulting in maximized optomechanical interaction and readout.

\section{STu4O - Spatial and Temporal Beam Control-Continued}

STu40.4 1 16:45

Selective Spatial Mode Excitation and Amplification in Ho:YAG Single Crystal Fiber, Yuan Li ${ }^{1}$, Wenzhe Li ${ }^{1}$, Keith Miller ${ }^{1}$, Eric Johnson ${ }^{1}$, Craig Nie ${ }^{2}$, James A. Harrington ${ }^{2}$. ${ }^{1}$ Clemson Univ., USA; ${ }^{2}$ Rutgers Univ., USA. Different spatial modes, $L P_{01}$ and $L P_{21}$, were successfully excited and amplified in a $0.5 \%$ Ho:YAG single crystal fiber (SCF) amplifier fabricated by the laser heated pedestal growth (LHPG) method at $2 \mu \mathrm{m}$.
STu4N.4 $17: 00$

Optomechanics with hybrid carbon nanotube resonators, Alexandros Tavernarakis ${ }^{1}$, Alexandros Stavrinadis', Alex Nowak', loannis Tsioutsios', Adrian Bachtold', Pierre Verlot ${ }^{1,2}$; ${ }^{1}$ CFO, Spain; ${ }^{2}$ Institut Lumiere Matiere, France. In this paper we report our first experimental results towards the establishement of a novel nanooptomechanical platform consisting of a hybrid, carbon nanotube-based mechanical resonator.
STu40.5 • 17:00

An Apodized-Imaged Hartmann Mask for Quantitative Wavefront Measurements in Laser Systems, Christophe Dorrer', Adam Kalb1, Kyle Gibney', Archana Sharma', Seung-Whan Bahk'; 'Univ. of Rochester, USA A Hartmann mask with apodized holes is demonstrated for in-situ wavefront characterization in laser systems with existing near-field imaging diagnostics. Spatially dithered binary pixelated apertures alleviate diffraction effects and improve the measurement accuracy.
STu4N.5 • 17:15

Optomechanical Nanobeam Cavity with High Q Factor due to Optical Spring Effect in Ambient Environment, Guoren Bai ${ }^{1}$, Kaiyu Cui', Zhilei Huang ${ }^{1}$, Xue Feng', Yidong Huang ${ }^{1}$, Fang Liu', Wei Zhang ${ }^{1}$; ${ }^{1}$ Dept. of Electronic Engineering, Tsinghua National Lab for Information Science and Technology, Tsinghua Univ., China. High Q optomechanical cavity is demonstrated based on silicon nanobeam structure with mechanical frequency of $4.52 \mathrm{GHz}$. The mechanical $Q$ can reach 24500 with the help of optical spring effect, which is record-high in ambient environment.
STu40.6 • 17:15

Gain-dependent Self-phasing in a Coherently Combined Fiber Laser with Imbalanced Losses, Mint Kunkel ${ }^{1}$, James R. Leger ${ }^{1}$. 'Univ. of Minnesota, USA. The individual beams in a coherently combined two-core fiber laser are influenced by mismatched loss and phase. Interplay between the gain dependent (Kramers-Kronig) phase and gain saturation determines the complex fields. Experiment and theory are compared. 
Executive Ballroom $210 \mathrm{~A}$

Executive Ballroom 210B

Executive Ballroom $210 \mathrm{C}$

\section{CLEO: Applications \& Technology}

ATu4A • Spectroscopic Sensing-Continued

ATu4A.7 - 17:30

Ultrasensitive Spectroscopy Based on Integrated Photonic Waveguides on $\mathrm{Al}_{2} \mathrm{O}_{3}$ / $\mathrm{SiO}_{2}$ Platform, Elham Heidari ${ }^{1}$, Xiaochuan $\mathrm{Xu}^{2}$, Lijun Huang ${ }^{1}$, Naimei Tang ${ }^{2}$, Ray T. Chen ${ }^{1,2} ;{ }^{1}$ Univ. of Texas at Austin, USA; ${ }^{2}$ Omega Optics Inc., USA. Integrated photonic waveguides on $\mathrm{Al}_{2} \mathrm{O}_{3} / \mathrm{SiO}_{2}$ platform are proposed to cover the 220 320nm wavelengthrange, which is of paramount significance in protein and nuclei acid quantification. The proposed system requires $500 x$ less volume of solutions compared with NanoDrop ${ }^{\mathrm{TM}}$.

\section{ATu4B • Information Transfer \& Precision Measurement Devices-Continued}

ATu4B.7 • 17:30

Extension of measurement range for Sagnac Loop Interferometer sensor based on birefringence interrogation, $\mathrm{Haijun} \mathrm{He}^{1}$, Liyang Shao', Lianshan Yan ${ }^{1}$, Xinpu Zhang ${ }^{1}$ Heng Qian', Jiawei Liang'; 'Southwest Jiaotong Univ., China. A novel birefringence measurement method was developed to interrogate the Sagnac loop interferometer based sensor. The response is linearized in full sensing range and not restricted by the free spectrum range.
ATu4A.8 • 17:45

A Small Aperture Terahertz Chip for Ultratrace Blood Glucose Level Measurement, Kazunori Serita', Kosuke Okada', Iwao Kawayama ${ }^{1}$, Hironaru Murakami', Masayoshi Tonouchi'; 'Osaka Univ., Japan. A small aperture terahertz chip was demonstrated for ultra-trace human blood measurement. The obtained refractive index decreases with an increase of blood glucose level and shows our chip can be worked as a terahertz glucometer.
ATu4B.8 • 17:45

Electrically tunable whispering gallery mode resonator based on liquid-crystalinfiltrated silica capillary, Chengkun Yang ${ }^{1}$ Bo Liu', Hao Zhang', Haifeng Liu'1; 'Inst. of Modern Optics, Nankai Univ., China. A tunable whispering gallery mode (WGM) resonator based on nematic-liquid-crystalinfiltrated silica capillary is presented in this paper. WGM wavelength shift is achieved by exploiting the birefringence effect of liquid crystal triggered by applied electric field.
ATu4C • Laser Interaction with Semiconductrors, Glasses and Metals-Continued

\section{ATu4C.5 • 17:30}

Comparison of Two-Temperature and Thermal Models for Prediction of the Optimal Femtosecond Laser-Material Processing of Silicon, Ryan E. Scott ${ }^{1}$, Lauren L. Taylor ${ }^{1}$, Jie Qiao': ' ${ }^{1}$ Rochester Inst. of Technology, USA A thermal and a two-temperature mode (TTM) describing femtosecond laser-materia interactions are compared. Both models accurately describe thermal response of silicon to multi-pulse irradiations, while the TTM distinguishes between thermal and nonthermal regimes.

Executive Ballroom 210D

\section{CLEO: QELS-} Fundamental Science

\section{FTu4D • On-chip Quantum} Optics-Continued

\section{FTu4D.7 • 17:30}

Demonstration of Local Teleportation Using Classical Entanglement, Diego GuzmanSilva ${ }^{1}$, Robert Brüning ${ }^{1}$, Felix Zimmermann ${ }^{1}$ Christian Vetter ${ }^{1}$, Markus Gräfe', Matthias Heinrich ${ }^{1}$, Stefan Nolte ${ }^{1}$, Michael Duparré ${ }^{1}$ Andrea Aiello², Marco Ornigotti', Alexande Szameit ${ }^{3} ;{ }^{1}$ Friedrich-Schiller-Universität Jena, Germany; ${ }^{2}$ Max Plank Inst. for the Science of Light, Germany; ${ }^{3}$ Univ. of Rostock, Germany. We report on an optical implementation of the teleportation protocol in the classical realm, solely based on entanglement between spatial and modal degrees of freedom of a purely classical light field.

ATu4C.6 • 17:45

The Coloring and Color Enhancement of Noble Metals via Multi-Burst Picosec ond Pulses, Jean-Michel Guay', Antonio Cala'Lesina', Joshua Baxter', Peter Gordon², Sean Barry², Lora Ramunno', Pierre Berini ${ }^{1}$ Arnaud Weck'; 'Univ. of Ottawa, Canada ${ }^{2}$ Carleton Univ. Canada. We report on the fast angle-independent coloring of noble metals. We show the passivation and colo tuning of the colored surfaces via the deposition of thin layers of aluminum oxide by atomic layer deposition (ALD)
FTu4D.8 • 17:45

Four-Wave-Mixing Comb Spectroscopy, Bachana Lomsadze ${ }^{1,2}$, Steven T. Cundiff', 'Univ. of Michigan, USA; ${ }^{2}$ JILA, Univ. of Colorado \& NIST, USA. We experimentally demonstrate four-wave-mixing spectroscopy based on optical frequency combs. We use a co-linear excitation geometry and demonstrate separation of the linear and four-wavemixing signals using heterodyne detection with a local oscillator comb. 


\section{Executive Ballroom $210 \mathrm{E}$}

Executive Ballroom
$210 \mathrm{~F}$
Executive Ballroom

210G
Executive Ballroom

$210 \mathrm{H}$

\section{CLEO: QELS-Fundamental Science}

\section{FTu4E • Quantum Dot Cavity QED-Continued}

\section{FTu4E.7 • 17:30}

Mode switching in bimodal microcavities and its connection to Bose condensation, Heinrich A. Leymann ${ }^{1,2}$, Daniel Vorberg', Thomas Lettau², Caspar Hopfmann ${ }^{3}$, Christian Schneider ${ }^{4}$, Martin Kamp ${ }^{4}$, Sven Höfling ${ }^{4,5}$ Roland Ketzmerick ${ }^{1,6}$, Jan Wiersig ${ }^{2}$, Stephan Reitzenstein ${ }^{3}$, Andre Eckardt'; ' Max Planck Inst. for the Physics of Complex Systems, Germany; ${ }^{2}$ Inst. for theoretical physics, Ottovon-Guericke Univ. Magdeburg, Germany; ${ }^{3}$ Institut für Festkörperphysik, Technical Univ. Berlin, Germany: ${ }^{4}$ Technische Physik, Univ. of Würzburg, Germany; ${ }^{5}$ Univ. of St Andrews, UK; ${ }^{6}$ Technical Univ. Dresden, Germany. We introduce an analytical theory for mode switching in a microcavity, which shows excellent agreement with experiment and numerics. This switching process is triggered by the intermode kinetics and resembles the physics of Bose condensation.

\section{FTu4E.8 • 17:45}

Giant Photon Bunching and Quantum Correlations in Superradiant QuantumDot Microcavity Lasers, Jan Wiersig ${ }^{1}$ Alexander Foerster ${ }^{1}$, Alexander Leymann ${ }^{2}$ Frank Jahnke ${ }^{3}$, Christopher Gies ${ }^{3}$, Marc Aßmann ${ }^{4}$, Manfred Bayer ${ }^{4}$, Christian Schneider $^{5}$, Martin Kamp ${ }^{5}$, Sven Höfling ${ }^{5}$; ${ }^{1}$ Inst. for Theoretical Physics, Otto-von-Guericke Univ. Magdeburg, Germany; ${ }^{2}$ Max Planck Inst. for the Physics of Complex Systems, Germany; 3 Inst. for Theoretical Physics, Univ. of Bremen, Germany; ${ }^{4}$ Experimentelle Physik II, Technische Universität Dortmund, Germany; ${ }^{5}$ Technische Physik, Julius-Maximilians-Universität Würzburg, Germany. Using semiconductor quantum dots in a cavity-quantum electrodynamics laser we show a direct connection between superradiant pulse emission and the photon correlations. This demonstrates the importance of quantum correlations in novel optoelectronic devices.

\section{FTu4F • Quantum \\ Communications-Continued}

FTu4F.6 • 17:30

Stabilization of Long, Deployed Optical Fiber Links for Quantum Networks, Matthew E. Grein', Mark L. Stevens', Nicholas D. Hardy' ${ }^{1}$ P. B. Dixon'; ${ }^{1}$ Massachusetts Inst of Tech Lincoln Lab, USA. We implemented an active feedback loop to compensate pathlength drift on a deployed $\sim 84-\mathrm{km}$-long optical fiber link between Lincoln Lab and MIT to enable quantum networking measurements and applications.

FTu4F.7 • 17:45

Directly Intensity-Modulated Quantum Key Distribution, George L. Roberts 1,2 Marco Lucamarini², James F. Dynes ${ }^{2}$, Seb J. Savory ${ }^{1}$ Zhiliang Yuan ${ }^{2}$, Andrew J. Shields ${ }^{2}$. ${ }^{1}$ Engineering Dept., Cambridge Univ., UK ${ }^{2}$ Toshiba Research Europe Limited, UK. The coherent one-way (COW) protocol is implemented using direct laser modulation, with security enabled by optical injection locking. This method generates secure keys at rates above $1 \mathrm{Mbit} / \mathrm{s}$ with interference visibilities over $98 \%$.

17:00-18:30 Meet the OSA Editors' Reception, Market Terrace

\section{FTu4G • Toward Applications of Metasurfaces II-Continued}

FTu4G.6 • 17:30

Effect of Strong Coupling on Photodegradation of the p3ht Semiconducting Polymer, Vanessa N. Peters', M. O. Faruk ${ }^{1}$, Rohan Alexander ${ }^{1,2}, D^{\prime}$ Angelo A. Peters ${ }^{3}$ Mikhail Noginov': ${ }^{1}$ Norfolk State Univ., USA ${ }^{2} \mathrm{School}$ of Engineering, Univ. of Michigan, USA; ${ }^{3}$ College of Science, Purdue Univ., USA. We have studied photodegradation of the semiconducting polymer p3ht in the resonant cavity and the control samples. The nearly three-fold reduction of the reaction rate is attributed to the strong polymercavity coupling.

\section{FTu4G.7 • 17:45}

Active Metamaterials Based on Monolayer Titanium Carbide MXene for Random Lasing, Zhuoxian Wang ${ }^{1}$, Xiangeng Meng ${ }^{1}$ Krishnakali Chaudhuri' ${ }^{1}$ Mohamed Alhabeb² Shaimaa I. Azzam ${ }^{1}$, Alexander V. Kildishev' Young L. Kim³', Vladimir M. Shalaev'1, Yury Gogotsi ${ }^{2}$, Alexandra Boltasseva'; ${ }^{1}$ Schoo of Electrical and Computer Engineering, and Birck Nanotechnology Center, Purdue Univ., USA; ${ }^{2}$ Dept. of Materials Science and Engineering, and A. J. Drexel Nanomaterials Inst., Drexel Univ., USA; ${ }^{3}$ Weldon Schoo of Biomedical Engineering, Purdue Univ. USA. We present an approach employing a random metamaterial constructed by dispersing monolayer $\mathrm{Ti}_{3} \mathrm{C}_{2}$ nanoflakes into the gain medium for achieving random lasing. The optical feedback is suggested to be provided by $\mathrm{Ti}_{3} \mathrm{C}_{2}$ nanoflakes through saturable absorption.
FTu4H • Functional Plasmonics with 2D and Novel MaterialsContinued

\section{FTu4H.6 • 17:30}

Broadband hot electron generation for solar energy conversion with plasmonic titanium nitride, Alberto Naldonii ${ }^{2,1}$, Urcan Guler $^{2}$, Zhuoxian Wang ${ }^{2}$, Marcello Marelli ${ }^{1}$ Francesco Malara ${ }^{1}$, Xiangeng Meng ${ }^{2}$, Lucas V. Besteiro ${ }^{3}$, Alexander O. Govorov ${ }^{3}$, Alexander V. Kildishev², Alexandra Boltasseva ${ }^{2}$ Vladimir M. Shalaev2; ${ }^{1}$ CNR-Istituto di Scienze e Tecnologie Molecolari, Italy; ${ }^{2}$ Schoo of Electrical \& Computer Engineering and Birck Nanotechnology Center, Purdue Univ. USA: ${ }^{3}$ Dept of Physics and Astronomy, Ohio Univ., USA. Plasmonic TiN decorated $\mathrm{TiO}_{2}$ nanowires support 4 times larger generation of over-barrier hot electrons than the $\mathrm{Au} / \mathrm{TiO}_{2}$ system resulting in an enhanced photoelectrochemical water splitting activity due to TiN broader plasmonic resonance and improved interface properties.

FTu4H.7 • 17:45

Temperature induced deviations to the optical responses of plasmonic materials Harsha Reddy Eragamreddy', Urcan Guler ${ }^{1}$, Krishnakali Chaudhuri', Aveek Dutta', Alexander V. Kildishev', Vladimir M. Shalaev ${ }^{1}$ Alexandra Boltasseva'; ' ${ }^{P}$ Purdue Univ., USA Temperature induced deviations to the optical responses of noble metals and refractory plasmonic metals were investigated to temperatures up to $900{ }^{\circ} \mathrm{C}$. The manifestation of these deviations in different plasmonic applications will be presented. 


\section{Meeting Room $211 \mathrm{~B} / \mathrm{D}$}

Meeting Room $212 \mathrm{~A} / \mathrm{C}$
Meeting Room 212 B/D

\section{CLEO: Science \& Innovations}

STu4I • Ultrafast Metrology IIContinued

STu4l.7 • 17:30

Bootstrap method for ultrabroad bandwidth carrier-envelope frequency noise analysis with superior detectivity, Haochen Tian', Youjian Song ${ }^{1}$, Nils Raabe ${ }^{2}$, Günter Steinmeyer', Ming-lie Hu'; 'Tianjin Univ., China; ${ }^{2}$ Max-Born-Institut, Germany. We demonstrate a novel carrier-envelope frequency noise power spectral density characterization method with $>70 \mathrm{~dB}$ dynamic range across 8-decade Fourier frequency range.

STu4I.8 • 17:45

Two-Dimensional Characterization of Spatiotemporal Coupling of Ultrashort Pulses Based on Chromatic Diversity, Seung-Whan Bahk', Christophe Dorrer', Jake Bromage"; 'Univ. of Rochester, USA. Two-dimensional spatiotemporal coupling is characterized by a single-shot scheme based on chromatic diversity, introduced by a quadratically distorted 2-D grid. Simultaneous and real-time measurement of pulse-front tilt and radial group delay is demonstrated.
STu4J • Microcomb Nonlinear Optical Technology-Continued

STu4J.6 • 17:30

Silicon Photonics as a Broadband Platform for Parametric Oscillation in the MidInfrared, Steven Miller ${ }^{1,2}$, Mengjie $\mathrm{Yu}^{3,4}$, Xingchen $\mathrm{Ji}^{1,2}$, Austin G. Griffith ${ }^{1}$, Jaime Cardenas 2,5 , Alexander L. Gaeta ${ }^{4}$, Michal Lipson"; ${ }^{2}$ School of Electrical and Computer Engineering, Cornell Univ. , USA; ${ }^{2}$ Dept. of Electrical Engineering, Columbia Univ., USA ${ }^{3}$ School of Applied and Engineering Physics, Cornell Univ., USA; ${ }^{4}$ Dept. of Applied Physics and Applied Mathematics, Columbia Univ. USA: ${ }^{5}$ The Inst. of Optics, Univ. of Rochester, USA. We demonstrate that silicon photonics can be leveraged for nonlinear optics in the mid-infrared range from 3-6 $\mathrm{mm}$. We fabricate an air-clad microresonator with $\mathrm{Q}=1$ million at $3.79 \mu \mathrm{m}$ wavelength and demonstrate $5.3 \mathrm{~mW}$ parametric oscillation threshold power.

STu4J.7 • 17:45

Octave Spanning Supercontinuum Generation in Silicon from $1.1 \mu \mathrm{m}$ to Beyond $2.4 \mu \mathrm{m}$, Neetesh Singh ${ }^{2}$, Ming Xin ${ }^{2}$, Diedrik Vermeulen², Katia Shtyrkova², Emir S. Mag den ${ }^{2}$, Patrick T. Callahan ${ }^{2}$, Nanxi Li ${ }^{2,3}$, Alfonso Ruocco ${ }^{2}$, Nicholas Fahrenkopf ${ }^{4}$, Douglas Coolbaugh' ${ }^{4}$, Bill Kuo', Stojan Radic ${ }^{1}$, Erich P. Ippen², Franz X. Kärtner ${ }^{2,5}$, Michael Watts ${ }^{2}$; ${ }^{1}$ Dept. of Electrical and Computer Engineering, Univ. of California San Diego, USA; ${ }^{2} R e-$ search Lab of Electronics, MIT, USA; ${ }^{3}$ John A Paulson School of Engineering and Applied Science, Harvard Univ., USA; ${ }^{4}$ College of Nanoscale Science and Engineering, Univ. at Albany, USA; ${ }^{5}$ Centre for Free Electron Lase Science, DESY, Germany. We demonstrate an octave spanning coherent supercontinuum generated in a silicon waveguide covering the near to shortwave IR (SWIR) region. The measured $-20 \mathrm{~dB}$ SC span ranges from 1.124 $\mu \mathrm{m}$ to $2.4 \mu \mathrm{m}$.

\section{STu4K • OAM \& Higher-Order Mode Fibers-Continued}

STu4K.7 • 17:30

Multimode Fibers for Mode Division Multiplexing, Pierre Sillard', Denis Molin ${ }^{1}$ Marianne Bigot-Astruc', Koen de Jongh' Frank Achten'; 'Prysmian Group, France. Graded-index-core multimode fibers can be appropriately rescaled in diameters and optimized to have low differential mode group delays for 6 to 36 spatial modes for multiple-input-multiple-output modedivision-multiplexed transmissions.

\section{Marriott \\ Salon I \& II}

\section{Joint}

JTu4L • Symposium on Ultrafast Laser Technology for X-ray Free Electron Lasers II-Continued
JTu4L.5 • 17:45

Operation of a seeded XUV free electron laser at DESY with high-gain harmonic generation seeding, Joern Boedewadt ${ }^{1}$, Christoph Lechner ${ }^{1}$, Ralph Assmann ${ }^{1}$, Armin Azima², Markus Drescher², Nagitha Ekanayake ${ }^{1}$, Bart Faaz ${ }^{1}$, Kirsten Hacker ${ }^{3}$, Mehdi Kazemi' ${ }^{1}$ Ingmar Hartl' ${ }^{1}$, Shaukat Khan ${ }^{3}$, Tim Laarmann', Theophilos Maltezopoulos², Tim Plath², Joerg Rossbach'; 'DESY, Germany; ${ }^{2}$ Dept. of Physics, Univ. Hamburg, Germany; ${ }^{3}$ Faculty of Physics, Technical Univ. Dortmunt, Germany. The XUV free electron laser FLASH has been recently operated in the high-gain harmonic generation $(\mathrm{HGHG})$ mode. We characterized the laser-induced energy modulation, as well as the temporal profile of the seeded FEL pulses. FEL saturation was reached for the 7 th harmonic of the 266 $\mathrm{nm}$ seed laser.

17:00-18:30 Meet the OSA Editors' Reception, Market Terrace 


$\begin{array}{llc}\text { Marriott } & \text { Marriott } & \text { Marriott } \\ \text { Salon III } & \text { Salon IV } & \text { Salon V \& VI }\end{array}$

\section{CLEO: Science \& Innovations}

STu4M • Nonlinear Impairments in Optical CommunicationsContinued
STu4N • OptomechanicsContinued

STu4N.6・17:30

Non-reciprocal Optomechanical Modulator, Donggyu B. Sohn'1, JunHwan Kim', Gaurav Bahl'; 'Univ. of Illinois, USA. We experimentally demonstrate non-reciprocal optical modulation using piezoelectric optomechanics. Non-reciprocity is achieved by breaking time-reversal symmetry for the light propagation in an optical resonator by unidirectionally traveling acoustic wave.
STu4O - Spatial and Temporal Beam Control-Continued

STu40.7 - 17:30

Multi-Joule, Sub-200ps Laser Pulse Generation via SBS Sub-Phonon Lifetime Pulse Compression, Chengyong Feng ${ }^{1}$, Xiaozhen $\mathrm{Xu}^{1}$, Jean-Claude Diels'; ' Univ. of New Mexico, USA. Multi-Joule level SBS sub-phonon lifetime pulse compression is demonstrated with a energy-scalable generator-amplifier setup to obtain 1.3J, 170ps (close to half of the phonon lifetime) laser pulses at $532 \mathrm{~nm}$.
STu4N.7 • 17:45

Direct Stabilization of Optomechanical Oscillators, Ke Huang' ${ }^{1}$ Mani Hossein-Zadeh'1. ${ }^{1}$ Univ. of New Mexico, USA. We demonstrate a simple and effective technique for stabilization of optomechanical oscillators. We show that using oscillation amplitude and laser detuning as the feedback and control parameters provide long term stability and reduce system complexity.

\section{STu40.8 • 17:45}

Coherent Enhancement of $10 \mu$ s BurstMode Ultraviolet Pulses at Megawatt Peak Power, Abdurahim Rakhman ${ }^{1,2}$, Yun Liu'; ' Oak Ridge National Lab, USA; ${ }^{2}$ Univ. of Tennessee, USA. A doubly-resonant optical cavity and its locking technique have been developed to achieve coherent enhancement of $402.5-\mathrm{MHz}, 50-$ ps, megawatt peak power ultraviolet $(355 \mathrm{~nm}$ ) laser pulses operating at a $10-\mu \mathrm{s} / 10-\mathrm{Hz}$ burst mode. 


\section{8:00-19:30 JTu5A - Poster Session I and Conference Reception}

JTu5A.1

An Ultra-High Sensitive Biosensor using Dual Resonance Long Period Grating in a Metal Clad Ridge Waveguide, Nabarun Saha', Arun Kumar'; ' ${ }^{1}$ Indian Inst. of Technology, Delhi, India. We propose and analyze an ultrahigh sensitive bio-sensor using long period grating near dispersion turning point in a metal clad ridge waveguide showing a refractive index sensitivity of $129 \mu \mathrm{m} / \mathrm{RIU}$ for watery solutions at telecommunication wavelength.

\section{JTu5A.2}

Coherent Raman spectroscopy with a graphene-synchronized all-fiber laser, Daniel Popa', Daniele Viola², Giancarlo Soavi ${ }^{1}$, Bo Fu' ${ }^{1}$, Lucia Lombardi', Stephen Hodge $^{1}$, Dario Polli2 ${ }^{2}$, Tullio Scopigno ${ }^{3}$, Giulio Cerullo', Andrea Ferrari'; ' 'Engineering, Univ. of Cambridge, UK; ${ }^{2}$ Physics, Politecnico di Milano, Italy; ${ }^{3}$ Physics, Univ. Sapienza, Italy. We demonstrate a wavelength-tunable graphene-synchronized all-fiber laser with $\mathrm{Yb}$ and Er cavities spanning 1040-1080 and $1535-1560 \mathrm{~nm}$, corresponding to $\sim 2750$ $3200 \mathrm{~cm}^{-1}$ frequency detuning. We apply the laser to coherent anti-Stokes Raman spectroscopy.

\section{JTu5A.3}

Ex vivo study of diffusion of indocyanine green (ICG) in cow retinal layers using optical coherence tomography, Changho Lee², Soohyun Lee ${ }^{2}$, J. Jeremy Chae', Gyeongwoo Cheon $^{2}$, Berk Gonenc' ${ }^{2}$, Peter L. Gehlbach ${ }^{1}$ Jin U. Kang ${ }^{2} ;{ }^{1}$ Wilmer Eye Inst., Johns Hopkins School of Medicine, USA; ${ }^{2}$ Dept. of electrical and computer engineering, Johns Hopkins Univ., USA. In this paper, we studied the diffusion of indocyanine green (ICG) into retinal layers by analyzing OCT images and showed that ICG diffuse all the way to RPE layer in ex vivo cow retina.

\section{JTu5A.4}

Biochemical Evaluation of Bone Submitted to lonizing Radiation by ATR-FTIR Spectroscopy, Pedro A. Castro ${ }^{1,2}$, Derly A. Dias ${ }^{1,2}$, Marcelo N. Veloso ${ }^{1}$, Denise M. Zezell ${ }^{1}$; 'IPEN - CNEN-SP, Brazil; ${ }^{2}$ Universidade de Sao Paulo, Brazil. FTIR spectroscopy associated with PC-LDA was able to discriminate bone samples receiving different ionizing radiation doses $(0,01 \mathrm{kGy}, 1 \mathrm{kGy}, 15 \mathrm{kGy})$, showing potential to the use of phosphate vibrational modes as a dose marker.

\section{JTu5A.5}

Structural Characterization of Dentin Irradiated with $\mathrm{Er}, \mathrm{Cr}$ :YSGG Laser and Fluoride for Caries Prevention, Patricia A. Ana $^{2}$, Carolina Benetti ${ }^{2}$, Luciano Bachmann ${ }^{3}$, Denise M. Zezell'; ${ }^{1}$ Center for Lasers and Applications, IPEN - CNEN-SP, Brazil; ${ }^{2}$ Center for Engineering, Modelling and Applied Social Sciences, Universidade Federal do ABC, Brazil; ${ }^{3}$ Faculdade de Filosofia, Ciencias e Letras de Ribeirao Preto, Universidade de Sao Paulo, Brazil. Er,Cr:YSGG laser induces the formation of bruxite and tetracalcium phosphate, as well as decreases the content of protein and water on dentin tissue, even when associated with a fluoride gel and at low energy densities.
JTu5A.6

Effects of Refractive Index Mismatch on Stimulated Raman Scattering And Coherent Anti-Stokes Raman Scattering Microscopy, Jarno N. van der Kolk', Antonino Cala Lesina', Lora Ramunno'; 'Univ. of Ottawa, Canada. SRS and CARS microscopy images are distorted by near-field enhancements and microlensing depending on object shape, producing micron shifts in image position and up to an order of magnitude signal enhancement.

\section{JTu5A.7}

A 3-D printed phantom for optical techniques in medicine, Jim Larsson', Peilang Liao', Märta Lewander Xu' ${ }^{4}$, Johannes Swartling ${ }^{4}$, Joakim Bood', Stefan Andersson-Engels $^{2}$, Patrik Lundin ${ }^{1,4}$, Emilie Krite Svanberg ${ }^{3}$. ${ }^{1}$ Dept. of Physics, Lund Univ., Sweden; ${ }^{2}$ Dept. of Physics, Univ. College Cork, Ireland; ${ }^{3}$ Dept. of Clinical Sciences, Lund Univ., Sweden ${ }^{4}$ GASPOROX AB, Sweden. Development of optical techniques in medical applications can be difficult due to limited access to realistic phantoms. Here a 3-D printed model based on CT-images of the thorax of an infant is developed and tested.

\section{JTu5A.8}

Photonic crystal slab sensor for monolayer detection, jingxing shi ${ }^{1}$, Michael Pollard ${ }^{2}$ ZiLong Wang ${ }^{1}$, Martin Charlton ${ }^{1}$, James Wilkinson'; 'Univ. of Southampton, UK ${ }^{2}$ Univ. of New South Wales, Australia. An asymmetric photonic crystal slab was shown the ability to detect monolayer molecule coating. A linear spectrum shift introduced by $0.8 \mathrm{~nm}$ level coating $\mathrm{ZnO}$ was observed. Self-assemble monolayer p-tolyltrichlosilane was detected.

\section{JTu5A.9}

Confocal Raman microscpectral imaging of human spinal cord sections employing uniand multi-variate methods for data analy sis, shuang wang ${ }^{1}$, Kaige Wang ${ }^{1}$, Zhuowen Liang'2, Yuze Gong', Xueyu Hu², Yaning Yin ${ }^{1}$ Qingli $\mathrm{He}^{3,1}$, Zhe Wang ${ }^{2}$, Jintao Bai ${ }^{1,3}$; ${ }^{1}$ Inst. of Photonics and Photon-Technology, Northwest Univ., China; ${ }^{2}$ Dept. of orthopaedics, Xijing Hospital, Fourth Military Medical Univ. China; ${ }^{3}$ Dept. of Physics, Northwest Univ. China. Precise correlations were established between the biochemical profile and histology architecture of human spinal cord tissue by confocal Raman micospectral imaging with $633 \mathrm{~nm}$ excitation. The constitution nature of both gray and white matter were revealed.

\section{JTu5A.10}

Improve the property of emission by honeycomb structure for excellent performance flexible light emitting diode, Chih-Hao Lin ${ }^{1}$, Lin Huang Yu ${ }^{1}$, Chien Chung Lin $^{1}$, Martin Charlton ${ }^{2}$, Hao-chung Kuo ${ }^{1}$ ${ }^{1}$ National Chiao Tung Univ., Taiwan; ${ }^{2}$ Univ. of Southampton, UK. This study presents the fabrication of flexible white-light-emitting diodes with nano-honeycomb-structured phosphor films and the 750-nm pattern exhibited the highest luminous efficiency $(7 \%)$ than a non-patterned phosphor film sample due to photonic crystal effect.
JTu5A.11

Femtosecond Laser Crystallization for Boosting the Conversion Efficiency of Flexible Ink-Printing $\mathrm{Cu}(\mathrm{In}, \mathrm{Ga}) \mathrm{Se}_{2}$ Thin Film Solar Cells, Shih-Chen Chen ${ }^{1}$, Nian-Zu She ${ }^{1}$ Jenh-Yih Juang', Yu-Ze Chen², Hao-chung $\mathrm{Kuo}^{3}$, Yu-Lun Chueh ${ }^{2}$, Kaung-Hsiung $\mathrm{Wu}^{1}$ ${ }^{1}$ Dept. of Electrophysics, National Chiao Tung Univ., Taiwan; ${ }^{2}$ Dept. of Materials Science and Engineering, National Tsing Hua Univ., Taiwan: ${ }^{3}$ Dept. of Photonics and Inst. of Electro-Optical Engineering, National Chiao-Tung Univ., Taiwan. We have successfully demonstrated that the femtosecond laser annealing treatment can give rise to significant improvements in both crystalline structure and defects reduction for the nonvacuum ink-printing CIGS thin films without introducing melting effect.

\section{JTu5A.12}

Deposition of $\mathrm{Al}$ and $\mathrm{Cu}$ nanoparticles on Silicon Wafer using a Picosecond Nd:YAG Laser: An Experiment-based Parameter Optimization Guide, MOHAMMAD HOSSEIN Azhdast ${ }^{1}$, Martin Kossatz ${ }^{2}$, Hans Joachim Eichler ${ }^{3}$, Klaus Lang ${ }^{4}$, Veronika Glaw ${ }^{4}$ ${ }^{1}$ PacTech \& TU-Berlin, Germany; ${ }^{2}$ PacTech GmBH, Germany; ${ }^{3}$ Optics, Technical Univ. of Berlin, Germany; ${ }^{4}$ IZM fraunhofer, Germany. The optimization of parameters for lase deposition of nanoparticles on Si-wafer is studied. The threshold of laser energy, pulses per laser shot and overlapping is crucial in order to achieve the best deposition results.

JTu5A.13

Single Photon Ge Vacancy Centers in Heteroepitaxial and Homoepitaxial Diamond Grown by HFCVD, Aaron D. Jackson ${ }^{1}$ Amber C. Wingfield ${ }^{1}$, Gary Harris ${ }^{1}$; ${ }^{\prime}$ Howard Univ., USA. Epitaxial diamond films containing single photon emitting Ge vacancies $(\mathrm{GeV})$ were grown on both diamond seeded Si substrates and single crystal (100) diamond wafers. Photoluminescence (PL) measurements showed a zero phonon line near $602 \mathrm{~nm}$.

\section{JTu5A.14}

Quantum Holographic Multiplexing and Its Application for Quantum Information, Anton Vetlugin', Ivan Sokolov'; 'St. Petersburg State Univ., Russia. We show that quantum holographic multiplexing allows one to use a single quantum memory device as a controllable multi-port beamsplitter for a time sequence of the quantized signals. The number of possible applications is considered.

\section{JTu5A.15}

Superradiant phase transition with graphene embedded in one dimensional optical cavity, Benliang $\mathrm{Li}^{1}{ }^{12}$, tao Liu', Danie Hewak ${ }^{2}$, qijie wang1: ' ${ }^{1}$ Nanyang technological Univ., Singapore; ${ }^{2}$ Univ. of Southampton UK. We theoretically investigate the cavity QED of graphene embedded in an optical cavity under perpendicular magnetic field this model exhibits a superradiant quantum phase transition. The complete excitation spectrum in both the normal phase and superradiant phase regimes is given.

JTu5A.16

A new study of on-demand emission of indistinguishable single photons from single quantum dots, Liron Gantz ${ }^{1,2}$, Dan Cogan' Ido schwartz', Emma Schmidgall1, Gad Bahir ${ }^{2}$ David Gershoni'; 'Physics Dept. and the Solid State Inst., Technion-Israel Inst. of Technology, Israel; ${ }^{2}$ Andrew and Erna Viterbi Dept. of Electrical Engineering, Technion-Israel Inst. of Technology, Israel. In this work we present a comprehensive theoretical and experimenta study of indistinguishable photon emission from various optical transitions and radiative cascades resulting from the same single QD.

JTu5A.17

Coherent Coupling Between Microwave and Optical Fields via Electron Spin Coherence in Diamond, Ignas Lekavicius ${ }^{1}$, Andrew Golter ${ }^{1}$, Thein Oo ${ }^{1}$, Hailin Wang ${ }^{1}$; ${ }^{1}$ Univ. of Oregon, USA. Using phase-dependent coherent population trapping, we demonstrate the conversion of microwave phase information into optical phase information via an electron spin coherence in diamond and vice versa.

\section{JTu5A.18}

Simultaneous filtering of the Mollow triplet sidebands via a Cs-based Faraday filter Simone Portalupi ${ }^{1}$, Matthias Widmann ${ }^{1}$ Cornelius Nawrath ${ }^{1}$, Michael Jetter ${ }^{1}$, Pete Michler ${ }^{1}$, Jörg Wrachtrup ${ }^{1}$, Ilja Gerhardt ${ }^{1}$ 'Univ. of Stuttgart, Germany. A Faraday filter is used to simultaneously allow the transmission of the Mollow triplet sidebands which are resonant with the Cs-D1 clock transition. The Mollow triplet is generated from a resonantlyexcited quantum dot.

\section{JTu5A.19}

Subwavelength Interference Based on Light Pulse Storage via Electromagnetically Induced Transparency, Jianji Liu', Zhixiang Li', Hongming Fan', Jiachen Liu', Guoquan Zhang ${ }^{1 ;}{ }^{1}$ The MOE Key Lab of Weak Light Nonlinear Photonics, School of Physics and TEDA Applied Physics Inst., Nankai Univ., China. By employing a light pulse storage and retrieval process based on the electromagnetically induced transparency effect, first-order subwavelength interference fringe with an effective wavelength equal to $\lambda / n$ was proposed and experimentally demonstrated with $n=2$ and a visibility $80 \%$.

\section{JTu5A.20}

Nano-second regime all optical switching in atomic cladding wave guides, Liron Stern ${ }^{1}$ Roy T. Zektzer ${ }^{1}$, Eliran Talker ${ }^{1}$, Noa Mazurski ${ }^{1}$ Uriel Levy'; 'Hebrew Univ. of Jerusalem, Is rael. We experimentally explore the spectra response of all optical switching in atomic cladding wave guides. As consequence of the fast transiting atoms, the roll-off frequency is enhanced and found to be in the $150 \mathrm{MHz}$ regime.

\section{JTu5A.21}


Exhibit Hall 1, 2 \& 3

\section{8:00-19:30 JTu5A - Poster Session I and Conference Reception}

\section{JTu5A.22}

Spin-sensitive Atom Mirror via Spinorbit Interaction, Lu Zhou', Ren-Fei Zheng ${ }^{1}$ Weiping Zhang2; 'East China Normal Univ., China: ${ }^{2}$ Shanghai Jiaotong Univ., China. We propose a scheme to realize spindependent scattering of cold atoms. The proposed system can act as a spin polarize or spin-selective atom mirror for the incident atomic beam.

JTu5A.23

Shortcut to Adiabaticity for an Electron Spin in Diamond, Mayra Amezcua' ${ }^{1}$, Andrew Golter ${ }^{1}$, Hailin Wang 1. 1 Univ. of Oregon, USA A strain-induced lambda-type three-level system in a nitrogen vacancy center in diamond is used for the implementation of shortcut to adiabatic passage through a dark state with counterdiabatic driving.

\section{JTu5A.24}

Robust Solid State Quantum System Operating at $800 \mathrm{~K}$, Mehran Kianinia', Sherif A. Tawfik', Blake Regan', TOAN T. TRAN' ${ }^{1}$ Mike J. Ford' ${ }^{1}$ Igor Aharonovich ${ }^{1}$, Milos Toth ${ }^{1} ;{ }^{1}$ Univ. of technology Sydney, Australia. Realization of Quantum information and communications technologies requires stable solid state single photon sources. However, existing sources cease to function above cryogenic or room temperature. we present an efficient source that is optically operate at elevated temperatures of up to $800 \mathrm{~K}$.

JTu5A.25

Experimental Investigation of Quantum Plasmonics in Subwavelength waveguide, Ming Li', Xi-Feng Ren'; 'Univ Sci \& Tech China, USA. We developed a fiber-integrated plasmonic probe, which can transport light in subwavelength scale. The probe provides a new method to collect fluorescence of quantum emitters efficiently and can transpor entangled state beyond the diffraction limit.

\section{JTu5A.26}

Strongly Extended Superradiance in Diamond Metamaterials, Olivia L. Mello', Yang Li', Phillip Camayd-Munoz', Cleaven Chia', I-Chun Huang1', Marko Lončar' ${ }^{1}$ Eric Mazur ${ }^{1,2} ;{ }^{1}$ Applied Physics, Harvard Univ., USA; ${ }^{2}$ Physics, Harvard Univ., USA. Zero index metamaterials (ZIM) experience near-perfect spatial coherence and infinite spatial wavelength. We model, through both analytical calculations and simulations, superradian emission of silicon vacancy centers in diamond ZIM that extends well beyond the emission wavelength near $737 \mathrm{~nm}$.

JTu5A.27

Quantum computing over the optical spatial mode comb with cluster states, Raphae C. Pooser ${ }^{1}$, Nick Black², Miller Eaton ${ }^{3}$, Benjamin Lawrie'; 'Quantum Information Science Group, Oak Ridge National Lab, USA; ${ }^{2}$ Univ. of Rochester, USA; ${ }^{3}$ Southern Illinois Univ. USA. We demonstrate multipartite entanglement in an optical spatial mode comb generated with an all-diode-laser optical pump and probe and four wave mixing in $\mathrm{Rb}$ vapor. This scalable scheme allows simultaneous access to all entangled modes.
JTu5A.28

A Hybrid Waveguide-Coupled Cavity Design for an Improved Spin-Photon Interface, Sara L. Mouradian', Dirk . Englund ${ }^{1}$ MIT, USA. This hybrid cavity design has an unloaded quality factor $(Q)>1 \times 10^{\wedge} 6$ and loaded Q of $5.5 \times 10^{\wedge} 4$ with $>75 \%$ of the emission coupled to a waveguide mode. Each cavity can be individually tuned onto resonance.

JTu5A.29

Ultra-high Compton Frequency, Parity Independent, Mesoscopic Schroedinger Cat Atom Interferometer with Heisenberg Limited Sensitivity, Resham Sarkar ${ }^{1}$, Renpeng Fang ${ }^{1}$, Selim M. Shahriar'; 'Northwestern Univ., USA. We show that collective-state detection combined with one-axis-twist squeezing and un-squeezing can produce a parity-independent, mesoscopic Schroedinger cat atom interferometer with ultra-high Compton frequency, yielding the ultimate (Heisenberg limited) sensitivity allowed by quantum mechanics.

\section{JTu5A.30}

Progress of the Self-sustaining Magnetom eter, Shiguang Wang ${ }^{1,2}$, Chi $\mathrm{Xu}{ }^{1,3}$, Yanying Feng ${ }^{1,2}$, Lu Zhao ${ }^{1,4}$, Lijun Wang ${ }^{1,2} ;{ }^{1}$ Joint Inst. for Measurement Science (JMI), Tsinghua Univ., China; ${ }^{2}$ State Key Lab of Precision Measurement Technology and Instrument, Dept. of Precision Instruments, Tsinghua Univ., China; ${ }^{3}$ Dept. of Physics, Tsinghua Univ., China; ${ }^{4}$ School of Physics, Beihang Univ., China. We report progress of the selfsustaining magnetometer. The sensitivity follows a faster $\mathrm{T}^{-1}$ rule rather than $\mathrm{T}^{-1 / 2}$ rule with time, and it can be close to the shot noise limit in a much shorter time.

\section{JTu5A.31}

Superradiance in a Two-Dimensiona Gas, Tyler Hill ${ }^{1}$, Barry C. Sanders ${ }^{2}, \mathrm{Hu}$ Deng'; ${ }^{1}$ Univ. of Michigan, USA; ${ }^{2}$ nstitute for Quantum Science and Technology, Univ. of Calgary, Canada. We examine the superradiant emission rate when the electromag netic field is confined to the plane. We find density-dependent power-law scaling of the superradiant emission rate, with powers rang ing from 0- 1/2 dependent on atom density.

\section{JTu5A.32}

Telecom-to-Near-Visible Frequency Translation via Bragg Scattering Four-Wave Mixing in a Rb Vapor Cell, Yun Zhao ${ }^{1}$, Prathamesh Donvalkar', Alexander L. Gaeta'; ${ }^{1}$ Columbia Univ., USA. We demonstrate telecom-tonear-visible frequency conversion, spanning over $181 \mathrm{THz}(734 \mathrm{~nm})$ via Bragg scattering four-wave mixing in a $\mathrm{Rb}$ vapor cell with a $0.15 \%$ energy conversion efficiency at $1 \mathrm{~mW}$ pump power.

\section{JTu5A.33}

Third Harmonic Light Control in Plasmonic Metasurfaces for Nonlinear Beam Shaping Antonino Cala Lesina ${ }^{1}$, Pierre Berini ${ }^{1,2}$, Lora Ramunno'; 'Dept. of Physics and Centre for Research in Photonics, Univ. of Ottawa, Canada; ${ }^{2}$ School of Electrical Engineering and Computer Science, Univ. of Ottawa Canada. Third harmonic generation in plasmonic monopole nanoantennas is highly controllable and, by arranging them in a metasurface, we numerically demonstrate the creation of highly pure vectorial vortex beams at frequencies not accessible by linear plasmonics.
JTu5A.34

Asymmetrical Diffusion through Time-Vary ing Material Parameters, Brian E. Edwards Nader Engheta'; 'Univ. of Pennsylvania, USA In this work we theoretically demonstrate rigorous mathematical analysis on how timevarying material parameters (capacitance and conductance) can yield an effective medium with asymmetric diffusion propertie with applications in thermodynamics and electrostatics.

\section{JTu5A.35}

Strong coupling in the novel dye / alumina membrane metamaterial, Cansu On ${ }^{1}$, Kevin E. Tanyi ${ }^{1}$, Mikhail Pashchanka ${ }^{2}$, Vanessa Peters' ${ }^{1}$, Jonathan R. Skuza ${ }^{1,3}$, Mikhail Noginov ${ }^{1}$ 'Center for Materials Research, Norfolk State Univ USA. ${ }^{2}$ Eduard-Zint-Institut, Technische Universität Darmstadt, Germany; ${ }^{3}$ Dept. of Physics \& Astronomy, Eastern Michigan Univ. USA We present the novel metamateria based on a nanoporous alumina membrane impregnated with R6G dye molecules. The splitting of the reflectance and emission bands at large dye concentration is discussed in terms of strong coupling.

\section{JTu5A.36}

Rigorous Diffraction Interface Theory, Christopher Roberts', Viktor A. Podolskiy ${ }^{1}$ 'Univ. of Massachusetts Lowell, USA. We present a formalism for analysis of optica properties of metasurfaces. Rigorous Diffraction Interface Theory provides a drastic speedup in computations and an analytica framework for understanding the transition between optically thin and thick structures.

\section{JTu5A.37}

Spin Induced Toroidal Dipole in Terahertz Metasurfaces, Longqing Cong 1,2, Yogesh K. Srivastava ${ }^{1,2}$, Ranjan Singh ${ }^{1,2} ;{ }^{1}$ Division of Physics and Applied Physics, School of Physical and Mathematical Sciences, Nanyang Technological Univ., Singapore; ${ }^{2}$ Centre for Disruptive Photonic Technologies, School of Physical and Mathematical Sciences, Nanyang Technological Univ., Singapore. We investigate the dominant role of toroida dipole in modulating the Fano resonance in planar terahertz metasurfaces by reducing the radiative loss from electric dipole.

\section{JTu5A.38}

A random metasurface for an all polarization flat lens, Matthieu Dupre ${ }^{1}$, Junhee Park' Boubacar Kante ${ }^{1} ;{ }^{1}$ Electrical and Computer Engineering, Univ. of California San Diego USA. Using full wave simulations and a transmission matrix approach, we design and then realize random metasurface lenses with anisotropic nanorods, and show that we can obtain a diffraction limited focal spot for al polarizations.

\section{JTu5A.39}

Long-range phase-free propagation in dielectric metasurface, Philip Camayd-Muñoz ${ }^{1}$, Shota Kita ${ }^{1}$, Daryl Vulis ${ }^{1}$, Orad Reshef ${ }^{2}$ Marko Lončar', Eric Mazur'; ' ${ }^{1}$ Harvard Univ. USA: ${ }^{2}$ Univ. of Ottawa, Canada. We present dielectric metasurface that supports in-plane guided waves with infinite wavelength, but suffers no material or radiation loss due to bound-state in the continuum. This design can be extended to large areas.

\section{JTu5A.40}

Time Domain Modeling of Lasing Dynamics in Hyperbolic Metamaterials, Shaima Azzam¹, Zhuoxian Wang', Shunsuke Murai², Satoshi Ishii ${ }^{3}$, Alexandra Boltasseva', Alexander V. Kildishev'; ${ }^{1}$ Purdue Univ., USA; ${ }^{2}$ Kyoto Univ., Japan; ${ }^{3}$ National Inst. for Materials Science, Japan. We use a semi-classical approach to model interaction of light wave with media and phenomenological approach to describe spontaneous emission that feeds the lasing modes to study a hyperbolic metamaterial coated with different gain materials.

\section{JTu5A.41}

Robust Edge States in Amorphous Gyromagnetic Photonic Lattices, shampy mansha', Yidong Chong'; 'Nanyang Technological Univ., Singapore. We show numerically that topological edge states can be realized in two dimensional (2D) gyromagnetic amorphous photonic crystals possessing short range order. These edge states are robust to disorder and show high unidirectional transmission.

\section{JTu5A.42}

Optical Weyl Points below the Light Line in Semiconductor Chiral Woodpile Photonic Crystals, Shun Takahashi ${ }^{1}$, Shuhei Oono ${ }^{2}$ Satoshi Iwamoto', Yasuhiro Hatsugai ${ }^{2}$, Yas uhiko Arakawa'; 'The Univ. of Tokyo, Japan Univ. of Tsukuba, Japan. Weyl points existing below the light line are numerically demonstrated in semiconductor-based simple chiral structures intended for near-infrared optical applications. Corresponding topologicallyprotected edge states are well-confined even at the vacuum interface.

JTu5A.43

Effect of Nonlocal Metal-Dielectric Environments on Concentration Quenching of HITC Dye, Srujana Prayakrao' Carl E. Bonner', Mikhail A. Noginov'; 'Norfolk State Univ., USA. We have experimentally demonstrated the inhibition of luminescence self-quenching in heavily doped HITC:PMMA polymeric films in vicinity of lamellar metal dielectric metamaterials with hyperbolic dispersion and metallic surfaces.

\section{JTu5A.44}

Quantum Features of Optical Metatronics, Yaakov Lumer ${ }^{1}$, Inigo Liberal ${ }^{1}$, Nader Engheta ${ }^{1} ;{ }^{1}$ Univ. of Pennsylvania, USA. We present a quantum circuit model for the description of charges and fields around a nanosphere in the context of optical metatronics. We calculate quantum charge fluctuations and note 


\section{8:00-19:30 JTu5A • Poster Session I and Conference Reception}

\section{JTu5A.46}

Statistical Measures of Spatial and Spectral Control with Binary Aperiodic Nanostructures, Yu-Chun Hsueh ${ }^{1}$, Kevin J. Webb 'Purdue Univ., USA. We describe the influence of the large number of degrees of freedom from a binary nanostructure using a multivariate statistical method. This analysis provides design guidelines to achieve spatial and spectral field control for applications.

JTu5A.47

Plasmon ultraviolet laser using patterned hyperbolic metamaterials, Kun-Ching Shen', Din-Ping Tsai ${ }^{1}$, Yuh-Jen Cheng ${ }^{1}$ : ${ }^{1}$ Academia Sinica, Taiwan. An ultraviolet plasmonic nanolaser was demonstrated using a patterned hyperbolic metamaterials (HMM) on AlGaN MOWs. The excited strong SPP resonance in the HMM structure provides a resonant feedback to the MOWs to reach lasing action.

JTu5A.48

Fiber-Metasurface for Wavefront Shaping, Zeba Naqvi', Christopher Rosenbury ${ }^{1}$ Michael Fiddy', Tsing-Hua Her'; 'Univ of North Carolina at Charlotte, USA. An array of embedded subwavelength fibers is proposed as a flexible, large-scale and mass-producible metasurface for wavefront shaping. As a proof of concept, we numerically demonstrate beam deflector, focusing optics, high reflector and sinusoidal wavefront shaping.

\section{JTu5A.49}

Longitudinal Shaping of Subwavelength Infrared Beams using Plasmonic Bull's-eye Structure with Concentric Slits, Ahmed Dorrah ${ }^{1,2}$, Arthur O. Montazeri², Hoi-Ying Holman', Mo Mojahedi'; ' 'Univ. of Toronto, Canada; ${ }^{2}$ Berkeley Synchrotron Infrared Structural Biology (BSISB) Program, Lawrence Berkeley National Lab, USA. We report on a bull's-eye gold nanostructure which focuses infrared beams into subwavelength scale with the ability to shape the longitudinal intensity profile and the focal length, thus addressing many challenges in label-free imaging, nanolithography, and biomedical applications.

\section{JTu5A.50}

Tailored Supercontinua via Spatial Beam Shaping, Alexandra Zhdanova', Yujie Shen', Jonathan Thompson", Marlan Scully ${ }^{1}$ Vladislav Yakovlev', Alexei Sokolov'; 'Texas A\&M Univ., USA. We show that programmable phase-only spatial optimization of the pump beam leads to significant broadening and flexible tunability of the supercontinuum spectrum without loss of input energy.

\section{JTu5A.51}

Observation of a Parity-Time-Symmetry Phase Transition in a Fiber Cavity, Ali Kazemi Jahromi' , Absar U. Hassan ${ }^{1}$, Demetrios Christodoulides ${ }^{1}$, Ayman F. Abouraddy ${ }^{1}$; 'Univ. of Central Florida, CREOL, USA. We predict and experimentally demonstrate that the lasing threshold can be lowered by virtue of PT-symmetry, and for the first time observe PT-symmetry-breaking in a long fiber cavity despite the presence of random phase fluctuations.
JTu5A.52

Observation of Coherent Perfect Absorption in a Short-Length Weakly Absorbing Fiber, Ali Kazemi Jahromi ${ }^{1}$, Ayman F. Abouraddy'; 'Univ. of Central Florida, CREOL, USA. We predict and experimentally confirm that a moderately-doped few-centimeter-long fiber can completely absorb an incoming beam, if the fiber is placed in a properly-designed cavity that satisfies the requirements for coheren perfect absorption.

\section{JTu5A.53}

Ferromagnetic-like Mode-locking Transition with Replica Symmetry Breaking in Nd:YAG Laser, Andre de Lima Moura ${ }^{1,2}$ Pablo Pincheira ${ }^{2}$, Ernesto Raposo ${ }^{3}$, Anderson Gomes ${ }^{2}$, Cid de Araújo ${ }^{2} ;{ }^{1}$ Campus Arapiraca Universidade Federal de Alagoas, Brazil: ${ }^{2} \mathrm{De}$ partamento de Física, Universidade Federa de Pernambuco, Brazil; 'Laboratório de Física Teórica e Computacional, Universidade Federal de Pernambuco, Brazil. We demonstrate the replica symmetry breaking (RSB) in the ferromagnetic-like mode-locking regime of multimode $\mathrm{Nd}$ :YAG lasers. This photonic phase, distinct of the RSB in random lasers, illustrates the universal character of the phenomenon in lasers.

\section{JTu5A.54}

Disorder driven spectral features of lasing in an Anderson localizing optical fiber Behnam Abaie ${ }^{1}$, Esmaeil Mobini', Salman karbasi ${ }^{3}$, Thomas Hawkins ${ }^{2}$, John Ballato ${ }^{2}$ Arash Mafi'; ' 'Univ. of New Mexico, USA ${ }^{2}$ Clemson Univ USA. ${ }^{3}$ Univ of California San Diego, USA. Spectral narrowing is reported in an Anderson localizing optical fiber lase when highly localized modes are pumped compared with the case where many modes including the less localized ones are excited.

\section{JTu5A.55}

Relative Performance of One-Dimensional Nonlinear Plasmonic Structures, C. Martijn de Sterke ${ }^{1}$, Guangyuan Li ${ }^{1}$, Stefano Palomba ${ }^{1}$ Univ. of Sydney, Australia. Plasmonic structures are promising for nonlinear optics because they strongly confine light. Many geometries were proposed but their relative merits remain under-explored. We compare different one-dimensional plasmonic structures and contrast with dielectric ones.

\section{JTu5A.56}

Nonperturbative Orbital Angular Momentum Buildup of Extreme-Ultraviolet Vortex Beams, Laura Rego', Julio San Roman ${ }^{1}$ Antonio Picon', Luis Plaja', Carlos HernandezGarcia'; 'Universidad de Salamanca, Spain Extreme-ultraviolet vortices are produced from the nonlinear conversion of infrared twisted beams through high-harmonic generation (HHG). The nonperturbative nature of $\mathrm{HHG}$ engenders an unexpectedly rich scenario for the orbital angular momentum buildup in extreme-ultraviolet vortices.

\section{JTu5A.57}

Localized Photonic Modes at Synthetic Gauge Lattice Interfaces, Artem Pankov' Ilya Vatnik', Dmitry V. Churkin', Andrey Sukhorukov2. 'Novosibirsk State Univ., Russia; ${ }^{2}$ Nonlinear Physics Centre, Research Schoo of Physics and Engineering, The, Australian National Univ., Australia. We predict nove localized modes supported by surface magnetic currents at interfaces between lattices with different synthetic gauge fields yet identical photonic band-gaps, and formulate their implementation in fiber loop mesh lattices with phase modulators.

JTu5A.58

Parity-Time Symmetric Fiber Ring Laser Sergey Smirnov ${ }^{1}$, Maxim Makarenko ${ }^{1}$, Sergey Suchkov², Ilya Vatnik', Dmitry V. Churkin ${ }^{1}$ Andrey Sukhorukov2; ' Novosibirsk State Univ., Russia; ${ }^{2}$ Nonlinear Physics Centre, Research School of Physics and Engineering The, Australian National Univ. , Australia. We propose a fiber laser composed of coupled ring cavities with gain and loss, featuring parity-time transition between symmetrybroken single-mode and bistable symmetric regimes, controllable by static phase shifters without active modulation.

\section{JTu5A.59}

One-way Nonlinear Mirror and Cancellation of Nonlinear Response via Multipola Interference From Metasurfaces, Ekaterina Poutrina ${ }^{1,2}$, Augustine Urbas'; ; ${ }^{1}$ Air Force Re search Lab, USA; ${ }^{2}$ UES, Inc., USA. We report examples of an image produced by difference frequency generation on one and the same side of a metasurface independently of the source location. Directionally-selective extinguishing of nonlinearly-generated multipolar modes is also shown numerically.

\section{JTu5A.60}

Modal Phase Matching in Nanostructured Zincblende Semiconductors for Second Harmonic Generation, Eleonora De Luca', Reza Sanatinia ${ }^{1}$, Mounir Mensi ${ }^{1}$, Srinivasan Anand ${ }^{1}$, Marcin Swillo'; ${ }^{1}$ KTH Royal Inst. of Technology, Sweden. Gallium phosphide nanowaveguide arrays, designed to fulfill the phase matching conditions and field-overlap are characterized by second-harmonic generation. The bandwidth of $30 \mathrm{~nm}$ with maximum conversion efficiency of $10^{-3}$ is measured for $150 \mathrm{fs}$ optical pulses.

\section{JTu5A.61}

Experimental Comparisons of P-T Symmetric Magneto-Electric Interactions in Molecular Liquids, Elizabeth F. Dreyer ${ }^{1}$, Alex ander A. Fisher' ${ }^{1}$ Stephen C. Rand'; ' $U$ Univ of Michigan, USA. Experimental comparisons of optically-induced magnetic scattering in fou liquids agree with recent quantum theoretica predictions of magneto-electric interactions obeying P-T symmetry.

\section{JTu5A.62}

Raman-Shift Suppression and Soliton Splitting in Photonic Crystal Fibers with Nonlinear Dispersion, Francisco Rodrigo Arteaga Sierra', Aku J. Antikainen', Govind P. Agrawal'; ' 'Univ. of Rochester, Mexico. We demonstrate numerically the cancellation of soliton self-frequency shift in photonic crystal fibers having nonlinear dispersion and positive linear dispersion slope. Fundamenta soliton fission across the zero-nonlinearity wavelength is also observed.
JTu5A.63

Realizing Ultra-Low Reflection and Reduced Dispersion of Slow Light, Fran Bello ${ }^{1,2}$, Freddie Page ${ }^{3}$, Andreas Pusch ${ }^{3}$ Joachim $\mathrm{Hamm}^{3}$, John Donegan ${ }^{1,2}$, Ortwin Hess $^{3}$; 'Trinity College Dublin, Ireland, ${ }^{2}$ Advanced Materials and Bioengineering Research, Ireland: ${ }^{3} / m p e r i a l$ College London, UK. We investigate hyperbolic, multilayered thin films which demonstrate epsilon-near zero behavior and contain stopped light energy bands. Together these two phenomena are able to radiatively excite slow ligh with reduced group velocity dispersion and perfect antireflection.

\section{JTu5A.64}

Simulation and Experimental Design of Saturated Excitation (SAX) Multiphoton Microscopy (MPM), Genevieve Vigil', Yide Zhang', Aamir Khan', Scott S. Howard'; ' Univ. of Notre Dame, USA. SAX is modeled and found to generate irregular PSF containing spatial frequency content beyond th diffraction limit. No special chemistry and minimal modification of MPM is needed toward super-resolved fluorescence imaging in scattering media.

\section{JTu5A.65}

Nonlinear Raman-Nath Second Harmonic Generation with Structured Fundamenta Wave, Haigang Liu', Jun $\mathrm{Li}^{2}$, Xiaohui Zhao' Yuanlin Zheng ${ }^{1}$, Xianfeng Chen'; 'Shanghai Jiao Tong Univ., China; ${ }^{2}$ College of Science and technology, Jiangxi Normal Univ. China. We proposed and experimentally demonstrated that nonlinear Raman-Nath second harmonic can be achieved when a fundamental wave with the phase periodically modulated, termed as structured fundamental wave, incident in a homogeneous nonlinear medium.

\section{JTu5A.66}

Demonstration of Non-accelerating SpaceTime Airy Beams, Hasan E. Kondakci' Ayman F. Abouraddy'; 'CREOL, Univ. of Central Florida, USA. We generate diffraction-free non-accelerating pulsed Airy beams having highly correlated spatio-temporal spectrum. Acceleration is controllably restored by introducing uncertainty in the spectral correlation.

JTu5A.67

Tunable Raman Solitons from $2.05 \mu \mathrm{m}$ to $2.25 \mu \mathrm{m}$ with High Conversion Efficiency, Honging Shi ${ }^{1}$, Xian Feng ${ }^{1}$, Fangzhou Tan Peng Wang ${ }^{1}$, Yuhang Shi', Jia Xu', Pu Wang '; 'Beijing Univ. of Technology, China. We report high conversion efficiency Raman solitons which possess $80 \%$ of the pulse energy and have wavelength tunable range from $2.05 \mu \mathrm{m}$ to $2.25 \mu \mathrm{m}$. 166-fs soliton pulses are obtained at wavelength of $2.25 \mu \mathrm{m}$ with an average power of $970 \mathrm{~mW}$, corresponding to a peak power of $140 \mathrm{~kW}$. 


\section{8:00-19:30 JTu5A - Poster Session I and Conference Reception}

\section{JTu5A.68}

Intracavity Phase Interferometry Enhanced with Resonant Linear Dispersion, James Hendrie $^{1}$, Matthias Lenzner ${ }^{2}$, Ladan Arissian', Jean-Claude M. Diels'; ${ }^{1}$ Univ. of New Mexico, USA; ${ }^{2}$ Lenzner Research, USA. Intracavity phase interferometry measures a beat frequency between two counter circulating pulses within a mode-locked cavity. A modification of sensitivity through insertion of resonant linear dispersion is experimentally demonstrated, while the pulse velocity remains unchanged.

\section{JTu5A.69}

On-chip Ultrafast Pulse Generator Based on Integrated Near-field Anapole Lasers, Juan Sebastian Totero Gongora', Andrey Miroshnichenko ${ }^{2}$,Yuri Kivshar ${ }^{2}$, Andrea Fratalocchi'; 'PRIMALIGHT, King Abdullah Univ. of Science and Technology, Saudi Arabia; ${ }^{2}$ Nonlinear Physics Centre, Research School of Physics and Engineering, Australian National Univ., Australia. We developed an all-dielectric integrated source of ultrafast optical pulses by exploiting the mutua interaction and synchronization of near-field nanolasers emitting at the anapole frequency.

\section{JTu5A.70}

Amorphous-Crystalline Micro- and Nanostructures in Silicon Fabricated Using Ultrashort Laser Pulses, Yasser I. Fuentes Edfuf ${ }^{1}$, Mario García Lechuga ${ }^{1}$, Daniel Puerto ${ }^{1}$, Camilo Florian Baron ${ }^{1}$, Adianez García Leis², Santiago Sánchez Cortés ${ }^{2}$, Javier Solis ${ }^{1}$ Jan Siegel1; 'Instituto de Óptica, Spanish National Research Council, Spain; ${ }^{2}$ Instituto de Estructura de la Materia, Spanish National Research Council, Spain. We demonstrate an innovative way to fabricate different types of amorphous-crystalline surface structures in silicon using ultrashort laser pulses. Fluencedependent solidification dynamics and interference of incident and scattered laser light are identified as underlying mechanisms.

JTu5A.71

A Full-wave Model for Laser-Induced Plasma emission from Metal Micro-Particles on Glass, Eyal Feigenbaum ${ }^{1}$, Omer Malik' Alexander Rubenchik', Manyalibo Matthews'; 'Lawrence Livermore National Lab, USA. A model based on full-wave simulations is used to evaluate laser-induced plasma emission from metal micro-particles on silica glass exit surface. The predicted deposited plasma energy distribution resulting from the light interference explains experimental observations.

\section{JTu5A.72}

Three-dimensional waveguide coupler/ beam splitter in lithium niobate crystals by femtosecond laser writing, Jinman $L v^{1}$, Xiaotao Hao', Feng Chen ${ }^{1}$; ${ }^{1}$ Shandong Univ., China. We report on the fabrication of threedimensional waveguide coupler in $\mathrm{LiNbO}^{3}$ crystal by using femtosecond laser writing This coupler is used to implement $1 \times 4$ beam splitting. The numerical simulations are in agreement with experimental results.
JTu5A.73

Chiral nearfield generation from chiral surface relief fabricated by optical vortex illumination with nano-imprinting technology, KEIGO MASUDA' ${ }^{1}$, Shogo Nakano', Guzhaliayi Juman', Itsuki Yoshida', Daisuke Sakai ${ }^{3}$, Kenji Harada ${ }^{3}$, Katsuhiko Miyamoto ${ }^{1,2}$, Takashige Omatsu㣙; ${ }^{1}$ Chiba Univ., Japan; ${ }^{2}$ Molecular Chirality Research Center, Chiba Univ., Japan; ${ }^{3}$ Kitami Inst. of Technology, Japan. We demonstrated the plasmon-enhanced nearfield induced chiral mass transport, in which the superimposed azo-polymer thin film is twisted, around the chiral surface relief formed by optical vortex illumination in combination with nanoimprinting technology.

JTu5A.74

Nonlinear above-threshold photoemission in single-wall carbon nanotube induced by fs-pulsed laser, Mark Green ${ }^{1}$, Jamie Gengler ${ }^{2}$, Robert Headrick ${ }^{3}$, Augustine Urbas $^{2}$, Junichiro Kono ${ }^{3}$, Matteo Pasquali ${ }^{3}$, Tsing-Hua Her'; ' 'Univ. of North Carolina at Charlott, USA: ${ }^{2}$ Air Force Research Lab, USA ${ }^{3}$ Rice Univ., USA. We study nonlinear abovethreshold photoemission (ATPE) in single-wall carbon nanotubes at two wavelengths. NIR photoemission demonstrates 5-photon ATPE, while UV ATPE is dominantly a 2 nd process. Two-pulse correlation exhibits enhanced photoemission with a very short lifetime less than $200 \mathrm{fs}$.

\section{JTu5A.75}

Vacuum-field Rabi Splitting at SWIR in Photocurrent of Quantum Cascade Infrared Photodetectors Coupled to Metamaterial Nano-antennas, Matias Katz ${ }^{1}$ Ofir Sorias ${ }^{1}$ Ben Dror ${ }^{1}$, Nicolas Grandjean², Meir Orenstein', Gad Bahir'; 'Dept. of Electrical Engineering, Technion-Israel Inst. of Technology, Israel; ${ }^{2}$ Inst. of Condensed Matter Physics, Ecole Polytechnique Fédérale de Lausanne, Switzerland. We present the design, realization, and characterization of room temperature optical and electrical strong light-matter coupling between intersubband transitions, at wavelength of 1.8 micron, in quantum cascade detector and planar metamaterials nano-cavity antenna.

\section{JTu5A.76}

Tunable Redox Property of Silver Deposited $\mathrm{TiO}_{2}$ Nanocomposite Synthesized by Pulsed Laser Ablation, Rui Zhou' Shengdong Lin': 'Xiamen Univ., China. Silver deposited $\mathrm{TiO}_{2}$ nanocomposites with tunable redox property were fabricated by laser ablation of silver and titanium targets in de-ionized water. This approach opens a route for one-step-synthesized redox system for potential applications in photocatalyst.

JTu5A.77

Infrared Absorption Spectroscopy of Monolayers with Thin Film Interference Coatings, sencer ayas ${ }^{1,2}$, Gokhan Bakan ${ }^{1,3}$ Erol Ozgur ${ }^{1}$, Kemal Celebi ${ }^{1}$, Aykutlu Dana ${ }^{1}$; ${ }^{1}$ Materials Science and Nanotechnology, Bilkent Univ., Turkey; ${ }^{2}$ Dept. of Radiology, Stanford Univ., USA; ${ }^{3}$ Electrical and Electronics Engineering, Atilim Univ., Turkey. We report high performance Infrared spectroscopy platforms based on interference coatings on metal using $\mathrm{CaF}_{2}$ dielectric films and $\mathrm{Ge}_{2} \mathrm{~S}$ $\mathrm{b}_{2} \mathrm{Te}_{5}$ (GST) phase-change films. IR vibrational bands of proteins and organic monolayers are also detected.
JTu5A.78

Phonon Chirality and Indirect Cooling in an Optomechanical System, Seunghwi Kim ${ }^{1}$ Xunnong $\mathrm{Xu}^{2}$, Jacob Taylor ${ }^{2,3}$, Gaurav Bahl ${ }^{1}$; ${ }^{1} M e c h a n i c a l$ Science and Engineering, Univ. of Illinois at Urbana-Champaign, USA; ${ }^{2}$ Join Quantum Inst., Univ. of Maryland, USA; ${ }^{3}$ Join Center for Quantum Information and Computer Science, National Inst. of Standards and Technology, USA. We demonstrate dynamical induction of chiral phonon transport in optomechanical resonators by means of traveling-wave acousto-optical interaction. The phenomenon results in unidirectiona defect tolerant transport and chiral cooling of high-Q phonon modes in the system.

\section{JTu5A.79}

Controllable Coupling of an Ultra-High-O Microtoroid Cavity with Monolayer Graphene, Xun Zhang ${ }^{1}$, Huibo Fan ${ }^{1,2}$, Xiaoshun Jiang', Min Xiao ${ }^{1,3} ;{ }^{1}$ College of Engineering and Applied Sciences, Nanjing Univ., China ${ }^{2}$ College of Physics Science and Technology, Yangzhou Univ., China; ${ }^{3}$ Dept. of Physics, Univ of Arkansas, USA. We have demonstrated controllable coupling between an ultrahigh-Q microcavity and monolayer graphene with tunability of the $\mathrm{Q}$-factor from $1.59 \times 10^{7}$ to $1.20 \times 10^{5}$. The $\mathrm{Q}$-factor has been finely tuned by adjusting the gap between them.

\section{JTu5A.80}

A hybrid system with highly enhanced graphene SERS for rapid and tag-free tumor cells detection, Yi Ningbo', Zonghui Duan', Qinghai Song', Shumin Xiao': 'Harbin Institude of Technology Shenzhen, China. Herein we demonstrate a facile device based on GSERS in a sandwich-structure of reduced graphene oxide between $\mathrm{Ag}$ and $\mathrm{Au}$, of which the coupling of localized surface plasmons demonstrated to realize huge enhanced G-SERS, the potential for detection and dentification for tag-free tumor cells.

\section{JTu5A.81}

Accurate Calculation of Modal Refractive Indices in Slightly Elliptical Optical Fibers Aku J. Antikainen ${ }^{2,1}$, René-Jean Essiambre Govind P. Agrawal1,3. "Univ of Rochester, USA; ${ }^{2}$ Nokia Bell Labs, USA; ${ }^{3}$ Lab for Laser Energetics, USA. We present a novel perturbation approach to accurately calculate the effects of core ellipticity on the modal propagation constants in step-index fibers. The method enables simple computation of the mode profiles and their effective indices.

\section{JTu5A.82}

All Reflective Multiphoton Microscope for use with Compact Multi-colored Broadband Femtosecond Fiber Lasers, Benjamin Cromey ${ }^{1}$, Robert Baker ${ }^{1}$, Babak Amirsolaimi' Soroush Mehravar' Khanh Q. Kieu' 'Univ. of Arizona, USA. We present and discuss a design for a multiphoton microscope that uses all reflective elements for beam shaping and expansion, which removes the effects of dispersion on femtosecond pulses as well as chromatic aberrations.

\section{JTu5A.83}

Gain Asymmetry in Saturated Raman-As sisted Fiber Optical Parametric Amplifiers Bofang Zheng', Chester Shu'; 'The Chinese Univ. of Hong Kong, Hong Kong. The spectral asymmetry in saturated Raman-assisted parametric amplification is investigated experimentally and numerically using a fivewave model. We identify that the interplay between dispersive waves and high-order four-wave mixing processes breaks the gain symmetry.

\section{JTu5A.84}

Pulse Generation from Laser Light using Temporal Talbot Array Illuminators, Carlos R. Fernandez-Pousa ${ }^{1,2}$, Reza Maram², Jose Azana2; ' ${ }^{1}$ Dep. of Communications Engineering, Universidad Miguel Hernandez de Elche, Spain; ${ }^{2}$ Inst. National de la Recherche Scientifique, Centre Énergie, Matériaux Télécommunications (INRS-EMT), Canada. Pulse generation from $\mathrm{cw}$ laser light with $>70 \%$ collection efficiency, $60-200$ ps pulse widths and repetition rates $1.1-2.5 \mathrm{GHz}$ is demonstrated using multilevel phase modulation and 1/2,1/6 and 1/10 fractional Talbot dispersive propagation.

\section{JTu5A.85}

Detrimental Effects in Brillouin Distributed Sensors Caused By EDFA Transient, Cheng Feng ${ }^{1}$, Haritz Iribas ${ }^{2}$ Jon Marielarena ${ }^{2}$, Thomas Schneider ${ }^{1}$, Alayn Loayssa ${ }^{2} ;{ }^{1}$ Institut fü Hochfrequenztechnik, Technische Universität Braunschweig, Germany; ${ }^{2}$ Departamento de Ingeniería Eléctrica y Electrónica, Universidad Pública de Navarra, Spain. We investigate the deleterious effect and the error in Brillouin optical time-domain analyzers induced by the combination of a low extinction ratio pulse generation with the transient behavio of erbium-doped fiber amplifiers.

\section{JTu5A.86}

Light Controlled Optical Fiber Comb Filte Enabled by Colloidal Quantum Dots, Gao Feng', Yang Wang ${ }^{1}$, Ming Tang', Huan Liu', 'Wuhan National Lab for Optoelectronics (WNLO) \& School of Optics and Electronic Information, Huazhong Univ. of Science and Technology (HUST), China. Utilizing the quantum confinement effect of colloidal quantum dots (CQD) embedded with exposed-core photonics crystal fiber interferometers, light-absorption controlled tunable optica fiber comb filter is achieved by simply applying $\sim \mathrm{mW}$ level pump power.

\section{JTu5A.87}

Optimizing Output Power Through Temporal Pulse Shaping, Graham R. Allan ${ }^{1}$, MarkA Stephen ${ }^{1}$, Anthony W. Yu' ', James B. Abshire ${ }^{1}$ Stewart T. Wu', Jeffery Chen', Kenji Numata', 'NASA Goddard Space Flight Center, USA We have doubled the output pulse energy to $550 \mu \mathrm{J}$ from a Raman Pumped VLMA EDFA 


\section{8:00-19:30 JTu5A • Poster Session I and Conference Reception}

JTu5A.88

High average power All-Fiber Superluminescent Pulse Amplifier with Tunable Repetition Rates and Pulse Widths, Haitao Zhang', He Hao', Xinglai Shen', Linlu He' Mali Gong'; 'Tsinghua Univ., China. We reported a high average power of $570 \mathrm{~W}$ laser generated by an all-fiber superluminescent pulse amplifier (SPA) structure, operating at variable repetition rates from $0.5 \mathrm{MHz}$ to 2 $\mathrm{MHz}$ and pulse widths from $100 \mathrm{~ns}$ to $220 \mathrm{~ns}$.

\section{JTu5A.89}

Compact and robust high-order random Raman fiber laser, Han $\mathrm{Wu}^{1}$, Zinan Wang ${ }^{1}$ Qiheng $\mathrm{He}^{1}$, Wei Sun ${ }^{1}$, Yunjiang Rao'; 'UESTC, China. We report a compact and robust cavity design for generating highorder random Raman fiber lasing. A pump combiner and broadband reflector are used to form forward pumping, providing a promising way to increase the output power significantly.

\section{JTu5A.90}

Tunable Multiwavelength Fiber Laser Based oNematic Liquid Crystal Device for Fiberoptic Electric Field Sensor, Hyun Ji Lee ${ }^{1}$, Sung-Jo Kim², Myeong Ock Ko' ${ }^{1}$, Jong-Hyun Kim ${ }^{1}$, Min Yong Jeon'; ' Chungnam Nationa Univ., South Korea; ${ }^{2}$ Center for Soft and Living Matter, Inst. for Basic Science, South Korea. We present a tunable multiwavelength fiber laser based on nematic liquid crystal device (NLC) for fiber-optic electric field sensor. The lasing multiwavelength could be tuned over $18 \mathrm{~nm}$ by applying electric field to the NLC device.

\section{JTu5A.91}

Image Transport Through Silica-Air Random Core Optical Fiber, JIAN ZHAO', Jose E. Antonio-Lopez', Rodrigo A. Correa', Arash Mafi ${ }^{2}$, Marie Windeck ${ }^{1}$, Axel Schülzgen ${ }^{1}$; 'CREOL, College of Optics and Photonics, Univ. of Central Florida, USA; ${ }^{2}$ Dept. of Physics and Astronomy, Univ. of New Mexico, USA. Optical image transport through lowloss silica-air based disordered fiber is reported for the first time. Transverse Anderson localization is confirmed by propagating a $976 \mathrm{~nm}$ laser beam through a 4.6-cm-long segment of random fiber.

JTu5A.92

Investigation of double-clad $\mathrm{Yb}^{3+}$-doped phosphate fiber for $976 \mathrm{~nm}$ single-frequency laser amplification, Jingwei $\mathrm{Wu}^{1}$, Xiushan Zhu', Valery Temyanko², LLOYD LACOMB ${ }^{2}$ Leonid Kotov', Kort Kiersma ${ }^{3}$, Jie Zong ${ }^{3}$, Arturo Chavez-Pirson ${ }^{3}$, Robert A. Norwood ', Nasser Peyghambarian'; ' ${ }^{1}$ nniv. of Arizona, USA; ${ }^{2}$ TIPD LLC, USA; ${ }^{3}$ NP Photonics, USA. $976 \mathrm{~nm}$ single-frequency ytterbium-doped double-clad phosphate fiber amplifiers were investigated and $3.41 \mathrm{~W}$ laser output with $\sim 41 \mathrm{~dB}$ optical signal-to-noise ratio was obtained. Further power scaling was studied with fiber amplifier modeling.
JTu5A.93

Stable Operation of Regeneratively and Harmonically Mode-locked Fiber Ring Laser Employing Clock Extraction from the Second Harmonic, Joji Maeda ${ }^{1}, \mathrm{Ka}$ Sakuma'; 'Tokyo Univ. of Sience, Japan. We experimentally demonstrate a regeneratively and harmonically mode-locked fiber laser employing clock extraction from the secondharmonic of the repetition frequency. The proposed laser showed much better stabil ty than did a laser employing conventional clock extraction.

\section{JTu5A.94}

Higher Gain of Single-Mode Cr-Doped Crystalline Core Fibers by Online Controlling Molten Zone, Liu Chun-Nien'1, TsungHau Wang ${ }^{1}$, Ting-Sou Rou ${ }^{1}$, Nan-Kuang Chen $^{2}$, Sheng-Lung. Huang ${ }^{3}$, Wood-Hi Cheng'; ' 'Graduate Inst. of Optoelectronic Engineering, National Chung Hsing Univ. Taiwan; ${ }^{2}$ Dept. of Electro-Optical Engineering, National United Univ., Taiwan; ${ }^{3}$ Graduate Inst. of Photonics and Optoelectronics, National Taiwan Univ. Taiwan. A single-mode Cr-doped crystalline core fiber (SMCDCCF) with longer fiber length by online controlling molten zone is demonstrated a gross gain of $4.2-\mathrm{dB}$. This gross gain is the highest yet reported for the SMCDCCF.

\section{JTu5A.95}

Squeezed Hollow Core Photonic Bragg fiber for surface sensing applications, Jingwen Li ${ }^{1}$, Hang Qu' ${ }^{1}$, Maksim Skorobogatiy ${ }^{1}$ ${ }^{1}$ Dept. Engineering physics, Ecole polytechnique de Montreal, Canada. We demonstrate theoretically and confirm experimentally that squeezing a section of the Bragg fiber core increases overlap between the optical fields of the core-guided modes and the modes bound to the sensing layer, which, in turn, enhances surface sensitivity of the fiber sensor.

\section{JTu5A.96}

Steering Lasing Beam in Topological Light Sources, Babak Bahari ${ }^{1}$, Junhee Park' ${ }^{1}$, Felipe Valini', Ricardo Tellez-Limon', Ashok Kodigala', Thomas Lepetit ${ }^{1}$, Yashaiahu Fainman ${ }^{1}$ Boubacar Kante 1. ${ }^{1}$ Univ of California San Diego, USA. We demonstrated the Bound State in the Continuum Surface Emitting Laser that can steer the beam with angles depending on the topology. This novel type of the topological light source operates at room temperature.

\section{JTu5A.97}

Carrier Dynamics of Ultrafast Semiconductor Disk Lasers, Cesare Alfieri', Dominik Waldburger ${ }^{1}$ Sandro M. Link ${ }^{1}$, Matthias Golling1, Ursula Keller'; ' $E T H$ Zürich, Switzerland. We theoretically and experimentally investigate gain dynamics of modelocked semiconductor disk lasers in the sub-200-fs regime. Spectral hole burning and shor carrier lifetime in the conduction band are limiting output power and optical-to-optical pump efficiency.
JTu5A.98

A novel dual-loop feedback scheme to reduce spurious tones in self-mode-locked two-section quantum Dash laser emitting at $\approx 1.55 \mu \mathrm{m}$, Haroon Asghar ${ }^{1,2}$, Ehsan Sooudi ${ }^{1,2}$, Pramod Kumar ${ }^{1,2}$, Alfonso Gonzalez, ${ }^{1,2}$, John Mclnerney ${ }^{1,2} ;{ }^{1}$ Univ. College Cork, Ireland: ${ }^{2}$ Tyndall National Inst., Ireland. We demonstrate a novel dual-loop scheme to suppress external cavity side-bands and modal overlaps induced in spectrum of self-mode-locked laser resulting from conventional single loop feedback and dual loop feedback configurations.

JTu5A.99

1.9 THz Difference-Frequency Generation in Mid-Infrared Quantum Cascade Laser with Grating Outcouplers, Jae Hyun Kim ${ }^{1}$ Seungyong Jung ${ }^{1}$, Yifan Jiang1', Kazuue Fujita2 ${ }^{2}$ Masahiro Hitaka², Akio Ito' ${ }^{2}$, Tadataka Masahiro ${ }^{2}$ Mikhail A Belkin'1.1 Univ of Texas at Austin, USA: ${ }^{2}$ Hamamatsu Photonics, Japan. We report terahertz quantum cascade laser sources based on intra-cavity difference-frequency generation. Devices are processed into double-metal waveguides with surface-grating outcouplers. Over 40 $\mathrm{mW}$ of power output at $1.9 \mathrm{THz}$ is produced at room temperature.

\section{JTu5A.100}

High-Power 1.65- $\mu \mathrm{m}$ Slab-Coupled Optical Waveguide Amplifiers, Jason Plant ${ }^{1}$ Dominic F. Siriani1, Toby Garrod², Antonio Napoleone ${ }^{1}$, Sara Mouser ${ }^{1}$, Paul Juodawlkis ${ }^{1}$ 'Lincoln Lab, MIT, USA; ${ }^{2}$ II-VI Epiworks, USA $1.65-\mu \mathrm{m}$ slab-coupled optical waveguide amplifiers (SCOWAs) that produce $>300$ $\mathrm{mW}$ output power at $20 \mathrm{~dB}$ gain are demonstrated. Such high-power devices could enable compact, large-standoff-distance methane sensors.

\section{JTu5A.101}

Stable and Narrow Linewidth Semiconduc tor Laser Assembly with Coherent Optical Negative Feedback, Konosuke Aoyama' Shuhei Kobayashi', Masashi Wada', Nobuhide Yokota', Hiroshi Yasaka'; 'Tohoku Univ., Japan. We demonstrate a stable and narrow-linewidth laser source based on an assembled coherent optical negative feedback system. The linewidth reduction from 13.5 $\mathrm{MHz}$ to $3.0 \mathrm{kHz}$ is kept for more than an hour.

\section{JTu5A.102}

Model for Frequency Comb Generation in Single-Section Quantum Well Diode Lasers, Mark Dong ${ }^{1}$, Niall Mangan², J. N. Kutz ${ }^{2}$, Steven T. Cundiff ${ }^{1}$, Herbert G. Winful': 'Univ. of Michigan, USA; ${ }^{2}$ Applied Mathematics, Univ. of Washington, USA We present a new, traveling-wave model for single-section quantum well diode lasers. We find that strong four-wave mixing based upon the gain grating, coupled with longitudinal spatial hole burning, allows for frequency comb generation.

\section{JTu5A.103}

CCD-based thermoreflectance measure ments of a multi-section slotted laser. David McCloskey ${ }^{1,2}$, Rudi O'Reilly Meehan ${ }^{3}$ Michael Wallace ${ }^{1,2}$, Ryan Enright ${ }^{3}$, John Donegan ${ }^{1,4}$; ${ }^{1}$ School of Physics, Trinity College Dublin, Ireland; 'Future Networks and Communications (CONNECT), Ireland ${ }^{3}$ Efficient Energy Transfer Dept. (nET), Bell Labs, Nokia, Ireland; ${ }^{4}$ Centre for Research on Adaptive Nanostructures and Nanodevices (CRANN), Trinity College Dublin, Ireland. CCD thermoreflectance microscopy is used to obtain high resolution images of surface temperature variations in a three section slotted single mode laser. These measurements indicate temperature gradients across the laser grating section, which can be used to better inform wavelength tuning models.

\section{JTu5A.104}

Voltage-Controlled Oscillators Based on Optically Injected Semiconductor Lasers, Nicholas G. Usechak', Joseph S. Suelzer ' Joseph W. Haefner'; ' 'US Air Force Research Lab, USA. A microwave voltage-controlled oscillator is demonstrated using an optically injected semiconductor laser; we achieve tuning from $5--15 \mathrm{GHz}$ in 10 ns with a voltage change of $600 \mathrm{mV}$; a tuning rate of $\sim 1 \times 10^{18} \mathrm{~Hz} / \mathrm{sec}$

\section{JTu5A.105}

Time delay signature suppression and complexity enhancement of chaos in laser with self-phase-modulated optical feedback, Chenpeng Xue ${ }^{1}$, Ning Jiang Guilan Li', Chao Wang ${ }^{1}$, Shuqing Lin ${ }^{1}$, Yunxin Lv', Kun Qiu'; ' Univ of Electronic Science \& Tech China, China. Chaos generated by a semiconductor laser subject to self-phase modulated optical feedback is proposed where the time-delay-signature is concealed and complexity is enhanced. Fascinatingly, the probability density distribution of its output intensity is close to symmetry.

JTu5A.106

Withdrawn

\section{JTu5A.107}

High Frequency and High Power Monolithic Mode-Locked Laser, Pengchao Zhao ${ }^{1,2}$, Anjin $\mathrm{Liu}^{2}$, Wanhua Zheng ${ }^{1,2}$; ${ }^{1}$ State Key Lab on Integrated Optoelectronics Lab, Inst. of Semiconductors, CAS, China; ${ }^{2}$ Lab of Solid State Optoelectronics Information Technology, Inst. of Semiconductors, CAS, China. We theoretically analyze the impact of a saturable absorber length and experimentally demonstrate a mode-locked laser with a pulse width of $1.75 \mathrm{ps}$, peak power of $188 \mathrm{~mW}$, and pulse energy of $0.33 \mathrm{pJ}$

\section{JTu5A.108}

$1.5 \mu \mathrm{m}$ Laser Diode on InP/Si substrate by Epitaxial Growth using Direct Bonding Method, Periyanayagam Gandhi Kallarasan ${ }^{1}$ Tetsuo Nishiyama', Naoki Kamada1, Yuya Onuki', Kazuhiko Shimomura'; 'Sophia Univ. Japan. we have demonstrated for the first time 1.5 $\mathrm{mm}$ GalnAsP laser diode on silicon substrate using direct wafer bonding and MOVPE growth. Our unique approach prio to the growth is that we do the adhesion of InP substrate and Si substrate using hydrophilic wafer bonding technique. 


\section{8:00-19:30 JTu5A - Poster Session I and Conference Reception}

\section{JTu5A.109}

Class-A Operation of InAs Quantum Dashbased Vertical-External-Cavity Surface Emitting Laser, Salvatore Pes, ${ }^{1,2}$ Kevin Audo ${ }^{2}$, Cyril Paranthoën ${ }^{1}$, Christophe Levallois ${ }^{1}$, Nicolas Chevalier', Goulch'hen Loas², Steve Bouhier'2, Cyril Hamel2, Carmen Go$\mathrm{mez}^{3}$, Jean-Christophe Harmand ${ }^{3}$, Sophie Bouchoule ${ }^{3}$, Hervé Folliot ${ }^{1}$, Mehdi Alouini ${ }^{2}$ FOTON, UMR CNRS 6082, INSA de Rennes, France: ${ }^{2}$ Institut de Physique de Rennes, UMR UR1-CNRS 6251, Université de Rennes 1 France; ${ }^{3}$ Centre de Nanosciences et de Nanotechnologies, CNRS, Université Paris-Sud, France. InAs Quantum Dash-based VerticalExternal-Cavity Surface-Emitting Laser on $\mathrm{InP}$ is demonstrated. Up to $163 \mathrm{~mW}$ and 7 $\mathrm{mW}$ have been obtained in multi-mode and single-mode operation, respectively. Class- $A$ behavior is demonstrated on such device.

JTu5A.110

Single-Defect Hexapole Mode GeSn Photonic Crystal Laser: Fabrication and Simulation, Shuyu Bao ${ }^{1,2}$, Haodong Qiu', Yeji Kim ${ }^{3}$ Yiding Lin ${ }^{1,2}$, Han-Youl Ryu ${ }^{3}$, Mee-Yi Ryu ${ }^{4}$ Yung Kee $\mathrm{Yeo}^{5}$, John Kouvetakis ${ }^{6}$, Eugene Fitzgerald ${ }^{2}$, Hong Wang ${ }^{1}$, Donguk $\mathrm{Nam}^{3}$ Chuan Seng Tan 1,2: ${ }^{1}$ Nanyang Technological Univ., Singapore; ${ }^{2}$ Singapore-MIT Alliance for Research and Technology (SMART), Singapore; ${ }^{3}$ Inha Univ., South Korea; ${ }^{4}$ Kangwon National Univ, South Korea; ${ }^{5}$ Air Force Inst. of Technology, USA; ' ${ }^{6}$ Arizona State Univ. USA. We fabricate a GeSn photonic crysta laser. The simulation shows a high Q-facto and wide spectral tunability. An improved ne gain and lowered power consumption are expected via strain-relaxation and reducing active mode volume, respectively.

\section{JTu5A.111}

Long Wavelength Single Mode GaSb Dide Lasers for Sensor Applications, Tobia Milde', Alvaro Jimenez' ${ }^{1}$ Joachim R. Sacher ${ }^{1,2}$, James O'Gorman',3; 'Sacher Lasertechnik $\mathrm{GmbH}$, Germany; ${ }^{2}$ Sensor Photonics $\mathrm{GmbH}$ Germany; ${ }^{3} X y l o p h o n e ~ O p t i c s ~ L t d$. , Ireland. New results in developing tunable single mode laser diodes based on GaSb material systems with emission in the wavelength (I) range $1.8 \mu \mathrm{m} \leq 1 \leq 4 \mu \mathrm{m}$ with application in absorption spectroscopy are presented.

JTu5A.112

Design of Dirac-point photonic crystal quantum cascade lasers, Yong Liang Zhixin Wang ${ }^{2}$, Xuefan Yin ${ }^{2}$, Chao Peng ${ }^{2}$ Weiwei $\mathrm{Hu}^{2}$, Jerome Faist'; ${ }^{1} \mathrm{ETH}$ Zurich, Switzerland; ${ }^{2}$ Peking Univ., China. We design photonic-crystals enabling laser operation at the Dirac-point, based on three-dimensional simulations of realistic quantum cascade laser structures. We demonstrate the feasibility of using the Dirac-point mode for higher-power single-mode photonic-crystal quantum cascade lasers.

JTu5A.113

Efficient Perovskite Light Emitting Diodes Based on Double Organic Cations, Bing $\mathrm{Xu}^{1}$, WanYu Cao', Xiaoli Zhang ${ }^{1}$, Weigao Wang', Junjie Hao', Shuming Chen', Kai Wang ${ }^{1}$, Xiao Wei Sun ${ }^{1}$; ${ }^{1}$ Electrical \& Electronic Engineering, Southern Univ. of Science and Technology, China. We report a FA and MA mixed hybrid perovskite light emitting diodes, which exhibited maximum luminescent of $6000 \mathrm{~cd} / \mathrm{A}$, maximum current efficiency of $6 \mathrm{~cd} / \mathrm{A}$ and maximum external quantum efficiency of $3.5 \%$.

\section{JTu5A.114}

$\mathrm{Pr}^{3+}$ Doped Ceramic Calcium Lanthanum Sulfide for Mid-IR Laser Gain Material Brandon Shaw ${ }^{1}$ Michael Hunt ${ }^{1}$, Woohong Kim', Shyam Bayya', Christopher Brown² Steve Bowman', Jasbinder Sanghera'; 'US Naval Research Lab, USA; ${ }^{2}$ Univ. Research Foundation, USA. We report on fabrication and spectroscopy of ceramic Praseodymium doped Calcium Lanthanum Sulfide for potential mid-IR laser gain material.

\section{JTu5A.115}

Stable quantum dots liquid-type white light emitting diode by laser-cut glass package Chih-Hao Lin' ', Lin Huang Y Y' ${ }^{1}$ Chin-wei Sher' Hao-chung Kuo'; ; National Chiao Tung Univ., Taiwan. The luminous efficiency and colo rendering index (CRI) of the LOD WLED can reach to $271 \mathrm{~lm} / \mathrm{W}_{\text {op }}$ and 95 respectively. Moreover, the glass box is employed to prevent from humidity and oxygen erosion and our device can survive over 1000 hours of on-self storage.

\section{JTu5A.116}

Carrier frequency interferometry for wavefront measurements of coated optics, Elzbieta Jankowska', Slawomir Drobczynski Carmen S. Menoni': 'Colorado State Univ. USA; ${ }^{2}$ Wroclaw Univ. of Technology, Poland. We apply carrier frequency interferometry to determine the wavefron deformation of thin films and multilayer dielectric coatings on thick substrates. The method allows to obtain the radius of curvature of the coated samples with high accuracy with a relatively simple setup.

\section{Ju5A.117}

Highly Transmittive Broadband Dielectric Nanoholes, Gauri Mangalgiri,1,2, Makars Siškins ${ }^{1}$, Alina Arslanova ${ }^{1}$ Martin Hammerschmidt ${ }^{2}$, Phillip Manley ${ }^{1,2}$, Wiebke Riedel ${ }^{3}$ Martina Schmid ${ }^{1,4} ;{ }^{1}$ Nanooptische Konzepte für die PV, Helmholtz Zentrum Berlin, Ger many; ${ }^{2}$ Computational Nano-Optics, Zuse Inst. Berlin, Germany; ${ }^{3}$ Chemistry, Freie Universität Berlin, Germany; ${ }^{4}$ Physics, Freie Universität Berlin, Germany. We demonstrate the fabrication and characterization of nanostructured transparent conductive oxides and via numerical simulation observe the parametric optical transmission trend. These structures can be used for customized ligh coupling to and from optoelectronic devices.

\section{JTu5A.118}

Optical Gain Characteristics of $\mathrm{Pb} / \mathrm{Bi} \mathrm{Co}$ doped Silica-based Optical Fiber, Haihong Zhan' Jianxiang Wen'. 'Shanghai Univ. China. A novel $\mathrm{Pb} / \mathrm{Bi}$ co-doped fiber has been fabricated. Its On/off gain without ASE is $12 \mathrm{~dB}$ at $1110 \mathrm{~nm}$ and $3 \mathrm{~dB}$ bandwidth is $180 \mathrm{~nm}$ by $0.2 \mathrm{~m}$ fiber, which indicates it is a promising active material.

\section{JTu5A.119}

Silica-based Inorganic Microdisk Cavity by the Ink-jet Printing Method, Hiroaki Yoshioka', Yuya Mikami', Soichiro Ryu', Shintaro Mitsui ${ }^{1}$, Cong Chen ${ }^{1}$, Naoya Nishimura ${ }^{2}$, Yuj Oki'; ' ${ }^{1}$ Kyushu Univ., Japan; ${ }^{2}$ Nissan Chemical Industries, Japan. We experimentally succeeded fabrication and WGM lasing of norganic microdisks by the ink-jet printing method. This silica-based microdisk cavity is the first demonstration in ordinary pressure and temperature among inorganic microdisks.

\section{JTu5A.120}

Chromatic dispersion fluctuation and optical parametric amplification performance in a tellurite hybrid microstructured optical fiber with buffer layer, Hoang Tuan Tong ${ }^{1}$, Trung Hoa Nguyen Phuoc ${ }^{1}$, Harutaka Kawamura', Takenobu Suzuki', Yasutake Ohishi'; 'Toyota Technological Inst., Japan. Chromatic dispersion fluctuation and optica parametric amplification performance in a tellurite hybrid microstructured optical fiber with buffer layer are studied when the fiber transverse geometry variation is taken into account to improve its practical meaning and performance.

\section{JTu5A.121}

High-Efficiency Single-Junction GaAs Sola Cell using ITO-Film as an Antireflection and Passivation Layer Deposited on AllnP layer by Thermally RF Sputtering, Jian-Cheng Lin', Wen-Jeng Ho', Jheng-Jie Liu', Shih-Ting Tseng', Cho-Chun Chiang', Bang-Jin You', Yun-Chie Yang', Wen-Bin Bai', Zong-Xian Lin ${ }^{1}$, Hung-Pin Shiao ${ }^{2} ;{ }^{1}$ National Taipei Univ. of Technology, Taiwan; ${ }^{2}$ Win Semiconductor Corp., , Taiwan. This study presents highefficiency of $23.52 \%$ single-junction $\mathrm{GaAs}$ solar cell using ITO-film as antireflection and passivation layer deposited by thermallyRF-sputtering. The impressive enhanced in efficiency of $8.94 \%$ was obtained, compared to the cell with $\mathrm{SiO}_{2}$-film.

\section{JTu5A.122}

Synthesis of Millimeter-Size Freestanding Perovskite Nanofilms from Single-Crysta Lead Bromide for Optoelectronic Devices Jian-Yao Zheng', Jing Jing Wang ${ }^{1}$, Hugh Manning ${ }^{1}$, Chuan Zhong ${ }^{1}$, Finn PurcellMilton', Rudi O'Reilly Meehan², Graeme Cunningham², Ryan Enright'ㄹ, Yurii K. Gun'ko' John Boland', John Donegan'; 'Trinity College Dublin, Ireland: ${ }^{2}$ Thermal Management Research Group, Efficient Energy Transfe (nET) Dept., Bell Labs Research, Nokia, Ireland. We developed a strategy for synthesizing perovskite nanofilms and demonstrate a high-performance nanofilm photodetector. This novel synthesis method provides a new platform for exploiting optoelectronic devices based on free-standing perovskite nanofilms with high crystal quality.

\section{JTu5A.123}

Chirality Transfers from Light to Surface Relief, Leila Mazaheri ${ }^{3}$, Olivier Lebel ${ }^{1}$, JeanMichel Nunzi'; ; Dept. of Chemistry and Chemical Engineering, Royal Military College of Canada, Canada; 'Dept. of Chemistry and physics, Queen's Univ. , Canada; ${ }^{3}$ Dept. of Physics, Queen's Univ. Canada. Distinct chiral structures were inscribed on a Disperse Red 1 glass-forming derivative using a single laser beam with different polarization states at $\lambda=532 \mathrm{~nm}$. The source of optical activity is attributed to the diffractive properties of the structures.

JTu5A.124

Harsh Environment Tests of Random Antireflective Surface Structures on Optics, Lynda E. Busse ${ }^{1}$, Jesse Frantz' ${ }^{1}$ Menelaos K. Poutous ${ }^{2}$, Ishwar Aggarwal ${ }^{3}$, Brandon Shaw', Jas Sanghera'; 'US Naval Research Lab, USA; ${ }^{2}$ Univ. of North Carolina, USA; ${ }^{3}$ Sotera Defense Solutions, USA. We show results for sustained optical performance of random antireflective surface structures on silica windows following sand and rain erosion and salt fog exposure, as a representative field test for laser windows in harsh environments.

\section{JTu5A.125}

Ge/SiGe Quantum-well Micro-bridges with High Tensile Strain, Muyu Xue Xiaochi Chen ${ }^{4}$, Junyan Chen ${ }^{2}$, Ming-Yen $\mathrm{KaO}^{3}$, Colleen Shang ${ }^{1}$, Kai Zang ${ }^{4}$, Yijie Huo ${ }^{4}$ Ching-Ying Lu ${ }^{4}$, Yusi Chen ${ }^{4}$, Huiyang Deng ${ }^{4}$ Theodore Kamins ${ }^{4}$, James Harris ${ }^{1,4}{ }^{1}{ }^{1}$ Materials Science and Engineering, Stanford Univ. USA; ${ }^{2}$ School of Physics, Peking Univ., China ${ }^{3}$ Electrical Engineering, National Taiwan Univ., Taiwan; ${ }^{4}$ Electrical Engineering, Stanford Univ., USA. Highly tensile-strained $\mathrm{Ge}$ $\mathrm{SiGe} \mathrm{MOW}$ structures on $\mathrm{Si}$ substrates are formed by introducing micro-bridge struc tures. Lattice matching and strain of epitaxia structures are analyzed by XRD. Large strain induced by micro-bridges is confirmed with Raman spectroscopy.

\section{JTu5A.126}

Process Design Kit and Modulator Simulation for Hybrid Silicon-Lithium Niobate Integrated Optics, Peter Weigel ${ }^{1}$, Shayan Mookherjea"; 'Univ. of California San Diego, USA. A set of optical components for the hybrid silicon-lithium niobate platform is designed and tabulated as robus parameterized-cells. Eye diagrams of an 10 electro-optic modulator are simulated at 25 and $50 \mathrm{Gbps}$.

\section{JTu5A.127}

Etch-tuning and Design of SiN Photonic Crystal Reflectors, Simon Bernard', Christoph Reinhardt ${ }^{1}$, Vincent Dumont ${ }^{1}$, Yves-Alain Peter ${ }^{2}$, Jack Sankey'; ${ }^{1}$ Physics, McGill Univ. Canada; ${ }^{2}$ Engineering Physics, Polytechnique, Canada. We tune a freestanding photonic crystal reflector resonance to within $0.15 \mathrm{~nm}$ (0.04 linewidths) of $1550 \mathrm{~nm}$ using iterative hydrofluoric acid etches, and provide design considerations for creating reflectors robust against beam collimation.

\section{JTu5A.128}




\section{8:00-19:30 JTu5A - Poster Session I and Conference Reception}

\section{JTu5A.129}

Spectral Hole Narrowing in $\mathrm{Er}^{3+} 4 \mathrm{f}$ Transitions by Isotope Separation, Takehiko Tawara ${ }^{1,2}$, Giacomo Mariani', Kaoru Shimizu', Hiroo Omi ${ }^{1,2}$, Satoru Adachi ${ }^{3}$, Hideki Gotoh ${ }^{1}$; ${ }^{1}$ NTT Basic Research Labs, Japan; ${ }^{2}$ NTT Nanophotonics Center, Japan; ${ }^{3}$ Hokkaido Univ., Japan. We report effect of isotope separation on the homogeneous linewidth in $\mathrm{Er}^{3+}$-doped $\mathrm{Y}_{2} \mathrm{SiO}_{5}$ measured by using spectral hole burning. Isotope ${ }^{167} \mathrm{Er}^{3+}$ provides a drastic reduction of the spectral hole width by strongly suppressing instantaneous spectral diffusion.

\section{JTu5A.130}

Continuously-chirped grating formation by low-cost laser interference lithography for achieving tunable guided mode resonance filter, Tzu-Chieh Kao', Jia-Jin Lin', Chia-Wei Huang', Yung-Jr Hung '; ' National Sun Yat-sen Univ., Taiwan. A guided-mode-resonance filter based on continuously-chirped gratings is realized by a modified Lloyd's mirror interferometer to provide a sharp transmission dip at the resonant wavelength that can be gradually swept across the visible spectral region.

\section{JTu5A.131}

Synthesis and Application of Metal Halide Perovskite Nanocrystals, Xiaoli Zhang ${ }^{1}$, Bing Xu' , Kai Wang ${ }^{1}$, Xiao Wei Sun ${ }^{1} ;{ }^{1}$ SUSTC, China. Metal halide perovskite nanocrystals are fabricated via all-solution method, which exhibit outstanding optoelectronic properties. The application in optoelectronic device demonstrates great improved performance, especially in light-emitting diodes, which display improved activity via proper composition adjustment and device construction modification.
JTu5A.132

Multifold enhancement of graphene interband absorption in a Salisbury screen, Xiangxiao Ying', Yang $\mathrm{Pu}^{1}$, Yi Luo', ${ }^{1} \mathrm{HaO}$

Peng', Zhe Li', Yadong Jiang', Zhijun Liu'; 'Univ of Electronic Sci \& Tech of China, China. An enhancement of graphene interband absorption by more than four-fold is demonstrated in a Salisbury screen configuration. For a monolayer graphene, peak absorptions between $9 \%$ and $40 \%$ are measured at different incident angles. 
Executive Ballroom 210A

Executive Ballroom 210B

\section{CLEO: Applications}

\& Technology

08:00-10:00

AW1A $\cdot$ Medical Devices and Systems

Presider: Xuan Liu; New Jersey

Inst. of Technology, USA

AW1A.1 • 08:00

All-Optical Pulse-Echo Ultrasound Imaging for Guiding Minimally Invasive Procedures Adrien Desjardins'. Charles Mosse', Richard Colchester', Sacha Noimark', Erwin Alles', Edward Zhang', Sebastien Ourselin', Ivan Parkin', loannis Papakonstantinou', Paul Beard', Malcolm Finlay',2, 'Univ. College London, UK; ${ }^{2}$ Queen Mary Univ. of London, UK. Recent advances in optical transmission and reception of ultrasound have enabled pulseecho imaging using fiber-optic probes that are suitable for guiding clinical intracardiac and intravascular procedures. In vivo images from pre-clinical models will be presented.
08:00-10:00

AW1B - Sensing in Fibers and

Free-space

Presider: Paul Williams; NIST, USA

AW1B.1 $\bullet$ 08:00

Fiber-Optic Current Sensor Immune to Polarization Cross-Talk at Polarization Maintaining Fiber Connectors, Klaus M. Bohnert', Chen-Pu Hsu', Lin Yang', Andreas Frank', Georg M. Mueller', Philippe Gabus ${ }^{2}$; ${ }^{1}$ Corporate Research, ABB Switzerland Ltd, Switzerland; ${ }^{2}$ High Voltage Products, ABB Switzerland Ltd, Switzerland. We investigate the effect of polarization cross-coupling at polarization maintaining fiber connectors on the accuracy of interferometric fiber-optic current sensors and demonstrate an optica circuit that is immune to polarization crosstalk at connectors.
Executive Ballroom $210 \mathrm{C}$

\section{CLEO: Science \&} Innovations

08:00-10:00

SW1C $\bullet$ Nanolasers and

Frequency Combs

Presider: Lan Fu; Australian

National Univ., Australia

SW1C.1 0 08:00

Focusing Metasurface Quantum-Cascade VECSEL, Luyao Xu $\mathrm{u}^{1,3}$, Christopher Curwen ${ }^{1,3}$ Daguan Chen', Tatsuo Itoh', John Reno Benjamin Williams ${ }^{1,3} ;{ }^{1}$ Dept. of Electrical Engineering, UCLA, USA; ${ }^{2}$ Center of Integrated Nanotechnologies, Sandia National Labs, USA; ${ }^{3}$ California NanoSystems Inst. (CNSI), UCLA, USA A terahertz quantum-cascade vertical-external-cavity surface-emitting laser (VECSEL) is demonstrated using an active focusing metasurface. This enables a hemispherical cavity with flat optics, which exhibits an excellent beam pattern and higher stability than a plano-plano cavity.
Executive Ballroom 210D

\section{CLEO: QELS- Fundamental Science}

08:00-10:00

FW1D • Non-diffracting Beams

Presider: Zhigang Chen; San

Francisco State Univ., USA
FW1D.1・08:00

Controlling Cherenkov Radiation Emission through Self-accelerating Wave-packets, $\mathrm{Yi} \mathrm{Hu}^{1}$, Zhili Li ${ }^{1}$, Benjamin Wetze ${ }^{3,2}$, Roberto Morandott ${ }^{3}$, Zhigang Chen ${ }^{1,4}$, Jingjun $\mathrm{Xu}^{1}$ 'Nankai Univ., China; ${ }^{2}$ Univ. of Sussex, UK: ${ }^{3} / n$ stitut National de la Recherche Scientifique Canada; ${ }^{4}$ San Francisco State Univ., USA. We demonstrate the control of dispersive waves (Cherenkov-like radiation) excited by selfaccelerating pulses propagating in optica fibers. We show that such dispersive waves can be effectively compressed/focused by simply tuning the pump pulse acceleration.

\section{AW1A.2 • 08:30 Invited}

Multimodal Stain-Free Imaging of the Retina with a Sub-35fs Yb-fiber Laser, Marcos Dantus'.; ${ }^{1}$ Michigan State Univ., USA Epi-directional imaging of the highly sensitive layers of the retina requires short pulse excitation to minimize thermal damage. We present multimodal differentiation of unstained retinal layers using a sub-40 fs Yb fiber laser.
AW1B.2 • 08:15

Optical Fiber Sensor-Fused Additive Manufacturing and Its Applications in Residual Stress Measurements, Ran Zou' Xuan Liang ${ }^{2}$, Rongtao Cao', Shuo Li', Albert $\mathrm{To}^{2}$, Paul Ohodnicki ${ }^{3}$, Michael Buric ${ }^{3}$, Kevin Chen'; ${ }^{1}$ Dept. of Electrical and Computer Engineering, Univ. of Pittsburgh, USA; ${ }^{2}$ Dept. of Mechanical Engineering and Materials Science, Univ. of Pittsburgh, USA; ${ }^{3}$ Nationa Energy Technology Lab, USA. This paper reports optical fiber embedded in Ti-6Al-4V components using active manufacturing process. Thermal-induced residual stress on the sensor-embedded parts was measured using Rayleigh scattering distributed sensing scheme with 5-mm spatial resolutions.

AW1B.3 $08: 30$

High Resolution Optical Fiber Sensor for Quasi-Static Strain Measurement by Strain-Temperature Discrimination, Jiageng Chen ${ }^{1}$, Qingwen Liu', Xinyu Fan ${ }^{1}$, Zuyuan $\mathrm{He}^{1}$. 'State Key Lab of Advanced Optical Communication Systems and Networks, Shanghai Jiao Tong Univ., China. A high resolution quasi-static strain sensor based on a nove scheme for strain and temperature discrimination has been developed. Within $16000 \mathrm{~s}$ measurement, resolutions of $0.025 \mu \varepsilon$ in strain and $0.0019^{\circ} \mathrm{C}$ in temperature are achieved.
SW1C.2 • 08:15

Optoelectronic Control of an External Cav ity Quantum Cascade Laser Using a Graphene Loaded Metamaterial Array, Stephen J. Kindness', David Jessop', Binbin Wei ${ }^{1}$ Robert Wallis ${ }^{1}$, Varun Kamboj', Long Xiao ${ }^{1}$ Yuan Ren ${ }^{1,2}$, Philipp Braeuninger-Weimer ${ }^{1}$ Stephan Hofmann', Harvey E. Beere', David A. Ritchie1, Riccardo Degl'innocenti'; ' 'Univ. of Cambridge, UK; ${ }^{2}$ Chinese Academy of Sciences, China. We present the optoelectronic frequency and amplitude modulation of a terahertz quantum cascade laser, achieved by implementing a graphene loaded split ring resonator array into an external cavity feedback set-up.

\section{SW1C. $3 \bullet 08: 30$ Invited}

Orbital Angular Momentum Microlaser, $\mathrm{Pe}$ Miao', Zhifeng Zhang ${ }^{1}$, Jingbo Sun', Wikto Walasik', Stefano Longhi², Natalia M. Litchinitser', Liang Feng'; ' SUNY Buffalo, USA ${ }^{2}$ Politecnico di Milano and Istituto di Fotonica e Nanotecnologie del Consiglio Nazionale delle Ricerche, Italy. By exploiting the emerging non-Hermitian photonics design at an exceptional point, we demonstrate a microring aser producing a single-mode OAM vortex lasing with the ability to precisely define the topological charge of the OAM mode.
FW1D.2 • 08:15

Nonlinear Interaction of Oppositely Charged Vortices Generating Hollow Gaussian Beams, Apurv Chaitanya Nellikka', Jabir M. V. ${ }^{1}$, J Banerji', Goutam Samanta'; 'Physical Research Lab, India. We demonstrate a novel experimental scheme generating high-power, higher-order hollowGaussian-Beam (HGB) through annihilation of orbital-angular-momentum of the interacting photons in nonlinear process. Also, report new and only method for characterizing the order of HGBs.

\section{FW1D. $3 \bullet 08: 30$}

Control of nonlinear instabilities in Bessel beams using shaped longitudinal intensity profiles, Ismail Ouadghiri Idrissi ${ }^{1}$ Remo Giust ${ }^{1}$, John Michael Dudley ${ }^{1}$, Francois Courvoisier'. 'FEMTO-ST, France. We show that tailored longitudinal intensity shaping of a non-diffracting Bessel beam can strongly reduce four wave mixing induced oscillations and stabilize nonlinear propagation at ablation-level intensities 
Executive Ballroom $210 \mathrm{E}$

Executive Ballroom $210 \mathrm{~F}$

\section{CLEO: QELS-Fundamental Science}

08:00-10:00

FW1E - Rare Earth Solid State

Quantum Memories

Presider: Elizabeth Goldschmidt;

US Army Research Lab, USA

\section{FW1E.1 08.00 Tutorial}

Introduction to the Spectroscopy of RareEarth Doped Crystals for Quantum Communications, Matthew Sellars ${ }^{1}$; ${ }^{1}$ Australian National Univ. Australia. Quantum memories for light are required elements for long-range quantum communication networks. Rareearth doped crystals are uniquely suited for this application. This tutorial will review the spectroscopy of these centers relating it to quantum storage protocols.

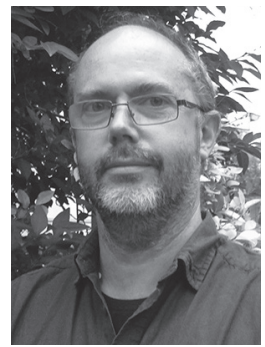

Matthew Sellars is internationally recognized for his foundational work in ultra-high resolution spectroscopy of rare-earth doped crystals and their use in quantum computing and quantum communication applications. $\mathrm{He}$ is currently the head of the Laser Physics Centre in the Research School of Physics and Engineering at the Australian National University.
08:00-10:00

FW1F • Quantum Entanglement Presider: Jeffrey Shapiro; MIT, USA

FW1F.1 • 08:00

Observation of Ten-photon Entanglement Using Thin $\mathrm{BiB}_{3} \mathrm{O}_{6}$ Crystals, Luo-Kan Chen', Zheng-Da Li', Xing-Can Yao', Miao Huang', Wei Li ${ }^{1}$, He Lu' ${ }^{1}$, Xiao Yuan², Yan-Bao Zhang ${ }^{3}$ Xiao Jiang', Cheng-Zhi Peng', Li Li', Nai-Le $\mathrm{Liu}^{1}$, Xiongfeng $\mathrm{Ma}^{2}$, Chao-Yang Lu', Yu-Ao Chen', Jian-Wei Pan ${ }^{1} ;{ }^{1}$ Univ. of Sci. and Tech. of China, China: ${ }^{2} T$ singhua Univ., China; ${ }^{3}$ Univ. of Waterloo, Canada. We demonstrate a ten-photon Greenberger-Horne-Zeilinger state using thin $\mathrm{BiB}_{3} \mathrm{O}_{6}$ crystals. The observed fidelity is 0.606 with a standard deviation of $3.6 \sigma$ and a p-value of $3.7 \times 10^{-3}$.

\section{FW1F. $2 \bullet 08: 15$}

Hyperentangled Photons Generation Using Crossed Quasi-Phase-Matched Superlattice, Salem F. Hegazy ${ }^{2,1}$, Salah S. Obayya ${ }^{3}$ Bahaa E. Saleh'; 'CREOL, The College of Optics \& Photonics, Univ. of Central Florida, USA; ${ }^{2}$ National Inst. of Laser Enhanced Sciences, Cairo Univ., Egypt; ${ }^{3}$ Centre for Photonics and Smart Materials, Zewail City of Science and Technology, Egypt. A superlattice structure featuring nonlinear layers with alternating orthogonal optic axes interleaved with orthogonal-poling directions, is shown to generate high-quality hyperentangled photons via orthogonal quasi-phase matching that corrects for phase- and group-velocity mismatching concurrently.

FW1F.3 • 08:30

Hyper-Entanglement of Photons Emitted by a Quantum Dot, Maximilian Prilmüller ${ }^{1}$, Tobias Huber 1,2, Markus Müller ${ }^{3}$, Peter Michler $^{3}$, Gregor Weihs', Ana Predojević ${ }^{4}$ IInstitut für Experimentalphysik, Universität Innsbruck, Austria; ${ }^{2}$ Joint Quantum Inst., NIST \& Univ. of Maryland, USA; ${ }^{3}$ nnstitut für Halbleiteroptik und Funktionelle Grenzflächen, Universität Stuttgart, Germany; ${ }^{4}$ Inst. for Quantum Optics, Universität Ulm, Germany. We report on demonstration of polarization and time-bin hyper-entanglement of photons emitted from a single quantum dot. We achieved this result by applying resonan excitation on a quantum dot system with absent fine structure splitting.
Executive Ballroom

210G

\section{Joint}

08:00-10:00

JW1G • Symposium on

Advances in Metaphotonic

Devices I

Presider: Xingijie Ni; Pennsylvania

State Univ.

\section{JW1G.1 08:00 Invited}

Quantum Plasmonics, Polaritons and Strong Light-Matter Interactions with 2d Material Heterostructures, Frank Koppens ${ }^{1,2}$; 'ICFO -The Inst. of Photonic Sciences, Spain; ${ }^{2}$ ICREA - Institució Catalana de Recerça i Estudis Avancats, Spain. In this talk, we will show several examples of $2 \mathrm{~d}$ materia heterostructure devices with novel ways of exciting, controlling and detecting polaritons. We challenge the limits of quantum light-matter interactions as well as extremes in propagating plasmon confinement, down to the scale of a few nanometers.

Executive Ballroom $210 \mathrm{H}$

\section{CLEO: QELS- Fundamental Science}

08:00-10:00

$\mathrm{FW} 1 \mathrm{H} \bullet$ Extreme

Electromagnetic Radiation - THZ to EUV: Generation, Detection \& Applications

Presider: Rohit Prasankumar; Los Alamos National Lab, USA

\section{FW1H.1 0 08:00}

Heisenberg vs. Stoner: Probing the Microscopic Picture of Ultrafast Demagnetization using High Harmonics, Dmitriy Zusin', Emrah Turgut ${ }^{1}$, Dominik Legut ${ }^{2,3}$, Karel Carva ${ }^{3}$ Christian Gentry', Phoebe Tengdin', Hans Nembach ${ }^{4}$, Justin Shaw ${ }^{4}$, Stefan Mathias ${ }^{5}$ Martin Aeschlimann ${ }^{6}$, Claus Schneider? Thomas Silva ${ }^{4}$, Peter Oppeneer ${ }^{8}$, Patrik Grychtol', Henry Kapteyn', Margaret Murnane'; 'JILA, Univ. of Colorado at Boulder, USA. ${ }^{2}$ IT4Innovations Center, VSB Technical Univ. of Ostrava, Czech Republic: ${ }^{3}$ Dept. of Condensed Matter Physics, Charles Univ. Czech Republic; ${ }^{4}$ Electromagnetics Division NIST, USA : ${ }^{5}$ Georg-August-Universitat Goettingen, Germany; 'Univ. of Kaiserslautern and Research Center OPTIMAS, Germany ${ }^{7}$ Peter-Grunberg-Institut PGI-6, Research Center Julich, Germany; ${ }^{8}$ Dept. of Physics and Astronomy, Uppsala Univ., Sweden. We uncover the multiple mechanisms underlying laser-driven demagnetization in cobalt using a tabletop high harmonic source. Ultrafast magnon excitation, as well as a transient reduction of the exchange splitting, are both important on femtosecond timescales.

FW1H.2 • 08:15

Real-time Measurement and Control of Multi-Petahertz Currents in Solids, Manish Garg ${ }^{1}$, Minjie Zhan ${ }^{1}$, Tran Trung Luu ${ }^{1}$, Harshit Lakhotia', Till Klostermann', Alexander Guggenmoss ${ }^{1}$, Eleftherios Goulielmakis ${ }^{1} ;{ }^{1}$ Max Planck Inst. for Quantum Optics, Germany. We used attosecond streaking to probe the temporal structure of isolated attosecond EUV pulses generated in a bulk solid using single-cycle optical driver. The encoded chirp connotes to intraband dynamics of electrons emitting EUV radiation.

\section{JW1G.2 • 08:30 Invited}

Metaoptics Technology in the Visible Federico Capasso'; 'Harvard Univ., USA $\mathrm{TiO}_{2}$ ALD based metaoptics with performance comparable to commercial optics has led to a host of applications of increased functionality and compactness. Advances in metalenses, miniature spectrometers, chira imaging, axicons, holograms, vortex plates and polarimeters will be presented.
FW1H.3 • 08:30

Extreme Nonlinear Carrier Dynamics Induced by Intense Quasi-half-cycle $\mathrm{THz}$ Pulses in n-doped InGaAs Thin Film, Xin Chai ${ }^{1}$, Xavier Ropagnol' ${ }^{1}$, Mohsen Raeiszadeh$^{2}$, Safieddin Safavi-Naeini ${ }^{2}$, Matthew Reid $^{3}$, Marc A. Gauthier', Tsuneyuki Ozaki' IINRS, Canada; ${ }^{2}$ Univ. of Waterloo, Canada ${ }^{3}$ Univ. of Northern British-Columbia, Canada. We report extreme nonlinear carrier dynamics in highly $n$-doped InGaAs thin film using terahertz time-domain spectroscopy. We attribute our observations to the dramatic reduction of terahertz photoconductivity induced by strong intervalley scattering effects. 


\section{CLEO: Science \& Innovations}

\section{8:00-09:45}

SW1I - Space-Division

Multiplexed Optical

Communications

Presider: Nicolas Fontaine; Nokia

Corporation, USA

\section{SW11.1 08:00 Invited}

MIMO-less Space Division Multiplexing Transmission over 1 km Elliptical Core Few Mode Fiber, Francesca Parmigiani', Yongmin Jung' ' Lars Grüner-Nielsen², Tommy Geisler', Periklis Petropoulos ${ }^{1}$, David Richardson 'Univ. of Southampton, UK; ${ }^{2}$ OFS, Denmark We experimentally demonstrate $10-\mathrm{Gbit} / \mathrm{s}$ OOK MIMO-less SDM transmission over 1 $\mathrm{km}$ of a three-spatial-mode elliptical-core fiber at $1550 \mathrm{~nm}$. Negligible power penalty is achieved thanks to the low modal crosstalk $(<-22 \mathrm{~dB})$ between any pair of the $L P_{01}, L P_{11}$ and $L P_{11 b}$ modes.

\section{SW11.2 • 08:30 Invited}

Novel Optical Fibers for Space Division Multiplexed Transmission Systems in Data Centers, Ming-Jun Li'; ${ }^{1}$ Corning Incorporated, USA. We review novel optical fibers for space division multiplexed short-reach transmission systems in data center applications. Both linear array multicore fibers and elliptical core few mode fibers are discussed and transmission results are presented.

\section{8:00-10:00}

SW1J • Precision References and Optical Synthesis

Presider: Tara Fortier; NIST, USA

\section{SW1J.1 08:00 Invited}

$1.5 \mu \mathrm{m}$ Lasers with Sub10 $\mathrm{mHz}$ Linewidth, Thomas Legero', Dan-Gheorghita Matei ${ }^{1}$, Sebastian Häfner ${ }^{1}$, Christian Grebing $^{2}$, Robin Weyrich', Fritz Riehle', Uwe Sterr ${ }^{1}$, Wei Zhang ${ }^{3}$, John Robinson ${ }^{3}$, Lindsay Sonderhouse ${ }^{3}$. Eric Oelker ${ }^{3}$, Jun $\mathrm{Ye}^{3}$. Germany; ${ }^{2}$ TRUMPF Scientific Lasers $\mathrm{GmbH}$, Germany: ${ }^{3}$ JILA, National Inst. of Standards and Technology and Univ. of Colorado, USA. We report on two ultrastable lasers stabilized to single-crystal silicon Fabry-Pérot cavities at $124 \mathrm{~K}$. The lasers show unprecedented thermal noise limited frequency instabilities of $4 \times 10^{-17}$ and linewidths below $10 \mathrm{mHz}$. ${ }^{1}$ Physikalisch-Technische Bundesanstalt,

08:00-10:00

SW1K • Flexible and Soft

Optoelectronics

Presider: Thomas Murphy; Univ. of Maryland at College Park, USA

SW1K.1 • 08:00 Tutorial

Recent Advances in Flexible/Stretchable Optoelectronics: From Next-Generation Displays to Skin-Mounted Wearables, John A. Rogers' ${ }^{1}{ }^{1}$ Materials Science, Northwestern Univ., USA. Advances in materials and device designs enable high performance optoelectronic systems that can flex, bend, twist and even stretch like a rubber band. This talk outlines the key ideas.

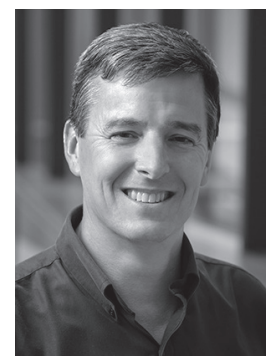

John A. Rogers is the Simpson/Querrey Professor of Materials Science and Engineering, Biomedical Engineering, Mechanical Engineering, Electrical Engineering and Computer Science, Chemistry and Medicine at Northwestern University, where he is Director of the Center on Bio-Integrated Electronics. He was previously at Bell Laboratories and the University of Illinois at Urbana/ Champaign

SW1J.2 $\bullet 08: 30$

A CW laser stabilized to a low expansion ceramic cavity with a $7 \mathrm{mHz} / \mathrm{s}$ frequency drift, Isao Ito', Alissa Silva', Takuma Nakamura', Yohei Kobayashi'; ' The Inst. for Solid State Physics, The Univ. of Tokyo, Japan. We developed a CW laser stabilized to a low expansion ceramic cavity with a frequency drift $7 \mathrm{mHz} / \mathrm{s}\left(\Delta \mathrm{f} / \mathrm{f} \sim 10^{-10} /\right.$ year), which is lower than those achieved with ultra-low expansion glass cavities.
08:00-10:00

SW1L - Sensing in Dynamic and Extreme Environments, Plasmas, and Explosions

Presider: Todd Stievater; US

Naval Research Lab, USA

\section{SW1L.1 08:00 Invited}

Optical Absorption Spectroscopy in Optically Dense Detonation Products, Nick Glumac'; 'Univ of Illinois at Urbana-Champaign, USA. Recent measurements of absorption of atomic and molecular species in optically thick fireballs generated by detonation of high explosives are presented. Tunable diode lasers, pulsed dye lasers, and broadband sources are used to probe UV and visible regions for critical intermediate species.
SW1L.2 • 08:30

Dual-Comb Spectroscopy of Laser-Induced Plasmas, Jenna Bergevin ${ }^{1}$, Tsung-Han $\mathrm{Wu}^{1}$, Jeremy Yeak $^{3}$, Brian Brumfield², Sivanandan S. Harila|2 ${ }^{2}$, Mark C. Phillips ${ }^{2}$, R. Jason Jones ${ }^{1}$ Univ. of Arizona, USA; ${ }^{2}$ Pacific Northwest National Lab, USA; ${ }^{3} P M \& A M$ Research, USA. We present the first results using broadband dual-comb spectroscopy in a laser-induced plasma. Preliminary results identifying ${ }^{85} \mathrm{Rb}$ and ${ }^{87} \mathrm{Rb}$ isotopes are shown using this technique. 


\section{CLEO: Science \& Innovations}

08:00-10:00

SW1M • Nonlinear Optics for Spectroscopy and Sensing

Presider: Michelle Sander; Boston Univ., USA

\section{SW1M.1 0 08:00 Tutorial}

Femtosecond 2D Spectroscopy of Nanomaterials and Photovoltaics, Martin Zanni'; ${ }^{1}$ Univ. of Wisconsin-Madison, USA. Femtosecond 2D spectroscopies reveal energy transfer, inhomogeneities, quantum coherences, and other photophysics. This talk will cover recent advances in the technology that enables easily obtained 2D spectra and highlight its application to nanomaterials and photovoltaics.

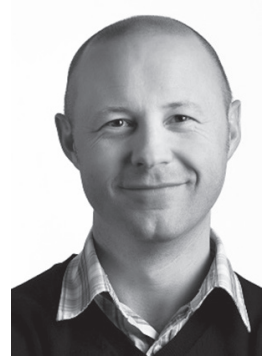

Martin T. Zanni is the Meloche-Bascom Professor of Chemistry at the University of Wisconsin-Madison. He is one of the major developers of ultrafast 2D Infrared and Electronic spectroscopies. His research program encompasses biophysics, photovoltaics, and surface science. He founded PhaseTech Spectroscopy, which is the first company to commercialize 2D spectroscopy.
08:00-10:00

SW1N • Silicon Photonic

Devices and Structures

Presider: Qiang Lin; Univ. of

Rochester, USA

\section{SW1N.1 • 08:00 Invited}

High-Radix Silicon Photonic Switches, Ming C. Wu', Tae Joon Seok'; 'Univ. of California Berkeley, USA. We review the state of the art of silicon photonic switches, with an emphasis on their scalabilities. We also describe a highradix MEMS-actuated silicon photonic switch that is scalable to hundreds of ports.

SW1N.2 • 08:30

Weakly-coupled Si waveguide Bragg reflector enabled by precisely-controlled graphene oxide gratings, Ya-Ching Liang ${ }^{1}$, Jyun-Fu Shih ${ }^{1}$, Chia-Wei Huang ${ }^{1}$, Tzu-Hsiang Yen', Jia-Jin Lin', Chun-Hu Chen', Yung-Jr Hung'; 'National Sun Yat-sen Univ., Taiwan. Enabled by atomically thickness control $(0.26$ $\mathrm{nm} / \mathrm{min})$ and loss engineering $(0.28 \mathrm{~dB} / \mathrm{cm} /$ $\mathrm{min}$ ) of graphene oxide (GO) integrated silicon waveguide via ozone treatment, a low-loss $(\sim 5 \mathrm{~dB} / \mathrm{cm})$ and narrowband (1.1-nm) $\mathrm{GO} /$ silicon hybrid waveguide Bragg reflector is demonstrated.
08:00-10:00

SW10 • Optical Comb \& Integrated Systems

Presider: Sasan Fathpour; CREOL, Univ. of Central Florida, USA

\section{SW10.1 0 08:00}

Micro-Integrated Extended Cavity Diode Laser with Integrated Optical Amplifier for Applications in Space, Christian Kürbis' Ahmad Bawamia', Mandy Krüger', Robert Smol', Andreas Wicht', Achim Peters', , Günther Tränkle'; 'Ferdinand-Braun-Institut, Leibniz-Institut für Höchstfrequenztechnik, Germany; ${ }^{2}$ Institut für Physik, HumboldtUniversität zu Berlin, Germany. We present a micro-integrated laser module consisting of an extended cavity diode laser and an optical amplifier. The fiber-coupled laser module emits $570 \mathrm{~mW}$ from a single mode, polarization maintaining fiber at $1064 \mathrm{~nm}$ with a FWHM linewidth of $26 \mathrm{kHz}(1 \mathrm{~ms})$.

SW10.2 • 08:15

Optical Frequency Synthesis by OffsetLocking to a Microresonator Comb, Shamsul Arafin', Arda Simsek', SeongKyun Kim', Sarvagya Dwivedi', Wei Liang ${ }^{2}$, Danny Eliyahu², Jonathan Klamkin', Andrey Matsko², Leif Johansson ${ }^{3}$, Lute Maleki², Mark J. Rodwell', Larry A. Coldren'; 'Univ. of California Santa Barbara, USA; ${ }^{2}$ EEwaves Inc., USA; ${ }^{3}$ Freedom Photonics LLC, USA. We report on the experimental demonstration of a chip-scale microresonator comb enabled optical frequency synthesizer using an agile and highly-integrated heterodyne optical phase-locked loop with InP-based photonic integrated circuit and commercial-off-theshelf electronic components.

\section{SW10.3 • 08:30}

Integratable Optical Comb Source for Coherent Communications Systems, Justin K. Alexander, ${ }^{1,2}$, Padraic E. Morrissey ${ }^{1}$, Ludovic Caro ${ }^{1,2}$, Mohamad Dernaika ${ }^{1,2}$, Niall Kelly,2, Frank Peters',2; 'Tyndall, Ireland; 'Physics, Univ. Collage Cork, Ireland. A coherent optical comb source is monolithically integrated. Optical combs were generated at $4 \mathrm{GHz}$ and $5 \mathrm{GHz}$, with the combs produced independent of cleaved facets. 
Executive Ballroom $210 \mathrm{~A}$

Executive Ballroom 210B

\section{CLEO: Applications \& Technology}

AW1A $\bullet$ Medical Devices and Systems-Continued
AW1B - Sensing in Fibers and Free-space-Continued
Executive Ballroom $210 \mathrm{C}$

\section{CLEO: Science \& Innovations}

SW1C $\bullet$ Nanolasers and Frequency Combs-Continued
Executive Ballroom 210D

CLEO: QELSFundamental Science

FW1D • Non-diffracting

Beams-Continued
AW1B.4 $\bullet$ 08:45

Dual-Core Optical Fibers for Simultaneous Measurements of Temperature and Strain Using Brillouin OTDA, Kevin P. Chen', Mohamed Zaghloul', Mohan Wang', Shenping $\mathrm{Li}^{2}$, Ming-Jun Li' ${ }^{2}$, Giovanni Milione ${ }^{3} ;{ }^{1}$ Univ. of Pittsburgh, USA: ${ }^{2}$ Corning Incorporate, USA ${ }^{3}$ NEC Labs America, Inc., USA. We report a dual-core fiber for simultaneous sensing of strain and temperature using BOTDA. By adjusting dopant compositions, 37\% difference in strain-optical coefficient was achieved between two cores to differentiate temperature and strain responses.

\section{AW1A.3 • 09:00}

Custom Thin Film Si Photodiode Arrays for Endoscopic Spatially Resolved Diffuse Reflectance Measurements, Benjamin LaRiviere'; ' ${ }^{2}$ CE Dept., Duke Univ., USA. The design, fabrication, and initial experimenta characterization of the first thin film Si photodiode spatially resolved DRS probe targeting endoscopic applications is reported.

\section{AW1A.4 $\bullet$ 09:15}

A demonstration of structured-illuminationbased technique using commercial surgical endoscope, Hanh N. Le', Hieu Nguyen ${ }^{2}$ Zhaoyang Wang', Jin U. Kang'; ' Johns Hopkins Univ., USA; ${ }^{2}$ Mechanical Engineering Catholic Univ. of America, USA. An integration of commercial surgical endoscope using structured illumination technique for threedimensional reconstruction was performed on biological samples with a depth of field of $20 \mathrm{~mm}$ and a relative accuracy of $0.1 \%$.

AW1A.5 • 09:30

Comparing digital and Shack-Hartmann wavefront sensing for in-vivo OCT imaging, Abhishek Kumar', Rainer A. Leitgeb', Wolfgang Drexler', Laurin Ginner', Matthias Pauliac', Lara Wurster'; 'Medical Univ. of Vienna, Austria. A small lateral field of view of $\sim 150 \times 150 \mu^{2}$ is scanned on human retina using a swept source OCT at a B-scan rate of $\sim 1.3 \mathrm{kHz}$ and used as a "guide star" to detect optical aberrations using subaperture based digital adaptive optics. The results are compared with Shack-Hartmann sensor measurements.

\section{AW1B.5 09.00 Invited}

The Application of Laser Off-Gas Analysis for Process Control in Harsh Industrial Environments, Doug Zuliani', Avishekh Pal'1. ${ }^{1}$ Tenova Goodfellow, Canada. Extractive or Insitu Lasers are traditionally employed to analyze process off-gas. Neither method offers a complete solution in harsh industria situations. A new hybrid method has been developed combining the best features of extractive \& lasers to provide multipoint analysis of hot, dirty gases.

AW1B.6 • 09:30

Towards a Scalable Ultrasensitive Optomechanical Magnetometer, Varun Prakash ${ }^{1}$ Beibei Li', Stefan Forstner', Douglas Bulla ${ }^{2}$ Scott Foster', Halina Rubinsztein-Dunlop', Warwick Bowen'; ' 'Univ. of Queensland, Australia; ${ }^{2}$ SES, Defense Science and Technology Group, Australia. Optomechanical magnetometers with reproducible sensitivity of $600 \mathrm{pT} / \mathrm{VHz}$ using a combination of standard photolithography and sputte deposition techniques which provides a scalable pathway for magnetometers for diverse applications has been realized.
SW1C.4 $\bullet$ 09:00

Non-Hermitian Aspects of Coherently Coupled Vertical Cavity Laser Arrays, Zihe Gao', Stewart T. Fryslie', Bradley Thompson' Harshil Dave', Katherine Lakomy', P. S. Carney', Kent D. Choquette1. 'Univ. of Illinois, USA. Coherently coupled $1 \times 2$ VCSEL arrays with intentional built-in asymmetry have been designed and fabricated. Preliminary characterization shows electrically controlled mode switching and indications of parity-time symmetry breaking.

\section{SW1C.5 $09: 15$}

Narrow Linewidth Frequency Comb Source based on Self-injected Quantum-Dash Passively Mode-Locked Laser, Kame Merghem', Vivek Panapakkam', Quentin Gaimard', Francois Lelarge ${ }^{2}$, Abderrahim Ramdane ${ }^{1} ;{ }^{1} \mathrm{CNRS}$, France; ${ }^{2}$ Almae technologies, France. An optical frequency comb generated by an InAs/InP quantum-dash-based passively mode-locked laser spans $\sim 1.5 \mathrm{THz}$ with $25 \mathrm{GHz}$ spacing, and less than $100 \mathrm{kHz}$ optical linewidth for all lines.

SW1C.6 $09: 30$

Time domain analysis of self-frequency modulated combs in quantum cascade lasers, Nathan C. Henry', Jacob Khurgin ${ }^{1}$ 'Johns Hopkins Univ., USA. We have developed a time domain model confirming that the most efficient mode operation of a free running $\mathrm{QCL}$ is a pseudo-random frequency modulated mode with constant intensity.
FW1D.4 • 08:45

Optimal energy confinement of optical Airy3 bullets, domenico bongiovanni', Benjamin Wetze ${ }^{1,2}, \mathrm{Yi} \mathrm{Hu}^{3}$, Zhigang $\mathrm{Chen}^{3,5}$, Roberto Morandottii',4; 'INRS, Canada; ${ }^{2}$ School of Mathematical and Physical Sciences, Univ. of Sussex, UK. ${ }^{3}$ TEDA Applied Physics Inst. and School of Physics, Nankai Univ., China; ${ }^{4}$ Inst. of Fundamental and Frontier Sciences, Univ. of Electronic Science and Technology of China, China; ${ }^{5}$ Dept. of Physics \& Astronomy, San Francisco State Univ., USA. We report on a numerical approach to improve the energy confinement of optical Airy3 bullets, based on compressing the Fourier spectrum. The resulting bullets exhibit a significant enhancement of the peak intensity.

\section{FW1D.5 • 09:00}

Abruptly Focusing and Defocusing Needles of Light, Liang Jie Wong', Ido Kaminer ${ }^{2}$ 'SIMTech, Singapore; ${ }^{2}$ MIT, USA. Fourier optics enforces a tradeoff between length and narrowness in electromagnetic wavepackets. We present a family of electromagnetic wavepackets that overcomes this tradeoff abruptly focusing to and defocusing from intensity hotspots of any aspect ratio.

FW1D.6 • 09:15

Nondiffracting Beams in a Thin Liquid Soap Films, Anatoly Patsyk', Miguel A. Bandres', Mordechai Segev'; 'TechnionIsrael Inst. of Technology, Israel. We observe non-diffracting beam channels propagating in liquid soap membranes and study this phenomenon experimentally. The channel's width is determined by the power of the beam and the thickness of the membrane.

FW1D.7 • 09.30

Demonstration of Diffraction-Free Beams with Correlated Spatio-Temporal Spectrum, Hasan E. Kondakci' Ayman F. Abouraddy'; 'CREOL, Univ. of Central Florida, USA. We experimentally demonstrate diffraction-free pulsed-beams with arbitrary spatial profile by introducing judicious correlations between the spatial and temporal degrees of freedom, which propagate 200 Rayleigh ranges of a comparable-sized Gaussian beam. 
Executive Ballroom $210 \mathrm{E}$

Executive Ballroom $210 \mathrm{~F}$

\section{CLEO: QELS-Fundamental Science}

FW1E - Rare Earth Solid State Quantum Memories-Continued
FW1F • Quantum

Entanglement-Continued
Executive Ballroom 210G

\section{Joint}

JW1G • Symposium on

Advances in Metaphotonic Devices I-Continued
FW1F.4 • 08:45

Effects of Entanglement in Optical Amplifiers, James D. Franson ${ }^{1}$ Richard A. Brewster ${ }^{1}$ 'Univ. of Maryland Baltimore County, USA Entanglement between the signal and the amplifying medium can produce a large amount of decoherence in an ideal optical amplifie even when the gain is arbitrarily close to unity and the added noise is negligible.

\section{FW1E.2 • 09:00}

Solid state source of non-classical multimode photon pairs with controllable delay, Kutlu Kutluer', Margherita Mazzera' Hugues de Riedmatten'; 'ICFO, Spain. We demonstrate for the first time a DLCZ-type memory with a rare-earth ion-doped crystal (REIC) with photon counting by combining the DLCZ and the AFC protocols. We find second order cross correlation values up to $21 \pm 4$ and violate Cauchy-Schwarz equality by $\mathrm{R}=44 \pm 20$. We store 11 temporal modes.

FW1E.3 • 09:15

Nanophotonic atomic-frequency-comb quantum memory based on a rare-earth doped photonic crystal cavity, Tian Zhong ${ }^{1}$, Jonathan M. kindem', Jake Rochman', John Bartholomew', Andrei Faraon ${ }^{1}$; ${ }^{1}$ California Inst of Technology, USA. We demonstrate an efficient atomic frequency comb quantum memory based on an impedance-matched nanophotonic cavity fabricated in a $\mathrm{Nd}$ doped YVO crystal. Storage of time-bin qubits with $97 \%$ fidelity is achieved.
FW1E.4 • 09:30

Toward all-optical control of rare-earth ions for on-chip quantum technology, John G. Bartholomew ${ }^{1}$, Raymond Lopez-Rios ${ }^{1}$ Jonathan M. kindem', Jake Rochman', Tian Zhong ${ }^{1}$, Andrei Faraon'; ${ }^{1}$ T. J. Watson Lab of Applied Physics, California Inst. of Technol ogy, USA. We present the characterization of the AC Stark shift in a rare-earth ion doped photonic crystal cavity. The strength of the interaction creates opportunities within previously inaccessible regimes of all-optical quantum memory protocols.

\section{FW1F.5 • 09:00}

Connecting two multipartite entangled states by entanglement swapping, Xiaolong $\mathrm{Su}^{1}$, Caixing Tian ${ }^{1}$, Xiaowei Deng ${ }^{1}$, Qiang Li ${ }^{1}$ Changde Xie', Kunchi Peng'; 'Shanxi Univ., China. We experimentally demonstrate the connection of two multipartite entangled states by quantum entanglement swapping The results provide a feasible technical reference for constructing more complicated quantum networks.

\section{FW1F.6 • 09:15}

Two-photon interference with frequencybin entangled photons, Poolad Imany ${ }^{1,2}$ Ogaga D. Odele $e^{1,2}$, Jose Jaramillo-Villegas ${ }^{1,2}$ Daniel Leaird ${ }^{1,2}$, Andrew Weiner ${ }^{1,2},{ }^{\prime}$ Purdue Univ., USA; ${ }^{2}$ Purdue Quantum Center, Purdue Univ., USA. We present a novel approach to demonstrate coherence between different bins of a frequency-bin entangled photonpair. The observed interference pattern provide a simple way to verify frequency-bin entanglement using slow single-photon detectors.

\section{FW1F.7 • 09:30 Invited}

Entangling Narrowband Photon Pairs, Shengwang Du ${ }^{1} ;{ }^{1}$ Hong Kong Univ of Science \& Technology, Hong Kong. We review our recent progress in generating and manipulating narrowband entangled photon pairs from spontaneous four-wave mixing in atomic ensembles. These photons are ideal for interacting with atomic quantum nodes in a photon-atom quantum network.
JW1G.3 • 09:00 Invited

Anti-Hermitian Metafilm-based Photode tector for Efficient Subwavelength Photonsorting, Mark Brongersma ${ }^{1}$; ${ }^{1}$ Stanford Univ USA. A metafilm-based photodetector is presented that affords spatial sorting of photons of different wavelengths below the free-space diffraction limit and extraction of useful photocurrent. An optimization of the metafilm leads to near-unity absorption and chipscale, anti-Hermitian optical coupling between the metafilm elements facilitates narrow spectral response $(\sim 30 \mathrm{~nm})$ for the different wavelength channels.

JW1G.4 • 09:30

Multilayered Metamaterials for Functiona Light Control, Euclides C. Almeida ${ }^{1}$ O Avayu $^{2}$, Tal Ellenbogen², Yehiam Prior ${ }^{1}$ 'Weizmann Inst. of Science, Israel; ${ }^{2}$ Dept. of Physical Electronics, Tel Aviv Univ., Israel. We demonstrate composite, multiplexed 3D metamaterials for functional light manipulation. Applications include multi-wavelength achromatic metalenses in the visible spectra range, integrated elements for STED microscopy, and nonlinear holography. Prospects for novel applications are discussed.
Executive Ballroom $210 \mathrm{H}$

\section{CLEO: QELS- Fundamental Science}

FW1 $\bullet$ Extreme

Electromagnetic Radiation - THZ to EUV: Generation, Detection \& Applications-Continued

FW1H.4 0 08:45

Emission of THz Radiation by GeS Nanosheets, Kateryna Kushnir' Mengjing Wang $^{2}$, Kristie Koski ${ }^{3,2}$, Lyubov Titova ${ }^{1}$ 'Worcester Polytechnic Inst., USA; ${ }^{2}$ Brown Univeristy, USA; ${ }^{3}$ Univ. of California Davis, USA. We have observed emission of terahertz radiation from photoexcited $\mathrm{GeS}$ nanosheets without external bias. We attribute the origin of terahertz pulse emission to the shift current resulting from inversion symmetry breaking in ferroelectric single- or few-layer $\mathrm{GeS}$ nanosheets.

\section{FW1H.5 09:00}

Hybrid Attosecond Pulse Generation, T J. Hammond ${ }^{1,2}$, David Villeneuve ${ }^{1}$, Paul B. Cor kum'; ' JAS Lab, U. Ottowa, Canada; ${ }^{2}$ Physics, Univ. of Central Florida, USA. We combine harmonics from solids and gases to generate isolated attosecond pulses with central frequency controlled by over an octave in the XUV. Spatio-temporal coupling and the carrier-envelope-phase significantly affect the generated half-cycle optical transient field.

\section{FW1 $16 \cdot 09 \cdot 15$}

Quantum-Interference Controlled High Harmonics in Semiconductors, Mackillo Kira', Ulrich Huttner ${ }^{2,1}$, Stephan W. Koch ${ }^{2}$ Fabian Langer $^{3}$, Matthias Hohenleutner ${ }^{3}$ Rupert Huber ${ }^{3} ;{ }^{1}$ Center for Ultrafast Optical Science, Univ. of Michigan, USA; ${ }^{2}$ Dept. of Physics, Univ. of Marburg, Germany; ${ }^{3}$ Dept. of Physics, Univ. of Regensburg, Germany. Theory--experiment comparison reveals how an electronic quantum interference introduces several macroscopic signatures to the high-harmonic emission in semiconductors, making it possible to shape temporal, spectral, and polarization-direction features of sources on a subcycle level.

FW1H.7 • 09:30

Affordable, ultra-broadband coherent detection of terahertz pulses via CMOS compatible solid-state devices, Alessandro Tomasino ${ }^{2,1}$, Anna Mazhorova², Matteo Clerici ${ }^{3}$, Marco Peccianti ${ }^{4}$, Sze Phing $\mathrm{Ho}^{5}$ Yoann Jestin ${ }^{2}$, Alessia Pasquazi ${ }^{4}$, Andrey Markov ${ }^{2}$, Xin Jin², Riccardo Piccoli², Sebastien Delprat ${ }^{2}$, Mohamed Chaker ${ }^{2}$, Alessandro Busacca', Jalil Ali ${ }^{5}$, Luca Razzari ${ }^{2}$, Roberto Morandotti i'; 'Univ. of Palermo, Italy: ${ }^{2} E M T$, INRS, Canada; ${ }^{3}$ Univ. of Glasgow, UK; ${ }^{4}$ Univ. of Sussex, UK; ${ }^{5}$ Universiti Teknologi Malaysia Malaysia. We demonstrate the first fully solidstate technique for the coherent detection of ultra-broadband $\mathrm{THz}$ pulses (0.1-10 THz), relying on the electric-field-induced secondharmonic generation attained in integrated CMOS-compatible devices. 
Meeting Room
$211 \mathrm{~B} / \mathrm{D}$
Meeting Room $212 \mathrm{~A} / \mathrm{C}$
Meeting Room

212 B/D
Marriott

Salon I \& II

CLEO: Science \& Innovations

\author{
SW1I - Space-Division \\ Multiplexed Optical \\ Communications-Continued
}

\section{SW1J • Precision References and Optical Synthesis- Continued}

SW1J.3 • 08:45

Er:fiber frequency comb for optical synthesis with $\mathrm{mHz}$ resolution, Holly F. Leopardi2,1 Josue Davila-Rodriguez ${ }^{1}$, Franklyn Quinlan' Scott Diddams ${ }^{1,2}$, Tara M. Fortier ${ }^{1} ;{ }^{1}$ National Inst of Science and Technology, USA; 'Physics, Univ. of Colorado Boulder, USA. We de scribe an erbium-fiber laser frequency comb that supports optical frequency synthesis at the millihertz level, or fractionally as $3 \times 10^{-18}$ $\mathrm{T}^{-1 / 2}$, by ensuring all critical fiber paths are within the servo-controlled feedback loop.

\section{SW1I.3 • 09:00}

Evaluation of the Elastic Optical Network Performance with Various Numbers of Spatial Modes, Wenbo Gao', Milorad Cvijetic ${ }^{1}$ Univ. of Arizona, USA. We have established a network model involving routing algorithms and assignment of modulation formats and spectral and spatial modes enabled to provide more insights on impacts of relevant signal and network parameters.

\section{SW11.4 09:15}

Experimental Demonstration of 20-Gbit/s Data Transmission Link using a $1.1 \mathrm{~km}$ Elliptical-Core Few-Mode Fiber assisted by Mapping from Conventional Amplitude Modulation to Spatial Mode Modulation, Long Zhu' ${ }^{1}$,i Mo ${ }^{1}$, Jian Wang ${ }^{1}$, Andong Wang'; 'Wuhan National Lab for Optoelectronics, China. We experimentally demonstrate $20 \mathrm{Gbit} / \mathrm{s}$ data transmission over a 1.1 $\mathrm{km}$ elliptical-core few-mode fiber (EC-FMF) link assisted by mapping from conventional amplitude modulation to spatial mode modulation, and achieve a $0.7 \mathrm{~dB}$ OSNR penalty improvement than single mode transmission at a BER of $3.8 \mathrm{e}-3$.

SW1I.5 • 09:30

4x10 Gb/s Polarization- and Mode GroupMultiplexing for Data Center Applications, Wei Wang ${ }^{1}$, Jian Zhao ${ }^{1}$, Lin Zhang ${ }^{1}$ Qi Mo², Zhiqun Yang', Chao Li', Zhenzhen Zhang', Cheng Guo', Guifang Li3;' 'Tianjin Univ., China; ${ }^{2}$ Fiberhome \& Fujikura Optics Co., China; ${ }^{3}$ Univ. of Central Florida, USA $4 \times 10 \mathrm{~Gb} / \mathrm{s}$ polarization- and mode groupmultiplexed transmission without MIMO equalization for data center applications is experimentally demonstrated, for the first time, over a panda-type polarization-maintaining few mode fiber.
SW1J.4 • 09:00

Multi-arm Ultra-low Noise Er:fiber Frequency Comb Comparison, Michele Giunta $^{1,2}$, Wolfgang Hänsel ${ }^{1}$, Marc Fischer ${ }^{1}$ Matthias Lezius ${ }^{1}$, Ronald Holzwarth', ${ }^{1,2}{ }^{1}$ Menlo Systems GmbH, Germany; ${ }^{2}$ Laser spectroscopy, Max-Planck-Institut für Quantenoptik, Germany. We have performed a multi-arm Er:fiber-based frequency comb comparison. The beat frequencies are simultaneously realized over $90 \mathrm{THz}$ (from 192 to $282 \mathrm{THz}$ ) showing an integrated phase noise below 55 $\operatorname{mrad}(1 \mathrm{~Hz}-1 \mathrm{MHz})$ and an overlapping ADEV of $5 \times 10^{-17}$ at $1 \mathrm{~s}$, averaging below $1 \times 10^{-18}$ at 100s (PI-mode).

\section{SW1J.5 09:15 Invited}

Optical Frequency References for Space, Thilo Schuldt' ${ }^{1}$, Klaus Döringshoff ${ }^{2}$, Markus Oswald $^{3}$, Evgeny Kovalchuk' ${ }^{2}$, Achim Peters ${ }^{2}$, Claus Braxmaier ${ }^{1,3}$; ${ }^{1}$ German Aerospace Center (DLR), Germany; ${ }^{2}$ Inst. of Physics, Humboldt-Univ. Berlin, Germany; ${ }^{3}$ Center of Applied Space Technology and Microgravity (ZARM), Univ. of Bremen, Germany. We present the development of optical frequency references with frequency instabilities at the $10^{-15}$ level for space applications. Special emphasis is put on compactness and rigidity of the optical systems.

\section{SW1K $\bullet$ Flexible and Soft Optoelectronics-Continued}

SW1K.2 • 09:00

Temperature Stable Electro-Optic Polymer Modulator using Ultra-Thin Silicon Waveguide, Shiyoshi Yokoyama', Hiroki Miura ${ }^{1}$ Feng Qiu', Andrew M. Spring ${ }^{1} ;{ }^{1}$ Kyushu Univ. Japan. We demonstrated the hybrid silicon and EO polymer modulator. The driving was $0.9 \mathrm{~V}$ at $1550 \mathrm{~nm}$, and the bandwidth of $40 \mathrm{GHz}$. The modulator showed excellent temperature stability at $85^{\circ} \mathrm{C}$ for 2000 hours.
SW1L • Sensing in Dynamic and Extreme Environments, Plasmas, and Explosions-Continued

SW1L.3 • 08:45

Two-Dimensional Fluorescence Spectroscopy for Measuring Uranium Isotopes in Femtosecond Laser Ablation, Mark C. Phillips ${ }^{1}$ Brian Brumfield ${ }^{1}$, Sivanandan S. Harilal' ${ }^{1}$, Kyle Hartig ${ }^{1,2}$, Igor Jovanovic ${ }^{3} ;{ }^{1}$ Pacific Northwest National Lab, USA; ${ }^{2}$ Dept. of Mechanical and Nuclear Engineering, The Pennsylvania State Univ., USA; ${ }^{3}$ Dept. of Nuclear Engineering and Radiological Sciences, Univ. of Michigan USA. We present the first two-dimensiona fluorescence spectroscopy measurements of uranium isotopes in femtosecond lase ablation plasmas. A new method of signal normalization is presented to reduce noise in absorption-based measurements of laser ablation.

\section{SW1L.4 • 09:00}

Spatio-Temporal Evolution of LIF in Laser Ablation Plumes, Kyle Hartig', Sivanandan Harilal ${ }^{1}$, Mark C. Phillips ${ }^{1} ;{ }^{1}$ Pacific Northwest National Lab, USA. Spatio-temporal mapping of LIF in laser-induced plasmas is studied. A tunable continuous wave frequency doubled Ti:sapphire laser was used to probe specific Al transitions in the expanding plasma at number of spatial positions.

\section{SW1K.3 • 09:15}

Nonlinear Refractive Index of Sulfur Copolymer Materials, Soha Namnabat', Masoud Babaeian', Laura E. Anderson², Michael S. Manchester ${ }^{2}$, Jeffery Pyun ${ }^{2}$, Robert A. Norwood'; ${ }^{1}$ College of Optical Sciences, Univ. of Arizona, USA: ${ }^{2}$ Chemistry and Biochemistry, Univ. of Arizona, USA. Nonlinear refractive in dices of novel sulfur copolymers are reported for the first time using a Z-scan setup. These values show a high nonlinearity compared to silica, demonstrating their potential for nonlinear optical applications.

\section{SW1K.4 09:30}

High-efficiency, Large-area and Color-stable Flexible Organic Light-emitting Diodes using an Ultra-thin Metal Electrode, Cheng Zhang ${ }^{1}$, Qingyu Huang ${ }^{1,2}$, Qingyu Cui ${ }^{1}$ Chengang $\mathrm{Ji}^{1}$, Zhong Zhang ${ }^{1}$, Suling Zhao ${ }^{2}$ L. Jay Guo ${ }^{1}$; Univ. of Michigan, USA; ${ }^{2}$ Beijing Jiaotong Univ., China. We demonstrate centimeter-size, color-stable flexible OLEDs using an ultra-thin Ag electrode. The device shows $\sim 35 \%$ enhanced current efficiencies compared to its ITO counterpart, stable emission colors even at large observation angles, and bending stability over 1000 circles.
SW1L.5 • 09:15

Continuous-Filtering Vernier Spectroscopy at $3.3 \mu \mathrm{m}$ Using a Femtosecond Optica Parametric Oscillator, Amir Khodabakhsh Lucile Rutkowski', Jerome Morville², Alexandra C. Johansson 1, Grzegorz Sobon ${ }^{1,3}$ Aleksandra Foltynowicz'; 'Umea Universitet, Sweden; ${ }^{2}$ Universite de Lyon, France; ${ }^{3}$ Wroclaw Univ. of Science and Technology, Poland. Using a cavity-enhanced continuous-filtering Vernier spectrometer based on a femtosecond optical parametric oscillator we measure broadband spectra of atmospheric water and $\mathrm{CH}_{4}$ around $3.3 \mu \mathrm{m}$ reaching $4 \mathrm{ppb}$ detection limit for $\mathrm{CH}_{4}$ in $15 \mathrm{~ms}$.

\section{SW1L.6 • 09:30}

Comparison of dual frequency comb absorption spectra of air-broadened wate vapor up to $1300 \mathrm{~K}$ with HITRAN Online and HITEMP2010 models, Paul Schroeder ${ }^{1}$ David J. Pfotenhauer ${ }^{1}$, Jinyu Yang ${ }^{1}$, Fabrizio Giorgetta ${ }^{2}$, William C. Swann², lan R. Coddington ${ }^{2}$, Nate Newbury ${ }^{2}$, Gregory B. Rieker ${ }^{1}$ ${ }^{1} \mathrm{CU}$ Boulder, USA; ${ }^{2}$ National Inst. of Standards and Technology, USA. We compare water-vapor absorption models generated with HITRAN Online and HITEMP2010 to measurements with a dual-comb spectrometer. For strong lines up to $1300 \mathrm{~K}$, HITRAN Online is a more accurate predictor of strengths, shapes, and positions. 


$\begin{array}{llc}\text { Marriott } & \text { Marriott } & \text { Marriott } \\ \text { Salon III } & \text { Salon IV } & \text { Salon V \& VI }\end{array}$

CLEO: Science \& Innovations

SW1M • Nonlinear Optics for Spectroscopy and SensingContinued

\section{SW1N • Silicon Photonic Devices and Structures- Continued}

SW1N.3 • 08:45

Dynamic Dispersion Tuning of Silicon Photonic Waveguides by Microelectromechanical Actuation, Carlos Errando-Herranz', Edinger Pierre ${ }^{1,2}$, Kristinn. Gylfason'; ${ }^{1} \mathrm{KTH}$ Royal Inst. of Technology, Sweden; ${ }^{2}$ Grenoble Inst. of Technology - INP Phelma, France. Efficient nonlinear silicon photonics rely on phase-matching through fine waveguide dispersion engineering. We experimentally demonstrate dynamic dispersion tuning of $800 \mathrm{ps} / \mathrm{nm} / \mathrm{km}$ in a silicon waveguide ring resonator, by using microelectromechanical actuation of an adjacent suspended waveguide rim.

\section{SW1N.4 • 09:00}

SW1M.2 • 09:00

Dual-comb Spectral focusing Coherent anti-Stokes Raman spectroscopy, Kun Chen', Tao Wu', Tao Chen', Haoyun Wei ${ }^{1}$ Yan Li'; 'Tsinghua Univ., China. High-speed coherent anti-Stokes Raman spectroscopy with two linearly chirped frequency combs is demonstrated. Multiplex Raman spectra covering most of the fingerprint region are acquired with high resolution on nanosecond measurement time scale.

SW1M.3 • 09:15

Transient Ring Opening and Closing of a Two-photon Photochromic Molecule Utilizing Energy Transfer, Peng Zhao', Raz Gvishi², Laura Bekere ${ }^{3}$, Vladimir Lokshin ${ }^{3}$, Vladimir Khodorkovsky', David Hagan', Eric Van Stryland'; ${ }^{1}$ CREOL Univ. of Central Florida, USA; ${ }^{2}$ Applied Physics Division," Soreq NRC, Israel; ${ }^{3}$ Aix Marseille Université, France. We have developed an efficient twophoton photochromic molecule by coupling a chromene with a donating 2PA chromophore via resonance energy transfer. The essential parameters of ring opening and closing are determined from transient absorption measurements.

\section{SW1M.4 09:30 Invited}

Nonlinear Optical Technologies for Frequency-Comb Based Molecular Sensing, Nathalie Picque'; 'Max-Planck-Institut fur Quantenoptik, Germany. State-of-the-art nonlinear optical technologies for frequency comb generation provide new powerful instruments for molecular physics and spectroscopy. Selected examples are given.
Integration of VCSEL on Silicon Photonics Using a Grating Coupler for Polarization Control and In-Plane Coupling, Yisu Yang ${ }^{1}$, Gligor Djogo', Moez Haque1, Peter R. Herman', Joyce Poon'; 'Dept. of Electrical and Computer Engineering, Univ. of Toronto, Canada. An O-band VCSEL is integrated with a silicon photonic chip using a grating coupler that provides feedback to maintain the emission polarization. The VCSEL-tochip coupling efficiency is $-5 \mathrm{~dB}$ at a current of $2.5 \mathrm{~mA}$.

SW1N.5 • 09:15

Correlation between Optical Return Loss and Transmission Fringe Amplitude in High-index-contrast Waveguides, $\mathrm{Chi}$ Xiong', Yves Martin 1', Marwan Khater', Jason Orcutt', Tymon Barwicz', Bo Peng', William Green': 'IBM TJ Watson Research Center, USA. We present a phenomenological amplitude of fringes in transmission spectrum due to distributed backscattering in highindex-contrast waveguides. The model is validated experimentally using four different waveguide cross sections.

\section{SW1N.6 • 09:30}

Silicon photonic add-drop microring filter banks with pinch-resistor characteristics, Xiaoxi Wang', Shayan Mookherjea ${ }^{1} ;{ }^{1}$ Univ. of California San Diego, USA. We design and demonstrate widely-tunable large-FSR dualmicroring add/drop filters. The integrated resistive tuning section in microrings included "pinched" p-n junctions, limiting the current at higher voltages and inhibiting damage under microsecond-scale pre-emphasized drive. model correlating optical return loss and

\section{SW10 - Optical Comb \& Integrated Systems-Continued}

SW10.4 0 08:45

Silicon Photonic OEIC for Memory Cell Information Sensing, Junfeng Song ${ }^{1,2}$, Xianshu Luo $^{1}$, Yanzhe Tang ${ }^{1}$, Qing Fang ${ }^{1}$, Chao Li', Lianxi Jia ${ }^{1}$, Xiaoguang Tu', Ying Huang ${ }^{1}$, Haifeng Zhout ${ }^{1}$ Eu-Jin Lim', Guogiang Lo ${ }^{1}$ Tsung-Yang Liow'; ' ${ }^{1}$ Inst. of Microelectronics, Singapore; ${ }^{2}$ College of Electronic Science and Engineering, Jilin Univ., China. We propose and experimentally demonstrate a novel scheme for simultaneous optical sensing of electric memory cell states. Results show that the effective sensing speed can be enhanced by 976 times with $100 \mathrm{~nm}$ spectrum ranges.

SW10.5 • 09:00

Advanced Path Mapping for Silicon Photonic Switch Fabrics, Qixiang Cheng', Meisam Bahadori', Keren Bergman ${ }^{1} ;{ }^{1}$ Columbia Univ., USA. We develop an advanced path mapping for photonic switch fabrics that improves the worst-case power-penalty and relaxes the receiver dynamic range requirements. Modeling shows a $6 \mathrm{~dB}$ power-penalty reduction for the equal-permutation of an $8 \times 8$ switch.

SW10.6 09:15

Optical Network Switch for Dynamically Reconfigurable Single- and Multi-cast Topologies, Gregory Steinbrecher ${ }^{1,2}$, Hemonth G. Rao ${ }^{2}$, Nicholas C. Harris' ${ }^{1}$ Jacob Mower', Michael Hochberg ${ }^{3}$, Tom Baehr-Jones ${ }^{3}$, Vincent Chan ${ }^{1}$, Dirk. Englund ${ }^{1}$, Scott A. Hamilton'; ${ }^{2}$ MIT, USA; ${ }^{2}$ MIT Lincoln Lab, USA; ${ }^{3}$ Coriant Technologies, USA. We present a network switch in the silicon-on-insulator platform capable of redistributing light to arbitrary outputs with continuously reconfigurable splitting ratios. We demonstrate software defined switching and physical-layer multicast of gigabit Ethernet.

SW10.7 • 09:30

Experimental Demonstration of On-chip $56 \times 56$ OXC Based on AWG Arrays, Zepeng Pan ${ }^{1}$, Minming Zhang ${ }^{1}$, Luluzi Lu', Feiya Zhou', Dongyu Li', Weijie Chang', Songnian $\mathrm{Fu}^{1}$, Deming Liu'; ' ${ }^{1}$ Huazhong Univ. of Science \& Technology, China. We experimentally demonstrated a large capacity OXC configuration based on a $56 \times 56$ monolithically integrated array waveguide grating (AWGs) by designing and fabricating several smallscale OXCs. 
Executive Ballroom

$210 \mathrm{~A}$

Executive Ballroom

210B

\section{CLEO: Applications \\ \& Technology}

AW1A $\bullet$ Medical Devices and Systems-Continued

AW1A.6 0 09:45

Experimental Demonstration of SparsityBased Single-Shot Fluorescence Imaging at Sub-wavelength Resolution, Maor Mutzafi', Yoav Shechtman 3,2, Or Dicker ${ }^{4}$, Lucien Weiss², Yonina C. Eldar, William E. Moerner

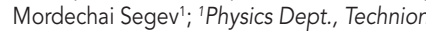
Israel Inst. of Technology, Israel; ' ${ }^{2}$ Dept. of Chemistry, Stanford Univ., USA; ${ }^{3}$ Faculty of Biomedical Engineering, Technion Israel Inst. of Technology, Israel; ${ }^{4}$ Electrical Engineering Dept., Technion Israel Inst. of Technology, Israel. We present, in experiments and simulations, a novel technique facilitating subwavelength resolution in a single-shot fluorescence imaging without capturing multiple frames, thereby enabling video-rate super-resolution imaging within living cells.
AW1B - Sensing in Fibers and Free-space-Continued

AW1B.7 • 09:45

All-optical ${ }^{4} \mathrm{He}$ Magnetometer Driven by Fictitious Oscillating Magnetic Field, Zaisheng Lin', Xiang Peng', Haidong Wang', Liang Shen', He Wang', Hong Guo'; 'State Key Lab of Advanced Optical Communication Systems and Networks, School of Electronics Engineering and Computer Science, and Center for Quantum Information Technology, Peking Univ. China; ${ }^{2}$ State Key Lab of Information Photonics and Optical Communications, Univ. of Posts and Telecommunications, China. We demonstrate an all-optical ${ }^{4} \mathrm{He}$ magnetometer driven by fictitious oscillating magnetic field achieved by the light shift. The intensity modulated detuning light creates the fictitious oscillating magnetic field to resonate with the Larmor precession.

\section{Executive Ballroom \\ $210 \mathrm{C}$}

\section{CLEO: Science \& Innovations}

SW1C $\bullet$ Nanolasers and

Frequency Combs-Continued

\section{SW1C.7 $\bullet 09: 45$}

Tunable Narrow Linewidth Microwave Oscillator based on an Optically Injected Semiconductor Laser using Opto-Electronic Feedback, Joseph S. Suelzer', Thomas B. Simpson², Preetpaul S. Devgan', Nicholas G. Usechak'; 'US Air Force Research Lab, USA 2L-3 Applied Technologies Inc., USA. Without a local oscillator or if amplifier, we exploit the fact that an optically injected semiconducto laser can be used to generate microwave signals to demonstrate a $<3 \mathrm{~Hz}$ microwave oscillator tunable over $18 \mathrm{GHz}$.

\section{Executive Ballroom 210D}

CLEO: QELSFundamental Science

FW1D $・$ Non-diffracting

Beams-Continued

\section{FW1D.8 • 09:45}

Investigation of Self-Healing Property of Composite Vector Vortex Beams, Pachava Srinivas ${ }^{1,2}$, Chithrabhanu Perumangatt ${ }^{2}$, Niji $\mathrm{ck}^{2}$, Shankar Pidishety ${ }^{1}$, Balaji Srinivasan ${ }^{1}$, Singh RP2; ${ }^{1}$ Indian Instisute of Technology Madras, India; ${ }^{2}$ hhysical Research Lab, India. We discuss the independent manipulation of the spin and orbital angular momentum of composite vortex beams and investigate their self-healing property. We demonstrate complete reconstruction of such beams within $50 \mathrm{~cm}$

10:00-18:30 Exhibition Open, Exhibit Hall 1, 2 \& 3

10:00-12:00 JW2A • Poster Session II, Exhibit Hall 1, 2 \& 3

10:00-10:30 Coffee Break, Exhibit Hall 1, 2 \& 3

10:00-12:45 OSA Members, Family and Friends Tour - Computer History Museum

Shuttle transportation will depart from the Hilton's Almaden Avenue entrance at 10:15 (Advanced Registration Required) 


\section{Executive Ballroom $210 \mathrm{E}$}

Executive Ballroom

210F

\section{CLEO: QELS-Fundamental Science}

FW1E • Rare Earth Solid State

Quantum Memories-Continued

FW1F • Quantum

Entanglement-Continued
Executive Ballroom

210G

Joint

JW1G • Symposium on

Advances in Metaphotonic

Devices I-Continued

JW1G.5 • 09:45

FW1E.5 • 09:45

Stoichiometric Rare-Earth Crystals for

Applications in Quantum Information, Mat-

thew Sellars' ${ }^{1}$, Rose Ahlefeldt' ${ }^{1}$ Michael Hush'²

${ }^{1}$ Australian National Univ., Australia; ${ }^{2}$ School

of Engineering and Information Technology,

Univ. of New South Wales, Australia. We

demonstrate that in stoichiometric rare-earth crystals the excitation induced interactions between ions can be resolved over the optical inhomogeneous linewidth, introducing the possibility of utilizing many-body effects

for quantum information applications.
Extreme Anisotropy, Spectral Modification and Intensity Enhancement in Luminescent Hyperbolic Metasurfaces, Joseph S. Smalley', Felipe Vallini', Sergio Montoya', Lorenzo Ferrari', Shiva Shahin'1, Conor T. Riley', Boubacar Kante' ${ }^{1}$ Eric E. Fullerton' ${ }^{1}$ Z Zhaowei Liu' Yeshaiahu Fainman'; ' 'Univ. of California San Diego, USA. We report the demonstration of luminescent hyperbolic metasurfaces (LuHMS) fabricated from deeply subwavelength multilayer metal-semiconductor nanostructures. The LuHMS exhibit extreme polarization anisotropy of absorption and emission, modified emission spectra, and enhanced emission intensity.
Executive Ballroom $210 \mathrm{H}$

\section{CLEO: QELS-} Fundamental Science

FW1 $\bullet$ Extreme

Electromagnetic Radiation - THZ to EUV: Generation, Detection \& Applications-Continued

\section{FW1H.8 • 09:45}

Nanoscale Imaging of Magnetic Domains using a High-Harmonic Source, Sergey Zayko ${ }^{2}$, Ofer Kfir ${ }^{2,1}$, Christina Nolte ${ }^{3}$, Murat Sivis², Marcel Möller ${ }^{2}$, Fabian Ganss' ${ }^{4}$, Birgit Hebler $^{4}$, Daniel Steil ${ }^{3}$, Sascha Schäfer ${ }^{2}$, Manfred Albrecht ${ }^{4}$, Oren Cohen ${ }^{1}$, Stefan Mathias ${ }^{3}$, Claus Ropers"; ' Solid State Inst. and Physics Dept., Technion Israel Inst. of Technology, Israel; ${ }^{2}$ IV. Physical Inst., Georg-August Univ. of Göttingen, Germany; ${ }^{3}$. Physical Inst. Georg-August Univ. of Göttingen, Germany ${ }^{4}$ Inst. of Physics, Univ. of Augsburg, Germany. We demonstrate the first implementation of magnetic imaging using high harmonic radiation. Out-of-plane magnetization patterns of worm-like domains in a $\mathrm{Co} / \mathrm{Pd}$ multilayer are imaged via Fourier transform holography with circularly polarized high harmonic radiation.

10:00-18:30 Exhibition Open, Exhibit Hall 1, 2 \& 3

10:00-12:00 JW2A • Poster Session II, Exhibit Hall 1, 2 \& 3

10:00-10:30 Coffee Break, Exhibit Hall 1, 2 \& 3

10:00-12:45 OSA Members, Family and Friends Tour - Computer History Museum

Shuttle transportation will depart from the Hilton's Almaden Avenue entrance at 10:15 (Advanced Registration Required)

10:30-12:00 Market Focus Session IV: How the Changing Political Landscape will Impact your Company, Exhibit Hall Theater 


\section{Meeting Room \\ $211 \mathrm{~B} / \mathrm{D}$}

Meeting Room
$212 \mathrm{~A} / \mathrm{C}$
Meeting Room
$212 \mathrm{~B} / \mathrm{D}$
Marriott

Salon I \& II

\section{CLEO: Science \& Innovations}

SW1I $\bullet$ Space-Division Multiplexed Optical Communications-Continued
SW1J • Precision References and Optical SynthesisContinued

SW1J.6 • 09:45

Dispersion-Engineered Silicon Nitride Supercontinuum for Frequency Comb Metrology at the $10^{-15}$ Level, David R. Carlson', Daniel Hickstein', Alex Lind', Judith B. Olson', Richard Fox ${ }^{1}$, Andrew Ludlow ${ }^{1}$ Qing Li², Daron Westly², Holly Leopardi ${ }^{1}$ Tara M. Fortier ${ }^{1}$, Kartik Srinivasan², Scott Diddams ${ }^{1}$, Scott Papp $;{ }^{1}$ NIST, USA; ${ }^{2}$ NIST, USA. Supercontinuum generation in dispersion engineered silicon nitride waveguides is used to perform frequency comb metrology by measuring the relative stability of two cavityreferenced optical clock lasers at $3.8 \times 10^{-15}$ at $\mathrm{T}=2$ seconds
SW1K • Flexible and Soft Optoelectronics-Continued

\section{SW1K.5 • 09:45}

All-carbon flexible photodetectors, Yujie Liu', Yuanda Liu', Shuchao Qin', Yongbing $\mathrm{Xu}^{1}$, Rong Zhang ${ }^{1}$, Frank (Fengqiu) Wang ${ }^{1}$; ${ }^{1}$ Nanjing Univ., China. We demonstrate a graphene-nanotube hybrid flexible photodetector with a high photoresponsivity ( 51 A/W) and a fast response ( $\sim 0 \mathrm{~ms}$ ) over the visible range. The devices remain stable under severe bending conditions and repetitive bending cycles.
SW1L • Sensing in Dynamic and Extreme Environments, Plasmas, and Explosions-Continued

\section{SW1L.7 • 09:45}

Resolving Gas Temperature Distributions with Single-beam Dual-comb Absorption Spectroscopy, Nathan Malarich', Gregory B. Rieker'; 'Univ. of Colorado Boulder, USA. We assess the potential to resolve line-of-sight gas temperature distributions with single-beam, broadband, dual-comb absorption spectroscopy. The technique shows promise for single-optical-port spatially-resolved temperature diagnostics for harsh environments.

10:00-18:30 Exhibition Open, Exhibit Hall 1, 2 \& 3

10:00-12:00 JW2A • Poster Session II, Exhibit Hall 1, 2 \& 3

10:00-10:30 Coffee Break, Exhibit Hall 1, 2 \& 3

10:00-12:45 OSA Members, Family and Friends Tour - Computer History Museum

Shuttle transportation will depart from the Hilton's Almaden Avenue entrance at 10:15 (Advanced Registration Required) 


$\begin{array}{llc}\text { Marriott } & \text { Marriott } & \text { Marriott } \\ \text { Salon III } & \text { Salon IV } & \text { Salon V \& VI }\end{array}$

\section{CLEO: Science \& Innovations}

SW1M • Nonlinear Optics for Spectroscopy and SensingContinued
SW1N • Silicon Photonic Devices and StructuresContinued

\section{SW1N.7 • 09:45}

Compact High-extinction-ratio Silicon Photonic Variable Optical Attenuators (VOAs)

Xiaoxi Wang', Shayan Mookherjea'; 'Univ. of California San Diego, USA. We design and demonstrate heat-harvesting two-part compact variable optical attenuators, which provide a high controllable extinction ratio at $\mathrm{C}$ - and L-band wavelengths, and multiplex the added channels into a single output port.

\begin{abstract}
SW10 - Optical Comb \& Integrated Systems-Continued
\end{abstract}

SW10.8 09:45

$47 \mathrm{~dB}$ Net on-chip Brillouin gain for true time delay applications, Iman Aryanfar ${ }^{1}$ Amol Choudhary', Yang liu', Khu Vu', Pan $\mathrm{Ma}^{2}$, Duk-Yong Choi ${ }^{2}$, Stephen Madden², David Marpaung ${ }^{1}$, Benjamin Eggleton'; ' Univ. of Sydney, Australia; ${ }^{2}$ Australian National Univ., Australia. A true-time-delay(TTD) element is demonstrated using on-chip stimulated Brillouin scattering. Record net-Brillouin-gain of $47 \mathrm{~dB}$ enabled $4 \mathrm{~ns}$ delay over $100 \mathrm{MHz}$ and phase-shift of $\sim 200^{\circ}$. The TTD element was validated by incorporating it in a twotap filter.

10:00-18:30 Exhibition Open, Exhibit Hall 1, 2 \& 3

10:00-12:00 JW2A • Poster Session II, Exhibit Hall 1, 2 \& 3

10:00-10:30 Coffee Break, Exhibit Hall 1, 2 \& 3

10:00-12:45 OSA Members, Family and Friends Tour - Computer History Museum

Shuttle transportation will depart from the Hilton's Almaden Avenue entrance at 10:15 (Advanced Registration Required)

10:30-12:00 Market Focus Session IV: How the Changing Political Landscape will Impact your Company, Exhibit Hall Theater

\section{CLEO Mobile App}

Use the conference app to plan your schedule; view program updates; receive special events reminders and access Technical Papers (separate log-in required).

- Go to www.cleoconference.org/app.

- Select the Apple App Store or Google Play link.

- Download the app.

- Log in to use app features such as contacting fellow conference attendeesusing your registration I.D. and email address. 


\section{JW2A.1}

Laser Vibrometer Interferometry for Speckle Patterns Tracking Systems, Arie Schwarz ${ }^{1}$, Amir Shemer ${ }^{1}$, Nisan Ozana ${ }^{1}$ Ran Califa ${ }^{3}$, Javier García ${ }^{2}$, Zeev Zalevsky' ${ }^{1}$ Faculty of Engineering, Bar Ilan Univ., Israel; ${ }^{2}$ Departamento de Óptica, Universitat de València, Spain; ${ }^{3}$ Continuse Biometrics, Israel. In this paper we propose a modulated laser system combining a speckle pattern tracking method for surface tilting changes sensing with an interferometer for surface z-axis changes sensing at the same scan time.

\section{JW2A.2}

Detection of strain induced temperature variations based on a four-core optical fiber, Belkis Gokbulut', Sema Guvenc' ${ }^{1}$, Naci Inci'; 'Bogazici Univ., Turkey. A four-core optical fiber is introduced as a strain based temperature sensor to explore the phase shift corresponding to the temperature variations. A phase shift of $20.4 \pm 0.29$ rad occurs for a temperature increment of $42 \mathrm{C}$

JW2A.3

Electro-optic measurement of averaged duty ratio for periodically poled crystals, Chia-Tsung Liuu', Chia-lun Tsai ${ }^{1}$, Jui-Yu Lai ${ }^{1}$ Yen-Hung Chen"2, Shang-Da Yang'; ' National Tsing Hua Univ., Taiwan; ${ }^{2}$ National Central Univ., Dept. of Optics and Photonics, Taiwan. A non-invasive electro-optic method is proposed to measure the averaged duty ratio for periodically poled crystals. The measured (expected) averaged duty ratios of two samples are $67.95 \% / 32.05 \%(75 / 25)$ and $55.02 \% / 44.98 \%(50 / 50)$

\section{JW2A.4}

Bound state operation of an all-polarization maintaining Er-doped fiber laser, Zhuang Zhao', Daniel Popa', Bo Fu'1, Syed Hussain ${ }^{1}$, Andrea Ferrari ${ }^{1}$; ${ }^{1}$ Engineering, Univ. of Cambridge, UK. We report single pulse and harmonic mode-locking of two-soliton bound states from an all-polarization maintaining mode-locked laser based on a nanotube saturable absorber that could aid increased telecom bandwidths.

JW2A.5

Self-starting, turn-key dual-comb modelocked fiber laser with a few-mode fibe filter, Jie Chen ${ }^{1}$, Ruliu Wang ${ }^{1}$, Ting Li ${ }^{1}$, Cu $\mathrm{Li}^{1}$, Yingling Pan ${ }^{1}$, Xin Zhao', Jiansheng Liu' Zheng Zheng 1,2; ${ }^{1}$ School of Electronic and Information Engineering, Beihang Univ. China; ${ }^{2}$ Collaborative Innovation Center of Geospatial Technology, China. A simple, self-starting, turn-key dual-wavelength-comb mode-locked fiber laser is realized with a dual-mode-fiber-based filter. The costeffective, multimode interference fiber filte with low spectral modulation depth enables relatively wide spectral bandwidths in the mode-locking windows

\section{0:00-12:00 JW2A • Poster Session II}

\section{JW2A.6}

Attosecond Synchronization of Passive Mode-locked Lasers Using Optical Heterodyne Techniques, Shijun Chen ${ }^{1}$, Jie Tian², Dawei Chen ${ }^{1}$, Qiang Chen ${ }^{1}$, Qingsong $\mathrm{Bai}^{3}$, Fuyu Sun ${ }^{3}$, Dong Hou ${ }^{3}$; ${ }^{\prime}$ ZTE Corporation, China; ${ }^{2}$ China Academy of Engineering Physics, China: ${ }^{3}$ Univ of Electronic Science \& Tech China, China. We demonstrate an attosecond synchronization of mode-locked lasers using optical heterodyne technique. The measured RMS timing fluctuation between two modelocked Er:fiber lasers with same color was about 800 attosecond within $60 \mathrm{~s}$.

JW2A.7

Remote Photo-Acoustic Spectroscopy (PAS) with an Optical Pickup Microphone Kazuhide Sato ${ }^{1}$, Kazuyoku Tei ${ }^{1}$, Shigeru Yamaguchi', Masaki Asobe', yoshito sonoda' ${ }^{1} T o k a i$ Univ., Japan. We propose a new gas detection method with optical wave microphone(OWM). It enables PAS remotely without installing conventional microphone where acoustic wave stands. We successfully detected acoustic signal from $\mathrm{C}_{2} \mathrm{H}_{2}$ with OWM.

\section{JW2A.8}

Optical-controlled Pencil-Beam Steering Phased-Array Based On FD-OP, Mutong Xie', Xinlu Gao', Mingyang Zhao' ${ }^{1}$, Wensheng Zhai', Wenjing X ${ }^{1}$, Jinwang Qian', Mingzheng Lei', Shanguo Huang ${ }^{1} ;{ }^{1}$ Beijing Univ of Posts \& Telecom, China. A pencil-beam steering phased-array based on optical-controlling scheme is proposed. FD-OP is used to gain flexibility and high tunablility. Experiments are conducted at $17 \mathrm{GHz}$ to validate the 2D beams-steering ability of the system.

\section{JW2A.9}

An 8x8 Heterodyne Lens-less OPA Camera, Reza Fatemi', Behrooz Abiri', Ali Hajimiri' ${ }^{1}$ Caltech, USA. This paper presents an $8 \times 8$ optical phased array (OPA) receiver that operates as a lens-less camera using a heterodyne architecture on a thin silicon-photonics integrated $\mathrm{SOI}$ substrate. It has a receiving beam width of $0.75^{\circ}$ and beam steering range of $8^{\circ}$.

\section{JW2A.10}

Highly Sensitive Back-Focal-Plane Interferometry for Tracking Nanoparticle Position Shuzo Masui ${ }^{1}$ Masaki Michihata², Kiyoshi Takamasu', Satoru takahashi²; 'Precision Engeneering, The Univ. of Tokyo, Japan; Research Center for Advanced Science and Technology, The Univ. of Tokyo, Japan. Recently, nanoparticles have played important roles in various fields. Back-focal-plane interferometry is a widely used method for nanoparticle tracking. We proposed and demonstrated a method to improve the sensitivity of back-focal-plane interferometry.

\section{JW2A.11}

High-order Suppression of Quasi-triangle Array Transmission Gratings, Tanchao $\mathrm{Pu}^{1,2}$, Ziwei Liu ${ }^{1,2}$, Lina Shi ${ }^{1}$, Changqing Xie ${ }^{1}$ Guanya Wang'; 'Inst. of Microelectronics of CAS, China; ${ }^{2}$ Univ. of Chinese Academy of Sciences, China. We propose a binary grating based on a membrane to solve the overlapping in the spectrum measurement. By optimizing the shape, size and position of the holes, a self-standing single-order diffraction gratings have been achieved.
JW2A.12

Thermally-induced optical bistability in $\mathrm{Cr}$ and Fe doped $\mathrm{ZnSe}$ mid-IR laser materials, Rick Watkins ${ }^{1}$, Ozarfar Gafarov', Chandle Bernard', Vladimir Fedorov'1, Sergey B. Mirov'; 'Univ. of Alabama at Birmingham, USA. Thermally-induced optical bistability and temperature broadening in spectroscopic bands in $\mathrm{Cr}(\mathrm{Fe}): \mathrm{ZnSe}$ mid-IR laser materials were studied in 1-6 $\mu \mathrm{m}$ spectral range to identify critical parameters to minimize thermally induced optical noise.

\section{JW2A.13}

Investigation of antirelaxation wall coatings beyond melting temperatures, Wenhao $\mathrm{Li}^{1,2}$, Mikhail Balabas ${ }^{3}$, Xiang Peng ${ }^{1}$, Szymon Pustelny ${ }^{4}$, Arne Wickenbrock ${ }^{5}$, Yucheng Yang ${ }^{1}$, Hong Guo ${ }^{1}$, Dmitry Budker $2,6 .{ }^{1}$ Peking Univ., China; ${ }^{2}$ Dept. of Physics, Univ. of California, Berkeley, USA; ${ }^{3}$ St. Petersburg State Univ., Russia; ${ }^{4}$ nst. of Physics, Jagiellonian Univ., Poland; ${ }^{5}$ Johannes Gutenberg-Univ. Mainz, Germany; ${ }^{6} \mathrm{Helmholtz} \mathrm{Inst.} \mathrm{Mainz,}$ Germany. We investigate vapor cells with antirelaxation wall coatings by measuring their relaxation properties beyond the melting temperatures and compare with the melting behavior of the coating material as observed with differential scanning calorimetry.

\section{JW2A.14}

A High Performance Optomechanical Mass Sensor, Yeping Zhang ${ }^{1}$, Jie Ai ${ }^{1}$, Yanjun Xiang ${ }^{1}$ Qinghua He1, Tao Li'; ' ${ }^{1}$ Inst. of Fluid Physics, China Academy of Engineering Physics, China. A mass sensor based on split-nanobeam optomechanical crystal cavity with effective motion mass below $10 \mathrm{fg}$ and mechanical frequency exceed $10 \mathrm{GHz}$ is proposed. Based on our simulation, exceed $10 \mathrm{MHz} / \mathrm{fg}$ detec tion sensitivity can be realized.

\section{JW2A.15}

Multi-parameter Sensing Platforms based on Plasmonic Structures and Planar Photonic Crystals, Yongyao Chen ${ }^{1}$, Miao Yu ${ }^{1}$ ${ }^{1}$ Univ. of Maryland Univ College, USA. In many complex environments, it is imperative to monitor various information and complex processes. We propose to use nanophotonic devices such as plasmonic structures and dielectric photonic crystals for simultaneous detection and discrimination of multiple parameters.

\section{JW2A.16}

Improved distributed optical fiber vibration sensor based on Mach-Zehnder-OTDR Yuheng Tong ${ }^{2}$, Zhengying $\mathrm{Li}^{1}{ }^{1,2}$, Jiaqi Wang ${ }^{2}$ Chun Zhang': ${ }^{1}$ National Engineering Lab for Fiber Optic Sensing Technology, China; ${ }^{2}$ Key Lab of Fiber Optic Sensing Technology and Information Processing, China. A highspeed distributed vibration sensor based on balanced Mach-Zehnder interference and optical time-domain reflectometer is introduced to distinguish multiple vibration simultaneously. The experimental results show a high spatial resolution and a fast frequency response.

JW2A.17

Improving the accuracy of dual-comb ranging system by suppressing the relative linewidth, Zebin Zhu' ${ }^{1}$ Kai Ni' ${ }^{1}$, Qian Zhou', Guanhao Wu'. 'Tsinghua Univ., China. We use an intra-cavity EOM to realize high speed synchronization of dual-comb. The relative linewidth is dramatically suppressed from $300 \mathrm{kHz}$ to sub-hertz without ultra-stable $\mathrm{CW}$ laser and the ranging accuracy is greatly improved.

JW2A.18

Reversible mapping of spin to orbital angular momentum degree of freedom of one photon of an entangled pair, Brian T. Kirby ${ }^{1}$ Michael Brodsky', Nenad Bozinovic 2,3, Siddharth Ramachandran'; ${ }^{1}$ US Army Research ab, USA: ${ }^{2}$ Dept. of Electrical \& Computer Engineering, Boston Univ., USA; ${ }^{3}$ Berkeley Lights Inc, USA. We demonstrate high fidelity conversion of a polarization-entangled photon pair into a hybrid OAM-polarization entangled pair by using a special multi-mod vortex fiber. A new model accounting for frequency-dependent modal loss fits the data.

\section{JW2A.19}

Heralded Photons for Quantum Teleportation, Francisco A. Dominguez Serna ${ }^{1,2}$, Karina Garay Palmett ${ }^{1}$, Fernando Rojas Iñiguez ${ }^{2}$ Optica, CICESE, Mexico; ${ }^{2}$ CNyN-UNAM, Mexico. We present a quantum teleportation protocol based on a hybrid entangled resource (HER) obtained with heralded photons and coherent states. High efficiency of teleportation can be obtained by adjusting spectral detection windows.

\section{JW2A.20}

Photon-pair Generation by Spontaneous Four-wave Mixing in Integrated Optical Waveguides: a Nonlinear Time-domain

Model, Gary Sinclair ${ }^{1}$ Mark Thompson 'Quantum Engineering Technology Labs, H.H. Wills Physics Lab, Univ. of Bristol, UK We model photon-pair generation in the presence of parasitic nonlinearities such as self- and cross-phase modulation. The effect of these nonlinearities on the pair production rate and heralded photon purity is explored.

\section{JW2A.2}

Two-qubits Controlled-unitary Quantum Gates for Quantum Computing by Silicon Photonic Chip, Jianguo Huang', Leong Chuang Kwek', Jiangbin Gong ${ }^{2}$, Weibo Gao', Yidong Chong ${ }^{1}$, Wee Ser ${ }^{1}$, Ai Qun Liu' 'Nanyang Technological Univ., Singapore; ${ }^{2}$ National Univ. of Singapore, Singapore. We demonstrate two-qubits controlled unitary quantum gates in a single silicon photonic chip. It can greatly reduce the size and complexity of the functional quantum circuits without decomposition it into plenty of elementary logic gates.

\section{JW2A.22}

An approach to the generation of $\mathrm{GHZ}$ states by interference of multiple integrated sources on a single chip, Nicola Bergamasco ${ }^{1}$, Matteo Menotti ${ }^{1}$, John Sipe ${ }^{2}$ 


\section{0:00-12:00 JW2A • Poster Session II}

\section{JW2A.23}

The effects of self- and cross- phase modulation in the generation of bright twin beams using SPDC, Nicolas Quesada², John Sipe'; ' Univ. of Toronto, Canada; ${ }^{2}$ Dept. of Physics \& Astronomy, Macquarie Univ., Australia. We introduce a simple methodology to calculate the effects of self- and cross-phase modulation in SPDC photon generation. We show that these processes make SPDC les efficient in the low spatio-temporal mode number limit.

\section{JW2A.24}

A cautionary tale: why you should not use the electric field to quantize in nonlinea optics, Nicolas Quesada22, John Sipe ${ }^{1} ;{ }^{1}$ Univ. of Toronto, Canada: ${ }^{2}$ Dept. of Physics \& Astronomy, Macquarie Univ., Australia. We show that using the electric field as a canonical quantization variable in nonlinear optics leads to incorrect expressions for the squeezing parameters in SPDC and conversion rates in frequency conversion.

\section{JW2A.25}

Coherent quantum control of on-chip time-frequency entangled photons, Poolad Imany 1,2, Ogaga D. Odele 1,2, Jose JaramilloVillegas ${ }^{1,2}$, Minghao $\mathrm{Qi}^{1}$, Daniel Leaird ${ }^{1}$ Andrew Weiner'; 'P ${ }^{1}$ urdue Univ., USA; ${ }^{2}$ Purdue Quantum Center, Purdue Univ., USA. We demonstrate a novel two-photon interference experiment for three coherent pump-lines generating Biphoton Frequency Combs (BFC) in a silicon nitride microring resonator.

\section{JW2A. 26}

Schrodinger Cat States and Quasiprobability Distributions, Richard A. Brewster' ${ }^{1}$, James Franson'; 'Univ. of Maryland, Baltimore County, USA. We consider the P-function of nonclassical states, such as the Schrodinger cat state, using the theory of distributions. It is seen that the P-function for this state can be given by a generalized Dirac delta function that admits complex arguments. Other nonclassical states are also considered.

\section{JW2A.27}

Probing the measurement process in DTOW via recurrence, Thomas Nitsche ${ }^{1}$ Regina Kruse ${ }^{1}$, Linda Sansoni", Martin Stefanak $^{2}$, Tamás Kiss ${ }^{3}$, Igor Jex², Sonja Barkhofen ${ }^{1}$ Christine Silberhorn"; ' Universität Paderborn, Germany; ${ }^{2}$ Czech Technical Univ. in Prague, Czech Republic; ${ }^{3}$ Inst. for Solid State Physics and Optics, Wigner Research Centre for Physics, Hungary. We investigate experimentally the measurement-induced transition from recurrent to a transient regime in a photonic time-multiplexed quantum walk. Introducing sinks and monitoring the evolution allows us to observe qualtitative differences between a restart- and a continue-regime.

\section{W2A.28}

Non-zero discord bipartite state generation via classical second-order interference, Yong-Su Kim ${ }^{1}$, Yujun Choi ${ }^{1,2}$, Hyang-Tag Lim ${ }^{3}$ Kanghee Hong ${ }^{3}$, Jiwon Yune ${ }^{1}$, Osung Kwon ${ }^{1}$ Sang-Wook Han ${ }^{1}$, Kyunghwan Oh², Yoon-Ho $\mathrm{Kim}^{3}$, Sung Mon ${ }^{1} ;{ }^{1}$ Center for Quantum Information, Korea Inst. of Science \& Technology South Korea; ${ }^{2}$ Dept. of Physics, Yonsei Univ. South Korea; ${ }^{3}$ Dept. of Physics, Pohang Univ. of Science and Technology (POSTECH) South Korea. We show that non-zero discord state can be generated via classical secondorder interference. Considering the fundamental importance of interference, this result provides a new insight to understand the physical interpretation of quantum discord.

\section{JW2A.29}

Diffractive Optics for the Generation and Detection of Dynamic Composite Optical Vortices, Kaitlyn Morgan' ${ }^{1}$ Wenzhe Li' ${ }^{1}$ Keith Miller ${ }^{1}$, Indumathi R. Srimathi' Eric Johnson ${ }^{1}$; ${ }^{1}$ Clemson Univ., USA. This paper introduce a novel method for the propagation and detection of temporally phase coupled Orbital Angular Momentum beams. Experimenta results are demonstrated for phase coupled OAM beams with arbitrary transient phase profiles.

\section{JW2A.30}

Direct Transverse Spatially-Resolved Characterization of Femtosecond Filaments, Milos Burger', Patrick Skrodzki', Igor Jovanovic': ' Univ. of Michigan, USA. We report direct experimental measurements of the spatial dependence of femtosecond filament spectrum by a versatile sampling and imaging approach. The method is appropriate for complete spatially resolved reconstruction of the filament electric field.

\section{JW2A.31}

Optimization of Parametric Comb Generation Using Interferometric Wavelength Selective Switch, Mohammad Al-Khateeb Mary E. McCarthy', M. Deseada Gutierrez Pascual ${ }^{2}$, Frank Smyth ${ }^{2}$, Andrew D. Ellis ${ }^{1} ;{ }^{1}$ As ton Univ., UK; ${ }^{2}$ Pilot Photonics, Invent Centre, Dublin City Univ., Ireland. We propose and demonstrate frequency comb regeneration using parametric mixer dispersion managed by interferometric wavelength selective switch. The results show a good control over the bandwidth/flatness of the comb generated by the parametric process.

\section{JW2A.32}

Reverse PT phase transition via adiabatic elimination, Mohammad Hosain Teimourpour', Ramy El Ganainy'; 'Michigan Technological Univ., USA. In PT dimers, we show that transitions between the symmetric and broken phases can be reversed through inclusion of strongly coupled passive dimers that act as two-mode adiabatic bridges between the gain and loss sites.

\section{JW2A.33}

Optomechanically-induced frequency combs, Mohammad-Ali Miri ${ }^{1}$, Giuseppe D'Aguanno', Andrea Alu'; 'The Univ. of Texas at Austin, USA. We investigate the classical nonlinear dynamics of a single mode optomechanical cavity, showing that a frequency comb made of equidistant spectral lines can be formed in the instability regime due to the excitation of multiple sidebands.

\section{JW2A.34}

Nonlinear imaging in photonic lattices Nikolaos K. Efremidis', Mihalis Barkas ${ }^{1}$; ${ }^{1}$ Univ. of Crete, Greece. We predict that nonlinear imaging is possible in periodic index configurations provided that we use two different segments of nonlinear media with opposite signs of the Kerr nonlinearity with no othe restriction about their magnitudes.

\section{JW2A.35}

Cross phase modulation mediated pulse control with Airy waves in optical fibers, Michael Goutsoulas', Vassilis Paltoglou" Nikolaos K. Efremidis': 'Univ. of Crete, Greece. We show that the frequency and velocity of a signal pulse can be controlably shifted by the use of an Airy pulse via cross-phase modulation. Furthermore, the signal diffraction is suppressed during the interaction.

\section{JW2A.36}

Curvature-assisted wave localization in Vertical Cavity Surface Emitting Lasers, Kuo-Bin Hong ${ }^{2}$, Chun-Yan Lin ${ }^{1}$, Tsu-Ch Chang ${ }^{2}$, Wei-Hsuan Liang ${ }^{2}$, Ying-Yu Lai ${ }^{2}$ Chien-Ming Wu ${ }^{1}$, You-Lin Chuang ${ }^{1}$, TienChang Lu ${ }^{2}$, Claudio Conti ${ }^{3}$, Ray-Kuang Lee ${ }^{1}$ ${ }^{1}$ National Tsing Hua Univ., Taiwan; ${ }^{2}$ Nationa Chiao Tung Univ., Taiwan; ${ }^{3}$ Inst. for Complex Systems, National Research Council, Italy. By implementing surface structures in vertical cavity surface emitting lasers as manifolds for curved space, we experimentally study the impacts of geometrical constraints on wave localization.

\section{JW2A.37}

Self Polarization of Stokes Waves in Twisted Fibers, Evgeny A. Kuzin', Mohammad Almanee ${ }^{2}$, Joseph Haus², Nikolay Koneev ${ }^{1}$, Baldemar lbarra-Escamilla', Manue Duran-Sanchez 1,3 Georgina Beltran-Perez ${ }^{4}$ Yazmin Bracamontes-Rodríguez ${ }^{5}$, Olivie Pottiez, Ivan Armas-Rivera ${ }^{4}$, Ricardo I. Alvarez Tamayo'; ${ }^{1}$ Inst Nat Astrofisica Optica Electronica, Mexico; ${ }^{2}$ Dayton Univ., USA ${ }^{3}$ CONACYT, Mexico; ${ }^{4}$ Benemerito Universidad Autonomo de Puebla, Mexico; ${ }^{5}$ Centro de Investigacion en Optica, Mexico. Polarization evolution of a single and coupled pulses in twisted fiber was studied experimentally and numerically. For coupled-pulse input, the output ellipticity evolves toward the circula polarization with abrupt shift at a linearly polarized input.

JW2A.38

Creation of Population Inversion Grating by Coherently Propagating Few-Cycle Optical Pulses, Nikolai N Rozanov1,3 Rostislav Arkhipov $^{1,2}$, Ihar babushkin ${ }^{4,5}$, Anton Pakhomov $^{6,7}$, Ayhan Demircan ${ }^{5,8}$, Uwe Morgner ${ }^{5,8}$ 'St Petersburg SU Info Tech Mech \& Optics, Russia; ${ }^{2}$ Max Planck Inst. for the Science of Light, Germany; ${ }^{3}$ Vavilov State Optical Inst. Russia; ${ }^{4}$ Max Born Inst., Germany; ${ }^{5}$ Inst. of Quantum Optics, Leibniz Univ. Hannover, Germany; ${ }^{6}$ Dept. of Physics, Samara National Research Univ., Russia; ${ }^{7}$ Dept. of Theoretical Physics, Lebedev Physical Inst., Russia ${ }^{8}$ Hannover Centre for Optical Technologies, Germany. We analyse theoretically effects of excitation of resonant two-level optical media by a sequence of few-cycle pulses. Periodic gratings of population inversion with subwavelength-scale spatial period are created by tuning the excitation pulse train.

JW2A.39

Terahertz repetition rate pulse generation in Erbium-Ytterbium co-doped fibe Sigang Yang ${ }^{1}$, Zhaohui Wu' ${ }^{1}$, Yi Yang ${ }^{1}$, Yu Li' Hongwei Chen', Minghua Chen'; 'Tsinghua Univ., China. We report generation of terahertz repetition rate pulse directly from Erbium-Ytterbium co-doped fiber based on the combination effect of amplification and nonlinear phase locking. The repetition rate is up to 2.75 terahertz and the pulse width is 100 femtosecond.

\section{JW2A.40}

Effect of Zero-nonlinearity Point on the Temporal Trajectory of Raman Soliton Inside a Silver Nanoparticle Doped Fiber Surajit Bose'; 'Fiber Optics and Photonics, Central Glass and Ceramic Research Inst India. We observe the temporal bending of Raman soliton at different distances by shifting zero-nonlinearity point, when an input pulse is launched close to zero dispersion wavelength in non-solitonic domain of selfdefocussing nonlinear medium.

\section{JW2A.4}

High Q silica nanobeam cavity for simultaneous resonance of TE- and TM-like modes, Tomohiro Tetsumoto ${ }^{1}$, Hajime Kumazaki' Kentaro Furusawa ${ }^{2}$, Norihiko Sekine ${ }^{2}$, Akifum Kasamatsu ${ }^{2}$, Takasumi Tanabe ${ }^{1}$; ${ }^{1}$ Keio Univ. Japan: ${ }^{2}$ National Inst. of Information and Communications Technology, Japan. We demonstrated the highest recorded Qs for a silica photonic crystal nanocavity of over 10 for both TE- and TM-like modes simultaneously. A Fano resonance was numerically observed due to TE-TM mode coupling.

\section{JW2A.42}

Second-Harmonic Induced Soliton Drifting and Annihilation in Microresonators, Xiaoxiao Xue ${ }^{1}$, Xiaoping Zheng ${ }^{1}$, Bingkun Zhou ${ }^{1}$ 'Tsinghua Univ., China. The fundamental soliton dynamics can be affected by secondharmonic generation in microresonators with simultaneous $X^{(2)}$ and $X^{(3)}$ nonlinearities. Soliton drifting and annihilation is observed which facilitates spectrally smooth singlesoliton comb generation.

\section{JW2A.43}

Experimental Investigation of Inverse Raman Scattering in a Single Mode Tellurite Fiber, Tonglei Cheng ${ }^{1}$, Xiaojie Xue', Tong H Tuan', Weiqing Gao', Takenobu Suzuki', Yasutake Ohishi': 1 ofmlab, Japan Inverse Raman scattering (IRS) effect is first investigated in a 2.5-m-long single mode tellurite fiber (SMTF) which induced a steadily growing 2 nd-order Raman Stokes that finally exceeded the 1storder Raman Stokes. 


\section{0:00-12:00 JW2A • Poster Session II}

\section{JW2A.45}

Nonlinear Propagation of 100 ps, UV Lase Pulses in Water with Strong Stimulated Raman Stokes Coupling, Yu-hsin Chen Alexander Stamm ${ }^{1}$, John Palastro', Bahman Hafizi', Theodore Jones' ${ }^{1}$, Dmitri Kaganovich ${ }^{1}$; 'Naval Research Lab, USA. Underwater UV laser pulse propagation experiments were performed at intensities spanning the linear and nonlinear regimes. Measurements and simulations show strong coupling to molecular Raman modes and suggest strong ionization-induced refraction near the beam focus

\section{JW2A.46}

Periodical Soliton Bunches in a Passively Mode-locked Fiber Laser by the Optomechanical Effect in Microfiber, Zhenhong Wang'; 'Nankai Univ., China. We experimentally observe periodical soliton bunches in a microfiber-based graphene saturable absorber mode-locked fiber laser. The optomechanical effect and dispersive wave are responsible for the inter- and intra-solitonbunch interaction in the laser, respectively.

\section{JW2A.47}

Robust Mid-Infrared Photothermal Imaging System for Characterization of Thin Films at High Spatial Resolution, Atcha Totachawattana $^{1,2}$, Di Huang ${ }^{1,2}$, Le Li ${ }^{2,3}$, Keith A Brown $^{3,4}$, Shyamsunder Erramilli, ${ }^{2,5}$, Michelle $Y$. Sander ${ }^{1,4},{ }^{1}$ Electrical and Computer Engineering, Boston Univ., USA; ${ }^{2}$ Photonics Center, Boston Univ., USA; ${ }^{3}$ Dept. of Mechanical Engineering, Boston Univ., USA; ${ }^{4}$ Division of Materials Science and Engineering, Boston Univ., USA; ${ }^{5}$ Dept. of Physics, Boston Univ. USA. We demonstrate robust performance of a mid-infrared photothermal imaging system with sensitive detection. Imaging of a patterned thin film shows a resolving powe of $3.1 \mu \mathrm{m}$, below the diffraction-limited spot size of the pump beam.

\section{JW2A.48}

Super-Resolution Diffusive Optical Imaging, Brian Z. Bentz ${ }^{1}$, Dergan Lin ${ }^{1}$, Justin Patel', Kevin J. Webb'; ${ }^{1}$ Purdue Univ., USA We demonstrate super-resolution diffusive optical imaging by localizing point fluorescent sources in highly scattering media. The method allows formation of higher resolution images of optical parameters in deep tissue than have previously been possible.

\section{JW2A.49}

Fourier domain optical coherence tomography and digital algorithm for dispersion compensation, Chia-Yun Lee ${ }^{1}$, Po Nien Yang ${ }^{1}$, Ling-Hsuan Tsai ${ }^{1}$, Hoang Yan Lin ${ }^{1}$ 'Graduate Inst. of Photonics and Optoelectronics, National Taiwan Univ., Taiwan. We combine the simplified numerical dispersion compensation technique and the Hamming window-based FIR filter as well as the Wiener filter to remove the dispersion effects and narrow the pulse width.
JW2A.50

Non-invasive diagnostic and monitoring of periodontal disease through optical coherence tomography: validation of the technique with animal model and patients, Cláudia C. Mota ${ }^{1,2}$, Luana O. Fernandes ${ }^{3}$ José K. Neves', Hugo O. Oliveira', Luciana S. Melo ${ }^{2}$, Tereza J. Dias ${ }^{3}$, Natália S. Pires ${ }^{3}$ Leógenes M. Santiago ${ }^{1}$, Anderson Gomes ${ }^{2,3}$ ${ }^{1}$ Faculty of Dentistry, Centro Universitário Tabosa de Almeida, Brazil; ${ }^{2}$ Dept. of Physics, Universidade Federal de Pernambuco, Brazil, ${ }^{3}$ Graduate Program in Dentistry, Universidade Federal de Pernambuco, Brazil. We propose the application of optical coherence tomog raphy as an auxiliary tool for periodonta disease diagnostic, and demonstrated in three stages: animal modeling, healthy patients and follow up of disease regression upon treatment.

\section{JW2A.51}

Labial mucosa evaluation in systemic sclerosis using optical coherence tomography, Natália S. Pires ${ }^{3}$, Andrea T. Dantas ${ }^{4}$, Angela L. Duarte ${ }^{4}$, Marcello M. Amaral ${ }^{5}$, Luana O. Fernandes ${ }^{3}$, Tereza J. Dias ${ }^{3}$, Luciana S. Melo ${ }^{2}$ Cláudia C. Mota ${ }^{1,2}$, Patricia F. Silva ${ }^{3}$, Ander son Gomes ${ }^{2,3}$; ${ }^{1}$ Faculty of Dentistry, Centro Universitário Tabosa de Almeida, Brazil ${ }^{2}$ Dept. of Physics, Universidade Federal de Pernambuco, Brazil; ${ }^{3}$ Graduate Program in Dentistry, Universidade Federal de Pernambuco, Brazil; ${ }^{4}$ Dept. of Rheumatology, Universidade Federal de Pernambuco, Brazil ${ }^{5}$ Lab of Biophotonics, IPEN-USP, Brazil. A clinical study was developed to evaluate the labial mucosa using optical coherence tomography in 33 systemic sclerosis patients and 35 healthy control. The mucosa presented statistically significant characteristics between the groups.

\section{JW2A.52}

Optical Clearing Agents Associated with Nanoparticles for Scanning Dental Structures with Optical Coherence Tomography, Vanda S. Carneiro ${ }^{2,1}$ Cláudia C. Mota ${ }^{1,3}$ Anderson Gomes ${ }^{3}$, Alex F. Souza', Natalia C. Araújo ${ }^{2}$, Rebeca F. Menezes ${ }^{2}$, Marleny E. Gerbi2: ${ }^{1}$ Centro Universitário Tabosa de Almeida, Brazil; ${ }^{2}$ Dept. of Restorative Dentistry, Universidade de Pernambuco, Brazil, ${ }^{3}$ Dept. of Physics, Universidade Federal de Pernambuco, Brazil. We evaluated the use of optical clearing agents, based on glycero associated to titanium dioxide and silver nitrate nanoparticles, to improve the optical coherence tomography imaging contrast of tooth hard tissues.

JW2A.53

Using speckles to recover different image planes of a bio-sample, Artur F. Sonsin Eduardo Fonseca ${ }^{1}$, Alcenísio Jesus-Silva', Danilo Pires ${ }^{1}$, Emiliano Barreto², Flávio Santos'; ' ${ }^{1}$ Physics, Federal Univ. of Alagoas, Brazil: ${ }^{2}$ biological sciences and health, Federal Univ. of Alagoas, Brazil. Exploring the self-reconfiguration effect, we show different layers of a biological sample. For this, we use the mesentery of a rat, a translucent materia covered by cell walls and irrigated with blood vessels inside.
JW2A.54

Withdrawn

JW2A.55

Three Dimensional Imaging of HeLa cells using light sheet based imaging flow cy tometry, Rasmi Cheloor Kovilakam', Sreedevi Padmanabhan², Rajan Kanhirodan' Ravi Manjithaya ${ }^{2}$, Partha Pratim Mondal' ${ }^{1}{ }^{1}$ Indian Inst. Of Science, Bangalore, India; ${ }^{2}$ Molecula Biology \& Genetics Unit (M.B.G.U.), Jawaharlal Nehru Center for Advanced Scientific Research (J.N.C.A.S.R.), India. Here we present a light sheet based imaging flow cytometry system to image 2D cross sections of the sample (HeLa cells) and these $2 \mathrm{D}$ sections in turn can be stitched togethe to form 3D image. The images are corrected for motion blur using Maximum a Posteriori algorithm (MAP)

\section{JW2A.56}

Refractive Index Sensor Based on Fano Resonance in Microcapillary Resonator Yuejiang Song ${ }^{1}$, Yunchong Peng', Yadong Miao', Mi Li' ${ }^{1}$ Y Yu Xiang ${ }^{1}$, Yu Lu' ${ }^{1}$, Qiang Chen ${ }^{2}$ ${ }^{1}$ College of Engineering and Applied Science, Nanjing Univ., China; ${ }^{2}$ School of Management and Engineering, Nanjing Univ. China. Fano resonance of high order WGMs with Lorentzian peak lineshape is generated and utilized for bulk RI sensor in optofluidic microcapillary resonator. And both high sensitivity $(\sim 800 \mathrm{~nm} / \mathrm{RIU})$ and FoM $(\sim 4100)$ can be achieved simultaneously.

\section{JW2A.57}

Ultra-narrow-linewidth Brillouin/Erbium Fiber Laser, Mo Chen ${ }^{1}$, Chenyu Wang ${ }^{1}$, Jianfe Wang', Hong Luo', Zhou Meng'. 'National Univ of Defense Technology, China. A Brillouin/erbium fiber laser of $1.8-\mathrm{Hz}$ linewidth and $-125 \mathrm{~dB} / \mathrm{Hz}^{1 / 2}$ phase noise at $1 \mathrm{kHz}$ (normalized to 1-m optical path difference) is demonstrated, utilizing 4-m erbium-doped fiber as both Brillouin and linear gain media.

JW2A.58

Self-pulsing Ring Cavity Ultra-long Raman Fiber Laser, Mohd Z. Zulkifli' kuen yao lau² Hani Kbashi" Mohd Adzir Mahdi², Serge Turitsyn'; 'Aston Univ., Aston Inst. of Photonic Technologies, UK; ${ }^{2}$ Wireless and Photonics Networks Research Centre, Faculty of Engineering, Universiti Putra Malaysia, Malaysia. We propose a self-pulsing ring cavity ultralong Raman fiber laser using TrueWave ${ }^{\circledR}$ REACH Fiber as the gain medium. This work shows its alternative prospect to linear laser cavity configuration or ring laser cavity employing other gain medium.
JW2A.59

Electrical polarization in micro optical fiber and its applications in kilovoltage sensing, Nan-Kuang Chen', Cheng Y. Li', Raman Kashyap ${ }^{1}$, Yi-Ning Chen ${ }^{1}$, Chinlon Lin', Xiaoguang . Zhang'; ' ${ }^{1}$ National United Univ., Taiwan. We demonstrate interesting electrical polarization phenomenon in silica micro fiber to make kilovoltage sensors. The micro fiber can be intrinsically polarized and then physically moved by an external kilovoltage source to change its spectral responses.

\section{JW2A.60}

All polarization maintaining optical frequency comb based on Er doped fiber lase with carbon nanotube, Motohiro Togashi' Takeru Nagaike1, Lei Jin', Youichi Sakakibara², Emiko Omoda ${ }^{2}$, Hiromichi Kataura ${ }^{2}$, Yasuyuk Ozeki', Norihiko Nishizawa'; ' Nagoya Univ. Japan; ${ }^{2}$ AIST, Japan; ${ }^{3}$ Univ. of Tokyo, Japan. All polarization maintaining optical frequency comb was demonstrated based on Er-doped ultrashort pulse fiber laser with carbon nanotube. The $f_{\text {ceo }}$ and $f_{\text {rep }}$ were stabilized and thei linewidths were compressed below $1 \mathrm{~Hz}$ by phase locking.

\section{JW2A.61}

Low Nonlinearity Yb-Doped Fluorosilicate Optical Fiber With Ultra-Flat Absorption Spectrum, Peter D. Dragic ${ }^{1}$, Maxime Cavil$\operatorname{lon}^{2}$, Courtney Kucera ${ }^{2}$, Thomas Hawkins ${ }^{2}$ John Ballato2; ${ }^{1}$ Univ of Illinois at UrbanaChampaign, USA; ${ }^{2}$ Clemson Univ., USA. W report on new $\mathrm{Yb}$-doped fluorosilicate optical fibers with reduced strength of Brillouin, Raman, and thermal Rayleigh scattering. $\mathrm{Yb}^{3+}$ absorption spectra strongly resemble borate glasses, with ultra-flat absorption in the $940 \mathrm{~nm}$ region.

\section{JW2A.62}

Time-Range-Extended Spatiotempora Measurement Technique for Multi-Mode Fiber Pulse, Ping Zhu ${ }^{1,2}$, Travis Jones ${ }^{2}$, Rick Trebino ${ }^{2}{ }^{1}$ National Lab on High Power Laser and Physics, Shanghai Inst. of Optics and Fine Mechanics, Chinese Academy of Science, China; ${ }^{2}$ School of Physics, Georgia Inst. of Technology, USA. A multi-delay spatiotemporal measurement technique is introduced to characterize multi-mode fiber long pulses without extra pulse pre-compression. Twomode fibers and four-mode fibers wer tested with extended temporal measurement range, indicating different modes experiencing different dispersion.

\section{JW2A.63}

Integrated chiral long period gratings in multicore fiber, Ruoxu Wang ${ }^{1}$, Ming Tang Songnian Fu', Hailiang Zhang ${ }^{2}$, Deming Liu', Perry Shum ${ }^{2} ;{ }^{1}$ Huazhong Univ. of Sci.\&Tech. China; ${ }^{2}$ School of Electrical and Electronics Engineering , Nanyang Technological Univ. Singapore. We developed a novel approach to inscribe integrated chiral long period gratings into multicore fiber with electrical arc discharge method. Transmission spectra and polarization characteristics are measured through spatial division multiplexing fan-in/ fan-out devices. 


\section{0:00-12:00 JW2A • Poster Session II}

\section{JW2A.64}

Extended linear cavity $2 \mu \mathrm{m}$ singlefrequency fiber laser using Tm-doped fiber saturable absorber, Shijie $\mathrm{Fu}^{1}$, We Shi', guannan shi', Quan Sheng ${ }^{1}$, Haiwei Zhang', jianquan yao'; 'Tianjin Univ., China. An extended linear cavity single-frequency fiber laser at $2 \mu \mathrm{m}$ was investigated using Tm-doped fiber as saturable absorber. More than $60 \mathrm{~mW}$ laser power was achieved with the linewidth of $\sim 40 \mathrm{kHz}$.

JW2A.65

Optical wavelength-swept source at $2.0 \mu \mathrm{m}$ and its application for ultrafast microscopy Sisi Tan ${ }^{1}$, Xiaoming Wei ${ }^{1}$, lingxiao yang ${ }^{1}$, can li', Nan Chen ${ }^{1}$, Kenneth Kin-Yip Wong ${ }^{1}$ 'Univ. of Hong Kong, China. We report an optical wavelength-swept source at $2 \mu \mathrm{m}$ at a sweeping rate of $\sim 18.926 \mathrm{MHz}$ over $30-\mathrm{nm}$ sweeping range based on a chirped fiber Bragg grating. Application in time-stretch microscopy is subsequently demonstrated.

\section{JW2A.66}

Talbot Laser with Tunable GHz Repetition Rate using an Electro-Optic Frequency Shifter, Lixian Wang ${ }^{1}$, Sophie LaRochelle ${ }^{1}$. Universite Laval, Canada. We use an electrooptic frequency shifter in a Talbot laser to demonstrate pulse multiplication factors up to five using temporal fractional Talbot effect and achieve pulse repetition rates of tens of $\mathrm{GHz}$.

\section{JW2A.67}

ow-noise Optical Multi-Carrier Generation using Brillouin Amplification in a Frequency-Shifted Recirculating Loop, Lixian Wang ${ }^{1}$ Jiachuan Lin'1, Leslie A. Rusch1', Sophie LaRochelle'; 'Universite Laval, Canada. Brillouin amplification is used in a frequency-shifted recirculating optical fiber loop to generate a low-noise multi-carrier optical signal. We minimize ASE noise through numerical simulations and we perform a proof-of-concept experimental demonstration.

\section{JW2A.68}

Elliptical-core Mode-selective Photonic Lanterns for MIMO-free Mode Division Multiplexing Systems, Xiaowei Sai ${ }^{1}$, Yan Li ${ }^{1}$, Xinglin Zeng ${ }^{1}$, lipeng feng ${ }^{1}$, Wei Li ${ }^{1}$, Jifang Qiu ${ }^{1}$, Xiaobin Hong ${ }^{1}$, Yong Zuo ${ }^{1}$, Hongxiang Guo', Jian Wu'; ' ${ }^{1}$ UUPT, China. We design a three mode elliptical-core mode-selective photonic lantern (EC-MSPL) which can match well with the elliptical core few mode fiber in MIMO-free MDM systems. The mode crosstalk of the EC-MSPL are below $-20 \mathrm{~dB}$ for all three modes.

\section{JW2A.69}

Small Core SiGe Alloy Optical Fibers by Templated Deposition, Subhasis Chaudhuri ${ }^{1}$, Xiaoyu Ji', Haw-Tyng Huang ${ }^{1}$, Todd Day', Venkatraman Gopalan'1, John Badding', 'The Pennsylvania State Univ., USA. Smal core Si-Ge alloy optical fibers have been fabricated by templated deposition inside silica capillaries using high-pressure chemical vapor deposition. These fibers can have a wide variety of applications in optics and optoelectronics.
JW2A.70

Measurements of Polarization Crosstalk in a Polarization-Maintaining Few-Mode Optical Fiber, Zhen Wang 2,1, Xiaolong $\mathrm{Hu}^{2,1}$ Muyang Lin 2,1 Qi Mo ${ }^{3,4}$, He Wen ${ }^{2,5}$, Guifang $\mathrm{Li}^{5,2}$; ${ }^{1}$ Key Lab of Optoelectronic Information Science and Technology, Ministry of Education, China: ${ }^{2}$ School of Precision Instrument and Optoelectronic Engineering, Tianjin Univ., China; ${ }^{3}$ Fiberhome \& Fujikura Optics Co., China; ${ }^{4}$ School of Optical and Electronic Information, Huazhong Univ. of Science and Technology, China; ${ }^{5}$ The College of Optics \& Photonics, Univ. of Central Florida, USA. We experimentally demonstrate the measurements of the intra- and inter-spatial-modal polarization crosstalk in a polarization-maintaining few-mode optical fiber using optica time-domain reflectometry.

\section{JW2A.71}

Temperature and Strain Sensing in BOTDA Fiber Sensor by Utilizing WavelengthSweeping BGS, Zonglei Li', Lianshan Yan ${ }^{1}$ Liyang Shao', Jiawei Liang', Wei Pan', Bin uo'. 'Southwest Jiaotong Univ., China. Instead of frequency-sweeping in the microwave domain, wavelength-sweeping is proposed for BGS measurement, based on which the decoded Brillouin wavelength shift exhibits sensing coefficients of $7.3^{\circ} \mathrm{C} / \mathrm{nm}$ and $160 \mathrm{me} / \mathrm{nm}$ in standard SMF.

JW2A.72

Dual-Mode Immunoassay using Photonic Crystal Biosilica, Alan X. Wang ${ }^{1} ;{ }^{1}$ Oregon State Univ., USA. We demonstrate an ultrasensitive immunoassay biosensor through photonic crystal enhanced fluorescence and surface-enhanced Raman scattering (SERS) using diatom biosilica. We experimentally achieved enhanced detection limit down to $10^{-16} \mathrm{M}$ and $10^{-13} \mathrm{M}$ respectively.

\section{JW2A.73}

Low-cost thermal infrared detector based on surface plasmon resonance imaging Brandon Hong ${ }^{1}$, Felipe Vallini ${ }^{1}$, Cheng-Y Fang $^{2}$, Amr Alassad ${ }^{3}$, Yeshaiahu Fainman' ${ }^{1}$ Electrical and Computer Engineering, Univ. of California San diego, USA; ${ }^{2}$ Materials Science and Engineering, Univ. of California San diego, USA; ${ }^{3}$ Center of Excellence for Telecommunication, King Abdulaziz City for Science and Technology, Saudi Arabia. We present an uncooled thermal infrared detector based on SPR imaging. IR induced thermo-optic shifts in polymer-clad metal gratings are detected by visible SPR readout, and an experimentally predicted NETD of 22 $\mathrm{K}$ is obtained.

\section{JW2A.74}

Silicon On-chip Ultracompact Integrated Sensor Array Based on High-O Photonic Crystal Nanobeam Cavities with Very Large Free Spectral Range, Daquan Yang', Bo Wang ${ }^{1}$, Xin Chen ${ }^{1}$, Chuan Wang ${ }^{1}$, Yuefeng Ji': ${ }^{1}$ Beijing Univ. of Posts and Telecommunications, China. We present a novel method for multiplexed on-chip integrated sensorarray, which are based on ultracompact one-dimensional photonic-crystal nanobeam cavities with ultrahigh- $Q$ and very large-freespectral-range (LFSR, $200 \mathrm{~nm}$ ). The high refractive-index sensitivities of $166.4 \mathrm{~nm} / \mathrm{RIU}$ can be achieved.
JW2A.75

Plasmonic Nanoantenna of Hole-Sphere Nanogaps for Surface Enhanced Raman Scattering Sensor, Jong M. Lee', Chanwoo Hong ${ }^{1}$ Samir Adhikari ${ }^{1}$, Hyuck Jeong ${ }^{1}$, Yu D. Jang', Jong S. Baek', Ilseon Yoon', Donghan Lee ${ }^{1}$; ${ }^{1}$ Chungnam National Univ., South Korea. We report gap-dependent strong SERS enhancements and plasmonic couplings of hole-sphere nanostructures. The structure shows uniform sensitivity of less than 10\% over the entire substrate, which comes from uniform narrow gaps over the substrate.

\section{JW2A.76}

Experimental Demonstration of Using Orbital Angular Momentum Based Spatia Spectrum Analysis for Object Paramete Estimation, Guodong Xie ${ }^{1}$, Haogian Song Zhe Zhao', Yongxiong Ren' ', Cong Liu', Runzhou Zhang', Long Li' ', Zhe Wang' ', Kai Pang Moshe Tur ${ }^{2}$ Alan E. Willner'. ' $U$. of Southern California, USA: ${ }^{2}$ Tel Aviv Univ., Israel. We demonstrate the use of orbital angula momentum based spatial spectrum analysis for the estimation of object parameters. The proposed approach is insensitive to object rotation or probing beam diffraction caused by object truncation.

JW2A.77

Exploiting Shock Wave and Self-Absorption for High Resolution Laser Induced Break down Spectroscopy, Ali Rastegari' ${ }^{1}$, Matthias Lenzner ${ }^{2}$, Chengyong Feng ${ }^{1}$, Ladan Arissian' Jean-Claude M. Diels ${ }^{1}$, Kristen Peterson 'Univ. of New Mexico, USA; ${ }^{2}$ Lenzner Research, USA; ${ }^{3}$ Southwest Sciences, Inc, USA The shock wave created by a high energy UV filament is sufficient to create a low pressure absorber enabling higher resolution laser induced breakdown spectroscopy, through reduction of pressure broadening. Isotopic selectivity is demonstrated.

\section{JW2A.78}

Low Q-factor Ring Resonators With UltraLow Limit of Detection Based on FFT Processing of Spectral Scanning Data, Lefteris Gounaridis', Panos Groumas ${ }^{1}$, Erik Schreuder ${ }^{2}$, George Tsekenis ${ }^{3}$, Rene Heideman $^{2}$, Hercules Avramopoulos', Christos Kouloumentas'; ${ }^{1}$ National Technical Univ. of Athens, Greece; ${ }^{2}$ LioniX B.V., Netherlands; ${ }^{3}$ Biomedical Research Foundation of the Academy of Athens, Greece. We extend the simulations for sensors with micro-ring resonators and FFT-based processing of the measurement data. We experimentally demonstrate a system with low $\mathrm{Q}$-factor resonators and coarse scanning steps, achieving limit of detection $7 \times 10^{-7} \mathrm{RIU}$.

\section{JW2A.79}

Time-Wavelength Optical Sampling Based on Laser Cavity Tuning, Lin Yang', Lingze Duan ${ }^{1} ;{ }^{1}$ Univ. of Alabama in Huntsville, USA We report a new time-wavelength mapping scheme based on optical sampling by cavity tuning and demonstrate its application in absorption spectroscopy.

JW2A.80

Computational Adaptive Sampling for Multiheterodyne Spectroscopy, Lukasz A. Sterczewski ${ }^{1,2}$, Jonas Westberg ${ }^{1}$, Link Patrick', Gerard Wysocki'; 'Dept. of Electrical Engineering, Princeton Univ., USA; ${ }^{2}$ Faculty of Electronics, Wroclaw Univ. of Science and Technology, Poland. We present a fas computational technique based on digital filtering, mixing, and linear resampling to enable high resolution multiheterodyne spectroscopy in any dual-comb system affected by frequency instabilities of the laser sources.

\section{JW2A.81}

Compressive sampling for spectral imaging, Luke Maidment ${ }^{1}$, Adam Polak ${ }^{2,3}$, Steve Marshall' ${ }^{2}$, Derryck T. Reid'; ${ }^{1}$ Inst. of Photonics and UK; ${ }^{2}$ Centre for Signal \& Image Processing Univ. of Strathclyde, UK; ${ }^{3}$ Fraunhofer Centre for Applied Photonics, UK. An iterative a gorithm is used to reconstruct the spectra of light passing through a scanning Michelson nterferometer, without using a Fourier transform, potentially allowing significantly fewe images to be recorded in spectral imaging.

\section{JW2A.82}

Design of a Band-selective Silicon Photonic Fourier Transform Spectrometer using Slow Light, Shayan Mookherjea'; 'Univ. of California San Diego, USA. A silicon-photonic Fourier Transform Spectrometer is designed based on coupled-microring resonators forming a slow-light waveguide, which scans the propagation delay electrically. The simulated device is compact, fast and achieves high spectral resolution in targeted bands.

JW2A.83

3D Temperature Mapping of Cellula Passive Cooling Structures Fabricated by Additive Manufacturing for Lasers, Shuo $\mathrm{Li}^{1}$, ran zou', aidong yan', lin cheng', Alber $\mathrm{To}^{2}$, Kevin P. Chen ${ }^{1} ;{ }^{1}$ Electrical and Computer Engineering, Univ. of Pittsburgh, USA ${ }^{2} \mathrm{Me}$ chanical Engineering \& Materials Science, Univ. of Pittsburgh, USA. 3D temperature mapping of passive cooling structures built y additive manufacturing was interrogated by in-fiber Rayleigh backscattering using optical frequency domain reflectometry for therm-mechanic structure optimization for laser and photonic systems.

\section{JW2A.84}

Tunable Resonant Graphene Plasmons for Mid-infrared Biosensing, Tingting $\mathrm{Wu}^{1}$, Le Wei'; 'Nanyang Technological Univ., Singapore. Tunable resonant surface plasmon based on graphene nanoribbon are studied to detect nanoscale protein molecules in mid-infrared region. High sensitivity in the detection of the refractive index and the protein chemical vibrations is achieved.

\section{JW2A.85}

All-fiber QEPAS Sensor and Its Application for Spatially Resolved Trace Gas Detection, 


\section{JW2A.86}

Explore on Inorganic Cladding of Neodymium Phosphate Glass used in Slab Amplifier, Bingyan Wang ${ }^{1}$, Jiangiang Zhu' ${ }^{1}$ Li Haiyuan', Xiong Huai'; 'SIOM, CAS, China. Polymer cladding is conventionally employed for neodymium phosphate glass in slab amplifier. But the organic polymer is easy to fracture under high flashlamp irradiation. An inorganic cladding is proposed, and the confirmatory experiment is accomplished.

JW2A.87

High Energy Pulse Recompression Tech niques for Petawatt Class Lasers, Efim A. Khazanov', Sergey Mironov', Vlad Ginzburg', Ivan Yakovlev', Anton Kochetkov', Andrey Shaykin', Gerard Mourou2; 'Inst. of Applied Physics, Russia; ${ }^{2}$ International Center for Zetta-Exawatt Science and Technology, France. Using of Thin Film Compressor for shortening of PW laser pulses was investigated in experiments. Cascade quadratic nonlinearity in a single KDP crystal can be applied for control temporal parameters of the laser pulses.

\section{JW2A.88}

High Pulse Energy Chirally-Coupled-Core Yb-Doped Fiber Amplifier System, Jinxu Bai', Jim Zhang'2, Joona Koponen², Manoj Kanskar ${ }^{2}$, Elias Towe ${ }^{1} ;{ }^{1}$ Carnegie Mellon Univ., USA: ${ }^{2} n$ Light, Inc, USA. A high pulse energy, chirally-coupled-core, Yb-doped fiber amplifier system is reported. This system has a pulse energy output of $1.2 \mathrm{~mJ}$ for 25 -ns pulses at a repetition rate of $100 \mathrm{kHz}$, with a slope efficiency of $82 \%$.

JW2A.89

Switchable High Polarization Purity Radially and Azimuthally Polarized Nd:YAG Microchip Laser, Hong-sen $\mathrm{He}^{1}$, Jun Dong ${ }^{1}$ Xiamen Univ., China. Watt-level switchable high polarization purity radially and azimuthally polarized laser beams have been generated in the Nd:YAG microchip laser pumped with a focused annular-shaped pump beam formed with a hollow focus lens.

\section{JW2A.90}

Amplifier for Optical Stochastic Cooling, Matthew B. Andorf', Valeri A. Lebedev², Philippe Piot ${ }^{1,2}$, Jinhao Ruan2.; ${ }^{1}$ Northern IIlinois Univ., USA; ${ }^{2}$ Fermi National Accelerato Lab, USA. The amplification of electromagnetic radiation emitted from charged-particle beams can be used for beam cooling. We discuss our progress to develop such a system using undulator radiation.

JW2A.91

Influence of crystal's nominal figure of merit on Ti:sapphire laser directly pumped by InGaN laser diodes, Naoto Sugiyama', Ryota Sawada', Hiroki Tanaka' ${ }^{1}$, Fumihiko Kannari' ${ }^{1}$; 'Keio Univ., Japan. A pump-induced loss in Ti:sapphire crystals of different figure of merit with 451-, 478- and 520-nm is investigated, and the loss with $451 \mathrm{~nm}$ is significant even for crystal of high figure of merit.

\section{0:00-12:00 JW2A • Poster Session II}

JW2A.92

Filter-driven four-wave mixing ultrafast all-fiber laser based on microfiber resonator, Qizhen Sun', Yanpeng Li'; 'Huazhong Univ of Science and Technology, China. we demonstrate a stable ultrafast all-fiber laser based on filter-driven four wave mixing using a microfiber resonator which serves both as filter and a high nonlinear element.

\section{JW2A.93}

A Burst-mode Nd:YVO,/Nd:YAG MOPA Laser with High-Repetiton-Rate and High-Pulse-Energy, XuDong Li ${ }^{1}$, renpeng yan ${ }^{1}$, yiping Zhou ${ }^{1}$, yufei ma ${ }^{1}$, deying chen ${ }^{1}$ zhongxiang zhou'; ${ }^{1}$ Harbin Inst. of Technology, China. We demonstrated a burst-mode $\mathrm{Nd}: \mathrm{YO}_{4} / \mathrm{Nd}$ :YAG MOPA laser. A LD pumped $\mathrm{Nd}: \mathrm{YVO}_{4} \mathrm{~A}-\mathrm{O} \mathrm{Q}$-switched laser works as oscillator and Nd:YAG modules as amplifiers. During $1 \mathrm{~ms}$ burst duration, maximum pulse burst energy of $730 \mathrm{~mJ}$ at $10 \mathrm{kHz}$ is obtained with single pulse energy of $73 \mathrm{~mJ}$ and a pulse width of $9.3 \mathrm{~ns}$.

\section{JW2A.94}

Path to Doubling the Efficiency of Mid-IR Erbium Lasers, Tigran Sanamyan'; ' ${ }^{\text {U U S Army }}$ Research Lab, USA. Absorption and emission spectra of $\mathrm{Cr}: \mathrm{ZnSe}$ host are perfectly positioned to amplify the output of the cascade $\mathrm{Er}: \mathrm{Y}_{2} \mathrm{O}_{3}$, or any cascade erbium laser, when pumped by $1.6 \mu \mathrm{m}$ and seeded by $2.7 \mu \mathrm{m}$ component of the laser.

\section{JW2A.95}

Double Layer Hollow Core Anti-resonant Fiber for Small Core and Low Loss Characteristics, Xiaosheng Huang ${ }^{1}$, Daryl $\mathrm{Ho}^{1}$ Wenliang Qi', Seong Yoo': 'Nanyang Technological Univ., Singapore. We study the function of second cladding layer of the tube lattice fiber (TLF) and experimentally demonstrate the important role of the second layer in the reduction of both confinement loss (CL) and bending loss.

\section{JW2A.96}

Triple-wave Diagnostics with Single Diffraction Pattern Based on Coherent Phase Modulation in High Power Laser Systems, Xingchen Pan', Cheng Liu', Jianqiang Zhu', ${ }^{1}$ Shanghai Inst of Optics and Fine Mech, China. A novel algorithm for on-line triplewave measurements based on phase modulation and coherent iterative engine with single diffraction pattern. It could be a potential technique for pulse laser diagnostic in high power laser systems.

JW2A.97

A 97-ps laser-pulse generation by twostage stimulated Brillouin and Raman scattering, Zhaohong Liu', Yulei Wang Hengkang Zhang ${ }^{1}$, Yirui Wang ${ }^{1}$, Hang Yuan ${ }^{1}$ Zhenxu Bai', Zhiwei Lu'; ${ }^{1}$ Harbin Inst. of technology, China. We demonstrate a pulse temporal compression technique based on a combination of Stimulated Brillouin scattering and subsequent Stimulated Raman scattering. A 97-ps pulse compressed from an 8-ns Q-switched $\mathrm{Nd}$ :YAG pulse laser is obtained.

\section{JW2A.98}

Functional Topological THz devices using Semiconductors, Babak Bahari', Ricardo Tellez-Limon', Boubacar Kante'; 'Univ. of California San Diego, USA. We showed that cyclotron resonance of semiconductors can be utilized in Topological devices to break the time-reversal symmetry for unidirectional propagation in $\mathrm{THz}$. To demonstrate, we proposed a tunable power splitter based on topological effect.

\section{JW2A.99}

Photo-thermal-acoustic $\mathrm{THz}$ detection based on 3-dimensional graphene, Mostafa Shalaby' ${ }^{1}$ C. Vicario", Flavio Giorgianni', Stefano lupi ${ }^{2}$, Christoph P. Hauri'; ' Paul scherre institut, Switzerland; ${ }^{2}$ Dept. of Physics, Sapienza Univ. Of Rome, Italy. We report on a novel, simple and efficient $\mathrm{THz}$ energy and intensity profile diagnostic tool which is based on the photo-thermo-acoustic (PTA) effect in a 3-dimensional graphene sponge.

\section{JW2A.100}

Enhanced Sensitivity of Terahertz Allergen Sensors Based on Complementary Metasurfaces, Guillermo A. Naranjo', Xomali G. Peralta ${ }^{1}$, Igal Brener ${ }^{2}$, Anthony James ${ }^{2}$ John Nogan"; ' Univ. of Texas at San Antonio, USA; ${ }^{2}$ Sandia National Labs, USA. We have designed, fabricated and characterized positive and complementary metasurfaces for biosensing. We demonstrate that complementary metasurfaces have a highe sensitivity than their positive counterparts with the largest increase in sensitivity occurring off resonance.

\section{JW2A.101}

3D printed hollow core terahertz Bragg waveguide for surface sensing applications, Jingwen $\mathrm{Li}^{1}$, Kathirvel Nallapan Hichem Guerboukha', Maksim Skorobogatiy'; 'Dept. Engineering physics, Ecole polytechnique de Montreal, Canada. We study the use of 3D-printed hollow-core Terahertz ( $\mathrm{THz}$ ) Bragg waveguides with defect layers operating in an effectively single mode regime for resonant surface sensing applications. The demonstrated sensitivity is found to be $0.12 \mathrm{GHz} / \mu \mathrm{m}$ to changes in the defect layer thickness.

\section{JW2A.102}

Broadband Terahertz Detection trough Plasmonic Photoconductive Nano-Antenn Arrays, Nezih Yardimci', Mona Jarrahi'; ' Univ. of California - Los Angeles, USA. We present a high-performance photoconductive terahertz detector based on plasmonic terahertz nano-antenna arrays. We demonstrate terahertz detection with record-high signalto-noise ratio levels as high as $107 \mathrm{~dB}$ over a $5 \mathrm{THz}$ detection bandwidth.

\section{JW2A.103}

Real-space and real-time imaging of $\mathrm{TH}$ wave confinement and standing wave in a Fabry-Perot resonator, Chongpei Pan', Yane Wang', Yao Lu', Qi Zhang', wenjuan zhao' Qiang $\mathrm{Wu}^{1}$, Jingjun $\mathrm{Xu}^{1}$; ${ }^{1}$ Nankai Univ., China. We fabricate a Fabry-Perot resonator in a $\mathrm{LiNbO}_{3}$ subwavelength slab and investigate the spatiotemporal evolution of terahertz pulses in the structure via time-resolved imaging system. The wave confinement and standing wave modes are clearly observed.

\section{JW2A.104}

Quad-Wavelength Multi-Focusing Lenses with Dual-Wavelength Meta-Atoms, sensong an ${ }^{1}$, jun ding ${ }^{1}$, bowen zheng ${ }^{1}$, yuankun in $^{2}$, weili zhang ${ }^{3}$, hualiang zhang ${ }^{1},{ }^{1} E C E$ Univ. of Massachusetts Lowell, USA; ${ }^{2}$ Physics, Univ. of North Texas, USA; ${ }^{3} E C E$, Oklahoma State Univ., USA. We proposed a novel quad-wavelength multi-focusing lens, in which four focusing spots could be arbitrarily controlled at four frequencies independently. A prototype quad-wavelength lens has been designed and numerically verified at terahertz frequencies.

JW2A.105

Graphene-based Metasurfaces for Multimode Tunable Terahertz Modulators Thomas A. Searles ${ }^{1}$, Mehdi Rezaee ${ }^{1}$, Amirhassan Shams-Ansari', Erin Strickland', Tina Brower-Thomas ${ }^{1}$, Gary Harris ${ }^{1}$, Riad Yahiaoui ${ }^{2}$ 'Howard Univ., USA; ${ }^{2}$ Universite Paris Ouest, France. We present a hybrid graphene metasurface and its modulation by electrostatically tuning the conductivity of the graphene. Through modification of unit cell symmetry, multiple Fano-like resonances arise for additional modes over a $300 \mathrm{GHz}$ range.

\section{JW2A.106}

3D Printed Hollow-Core Terahertz Optical Waveguides with Hyperuniform Disordered Dielectric Reflectors, Tian $\mathrm{Ma}^{1}$, Hichem Guerboukha', Maksim Skorobogatiy'; 'Dept. of Engineering Physics, Ecole Polytechnique de Montréal, Canada. Novel hollow-core $\mathrm{THz}$ waveguides featuring hyperuniform disordered reflectors are proposed, fabricated, and characterized. The results confirm that proposed waveguide exhibit sizable photonic band gaps (20\%) even when relatively low refractive index contras used (resin/air).

\section{JW2A.107}

Terahertz Emission in One-Dimensiona Disordered Systems, Yongquan Zeng ${ }^{1}$ Guozhen Liang ${ }^{1}$, Hou Kun Liang ${ }^{2}$, Bo Qiang' Bo Meng', shampy mansha ${ }^{3}$, Lianhe $\mathrm{Li}^{4}$ Alexander Giles Davies ${ }^{4}$, Edmund Harold Linfield ${ }^{4}$, Ying Zhang ${ }^{2}$, Yidong Chong ${ }^{3}$, qijie wang'; ' School of Electrical and Electronic Engineering, Nanyang Technological Univ. Singapore; ${ }^{2}$ Singapore Inst. of Manufacturing Technology, Singapore; ${ }^{3}$ School of Physical and Mathematical Sciences, Nanyang Technological Univ., Singapore; ${ }^{4}$ School of Electronic and Electrical Engineering, Univ. of Leeds, UK. Multimode terahertz (THz) lase emission around $3.2 \mathrm{THz}$ in one-dimensiona (1-D) disordered systems with $20 \%$ disorde Simulation and experimental work provide evidence for spatial localization of light of defect modes within the bandgap frequency window.

\section{JW2A.108}




\section{0:00-12:00 JW2A • Poster Session II}

JW2A.109

High Efficient Terahertz Generation Us ing Tilted-Pulse-Front Photoexcitation of Semiconductor Surface, Yuri Avetisyan ${ }^{1}$ Armen Makaryan ${ }^{1}$, Masayoshi Tonouchi ${ }^{2}$. 'Microwave Eng. Dept., Yerevan State Univ., Armenia; ${ }^{2}$ Inst. of Laser Engineering, Osaka Univ, Japan. It is shown that photoexcitation of InAs surface with tilted-front laser pulses allows controlling the direction of terahertz emission and by that way achieving significant increase in the generated power.

\section{JW2A.110}

Highly Efficient GeSn Electroabsorprtion Modulator Using Higher-order-mode for Mid-infrared Ge-on-Si Platform, Takanor Sato', Minami Akie ${ }^{1}$, Masakazu Arai ${ }^{2}$, Takesh Fujisawa', Kunimasa Saitoh'. ${ }^{1}$ Hokkaido Univ. Japan; ${ }^{2}$ Univ. of Miyazaki, Japan. A highly efficient $\mathrm{GeSn}$ electroabsorption modulator using higher-order-mode is proposed for mid-infrared photonics. Proposed structure is suitable for monolithic integration on $\mathrm{Ge}$ on-Si platform and $>10 \mathrm{~dB}$ enhancement of the extinction ratio is possible.

\section{JW2A.111}

Silicon photonics multi-channel Bragg reflectors based on narrowband claddingmodulated gratings, Tzu-Hsiang Yen ${ }^{1}$ Chong-Jia Wu'1, Chia-Ju Yu', Yung-Jr Hung ${ }^{1}$ National Sun Yat-sen Univ. Taiwan. Silicon photonics multi-channel Bragg reflectors with equal and narrow linewidths are demonstrated by connecting multiple gratings in series or parallel through waveguide effective index engineering and/or grating coupling strength balance.

JW2A.112

Withdrawn

JW2A.113

Hybrid Grating Assisted Contra-Directional Coupler with Two Mode Channels, Xiangjie Zhao ${ }^{1}$, Yuxi Wang ${ }^{1}$, Qingzhong Huang ${ }^{1}$, Jinsong Xia'; ' 'Wuhan National Lab for Optoelectronics, China. A novel two-mode-channe contra-directional coupler based on hybrid grating is proposed and experimentally demonstrated. By applying apodization on the hybrid grating, high filtering contrast of about $30 \mathrm{~dB}$ are achieved for both modes channels.

\section{JW2A.114}

Highly Efficient IR Transparent Perovskite Solar Cells, Xianqiang $\mathrm{Li}^{1,2}, \mathrm{Tao}^{3}{ }^{3}$, Xizu Wang ${ }^{2}$, Xiaohong Tang ${ }^{1} ;{ }^{1}$ Nanyang Technological Univ., Singapore; ${ }^{2}$ Inst. of Materials Research and Engineering, Singapore; ${ }^{3}$ National Univ. of Singapore, Singapore. We designed and fabricated a highly efficient infrared (IR) transparent perovskite solar cells (PSCs). The optimized PSCs have a power conversion efficiency (PCE) more than $15 \%$ and IR transmittance of around $40-50 \%$.

\section{JW2A.115}

Dual-Cavity Optically and Electrically Resonant Modulators for Efficient Narrowband RF/Microwave Photonics, Yosse Ehrlichman', Milos A. Popovic2. ${ }^{1}$ Electrical, Computer and Energy Engineering, Univ. of Colorado, USA; ${ }^{2}$ Dept. of Electrical and Computer Engineering, Boston Univ., USA We propose doubly and triply resonant electro-optic modulators for efficient narrowband RF-to-optical signal conversion. Using a dual-optical-cavity design, we predict $30 \mathrm{~dB}$ higher conversion gain than single-cavity modulators, and another 5-10dB using a realistic electrical resonance.

\section{JW2A.116}

Broadband dispersion engineering for integrated photonics just by tuning the width of the waveguide, Yu Li', Le An ${ }^{1}$, Yuandong Huo', Minghua Chen', Hongwei Chen ${ }^{1}$ Sigang Yang'; ${ }^{1} T$ singhua Univ., China. We develop a dispersion engineering technique based on tailoring the width of waveguide. It achieves anomalous dispersion with a range of $70 \mathrm{~nm}$ numerically and alters the dispersion from $-820 \pm 30 \mathrm{ps} / \mathrm{nm} / \mathrm{km}$ to $-140 \pm 40 \mathrm{ps} / \mathrm{nm} /$ $\mathrm{km}$ experimentally.

\section{JW2A.117}

Painting silk inverse opals by tuning their structural colour, Yu Wang ${ }^{1}$, Daniele Aurelio ${ }^{2}$, wenyi li', Peter Tseng ${ }^{1}$, Zhaozhu Zheng ${ }^{1}$, Meng Li', David L. Kaplan', Marco Liscidini², Fiorenzo Omenetto'; 'Tufts Univ. USA; ${ }^{2}$ Università degli Studi di Pavia, Italy. Opals owe their astonishing colours to light interference from their periodic features. Exposing silk inverse opals to water vapou or ultraviolet light modifies their molecula structure on the nanoscale scale and tunes their spectral response.

\section{JW2A.118}

Optical Simulations on Silicon Microstruc ture Chips: Quantum Walk and Its Applications, Yufei Wang ${ }^{1,2}$, Fan $\mathrm{Qi}^{1,2}{ }^{1}$, Qingyan $\mathrm{Ma}^{1,2}$ Zhishuang Liu', ${ }^{1,2}$, Wanhua Zheng ${ }^{1,2}$; ${ }^{1}$ Lab of Solid State Optoelectronics Information Technology, Inst. of Semiconductors, CAS,

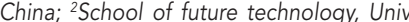
of Chinese Academy of Sciences, China. We propose a photonic crystal self-collimation chip for experimentally simulating discretetime quantum walk (OW), and also demonstrate simulation of second-order coupling Heisenberg equation and $\mathrm{OW}$-based search on improved-glued-binary-tree on different silicon artificial microstructures.

\section{JW2A.119}

Silicon Based Photonic Crystal Nanobeam Cavity with Polymer Cladding, Yuguang Zhang', Yaocheng Shi'; 'Zhejiang Univ., State Key Lab for Modern Optical Instrumentation, China. Silicon based photonic crystal nanobeam cavities with polymer cladding have been investigated. We experimentally demonstrate the temperature insensitive photonic crystal (PhC) nanobeam cavity, the post-trimming of the $\mathrm{PhC}$ cavity and a highsensitivity temperature sensor, respectively.
JW2A.120

Superconducting Nanowire Single-Photon Detectors Integrated with Current Reservoirs, Yuhao Cheng ${ }^{1}$, Haiyi Liu', Chao Gu', Xiaotian Zhu', Xiaolong Hu'; 'Tianjin Univ., China. We integrate superconducting nanowire single-photon detectors with wider superconducting wires that behave as current reservoirs to provide gain for signal amplification and increase the slew rate for reducing timing jitter without affecting the detection efficiency.

\section{JW2A.121}

Enhanced Thermo-Optic Bistability in Graphene-on-Silicon Nitride Ring Resonators, Yun Gao', Wen Zhou', Chester Shu' Hon Ki Tsang $1{ }^{1}{ }^{1}$ Chinese Univ. of Hong Kong, Hong Kong. We observed enhancement of optical bistability and hysteresis in grapheneon- $\mathrm{Si}_{3} \mathrm{~N}_{4}$ ring resonators. Stronger thermooptic effect results in twofold resonance shift rate and 17-fold increase in the effective nonlinear refractive index compared to bare $\mathrm{Si}_{3} \mathrm{~N}_{4}$ resonator.

JW2A.122

Low-power optical logic gate in a silicon waveguide, Yun Zhao ${ }^{1}$, David Lombardo ${ }^{1}$ Jay Mathews', Imad Agha'; ' 'Univ. of Dayton, USA. We experimentally demonstrate a transistor-like all-optical logic gate in a silicon waveguide via four-wave mixing Bragg scattering, and evaluate the eye diagrams using pseudo-random data.

\section{JW2A.123}

Design and fabrication of subwavelength grating (SWG) slot waveguide at shortwave infrared wavelength of $2 \mu \mathrm{m}$, Zhengsen Ruan', Li Shen', Shuang Zhen', Andong Wang ${ }^{1}$, Jun Liu'; ${ }^{\top}$ Huazhong Uni. of Science and Technology, China. We propose and fabricate high-performance subwavelength grating (SWG) slot waveguides on an SO platform for the short-wave infrared (SWIR) We further demonstrate 5-Gbit/s direct modulation data transmission in the emerging $2 \mu \mathrm{m}$ communications waveband.

\section{JW2A.124}

Zigzag grating with quasi-random array for single order diffraction, Ziwei Liu ${ }^{1,2}$ Tanchao Pu ${ }^{1,2}$, Lina Shi ${ }^{1}$, Changqing $\mathrm{Xie}^{1}$ Guanya Wang'; '1/nst. of Microelectronics, China; 'Univ. of Chinese Academy of Sciences, China. We present a novel quasirandom array of zigzag gratings for high order diffraction suppression in $\mathrm{x}$-ray region. By optimizing the structure parameters with Kirchhoff's diffraction theory, the 2 to 6 order can be completely suppressed.

\section{JW2A.125}

Simulations of Taper Designs for Integrated Ge/SiGe Waveguide System, Ching-Ying Lu$^{1}$, Kai Zang1, Yijie Huo', Xiaochi Chen ${ }^{1}$ Edward T. Fei ${ }^{1}$, Muyu Xue ${ }^{2}$, Theodore Kamins ${ }^{1}$, James Harris ${ }^{1}$; ${ }^{1}$ Electrical Engineering, Stanford Univ., USA; ${ }^{2}$ Material Science and Engineering, Stanford Univ., USA. We simulated different taper designs and demonstrated that 3D tapers improved the coupling efficiencies and maintained the fundamenta mode as they provided a more gradual optical transition between a Si waveguide and $\mathrm{Ge} / \mathrm{SiGe}$ device layers.
JW2A.126

On-chip Low-threshold Silicon Nitride Distributed Feedback Colloidal Quantum Do Laser, Yunpeng Zhu' ${ }^{1}$, Weiqiang $\mathrm{Xie}^{1}$, Piete Geiregat ${ }^{1}$ Suzanne Bisschop ${ }^{1}$ Tangi Aubert ${ }^{1}$, Edouard Brainis'1, zeger hens' ${ }^{1}$, Dries Van Thourhout ${ }^{1} ;{ }^{1}$ Ghent Univ., Belgium. We report on hybrid integrated distributed feedback (DFB) lasers based on silicon nitride waveguide stacks containing a layer of embedded colloidal quantum dots. The DFB laser shows a low optical pumping threshold of $188 \mathrm{~kW} /$ $\mathrm{cm}^{2}$ and operates in a single mode regime.

\section{JW2A.127}

Integrated silicon photonic reflective modulator for passive optical networks Fatemah Soltani ${ }^{1}$, Michael Menard ${ }^{2}$, andrew kirk': ${ }^{1}$ Dept. of Electrical and Computer Engineering, McGill Univ., Canada; ${ }^{2} 2 \mathrm{Co}$ FaMic Research Center, Univ. of Quebec at Montreal, Canada. The analysis of the bandwidth of loop mirror Mach-Zehnder modulators (LMM) is reported. We demonstrated experimentally that these modulators can provide $20 \mathrm{~Gb} / \mathrm{s}$ modulation. LMM could be used for upstream transmission in passive optical networks.

\section{JW2A.128}

Self-electro-optic bistability in hybrid silicon photonic microring resonators Jiajiu Zheng ${ }^{1}$, Dan Guo ${ }^{1}$, Arka Majumdar ${ }^{1,2}$ 'Dept. of Electrical Engineering, Univ. of Washington, USA; ${ }^{2}$ Dept. of Physics, Univ. of Washington, USA. We propose an on-chip self-electro-optic bistable device based on hybrid silicon microring resonators. The device enables bistability at low optical powe with the switching energy of only $\sim 5 \mathrm{fJ}$ and can potentially operate at $\sim 100 \mathrm{GHz}$.

\section{JW2A.129}

Performance Comparison between SerialConnected and Parallel-Connected Photodiode Array, Jiarui Fei', Yongqing Huang ${ }^{1}$ Tao Liu, Xiaokai Ma', Xiaofeng Duann ${ }^{1}, \mathrm{Ka}$ Liu', Xiaomin Ren'; 'IPOC, BUPT, China. Two-element serial-connected photodiode array (SC-PDA) and parallel-connected photodiode array (PC-PDA) were fabricated and tested. The novel PC-PDA shows a higher responsivity, a smoother frequency response and a higher saturation current comparing to the SC-PDA.

\section{JW2A.130}

Design and fabrication quasiperiodic photonic crystals for simultaneous slab waveguide coupling and splitting, jingxing shi ${ }^{1}$, Michael Pollard ${ }^{2}$, James Gates Martin Charlton': ${ }^{1}$ Univ. of Southampton, UK 2UNSW, Australia. A quasi-crystal structure yields coupling to 12 modes from single chromatic color laser source. The device was fabricated in thin film of $\mathrm{SiON}$ on glass substrate, and simultaneously acts as both coupler and beam splitter. 


\section{0:00-12:00 JW2A • Poster Session II}

\section{JW2A.131}

Experimental demonstration of silicon strip and slot waveguides for $2 \mu \mathrm{m}$ chip-scale optical data transmission, Li Shen' ${ }^{1}$, Zhengsen Ruan', Shuang Zhen ${ }^{1}$, Andong Wang', Jun Liu', Shuhui Li', Jian Wang ${ }^{1}$; ${ }^{1}$ Wuhan National Lab for Optoelectronics, Huazhong Univ. of Science and Technology, China. Silicon strip and slot waveguides are fabricated and 5 $\mathrm{Gb} / \mathrm{s}$ directly-modulated data transmission is demonstrated in $2 \mu \mathrm{m}$ waveband. The results indicate the suitability of SOI platform for data transmission applications in this midinfrared wavelength.

\section{JW2A.132}

Experimental Study of Electro-Optic Crosstalk in Parallel Silicon Photonic MachZehnder Modulators, Lingjun Jiang ${ }^{1,2}, \mathrm{Xi}_{\mathrm{i}}$ Chen ${ }^{1}$, Kwangwoong Kim ${ }^{1}$, Guilhem de Valicourt', Zhaoran R. Huang ${ }^{2}$, Po Dong'; ${ }^{1}$ Nokia Bell Labs, USA; ${ }^{2}$ Dept. of Electrical, Computer and Systems Engineering, Rensselaer Polytechnic Inst., USA. The electrooptic crosstalk between two parallel silicon Mach-Zehnder modulators is characterized. Up to $1.6 \mathrm{~dB}$ power penalty is observed for $36-\mathrm{Gb} / \mathrm{s}$ on-off-keying signals with a $\sim-20 \mathrm{~dB}$ crosstalk, posing challenge to dense photonic integration.

\section{JW2A.133}

Frequency and stability analysis of two mutually delay-coupled semiconductor lasers in photonic integrated circuits Masoud Seifikar ${ }^{1,2}$, Andreas Amann ${ }^{3,1}$, Frank Peters ${ }^{1,2 ;}{ }^{1}$ Tyndall National Inst.,, Ireland; ${ }^{2}$ Dept. of Physics, Univ. College Cork, Ireland; ${ }^{3}$ School of Mathematical Sciences, Univ. College Cork, Ireland. A system of two mutually delay-coupled semiconductor lasers for integration in a photonic integrated circuit is investigated. Multi-stabilities and bifurcation scenarios are presented, followed by a comprehensive frequency analysis of the symmetric and symmetry-broken, 1-colour and 2-colour states.

\section{JW2A.134}

Silicon Nitride Polarization Beam Splitter Based on MMI with Phase Delay Line, Min TENG ${ }^{1}$, Sangsik Kim ${ }^{1}$, Kyunghun Han ${ }^{1}$, Ben Niu', Yunjo Lee ${ }^{1}$, Minghao Qi'; ' ${ }^{1}$ Purdue Univ. USA. We purpose a nitride polarization beam splitter which shows $21 \mathrm{~dB}$ extinction over $100 \mathrm{~nm}$ bandwidth and $30 \mathrm{~dB}$ at $\mathrm{C}$ band. With two-stage cascade, $38 \mathrm{~dB}$ flat-band extinction can be achieved for both polarizations.
JW2A.135

Scalable, Low-Power-Penalty Nanosecond Reconfigurable Hybrid Optical Switches for Data Centre Networks, Minsheng Ding ${ }^{1}$ Adrian Wonfor ${ }^{1}$, Qixiang Cheng ${ }^{1}$, Richard V. Penty', Ian White ${ }^{1 ;}{ }^{1}$ Univ. of Cambridge, UK. A quantitative analysis shows for the first time that a Clos-Beneš MZI-SOA based switch with a hybrid fibre-integration approach can achieve a record 2048 port count with 1.15 $\mathrm{dB}$ penalty when using soft decision FEC.

\section{JW2A.136}

Low-loss Arbitrary-ratio $1 \times \mathrm{N}$ Power Splitter, Ping Xue ${ }^{1}$, Zhixin Wang ${ }^{1}$, Che Zhao ${ }^{1}$, Te Chen'ㄹ, Weiwei Hu'; ' ${ }^{2}$ Peking Univ., China; ${ }^{2}$ Inst. of Telecommunication Satellites, China Academy of Space Technology, China. An arbitrary-ratio $1 \times \mathrm{N}$ power splitter is demonstrated. The power distribution of $\mathrm{N}$-way outputs can be freely customized based on demand. It also has the features of low loss, compact size, easy configuration and wide band.

\section{JW2A.137}

Fabrication of Lightwave Circuits on Flat Fibers: System-in-Fiber, Sheng Huang ${ }^{1}$, Mohan Wang ${ }^{1}$, Ya-wen Huang ${ }^{1}$, Rongtao $\mathrm{Cao}^{1}$, Shuo Li', Ran Zou', Aidong Yan', Ming-Jun $\mathrm{Li}^{2}$, Kevin Chen'; 'Univ. of Pittsburgh, USA ${ }^{2}$ Corning Inc., USA. This paper reports ultrafast laser fabrication of waveguide circuits in optical fibers with rectangular cross-section and large flat surface area designed for on/ in fiber integration. Low-loss coupler and WDM devices are directly integrated in fibers.

\section{JW2A.138}

Chasing Moore's Law with CLEAR, Shua Sun', Vikram Narayana', Tarek El-Ghazawi ${ }^{1}$ Volker J. Sorger ${ }^{1}$; ' George Washington Univ., USA. We introduce a multi-hierarchica FOM termed CLEAR (Capability-to-LatencyEnergyAmount-Resistance) applying to the device, interconnect, and system levels which accurately postdicts technology developments since 1940s, and predicts photonics as technology extension to keepup the pace of evolution.

\section{JW2A.139}

Robust photonic differentiator employing slow light effect in photonic crysta waveguide, Siqi Yan', Ziwei Cheng ${ }^{1}$, Lars H. Frandsen'2, Yunhong Ding' ${ }^{2}$, Feng Zhou', Jianj Dong', Xinliang Zhang'; ' Wuhan National Lab for Optoelectronics, China: ${ }^{2}$ DTU Fotonik, Denmark. A robust photonic DIFF exploiting the slow light effect in a photonic crystal waveguide is proposed and experimentally demonstrated. Input Gaussian pulses with full-width half-maximums ranging from 2.7 ps to 81.4 ps can be accurately differentiated.
JW2A.140

Full Control of Far-field Radiation via Photonic Integrated Circuits Decorated with Plasmonic Nano-antennae, Yi-Zhi Sun ${ }^{1,2}$ Renaud Bachelot ${ }^{3}$, Sylvain Blaize ${ }^{3}$, Lishuang Feng', Wei Ding ${ }^{2}$; ${ }^{1}$ School of Instrumentation Science and Opto-electronics Engineering Beihang Univ., China; ${ }^{2}$ CAS Inst. of Physics, China; ${ }^{3}$ Laboratoire de Nanotechnologie et $d^{\prime}$ Instrumentation Optique (LNIO), Université de Technologie de Troyes, France. We introduce a PIC/plasmonic-antenna hybrid structure to fully control phase, amplitude, and polarization of far-field radiation. Out-ofplane light collimating ( $\varnothing \Phi \sim 0.23 \mathrm{rad}$ ), highNA ( 0.65) beam focusing, and high-purity ( 99\%) vector/vortex mode generation are demonstrated in simulation.

\section{JW2A.141}

Fast circuit modeling of heat transfer in photonic integrated circuits., Xiaoxi Wang Shayan Mookherjea ${ }^{1}{ }^{1}$ 'U.C. San Diego, USA We demonstrate a fast method of modeling heat transfer in photonic integrated circuits by simple thermal resistance circuits that use a conical surface area approximation for effective thermal resistance. We show with Spectre simulations accuracy with Lab measurements within $1 \sim \mathrm{K}$ to $2 \sim \mathrm{K}$.

\section{JW2A.142}

Microwave Photonic Interrogation of a High-resolution and Temperature insensitive Refractive Index Sensor, Yuan Cao ${ }^{1}$, Xudong Wang ${ }^{1}$, Xinhuan Feng Bai-Ou Guan', Jianping Yao'; 'Jinan Univ. China; ${ }^{2}$ Univ. of Ottawa, Canada. Microwave photonic interrogation of a high-resolution and temperature-insensitive refractive index sensor is proposed. The Rl information is translated to microwave-spectral-response change, providing a resolution that is threeorder-of-magnitude higher than that based on wavelength interrogation.

\section{JW2A.143}

Low-loss Two-dimensional Grating Coupler on SOI Platform with Bonded Metal Mirror, Zhichao Nong', Siyuan Yu', Yan n. Luo ${ }^{1}$ Shengqian Gao', Xinlun Cai'; 'Sun Yat-Sen Univ., China. We design and fabricate low loss 2D grating couplers on the silicon-oninsulator platform with bonded metal mirror. The coupling efficiencies of $-1.8 \mathrm{~dB}$ and with $1 \mathrm{~dB}$ bandwidth of $32 \mathrm{~nm}$ was achieved.

\section{JW2A.144}

A Microwave Photonics-based Inverse Synthetic Aperture Radar System, Xuedi Xiao ${ }^{2,}$ Shangyuan $\mathrm{Li}^{2,1}$, Boyu Chen ${ }^{2,1}$, Xiao Yang ${ }^{2,1}$ Dexin Wu ${ }^{2,1}$ Xiaoxiao Xue ${ }^{2,1}$ Xiaoping Zheng ${ }^{2,1}$, Bingkun Zhou', , ' 'Tsinghua National Lab for Information Science and Technology, China; ${ }^{2}$ Dept. of Electronic Engineering, Tsinghua Univ., China. We demonstrate a microwave imaging radar experimenta platform, using photonic generation of linear frequency modulated waveform centered at $10 \mathrm{GHz}$ with $4 \mathrm{GHz}$ bandwidth. Distance and velocity resolution of about $5 \mathrm{~cm}$ and $2 \mathrm{~m} / \mathrm{s}$ are obtained experimentally.

\section{JW2A.145}

High performance light emitting memories: multifunctional devices for unveiling information by optical and electrical detection Yi-Rou Liou', Golam Haider ${ }^{1}$, Shu-Yi Ca 1, Chia-Lin Wu', Tai-Yuan Lin ${ }^{2}$, Yang-Fang Chen'; 'Dept. of Physics, National Taiwan Univ., Taiwan; ${ }^{2}$ Optoelectronic Sciences, $\mathrm{Na}$ tional Taiwan Ocean Univ., Taiwan. The LEM can read the encoded signal electrically and enable the signal communication via optical detection and provides the capability for parallel reading process, which enables to raise the transmission rate of signal dramatically.

\section{JW2A.146}

Fully Compressible Wideband Radar Signal Generation with Photonic Frequency Multiplication, Yu Zha', Xiaoxiao Xue', Haojie Wang ${ }^{1}$, Xiaoping Zheng ${ }^{1}$, Shangyuan $\mathrm{Li}^{1}$, Bingkun Zhou': 'Tsinghua Univ., China. We demonstrate wideband radar signal generation by combining the advantages of direct digital synthesis and photonic frequency multiplication. Linear frequency modulated waveforms with a time-bandwidth product as high as 3200 are generated experimentally.

\section{JW2A.147}

On-chip Microring Resonator Based Elec tro-optic Full Adder for Optical Computing Zhoufeng Ying ${ }^{1}$, Zheng Wang ${ }^{1}$, Shounak Dhar', Zheng Zhao', David Z. Pan', Ray T. Chen'; ' the Univ. of Texas at Austin, USA. We propose a ripple-carry electro-optic full adder using microring resonators, taking advantage of unique properties of light on chip. This proposed design with larger bandwidth and lower power consumption paves the way to future optical computing

13:00-15:00 JW3A • Plenary Session II and Awards, Grand Ballroom

15:00-15:30 Coffee Break and Unopposed Exhibit Only Time, Exhibit Hall 1, 2 \& 3

15:00-16:30 Market Focus Session V: Bright Ideas Competition, Exhibit Hall Theater 
Executive Ballroom 210A

Executive Ballroom 210B

\section{CLEO: Applications \& Technology}

15:30-17:15

AW4A • Biomedical Imaging I

Presider: Jin Kang; Johns Hopkins Univ., USA

Listening to Light and Seeing Through: In Vivo Multiscale Photoacoustic Imaging, Chulhong Kim"; "POSTECH, Korea (the Republic of). In this presentation, the following topics of photoacoustic imaging will be discussed; (1) multi-scale photoacoustic imaging systems, (2) morphological, functional, and molecular photoacoustic imaging, (3) potential clinical applications, and (4) contrast agents for photoacoustic imaging.

15:30-17:15

Presider: Boon Ooi; King

Abdullah Univ. of Science \&

Tech., Saudi Arabia

AW4B. 1 - 15:30
AW4B - Lasers and Applications

Highly Stretchable Label-like Random Laser on Universal Substrates, Yu-Ming

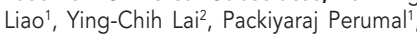
Wei-Cheng Liao', Chi-Yuan Chang', ChiShiun Liao", Shih-Yao Lin ${ }^{1}$, Yang-Fang Chen ${ }^{1}$ ${ }^{1}$ National Taiwan Univ., Taiwan; ${ }^{2}$ Dept. of Materials Science and Engineering, National Chung Hsing Univ., Taiwan. Stretchable labellike random laser can be easily transferred on any unconventional substrates, and function stably under $100 \%$ strain with many cycles. We believe our device can serve as advanced photonics modules.

AW4B.2 • 15:45

Isotope Identification with Swept-Wavelength Raman Signatures, Calvin A. Zulick ${ }^{1}$ Nagapratima Kunapareddy', Jacob Grun"; ${ }^{1}$ Naval Research Lab, USA. Swept-wavelength Raman signatures have been measured for isotopic variants of polyethylene and acetic acid. Wavenumber shifts, peak amplitude variation, and wavelength dependent amplitudes have been identified as isotope identification mechanisms

AW4B.3 • 16:00

Broadly Tunable Semiconductor Laser with Self-Imaging Three-Branch Multi-Mode Interferometer, Guan-Lin Su' ${ }^{1}$ Ming C. Wu ${ }^{1}$ UC Berkeley, USA. Using the Vernier effect and the interference between its three branches, the proposed self-imaging multimode-interferometer (MMI)-based tunable laser can access all ITU channels across $\mathrm{C}$-band with high side-mode suppression ratios (SMSRs).

\section{Executive Ballroom $210 \mathrm{C}$}

\section{CLEO: Science \& Innovations}

15:30-17:30

SW4C • Semiconductor Lasers on Silicon

Presider: Boon Ooi; King

Abdullah Univ. of Science \&

Tech., Saudi Arabia

SW4C.1 • 15:30

Optically pumped Si-based edge-emitting GeSn laser, Sattar H. Al-Kabi' Seyed Ghetmiri $^{1}$, Joe Margetis ${ }^{2}$, Thach Pham ${ }^{1,3}$, Yiyin Zhou ${ }^{1}$ Wei Dou', Wei Du1,4, Aboozar Mosleh"1, Jifeng Liu', Gregory Sun ${ }^{7}$, Richard Soref' John Tolle ${ }^{2}$, Baohua Li ${ }^{3}$, Mansour Mortazavi ${ }^{4}$ Hameed Naseem 1 , Shui-Qing Yu'; ' ${ }^{1}$ Electrical Engineering, Univ. of Arkansas, USA : ${ }^{2}$ ASM USA; ${ }^{3}$ Arktonics LLC, USA; ${ }^{4}$ Chemistry and Physics, Univ. of Arkansas, USA; ${ }^{5}$ Electrical Engineering, Arkansas Tech, USA; ${ }^{6}$ Thayer School of Engineering, Dartmouth College USA; 'Dept. of Engineering, Univ. of Mas sachuaswtts, USA. We present double heterostructure GeSn edge emitting laser. The structure was grown on a Si substrate using a commercial chemical vapor deposition with $\mathrm{GeH}_{4}$ and $\mathrm{SnCl}_{4}$. The lasing threshold of 68 $\mathrm{KW} / \mathrm{cm}^{2}$ at $10 \mathrm{~K}$ and maximum laser operating temperature of $110 \mathrm{~K}$ was achieved.

\section{SW4C.2 ・ 15:45}

InAs/InAIGaAs Quantum Dot-on-Silicon Microdisk Lasers Operating at $1.55 \mu \mathrm{m}, \mathrm{Be}$ Shi', Si Zhu' ${ }^{1}$, Qiang Li', Yating Wan', Evelyn $\mathrm{Hu}^{2}$, Kei M. Lau'; ${ }^{1}$ Hong Kong Univ. of Sci. \& Tech., Hong Kong; ${ }^{2}$ Harvard Univ., USA. InAs/ InAlGaAs quantum dot microdisk lasers were epitaxially grown on Si (001) substrates by MOCVD. CW lasing at $1544 \mathrm{~nm}$ was achieved at $4.5 \mathrm{~K}$, with a low threshold of $230 \mu \mathrm{W}$ and quality factor of 2200 .

\section{SW4C.3・16:00}

Heterogeneous silicon widely-tunable lasers with monolithically integrated highQ ring, Songtao Liu, ${ }^{1,2}$, Tin Komljenovic Michael Davenport', Erik Norberg ${ }^{3}$, Greg $\mathrm{Fish}^{3}$, Lingjuan Zhao ${ }^{2}$, Chen $\mathrm{Ji}^{2}$, John Bowers ${ }^{1}$; ${ }^{1}$ Electrical and Computer Engineering, Univ. of California, Santa Barbara, USA; ${ }^{2}$ Key Lab of Semiconductor Materials Science, Inst. of Semiconductors, CAS, China; ${ }^{3} J u n i p e r$ Networks, USA. We present preliminary results on a widely-tunable laser with monolithically integrated high-Q ring based on heterogeneous silicon integration platform. The laser exhibits $>43 \mathrm{~nm}$ tuning range with side mode suppression ratio larger than 40 $\mathrm{dB}$ in the O-band.
Executive Ballroom 210D

\section{CLEO: QELS- Fundamental Science}

15:30-17:30

FW4D • Nonlinear Optical

Sources

Presider: Govind Agrawal; Univ.

of Rochester, USA

\section{FW4D.1 1 15:30 Invited}

Harmonically Mode-Locked Quantum Cascade Lasers for Mid-Infrared Frequency Comb Generation, Marco Piccardo ${ }^{1}$, Dmitry Kazakov ${ }^{1}$ Paul Chevalier ${ }^{1}$, Tobias Mansuripur ${ }^{2}$, Feng $\mathrm{Xie}^{3}$, Kevin Lascola ${ }^{3}$, Chung-en $Z^{2}{ }^{3}$, Alexey Belyanin ${ }^{4}$, Federico Capasso 'Harvard Univ USA. '2Pendar Technologies, USA; ${ }^{3}$ Thorlabs Quantum Electronics, USA ${ }^{4}$ Texas A\&M Univ., USA. The single-mode to multimode transition in standing-wave quantum cascade lasers exhibits a harmonically mode-locked regime skipping cavity modes. The large sideband separation unravels a new mechanism for frequency comb generation seeded by the RNGH instability. ent tomography, this compression enables We describe this concept and a novel highspeed laser based on stretched-pulse mode locking (SPML).
FW4D.2 • 16:00

An ultra-narrow spectral width passively mode-locked laser, Michael Kues ${ }^{1,2}$, Christian Reimer ${ }^{1}$, Benjamin Wetzel 1,3 , Piotr Roztocki Brent Little ${ }^{4}$, Sai Chu ${ }^{5}$, Tobias Hansson", Evgeny Viktorov ${ }^{6}$, David J. Moss', Roberto Morandotti'; ${ }^{1}$ INRS-EMT, Canada; ${ }^{2}$ School of Engineering, Univ. of Glasgow, UK; ${ }^{3}$ School of Mathematical and Physical Sciences, Univ. of Sussex, UK; ${ }^{4} X_{i}$ an Inst. of Optics and Precision Mechanics, Chinese Academy of Science, China: ${ }^{5}$ Dept. of Physics and Materials Science, City Univ. of Hong Kong, Hong Kong; ${ }^{6}$ National Research Univ. of Information Technologies, Mechanics and Optics, Russia; Center for Micro-Photonics, Swinburne Univ. of Technology, Australia. We demonstrate a passively mode-locked laser emitting 4.3 nanosecond Fourier-limited pulses with record low spectral bandwidth of $104.9 \mathrm{MHz}$ (more than 100 times narrower than previous realizations), allowing its full characterization in the radio-frequency domain. 
Executive Ballroom

$210 \mathrm{E}$

\section{CLEO: QELS-Fundamental Science}

15:30-17:30

FW4E • Quantum

State Generation and Characterization

Presider: Shengwang Du;

Hong Kong Univ of Science \&

Technology, Hong Kong

FW4E.1 • 15:30

Generation and characterization of factorable biphotons with $99 \%$ spectral purity, Changchen Chen', Bo $\mathrm{CaO}^{2}$, Yuezhen Niu', Feihu $\mathrm{Xu}^{1}$, Zheshen Zhang ${ }^{1}$, Jeffrey $\mathrm{H}$ Shapiro', Franco Wong ${ }^{1} ;{ }^{1}$ MIT, USA; ${ }^{2}$ Electrical Engineering, Tohoku Univ., Japan. We generate biphotons via pulsed spontaneous parametric downconversion under extended Gaussian phase matching, and measure their joint spectral intensity at high resolution using a low-loss dispersion compensation module to obtain a $99 \%$ heralded-state spectral purity.

FW4E.2 • 15:45

Joint Spectral Intensity of $1.55 \mu \mathrm{m}$ photon-pairs generated by Si microrings, Shayan Mookherjea', Marc Savanier ${ }^{1,2}$, Nikhil Mathur'; ' 'Univ. of California San Diego, USA; '2 Luxtera, Inc., USA. The two-photon spectrum of photon pairs generated at room-temperature in optically-pumped silicon microrings was measured using tunable filters, InGaAs single-photon avalanche detectors and deconvolution, and an image-processing artifact-reduction algorithm is discussed.

FW4E.3 - 16:00 Invited

Gaussian and Non-Gaussian Highly Multimode Quantum Light, Claude Fabre ${ }^{1,2}$ Francesco Arzani ${ }^{1,2}$, Valentin Averchenko ${ }^{1,3}$, Adrien Dufour ${ }^{1,2}$, Clement Jacquard ${ }^{1,2}$, YungSik Ra ${ }^{1,2}$, Valerian Thiel ${ }^{1,4}$, Nicolas Treps ${ }^{1,2}$ 1 Universite Pierre et Marie Curie, Paris, France; ${ }^{2}$ Laboratoire Kastler Brossel, France; ${ }^{3}$ Max Planck Inst. for the Science of Light, Germany; ${ }^{4}$ Oxford Univ., UK. We show that non-linear processes pumped by ultrashort pulses of adjustable spectral shape are ideal tools to produce highly multimode quantum states in specific light modes, featuring either Gaussian squeezed, or non-Gaussian negative, Wigner functions estimation of an arbitrary force.
Executive Ballroom

210F

Executive Ballroom

210G

\section{Joint}

16:00-17:30

FW4F • Quantum States and Sensing with Optomechanical Systems

Presider: Thomas Purdy; NIST, USA

FW4F.1 1 16:00

Quantum correlations of light due to a room temperature mechanical oscillator Hendrik Schütz ${ }^{1}$, Vivishek Sudhir ${ }^{1}$, Ryan Schilling ${ }^{1}$, Sergey Fedorov', Dalziel Wilson' ${ }^{1}$ Tobias J. Kippenberg'; ${ }^{1}$ Ecole Polytechnique Federale de Lausanne, Switzerland. We observe quantum correlations imprinted on an optical beam interacting with a room temperature nanomechanical oscillator, and show how this leads to an enhancement in the relative signal-to-noise ratio for the

\section{5:30-17:30}

JW4G • Symposium on

Advances in Metaphotonic Devices II

Presider: Andrea Alu; Univ. of Texas at Austin, USA

\section{JW4G.1 • 15.30 Invited}

Extreme Platforms for Metaphotonics Nader Engheta' ${ }^{1}$ Brian E. Edwards' ${ }^{1}$ Inigo Liberal' ${ }^{1}$ Nasim Mohammadi Estakhri', Ahmed M. Mahmoud', Yaakov Lumer'; ' 'Univ. of Pennsylvania, USA. We have been investigating light-matter interaction in extremeparameter structures. We have found that metastructures with unconventional material parameters provide us with unique platforms exhibiting unprecedented classical and quantum metaphotonic features with various potential applications.
JW4G.2 • 16:00 Invited

Scalably Manufactured Metafilms for Effective Day-time Radiative Cooling, Xiaobo Yinn ${ }^{1,2}$; ${ }^{1}$ Dept. of Mechanical Engineering, Univ. of Colorado at Boulder, USA; ${ }^{2}$ Materials Science and Engineering Program, Univ. of Colorado at Boulder, USA. We demonstrate a 300-mm-wide, amorphous, organic-inorganic hybrid metamaterial manufactured on a rollto-roll extruder for effective day-time radiative cooling.
Executive Ballroom $210 \mathrm{H}$

\section{CLEO: QELS} Fundamental Science

15:30-17:30

FW4H $\bullet$ Ultrafast Optics and

Plasmonics in Nanostructures Presider: Christopher Petoukhoff; Okinawa Inst. of Science and Technol, Japan

\section{FW4H 1 15.30 Invited}

Active Upconverters for Biological Force and Field Sensing, Jennifer Dionne'; 'Stanford Univ., USA. Most upconverters suffe from low efficiencies and minimal active tunability with external forces or fields. Here, we develop novel upconverting materials that address these limitations, and utilize them for in-vivo biological force and field sensing.
FW4H.2 • 16:00

Broadband Pump-Probe Ultrafast Spectroscopy of Plasmonic Nanostructure Michael Mrejen', Uri Arieli', Assaf Levanon', Haim Suchowski'; 'School of Physics and Astronomy, Tel Aviv Univ., Israel. We experimentally measure the ultrafast spatio-temporal near-field response of a single plasmonic nanostructure over a broad spectral range, using a pump-probe setup combined with a nano-FTIR SNOM illuminated by ultrabroadband few-cycle femtosecond source. 


\section{CLEO: Science \& Innovations}

\section{5:30-17:00 \\ SW4I • Orbital Angular Momentum Based Optical Communications Presider: Francesca Parmigiani; Univ. of Southampton, UK}

\section{SW4I.1 • 15:30}

Demonstration of Hybrid Orbital Angular Momentum (OAM) and Gaussian Mode Encoding/Decoding for 10-Gbit/s Data Transmission through a $2.6-\mathrm{km}$ Conventional Graded-Index Multimode (OM3) Fiber, Long Zhu' ${ }^{1}$, Jian Wang ${ }^{1}$, Andong Wang ${ }^{1}$. 'Wuhan National Lab for Optoelectronics, China. We experimentally demonstrate hybrid orbital angular momentum (OAM) Mode and Gaussian mode encoding/decoding for 10-Gbit/s data transmission through a 2.6$\mathrm{km}$ conventional graded-index multimode Fiber (OM3), and achieve bit-error rate (BER) below 3.8e-3.

\section{SW4I.2 • 15:45}

Parasitic Effect of TE and TM modes in OAM-MDM Transmission Systems, Reza Mirzaei Nejad', Lixian Wang ${ }^{1}$, Jiachuan Lin ${ }^{1}$ Sophie LaRochelle ${ }^{1}$, Leslie Rusch ${ }^{1} ;{ }^{1}$ Universite Laval, Canada. We characterize an OAMMDM link by measuring the channel impulse response using two OAM fiber designs investigating the impact of $\mathrm{TE}_{01}, \mathrm{TM}_{01}$ modes on receiver performance and required equalizer taps in separate mode detection schemes.
15:30-17:30

SW4J • Precision Spectroscopy

Presider: lan Coddington; NIST, USA

15:30-17:30

SW4K・2D Materials \& Devices I

Presider: Thomas Murphy; Univ. of Maryland at College Park, USA

SW4J.1 1 15:30

Terahertz dual-comb spectroscopy with a free-running, dual-wavelength-comb fiber laser, Guoging $\mathrm{Hu}^{3,1}$, Tatsuya Mizuguchi ${ }^{1}$, Xin Zhao $^{3}$, Takeo Minamikawa ${ }^{1,2}$, Ting Li ${ }^{3}$, Zheng Zheng ${ }^{3,4}$, Takeshi. Yasuil,2, ' $T$ Tokushima Univ., Japan: ${ }^{2}$ JST, ERATO MINOSHIMA Intelligent Optical Synthesizer (IOS), Japan; ${ }^{3}$ Beihang Univ., China; ${ }^{4}$ Collaborative Innovation Center of Geospatial Technology, China. $\mathrm{THz}$ dual-comb spectroscopy using a freerunning, dual-wavelength-comb fiber laser is demonstrated for highly cost-effective and compact $\mathrm{THz}$ spectroscopy systems while maintaining its high spectroscopic performance.

\section{SW4K.1 $15: 30$}

Long term stable black phosphorus saturable absorber for mode-locked fiber laser Xinxin Jin' ${ }^{1}$ Guohua $\mathrm{Hu}^{2}$, Meng Zhang ${ }^{1}$, Yuwei $\mathrm{Hu}^{1}$, T Albrow-Owen², R Howe ${ }^{2}$, Tien-Chun $\mathrm{Wu}^{2}$, Xuekun Zhu', Zheng Zheng', Tawfique Hasan'; 'Beihang Univ., China: ${ }^{2}$ Univ. of Cambridge, UK. We demonstrate a long-term stable fiber laser mode-locked by a solution deposited and encapsulated black phosphorus (BP) saturable absorber. Our work highlights the potential of BP-based devices for photonic applications when operating under ambient conditions.
SW4J.2 $\bullet 15: 45$

A Carrier Offset Tunable, Highly Integrated, Fiber-Based Comb in the $7-10$ $\mu \mathrm{m}$ Range via DFG in OP-GaP, Kevin F. Lee ${ }^{1}$, Christopher J. Hensley', P. G. Schunemann², Martin E. Fermann'; 'IMRA America, Inc., USA; ${ }^{2} B A E$ Systems, USA. We demonstrate a carrier offset frequency tunable comb producing $25 \mathrm{~mW}$ in the midinfrared via DFG in OP-GaP between Er and Tm fiber amplifiers seeded with a common Er fiber comb.

\section{SW4K.2 • 15:45}

Broadband Third-Harmonic Generation in Black Phosphorus, Yigit Aytac', Martin Mittendorff', Thomas E. Murphy'; 'Univ. of Maryland, USA. We measure third-harmonic generation in mechanically exfoliated black phosphorus flakes using a tunable ultrafast infrared laser. Visible microscopy of the optically pumped flake shows third-harmonic generation in transmission that is localized to the black phosphorus flake.

\section{SW41.3 - 16:00 Invited}

3.36-Tbit/s OAM and Wavelength Multiplexed Transmission over an InverseParabolic Graded Index Fiber, Jiangbo Zhu', Xuyang Wang', Shuangyi Yan', Yanni $\mathrm{Ou}^{1}$, Ziyang $\mathrm{Hu}^{1}$, Younès Messaddeq ${ }^{2}$ Sophie LaRochelle², Leslie Rusch², Dimitra Simeonidou', Siyuan Yu'; ' 'Univ. of Bristol, UK; 'Universite Laval, Canada. We demonstrate MIMO-free two-dimensional multiplexing, transmission and de-multiplexing over 4 OAM modes (including two modes of $\| \mid=$ 2) and 15 wavelengths through 100-meter inverse-parabolic graded-index fiber with aggregated total capacity of 3.36-Tbit/s.
SW4J $3 \cdot 16: 00$

Widely Tunable Mid-IR, High Signal-toNoise Frequency Comb based Fourier Transform Spectrometer, Vinicius Silva de Oliveira ${ }^{1}$, Axel Ruehl ${ }^{1}$, Piotr Maslowski ${ }^{2}$, Ingmar Hartl'; ${ }^{1}$ Deutsches ElektronenSynchrotron, Germany; ${ }^{2}$ Inst. of Physics, Nicolaus Copernicus Univ., Poland. We present an optical frequency comb based Fourier Transform Spectrometer, covering 3.0 to $5.2 \mu \mathrm{m}$ with $2 \mathrm{~m}$ optical path delay and a signal-to-noise ratio of 800 in $40 \mathrm{~s}$ acquisition time at $4.6 \mu \mathrm{m}$.

\section{SW4K.3 • 16:00 Invited}

Nanoscale Nonlinear Optics with Lowdimensional Nanomaterials, Zhipei Sun ${ }^{1}$. ${ }^{1}$ Dept. of Micro- and Nanosciences, Aalto Univ., Finland. I will discuss our recent nanoscale nonlinear optic experiments (e.g. quantum emitters, wavelength converters, ultrafast lasers) with one-dimensional (e.g., carbon nanotubes), two-dimensional (e.g., graphene, MoS 2 , phosphorene) nanomaterials and their hybrid structures (e.g., heterostructures, plasmonic structures, silicon/fibre integrated structures).
15:30-17:30

SW4L - Standoff and Remote Sensing

Presider: Adam Fleisher; NIST, USA

\section{SW4L.1 • 15:30 Tutorial}

Lidar Instruments and Applications, Joseph Shaw': 'Montana State Univ., USA. This tutorial will review the basic design of LIDAR systems for elastic and inelastic scattering in remote sensing applications ranging from airborne mapping of fish in lakes to differential-absorption measurements of gas concentration in the atmosphere.

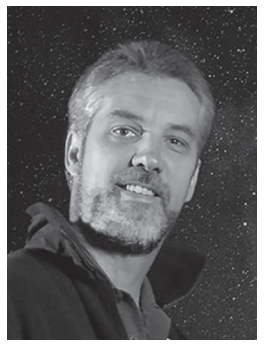

Joseph Shaw is the director of the Optical Technology Center and professor of electrical engineering, physics, and optics and photonics at Montana State University in Bozeman Montana. He has been developing optical and infrared remote sensing instruments for nearly three decades for a wide range of applications ranging from measuring atmospheric clouds and aerosols to mapping insects and fish. He is a Fellow of the OSA and SPIE. 


$\begin{array}{llc}\text { Marriott } & \text { Marriott } & \text { Marriott } \\ \text { Salon III } & \text { Salon IV } & \text { Salon V \& VI }\end{array}$

\section{CLEO: Science \& Innovations}

15:30-17:30

SW4M • Concepts \& Advances

in Quasi-phase Matching

Presider: Irina Sorokina; Norges

Teknisk Naturvitenskapelige Univ., Norway

\section{SW4M.1 • 15:30}

Simultaneous Second and Fourth Harmonic Generation of a Carbon dioxide laser in a single Orientation-Patterned Gallium Phosphide Crystal, Shekhar Guha', Joel Murray ${ }^{2}$, Jean Wei ${ }^{3}$, Jacob Barnes ${ }^{4}$, P. G. Schunemann ${ }^{5}$ ' ${ }^{1}$ US Air Force Research Lab, USA; ${ }^{2}$ UES, Inc., USA; ${ }^{5}$ BAE Systems, USA. First demonstration of simultaneous frequency doubling and quadrupling of a pulsed carbon-dioxide laser in a single-grating orientation-patterned Gallium Phosphide crystal grown by hydride vapor phase epitaxy is reported.
SW4M.2 • 15:45

Mid-Infrared Picosecond Difference Frequency Generation in OrientationPatterned Gallium Phosphide, Josep C. Casals ${ }^{1}$, Shahrzad Parsa ${ }^{1}$, Chaitanya Kumar Suddapalli ${ }^{1,2}$, Kavita Devi', P. G. Schunemann ${ }^{3}$, Maijid Ebrahim-Zadeh",4: ${ }^{1} /$ CFO-The Inst. of Photonic Sciences, Spain; ${ }^{2}$ Radiantis, Spain; ${ }^{3} B A E$ Systems, Incorporated, USA; ${ }^{4}$ Institucio Catalana de Recerca i Estudis Avancats (ICREA), Spain. We report the first picosecond mid-infrared source based on OP-GaP using single-pass DFG, providing tunable radiation across $3040-3132 \mathrm{~nm}$, with $57 \mathrm{~mW}$ of average-power at $3044 \mathrm{~nm}, 3.2 \%$ rms stability over $1 \mathrm{~h}$, in good beam-quality.

SW4M.3 • 16:00

Temperature Dependent Sellmeier Equation for the Refractive Index of GaP, Shekhar Guha', Jean $\mathrm{Wei}^{3}$, Joel Murray ${ }^{2}$, Jacob Barnes $^{2}$, P. G. Schunemann ${ }^{4}$, Douglas Krein ${ }^{5}$; 'US Air Force Research Lab, USA; ${ }^{2}$ UES, Inc., USA; ${ }^{3}$ UES, Inc, USA; ${ }^{4}$ BAE Systems, USA; ${ }^{5} \mathrm{GDIT}$, USA. The first temperature-dependent Sellmeier equation for $\mathrm{GaP}$ was fit to extensive refractive index data measured on thin $\mathrm{GaP}$ plates for wavelengths between 1 and 12 microns over a temperature range of 200 to $450 \mathrm{~K}$
15:30-17:30

SW4N • Microresonator

Frequency Comb

Presider: Takasumi Tanabe; Keio

Univ., Japan

SW4N.1 $15: 30$

Experimental Demonstration of Dual-Comb Generation by XPM Between Two Polarization States in a Microresonator, Changjing $\mathrm{Bao}^{1}$, Peicheng Liao ${ }^{1}$, Arne Kordts ${ }^{2}$, Lin Zhang $^{3}$, Maxim Karpov' ${ }^{2}$ Martin Pfeiffer ${ }^{2}$, Andrey Matsko ${ }^{4}$, Guodong Xie ${ }^{1}$, Yinwen Cao ${ }^{1}$, Yan Yan', Ahmed Almaiman', Morteza Ziyadi', Amirhossein Mohajerin-Ariaei ${ }^{1}$, Ahmad Fallahpour ${ }^{1}$, Fatemeh Alishahi ${ }^{1}$, Moshe Tur ${ }^{5}$, Lute Maleki ${ }^{4}$, Tobias J. Kippenberg ${ }^{2}$, Alan E. Willner'; ' Univ. of Southern California, USA; ${ }^{2}$ Ecole Polytechnique Federale de Lausanne (EPFL) Lausanne, Switzerland; ${ }^{3}$ School of Precision Instrument and Opto-electronics Engineering, Tianjin Univ., China; ${ }^{4}$ OEwaves Inc., USA; ${ }^{5}$ School of Electrical Engineering, Tel Aviv Univ., Israel. We demonstrate dual-comb generation by XPM between TE polarized continuous wave and TM polarized soliton pulse. A TE polarized light with only $0.9 \mathrm{~mW}$ power could excite a comb with the assistance of a soliton.

SW4N.2 • 15:45

Single mode dispersive waves and soliton microcomb dynamics, $\mathrm{Xu} \mathrm{Yi}{ }^{1}$, Qifan Yang ${ }^{1}$, Xueyue Zhang', Ki Y. Yang', Kerry Vahala ${ }^{1} ;{ }^{1}$ California Inst. of Technology, USA. Dispersive-wave scattering from dissipative Kerr solitons is induced by spatialmode interactions within a high-Q micro-resonator. A limiting case, single-mode dispersive waves, are observed and their interaction with the soliton causes hysteretic behavior.

\section{SW4N.3 • 16:00 Invited}

Optical Frequency Synthesis Using a DualKerr-Microresonator Frequency Comb, Travis C. Briles'1, Tara Drake', Daryl Spencer', Jordan R. Stone ${ }^{1}$, Connor Fredrick', Qing Li ${ }^{2}$, Daron Westly², B. R. Ilic ${ }^{2}$, Xu Yi ${ }^{3}$, Ki Y. Yang ${ }^{3}$, Kerry Vahala ${ }^{3}$, Kartik Srinivasan ${ }^{2}$, Scott Diddams', Scott Papp'; 'Time and Frequency, NIST, USA; ${ }^{2}$ Center for Nanoscale Science and Technology, NIST, USA: ${ }^{3}$ California Inst. of Technology, USA. We report a photonicchip optical-frequency synthesizer using interlocking $1 \mathrm{THz}$ and $22 \mathrm{GHz}$ Kerr microcombs, which provide $f-2 f$ self-referencing and a dense comb spanning the C-band, respectively. We demonstrate the optical synthesizer with $\mathrm{Hz}$ resolution.
15:30-17:30

SW4O • Laser Induced

Excitations in Matter

Presider: Emmanuel Haro-

Poniatowski; UAM-Iztapalapa,

Mexico

SW40.1 • 15:30

Laser-based Noble-gas Metastable Excitation Techniques with Application to Atom Trap Trace Analysis, Philip S. Light ${ }^{1}$, Milad A. Dakka', Rohan Glover'2, Robert Sang ${ }^{2}$, Andre N. Luiten'; 'School of Physical Sciences, Univ of Adelaide Australia: ' ${ }^{2}$ riffith Univ. Australia. Atom-trap trace analysis (ATTA) is an ultra-sensitive technique for measurement of noble-gas radio-nuclide ratios at the $10^{-17}$ level, with application to environmental science. We explore laser-based metastable excitation techniques to improve measurement efficiency, accuracy and speed.

SW40.2 • 15:45

Generation of sub-THz surface wave on a metal wire by intense laser interaction with a foil target, Kensuke Teramoto ${ }^{1}$, Shunsuke Inoue ${ }^{1}$, Shigeki Tokita ${ }^{2}$, Ryo Yasuhara $^{3}$, Takeshi Nagashima ${ }^{4}$, Yoshihide Nakamiya ${ }^{1}$ Kazuaki Mori', Masaki Hashida ${ }^{1}$ Shuji Sakabe'; ${ }^{1}$ Kyoto Univ., Japan; ${ }^{2}$ Osaka Univ., Japan; ${ }^{3}$ National Inst. for Fusion Science, Japan; ${ }^{4}$ Setsunan Univ., Japan. It has been demonstrated that the electric field associated with electrons generated by an intense femtosecond laser pulse in a foil can induce intense sub-THz surface wave on a wire waveguide adjacent to the foil.

SW40.3 • 16:00

Pulse-induced permanent group-velocity matching in a dual-core $\mathrm{As}_{2} \mathrm{Se}_{3} / \mathrm{PMMA}$ fiber, Chams Baker', Song Gao', Liang Chen', Xiaoyi Bao'; 'Univ. of Ottawa, Canada. We report that transmission of pulses in tapered dual-core $\mathrm{As}_{2} \mathrm{Se}_{3}$-PMMA fibers induces permanent effective group-velocity matching between the field propagating in the fiber. 
Executive Ballroom

210A

Executive Ballroom

210B

\section{CLEO: Applications \\ \& Technology}

AW4A • Biomedical Imaging IContinued

\author{
AW4B - Lasers and \\ Applications-Continued
}

AW4B.4 1 16:15

Pulsed-Laser Induced Rayleigh-Taylor Instabilities of Ultrathin Metal Films Inside Homogeneous Liquid Mixtures, Ramki Kaly anaraman 2,1 Venkatanarayana Prasad P. Sandireddy', Sagar p. Yadavali'; ' ${ }^{1}$ Chemical and Biomolecular Eng., Univ. of Tennessee, USA ${ }^{2}$ Dept. of Materials Science and Engineering Univ. of Tennessee, USA. Nanosecond pulsed laser melting of ultrathin gold films immersed inside glycerol-water mixtures assemble into monomodal sized nanoparticles with small spacing. This is a result of the large vapo pressure gradient created at the metal-fluid interface.

AW4A.3 • 16:30

High-Sensitivity Contrast-Enhanced in vivo Imaging with Optical Coherence Tomography (OCT), Orly Liba ${ }^{1,2}$, Elliott SoRelle ${ }^{3,2}$, Debasish $\mathrm{Sen}^{2}$, Adam de la Zerdaa,4; ${ }^{1}$ Electrical Engineering, Stanford Univ., USA; ${ }^{2}$ Structural Biology, Stanford Univ., USA; ${ }^{3}$ Biophysics, Stanford Univ., USA; ${ }^{4}$ Molecular Imaging Program, Stanford Univ., USA. We developed custom spectral detection algorithms and highly-scattering large gold nanorods for sub-nanomolar sensitivity contrast-enhanced optical coherence tomography (OCT). We used this approach for noninvasive 3D imag-
AW4B.5 $\bullet 16: 30$

Integration of Ultrafast Laser-inscribed Optical Waveguides and Renewable Ring Lasers, Hengky Chandrahalim', Stephen C. Rand' ${ }^{1}$ Xudong Fan' ${ }^{1}$ ' Univ. of Michigan, USA We demonstrated the monolithic integration of renewable and wavelength reconfigurable ring lasers and waveguides of arbitrary shapes. This work enables reconfigurable optical devices for on-chip lasers, flexible optical processing, and the investigation of new optical phenomena.
AW4B.6 6 16:45

Novel Long-Period Fiber Gratings: Fabrication and Sensing Applications, Liyong Ren ${ }^{1}$, Kaili Ren ${ }^{1}$, Xudong Kong ${ }^{1}$, Jian Liang ${ }^{1}$ Haijuan $\mathrm{Ju}^{1}$, Zhaoxin $\mathrm{Wu}^{2}{ }^{2}{ }^{1} \mathrm{Xi}^{\prime}$ an Inst. of Opt. \& Precision Mech., China; ${ }^{2} \mathrm{Xi}^{\prime}$ an Jiaotong Univ., China. We presented two
Executive Ballroom $210 \mathrm{C}$

\section{CLEO: Science \&} Innovations

SW4C • Semiconductor Lasers on Silicon-Continued

SW4C.4 • 16:15

Difference-Frequency Generation Quantum Cascade Laser Sources on Silicon, Seungyong Jung ${ }^{1}$, Jae Hyun Kim ${ }^{1}$, Yifan Jiang ${ }^{1}$, Karun Vijayraghavan ${ }^{1}$, Mikhail A Belkin'; ' ${ }^{1}$ niv. of Texas at Austin, USA. We demonstrate that a heterogeneous integration of Cherenkov terahertz quantum cascade laser sources on a high-resistive silicon substrate enables 5 times improvement in terahertz power compared to that of devices on a native $\ln P$ substrate.

SW4C.5 $\bullet 16: 30$

A $20 \mathrm{GHz}$ colliding pulse mode-locked heterogeneous InP-silicon laser, Songtao Liu $^{1,2}$, Michael Davenport ${ }^{1}$, John Bowers ${ }^{1}$ 'Electrical and Computer Engineering, Univ. of California, Santa Barbara, USA; ${ }^{2}$ Key Lab of Semiconductor Materials Science, Inst. of Semiconductors, CAS, China. We demonstrate a colliding pulse mode-locked laser based on heterogeneous InP-silicon platform, working in the passive mode locking regime with a $20 \mathrm{GHz}$ repetition rate. The lase outputs nearly transform limited pulses with record narrow pulse width of $1.37 \mathrm{ps}$ on the silicon platform novel schemes for fabricating micro-tapered long-period fiber gratings (LPFGs) and helica LPFGs, respectively, by periodically tapering and by directly twisting single mode fibers. Superior sensing characteristics of them are also demonstrated experimentally.
SW4C.6 $16: 45$

Differential Frequency Tunable Dual-Mode Heterogeneous QD Laser with Si PIC Atsushi Matsumoto ${ }^{1}$, Toshimasa Umezawa ${ }^{1}$ Kouichi Akahane', Naokatsu Yamamoto' Hirohito Yamada ${ }^{2}$, Tomohiro Kita2. ${ }^{1}$ NICT, Japan: ${ }^{2}$ Tohoku Univ., Japan. We proposed a tunable dual-mode heterogeneous quantum dot laser diode with a Si-photonics-based photonic integrated circuit, and successfully demonstrated dual-mode lasing oscillation by tuning the differential frequency from approximately $20 \mathrm{GHz}$ to $200 \mathrm{GHz}$.

Executive Ballroom 210D

CLEO: QELSFundamental Science

FW4D • Nonlinear Optical Sources-Continued

\section{FW4D.3 • 16:15}

Yb:YAG regenerative thin-disk amplifiers as an ideal pump and seed source for OPCPA Joerg Neuhaus ${ }^{1}$, Florian Fink', Gregor Hehl' ${ }^{1}$ Mikhail Larionov', Robert Riedel², Michael Schulz2: ${ }^{1}$ Dausinger + Giesen GmbH, Germany; ${ }^{2}$ Class 5 Photonics GmbH, Germany. We present experimental and theoretical results for parallel amplification of $350 \mathrm{fs}$ and $2.7 \mathrm{ps}$ in the same Yb:YAG regenerative thin-disk amplifier for high energy OPCPA pumping and stable supercontinuum generation for OPCPA seeding.

FW4D.4 • 16:30

Narrowband Terahertz Generation with Broadband Chirped Pulse Trains in Periodically Poled Lithium Niobate, Spencer W. Jolly ${ }^{1,2}$, Frederike Ahr ${ }^{3,4}$, Nicholas H. Matlis ${ }^{3}$, Sergio Carbajo ${ }^{3}$, Koustuban Ravi ${ }^{3,5}$, Tobias Kroh $^{3}$, Jan Schulte ${ }^{3}$, Damian N. Schimpf ${ }^{3}$ Andreas R. Maier ${ }^{2}$, Franz X. Kärtner ${ }^{3,5}$; ' ${ }^{1} \mathrm{ELI}$ Beamlines project, Institue of Physics of the ASCR, Czech Republic; ${ }^{2}$ Center for Free Electron Laser Science \& Dept. of Physics, Univ. of Hamburg, Germany; ${ }^{3}$ Center for Free-Electron Laser Science, Deutsches Elektronen Synchrotron (DESY), Germany; ${ }^{4}$ Dept of Physics, Univ. of Hamburg, Germany ${ }^{5}$ Research Lab of Electronics, MIT, USA. We generate narrowband terahertz radiation in periodically poled lithium niobate crystals using a train of chirped-and-delayed pulses from a broadband high energy Ti:sapphire laser. We achieve a multi-cycle $\mathrm{THz}$ energy of $40 \mu \mathrm{J}$ at $0.544 \mathrm{THz}$.

\section{FW4D.5 • 16:45}

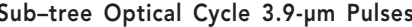
Through Hollow-Core-Waveguide Compression, Tadas Balciunas ${ }^{1}$, Guangyu Fan ${ }^{1}$ Skirmantas Alisauskas', Valentina Shumakova ${ }^{1}$, Alexander Mitrofanov ${ }^{2}$, Dmitriy Sidorov-Biryukov², Aleksei Zheltikov² Bruno E. Schmidt ${ }^{3}$, Francois Legare ${ }^{4}$, Andrius Baltuska'; 'Vienna Univ. of Technology, Austria; ${ }^{2}$ Moscow State Univ., Russia; ${ }^{3}$ few-cycle, Canada; ${ }^{4}$ Inst. National de la Recherche Scientifique, Canada. Sub-three-optical-cycle $3.9-\mu \mathrm{m},>13 \mathrm{~mJ}$ pulses are generated via spectral broadening in a noble-gas-filled capillary and subsequent dispersion compensation in bulk. The use of large, 1-mm-diameter capillary reduces losses and prevents unwanted ionization. 
Executive Ballroom $210 \mathrm{E}$

Executive Ballroom 210F

\section{CLEO: QELS-Fundamental Science}

FW4E • Quantum

State Generation and Characterization-Continued Systems-Continued
FW4F • Quantum States and Sensing with Optomechanical

FW4F.2 $\bullet$ 16:15

Torsional optomechanics and quantum simulation with a levitated nanodiamond Tongcang Li', Thai Hoang ${ }^{1}$, Yue Ma ${ }^{2}$, Ming Gong $^{3}$, Jonghoon Ahn', Jaehoon Bang ${ }^{1}$ Zhang-Oi Yin'2: 'Purdue Univ., USA; ${ }^{2}$ Tsinghua Univ., China; ${ }^{3}$ Univ. of Science and Technology of China, China. We report the observation of the torsional vibration of an optically levitated nanodiamond in vacuum. We propose a scheme to achieve torsiona ground state cooling, and utilize the electron spin-torsional coupling to do quantum simulation.

FW4E.4 • 16:30

Pulsed Quantum Frequency Combs from an Actively Mode-Locked Intra-Cavity Generation Scheme, Piotr Roztocki', Michael Kues ${ }^{1,2}$ Christian Reimer ${ }^{1}$, Benjamin Wetzel 1,3 , Brent Little $^{4}$, Sai Chu ${ }^{5}$, David J. Moss ${ }^{1,6}$, Roberto Morandotti ${ }^{1,7} ;{ }^{1}$ INRS-EMT, Canada; ${ }^{2}$ Univ. of Glasgow, UK; ${ }^{3}$ Univ of Sussex, UK: ${ }^{4} \mathrm{Xi}^{\prime}$ an Inst. of Optics and Precision Mechanics of CAS, China; ${ }^{5}$ City Univ. of Hong Kong, China; ${ }^{6}$ Swinburne Univ. of Technology, Australia; ${ }^{7}$ National Research Univ. of Information Tech nologies, Russia. We introduce an intra-cavity actively mode-locked excitation scheme for nonlinear microring resonators that removes the need for external laser excitation in the generation of pulsed two-photon frequency combs.
Executive Ballroom

210G

Joint

JW4G • Symposium on Advances in Metaphotonic Devices II-Continued
FW4F.3 • 16:30

A Near-Unity Efficiency Source of Entangled Surface Phonon Polaritons, Nicholas Rivera ${ }^{1}$, Ido Kaminer ${ }^{1}$, Marin Soljacic ${ }^{1} ;{ }^{1}$ MIT, USA. We show that mid-IR polaritons in polar dielectrics can be used to develop an atomic emitter of photon pairs at quantum efficiencies over $90 \%$, suggesting a new route towards the production of entangled light.

\section{FW4F.4 1 16:45}

Single Atom Sub Atto-Newton Force Sensor in Three-Dimensions, Erik W. Streed ${ }^{1}$, Valdis Blums ${ }^{1}$, Marcin Piotrowski ${ }^{1,2}$ Mohammed I. Hussain', Benjamin Norton' Steven Connell ${ }^{1,2}$, Steven Gensemer ${ }^{1,2}$, Mirko Lobino'; 'Griffith Univ., Australia; ${ }^{2} \mathrm{CSIRO}$ Australia. Ultra-sensitive force measurements are crucial for physics. Nanometer precision displacement measurements of a Pau trapped ${ }^{174} \mathrm{Yb}^{+}$ion provides force sensitivities below aN per $\sqrt{ } \mathrm{Hz}$. Accuracy was verified by measuring the $95 \mathrm{zN}$ cooling laser light force pressure.
JW4G.3 • 16:30 Invited

Low-Threshold Surface-Plasmon-Polariton Laser Pumped by Surface Plasmon Polaritons, Wengi Zhu ${ }^{1}$, Cheng Zhang ${ }^{1}$, Ting $\mathrm{Xu}^{1}$, Amit K. Agrawal' ${ }^{1}$, Henri J. Lezec'; ; ${ }^{1}$ NIST, USA, USA. We demonstrate a narrow-linewidth, low-threshold surface-plasmon-polariton (SPP) laser based on a low-loss open cavity resonator leveraging grating-coupled SPPS to pump the lasing SPPs with strong spatia overlap and minimum perturbation.
Executive Ballroom $210 \mathrm{H}$

\section{CLEO: QELS-} Fundamental Science

FW4H $\bullet$ Ultrafast Optics and Plasmonics in NanostructuresContinued

\section{FW4H.3 $16: 15$}

Spin Contrast of Purcell-Enhanced Nitrogen-Vacancy Centers in Diamond, Simeon Bogdanov', Mikhail Y. Shalaginov', Alexey V. Akimov'2, Alexei Lagutchev', Jing Liư ${ }^{3}$, Dewan Woods ${ }^{1}$, Marcello Ferrera ${ }^{4}$, Polina Kapitanova ${ }^{5}$, Pavel Belov ${ }^{5}$, Joseph Irudayaraj ${ }^{1}$ Alexandra Boltasseva' ${ }^{1}$, Vladimir M. Shalaev 'Purdue Univ., USA; ${ }^{2}$ Texas A\&M Univ., USA ${ }^{3}$ South Dakota School of Mines \& Technology, USA; ${ }^{4}$ Heriott Watt Univ., UK; ${ }^{5}$ ITMO Univ., Russia. A novel method for measuring the spin contrast in large nitrogen-vacancy ensembles in diamond is introduced. We use this method to study how the photonic density of states must be engineered in order to minimize the uncertainty of spin readout for nanoscale sensing applications.

FW4H.4 • 16:30

Lifetime Shortening and Photoluminescence Emission Enhancement of Single CdSe/ CdS/PMMA Quantum Emitters Coupled to Plasmonic Bullseye Resonators, Florian Werschler ${ }^{1}$, Benjamin Lindner ${ }^{1}$, Christopher Hinz', Tjaard de Roo', Stefan Mecking ${ }^{1}$, De nis Seletskiy', Alfred Leitenstorfer'; ' 'Univ. of Konstanz, Germany. An order-of-magnitude enhancement in the radiative recombination rate is observed in single $\mathrm{CdSe} / \mathrm{CdS} / \mathrm{PMMA}$ colloidal quantum dots when coupled to gold plasmonic resonators, consistent with the measured increase in the excitonic photoluminescence signal.
Quantum State Tomography with a Single Observable, Dikla Oren ${ }^{1}$, Maor ogy, Israel; ${ }^{2}$ Electrical Engineering, Technion from a single observable, corresponding single setup, by adding a known anc state, introducing mixing between degrees of freedom, and utilizing structure in the states.
FW4H.5 • 16:45

Polarization-dependent interference of coherent scattering from orthogonal dipole moments of a resonantly excited quantum dot, Disheng Chen', Gary Lander ${ }^{1}$, Glenn S Solomon ${ }^{2,3}$, Edward Flagg'; 'West Virginia Uiversity, USA; ${ }^{2}$ Univeristy of Maryland, USA ${ }^{3}$ NIST, USA. Interference between coherent scattering from the two fine structure split exciton states in a neutral InGaAs quantum dot causes an unconventional excitation line shape. Analysis allows the extraction of steady-state coherence between the exciton states 


\section{CLEO: Science \& Innovations}

\section{SW4I • Orbital Angular Momentum Based Optical Communications-Continued}

\author{
SW4J • Precision \\ Spectroscopy-Continued
}

SW4J.4 1 16:15

Line-shapes and intensities of carbon monoxide transitions in the $(3 \leftarrow 0)$ and $(4 \leftarrow 1)$ bands, Zachary D. Reed ${ }^{1}$, Joseph T. Hodges', Oleg Polyansky ${ }^{2} ;{ }^{1}$ NIST, USA; ${ }^{2}$ Univ. College London, UK. We present line shape parameters and intensities of air-broadened $\mathrm{CO}(3 \leftarrow 0)$ and $(4 \leftarrow 1)$ band transitions using frequency-stabilized cavity ringdown spectroscopy at room temperature. Measurements are compared to ab initio calculations.

\section{SW4I.4 16:30 Invited}

Experimental Demonstration of an OrbitalAngular-Momentum Encoded Quantum Communication Link Co-propagating with a Classical Channel, Yongxiong Ren', Cong Liu', Kai Pang', Jiapeng Zhao², Yinwen Cao', Guodong Xie', Long Li', Zhe Zhao', Zhe Wang ${ }^{1}$, Moshe Tur ${ }^{3}$, Robert Boyd ${ }^{2}$, Alan E. Willner'; ' Univ. of Southern California, USA; 2Dept. of Physics and Astronomy, The Inst. of Optics, Univ. of Rochester, Rochester, USA; ${ }^{3} \mathrm{Tel}$ Aviv Univ., Israel. We experimentally demonstrate an OAM-based quantum communication link populated by a classical channel. OAM enables an up to 100-Mbit/s quantum data rate as well as an additional 18.5- $\mathrm{dB}$ separation between the quantum and classical channels.

\section{SW4J.5 • 16:30 Invited}

Optical Frequency Comb Spectroscopy for Gas Metrology and Trace Gas Detection, Piotr Maslowski', Grzegorz Kowzan' Dominik Charczun', Daniel Lisak', Ryszard Trawinski ${ }^{1}$, Lucile Rutkowski², Alexandra C. Johansson², Amir Khodabakhsh², Aleksandra Foltynowicz ${ }^{2}$, Kevin F. Lee ${ }^{3}$, Martin E. Fermann ${ }^{3} ;{ }^{1}$ Nicolaus Copernicus Univ., Faculty of Physics, Astronomy and Informatics, Nicolaus Copernicus Univ., Poland; ${ }^{2}$ Dept. of Physics, Umeå Univ., Sweden; ${ }^{3}$ MRA America, Inc. USA. We report recent developments of comb-based broadband absorption spectroscopy. The comb-line resolving approaches and Fourier transform spectroscopy with subnominal resolution overcome the frequency resolution limits of conventional techniques. Advantages for various applications will be discussed.

\section{SW4K・ 2D Materials \& \\ Devices I-Continued}

SW4L - Standoff and Remote Sensing-Continued
SW4K.4 • 16:30

Near Infrared Emission from Defect States of Atomically Thin Phosphorene, Shahriar Aghaeimeibodi', Jehyung Kim', Edo Waks ${ }^{1}$; ${ }^{1}$ Univ. of Maryland, USA. We demonstrate a new class of near infrared localized defects in few layer phosphorene. This work highlights the significance of defect states of phosphorene for near infrared optoelectronic applications.

SW4K.5 $\bullet 16: 45$

Graphene Flakes Controlled by Magnetic Fields for a Display Application, Chao $\mathrm{Niu}^{1}$ Feng Lin ${ }^{2,4}$, Zhuan Zhu ${ }^{2}$, Xufeng Zhou ${ }^{3}$ Zhaoping Liu $^{3}$, Zhiming Wang ${ }^{4}$, Jiming $\mathrm{Bao}^{2,4}$, Jonathan $\mathrm{Hu}^{1}$; 'Baylor Univ., USA; ${ }^{2}$ Univ. of Houston, USA; ${ }^{3}$ Chinese Academy of Sciences, China; ${ }^{4}$ Univ. of Electronic Science and Technology of China, China. We study optical transmission and reflection of graphene flakes suspended in liquid with external magnetic fields in both experiment and simulation. We show that graphene flakes controlled by magnetic fields can be used for display.
SW4L.2 • 16:30

Nonmechanical Beam Steering Using Tunable Lenses, Mo Zohrabi ${ }^{1}$ Robert $\mathrm{H}$. Cormack', Juliet T. Gopinath'; ' 'Dept. of Electrical, Computer, and Energy Engineering, Univ. of Colorado, USA. We have used three tunable liquid lenses to demonstrate nonmechanical beam steering of $\pm 75^{\circ}$ using a fisheye lens, in two dimensions. The system can control the beam divergence and provide two-dimensional beam steering.

\section{SW4L.3 • 16:45}

Random Access Optical Scanning Using a MEMS Phased Array, Stephen Hamann 1 Ryosuke Itoh ${ }^{2}$, Lars Eng ${ }^{3}$, Jim Hunter ${ }^{3}$, Alexander Payne ${ }^{3}$, Olav Solgaard'; ' ${ }^{2}$ electrical Engineering, Stanford, USA; ${ }^{2} R \& D, S C R E E N$ Holdings, Japan; ${ }^{3}$ Silicon Light Machines, USA. We introduce a 1088 element MEMS phased array capable of random access scanning, such that any state can be accessed at high speed. The scanning characteristics of the phased array are demonstrated. 


$\begin{array}{llc}\text { Marriott } & \text { Marriott } & \text { Marriott } \\ \text { Salon III } & \text { Salon IV } & \text { Salon V \& VI }\end{array}$

\section{CLEO: Science \& Innovations}

SW4M • Concepts \& Advances in Quasi-phase MatchingContinued

\section{SW4M.4 $16: 15$}

Highly-Efficient Cascaded Mirrorless OPO in Sub- $\mu \mathrm{m}$ Periodically Poled RKTP Crystals, Andrius Zukauskas', Charlotte Liljestrand', Anne-Lise Viotti', Valdas Pasiskevicius ${ }^{1}$, Carlota Canalias ${ }^{1}{ }^{1}$ Kungliga Tekniska Hogskolan, Sweden. We demonstrate low threshold mirrorless optical parametric oscillation in sub-um periodically poled RKTP crystals reaching an efficiency of $43 \%$. The generated signal serves as a pump in a cascaded MOPO process in the same crystal.

\section{SW4M.5 $16: 30$}

Hybrid Lithium Niobate Waveguide for Efficient Quasi-Phase-Matched Optical Frequency Conversion, Peter Weigel ${ }^{\prime}$, Marc Savanier ${ }^{2}$, Shayan Mookherjea'; ' Univ. of California San Diego, USA; ${ }^{2}$ Luxtera, USA. We design a nonlinear waveguide for $2 f$-to3f optical frequency conversion based on silicon nitride-lithium niobate and calculate high nonlinear conversion efficiencies of 898 , 623 , and $3169 \% /\left(\mathrm{W}-\mathrm{cm}^{\wedge} 2\right)$ for the processes discussed.

\section{SW4M.6 $16: 45$}

Ultrafast Adiabatic Second Harmonic Generation, Asaf Dahan', Assaf Levanon', Mordechai Katz ${ }^{2,1}$, Haim Suchowski'; 'Raymond and Beverly Sackler School of Physics and Astronomy Tel Aviv Univ., Israel; ${ }^{2}$ Solid State Physics Dept., Electro-Optics Division, Soreq NRC, Israel. We report an efficient and robust frequency doubling for $75 \mathrm{~nm}$ bandwidth with thermal acceptance of $100^{\circ} \mathrm{C}$ and chirp variation of $300 \mathrm{fs}-3.5 \mathrm{ps}$. This was achieved by adiabatic SHG crystal, designed by a generalized nonlinear spatiotemporal simulation.

\section{SW4N • Microresonator Frequency Comb-Continued}

\section{SW4N.4 $16: 30$}

Globally stable Turing pattern formation in $\mathrm{Si}_{3} \mathrm{~N}_{4}$ microresonator, Shu-Wei Huang ${ }^{1}$, Jinghui Yang', Shang-Hua Yang ${ }^{1}$, Mingbin $Y^{2}{ }^{2}$, Dim-Lee Kwong2, Tanya Zelevinsky ${ }^{3}$, Mona Jarrahi', Chee Wei Wong'; ' Univ. of California Los Angeles, USA; ${ }^{2}$ Inst. of Microelectronics, Singapore; ${ }^{3}$ Columbia Univ., USA. We report efficient on-chip Turing pattern formation, uniquely enabled by mode-hybridization induced phase matching. Destabilization of Turing pattern is circumvented, thereby achieving unprecedented pump depletion and record high external pump-to-comb conversion efficiency of $45 \%$.

\section{SW4N.5 $16: 45$}

Normal Dispersion High Conversion Efficiency Kerr Comb with $50 \mathrm{GHz}$ Repetition Rate, Cong Wang ${ }^{1}$, Chengying Bao ${ }^{1}$, Yi Xuan', Kyunghun Han¹, Daniel Leaird', Minghao $\mathrm{Qi}^{1}$, Andrew Weiner ${ }^{1}$; ${ }^{1}$ Purdue Univ., USA. We demonstrate a $50 \mathrm{GHz}$, low noise Kerr comb with $34 \%$ conversion of the pump light into the comb based on a normal dispersion, silicon nitride microring resonator.

\section{SW4O • Laser Induced Excitations in Matter- Continued}

SW40.4 • 16:15

Liquid crystal-modulated spontaneous emission via plasmonic waveguide cladded with low index metamaterials, $\mathrm{He} \mathrm{Hao}{ }^{1}$, Ying Gu', Juanjuan Ren', Hongyi Chen', lam Choon Khoo', Qihuang Gong'; 'Peking Univ., China; ${ }^{2} P e n n s y l v a n i a$ State Univ.,, USA. Using the liquid crystal-metal-low index metamaterial waveguide which supports surface plasmon polaritons, we theoretically demonstrate an active modulation of spontaneous emission. It can be modulated from 131g0 to $327 \mathrm{~g} 0$ by varying optical axis.

SW40.5 • 16:30

Enhancement of light-2D material interaction envisioned for energy harvesting applications, Hossein Taghinejad' 1 , Mohammad Taghinejad', Alexey Tarasov', Ami Hossein Hosseinnia ${ }^{1}$, Hesam Moradinejad', Ali A. Eftekhar ${ }^{1}$, Eric Vogel ${ }^{1}$, Ali Adibi ${ }^{1} ;{ }^{1}$ Georgia Inst. of Technology College of Engineering USA. Strong enhancement of Raman signal upon integration of trilayer $\mathrm{MoS}_{2}$ with FabryPerot (FP) optical cavities is reported. We also discuss prospective application of enhanced light matter interaction in 2D materials for energy harvesting purposes.

SW40.6 - 16:45

Elastic and thermal properties of straintailored air-gap heterostructures, Peter Gaal ${ }^{1,2}$, Roman Bauer ${ }^{1}$, Mathias Sander ${ }^{3}$, Taras Slobodskyy ${ }^{1}$, Wolfgang Hansen ${ }^{1}$; ${ }^{1}$ Instiute for Solid-State and Nanostructure Physics, Universität Hamburg, Germany; ${ }^{2}$ Helmholtz-Zentrum for Materials and Energy Berlin, Germany; ${ }^{3}$ nst. of Physics and Astronomy, Universität Potsdam, Germany. The temperature-dependent expansion coefficient in strain-tailored semiconductor air-gap heterostructures (AGHs) has be measured via static $x$-ray diffraction (XRD). Time-dependent XRD measurements reveal different thermal transport regimes on picosecond and nanosecond timescales. 
Executive Ballroom

$210 \mathrm{~A}$

Executive Ballroom

210B

\section{CLEO: Applications \\ \& Technology}

AW4A - Biomedical Imaging $1-$ Continued

AW4A.5 • 17:00

Characterizing Cardiomyocytes Motion with Quantitative Phase Imaging, Christine E. Cordeiro', Oscar Abilez', Tushar Gupta', Georges Goetz', Olav Solgaard', Daniel Palanker'; 'Stanford Univ., USA. Characterizing cardiomyocytes' activity is important for drug development, but traditional patch clamping analysis is destructive and slow. A label-free method for extracting timing and motion characteristics of cardiomyocytes using quantitative phase imaging is presented.

\section{AW4B - Lasers and Applications-Continued}

AW4B.7 • 17:00

Design and Deployment of Mobile FSO Communication System, Wael Alheadary Yujian Guo', Edgars Stegenburgs', Ki-Hong Park', Tien Khee Ng', Boon S. Ooi', Mohamed-Slim Alouini''; 'KAUST, Saudi Arabia. As a potential solution to many applications, we developed a mobile free-space optical (FSO) system that achieves 1Gbps with transmission distance of $70 \mathrm{~m}$. This system needs minimal preparation to be deployed within an hour.

\section{Executive Ballroom $210 \mathrm{C}$}

\section{CLEO: Science \& Innovations}

\section{SW4C • Semiconductor Lasers on Silicon-Continued}

SW4C.7 • 17:00

Optical Feedback Sensitivity of Heterogeneously Integrated Silicon/III-V Lasers, Mark Harfouche', Dongwan Kim², Huolei Wang ${ }^{2}$, Naresh Satyann, George Rakuljic ${ }^{3}$, Amnon Yariv ${ }^{2,1}$; ${ }^{1}$ Electrical Engineering, California Inst. of Technology, USA; ${ }^{2}$ Applied Physics and Materials Science, California Inst. of Technology, USA: ${ }^{3}$ Telaris Inc., USA. The feedback sensitivity of a high coherence silicon/III$\checkmark$ laser is quantified using an interferometer. High fringe visibility is maintained up to at a reflectivity of $-21 \mathrm{~dB}$, a $10 \mathrm{~dB}$ improvement compared to a high end commercially available DFB laser.

\section{SW4C.8・17:15}

Suppression of Linewidth Enhancement Factor in High-coherence Heterogeneously Integrated Silicon/III-V Lasers, Dongwan Kim ${ }^{1}$, Mark Harfouche ${ }^{1}$, Huolei Wang ${ }^{1}$ Naresh Satyan², George Rakuljic², Amnon Yariv'; 'California Inst. of Technology, USA ${ }^{2}$ Telaris Inc., USA. We observe a relaxation resonance frequency of hundreds of $\mathrm{MHz}$ in high-coherence Si/III-V lasers, up to $5 x$ less than commercial III-V lasers. This results in very noise frequency noise PSD of $720 \mathrm{~Hz}^{2}$ / $\mathrm{Hz}$ above the relaxation resonance frequency due to the suppression of linewidth enhancement factor.
Executive Ballroom 210D

CLEO: QELSFundamental Science

FW4D • Nonlinear Optical Sources-Continued

FW4D.6 • 17:00

Thermal equilibrium of Photons and Lasing without an Overall Inversion in Standard Erbium-Doped Fibers, Rafi Weill' ${ }^{1}$, Alexander Bekker ${ }^{1}$, Boris Levit ${ }^{1}$, Michael Zhurahov', Baruch. Fischer'; 'Technion Israel Inst. of Technology, Israel. We show thermal-equilibrium (TE) and Bose-Einstein distribution of photons in standard erbium-doped fibers. We also find a coexistence of TE with oscillation without an overall inversion that can be attributed to lasing or BEC.

\section{FW4D.7 • 17:15}

Synchronization of Mutually Coupled High- $B$ Quantum Dot Microlasers, Sören Kreinberg ${ }^{1}$, Felix Krüger ${ }^{1}$, Steffen Holzinger ${ }^{1}$ Elisabeth Schlottmann ${ }^{1}$, Martin Kamp ${ }^{2}$ Christian Schneider ${ }^{2}$, Sven Höfling ${ }^{2,3}$, Xavier Porte ${ }^{1}$, Stephan Reitzenstein ${ }^{1}$; ${ }^{1}$ Technische Universität Berlin, Germany: ${ }^{2}$ Julius-Maximiians-Universität Würzburg, Germany; ${ }^{3}$ Univ. of St Andrews, UK. We perform experiments on mutual coupling and synchronization of high- $\beta$ microlasers and show phenomena like partial, unidirectional and mutual locking, as well as synchronization of mode switching dynamics. We demonstrate qualitative deviations from classical expectations. 
Executive Ballroom
$210 \mathrm{E}$

\section{CLEO: QELS-Fundamental Science}

FW4E • Quantum

State Generation and

Characterization-Continued

FW4E.6 • 17:00

Tomography of Mode-Tunable Coherent Single-Photon Subtractor, Young-Sik Ra', Clément Jacquard', Adrien Dufour', Claude Fabre', Nicolas Treps'; 'Laboratoire Kastler Brossel, France. We implement a singlephoton subtractor that can be tuned to subtract a single photon exclusively from one mode or coherently from multiple modes. We experimentally characterize the device by employing coherent-state quantum process tomography.

FW4E.7 • 17:15

Generation and characterization of energyentangled W states, Matteo Menotti', Bin Fang ${ }^{2}$, Virginia O. Lorenz ${ }^{2}$, John Sipe ${ }^{3}$, Marco Liscidini'; 'Dept. of Physics, Univ. of Pavia, Italy; ${ }^{2}$ Dept. of Physics and Astronomy, Univ. of Delaware, USA; ${ }^{3}$ Dept. of Physics, Univ. of Toronto, Canada. We demonstrate the generation of W states entangled in the energy degree of freedom. Using a reduced density matrix approach, these states are characterized without the need for frequency conversion.
Executive Ballroom

210F

Executive Ballroom

210G

Joint

FW4F • Quantum States and Sensing with Optomechanical Systems-Continued

\section{FW4F.5 • 17:00 Invited}

Multimode Quantum Optomechanics with Ultra-coherent Nanomechanical Resonators, Yeghishe Tsaturyan', William H. Nielsen', Christoffer B. Møller ${ }^{1}$, Andreas Barg', Junxin Chen', Yannick Seis ${ }^{1}$, Eugene S. Polzik', Albert Schliesser'; 'Niels Bohr Inst. Univ. of Copenhagen, Denmark. Mechanical resonators with "soft" phononic clamping dilute the material's intrinsic dissipation by five orders of magnitude. Decoherence rates comparable to trapped ions ensue, enabling quantum optomechanical experiments with multimode hybrid systems.

\section{JW4G • Symposium on Advances in Metaphotonic Devices II-Continued}

JW4G.4 • 17:00

Enhancing Light-Matter Interaction with high-Q Fano Dielectric Metasurfaces, Sheng Liu', Sadhvikas Addamane ${ }^{2}$, Michael Sinclair ${ }^{1}$, Gordon Keeler ${ }^{1}$, Ganesh Balakrishnan', Igal Brener'; 'Sandia National Labs, USA; ${ }^{2}$ Electrical engineering, Univ. of New Mexico, USA. We experimentally demonstrate largely enhanced light-matter interaction using high-Q Fano dielectric metasurfaces. We observe large enhancements, spectral tailoring and lifetime shortening of the photoluminescence of single photon emitters embedded inside III-V all-dielectric metasurfaces.

\section{JW4G.5 • 17:15}

Quantum Imaging with Dielectric Metasurfaces for Multi-Photon Polarization Tomography, Kai Wang ${ }^{1}$, Sergey S. Kruk ${ }^{1}$ Lei $\mathrm{Xu}^{1,2}$, Matthew Parry ${ }^{1}$, Hung-Pin Chung ${ }^{1,3}$, Alexander S. Solntsev' ${ }^{1}$, James G. Titchener ${ }^{1}$, Ivan Kravchenko ${ }^{4}$, Yen-Hung Chen ${ }^{3}$, Yuri Kivshar ${ }^{1}$, Dragomir N.Neshev ${ }^{1}$, Andrey A. Sukhorukov ${ }^{1} ;{ }^{1}$ Nonlinear Physics Centre, Research School of Physics and Engineering, The Australian National Univ., Australia; ${ }^{2}$ The MOE Key Lab of Weak Light Nonlinear Photonics, School of Physics and TEDA Applied Physics Inst., Nankai Univ., China; ${ }^{3}$ Dept. of Optics and Photonics, National Central Univ., Taiwan; ${ }^{4}$ Center for Nanophase Materials Sciences, Oak Ridge National Lab, USA. We suggest and realize experimentally dielectric metasurfaces with high transmission efficiency for quantum multi-photon tomography, allowing for full reconstruction of pure or mixed quantum polarization states across a broad bandwidth.
Executive Ballroom $210 \mathrm{H}$

\section{CLEO: QELS} Fundamental Science

FW4H $\bullet$ Ultrafast Optics and Plasmonics in NanostructuresContinued

FW4H.6 • 17:00

Double Quantum Coherence in Individual Quantum Dots Enhanced by Weak Excitation of Delocalized States, Eric Martin ${ }^{1}$ Steven T. Cundiff'; 'Univ. of Michigan, USA Two-quantum multidimensional coherent spectroscopy is used to probe few quantum dots in a diffraction limited spot. Creating a small number of carriers in the quasicontinuum levels enhances the signal and controls coherent coupling between states.

FW4H.7 • 17:15

Mid-Infrared Electro-Optic Modulation in Black Phosphorus, Ruoming Peng ${ }^{1}$ Nathan Youngblood ${ }^{1}$, Mo Li'; ' Univ. of Min nesota, USA. An out of plane electric field can modulate the optical absorption of black phosphorus due to a combination of Pauli blocking and quantum-confined FranzKeldysh effects. Optical transitions between different sub-bands are explored and over $5 \%$ modulation is demonstrated in $13 \mathrm{~nm}$ black phosphorus.

16:30-18:30 Happy Hour in Exhibit Halll, Exhibit Hall 1, 2 \& 3

18:00-19:00 OSA Nanophotonics Technical Group 20x20, Executive Ballroom 210 A 


\section{CLEO: Science \& Innovations}

\section{SW4I • Orbital Angular Momentum Based Optical Communications-Continued}

\author{
SW4J • Precision \\ Spectroscopy-Continued
}

SW4J.6 $17: 00$

Mechanical Fourier Transform Spectrometer with $\mathrm{kHz}$ Resolution, Lucile Rutkowski ${ }^{1}$ Alexandra C. Johansson', Amir Khodabakhsh', Aleksandra Foltynowicz'; ${ }^{1}$ Umea Univ., Sweden. We measure simultaneously 11000 resonances of a high-finesse cavity with $\mathrm{kHz}$ level resolution using optical frequency comb Fourier transform spectroscopy and retrieve the dispersion of the cavity mirrors from the cavity mode spacing.
SW4J.7 • 17:15

A 1000-fold contrast enhancement in Fabry-Perot interferometers, Giuseppe Antonacci' ${ }^{1}$, Simone De Panfilis', Giuseppe Di Domenico', Eugenio DelRe², Giancarlo Ruocco"; "Istituto Italiano di Tecnologia, Italy; 'Universita' di Roma "Sapienza", Italy. Spectral contrast in Fabry-Perot interferometers is key to measure weak signals. Using a high-resolution, high-throughpu VIPA spectrometer, we demonstrate an intensity-equalization method to achieve an unprecedented 1000 -fold increase in spectral contrast in a single-pass configuration.

\section{SW4K・ 2D Materials \& \\ Devices I-Continued}

SW4K.6 • 17:00

Demonstration of a New Technique for the Transfer Printing of Graphene on Photonic Devices, Leili Abdollahi Shiramin ${ }^{1}$, Alexander Bazin ${ }^{1}$, Steven Verstuyft', Sylvia Lycke ${ }^{2}$, Peter Vandenabeele ${ }^{2}$, Gunther Roelkens ${ }^{1}$, Dries Van Thourhout'; ' Ghent Univ.-IMEC, Belgium; ${ }^{2}$ Ghent Univ., Belgium. We demonstrate an automated method for transfer printing of micron sized graphene patterns on predefined sites on a photonic chip. Silicon nitride waveguides with graphene transferred on top exhibit an absorption loss of $0.054 \mathrm{~dB}$ $\mu \mathrm{m}$, in line with simulation results.

\section{SW4K.7 • 17:15}

Photonic synaptic device capable of optical memory and logic operations, Shuchao Qin', Yujie Liu', Xiaomu Wang', Yongbing $X^{\prime}$ ', Yi Shi', Rong Zhang', Frank (Fengqiu) Wang'; 'Nanjing Univ., China. We demonstrate an optically-driven artificial synapse based on a graphene hybrid phototransistor. Both optical memory function (long-term plasticity) and logic operations are achieved, which adds important new capabilities to photonics enabled neuromorphic computing.

\section{SW4L - Standoff and Remote Sensing-Continued}

SW4L.4 • 17:00

Wide Field-of-View and Mid-Range Distance Imaging LIDAR by Digital MicroMirror Device, Brandon Hellman', Braden Smith', Adley Gin', Young-sik Kim', Guanghao Chen ${ }^{1}$, Paul Winkler', Yuzuru Takashima' 'Univ. of Arizona, USA. A Digital Micro-mirro Device enables a fast, wide field-of-view, mid-range distance mapping while minimizing requirements for mechanical scanning components. The optical architecture offers a compact, low-cost solution to an imaging LIDAR with a single detector.

16:30-18:30 Happy Hour in Exhibit Halll, Exhibit Hall 1, 2 \& 3

SW4L.5 • 17:15

Heterodyne Efficiency in Chirped Laser Dispersion Spectroscopy, Yifeng Chen ${ }^{1}$ Genevieve Plant ${ }^{1,2}$, Gerard Wysocki ${ }^{1}$; ${ }^{1}$ Princeton Univ., USA; ${ }^{2}$ Electrical and Computer Engineering, Univ. of Michigan, USA. We present an analysis of diffusive reflection and its effect on heterodyne efficiency in conventional and heterodyne-enhanced chirped laser dispersion spectroscopy motivated by applications in stand-off chemical detection.

18:00-19:00 OSA Nanophotonics Technical Group 20x20, Executive Ballroom $210 \mathrm{~A}$ 


$\begin{array}{llc}\text { Marriott } & \text { Marriott } & \text { Marriott } \\ \text { Salon III } & \text { Salon IV } & \text { Salon V \& VI }\end{array}$

\section{CLEO: Science \& Innovations}

SW4M • Concepts \& Advances in Quasi-phase MatchingContinued

SW4M.7 • 17:00 Invited

Large Aperture Quasi-phase Matched Nonlinear Material for Functional Power Lasers, Takunori Taira ${ }^{1}$, Hideki Ishizuki'; 'Laser Research Center, Inst. for Molecular Science, Japan. High-power and high-energy nonlinear optics, which includes optical parametric process and based on large-aperture periodically poled $\mathrm{Mg}$-doped $\mathrm{LiNbO}_{3}$ for mid-infrared-to-terahertz wave region are discussed. Topics include few cycles ultrafast pulse treatments with regarding $\mathrm{HHG}$ sources

\section{SW4N • Microresonator Frequency Comb-Continued}

SW4N.6 $17: 00$

Competition Between Raman and Kerr Effects in Microresonators, Yoshitomo Okawachi', Mengjie Yu ${ }^{1,2}$, Vivek Venkataraman $^{3}$, Pawel M. Latawiec ${ }^{3}$, Marko Lončar ${ }^{3}$, Alexander L. Gaeta'; ${ }^{1}$ Columbia Univ., USA; ${ }^{2}$ Cornell Univ., USA; ${ }^{3}$ Harvard Univ., USA. We investigate competing effects of Raman and Kerr gain in diamond microresonators. Strong, narrowband Raman gain inherent in crystalline materials determines a maximum microresonator size allowable to achieve Kerr combs.
SW4N.7 • 17:15

$10 \mathrm{GHz}$ Frequency Comb Spectral Broadening in AlGaAs-On-Insulator NanoWaveguide with Ultra-Low Pump Power, $\mathrm{Hao} \mathrm{Hu}{ }^{1}$, Minhao Pu${ }^{1}$, Kresten Yvind ${ }^{1}$, Leif K. Oxenløwe'; 'DTU Fotonik, Denmark. We experimentally demonstrated $10 \mathrm{GHz}$ frequency comb spectral broadening with a 30-dB bandwidth of $238 \mathrm{~nm}$ in an $11-\mathrm{mm}$ long AlGaAsOI nano-waveguide. The $10-\mathrm{GHz}$ 230 -fs pump pulse has an average power of only $12 \mathrm{~mW}$.

\section{SW4O • Laser Induced Excitations in Matter- Continued}

SW40.7 • 17:00

Plasmonically Induced Coherent and Polarized Random Laser Emissions in Colloidal $\mathrm{CdSe} / \mathrm{ZnS}$ Quantum Dots with Ellipsoidal Ag Nanoparticles, Yung-Chi Yao', Zu-Po Yang $^{2}$, Jing-Yu Haung ${ }^{1}$, Min-Hung Lee ${ }^{1}$ Meng-Tsan Tsai ${ }^{3}$, Ya-Ju Lee ${ }^{1} ;{ }^{1}$ Inst. of ElectroOptical Science and Technology, National Taiwan Normal Univ. Taiwan; ${ }^{2}$ Inst. of Photonic System, National Chiao-Tung Univ., Taiwan; ${ }^{3}$ Dept. of Electrical Engineering, Chang Gung Univ., Taiwan. We demonstrate the capability of controlling the optical anisotropy by manipulating the coupling strength between the oscillated electric field and the localized surface plasmon resonance for a random lasing medium composed of $\mathrm{CdSe} / \mathrm{ZnS}$ quantum dots and ellipsoidal Ag nanoparticles.

\section{SW40.8 • 17:15}

Optical vortex illumination to form polymeric twisted fiber, Junhyung Lee ${ }^{1}$, Shunsuke Toyoshima', Katsuhiko Miyamoto ${ }^{1,2}$, Yoshihiko Arita 2,3 Kishan Dholakia ${ }^{3}$, Takashige Omatsu1,2; ${ }^{1}$ Chiba Univ., Japan; ${ }^{2}$ Molecular Chirality Research Center, Chiba Univ., Japan; Univ. of St. Andrews, UK. We fabricated the twisted fiber by $405 \mathrm{~nm}$ optical vortex illumination onto ultraviolet curing resin. Twisted direction and branching number of the fiber were assigned based on the handedness and topological charge of illuminated optical vortex.

16:30-18:30 Happy Hour in Exhibit Halll, Exhibit Hall 1, 2 \& 3

18:00-19:00 OSA Nanophotonics Technical Group 20x20, Executive Ballroom 210 A 
Executive Ballroom $210 \mathrm{~A}$

Executive Ballroom 210B

\section{CLEO: Applications \& Technology}

08:00-10:00

ATh1A • Biomedical Imaging II Presider: Jin Kang; Johns Hopkins Univ., USA

\section{ATh1A.1 • 08:00 Invited}

In Vivo Cellular Imaging with Spectrally Encoded Confocal Microscopy, DongKyun Kang'; ' ${ }^{1}$ Massachusetts General Hospital, USA. Spectrally encoded confocal microscopy (SECM) is a high-speed confocal microscopy technique. In this presentation, recent development on endoscopic SECM and smartphone SECM and their uses in in vivo human imaging will be presented.

08:00-10:00

ATh1B • Active Remote

Environmental Sensing

Presider: To be Determined

\section{ATh1B.1 • 08:00}

ATh1A.2 • 08:30 Invited

Mirror Enhanced STED Super-resolution Microscopy, Peng Xi'; ' Peking Univ., China. Through reflective interference, the axial thickness of confocal point spread function can be easily improved to $100 \mathrm{~nm}$. Six-fold of axial resolution and two-fold of lateral resolution can be obtained for STED nanoscopy.
Depolarization Studies of Atmospheric Particles during Rush Hour by Employing the Scheimpflug Lidar Technique, Liang Mei ${ }^{1}$, Peng Guan', Yang Yang ${ }^{1}$; ${ }^{1}$ Dalian Univ. of Technology, China. Depolarization ratio is highly relevant to geometries of atmospheric particles. A polarization Scheimpflug lida system has been developed for atmospheric particle depolarization studies. Atmospheric measurements were carried out during rush hour for urban particle studies.

\section{ATh1B.2・08:15}

Multispectral polarimetric modulation spectroscopy for species and sex determination of Malaria disease vectors, Alem K. Gebru' ${ }^{1,4}$, Samuel Jansson 1 , Rickard Ignell ${ }^{2}$ Carsten Kirkeby ${ }^{3,4}$, Mikkel Brydegaard ${ }^{1,4}$ 'Lund Laser Centre, Dept of Physics, Lund Univ., Sweden; ${ }^{2}$ Chemical Ecology Unit, Dept. of Plant Protection Biology, Swedish Agricultural Sciences Univ., Sweden: ${ }^{3}$ National Veterinary Inst., Denmark Technical Univ., Denmark; ${ }^{4}$ FaunaPhotonics IVS, Denmark. A multispectral polarimetric optical detection system with $\mathrm{kHz}$ sample rates was implemented to determine mosquitos species and sex based on their wing-beat frequency (WBF), harmonic overtones, optical crosssection and melanization in flight.

\section{ATh1B.3 • 08:30}

UV-Vis-NIR white light LIDAR using polarization-controlled laser filamentation Shermineh Rostami ${ }^{2}$, Matthieu Baudelet ${ }^{2,1}$ Martin Richardson'; ${ }^{1}$ National center for Forensic science, Univ. Of Central Florida USA; ${ }^{2}$ Laser Plasma Lab, CREOL, Univ. O Central Florida, USA. White-Light LIDAR was performed using a unique polarizationcontrolled white light source generated by filamentation. The design and performance of this supercontinuum source will be shown as well as its importance for a novel LIDAR approach.

\section{Executive Ballroom $210 \mathrm{C}$}

\section{CLEO: Science \& Innovations}

08:00-10:00

STh1C • III-V Lasers

Presider: Dominic Siriani; MIT Lincoln Lab Periodical Library, USA

\section{STh1C.1・08:00}

Electrically Driven Deep Ultraviolet Lasers based on MgZnO Thin Films at Room Temperature, Mohammad Suja', Sunayna Bashar', Wenhao Shi', Jianlin Liu'; 'Electrical and Computer Engineering, Univ. Of California, USA. Metal-semiconductor-metal devices were fabricated by utilizing $\mathrm{MgZnO}$ thin films to demonstrate random lasing in the deep ultraviolet wavelength range tuned by Mg content in the film. Room temperature lasing is realized in the wavelength range down to $284 \mathrm{~nm}$.

\section{STh1C.2 $\bullet 08: 15$}

Monolithic GaN-InGaN Core-shell Lasers in Submicron Scale, Chia-Yen Huang ${ }^{1}$, Jing-Jie Lin', Tsu-Chi Chang', Che-Yu Liu', Tzu-Ying Dai', Kuo-Bin Hong ${ }^{1}$, Tien-Chang Lu', Haochung Kuo"; ${ }^{1}$ Dept. of Photonics, NCTU, Taiwan. We demonstrated a GaN-InGaN core-shell nanorod periodic array lasing under room temperature. Optical simulations and photoluminescence measurement revealed optically-coupled whisper gallery modes. The threshold pumping density was $80 \mathrm{~kW} / \mathrm{cm}^{2}$ with a quality factor of 1940 .

\section{STh1C. $3 \bullet 08: 30$ Invited}

Growth and Characterization of III-N Ultraviolet Lasers and Avalanche Photodiodes by MOCVD, Russell D. Dupuis ${ }^{1}$, Mi-Hee Ji ${ }^{1}$ Yuh-Shiuan Liu', Jeomoh Kim', Young-Jae Park', Theeradetch Detchprohm', Tsung-Ting Kao ${ }^{1}$, Shyh-Chiang Shen ${ }^{1}$, Karan Mehta ${ }^{1}$ P. Douglas Yoder ${ }^{1}$, Hongen $\mathrm{Xie}^{2}$, Fernando Ponce ${ }^{2}$, Ashok Sood ${ }^{3}$, Nibir Dhar', Jay Lewis ${ }^{5}$. ${ }^{1}$ Georgia Inst. of Technology, USA; ${ }^{2}$ Dept. of Physics and Astronomy, Arizona State Univ. USA; ${ }^{3}$ Magnolia Optical Technologies, USA ${ }^{4}$ Night Vision Sensors and Electronic Division, USA; ${ }^{5}$ DARPA MTO, USA. III-N UV lasers and APDs are demonstrated operating at wavelengths $240 \mathrm{~nm}<\lambda<370 \mathrm{~nm}$. These device structures are grown by MOCVD on single-crystal substrates. The growth and properties of these devices will be described.
Executive Ballroom 210D

\section{CLEO: QELS- Fundamental Science}

08:00-10:00

FTh1D • Topological Photonics

Presider: Mikael Rechtsman:

Pennsylvania State Univ., USA

\section{FTh1D.1 08:00 Invited}

Embedded Photonic Topological Insulators, Miguel A. Bandres ${ }^{1}$, Mordechai Segev 'Technion, Israel. We show that, counterintuitive, it is possible to control the properties of photonic topological insulators by tailoring defects. In the extreme case, a lattice of defects inside a topological insulator creates a totally new topological insulator.
FTh1D.2 • 08:30

Observation of Photonic Topological Valley Transport, Jiho Noh ${ }^{1}$, Sheng Huang ${ }^{2}$, Kevin P. Chen', Mikael C. Rechtsman'; 'Dept. of Physics, The Pennsylvania State Univ., USA; ${ }^{2}$ Dept. of Electrical and Computer Engineering, Univ. of Pittsburgh, USA. We present the experimental realization of valley Hall topological edge states in armchair and bearded edge domain walls of inversion symmetry broken honeycomb lattices. 
Executive Ballroom
$210 \mathrm{E}$

\section{Joint}

08:00-10:00

JTh1E • Quantum Photonics I

Presider: Todd Pittman; Univ. of

Maryland Baltimore County, USA

\section{JTh1E.1 • 08:00 Tutorial}

Photonic Quantum Computing, Jeremy L. O'Brien'; 'Univ. of Bristol, UK. Of the various approaches to quantum computing, photons are appealing for their low-noise properties and ease of manipulation at the single photon level; while the challenge of entangling interactions between photons can be met via measurement induced non-linearities. However, the real excitement with this architecture is the promise of ultimate manufacturability: All of the components have been implemented on chip, and increasingly sophisticated integration of these components is being achieved. We will discuss the opportunities and challenges of a fully integrated photonic quantum computer.

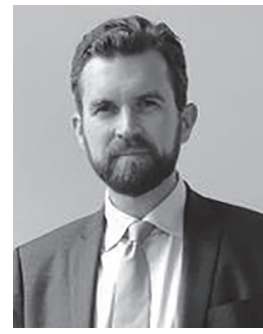

Jeremy O'Brien is the director of the Centre for Ouantum Photonics (COP) at the University of Bristol. CQP's efforts are focused on the fundamental and applied quantum mechanics at the heart of quantum information science and technology, ranging from prototypes for scalable quantum computing to generalised quantum measurements, quantum control, and quantum metrology. He received his $\mathrm{PhD}$ in physics from the University of New South Wales in 2002 for experimental work on correlated and confined electrons in organic conductors, superconductors and semiconductor nanostructures, as well as progress towards the fabrication of a phosphorus in silicon quantum computer. As a research fellow at the University of Queensland (2001-2006) he worked on quantum optics and quantum information science with single photons. He is currently Professor in Physics and Electrical Engineering at the University of Bristol.
Executive Ballroom 210F
Executive Ballroom

210G
Executive Ballroom

$210 \mathrm{H}$

\section{CLEO: QELS-Fundamental Science}

\section{8:00-10:00}

FTh1F • Ultrafast Exciton

Dynamics in Van Der Waals

Materials

Presider: Lyubov Titova;

Worcester Polytechnic Inst., USA

\section{FTh1F.1 08:00 Invited}

Exciton Spectroscopy in Monolayer Transition Metal Dichalcogenides and Van der Waals Heterostructures, Bernhard Urbaszek'; ${ }^{1}$ CNRS - Toulouse Univ., France. Excitons dominate the strong light-matter interaction in transition metal dichalcogenide monolayers and present exciting opportunities for applications and new physics. Here we investigate carrier dynamics and valley properties in optical spectroscopy on highquality samples.

FTh1F.2 • 08:30

Optical Tuning of Interlayer Excitonic Systems in Trilayer Transition Metal Dichalcogenides Heterostructures, Chanyeol Choi ${ }^{1,2}$ Hung-Chieh Cheng ${ }^{3,4}$, Hyunseok Kim² Abhinav K. Vinod ${ }^{1,2}$, Sang-Hoon Bae ${ }^{3,4}$, Javad Azadani ${ }^{5}$, Jongjae Chae ${ }^{3}$, Shu-Wei Huang ${ }^{1,2}$ Xiangfeng Duan ${ }^{4,6}$, Tony Low ${ }^{5}$, Cheewei Wong 1,2. ' ${ }^{1}$ Fang Lu Mesoscopic Optics and Quantum Electronics Lab, Univ. of California USA; ${ }^{2}$ Dept. of Electrical Engineering, Univ. of California, USA; ${ }^{3}$ Dept. of Material Science and Engineering, Univ. of California USA; ${ }^{4}$ California Nanosystems Inst., Univ. of California, USA; ${ }^{5}$ Dept. of Electrical and Computer Engineering, Univ. of Minnesota, USA ${ }^{6}$ Dept. of Chemistry and Biochemistry, Univ. of California, USA. We report enhanced interlayer excitonic systems, excitons and trions, in trilayer transition metal dichalcogenides heterostructrues with photoluminescence spectroscopy. We investigate interlayer trion binding energy and carrier lifetime of interlayer excitons and trions.
08:00-10:00

FTh1G • Nonlinear and Hyperbolic Metamaterials Presider: Viktor Podolskiy; Univ. of Massachusetts Lowell, USA

\section{FTh1G.1 • 08:00 Invited}

Engineering Optical Density of States with Nonlocal Metamaterials, Viktor A Podolskiy', Pavel Ginzburg, ${ }^{2,3}$, Diane Roth ${ }^{2}$, Alexey Krasavin ${ }^{2}$, Brian Wells ${ }^{1,4}$, Anatoly Zayats". ${ }^{1}$ Univ. of Massachusetts Lowell, USA ${ }^{2}$ Dept of Physics, Kings College London, UK; ${ }^{3}$ Tel Aviv Univ., Israel; ${ }^{4}$ Univ. of Hartford, USA. We present theoretical, numerical, and experimental analysis of optical density of states in plasmonic nanowire metamaterials. Averaged Purcell factors of the order of 30 , attributed to nonlocal electromagnetic response of metamaterials, are reported.

FTh1G.2 • 08:30

Solving Integral Equations with Optical Metamaterial-Waveguide Networks, Nasim Mohammadi Estakhri ${ }^{1}$, Brian E. Edwards ${ }^{1}$ Nader Engheta'; 'Univ. of Pennsylvania, USA We propose a wave-based technique to solve integral equations of general format. We numerically show that designer metamaterial kernels combined with proper waveguide feedback networks operate as compact equation solvers, demonstrated via solving Fredholm equations.

\section{8:00-10:00 \\ FTh1H • Nanoscale \\ Optomechanics}

Presider: Ido Kaminer; MIT, USA

\section{FTh1H.1 • 08:00 Tutorial}

Levitation Nano-Optomechanics, Romain Quidant ${ }^{1,2}$; ${ }^{1}$ CFO -The Inst. of Photonic Sciences, Spain; ${ }^{2}$ CCREA, Spain. In this tutorial talk we introduce the use of a levitated nanoparticle in vacuum as a nanooptomechanical system with unprecedented performances.

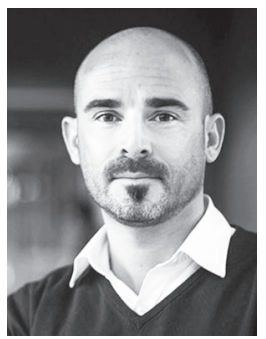

Romain Quidant is an ICREA Professor at ICFO in Barcelona. The activities of his group cover both fundamental and applied research. The fundamental part of his work is mainly directed towards enhanced light/ matter interaction for quantum optics. From a more applied viewpoint, his group investigates the use of light and heat control at the nanometer scale for biomedical applications, including early detection and less invasive therapies. 


\section{Meeting Room $211 \mathrm{~B} / \mathrm{D}$}

Meeting Room
$212 \mathrm{~A} / \mathrm{C}$
Meeting Room
$212 \mathrm{~B} / \mathrm{D}$
Marriott

Salon I \& II

\section{CLEO: Science \& Innovations}

08:00-10:00

STh1I $\cdot 2 \mathrm{D}$ Materials and

Devices II

Presider: Matthew Escarra; Tulane Univ., USA

\section{STh1I.1 08:00 Invited}

Optical Properties of Atomically Thin Two-dimensional Materials, Tony F. Heinz ${ }^{1,2}$; 'Stanford Univ., USA; '2SLAC National Accelerator Lab, USA. We review our understanding of the distinctive optical properties of monolayer semiconductors in the transition metal dichalcogenide family, including their strong and anomalous excitonic effects, their valley circular dichroism, and their interlayer interactions.
STh1l.2 • 08:30

High-Q optical microresonators functionalized with two-dimensional material, Clément Javerzac-Galy ${ }^{1}$, Nicolas Piro ${ }^{1}$, Ryan Schilling ${ }^{1}$, Anshuman Kumar ${ }^{1}$, Matteo Barbone2, Ilya Goykhman², Andrea Ferrari², Tobias J. Kippenberg1; ' ${ }^{1}$ Ecole Polytechnique Federale de Lausanne, Switzerland; ${ }^{2} \mathrm{Cam}$ bridge Graphene Centre, Univ. of Cambridge, UK. We report the functionalization of high-O silica microdisks with WSe2 and their optical characterization. Backgroundfree cavity enhanced photoluminescence and photoluminescence saturation are observed at room temperature. We show precise measurements of the quantum yield of WSe2.

\section{8:00-10:00}

STh1J • Laser Ablation

Fundamentals and Applications

Presider: Tsing-Hua Her; Univ of

North Carolina at Charlotte, USA

\section{STh1J.1 • 08:00 Tutorial}

Laser Printing of Functional Materials, Pere Serra'; ${ }^{1}$ Universitat de Barcelona, Spain. In this tutorial we review laser induced forward transfer, a technique which uses laser radiation to print functional inks of the most diverse materials with the aim of manufacturing miniaturized devices for high technology applications (printed electronics, smart systems, tissue engineering).

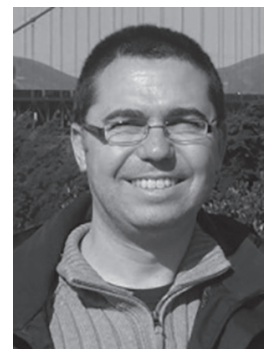

Pere Serra is professor of Applied Physics at the University of Barcelona. His research career has been almost entirely focused on the field of laser microfabrication, particularly on laser printing applications. He has coauthored 90 publications in international journals, has given 20 invited talks, and served as co-chair and committee member in numerous international conferences.
08:00-10:00

STh1K • Nonlinear Fiber

Photonics I

Presider: Kenneth Kin-Yip Wong; Univ. of Hong Kong, Hong Kong

\section{STh1K.1・08:00}

Temperature dependence of polarized GAWBS spectrum in high nonlinear fibers Neisei Hayashi', Kohei Suzuki ${ }^{1}$, Sze Y. Set ${ }^{1}$ Shinji Yamashitaa'; 'The Univ. of Tokyo, Japan. We measured polarized guided acoustic wave Brillouin scattering (GAWBS) spectrum dependence on temperature using a high nonlinear fiber. The temperature coefficient was $168 \mathrm{kHz} / \mathrm{K}$, which is 1.7 times larger than that of small-core photonic crystal fibers.

\section{STh1K.2 • 08:15}

Role of Correlated Photon Pairs in the Spectrum Compression in Optical Fibers with Normal Dispersion, Serguei Papernyi ${ }^{1}$ Wallace Clements', Vladimir Ivanov'; ${ }^{1} \mathrm{MPB}$ Communications Inc, Canada. We show experimentally that the presence of correlated photon pairs generated by Four Wave Mixing in Raman fiber lasers significantly increase the efficiency of spectrum compression of continuous-wave multimode waves propagating in normal dispersion fiber.

\section{STh1K.3・08:30}

Deterministic transverse mode conversion at the single-photon level, Daniel Cruz-Delgado ${ }^{1}$, Juan Carlos Alvarado Zacarias ${ }^{2}$, Hector Cruz-Ramirez', Jose E. Antonio-Lopez² Sergio Leon-Saval ${ }^{3}$, Rodrigo A. Correa ${ }^{2}$, Alfred U'Ren'; ' UNAM, Mexico; ${ }^{2}$ CREOL, USA ${ }^{3}$ IOPS, Australia. We demonstrate the use of a 6-terminal photonic lantern in order to achieve deterministic conversion of the transverse structure of heralded single photons, generated through the spontaneous four wave mixing process in a birefringent fiber.
08:00-10:00

\section{STh1L • High Average Power} Lasers

Presider: Hiromitsu Kiriyama; National Inst. Quantum \& Rad Sc \& Tech, Japan

\section{STh1L.1 • 08:00 Invited}

1 kW Ultrafast Thin-Disk Amplifier System Christoph Wandt ${ }^{1}$, Sandro Klingebiel ${ }^{1}$, Marce Schultze ${ }^{1}$, Stephan Prinz', Catherine Y. Teisset $^{1}$, Sebastian Stark', Christian Grebing ${ }^{1}$ Matthias Häfner ${ }^{1}$, Robert Bessing ${ }^{1}$, Tobias Herzig ${ }^{1}$, Aleksander Budnicki², Dirk Sutter ${ }^{2}$ Knut Michel ${ }^{1}$, Thomas Nubbemeyer ${ }^{3}$, Ferenc Krausz ${ }^{4,3}$, Thomas Metzger'; 'TRUMPF Scientific Lasers GmbH + Co. KG, Germany ${ }^{2}$ TRUMPF Laser $\mathrm{GmbH}$, Germany; ${ }^{3}$ Dept für Physik, Ludwig-Maximilians-Universität München, Germany; ${ }^{4}$ Max-Planck Inst. of Quantum Optics, Germany. A thin-disk based regenerative amplifier with a compressed output power of more than $1 \mathrm{~kW}$ is presented. At a wavelength of $1031 \mathrm{~nm}$ pulse energies $\geq 100 \mathrm{~mJ}$ are demonstrated at a repetition rate of $10 \mathrm{kHz}$ with pulse durations of $<1.0 \mathrm{ps}$.

\section{STh1L.2 • 08:30 Invited}

All Diode-Pumped, High-repetition-rate Advanced Petawatt Laser System (HAPLS), Emily F. Sistrunk', Thomas Spinka', Andrew Bayramian', Paul Armstrong', Salmaan Baxamusa ${ }^{1}$ Shawn Betts' ${ }^{1}$ Darrell Bopp ${ }^{1}$, Samuel Buck², Ken Charron', Josef Cupal', Robert Demaret ${ }^{1}$, Robert Deri ${ }^{1}$, Jean-Michelle D Nicola', Marc Drouin², Al Erlandson', Steve Fulkerson ${ }^{1}$, Chris Gates ${ }^{1}$, Jeff Horner ${ }^{1}$, Jakub Horacek2, Jeff Jarboe', Karel Kasl2, Danny Kim', Edward Koh1', Lucia Koubikova², Rod Lanning ${ }^{1}$, Jeremy Lusk' ${ }^{1}$. William Maranville ${ }^{1}$ Chris Marshall', Dan Mason', Petr Mazurek², Joe Menapace', Phil Miller', Jack Naylon² James Nissen", Jakub Novak², Davorin Peceli ${ }^{2}$, Paul Rosso ${ }^{1}$, Kathleen Schaffers ${ }^{1}$ Tara Silva', Daniel Smith ${ }^{1}$, Joel Stanley ${ }^{1}$ Rusty Steele ${ }^{1}$, Chris Stolz', Steve Telford', Jiri Thoma ${ }^{2}$, Diana VanBlarcom ${ }^{1}$, Jiri Weiss ${ }^{2}$, Paul Wegner ${ }^{1}$, Bedrich Rus ${ }^{2}$, Constantin Haefner ${ }^{1}$ 'Lawrence Livermore National Lab, USA; 2ELI-Beamlines, Czech Republic. The HAPLS laser system has been commissioned to its first integrated performance milestone, delivering laser pulses with $16 \mathrm{~J}$ sub-30fs duration at a $31 / 3 \mathrm{~Hz}$ repetition rate. This first all-diode-pumped petawatt-class laser offers the average powers required for secondary source applications. 
Marriott

Salon III
Marriott

Salon IV
Marriott

Salon V \& VI

\section{CLEO: Science \& Innovations}

08:00-10:00

STh1M • Phased Arrays Related

Device

Presider: Wei Jiang; Rutgers

Univ., USA

\section{STh1M.1 • 08:00}

Large-Scale Visible and Infrared Optical Phased Arrays in Silicon Nitride, Christopher V. Poulton', Matthew Byrd', Manan Raval', Zhan Su ${ }^{1}$, Nanxi Li ${ }^{1}, 3$, Erman Timurdogan', Douglas Coolbaugh'², Diedrik Vermeulen ${ }^{1}$, Michael Watts ${ }^{1} ;{ }^{1}$ MIT, USA; ${ }^{2}$ College of Nanoscale Science and Engineering, Univ. at Albany, USA; ${ }^{3}$ Harvard Univeristy, USA Large-scale optical phased arrays at $635 \mathrm{~nm}$ and $1550 \mathrm{~nm}$ wavelengths are demonstrated with aperture sizes up to $4 \times 4 \mathrm{~mm}^{2}$. A diffraction limited spot with a record $0.021^{\circ} \times 0.021^{\circ}$ divergence and output powers as high as $400 \mathrm{~mW}$ are shown.

\section{STh1M.2 • 08:15}

$1 \times 256$ Multi-layer, low-loss, $\mathrm{Si}_{3} \mathrm{~N}_{4}$ waveguide optical phased arrays with 0.050 Instantaneous-Field-of-View, Chuan Qin ${ }^{1}$ Kuanping Shang', Shaogi Feng', Gengchen Liu', Guangyao Liu'1, Shibnath Pathak' S. J. Ben Yoo'; 'Univ. of California Davis, USA. We report multilayer $1 \times 256 \mathrm{Si}_{3} \mathrm{~N}_{4}$ optical phased arrays with 8-stage multimode interferometer (MMI) tree. The device shows $4.5 \mu \mathrm{m}$ mode size, $3 \mathrm{~dB}$ excess loss and far field pattern with 0.050 beam width.

STh1M.3・08:30

Fresnel-Lens-Inspired Focusing Phased Arrays for Optical Trapping Applications, Jelena Notaros ${ }^{1}$, Christopher V. Poulton ${ }^{1,3}$ Manan Raval', Matthew. Byrd', Douglas Coolbaugh'2, Michael Watts'; 'Research Lab of Electronics, MIT, USA; ${ }^{2}$ College of Nanoscale Science and Engineering, Univ. at Albany, USA; ${ }^{3}$ Analog Photonics, USA. An integrated optical phased array which focuses radiated light in one dimension to a tightly confined spot in the near field is demonstrated for the first time and proposed for chip-scale optical trapping applications.
08:00-10:00

STh1N • Optical Computing \&

Communications using Photonic

Nanostructures

Presider: Dirk Englund; MIT, USA

STh1N.1 • 08:00 Tutorial

Integrated Nanophotonics for Optical Computation, Masaya Notomi ${ }^{1,2},{ }^{1}$ NTT Basic Research Labs, Japan; ${ }^{2}$ NTT Nanophotonics Center, Japan. Recent advances of integrated nanophotonic devices in terms of energy consumption and latency, especially about OE/EO conversion, are reviewed, and their potential application to ultralow-latency optical computing based on optical pass-gate logic will be discussed.

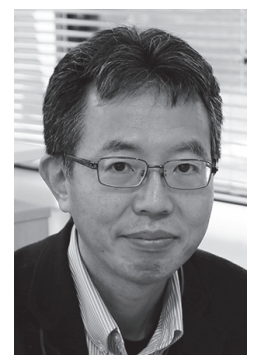

Masaya Notomi received B.E., M.E. and Ph. D. degrees in applied physics from University of Tokyo in 1986, 1988, and 1997. He has been working on physics and devices of nanophotonics. He is currently Senior Distinguished Scientist of NTT Basic Research Laboratories and heading NTT Nanophotonics Center. He is an IEEE Fellow.

\section{8:00-10:00 \\ STh10 - Direct Detection}

Multicarrier Optical

Communications

Presider: Yue-Kai Huang; NEC

Labs America Inc, USA

STh10.1 • 08:00

The High Power Budget IMDD OFDM-PON Down-stream Scheme Employing Sparse Volterra Filter-based Nonlinear Impairment Mitigation, Nan Feng', Nan liu', Chang Liu', Xue Chen', Pengfei Yang'; 'Beijing Univ. of Posts and Telecommunications, China. This paper focuses on sparse Volterra filter in high power budget IMDD OFDM-PON. The simulation results show that the power budget @ $50 \mathrm{Gbps}$ is up to $32 \mathrm{~dB}$ with low complexity equalizer in ONU receivers. The experimental results show that this scheme could achieve 20 Gbps transmission over $95-\mathrm{km}$ SMF.

STh10.2 • 08:15

60-Gbit/s QAM-OFDM Direct-Encoded Colorless Laser Diode Uniform Transmitter for DWDM-PON Channels, Zu-Kai Weng', Huai-Yung Wang', Hsuan-Yun Kao', Cheng-Ting Tsai', Yu-Chieh Chi', Gong-Ru Lin'; 'Graduate Inst. of Photonics and Optoelectronics, National Taiwan Univ., Taiwan. Directly 64-QAM OFDM encoding the colorless laser diode with power-to-frequency pre-leveling is demonstrated for 60-Gbit/s $\mathrm{BtB}$ and 55.8-Gbit/s $25-\mathrm{km}$ fiber transmissions with its selectable channel wavelength to construct uniform transmitter for DWDMPON channels.

STh10.3 • 08:30

Sensitivity Improvement in IM-DD OFDMPON by Amplitude Scaling and Subcarrier Enabled PAPR Reduction, Jizong Peng ${ }^{1}$ Shaohua An', Qingming Zhu', Ciyuan Qiu', Yong Zhang', Yikai Su'; 'Shanghai Jiao Tong Univ., China. An effective sensitivity-improving scheme combining symbol-amplitude scaling and peak-amplitude reduction is proposed for IM-DD OFDM-PONs. Sensitivity improvements of $3.8 \mathrm{~dB}$ and $4 \mathrm{~dB}$ are observed in two ONUs with 40-km and 80-km transmission distances, respectively. 
Executive Ballroom

$210 \mathrm{~A}$

Executive Ballroom

210B

\section{CLEO: Applications}

\& Technology

ATh1A • Biomedical Imaging II-Continued

ATh1A.3 • 09:00

Sparsity-based On-chip Holographic Microscopy, Yair Rivenson ${ }^{1}$, Yichen $\mathrm{Wu}^{1}$, Hongda Wang ${ }^{1}$, Yibo Zhang ${ }^{1}$, Alborz Feizi ${ }^{1}$, Aydogan Ozcan'; ' ${ }^{1}$ Univ. of California Los Angeles, USA. We demonstrate a sparsity-based phase reconstruction technique implemented in wavelet domain to achieve at least 2-fold reduction in the number of holographic measurements for coherent imaging of samples.

\section{ATh1B - Active Remote Environmental Sensing- Continued}

ATh1B.4 • 08:45

High precision $2.0 \mu \mathrm{m}$ Photoacoustic Spectrometer for Determination of the ${ }^{13} \mathrm{CO}_{2} /{ }^{12} \mathrm{CO}_{2}$ Isotope Ratio, Zachary D Reed ${ }^{1}$, Joseph T. Hodges ${ }^{1} ;{ }^{1}$ NIST, USA. A photoacoustic spectrometer operating near $2.0 \mu \mathrm{m}$ has been developed for high precision measurements of the ${ }^{13} \mathrm{CO}_{2} /{ }^{12} \mathrm{CO}_{2}$ isotope ratio. The instrument performance and effects of water vapor on the photoacoustic signal are discussed.

\section{ATh1B.5 • 09:00 Invited}

Locating Methane Leaks Across Large Areas with Frequency Comb Lasers, Gregory B. Rieker ${ }^{1}$, Sean Coburn ${ }^{1}$, Caroline Alden ${ }^{1}$ Robert Wright ${ }^{1}$, Kuldeep Prasad ${ }^{2}$, Subhomoy Ghosh $^{2}$, Garwing Truong ${ }^{3}$, Kevin Cossel ${ }^{3}$ Esther Baumann ${ }^{3}$, lan Coddington ${ }^{3}$, Nate Newbury3: 'Univ. of Colorado at Boulder, USA; ${ }^{2}$ National Inst. of Standards and Tech nology, USA; ${ }^{3}$ National Inst. of Standards and Technology, USA. Recent advancements in mobile frequency comb technology and inverse methods are enabling the location and sizing of small methane leaks across large regions.
Executive Ballroom

$210 \mathrm{C}$

\section{CLEO: Science \&} Innovations

STh1C • III-V Lasers-Continued

\section{FTh1D • Topological}

\section{CLEO: QELS-} Fundamental Science

FTh1D.3 • 08:45

Towards the Experimental Realization of the Topological Insulator Laser, Steffen Wittek ${ }^{2}$, Gal Harari ${ }^{1}$, Miguel A. Bandres ${ }^{1}$ Hossein Hodaei², Midya Parto², Parinaz Aleahmad ${ }^{2}$, Mikael C. Rechtsman ${ }^{3}$, Yidong Chong $^{4}$, Demetrios Christodoulides², Mercedeh Khajavikhan², Mordechai Segev ${ }^{1}$ ${ }^{1}$ Technion, Israel; ${ }^{2}$ Univ. of Central Florida, USA; ${ }^{3}$ The Pennsylvania State Univ., USA ${ }^{4}$ Nanyang Technological Univ., Singapore. We propose a practical design to implement of a topological insulator laser. Due to the topological protection, the topological lase maintains a high slope efficiency and single mode lasing even in the presence of defects and disorder.
STh1C.4 • 09:00

Circular Polarized Lasing Characteristics in Metal/GaN Double-Spiral Nanowire Cavity, Cheng L. Yu', Shu-Wei Liao', Yu-Hao Hsiao', Hao-chung Kuo', Min-Hsiung Shih', ${ }^{1,2}$ ${ }^{1}$ National Chiao Tung Univ., Taiwan: ${ }^{2}$ Research Center for Applied Sciences, Academia Sinica, Taiwan. Room temperature highly circular polarized laser was demonstrated with a compact metal/GaN double-spiral nanowire cavity. The lasing action was observed with a UV wavelength of $363 \mathrm{~nm}$ and a high dissymmetry factor of +1.05 .

\section{FTh1D.4 • 09:00}

Topological Aharonov-Bohm Suppression of Optical Tunneling in Twisted Nonlinear Multicore Fibers, Midya Parto', Helena E. Lopez Aviles', Mercedeh Khajavikhan', Rodrigo A. Correa' ${ }^{1}$ Demetrios Christodoulides ${ }^{1}$ ${ }^{\prime}$ CREOL, USA. We show that the AharonovBohm-like suppression of optical tunneling in twisted multicore fibers can persist unde highly nonlinear conditions. The energy exchange dynamics are analyzed and possible arrangements to experimentally observe this effect are presented.
ATh1A.4 • 09:15

Fiber supercontinuum source for broadband-CARS microscopy based on an allnormal-dispersion mode-locked laser, Yan $\mathrm{Li}^{1}$, Xiaosheng Xiao ${ }^{1}$, Lingjie Kong', Changxi Yang'; 'Tsinghua Univ., China. We propose and demonstrate a fiber supercontinuum source for broadband coherent anti-Stokes Raman scattering microscopy, seeded by an all-normal-dispersion mode-locked laser. We achieve $700-1900 \mathrm{~cm}^{-1}$ spectral range, and show its application in analyzing complex mixture.
STh1C.5 • 09:15

17.6-Gbps Universal Filtered Multi-Carrier Encoding of GaN Blue LD for Visible Light Communication, Yu-Fang Huang', ChengTing Tsai ${ }^{1}$, Hsuan-Yun Kao', Yu-Chieh Chi ${ }^{1}$ Huai-Yung Wang ${ }^{1}$, Tien-Tsorng Shih², Gong$\mathrm{Ru}$. Lin"1; ${ }^{1}$ Graduate Inst. of Photonics and Optoelectronics, National Taiwan Univ., Taiwan ${ }^{2}$ Dept. of Electronic Engineering, National Kaohsiung Univ. of Applied Sciences, Taiwan. TO-can packed GaN LD connected with impedance-matched transmission-line circuit board enhances the UFMC additive 16-QAM OFDM bandwidth to enable 17.6-Gbps VLC at 4.4-bit/s/Hz spectral-usage efficiency with suppressed inter-OFDM-carrier interference and improved BER of $1.07 \times 10^{-4}$.
FTh1D.5 • 09:15

Recasting Hamiltonians with gaugeddriving, Hanan H. Herzig Sheinfux' ${ }^{1}$, Stella Tallulah Schindler', Yaakov Lumer², Mordechai Segev". 'Technion Israel Inst. of Technology, Israel; ${ }^{2}$ Dept. of Electrical and Systems Engineering, Univ. of Pennsylvania, USA. We show how to modify the effective Hamiltonian of a dynamic system in an almost arbitrary fashion, using periodic gauge and driving As an example, we generalize dynamic localization; counteract disorder effects in waveguide lattices. 


\section{Executive Ballroom $210 \mathrm{E}$}

Joint

JTh1E • Quantum Photonics IContinued
Executive Ballroom $210 \mathrm{~F}$
Executive Ballroom

210G
Executive Ballroom $210 \mathrm{H}$

\section{CLEO: QELS-Fundamental Science}

\section{FTh1F • Ultrafast Exciton Dynamics in Van Der Waals Materials-Continued}

FTh1F.3 • 08:45

Ultrafast anisotropic dynamics of nondegenerated excitons in atomically-thin $\operatorname{ReS}_{2}$, Doeon Lee', Sangwan Sim', Sungjun $\mathrm{Cho}^{2}$, Wooyoung Shim ${ }^{2}$, Hyunyong $\mathrm{Choi}^{1}$ ${ }^{1}$ School of Electrical and Electronics Engineering, Yonsei Univ., Korea (the Republic of); ${ }^{2}$ Materials science and Engineering, Yonse Univ., Korea (the Republic of). We report the first anisotropic two excitons dynamics in bulk $\operatorname{ReS}_{2}$. Both exciton states show anisotropic dynamics upon carrier injection with stronger response in higher exciton state than the lower state.

FTh1F.4 • 09:00

Exciton-selective optical Stark effect in two-dimensional group-VII transition metal dichalcogenide $\operatorname{ReS}_{2}$, Sangwan Sim', Doeon Lee ${ }^{1}$ Minii Noh'1 Soonyoung Cha' ${ }^{1}$ Chan $\mathrm{Ho}$ Soh ${ }^{1}$, Ji Ho Sung ${ }^{2}$, Sungjun Choi', Wooyoung Shim', Moon-Ho Jo2, Hyunyong Choi'; ' $Y$ Yonsei Univ., Korea (the Republic of); ${ }^{2}$ Postech, Korea (the Republic of . We present ultrafast optical Stark effect of anisotropic excitons in few layer $\mathrm{ReS}_{2}$. With varying pump-probe polarization configuration, we selectively shift the excitons, based on their anisotropic optical selection rules.

of fully uncorrelated photon pairs in an integrated device. A fully separable state is obtained by independent control over the quality factors of the resonances involved in the parametric fluorescence process.

\section{JTh1E.3 • 09:15}

Hybrid Quantum Photonics, Ali W. Elshaari ${ }^{1}$ Iman Esmaeil Zadeh², Andreas Fognini², Michael E. Reimer ${ }^{3}$, Dan Dalacu ${ }^{4}$, Philip J. Poole ${ }^{4}$ Val Zwiller ${ }^{1}$ Klaus D. Jöns'. ${ }^{1}$ Quantum Nano Photonics Group, Applied Physics, Kungliga Tekniska Högskolan (KTH), Sweden: ${ }^{2}$ Kavli Inst. of Nanoscience, TU Delft, Netherlands; ${ }^{3}$ Inst. for Quantum Computing and Dept. of Electrical \& Computer Engineering, Univ. of Waterloo, Canada; ${ }^{4}$ Nationa Research Council Canada, Canada. We deterministically integrate nanowire quantumemitters in SiN photonic circuits. We generate single-photons, suppress excitation-laser, and isolate specific transitions in the quantumemitter all on-chip with electrically-tunable filter. Finally, we demonstrate a novel Quantum-WDM channel on-chip.

\section{FTh1G • Nonlinear and Hyperbolic Metamaterials- Continued}

\section{FTh1G.3 • 08:45}

Magneto-optical Nanowire Metamaterials, Bo Fan', Mazhar Nasir ${ }^{2}$, Anatoly Zayats², Viktor A. Podolskiy'; ' Univeristy of Massachusetts at Lowell, USA; ${ }^{2}$ hyssics, King's College London, UK. We present effective medium description of nanowire composites that comprise plasmonic and magneto-optical components, a new material platform that may find applications in optical insulator and polarization control technology.

\section{FTh1G.4 • 09:00}

Pulse Shaping for Super-Resolution Imag ing, Andrei Rogov', Evgenii E. Narimanov ${ }^{1}$, 'Purdue Univ., USA. We present a new approach to metamaterial-based superresolution imaging, where optical pulse shaping allows to dramatically reduce the influence of material loss.
FTh1 $\bullet$ Nanoscale Optomechanics-Continued
FTh1F.5 • 09:15

A Fine Structure of Strongly-Bound Interlayer Exciton States in Twisted $\mathrm{Bi}$ layer Graphene, Hiral Patel', Lujie Huang ${ }^{3,2}$ Cheol-Joo Kim,2, Jiwoong Park ${ }^{3,2}$, Matthew W. Graham'; 'Physics, Oregon State Univ. USA; ${ }^{2}$ Chemistry, Univ. of Chicago, USA ${ }^{3}$ Kavli Inst. at Cornell for Nanoscale Science USA. We uncover excitonic states in twisted bilayer graphene using two-photon emission and transient absorption microscopy at the single-grain level. The observed intraband and two-photon spectral peaks imply angledependent exciton binding energies ranging from 0.45 to $0.76 \mathrm{eV}$.
FTh1G.5 09:15

Artificial magnetism in one-dimensional multilayer metamaterials, Georgia Theano Papadakis ${ }^{2}$, Dagny Fleischman ${ }^{3}$, Artur Davoyan ${ }^{1}$, Pochi Yeh ${ }^{4}$, Harry A. Atwater ${ }^{1}$ ${ }^{1}$ California Inst. of Technology, USA; ${ }^{2}$ Applied Physics, California Inst. of Technology, USA; ${ }^{3}$ Material Science, California Inst. of Technology, USA; ${ }^{4}$ Electrical Engineering, Univ. of Santa Barbara, USA. Artificial magnetism is usually investigated in rather complex twoand three-dimensional metamaterials. We propose and experimentally demonstrate non-unity permeability in planar multilayer structures. We further demonstrate the existence of TE hyperbolic modes and magnetic plasmons
FTh1H.2 • 09:00

Near-field, on-chip Optical Brownian Motors, Shao-Hua Wu', Ningfeng Huang', Eric Jaquay', Michelle Povinelli'; ' 'Univ. of Southern California, USA. We show the first demonstration of all-dielectric near-field optical Brownian ratchets of $520 \mathrm{~nm}$ particles with a transport speed of $\sim 1 \mu \mathrm{m} / \mathrm{s}, 10-100$ times greater than other optical ratchets.
FTh1H.3・09:15

Topologically Enabled Optical Nanomotors, Ognjen Ilic', Ido Kaminer', Bo Zhen Owen Miller ${ }^{2}$, Hrvoje Buljan ${ }^{3}$, Marin Soljacic 'Dept. of Physics, Massachusetts Inst. of Techonology, USA; ${ }^{2}$ Dept. of Applied Physics, Yale Univ., USA; ${ }^{3}$ Dept. of Physics, Univ. of Zagreb, Croatia. We show that tailoring the topology of the phase space of the lightparticle interaction is a powerful approach to manipulate particle dynamics. In this manner, we find that optically asymmetric (Janus) particles can become stable nanoscale motors in a light field with zero angular momentum. 


\section{Meeting Room $211 \mathrm{~B} / \mathrm{D}$}

Meeting Room
$212 \mathrm{~A} / \mathrm{C}$
Meeting Room

212 B/D
Marriott

Salon I \& II

CLEO: Science \& Innovations

\section{STh1I $・ 2 \mathrm{D}$ Materials and Devices II-Continued}

STh11.3 • 08:45

Tungsten disulphide saturable absorber for ultrashort pulse generation in all-fiber lasers, Wen Liu',2, Zhiyi Wei2; 'School of Science, Beijing Univ. of Posts and Telecommunications, China; ${ }^{2}$ Chinese Academy of Sciences, China. The $\mathrm{WS}_{2} \mathrm{SA}$ is manufactured with large nonlinearity and high reliability. The modulation depth, SNR and bandwidth are $25.48 \%, 92 \mathrm{~dB}$ and $57 \mathrm{~nm}$, respectively. The pulse duration is $246 \mathrm{fs}$, which is the shortest pulse duration among all-fiber lasers with $\mathrm{WS}_{2} \mathrm{SAs}$.

\section{STh11.4 09:00}

Inkjet-Printing of Graphene Saturable Absorbers for $\sim 2 \mu \mathrm{m}$ Bulk and Waveguide Lasers, Xavier Mateos ${ }^{1,2}$, Pavel Loiko ${ }^{3}$, Josep M. Serres' ${ }^{1}$, Szymon S. Delekta ${ }^{4}$, Esrom Kifle ${ }^{1}$, Alexander Baranov ${ }^{3}$, Magdalena Aguiló ${ }^{\prime}$ Francesc Díaz', Uwe Griebner², Valentin Petrov' ${ }^{2}$ Sergei Popov ${ }^{4}$, Jiantong Li ${ }^{4}$, Mikael Östling ; ${ }^{1}$ Universitat Rovira i Virgili, Spain: ${ }^{2}$ Max Born Inst., Germany; ${ }^{3}$ TMO Univ., Russia; ${ }^{4} \mathrm{KTH}$ Royal Inst. of Technology, Sweden. We report on inkjet-printing of graphene saturable absorbers (SAs) suitable for passive Q-switching of $\sim 2-\mu \mathrm{m}$ bulk and waveguide lasers. Using graphene-SA in a microchip $\mathrm{Tm}: \mathrm{KLu}\left(\mathrm{WO}_{4}\right)_{2}$ laser, $1.2 \mu \mathrm{J} / 136 \mathrm{~ns}$ pulses are generated at $1917 \mathrm{~nm}$.

\section{STh11.5 • 09:15}

Influence of substrates on photocarrier dynamics in monolayer TMDs, Zhonghui $\mathrm{Nie}^{1}$, Yang Cui ${ }^{1}$, Yuze Meng ${ }^{1}$, Yongbing $\mathrm{Xu}^{1}$, Frank (Fengqiu) Wang'; ' ${ }^{2}$ Nanjing Univ., China. We demonstrate that dielectric screening from substrates has a strong influence on photocarrier dynamics in monolayer TMDs. In particular, the interband recombination time is found dramatically shortened with the increase of substrate dielectric constants.
STh1J • Laser Ablation

Fundamentals and Applications-Continued

\section{STh1J.2 • 09:00}

Strong Enhancement of Nanosecond Laser Ablation of Silicon by Axial Magnetic Field Hamid Farrokhi ${ }^{1,2}$, Vitaly Gruzdev ${ }^{3}$, Hongyu Zheng', Wei Zhou'; 'School of Mechanical and Aerospace Engineering, Nanyang Technological Univ., Singapore; ${ }^{2}$ Wellman Center for Photomedicine, Massachusetts General Hospital and Harvard Medical School, USA ${ }^{3}$ Dept. of Mechanical \& Aerospace Engineering, Univ. of Missouri, USA; ${ }^{4}$ Machining Technology Group, Singapore Inst. of Manufacturing Technology, Singapore. Axia constant magnetic field produces 69-fold increase of ablation-crater depth on silicon surface ablated by ultraviolet nanosecond laser pulses. Analysis of potential mechanisms suggests strong combined effects from magneto-absorption and propagation through magnetized ablation plasma.

STh1J.3 • 09:15

High Frequency Core Inductor Using Sintered Aluminum Nano-paste with Aluminum Nano-polycrystalline Structure Shinichiro Masuda', Taku Saiki', Yukio lida', Mitsuru Inada'; ${ }^{1}$ kansai Univ., Japan. We fabricated core inductors with an aluminum nano-polycrystalline structure, which is a ferromagnetic body, for the first time. Al nano-polycrystalline structure using $A$ nanoparticles synthesized by pulse laser worked as inductor cores at 5-MHz frequency.
STh1K $\bullet$ Nonlinear Fiber
Photonics I-Continued

STh1K.4 • 08:45

All-fibered chalcogenide based continuouswave parametric amplification in the mid-infrared, Sida Xing ${ }^{1}$, Davide Grassani ${ }^{1}$, Svyatoslav Kharitonov ${ }^{1}$, Camille-Sophie Bres ${ }^{1}$. ${ }^{1}$ Ecole Polytechnique Federale de Lausanne, Switzerland. We demonstrate parametric amplification around $2 \mu \mathrm{m}$ in a dispersion engineered tapered microstructured chalcogenide fiber. Almost $5 \mathrm{~dB}$ of signal amplification was achieved by $125 \mathrm{~mW}$ coupled power from a thulium-doped fiber pump laser.

\section{STh1K.5 • 09:00 Invited}

Efficient Mid-Infrared Supercontinuum Generation in Tapered Large Mode Area Chalcogenide Photonic Crystal Fibers, Christian R. Petersen ${ }^{4}$, Rasmus E. Dybbro ${ }^{4}$ Christos Markos ${ }^{4}$, Laurent Brilland', Johann Troles ${ }^{3}$, Ole Bang ${ }^{4,2}$; 'Selenoptics, France; ${ }^{2} N K T$ Photonics A/S, Denmark; ${ }^{3}$ Equipe Verres et Céramiques, Université Rennes, France; ${ }^{4}$ DTU Fotonik, Technical Univ. of Denmark, Denmark. Mid-infrared supercontinuum spanning from 1.8-9 $\mu \mathrm{m}$ with an output power of $41.5 \mathrm{~mW}$ is demonstrated by pumping tapered large mode area chalcogenide photonic crystal fibers using a $4 \mu \mathrm{m}$ optical parametric source.
STh1L.3 • 09:00

A Compressor for High Average Power Ultrafast Laser Pulses with High Energies David Alessi', Emily F. Sistrunk ${ }^{1}$, Hoang Nguyen', Paul Rosso', Thomas Spinka1, Michae Aasen ${ }^{1}$, Sandrine Herriot ${ }^{1}$, Jerald Britten ${ }^{1}$ Constantin Haefner'; 'Lawrence Livermore National Lab, USA. We have developed a high-efficiency ( 90\%), broad-bandwidth low-absorption pulse compressor suitable for high energy pulses. This technology is a significant step in enabling high peak power laser systems to operate at high repetition rates.
STh1L.4 09:15

64J Output Energy in 10ns Pulse from Cryogenic Yb:YAG Ceramics Laser, Takashi Sekine', Yasuki Takeuchi' ${ }^{1}$ Yuma Hatano ${ }^{1}$, Yuki Muramatsu', Takashi Kurita', Takaaki Morita' Yoshio Mizuta', Yuki Kabeya', Masateru Kurata', Kazuki Kawai ${ }^{1}$, Takuto Iguchi ${ }^{1}$, Yoshinori Tamaoki ${ }^{1}$, Koichi lyama', Yujin Zheng', Yoshinori Kato'; ${ }^{1}$ Hamamatsu Photonics K. K., Japan. A 64J at 10 ns output was demonstrated by diode-pumped cryogenically cooled Yb:YAG ceramic laser amplifier. An extraction efficiency was evaluated $43.3 \%$ at stored energy of $148 \mathrm{~J}$ with small-signalgain of 20.4 . 


$\begin{array}{llc}\text { Marriott } & \text { Marriott } & \text { Marriott } \\ \text { Salon III } & \text { Salon IV } & \text { Salon V \& VI }\end{array}$

CLEO: Science \& Innovations

STh1M • Phased Arrays Related Device-Continued

\section{STh1M.4 $\bullet$ 08:45}

Star coupler for high-etendue LIDAR, Eric J. Stanton ${ }^{1}$, Nicolas Volet ${ }^{1}$, Tin Komljenovic', John Bowers'; 'Univ. of California Santa Barbara, USA. A 1:480 star coupler is designed for high-etendue LIDAR applications and demonstrated using silicon waveguides. A large 0.96-mm output aperture and dense output waveguide spacing of $2 \mu \mathrm{m}$ allows for a narrow $0.072^{\circ}$ far-field spot.

\section{STh1M.5 • 09:00}

Unidirectional Waveguide Grating Antennas for Nanophotonic Phased Arrays, Manan Raval', Christopher V. Poulton ${ }^{1,2}$, Michael Watts'; ${ }^{1}$ Research Lab of Electronics, MIT, USA $;{ }^{2}$ Analog Photonics, USA. Unidirectional waveguide grating antennas for nanophotonic phased arrays are demonstrated with over $90 \%$ directionality. Unidirectional emission eliminates the fundamental problem of element factor blind spots due to reflections of the antenna radiation within the substrate.

STh1M.6・09:15

Millimeter Long Grating Coupler with Uniform Spatial Output, Moshe Zadka', You-Chia Chang', Aseema Mohanty ${ }^{1,2}$ Christopher Phare ${ }^{1,2}$, Samantha P. Roberts', Michal Lipson'; 'Electrical Enigneering, Columbia Univ., USA; ${ }^{2}$ Cornell Univ., USA. We demonstrate a millimeter long grating coupler with uniform spatial output based on a platform formed by both silicon and $\mathrm{Si}_{3} \mathrm{~N}_{4}$ and engineered grating duty cycle to match a desired profile of the output beam.
STh1N • Optical Computing \& Communications using Photonic Nanostructures-Continued

\section{STh1N.2 • 09:00}

Optical switches based carry-ripple adder for future high-speed and low-power consumption optical computing, Zheng Wang ${ }^{1,2}$, Zhoufeng Ying ${ }^{2}$, Shounak Dhar ${ }^{2}$, Zheng Zhao ${ }^{2}$, David Z. Pan ${ }^{2}$, Ray T. Chen ${ }^{1,2}$; ${ }^{1}$ Texas Materials Inst., Univ. of Texas at Austin, USA; ${ }^{2}$ Electrical and Computer Engineering, The Univ. of Texas at Austin, USA. We report an optical switch based architecture for realizing optical computing. Numerical simulation has been performed to validate the architecture by adopting CMOS compatible PN depletion micro-ring resonator as the approach for optical switching.

STh1N.3・09:15

Superconducting Optoelectronic Platform for Neuromorphic Computing, Sonia M. Buckley', Adam N. McCaughan', Jeffrey Chiles ${ }^{1}$, Richard Mirin ${ }^{1}$, Sae Woo Nam", Jeffrey Shainline'; ${ }^{1}$ NIST, USA. We propose a hybrid semiconductor-superconductor hardware platform for large-scale neuromorphic computing. The platform combines semiconducting few-photon light-emitting diodes with superconducting-nanowire single-photon detectors to behave as spiking neurons.
STh10 • Direct Detection

Multicarrier Optical

Communications-Continued

STh10.4 • 08:45

First Investigation of Set-Partition Format based IM/DD OFDM for Fiber Communications, Jian Zhao', Lian-Kuan Chen'; ' 'Tyndall National Inst., Ireland; ${ }^{2}$ Dept. of Information Engineering, The Chinese Univ. of Hong Kong, Hong Kong. We propose, for the first time, set-partition (SP) format based optical OFDM and experimentally show that SP64QAM/SP-128QAM intensity-modulation direct-detection OFDM exhibits greatly improved performance over conventional 16QAM and 8QAM OFDM.

STh10.5 • 09:00

Simplified Self-Homodyne Detection for Optical OFDM with Inserted Pilot SubSamples and its Application in Downstream of Optical Access Networks, Guo-Wei Lu', Xun Guan ${ }^{3}$, Takahide Sakamoto', Naokatsu Yamamoto ${ }^{1}$, Calvin C. Chan ${ }^{3}$. ${ }^{1}$ National Inst of Info. and Comm Tech, Japan; ${ }^{2}$ Tokai Univ., Japan; ${ }^{3}$ The Chinese Univ. of Hong Kong, Hong Kong. A cost-effective and energyefficient self-homodyne downstream scheme is proposed for WDM-PON with inserted pilot sub-samples and interferometer-based detection. A 10-Gbps self-homodyne OFDM downstream transmission is experimentally demonstrated over 20-km SSMF with errorfree operations.

STh10.6 09:15

Experimental Demonstration of Adaptive 3-D Optimization for Optical Direct-detection OFDM, Yu Zhenming ${ }^{1}$, Yiming Lou', Minghua Chen', Hongwei Chen ${ }^{1}$, Sigang Yang ${ }^{1}$, Shizhong Xie ${ }^{1} ;{ }^{1}$ Tsinghua Univ., China. We propose an adaptive 3-D optimization including bit loading, power loading and TCM for optical direct-detection OFDM in the fading channel. This method is experimentally demonstrated to improve both the receiver sensitivity and capacity. 
Executive Ballroom

$210 \mathrm{~A}$

Executive Ballroom

210B

\section{CLEO: Applications \\ \& Technology}

ATh1A • Biomedical Imaging II-Continued

ATh1A.5 • 09:30

On-chip Microscopy and Nano-particle Detection Using Ultraviolet Light, Mustafa Daloglu', Aniruddha Ray', Zoltán Göröcs', Matthew Xiong', Ravinder Malik', Gal Bitan' Euan McLeod', Aydogan Ozcan'; 'Univ. of California, Los Angeles, USA; ${ }^{2}$ The Univ. of Arizona, USA. Ultraviolet light enhances particle imaging capabilities of on-chip microscopy, increasing the detection sensitivity to sub-30 $\mathrm{nm}$ particles. Ultraviolet illumination also enables high contrast imaging of biomolecules, e.g., protein aggregates, over a large field-of-view.

ATh1A.6 • 09:45

Pixel Super-Resolution in Coherent Microscopy Systems Through Out-of-Focus Imaging, Hongda Wang', Zoltán Göröcs', Wei Luo', Yibo Zhang', Yair Rivenson', Aydogan Ozcan'; 'UCLA, USA. We introduce a widefield pixel super-resolution method that uses a stack of out-of-focus images to provide better utilization of the space-bandwidthproduct of an objective-lens and improve the throughput of lens-based coherent imaging and holography systems.
ATh1B - Active Remote Environmental SensingContinued

\section{ATh1B.6 09.30 Invited}

Chirped-laser Dispersion Spectroscopy for Large-area Methane Detection, Gerard Wysocki'; ${ }^{1}$ Electrical Engineering, Princeton Univ., USA. This work presents an overview of Chirped Laser Dispersion Spectroscopy (CLaDS) implementations to large area methane monitoring and recent developments in sensitivity enhancement techniques.

10:00-15:00 Exhibition Open, Exhibit Hall 1, 2 \& 3

10:00-11:30 Coffee Break (10:00-10:30) and Unopposed Exhibit Only Time, Exhibit Hall 1, 2 \& 3

10:15-13:00 Technology Transfer Program, Exhibit Hall Theater 
Executive Ballroom
$210 \mathrm{E}$

Joint

JTh1E • Quantum Photonics IContinued

JTh1E.4 • 09:30

Time-Domain Observation of Vacuum Rabi Oscillations in a Strongly Coupled Quantum Dot-Nanocavity System, Kazuhiro Kuruma', Yasutomo Ota', Masahiro Kakuda' Satoshi Iwamoto', Yasuhiko Arakawa'; ' $U$ Univ. of Tokyo, Japan. We report the time-domain observation of vacuum Rabi oscillations in a single quantum dot-photonic crystal nanocavity system under optical carrier injection. A significantly-improved cavity $Q$-factor facilitates the direct access to the ultrafast exciton-photon dynamics.

JTh1E.5 • 09:45

Determining the Optical Nonlinearity of Silicon at Cryogenic Temperatures for Applications in Integrated Photonics, Nicola A. Tyler ${ }^{1}$, Gary Sinclair ${ }^{1}$, Gerardo E. Villarreal ${ }^{1}$ Geraint Gough1, Jorge Baretto', Döndü Sahin', Mark Thompson'; ' ${ }^{1} \mathrm{HH}$ Wills Physics Lab, Bristol, UK. We determine the nonlinear coefficient of a silicon wire waveguide from room temperature down to $3.8 \mathrm{~K}$. Measurements are taken of the self-phase modulation and two-photon absorption.

\section{Executive Ballroom 210F}

Executive Ballroom

210G
Executive Ballroom

$210 \mathrm{H}$

\section{CLEO: QELS-Fundamental Science}

FTh1F • Ultrafast Exciton Dynamics in Van Der Waals Materials-Continued

FTh1F.6 • 09:30 Invited

Ultrafast XUV ARPES Studies of Electron and Exciton Dynamics in the Transition Metal Dichalcogenide $\mathrm{MoSe}_{2}$, Jan H. Buss ${ }^{1}$ Frederic Joucken ${ }^{1}$, Julian Maklar ${ }^{1}$, He Wang ${ }^{1}$ Yiming $\mathrm{Xu}^{1}$, Rohit Unni', Changhyun $\mathrm{Ko}^{2}$ Sefaattin Tongay ${ }^{3}$, Junqiao $\mathrm{Wu}^{2}$, Robert A Kaindl': ${ }^{1}$ Materials Sciences Divison, Lawrence Berkeley National Lab, USA; ${ }^{2}$ Dept. of Materials Science and Engineering, UC Berkeley, USA; ${ }^{3}$ School for Engineering of Matter, Transport and Energy, Arizona State Univ., USA. We exploit extreme-UV angle-resolved photoemission spectroscopy (XUV ARPES) for sensitive ultrafast studies of non-equilibrium carriers in $\mathrm{MoSe}_{2}$. The experiments reveal inter-valley scattering and provide the first evidence for the observation of band-gap excitons in ARPES.
FTh1G • Nonlinear and Hyperbolic MetamaterialsContinued

\section{FTh1G.6 • 09:30 Invited}

Nonlinear Optics of Plasmonic Metamaterials, Anatoly Zayats ${ }^{1} ;{ }^{1}$ King's College London, UK. Nonlinear optical properties of plasmonic metamaterials will be overviewed focusing on achieving enhanced Kerr-type nonlinearity in a desired spectral range and with controlled ultrafast response. Nonlinear control of light polarization will be discussed.

10:00-15:00 Exhibition Open, Exhibit Hall 1, 2 \& 3

10:00-11:30 Coffee Break (10:00-10:30) and Unopposed Exhibit Only Time, Exhibit Hall 1, 2 \& 3

10:15-13:00 Technology Transfer Program, Exhibit Hall Theater 


\section{Meeting Room $211 \mathrm{~B} / \mathrm{D}$}

Meeting Room
$212 \mathrm{~A} / \mathrm{C}$
Meeting Room
$212 \mathrm{~B} / \mathrm{D}$
Marriott

Salon I \& II

\section{CLEO: Science \& Innovations}

\section{STh1I • 2D Materials and Devices II-Continued}

\section{STh11.6 09:30}

Realizing thermal strain of patterned sapphire substrates dominate the bandgapshifted of bilayer $\mathrm{MoS}_{2}$, Sheng-Wen Wang ${ }^{1}$, Henry Medina ${ }^{2}$, Kuo-Bin Hong', Chun-Chia $\mathrm{Wu}^{1}$, Manikandan Arumugam ${ }^{2}$, Teng-Yu Su ${ }^{2}$ Po-Tsung Lee', Yu-Lun Chueh², Hao-chung Kuo'; ${ }^{1}$ Dept. of Photonics \& Inst. of ElectroOptical Engineering, National Chiao Tung Univ., Taiwan; ${ }^{2}$ Dept. of Materials Science and Engineering, National Tsing Hua Univ., Taiwan. Using thermal strain concept, we can tune the bandgap of bilayer $\mathrm{MoS}_{2}$ through the two different thermal expansion coefficients of sapphire. Also, we propose a simple model to explain and precisely predict the bandgap-shifted behavior.

\section{STh11.7 • 09:45}

Dipole aligned energy transfer between excitons in 2D semiconductors and organic

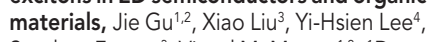
Stephen Forrest ${ }^{3}$, Vinod M. Menon ${ }^{1,2} ;{ }^{1}$ Dept. of Physics, City College of New York of the City Univ. of New York, USA; ${ }^{2}$ Dept. of Physics, Graduate Center of the City Univ. of New York, USA; ${ }^{3}$ Dept.s of Electrical Engineering and Computer Science and Physics, Univ. of Michigan, USA; ${ }^{4}$ Dept. of Materials Science and Engineering, National Tsing Hua Univ. Taiwan. Energy transfer from low mobility material to high mobility material is essential for optoelectronic application. We demonstrate Foster energy transfer from organic material (PTCDA) to monolayer MoSe2 though steady state and transient photoluminescence spectroscopy.

\author{
STh1J • Laser Ablation \\ Fundamentals and \\ Applications-Continued
}

STh1J.4 • 09:30

Fabrication of Superconducting Micro Particles by Laser Ablation in Superfluid Helium, Masaaki Ashida', Yosuke Minowa' Mitsutaka Kumakura ${ }^{2}$, Yuta Takahashi ${ }^{3}$ Fusakazu Matsushima ${ }^{3}$, Yoshiki Moriwaki ${ }^{3}$ ${ }^{1}$ Graduate School of Engineering Science, Osaka Univ., Japan; ${ }^{2}$ Dept. of Applied Physics, Univ. of Fukui, Japan; ${ }^{3}$ Dept. of Physics, Univ. of Toyama, Japan. Superconducting micro particles have been selectively trapped using a quadrupole magnetic field after the production of metallic micro particles by laser ablation in superfluid helium. Size dependence of the superconducting transition temperature will be discussed.

\section{STh1 J.5 • 09:45}

Imaging Nanosecond Ablation of Copper at Low Ambient Pressure, Alexander W. Raymond ${ }^{1}$, Eric Mazur ${ }^{1} ;{ }^{1}$ Harvard Univ., USA. The ablation of copper at low pressure by nanosecond pulses is imaged in a shadowgraph experiment. The ambient pressure affects the expansion dynamics as evidenced by the shock wave and damage spot.

\section{STh1K • Nonlinear Fiber Photonics I-Continued}

\section{STh1K.6 • 09:30}

Ultrafast Kerr-Driven Beam Cleanup in Graded-Index Multimode Fiber, Zhanwe Liu', Logan Wright', Demetrios Christodoulides ${ }^{2}$, Frank W. Wise ${ }^{1} ;{ }^{1}$ Cornell Univ., USA ${ }^{2}$ Univ. of Central Florida, USA. We observe a nonlinear spatial self-cleaning process with ultrashort pulses in multimode fiber. Experiments and simulations demonstrate its origin to be nonlinear interactions between the fiber spatial modes, due exclusively to the Kerr effect.

\section{STh1L $\bullet$ High Average Power Lasers-Continued}

STh1L.5 • 09:30

Multi-mJ, 1kHz, $3.1 \mu \mathrm{m}$ OPCPA, Susannah Wang ${ }^{1}$, Michael Gerrity', Sterling J. Backus ${ }^{2}$, Margaret Murnane ${ }^{1,2}$, Henry Kapteyn ${ }^{1,2}$, Seth L. Cousin'; ' JILA, Univ. of Colorado, Boulder, USA; ${ }^{2} K M L a b s$, USA. We present a mid-IR OPCPA laser producing multi-mJ femtosecond pulses at $1 \mathrm{kHz}$. The beam profile of the $3.1 \mu \mathrm{m}$ idler is excellent, enabling efficient coupling into high-gas-pressure waveguides, required for phase matched $1 \mathrm{keV}$ high harmonic generation.

\section{STh1K.7 • 09:45}

Broadband supercontinuum generation in tapered multimode graded-index optical fibers, Mohammad Amin Eftekhar ${ }^{1}$, Z. Sanjabi Eznaveh', Jose E. Antonio-Lopez', Juan Carlos Alvarado Zacarias', Axel Schülzgen' Miroslav kolesik², Frank W. Wise ${ }^{3}$, Rodrigo A. Correa', Demetrios Christodoulides'; ' 'Univ. of Central Florida, USA; ${ }^{2}$ College of Optical Sciences, The Univ. of Arizona, USA; ${ }^{3}$ School of Applied and Engineering Physics "Cornell Univ., USA. We experimentally demonstrate for the first time uniform and broadband supercontinuum generation in long tapered multimode fibers. This is achieved through an accelerated geometric parametric instability that forces the sidebands towards higher/ lower frequencies.

\section{STh1L.6 • 09:45}

High Power (9.2 W) CW 4.15 $\mu \mathrm{m}$ Fe:ZnSe laser, Dmitry V. Martyshkin', Vladimir V. Fedorov', Mike Mirov', Igor Moskalev' ${ }^{1}$, Sergey Vasilyev', Viktor Smolski ${ }^{1}$, Andrey Zakrevskiy ${ }^{1}$ Sergey B. Mirov'; ' ${ }^{1}$ PG Photonics Mid-IR Lasers, USA. We report to the best of our knowledge the highest output power of 9.2 W Fe:ZnSe laser operating in CW regime. The lasing wavelength was at $4.15 \mu \mathrm{m}$ in non-selective cavity at 77K.

10:00-15:00 Exhibition Open, Exhibit Hall 1, 2 \& 3

10:00-11:30 Coffee Break (10:00-10:30) and Unopposed Exhibit Only Time, Exhibit Hall 1, 2 \& 3 


$\begin{array}{llc}\text { Marriott } & \text { Marriott } & \text { Marriott } \\ \text { Salon III } & \text { Salon IV } & \text { Salon V \& VI }\end{array}$

CLEO: Science \& Innovations

STh1M • Phased Arrays Related Device-Continued

STh1M.7 • 09:30

Low-loss arrayed waveguide grating at 2.0 um, Eric J. Stanton ${ }^{1}$, Nicolas Volet ${ }^{1}$, John Bowers'; ' 'Univ. of California Santa Barbara, USA. A low-loss arrayed waveguide grating operating at 2.0- $\mathrm{mm}$ wavelength is demonstrated with an average on-chip loss of $0.5 \mathrm{~dB}$ and crosstalk per channel of $-30.2 \mathrm{~dB}$. These are the lowest reported values for a silicon AWG at 2.0- $\mu \mathrm{m}$ wavelength.
STh1M.8 09:45

Nanotriangle Decorated Silicon Nitride Waveguides for Integrated SurfaceEnhanced Raman Spectroscopy, Piete Wuytens ${ }^{1,3}$, Andre G. Skirtach ${ }^{2,3}$, Roel Baets ${ }^{1,3}$ ${ }^{1}$ INTEC - Photonics Research Group, Ghent Univ. - imec, Belgium; ${ }^{2}$ Dept. of Molecular Biotechnology, Ghent Univ., Belgium; ${ }^{3}$ Center for Nano- and BioPhotonics, Ghent Univ., Belgium. Nanosphere lithography provides an e-beam free method for patterning gold nanoplasmonic antennas. By combining this technique with deep-UV photolithography, we fabricate $\mathrm{Si}_{3} \mathrm{~N}_{4}$ waveguides interfaced to plasmonic antennas capable of exciting and collecting surface-enhanced Raman spectra.
STh1N • Optical Computing \& Communications using Photonic Nanostructures-Continued

STh1N.4 • 09:30

Photonic Physical Unclonable Functions using Silicon Nitride Spiral Cavities, Bryan Bosworth', Brian C. Grubel', A. Brinton Cooper ${ }^{1}$, Mark A. Foster ${ }^{1}$, Amy Foster ${ }^{1}$; ${ }^{1}$ The Johns Hopkins Univ., USA. We demonstrate an on-chip photonic physical unclonable function using integrated evanescently coupled multimode spiral cavities formed in silicon nitride. Hongcheng Sun', Milad Alemohammad",

\section{STh10 • Direct Detection \\ Multicarrier Optical}

Communications-Continued

STh10.7 • 09:30

$80 \mathrm{Gbit} / \mathrm{s}$ Single-Channel Direct Detection Optical FBMC Signal Generation and Transmission at 2- $\mu \mathrm{m}$, Qiong $W u^{2}$, Yongqiang $\mathrm{Xie}^{1}$, Ke Xu1, Ruoxu Wang ${ }^{2}$, Ming Tang ${ }^{2}$, Songnian $\mathrm{Fu}^{2}$, Deming Liu2. ${ }^{1}$ Harbin Inst. of Technology, Shenzhen, China; ${ }^{2}$ Huazhong Univ. of Science and Technology, China. We experimentally generated single wavelength $80 \mathrm{Gbit} / \mathrm{s}$ signals at $1952 \mathrm{~nm}$ with intensity modulated filter bank multicarrier scheme. Its transmission over $100 \mathrm{~m}$ solid core SMFs was demonstrated with BER below FEC limit of $3.8 \times 10^{-3}$.

\section{STh1N.5 • 09:45}

Encrypted Communication using Chaotic Silicon Photonic Microcavities, Brian C. Grubel', Bryan Bosworth', Michael Kossey', Amy Foster ${ }^{1}$, A. Brinton Cooper ${ }^{1}$, Mark A. Foster'1: 1 Johns Hopkins Univ., USA. We demonstrate encrypted two-party communications using a physically-secure one time pad incorporating two CMOS-compatible chaotic silicon photonic microcavities.

10:00-15:00 Exhibition Open, Exhibit Hall 1, 2 \& 3

10:00-11:30 Coffee Break (10:00-10:30) and Unopposed Exhibit Only Time, Exhibit Hall 1, 2 \& 3

10:15-13:00 Technology Transfer Program, Exhibit Hall Theater 


\section{0:00-12:00 JTh2A • Poster Session III}

\section{JTh2A.1}

Monitoring Aerosol Profiles in the Bahamas Using a Portable Bistatic Camera Lidar Amin S. Kabir ${ }^{1}$, Nimmi Sharma ${ }^{2}$, John E. Barnes $^{3}$, Jalal Butt2. 'Univ. of the Bahamas, Bahamas; ${ }^{2}$ Central Connecticut State Univ., USA; ${ }^{3}$ NOAA/ESRL/Global Monitoring Division, USA. A wide-angle camera based bistatic lidar is used to profile aerosols in the Bahamas. Aerosol extinction was derived and boundary layer top agrees with radiosonde relative humidity demonstrating CLidar utility for in-situ environmental characterization.

\section{JTh2A.2}

Refillable and Reconfigurable Dye-doped Ring Lasers, Hengky Chandrahalim ${ }^{1}$, Stephen C. Rand', Xudong Fan'; 'Univ. of Michigan, USA. We present refillable, ultralow-thresholds, and wavelength reconfigurable ring lasers on a fused-silica chip. These devices will enable various photonic and biochemical sensing applications that require sustainable, configurable, and lowthreshold coherent light sources on a chip.

\section{JTh2A.3}

Non-Imaging Perceptual Hashing Recognition Based on Ghost Imaging System. Hui c. Chen ${ }^{1}$, Jianhong Shi', Guihua Zeng ${ }^{1}$ 'Shanghai Jiao Tong Univ., China. We propose and experimentally demonstrate non-imaging perceptual hashing algorithm based on ghost imaging system can realize non-imaging recognition of image. This technique could find applications in image recognition.

\section{JTh2A.4}

Polarization invariance in beam propagation for space-to ground optical communication downlink, Jiajie $\mathrm{Wu}^{1}$, Jing $\mathrm{Ma}^{1}$, Liying Tan', Siyuan Yu'; ${ }^{1}$ Harbin Inst. of Technology China. The sufficient condition for Gaussian Schell-model beam to keep the polarization properties unchanged for a space-to-ground downlink is derived. The results indicate when three coherence lengths $\left(\delta_{x x \prime} \delta_{y y}\right.$ and $\left.\delta_{x y}\right)$ are equal to each other, the beam maintains the polarization properties on propagation

\section{JTh2A.5}

Probing Temperature Gradient inside SOFC using Fiber with Enhanced Rayleigh Scattering Profiles, Kevin P. Chen', sheng huang', Aidong Yan', Michael Buric ${ }^{2}$, Paul Ohodnicki ${ }^{2}$, Shiwoo Lee ${ }^{2} ;{ }^{1}$ Univ. of Pittsburgh USA; ${ }^{2}$ National Energy Technology Lab, USA Temperature gradient inside a solid-oxide fuel-cell interconnect channel was measured using a distributed fiber sensor with enhanced Rayleigh scattering profile. Reliable temperature measurements were achieved during the operation of a SOFC with 4-mm spatial resolution.

\section{JTh2A.6}

Laser threshold estimation for organic VCSEL, LEI ZENG ${ }^{1}$, Mahmoud Chakaroun ${ }^{1}$ Azzedine Boudrioua'; ' 'Laboratoire de Physique des Lasers, France. The organic VCSEL is modeled by the transfer matrix method. This method is capable to calculate the eigenmode and estimate the laser threshold. The exciton population density at threshold for a typical organic VCSEL is $1.4 \times 1017 \mathrm{~cm}-3$. With a complete OLED structure, the threshold rises to $6 \times 1018 \mathrm{~cm}-3$ due to the absorption of charge injection and transport layers.
JTh2A.7

Particle Emission Source Tracking by a Scanning Scheimpflug Lidar System, Liang Mei ${ }^{1}$, Yang Yang ${ }^{1}$, Peng Guan ${ }^{1}$; ${ }^{1}$ Dalian Univ. of Technology, China. A scanning Scheimpflug lidar system is developed for in situ monitoring of particle emission source. Field campaigns were performed in by scanning the near-ground atmosphere of the urban area in a haze weather condition.

\section{JTh2A.8}

Development of Double-Pulsed Two-Micron Laser for Atmospheric Carbon Dioxide Measurements, Mulugeta Petros ${ }^{1}$, Tame Refaat ${ }^{1}$, Jirong $\mathrm{Yu}^{1}$, Upendra Singh ${ }^{1} ;{ }^{1} \mathrm{NASA}$ Langley Research Center, USA. A CO ${ }_{2}$ lidar double-pulse two-micron high-energy transmitter, tuned to on- and off-line absorption wavelengths, has been developed. Transmitter operation and performance has been verified on ground and airborne platform.

\section{JTh2A.9}

Temperature sensor based on a Core-offset Mach-Zehnder Interferometer with single mode fiber, Rosario Penaloza'; 'UGTO, Mexico. The MZI was implemented by coreoffset fusion. The output power spectrum of the MZI showed a stripe contrast around $20 \mathrm{~dB}$. The experimental results showed a change in wavelength due to a temperature change applied.

\section{JTh2A.10}

Application of TMDs in Nano-Absorbers: An Impression, Sajal Agarwal ${ }^{1}$, Yogendra Kumar Prajapati'; ' Motilal Nehru National Inst. of Tech, India. This study provides an insight of TMDs to be used as nano-absorber. Individual 2-D materials have been studied for their absorbance. It is seen that 2-D materials are good absorber for certain wavelength region.

\section{JTh2A.11}

Recyclable Air Fuel Cells Using Sintered Nanopastes with reduced $\mathrm{Mg}$ nanoparticles prepared by Pulse Laser Ablation in Liquids for Solar Energy Cycle, Taku Saiki Shigeaki Uchida ${ }^{2}$, Seiji Taniguchi ${ }^{3}$; ${ }^{1}$ Kansai Univ., Japan; ${ }^{2}$ Osaka Univ., Japan; ${ }^{3}$ Inst. for Laser Technology, Japan. MgO particles were reduced to $\mathrm{Mg}$ nanoparticles by using ns-pulse lasers, which is converted from solar light. Pastes with reduced Mg nanoparticles were sintered. We succeeded in generating electricity by air cells using the pastes.

\section{JTh2A.12}

Optional output mode based on doublering external cavity lasers, Chao Wang ${ }^{1}$, Xia Li', Changyun Zhao', Hao Jin', Qiang Zhou', Hui Yu', Jianyi Yang', Xiaoging Jiang1; 'Zhejiang Univ., China. An external cavity laser based on double-ring mirror which has optional output mode, has been presented. Double-crest curve and two single-crest curves are observed. They are corresponding to hybrid mode, TE and TM mode, respectively.

\section{JTh2A.13}

Nonlinear core-shell Yagi-Uda nanoantenna for highly tunable directive emission, $\mathrm{Al}$ exandr Krasnok ${ }^{1}$, Olga Sergaeva ${ }^{2}$, Roman Savelev ${ }^{3}$, Denis Baranov ${ }^{4}$, Andrea Alu ${ }^{1}$ 'The Univ. of Texas at Austin, USA; ${ }^{2}$ Dept. of Mechanical \& Aerospace Engineering Univ. of Missouri, USA; ${ }^{3}$ ITMO Univ., Russia; ${ }^{4}$ Chalmers Univ. of Technology, Sweden. We propose a novel type of tunable Yagi-Uda nanoantenna composed of metal-dielectric core-shell nanoparticles and suggest method for flexible dynamical tuning of the nanoantenna emission pattern via electron hole plasma excitation.

\section{JTh2A.14}

Ultrafast Time-Resolved Studies on the Influence of Spin Excitations in Dysprosium Thin Film, Azize Koc', Alexander von Reppert ${ }^{2}$, Jan-Etienne Pudell2, Matthias Reinhardt ${ }^{1}$, Karine Dumesnil ${ }^{3}$, Daniel Schick ${ }^{1}$ Cristian Schüßler-Langeheine ${ }^{1}$, Marc Herzog ${ }^{2}$ Flavio Zamponi', Matias Bagheer ${ }^{2,1}$; ${ }^{1} \mathrm{Helm}$ holtz-Zentrum Berlin, Germany; ${ }^{2}$ Institut für Physik \& Astronomie, Universität Potsdam, Germany; ${ }^{3}$ nstitut Jean Lamour, Universite Lorraine, France. We investigate the struc tural and thermal nonequilibrium dynamics including the spin-reordering dynamics of an antiferromagnetic Dysprosium (Dy) nanolayer after excitation with fs-laser pulses by means of ultrafast structural and magnetic x-ray diffraction experiments.

\section{JTh2A.15}

Robust density matrix modeling of quantum cascade laser kinetics, Benjamin A Burnett ${ }^{1,2}$, Andrew Pan ${ }^{2}$, Prineha Narang ${ }^{1,3}$ Benjamin Williams ${ }^{2} ;{ }^{1}$ Northrop Grumman Corporation, USA; ${ }^{2}$ Electrical Engineering, Univ. of California Los Angeles, USA; ${ }^{3}$ Faculty of Arts and Sciences, Harvard Univ., USA We present an electron transport model for quantum-cascade lasers which naturally captures scattering-induced localization without a phenomenologically localized basis. Its numerical lightness enables the study of nonperturbative optical excitation and optical nonlinearity.

\section{JTh2A.16}

Carrier-Envelope Phase-Dependent Coherence in Two-Level Systems Interacting with Few-Cycle Pulse Pairs, Bing Zeng ${ }^{1}$, Lingze Duan ${ }^{1 ;}{ }^{1}$ Univ. of Alabama in Huntsville, USA We report a numerical study of the carrierenvelope phase-sensitive population inversion and polarization in two-level systems interacting with few-cycle optical pulse pairs.

\section{JTh2A.17}

Ultrafast Spectroscopy Using Coherent Wavepackets, Brian Kamer ${ }^{1}$, Jean-Claud M. Diels', Ladan Arissian",2; 'Univ. of New Mexico, USA; ${ }^{2}$ National Research Council of Canada, Canada. We present the signature of coherent wavepacket induced by strong field laser in the emission spectrum of plasma. Time dependent contribution of rotational states and phase relation between " $\mathrm{P}$ " and " $\mathrm{R}$ " branch is presented.

JTh2A.18

Withdrawn

JTh2A.19

High energy-resolution measurements of $x$-ray into ultraviolet parametric downconversion with an $\mathrm{x}$-ray tube source, Denis Borodin', Shiran Levy', Sharon Shwartz' 'Bar Ilan Univ., Israel. We describe first high energy-resolution measurements of parametric down-conversion of $x$-rays into ultraviolet using an $\mathrm{x}$-ray tube. The technique is atomic selective and can be used for the study of electronic properties with atomicscale resolution.

\section{JTh2A.20}

Ultrafast Phonon Dynamics in GraphenehBN Structures, Dheeraj Golla1, Alexandra Brasington', Brian LeRoy', Arvinder Sandhu', 'Physics, Univ. of Arizona, USA. We present ultrafast pump-probe measurements that show fast relaxation of carriers in graphenehexagonal Boron Nitride (hBN) heterostruc tures due to the high interfacial therma conductance of the graphene-hBN interface. This warrants the use of $h B N$ as a substrate for high powered graphene devices.

\section{JTh2A.21}

Charging Dynamics of Single InGaAs Quantum Dots under Resonant Excitation Gary R. Lander ${ }^{1}$, Disheng Chen ${ }^{1}$, Samantha D. Isaac', Glenn S. Solomon², Edward Flagg'; ${ }^{1}$ West Virginia Univ., USA; ${ }^{2}$ National Inst. of Standards and Technology, USA. We investigate the rates of charge state fluctuation in single InGaAs quantum dots under resonant excitation and with an additional low-power above-band laser. Multiple charging processes are identified.

\section{JTh2A.22}

Interband Effects on Hot Carrier Relaxation in Titanium Nitride Films, Heather Ferguson ${ }^{1}$, Urcan Guler ${ }^{2}$, Nathaniel Kinsey ${ }^{2}$, Vladimir M. Shalaev², Theodore Norris ${ }^{1}$, Alexandra Boltasseva ${ }^{2} ;{ }^{1}$ Univ. of Michigan, USA; ${ }^{2}$ Purdue Univ., USA. Hot carrier cooling rates were measured using transmission pump-probe on $30 \mathrm{~nm}$ TiN films. Experiments used a 400nm pump/800nm probe and a $650 \mathrm{~nm}$ pump/ $800 \mathrm{~nm}$ probe. The bandstructure gives insight into the long cooling times observed.

\section{JTh2A.23}

The Influence of the Substrate Material on the optical properties of Tungsten Diselendide Monolayers, Lorenz M. Schneider ${ }^{1}$ Sina Lippert ${ }^{1}$, Dylan Renauld ${ }^{1}$, Jan Kuhnert ${ }^{1}$ Kyung Nam Kang ${ }^{2}$, Obafunso Ajayi ${ }^{3}$, Marc Uwe Halbich ${ }^{1}$, Oday M. Abdulmunem ${ }^{1}$ Xing Lin ${ }^{1}$, Khaleel Hassoon', Saideh EdalatiBoostan', Young Duck Kim³ ${ }^{3}$, Wolfram Heimbrodt', Eui-Hyeok Yang ${ }^{2}$, James C. Hone ${ }^{3}$ 


\section{0:00-12:00 JTh2A • Poster Session III}

\section{JTh2A.24}

Photo-Induced Correlated Spin-Density Wave State Formation in Overdoped Pnictide Superconductors, Martin Mootz ${ }^{1}$ \|lias E. Perakis', Liang Luo ${ }^{2,3}$, Aaron Patz ${ }^{2,3}$ Xu Yang ${ }^{2,3}$, Sergey L. Bud'ko ${ }^{2,3}$, Paul C. Canfield ${ }^{2,3}$, Jigang Wang ${ }^{2,3}$; 'Dept. of Physics, Univ. of Alabama at Birmingham, USA ${ }^{2}$ Dept. of Physics and Astronomy, lowa State Univ., USA; ${ }^{3}$ Ames Lab, U.S. DOE, USA. The non-equilibrium dynamics of superconducting order after the ultrafast gap quenching is analyzed. Evidence is shown for the formation of a photo-excited correlated spin-density wave state that can be controlled by adjusting pump laser's intensity.

\section{JTh2A.25}

Crystallographic Orientation-Dependent Dynamics in Individual Silicon Nanowires, Michael R. Williams ${ }^{1}$, Mel F. Hainey Jr. ${ }^{2}$ Aidan L. O'Beirne ${ }^{1}$, Joan M. Redwing ${ }^{2}$, Rohit P. Prasankumar'; ' 'Los Alamos National Lab, USA; ${ }^{2}$ Dept. of Materials Science, Materials Research Inst., The Pennsylvania State Univ. USA. Ultrafast optical microscopy was used to study carrier dynamics in individual Si nanowires with different crystallographic orientations. We found that the carrier lifetime in $\langle 110\rangle$-oriented nanowires increased with decreasing diameter, in contrast with previous observations.

\section{JTh2A. 26}

First-principles calculations for saturable absorption in graphite, Mitsuharu Uemoto ${ }^{1}$ Shintaro Kurata ${ }^{2}$, Norihito Kawaguchi ${ }^{2}, \mathrm{Ka}$ zuhiro Yabana ${ }^{1} ;{ }^{1}$ Center for Computational Sciences, Univ. of Tsukuba, Japan; ${ }^{2}$ Advanced Applied Science Dept., Research Lab, IH Corporation, Japan. We present first-principles calculations for saturable absorption in graphite based on time-dependent density functional theory. It is found that the saturation takes place for ultrashort pulses stronger than $10^{10} \mathrm{~W} / \mathrm{cm}^{2}$

JTh2A.27

Tunable Magnonic spectra in two dimensional $\mathrm{Ni}_{80} \mathrm{Fe}_{20}$ annular lattices, Prasanta $\mathrm{K}$. Datta ${ }^{1}$, Nikita Porwal ${ }^{1}$; 1 Physics and Meteorology, Indian Inst. of Technology Kharagpur, India. Investigation on ultrafast time resolved spin wave (SW) dynamics of 2-D Ni ${ }_{20} \mathrm{Fe}_{20}$ (Py) annular antidot lattice with varying externa magnetic field has been performed. Multiple frequency modes observed in experiment are verified by micromagnetic simulations.

\section{JTh2A.28}

Modification of Energy Bands of a Dielectric Crystal by Pondermotive Potential of Gaussian Ultrashort Laser Pulse, Olga N. Sergaeva', Vitaly Gruzdev'1; ' Univ. of Missouri, USA. Ultrafast modification of direct-gap parabolic energy bands of a dielectric crystal by high-intensity Gaussian ultrashort laser pulse is theoretically studied. Non-trivia dynamics of laser-driven Bloch oscillations of electrons result in formation of transient indirect bands.
JTh2A.29

Generation of Cooler, Ultrafast Electron Packets Via Mid-IR Driven Nanostructures Phillip D. Keathley ${ }^{1}$, Peter Krogen ${ }^{1}$, Wil liam Putnam ${ }^{1}$, Michael Swanwick ${ }^{2}$, Jeffrey A. Moses², Luis Velasquez-Garcia², Franz Kaertner ${ }^{1,3}$; ${ }^{1}$ Electrical Eng. and Computere Science, MIT and Research Lab of Electronics, USA; ${ }^{2}$ Massachussets Inst. of Technology Mictosystems and Tech. Lab., USA; ${ }^{3}$ Cente for Free Electron Laser Science, DESY, Germany. A detailed comparison of ultrafast electron emission from structured, silicon nano-tips driven by $800 \mathrm{~nm}$ and $2.1 \mu \mathrm{m}$ pulses was performed. In the low energy portion of the spectrum, a saturation of the direct electron energy bandwidth to $\sim 1.6 \mathrm{eV}$ fo $800 \mathrm{~nm}$ and $\sim 1 \mathrm{eV}$ for $2.1 \mathrm{um}$ was observed.

\section{JTh2A.30}

Excited-state nonlinearities of $\operatorname{Ir}(\mathrm{III}) \mathrm{com}$ plexes, Salimeh Tofighi', Himansu S. Pattanaik', Peng Zhao', Mykhailo Bondar ${ }^{2}$, Ryan M. $\mathrm{O}^{\prime}$ Donnell ${ }^{3}$, Jianmin Shi ${ }^{3}$, David Hagan ${ }^{1}$, Eric Van Stryland $1 ;{ }^{1}$ CREOL, USA; ${ }^{2}$ Inst. of physiscs NASu, Ukraine: ${ }^{3}$ US Army research Lab, USA. An investigation of $\mathrm{Ir}(\mathrm{III})$ complexes has been performed using double-pump probe (DPP) experiments to decouple the triplet quantum yield and triplet cross-section of these complexes. Both femtosecond and picosecond DPP measurements are presented.

JTh2A.31

Predictive theory of optical nonlinearity due to conduction electrons, Shukai $Y u$ Kate H. Heffernan', Diyar Talbayev'; 'Tulane Univ., USA. An experimental and computational study of optical nonlinearity due to intense terahertz electric field is presented. We propose a model based on Drude parameters, realistic band structure, and semiclassical electron dynamics to describe the nonlinearity.

\section{JTh2A.32}

Laser-Excited Spin Waves and Special Extrinsic Damping in $\mathrm{CO}_{2} \mathrm{FeAl}_{0.5} \mathrm{Si}_{0.5}$ Alloy Films, Zhifeng Chen ${ }^{1,2}$, Feipeng $\mathrm{Pi}^{1}$, Yong Yan², Wenan Li', Jun Peng'; ' ${ }^{1}$ Guangzou Univ. China; ${ }^{2}$ Sun Yat-Sen Univ., State-Key Lab of Optoelectronic Materials and Technologies, China. Backward volume and perpendicula standing spin waves are simultaneously excited and observed in full-Heusler $\mathrm{Co}_{2} \mathrm{FeAl}_{0.5} \mathrm{Si}_{0.5}$ films using TR-MOKE spectroscopy. The dispersion relations and the special extrinsic damping are analyzed.

\section{JTh2A.33}

Exceptional Points in Hybridized Plasmonic Systems, Ashok Kodigala', Thomas Lepetit ${ }^{1}$ Boubacar Kante'; 'Univ. of California, San Diego, USA. We present evidence of the existence of exceptional points (EPs) in three-dimensional (3D) plasmonic nanostructures. The systems are composed of coupled plasmonic nanoresonators and can be judiciously driven to EPs by controlling symmetry-compatible modes.

\section{JTh2A.34}

Polarized SHG spectroscopy for three-fold rotationally symmetric Au triangular nanoprism at LSP resonances, Atsushi Sugita Hirofumi Yogo', Atsushi Ono', Yoshimasa Kawata'; 'Shizuoka Univ., Japan. Polarized SHG spectroscopies were performed for three-fold rotationally symmetric Au triang lur nanoprisms. The LSP-enhanced SHG radiations arose from excitations due to not only the near-fields oscillating within the triangula surface but those perpendicular to it.

\section{JTh2A.35}

The unique characteristics of SHG from extreme nano-sized bi-metal structure, Avi Nivi ${ }^{1}{ }^{1}$ Inst.s for desert research, Ben-Gurion Univ. of the Negev, Israel. New type of noninear optics from nanosized metal structure is presented. This source has the potential of surpassing anything known to date and may even lead to high harmonics, sub harmonics, and chaos.

\section{JTh2A.36}

Mid-Infrared Plasmonic Coaxial Nanoring for Surface Enhanced Infrared Absorption (SEIRA) Spectroscopy, Che Chen'1, Daehan Yoo' Nathan Youngblood' Sang-Hyun Oh

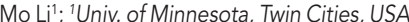
The plasmonic resonance of metallic coaxia nanorings were measured in mid-IR spectra range. Benefiting from a coherent lase source and lock-in measurement scheme, the resonance peaks from as few as 13 apertures were observed.

\section{JTh2A.37}

A Graphene Based Plasmonic Antenna Design for Communication in the $\mathrm{THz}$ Regime, Christoph Suessmeier ${ }^{1}$, Stephan Schaeffer ${ }^{1}$, Sergi Abadal², Eduard Alarcón² Seyed Ehsan Hosseininejad ${ }^{2}$, Anna Katharin Wigger ${ }^{1}$, Daniel Stock ${ }^{1}$, Stefan Wagner ${ }^{1}$ Albert Cabellos-Aparicio², Max Lemme Peter Haring Bolivar'; ' 'Universität Siegen, Germany; ${ }^{2}$ N3Cat (NaNoNetworking Center in Catalunya), Spain. We show the first $\mathrm{TH}$ emission of a graphene based plasmonic antenna structure. Furthermore we present the minimum material requirements for an operational graphene antenna in terms of chemical potential $\mu_{\mathrm{c}}$ and relaxation time $\mathrm{T}$.

JTh2A.38

Directional and enhanced emission by single gold nanorod, Guowei Lu', Hongming Shen', Qihuang Gong1; 'Peking Univ. China. We demonstrated unidirectiona enhanced fluorescence from a nanodiamond modulated by a gold nanorod. The hybrid system was assembled via atomic force microscope nanomanipulation and directiona emission can be controlled by adjusting the configuration.

\section{JTh2A.39}

Focusing Properties of a Cascaded Asymmetric Microstructure under Gaussian Beam Illumination, Jinlong Zhu', Lynford Goddard"; 'Univ. of Illinois at UrbanaChampaign, USA. We compare the focusing of Gaussian and plane wave illumination for a cascaded asymmetric microstructure. Although the Gaussian beam generates onger and wider photonic nanojet, it can still focus efficiently for non-optimal microstructure geometries.

JTh2A.40

Evanescent-Vacuum-Enhanced Reversible Photon-Exciton Interaction and fluorescence collection efficiency, Ying $\mathrm{Gu}^{1}$, Juanjuan Ren', dongxing Zhao', Fan Zhang', Tiancai Zhang', Qihuang Gong'; ' Peking Univ. China: ${ }^{2}$ Shangxi Univ, China Taking the evanescent vacuum provided by a silver or dielectric single-mode nanowire as electromagnetic background, we theoretically demonstrated the enhanced reversible photon-exciton interaction and fluorescence collection efficiency through the careful optical mode design.

\section{JTh2A.41}

Withdrawn.

\section{JTh2A.42}

A Variable Transmission Thin Film for Vis ible Light, Brian Roberts ${ }^{1}$, Megha Ghosh Pei-Cheng Ku'; ' Univ. of Michigan, Ann Arbor, USA. A variable transmission system for visible light is proposed. The $(0 \sim 100 \%)$ transmission filter with negligible image distortion is realized with a multiscale architecture of an array of metal rods on a corrugated actuating surface.

\section{JTh2A.43}

Disordered Nanoantennas : A New Paradigm in Space-Division Multiplexing, Mohammad Haghtalab', Safieddin SafaviNaeini'; ' 'Univ. of Waterloo, Canada. A nove approach for information multiplexing is in troduced. The degrees of freedom provided by disordered structures can be employed for realizing information processing devices. Disordered highly coupled nanoantennas are investigated for this purpose.

\section{JTh2A.44}

Optical Antenna Enhanced Spontaneous Emission from CVD-Grown Monolayer WS $_{2}$, Mohammad H. Tahersima', Muhammad Danang Birowosuto ${ }^{2}$, Zhizhen Ma' William C. Coley $^{3}$, Michael Valentin33, I-Hsi Lư ${ }^{3}$ Ke Liu', Yao Zhou ${ }^{4}$, Amy Martinez ${ }^{3}$, Ingrid Liao ${ }^{3}$, Brandon Davis ${ }^{3}$, Joseph Martinez ${ }^{3}$, Sahar Naghi$\mathrm{bi}^{3}$, Dominic Martinez-Ta ${ }^{3}$ Allison Guan ${ }^{3}$ Ariana E. Nguyen ${ }^{3}$, Cesare Soci ${ }^{2}$, Evan Reed ${ }^{4}$ Ludwig Bartels ${ }^{3}$, Volker J. Sorger'; ' 1 Dept. of Electrical and Computer Engineering, The George Washington Univ., USA; ${ }^{2}$ Division of Physics and Applied Physics, Nanyang Technological Univ., Singapore; ${ }^{3}$ Chemistry Materials Science \& Engineering and Electrical Engineering, Univ. of California, Riverside, USA; ${ }^{4}$ Dept. of Materials Science and Engineering, Stanford Univ., USA. We report on efficacy of light emission enhancement of CVD-grown $\mathrm{WS}_{2}$ films under monomer and dimer plasmonic nanoantennas separated by a spacer layer from the $\mathrm{WS}_{2}$ emitter.

\section{JTh2A.45}

Hybrid Coupling of Excitons, Plasmonic and Photonic Modes in Organic-dyedoped Nanostructures, Ruwen Peng ${ }^{1}$, Kun Zhang ${ }^{1}$, Yue Xu' ${ }^{1}$, Wen-Bo Shi ${ }^{1}$, Ren-Hao 


\section{0:00-12:00 JTh2A • Poster Session III}

\section{JTh2A.46}

Low Loss Volume Modes in a Slab of Lamellar Hyperbolic Metamaterial, Samantha R. Koutsares ${ }^{1}$, Kevin E. Tanyi ${ }^{1}$ Michael Admassu', Ilya Shadrivov², Roman S. Savelev ${ }^{3}$, Mikhail Noginov'; ${ }^{1}$ Norfolk State Univ., USA; ${ }^{2}$ Nonlinear Physics Center Australian National Univ., Australia; ${ }^{3}$ Lab of Nanophotonics and Metamaterials, ITMO Univ., Russia. We have studied, theoretically and experimentally, coupling to propagating volume modes in a lamellar metal/dielectric metamaterial with hyperbolic dispersion. Highly efficient light penetration though tens of metamaterial's layers suggests reasonably low propagation loss.

\section{JTh2A.47}

Spontaneous Emission of Electric and Magnetic Dipole Transitions in Plasmonic Gratings and Strips Arrays, Soheila Mashhadi', Marvin Clemmons', Danielle Gable ${ }^{1}$ Jade Griffin ${ }^{1}$, Natalia Noginova'; ${ }^{1}$ Norfolk State Univ., USA. Spontaneous emission of $\mathrm{Eu}^{3+}$ is studied in the vicinity of gold gratings and nanostrip arrays. Radiation patterns of magnetic and electric transitions show features associated with plasmonic excitations and a narrow reflection anomaly in the strips.

\section{JTh2A.48}

Photo-acoustic Spectroscopy of Resonant Absorption in III-V Semiconductor Nanowires, Teemu Hakkarainen', Grigore Leahu², Emilija Petronijevic ${ }^{2}$, Alessandro Belardini ${ }^{2}$ Marco Centini², Roberto Li Voti ${ }^{2}$, Eero Koivusalo', Marcelo Rizzo Piton', Mircea Guina' Concita Sibilia2; ${ }^{1}$ Optoelectronics Research Centre, Tampere Univ. of Technology, Finland; ${ }^{2}$ SBAl Dept., Sapienza Univ. of Rome Italy. We show that photo-acoustic spectroscopy allows determination of the nanowire absorbance properties including resonan peak positions and peak broadening due to collective ensemble properties. Furthermore, we demonstrate chiral optical response in asymmetrically Au-coated nanowires.

\section{JTh2A.49}

Modification of UV Surface Plasmon Resonances in Aluminum Hole-Arrays with Graphene, Yunshan Wang', Sourangsu Banerji', Jieying Mao', Sara Arezoomandan', Berardi Sensale-Rodriguez', Steve Blair'; 'Univ. of Utah, USA. In this work we study the UV transmission through monolayer graphene films transferred on top of aluminum holearrays. Interaction of graphene pi-plasmons with surface plasmon resonances leads to strong wavelength shifts.

\section{JTh2A.50}

Enhanced soft X-ray high-harmonic generation driven by two-color $(\omega+3 \omega)$ mid-IR laser pulses, Anne-Laure Calendron², Jonathas Siquiera ${ }^{3,1}$, Cheng Jin ${ }^{4}$, Peter Krogen ${ }^{1}$, Tobias Kroh $^{1,2}$, Phillip D. Keathley', Houkun Liang ${ }^{1}$, Edison Falcao-Filho ${ }^{5}$, Chii-Dong Lin ${ }^{4}$, Kyung Han Hong ${ }^{1}$, Franz X. Kärtner 1,2, ${ }^{1}$ Research Lab of Electronics, MIT, USA; ${ }^{2}$ Center for FreeElectron Laser Science, Deutsches Elektronen Synchrotron, Germany; ${ }^{3}$ Grupo de Fotônica, Instituto de Fisica de São Carlos, Brazil: ${ }^{4} \mathrm{~J}$. R. Macdonald Lab, Dept. of Physics, Kansas State Univ., USA; ${ }^{5}$ Departamento de Fisica Universidade Federal de Pernambuco, Brazil. We demonstrate efficiency enhancement of soft X-ray high-harmonic generation from $\mathrm{A}$ in the range of 40-200 eV using two-colo $(\omega+3 \omega)$ mid-infrared to visible pulses with relative phase control, which is in qualitative agreement with $3 \mathrm{D}$ simulations.

\section{JTh2A.51}

Control of Laser Induced Couplings in Autoionizing States by XUV Transient Absorption, Chen-Ting Liao ${ }^{1}$, Nathan Harkema ${ }^{1}$, Arvinder Sandhu'; ; ${ }^{1}$ Univ. of Arizona, USA. Using XUV transient absorption, we investigate and control the excitations between autoionizing states of argon with a femtosecond pulse. We observed and calculated a lineshape transition between Breit-Wigner to Beutler-Fano profiles via varying laser polarization.

\section{JTh2A.52}

Gouy Phase Shift for Annularly Truncated Beam Profiles in Attosecond Pump-Probe Measurements, Fabian Schlaepfer ${ }^{1}$, André Ludwig', Matteo Lucchini', Lamia Kasmi' Mikhail Volkov', Lukas Gallmann", Ursula Keller'; ' 'ETH Zurich, Switzerland. We performed a detailed measurement of the Gouy phase of an infrared beam reflected on a drilled mirror and demonstrated its influence on attosecond measurements with two spatially separated targets.

JTh2A.53

Strong Field Double-lonization of Water Greg A. McCracken², Chelsea LiekhusSchmaltz ${ }^{1}$, Andreas Kaldun ${ }^{1}$, Philip Bucksbaum 1,2; 'Physics, Stanford Univ., USA; ${ }^{2}$ Applied Physics, Stanford Univ., USA. The dissociation of water into $\mathrm{OH}+-\mathrm{H}+$ in $40-\mathrm{fs}$ laser pulses shows tunnel ionization out of lower- lying orbitals. Our kinematically complete experiment identifies ionization pathways by scanning wavelength $(266 \mathrm{~nm}$ to $800 \mathrm{~nm}$ ) and intensity.

\section{JTh2A.54}

Petahertz optical switching in air-plasma, Hao Teng ${ }^{1}$, Xin Lu', Lifeng Wang ', shiyou chen', xinkui He' ${ }^{1}$, Zhiyi Wei ${ }^{1}$; ${ }^{1}$ Inst. of Physics, Chinese Academy of Sciences, China. The modulation of electron conductivity of air-plasma with carrier-envelope phase (CEP) stabilized 7-fs laser pulses was demonstrated which originates from the CEP-induced modification of electric field of laser pulses which is nonlinear amplified during its propagation.

\section{JTh2A.55}

Accelerating Beam-Driven Generation of Isolated Few-cycle EUV and X-ray Pulses, Liang Jie Wong', Ido Kaminer';' 'SIMTech, Singapore; ${ }^{2}$ MIT, USA. We propose a scheme that leverages the spatial structure of accelerating beams to achieve isolated and highly-directional few-cycle EUV and X-ray pulses, circumventing the need for extremely short driver pulse durations.

\section{JTh2A.56}

Undulators from Hyperbolic Phonon Polaritons, Nicholas Rivera', Liang Jie Wong ${ }^{2}$ Ido Kaminer', Marin Soljacic'; ${ }^{1} M I T$, USA ${ }^{2}$ SimTECH, Singapore. We show that mid-IR polaritons in polar dielectrics can be used as a nanoscale undulator for extreme optical up-conversion. We identify unique regimes where quantum recoil significantly influences the properties of the output photon.

\section{JTh2A.57}

General Formalism for Dynamical Sym metries and Selection Rules in High Harmonic Generation, Ofer Neufeld ${ }^{1,2}$, Oren Cohen ${ }^{1,2}$; 'Physics Dept., Technion - Israel Inst. of Technology, Israel; ${ }^{2}$ Solid State Inst. Technion - Israel Inst. of Technology, Israel. We formulate a group theory for (2+1)D Flo quet systems and utilize it for mapping the dynamical symmetries and selection rules in high harmonic generation, including elliptica dynamical symmetry that controls the high harmonics ellipticity.

\section{JTh2A.58}

Towards a High-Energy Sub-Cycle 4-12 $\mu \mathrm{m}$ Laser, Yanchun Yin ${ }^{1}$, Andrew Chew ${ }^{1}$, Xiaom ing Ren ${ }^{1}$, Jie Li' ${ }^{1}$ Yang Wang ${ }^{1}$, Yi Wu ${ }^{1}$, zenghu chang'; ${ }^{1}$ Univ. of Central Florida, USA. We present an approach for generation, amplification, and compression of 4-12 $\mu \mathrm{m}$ pulses by tailoring the phase matching of ZGP in broadband-pumped dual-chirped optical parametric amplification and by indirect pulse shaping

\section{JTh2A.59}

Experimental Generation of a 64-QAM by Optically Aggregating Three Independent QPSK Channels using Nonlinear Wave Mixing of Multiple Kerr Comb Lines, Ahmad Fallahpour ${ }^{1}$, Morteza Ziyadi', Amirhossein Mohajerin-Ariaei ${ }^{1}$, Arne Kordts ${ }^{2}$ Maxim Karpov², Martin Pfeiffer ${ }^{2}$, Changjing Bao ${ }^{1}$, Peicheng Liao ${ }^{1}$, Yinwen $\mathrm{Cao}^{1}$, Ahmed Almaiman ${ }^{1}$, Fatemeh Alishahi ${ }^{1}$, Bishara Shamee ${ }^{1}$, Loukas Paraschis ${ }^{6}$, Moshe Tur ${ }^{5}$ Carsten Langrock ${ }^{4}$, Martin M. Fejer ${ }^{4}$, Joe Touch $^{3}$, Tobias J. Kippenberg ${ }^{2}$, Alan E. Willner': ' ${ }^{1}$ niv. of Southern California, USA ${ }^{2} E$ cole Polytechnique Federale de Lausanne, Switzerland; ${ }^{3}$ Information Sciences Inst., USA ${ }^{4}$ Stanford Univ., USA; ${ }^{5} \mathrm{Tel}$ Aviv Univ., Israel, Infinera Corporation, USA. We experimentally demonstrate an arbitrary optical higher order QAM generation using single stage nonlinear element and Kerr frequency comb. We successfully generated $80-\mathrm{Gbit} / \mathrm{s}$ 16-QAM and $120 \mathrm{Gbit} / \mathrm{s} 64-\mathrm{QAM}$ at EVM of $6.5 \%$ and $5.5 \%$ by multiplexing two and three 40-Gbit/s QPSK signals, respectively.

\section{JTh2A.60}

Estimating the Performance of Fully Loaded DWDM Systems with Multidimensional Modulation, Ahmed I. Abd El-Rahman

John C. Cartledge'; 'Queen's Univ. at Kingston, Canada. An efficient procedure is presented for estimating the performance of multidimensional modulation formats. It allows the explicit properties of signal constellations to be captured and is applicable to fully loaded DWDM transmission systems.

\section{JTh2A.61}

Analog and Digital Performance of Multiple Discrete Time Delays based on a Fibe Loop with an Internal Frequency Shifter, Fatemeh Alishahi', Amirhossein MohajerinAriaei', Ahmed Almaiman', Morteza Ziyadi' Yinwen $\mathrm{CaO}^{1}$, Peicheng Liao ${ }^{1}$, Ahmad Fallahpour', Changjing Bao ${ }^{1}$, Bishara Shamee ${ }^{1}$ Shlomo Zach², Nadav Cohen², Moshe Tur ${ }^{2}$ Alan E. Willner'; ' 'Univ. of Southern California USA; ${ }^{2} T e l$ Aviv Univ., Israel. A fiber loop with an internal frequency shifter is used to produce the delayed copies of an incoming data signal. The linear phase response of the system is verified and the spurious free dynamic range (SFDR) is measured. Also, the BER and constellation diagrams of $10 \mathrm{Gbaud}$ QPSK signals are obtained.

\section{JTh2A.62}

Multi-Dimensional Formats for Flexible Optical Networks with Cascaded Optical Add/Drop Nodes, Yukui Yu ${ }^{1,2}$, Wei Jia ${ }^{3}$ Ning Deng ${ }^{3}$, Wei Wang ${ }^{2}$, Jian Zhao'; ${ }^{1}$ Tyndall National Inst., Ireland; ${ }^{2}$ Harbin Engineering Univ., China; ${ }^{3}$ Huawei Technologies Co. Ltd. China. We propose 8-dimensional 81920AM to balance the nonlinear transmission performance and add/drop induced optical filtering. Simulations show that this format outperforms conventional PDM-160AM PDM-8QAM, and 4-dimensional 1280AM for 200-Gbit/s/channel optical networks with cascaded add/drop nodes.

\section{JTh2A.63}

Implementation of Nyquist OTDM Signal Demultiplexing Using a Single IQ Modulator, Lei Yue ${ }^{1}$, Deming Kong ${ }^{1}$, Yan Li ${ }^{1}$, Jian $\mathrm{Wu}^{1}$; 'State Key Lab of Information Photonics and Optical Communications, Beijing Univ. of Posts and Telecommunications, China. High-performance demultiplexing of Nyqusit OTDM signal is demonstrated using a single commercially-available $\mathrm{IQ}$ modulator. $6.8 \mathrm{~dB}$ required-OSNR improvement (@BER=1E-4) is observed compared with Gaussian sampling with the same pulse-width.

\section{JTh2A.64}

Experimental Verification of Four Wave Mixing in Lumped Optical Transmission Systems that Employ Mid-Link Optical Phase Conjugation, Mohammad Al-Khateeb', Mary E. McCarthy ${ }^{1}$, Andrew D. Ellis'; 'Aston Univ. UK. We experimentally validate an analytica description of four wave mixing generated in lumped amplification systems that employ optical phase conjugation. The experimenta results show good agreement with theoretica predictions within an error margin of $0.5 \mathrm{~dB}$. 


\section{0:00-12:00 JTh2A • Poster Session III}

\section{JTh2A.65}

Optical and Electrical Equalizers for Fiber Optic Links, Xuhan Guo ${ }^{1}$, David Cunning ham1, Richard V. Penty', lan White'; 'Univ. of Cambridge, UK. Optical equalizers and electrical equalizers for a fiber link has been explicitly studied and compared. The results demonstrate that both can improve optical links significantly but optical equalizers can perform better in terms of noise enhancement.

\section{JTh2A.66}

Mode-Dependent Loss Mitigation Scheme for PDM-640AM Few-Mode Fiber SpaceDivision-Multiplexing Systems via STBCMIMO Equalizer, Yi Weng ${ }^{1}$, Xuan $\mathrm{He}^{1}$, Wang Yao', Michelle Pacheco', Junyi Wang ${ }^{2}$ Zhongqi Pan'; ' Univ. of Louisiana at Lafayette, USA; ${ }^{2}$ Qualcomm Technologies, USA. A space-time block-coding algorithm is purposed for mode-dependent loss mitigation in few-mode communication systems with PDM-64QAM, which increase convergence speed by $34.1 \%$ over conventional leastmean-squares method, with $71.6 \%$ hardware complexity reduction over recursive-leastsquares approach.

\section{JTh2A.67}

Kerr Superoscillator Model for Microresonator Frequency Combs, Jonathan M. Silver ${ }^{1}$, Changlei Guo ${ }^{1,2}$, Leonardo Del Bino', Pascal Del'Haye'; 'National Physical Lab, UK; ${ }^{2}$ Xiamen Univ., China. We present an intuitive frequency-domain model of microresonator-based frequency combs in which large numbers of comb modes act as a few superoscillators. Our model is linked to a recently-developed description of periodic pulse patterns (soliton crystals)

\section{JTh2A.68}

Comb offset frequency measurement using two-photon-three-photon quantum interference control, Kai Wang ${ }^{2}$, rodrigo A. Muniz', John Sipe ${ }^{1}$, Steven T. Cundiff2; ' Univ. of Toronto, Canada; ${ }^{2}$ Physics, Univ. of Michigan, USA. Using the directional photocurrent induced by quantum interference between two- and three-photon absorption in a semiconductor ( $\mathrm{AlGaAs})$, we measure the comb offset frequency of a mode-lock fiber laser.

\section{JTh2A.69}

Modulation-Free Frequency-Stabilized Laser at $1.5 \mu \mathrm{m}$ Using a Narrow-Linewidth Diode Laser, Kazumichi Yoshii ${ }^{1,2}$, Takuya Inamura', Hiroyuki Sagawa', Yu Asahina', Ken'ichi Nakagawa ${ }^{3}$, Feng-Lei Hong ${ }^{1,2}$; ${ }^{1}{ }$ Okohama National Univ., Japan; ${ }^{2} J S T$, ERATO MINOSHIMA Intelligent Optical Synthesizer Project, Japan; ${ }^{3}$ Inst. of Laser Science, Univ. of Electro-Communications, Japan. We demonstrate high-precision spectroscopy of acetylene using a narrow-linewidth diode laser at $1.5 \mu \mathrm{m}$. Modulation-free laser output is obtained with a linewidth of a few $\mathrm{kHz}$ and a stability of $3.6 \times 10^{-12}$ (averaging time $=1 \mathrm{~s}$ ).
JTh2A.70

Study of AM-to-PM Conversion in p-i-n Photodiodes with Varying Beam Radius and Spot Position, Lanbing Kang ${ }^{1}$, Brian H. Kolner ${ }^{1,2} ;{ }^{1}$ Electrical and Computer Engineering, Univ. of California, Davis, USA; ${ }^{2}$ W. W. Hansen Experimental Physics Lab, Physics Dept. Stanford Univ., USA. The AM-to-PM conversion gain of a silicon $\mathrm{p}$-i-n photodiode was found to be dependent on laser beam radius and spot position on the photosensitive area.

JTh2A.71

Digital holography using multiple synthesized wavelengths cascaded by optical frequency synthesizer, Masatomo Yamagiwa' Takayuki Ogawa', Yusuke Kawahito', Takeo Minamikawa' ${ }^{1}$, Hirotsugu Yamamoto², Takesh . Yasui', Clement Torovato3. 'Tokushima Univ. Japan; ${ }^{2}$ Utsunomiya Univ., Japan; ${ }^{3}$ Univ. of Bordeaux, France. To expand the dynamic range of depth in digital holography, 4 synthesized wavelengths were cascaded within the range of $1.5 \mu \mathrm{m}$ to $3 \mathrm{~mm}$ by an optical frequency synthesizer phase-locked to an optical frequency comb.

\section{JTh2A.72}

Dual-comb mid-infrared spectroscopy with free-running oscillators and complete optical calibration from a radio-frequency reference, OGUZHAN KARA' ${ }^{1}$, Zhaowei Zhang ${ }^{1,3}$ Tom Gardiner ${ }^{2}$, Derryck T. Reid ${ }^{1}$; ${ }^{1}$ Heriot-Watt Univ., UK; ${ }^{2}$ Emissions and Atmospheric Metrology Group, National Physical Lab, UK; ${ }^{3}$ School of Optical and Electrical Information, China. By using free-running femtosecond $\mathrm{OPOs}$ with a repetition-rate difference of $\sim 500 \mathrm{~Hz}$ we demonstrate methane absorption spectroscopy at a resolution $<0.08 \mathrm{~cm}^{-1}$, fully calibrated in the optical domain from repetition-rate and carrier-envelope-offse frequency measurements.

JTh2A.73

Phase Sensing Beyond Standard Quantum Limit with a Truncated SU(1,1) Interferometer, Prasoon Gupta ${ }^{1}$, Brian Anderson ${ }^{1}$ Bonnie Schmittberger ${ }^{1}$, Travis Horrom ${ }^{1}$ Carla Hermann Avigliano', Kevin Jones ${ }^{2}$ Paul Lett1,3: ' Joint Quantum Inst., Univ. of Maryland, College Park, USA; ${ }^{2}$ Dept. of Physics, Williams College, USA; ${ }^{3}$ Quantum Measurement Division, National Inst. of Standards and Technology, USA. We present an SU $(1,1)$ interferometer variation where the second nonlinear interaction is replaced with homodyne detection. Sensitivity measure ments as a function of phase demonstrate that our device beats the standard quantum limit by $4 \mathrm{~dB}$.

JTh2A.74

Nitrogen-Vacancy Ensemble Magnetometry Based on Pump Absorption, Sepehr Ahmadi', Haitham A. R. El-Ella', Jørn B. Hansen ${ }^{1}$, Alexander Huck', Ulrik L. Andersen'1 'Danmarks Tekniske Universitet, Denmark. We demonstrate magnetic field sensing by recording the variation in the pump light absorption with nitrogen-vacancy center en semble. At a frequency of $10 \mathrm{mHz}$ we obtain a noise floor of $\sim 30 \mathrm{nT} / \sqrt{ } \mathrm{Hz}$.
JTh2A.75

Absolute Group Refractive Index Measurement of Air Using Frequency-sweeping Interferometry Calibrated by Frequency Comb, Yang Lijun', Haoyun Wei', Honglei Yang', Yan Li'; ' ${ }^{1}$ Tsinghua Univ., China. Realtime absolute group refractive index of air a $1545 \mathrm{~nm}$ is measured using frequency comb calibrated frequency-sweeping interferometry by recording the calibration peaks and interference signal simultaneously. For ambient air, a combined uncertainty of $8.6 \times 10^{-8}$ is achieved.

\section{JTh2A.76}

Formation of a Real-Time Time Scale with Fiber-Based Frequency Transfer Network Yichen Guo ${ }^{1}$, Bo Wang ${ }^{1}$, Hongwei Si ${ }^{1}$, Jingwen Dong', Lijun Wang',2: 'State Key Lab of Precision Measurement Technology and Instruments, Dept. of Precision Instrument Tsinghua Univ., China; ${ }^{2}$ Dept. of Physics, Tsinghua Univ., China. This paper introduces the formation of a physical real-time time scale using data of remote clocks from different time Labs in Beijing region linked via fiber-based high-precision frequency transfer network

\section{JTh2A.77}

Repetition rate multiplication of fiberbased optical frequency comb with a long fiber-based ring cavity, Yoshiaki Nakajima ${ }^{1,2}$ Akiko Nishiyama ${ }^{1,2}$, Satoru Yoshida ${ }^{1,2}$, Takuya Hariki', Kaoru Minoshima ${ }^{1,2}$; ${ }^{1}$ The Univ. of Electro-Communications, Japan: ${ }^{2} J a p a n ~ S c i-$ ence and Technology Agency (JST), ERATO MINOSHIMA Intelligent Optical Synthesizer (IOS) Project, Japan. A long-fiber-based ring cavity has been applied to repetition rate multiplication of a fiber-based optical frequency comb with a multiplication factor of 5 Mode filtering of a 48.73- $\mathrm{MHz}$ repetition rate fiber comb generates a 243.65-MHz-comb.

\section{JTh2A.78}

$\mathrm{Nd}_{\mathrm{x}} \mathrm{Y}_{1.00-\mathrm{x}} \mathrm{Al}_{3}\left(\mathrm{BO}_{3}\right)_{4}$ Nanocrystalline Powders: Multi-functional Materials for Random Laser Tunability and Sensor Applications, Andre de Lima Moura ${ }^{1}$, Vladimir Jerez ${ }^{2}$ Sandra Carreño ${ }^{3}$, Pablo Pincheira ${ }^{3}$, Zanine Fabris ${ }^{4}$, Lauro Maia ${ }^{4}$, Anderson Gomes ${ }^{3}$, Cid de Araújo ; ; ${ }^{1}$ Campus Arapiraca, Universidade Federal de Alagoas, Brazil; ${ }^{2}$ Grupo FIELDS, Universidad de Investigacion y Desarrollo, Colombia; ${ }^{3}$ Departamento de Física, Universidade Federal de Pernambuco, Brazil; ${ }^{4}$ Grupo Física de Materiais, Instituto de Física, Universidade Federal de Goiás, Brazil. The use of $\mathrm{Nd}_{x} \mathrm{Y}_{1.00-x} \mathrm{Al}_{3}\left(\mathrm{BO}_{3}\right)_{4}$ nanocrystalline powders as Random Lasers allowed observation of selfsecond-harmonic and self-sum-frequency generation of tunable UV-blue light. An optical thermometer operation based on a twocolor random laser was also demonstrated.

\section{JTh2A.79}

Cascaded Soliton Self-Compression and Mid-Infrared Supercontinuum Generation in $X^{(2)}$-Structured KTP and KTA, Anne-Lise Viotti ${ }^{1}$, Robert Lindberg ${ }^{1}$, Fredrik Laurell ${ }^{1}$ Valdas Pasiskevicius'; ' ${ }^{1}$ Royal Inst. of Technology, Sweden. Periodically poled KTP and KTA were numerically investigated fo nonlinear pulse compression in the norma and anomalous dispersion regimes using cascading $X^{(2)}$ interactions. The generation of sub-10 fs octave-spanning supercontinuum pulses is shown.

JTh2A.80

Nitrogen Laser Guide Star Using Four Wave Mixing, Brian Kamer' ${ }^{1}$, Ali Rastegari M Rasoulof', Ladan Arissian ${ }^{1,2} ;{ }^{1}$ Univ. of New Mexico, USA: ${ }^{2}$ National Research Council of Canada, Canada. We present stimulated emission from nitrogen molecular cation using $800 \mathrm{~nm}$ ultrashort pulses. The 427 $\mathrm{nm}$ lines are dominated by strong field ionization and the $391 \mathrm{~nm}$ are driven by four wave mixing.

\section{JTh2A.81}

Enhanced Four-Wave-Mixing of Inversely Tapered Asymmetric C-rich SiC Channe Waveguide, Cai-Syuan $\mathrm{Fu}^{1}$, Chih-Hsien Cheng ${ }^{1}$, Yu-Chieh Chi ${ }^{1}$, Gong-Ru . Lin ${ }^{1}$ 'Graduate Inst. of Photonics and Optoelectronics, and Dept. of Electrical Engineering National Taiwan Univ., Taiwan. Inversely tapered asymmetric $\mathrm{C}$-rich $\mathrm{SiC}$ waveguide with $10-\mathrm{dB}$ insertion loss in 9-mm length reveals strong nonlinear refractive index of $2.5 \times 10^{-12} \mathrm{~cm}^{2} / \mathrm{W}$ to enhance four-wave mix ing at a conversion efficiency up to $-49 \mathrm{~dB}$.

\section{JTh2A.82}

Wideband Tunable Low Noise Microwave Generation Utilizing an Optoelectronic Oscillator Based on the Deamplification of Stimulated Brillouin Scattering, Huanfa Peng ${ }^{1}$, Yongchi $\mathrm{Xu}^{1}$, Xiaofeng Peng ${ }^{1}$, Yuanxiang Chen' ' Cheng Zhang', Lixin Zhu', Weiwei $\mathrm{Hu}^{1}$, Zhangyuan Chen ${ }^{1} ;{ }^{1}$ Peking Univ., China. A wideband tunable low-noise microwave generation system utilizing optoelectronic oscillator based on the deamplification of stimulated brillouin scattering is demonstrated. Tunable $12-20 \mathrm{GHz}$ microwave signals with phase noise of $-120 \mathrm{dBc} / \mathrm{Hz}$ at $10 \mathrm{kHz}$ offset are achieved.

\section{JTh2A.83}

Noise Characteristics of Fiber-Laser Pumped Femtosecond Optical Parametric Generation, Jingtao Fan ${ }^{1}$, Wei Chen' Chenglin $\mathrm{Gu}^{2}$, Jun Zhao', Youjian Song ${ }^{1}$, Lu Chai ${ }^{1}$, Chingyue Wang ${ }^{1}$, Ming-lie Hu' ${ }^{1}{ }^{1}$ Tianjin Univ., China; ${ }^{2}$ East China Normal Univ., China. We characterize, for the first time, relative intensity noise and timing jitter properties of the OPG output pulses based on the balanced optical cross-correlator technique, which is in a fairly good agreement with numerical simulation.

\section{JTh2A.84}

Passive generation of parabolic similaritons in tapered hydrogenated amorphous silicon photonic wires, Chao Mei ${ }^{1}$, Jinhui Yuan ${ }^{1}$, Zhe Kang ${ }^{1}$, Feng Li ${ }^{2}$, Xianting Zhang ${ }^{1}$ Binbin Yan ${ }^{1}$, Xinzhu Sang ${ }^{1}$, Xian Zhou ${ }^{2}$ Kangping Zhong ${ }^{2}$, Liang Wang ${ }^{3}$, Kuiru Wang ${ }^{1}$ Chongxiu Yu', Chao Lu², Hwa Yaw Tam², P. K. A. Wai ${ }^{2} ;{ }^{1}$ Beijing Univ. of Posts and Telecomm, China; ${ }^{2}$ Dept. of Electronic and Information Engineering, The Hong Kong Polytechnic Univ., Hong Kong: ${ }^{3}$ Dept. of Electronic Engineering, The Chinese Univ. of Hong Kong Hong Kong. We numerically study parabolic 


\section{JTh2A.85}

Optical angular momentum doubling of continuous-wave optical vortices in telecommunication wavelength, Junich Hamazaki', Guo-Wei Lu², Keizo Inagaki', Tadashi Kishimoto ${ }^{3,1}$, Yoh Ogawa', Norihiko Sekine', Akifumi Kasamatsu', Naokatsu Yamamoto', Shigeru Yamaguchi², Iwao Hosako'; ${ }^{1}$ NICT, Japan; ${ }^{2}$ Tokai Univ., Japan ${ }^{3}$ Oki Electric Industry Co., Ltd., Japan. Optical angular momentum (OAM) doubling of continuous-wave optical-vortices (OVs) in telecommunication wavelength is demonstrated via single-pass SHG in a PPLN. By measuring SHG-OVs output power, opticaloptical conversion efficiencies depending on OAMs are investigated.

\section{JTh2A.86}

Crosstalk Mitigation in Polychromatic Sampling via Backward Raman Amplification Junying Ru', Chaoran Huang', Chester Shu' 'CUHK, Hong Kong. By applying backward Raman amplification and reduced input signal power in polychromatic parametric sampling we obtain $12.5 \mathrm{~dB}$ and $18.0 \mathrm{~dB}$ reduction of second-harmonic distortion in two channels caused by inter-channel crosstalk.

\section{JTh2A.87}

Stable Supercontinuum Generation in YAG with Picosecond Pulses, Lukáš Indra ${ }^{1,2}$, Frantisek Batysta ${ }^{1,2}$, Petr Hribek ${ }^{1}$ Jakub Novak',2, Jonathan T. Green', Roman Antipenkov', Jack Naylon', Pavel Bakule', Bedrich Rus'; 'ELI Beamlines, Czech Republic; ${ }^{2}$ CTU FNSPE, Czech Republic. We present a stable supercontinuum generation in a YAG crystal, driven by 3 ps pulses at $1030 \mathrm{~nm}$. The supercontinuum is demonstrated to be coherent and compressible by Dazzler and prism compressor to below $15 \mathrm{fs}$.

\section{JTh2A.88}

Towards Actively Stabilized Micro Ring Resonator Based Frequency Combs, Martin R. Henriksen ${ }^{1}$, Ayman N. Kamel2 , Minhao Pu ${ }^{2}$ Kresten Yvind ${ }^{2}$, Jan Westenkær Thomsen ${ }^{1}$; ${ }^{1}$ Niels Bohr Inst., Denmark; ${ }^{2}$ DTU Fotonik, Technical Univ. of Denmark, Denmark. We present a simple and versatile scheme for active locking of a micro ring resonance to a highly stable fibre laser allowing continuous resonance tuning and locking on a time scale of 10 microseconds.

\section{JTh2A.89}

Phase-Matched Second Harmonic Generation With On-Chip GaN-On-Si Microdisks, lannis Roland', Maksym Gromovyi², yijia Zeng', Moustafa El Kurdi³, Sébastien Sauvage', Farsane Tabataba-Vakili', Christelle Brimont $^{4}$, Thierry Guillet ${ }^{4}$, Bruno Gayral ${ }^{5}$, Stéphanie Rennisson², Fabrice Semond ${ }^{2}$ Jean-yves Duboz², Marc de Michelí, Xavier Checoury ${ }^{3}$, Philippe Boucaud'; ' $C$ CNRS-Univ. Paris Sud, France; ${ }^{2}$ CNRS-CRHEA, France; ${ }^{3}$ Univ. Paris Sud, France; ${ }^{4}$ Univ. Montpellier, France; ${ }^{5}$ nac, CEA, France; ${ }^{\circ} L P M C$, Univ. Nice, France. We demonstrate phasematched second harmonic generation in GaN microdisks integrated on a photonic circuit on silicon.

\section{0:00-12:00 JTh2A • Poster Session III}

JTh2A.90

Multi-GHz Femtosecond Airy Beam Radiation in Higher-harmonic Fractional Cavity OPO, Varun Sharma ${ }^{1}$, Aadhi $A^{1}, N$ Apurv Chaitanya ${ }^{1}$, Goutam Samanta'; ${ }^{1} P R L$ Ahmedabad, India. We report multi-GHz repetition-rate femto-second Airy beam radiation in singly-resonant optical parametric oscillator. The OPO generate $680 \mathrm{~mW}$ of signal radiation in Airy intensity profile with $639 \mathrm{fs}$ pulse-width and $510 \mathrm{~mW}$ of broad-band idler in Gaussian profile.

\section{JTh2A.91}

Multi-Channel Phase-Sensitive Amplification in Nonlinear Waveguides, Young Zhang ${ }^{1}$, Christian Reimer ${ }^{1}$, Jenny Wu' ${ }^{1}$, Piotr Roztocki ${ }^{1}$, Benjamin Wetzel ${ }^{1,2}$, Brent Little ${ }^{3}$ Sai Chu ${ }^{4}$, David J. Moss ${ }^{5}$, Michael Kues ${ }^{1,6}$ Roberto Morandotti 1,7, ' $E M T$ TINRS, Canada, ${ }^{2}$ Dept. of Physics and Astronomy, Univ. of Sussex, UK: ${ }^{3}$ State Key Lab of Transient Optics and Photonics, Chinese Academy of Science, China; ${ }^{4}$ Dept. of Physics and Material Science, City Univ. of Hong Kong, Hong Kong: ${ }^{5}$ Center for Micro-Photonics, Swinburne Univ. of Technology, Australia ${ }^{6}$ School of Engineering, Univ. of Glasgow, UK Inst. of Fundamental and Frontier Sciences, Univ. of Electronic Science and Technology of China, China. We demonstrate on-chip multi-channel phase-sensitive amplification in a nonlinear waveguide, achieving $5 \mathrm{~dB}$ net gain and $15 \mathrm{~dB}$ extinction ratio. We show the manipulation of individual channels in a multi-channel scheme through controlling the initial phases.

\section{JTh2A.92}

Raman Amplifier in Ethane-Filled Hollow Core Fiber, Zefeng Wang ${ }^{1}$, yubin chen ${ }^{1}$, bo $\mathrm{gu}^{1}$, jianqiu $\mathrm{cao}^{1}$, xiaoming $\mathrm{xi}^{1}$, jinbao chen ${ }^{1}$. ${ }^{1}$ National Univ of Defense Technology, China. A C-band efficient fiber gas Raman laser amplifier is demonstrated for the first time in ethane-filled hollow-core fiber seeded by a $1.5 \mu \mathrm{m}$ tunable CW DFB laser, and the maximum Raman conversion efficiency is $47.5 \%$.

\section{JTh2A.93}

Collinear Chiral Sum Frequency Generation Microscopy by Using Vectorial Beam, Ziheng $\mathrm{Ji}^{1}$, Wentao Yu', Yanhui Caii ${ }^{1}$, Hong yang1, Kaihui Liu', Qihuang Gong ${ }^{1}$, Zhiwen $\mathrm{Liu}^{2}$, Kebin Shi'; 'Peking Univ., China; ${ }^{2}$ Pennsylvania State Univ., USA. We report a collinear-chiral-SFG microscope for mapping chirality by utilizing longitudinally polarized optical field generated from vectorial beam, diffraction limited spatial resolution is demonstrated which conventional non-collinear chiral SFG imaging could not reach.

\section{JTh2A.94}

ENZ Conducting Oxide Broadband Perfect Absorbers with Deep Sub-Wavelength Thicknesses, Aleksei Anopchenko', Ho Wa Howard Lee 1,2; 'Physics, Baylor Univ., USA; ${ }^{2}$ The Inst. for Quantum Science and Engineering, Texas A\&M Univ., USA. Absorption due to bound and radiative modes in ultrathin epsilon-near-zero (ENZ) conducting oxide multilayers is computed. Multilayers with 11-nm-thin ENZ absorb >95\% of light in 1-1.5 um wavelength range by exciting ENZ bound or leaky modes.

\section{JTh2A.95}

Programmable Infrared Steganography Using Photoinduced Heating of Nanostructured Metallic Glasses, Ceren Uzun ${ }^{1,2}$ Niloofar Kahler ${ }^{3}$, Luis Grave de Peralta ${ }^{2}$ Golden Kumar ${ }^{3}$, Ayrton Bernussi ${ }^{1,4},{ }^{1}$ NanoTech Center, Texas Tech Univ., USA; ${ }^{2}$ Dept. of Physics and Astronomy, Texas Tech Univ. USA; ${ }^{3}$ Dept. of Mechanical Engineering Texas Tech Univ., USA; ${ }^{4}$ Dept. of Electrical and Computer Engineering, Texas Tech Univ., USA. We demonstrate a new steganography approach that combines an infrared laser, nanopatterned metallic glasses, and a thermal camera. The marked photoinduced heat localization allows for generating welldefined programmable patterns at low powe laser excitation conditions.

\section{JTh2A.96}

Spectral Model of Multiple-Layer of Hybrid Colloidal Quantum Dots for Light Emitting Diodes, Che-Hsuan Huang', Shun-Chieh $\mathrm{Hsu}^{1}$, Li-Ann Ke${ }^{1}$, Meng-Ting Chung ${ }^{1}$, Teng Ming Chen"1, Hao-chung Kuo ${ }^{1}$, Chia-We Sun', Chien Chung Lin'; ' National ChiaoTung Univ., Taiwan. A semi-empirical mode is developed to model the multiple-layer structure of hybrid colloidal quantum dot light emitting diodes. The layer-sequence effect of the quantum dots are matched with the measured data and the optimal condition is obtained.

\section{JTh2A.97}

Backside-illuminated CMOS photodiodes with polysilicon grating back-reflectors Chen-Han Lu', Hsiu-Wei Su'1, Yung-Jr Hung ${ }^{1}$ ${ }^{1}$ National Sun Yat-sen Univ., Taiwan. Polysilicon grating reflectors are employed in thin backside-illuminated CMOS photodiodes to not only improve its responsivity for near-infrared light but also allow polarizationsensitive photocurrent generation. A 1.14x photocurrent enhancement is obtained for TE-polarized incidence.

\section{JTh2A.98}

Charge Transfer and Enhanced Absorp tion in $\mathrm{MoS}_{2}$ - Organic Heterojunction Using Plasmonic Metasurfaces, Christopher Petoukhoff1,2, M Bala Murali Krishna', Damien Voiry², Ibrahim Bozkurt², Skylar Deckoff-Jones ${ }^{1}$, Manish Chhowalla ${ }^{2}$, Deirdre M. O'Carroll2,3, Keshav Dani' ${ }^{1}{ }^{1}$ Femtosecond Spectroscopy Unit, Okinawa Inst. of Science and Technology Graduate Univ., Japan ${ }^{2}$ Materials Science and Engineering, Rutgers Univ., USA; ${ }^{3}$ Chemistry and Chemical Biology, Rutgers Univ., USA. We observed ultrafast hole transfer from monolayer $\mathrm{MoS}_{2}$ to a conjugated polymer using pump-probe measurements. We show that plasmonic metasurfaces lead to enhanced absorption in in the heterojunction, with a 90-nm absorption bandwidth increase.

\section{JTh2A.99}

Integrated zero-index supercouplers, Dary Vulis ${ }^{1}$, Phillip Camayd-Munoz ${ }^{1}$, Yang Li', Orad Reshef', Marko Lončar', Eric Mazur'; ${ }^{1}{ }^{1}$ Harvard Univ., USA. Zero-index supercoupling offers a mechanism through which light is confined to sub-diffraction limited length scales and efficient coupling between disparate modes is achieved. We explore this phenomena in an integrated metamaterial platform toward experimental demonstration.

JTh2A.100

Withdrawn

JTh2A.101

Ultra-compact, Low-loss and Low-crosstalk Wavelength Demultiplexer for CWDM System Based on the Photonic-Crystal-Like Metamaterial Structure, Feiya Zhou', Luluz Lu', Minming Zhang', Weijie Chang ', Dongyu Li', Lei Deng', Deming Liu'; 'School of Optical and Electrical Information, Huazhong Univ. of Science and Technology, China. A wavelength demultiplexer for CWDM system is designed, fabricated and characterized based on metamaterial structure. The device experimentally displays low loss $(-2.3 \mathrm{~dB})$, low crosstalk $(-16.4 \mathrm{~dB})$ and broad $1-\mathrm{dB}$ bandwidth $(>18 \mathrm{~nm})$ with compact size of $2.6 \mu \mathrm{m} \times 5 \mu \mathrm{m}$

\section{JTh2A.102}

Withdrawn.

\section{JTh2A.103}

TM Grating Couplers for Low-Loss LPCVD based $\mathrm{Si}_{3} \mathrm{~N}_{4}$ Waveguide Platform, George Dabos ${ }^{1}$, Athanasios Manolis ${ }^{1}$, Anna Lena Giesecke $^{2}$, Caroline Porschatis², Bartos Chmielak $^{2}$, Thorsten Wahlbrink², Nikos Pleros $^{1}$, Dimitris Tsiokos'; 'Dept. of Informatics, Center for Interdisciplinary Research and Innovation, Aristotle Univ. of Thessaloniki, Greece: ${ }^{2} \mathrm{AMO} \mathrm{GmbH}$, Germany. We demonstrate TM grating couplers for LPCVD silicon nitride platform with coupling loss of $6.5 \mathrm{~dB}$ at $1541 \mathrm{~nm}$ and $1 \mathrm{~dB}$ bandwidth of $55 \mathrm{~nm}$ employing optical projection lithography fo low-cost and mass manufacturing of photonic integrated circuits.

\section{JTh2A.104}

Synchronization of thermal-carrier oscillations in coupled silicon microcavities, Gustavo de Oliveira Luiz' ${ }^{1}$ Thiago P. M. Alegre Gustavo S. Wiederhecker'; ; Instituto de Fisica Gleb Wataghin, Universidade Estadual de Campinas, Brazil. We report on the synchronization of thermal-carrier self-sustaining oscillations in coupled silicon microdisks. Time and frequency domain signatures of synchronization are observed.

\section{JTh2A.105}

Enhanced and directional photoluminescence from doubly-resonant $\mathrm{WSe}_{2}-\mathrm{S}$ hybrid structure, Haitao $\mathrm{Chen}^{4}$, Stefan $\mathrm{Nanz}^{1}$, Aimi Abass ${ }^{3}$, Jingshi Yan ${ }^{4}$, Tingge $\mathrm{GaO}^{4}$, Duk-Yong $\mathrm{Choi}^{2}$, Carsten Rockstuhl ${ }^{1,3}$ Yuri S. Kivshar ${ }^{4}$, Dragomir N.Neshev ${ }^{4} ;{ }^{1}$ Inst. of 


\section{0:00-12:00 JTh2A • Poster Session III}

\section{JTh2A.106}

Light generated bubble for microparticle propulsion, Ido Frenkel'1, Avi Niv'; ' 'Ben Gurion Univ. of the Negev, Israel. Light activated motion of micron-sized objects with exceptional effective forces (micro-Newtons) and unprecedented speed $(\sim 1 \mathrm{~m} / \mathrm{s})$ is demonstrated with propulsion generated by short lifetime micro vapor bubbles transforming accumulated heat to mechanical motion.

\section{JTh2A.107}

A Butt-Coupled 3D-Bulk Si CMOS Photodetector Array Integrated with a Monolithic U-Groove Array on a Single Chip, Iman SabriAlirezaei ${ }^{1}$, Jörg Vierhaus ${ }^{1}$, Edmund P. Burte ${ }^{1}$; Otto-von-Guericke Univ. of Magdeburg,Electrical Engineering and Information Technology, Inst. of Micro and Sensor Systems (IMOS), Germany. An integrated butt-coupled 3D bulk-CMOS Si photodetector array including a large passivated photoactive area for visible-light is presented. The device shows a leakage current of 78.5nA and a capacitance of $\sim 30 \mathrm{fF}$ at $5 \mathrm{~V}$ reverse bias.

\section{JTh2A.108}

Tunable Photoresponse in InAs Nanowire Photodetectors Through Surface-State Engineering, Jack A. Alexander-Webber', Catherine Groschner ${ }^{1}$, Abhay Sagade ${ }^{1}$, Stephan Hofmann ${ }^{1}$, Hark Hoe Tan², Chennupati Jagadish', Hannah Joyce ${ }^{1} ;{ }^{1}$ Univ. of Cambridge, UK; ${ }^{2}$ Dept. of Electronic Materials Engineering, Research School of Physics and Engineering, The Australian National Univ., Australia. We report on individualInAs nanowire optoelectronic devices which, through surface-state engineering, can be tailored to exhibit either negative- or positive-photoconductivity, opening pathways towards engineering semiconductor nanowires for novel optical-memory and photodetector applications.

\section{JTh2A.109}

Withdrawn.

\section{JTh2A.110}

Wavelength-tunable thermal sources with nonvolatile phase changing material, Kaikai $\mathrm{Du}^{1}$, Yue Lu', Yanbiao Lyu², Zhiyuan Cheng², Min Qiu', Qiang Li'; ' 'State Key Lab of Modern Optical Instrumentation, College of Optical Science and Engineering, Zhejiang Univ. China; ${ }^{2}$ Inst. of Microelectronics and Nanoelectronics, College of Information Science \& Electronic Engineering, Zhejiang Univ., China. Two kinds of wavelength-tunable thermal sources at 3-5 $\mu \mathrm{m}$ mid-infrared atmospheric window are experimentally demonstrated by simple metallic and $\mathrm{Ge}_{2} \mathrm{Sb}_{2} \mathrm{Te}_{5}$ (GST) layered structures.

\section{JTh2A.111}

Bandwidth Tunable SOI Add-Drop Filter, Mehrnoosh Taghi Boroojerdi', Michael Menard ${ }^{2}$, Andrew Kirk'; ${ }^{1}$ McGill Univ., Canada; ¿UQAM, Canada. A new fully reconfigurable integrated add-drop filter is proposed, overcoming the limitations in operational range exhibited by previous designs. It provides a $1.6 \mathrm{~nm}$ wavelength tuning range with $0.49 \mathrm{~nm}$ to $3.36 \mathrm{~nm}$ bandwidth tunability.
JTh2A.112

Towards picoliter microsensing in index and temperature using hundreds-micronscale fiber Michelson interferometers, Nan-Kuang Chen' ${ }^{1}$ Shih-Hsin Lo', chialung tsai ${ }^{1}$, Santosh Kumar ${ }^{2}$, Wood-Hi Cheng ${ }^{4}$, Raman Kashyap ${ }^{3} ;{ }^{1}$ National United Univ. Taiwan: ${ }^{2}$ DIT Univ., India: ${ }^{3}$ Polytechnique Montreal, Canada; ${ }^{4}$ National Chung Hsing Univ., Taiwan. We demonstrate index and temperature microsensing at picoliter liquid sample volume using hundreds-micron-scale fiber Michelson interferometer with multiple small foci in space. The index and temperature sensitivity can reach $59.4 \mathrm{~nm} / \mathrm{RIU}$ and 23 $\mathrm{pm} /{ }^{\circ} \mathrm{C}$, respectively.

\section{JTh2A.113}

Exciting Localized Modes in Polar EpsilonNear-Zero Materials, Owen Dominguez', Leland J. Nordin², Kaijun Feng', Junchi Lu' Dan Wasserman ${ }^{2}$, Anthony J. Hoffman ${ }^{1}$ 'Electrical Engineering, Univ. of Notre Dame USA; ${ }^{2}$ Electrical Engineering, Univ. of Texas a Austin, USA. We design, fabricate and characterize nanoantennas on epsilon-near-zero polar thin films. Reflection measurements and simulations indicate a localized mode at 11.2 $\mathrm{mm}$ that has a strong electric field in the AIN epsilon-near-zero film.

\section{JTh2A.114}

Infrared Detection Using Plasmonically Enhanced Thermomechanically Actuated Nanowire Arrays, Qiancheng Zhao ${ }^{1}$ Parinaz Sadri-Moshkenani ${ }^{1}$, Mohammad W. Khan', Rasul Torun', Imam-Uz Zaman'1, Ozda Boyraz'; 'Univ. of California, Irvine, USA A plasmo-thermomechanical IR detector based on suspended bilayer nanowire array is demonstrated. The detector exhibits an absorption coefficient of 0.0494 . The maximum difference in $\mathrm{S}_{21}$ between the radiation on and off states is 0.013 with a radiation intensity of $0.1 \mathrm{~mW} / \mu \mathrm{m}^{2}$.

JTh2A.115

On-chip Eletromechanically Induced Brillouin Scattering on Suspended Aluminum Nitride Waveguides, Qiyu Liu', Mo Li ${ }^{1}$ Huan Li ${ }^{1}$; ${ }^{1}$ Univ. of Minnesota, USA. We propose and have theoretically investigated on-chip eletromechanically induced Brillouin scattering on suspended aluminum nitride waveguides by simulation of its acoustic and optical properties. Prototype devices have been fabricated.

JTh2A.116

Reconfigurable Integrated MIMO Optical Mode Demultiplexer using MMI Couplers, Rui Tang1', Takuo Tanemura', Yoshiak Nakano'; 'The Univ. of Tokyo, Japan. We propose a novel reconfigurable integrated MIMO optical mode demultiplexer consisting of cascaded MMI couplers. By configuring phase shifters adaptively, large-scale $(>10$ modes), low-crosstalk ( $<-20 \mathrm{~dB}$ ) demultiplexing can be realized on chip.

\section{JTh2A.117}

Withdrawn.
JTh2A.118

Multispectral Plasmonic Structures Using Native Aluminum Oxide and Aluminum sencer ayas ${ }^{1,2}$, Gokhan Bakan 1,3, Aykutlu Dana': 'Material Science and Nanotechnology, Bilkent Univ., Turkey; ${ }^{2}$ Dept. of Radiology, Stanford Univ., USA; ${ }^{3}$ Electrical and Electronics Engineering, Atilim Univ., Turkey. We report the use of native aluminum oxide to fabricate periodic metal-insulator-metal resonators with simultaneous resonances in the visible and IR wavelengths. The cavity size is in the order of $\lambda^{3} / 25000$ in the NIR.

\section{JTh2A.119}

On-chip Spectrometer Enhanced by Ring Resonator Cavity: High-resolution and Large-bandwidth, Shaonan Zheng ${ }^{1}$, L. K. Chin', Ai Qun Liu'; 'Nanyang Technological Univ., Singapore. A high-resolution $(0.15 \mathrm{~nm})$ and large-bandwidth (100 nm) on-chip spectrometer is demonstrated with a microring resonator-enhanced Mach-Zehnder interferometer. It is promising to develop integrated photonic sensing systems for environmental and water quality monitoring.

\section{JTh2A.120}

Dispersion immune change of soliton repetition rate in a silicon-nitride microresonator, Chengying Bao', Andrew Weiner ${ }^{1}$, Jose Jaramillo-Villegas ${ }^{1}$, Yi Xuan', Cong Wang ${ }^{1}$ Daniel Leaird', Minghao Qi'; 'Purdue Univ. USA. We show soliton-self-frequency-shift (SSFS) in a SiN microresonator dominates the change of soliton repetition rate relative to thermal effects. The SSFS-induced repetition rate change with detuning is not directly dependent on the dispersion.

JTh2A.121

Mechanically Exfoliated $\mathrm{MoS}_{2}$ onto D shaped Optical Fiber for Erbium Doped Fiber Laser Mode-locking, Eduardo J. Aiub', David Steinberg', Eunezio A. Thoroh de Souza', Lucia A. Saito'; 'Universidade Presbiteriana Mackenzie, Brazil. For the first time, we report an ultrashort pulse generation of $266 \mathrm{fs}$ obtained by mode-locked Erbium doped fiber laser using a mechanically exfoliated $\mathrm{MoS}_{2}$ deposited onto the side polished surface of D-shaped optical fiber.

\section{JTh2A.122}

Temporal Stability Performance of the Parametric Spectro-temporal Analyzer (PASTA) System, Haidong Zhou', Liao Chen ${ }^{1}$, Xi Zhou' ${ }^{1}$, Chi Zhang ${ }^{1}$, Kenneth Kin-Yip Wong', Xinliang Zhang ${ }^{1}$; ${ }^{1}$ Wuhan National Lab for Optoelectronics, Huazhong Univ. of Science and Technology, China; ${ }^{2}$ Dept. of Electrical and Electronic Engineering, The Univ. of Hong Kong, Hong Kong. Tempora stability of the parametric spectro-tempora analyzer (PASTA) system is thoroughly explored and significantly enhanced, with the spectral accuracy improved from $1.6 \mathrm{~nm}$ to $0.04 \mathrm{~nm}$, leveraging the active phase-lock loop and temperature feedback control.

\section{JTh2A.123}

High-Speed Compressive Measurement using a Time-Lens Spectral Shaper, Jaspe R. Stroud', Mark A. Foster'; ' 'Johns Hopkin Univ., USA. We present a method for highspeed pseudorandom spectral patterning using a time lens. When combined with compressed sensing we can measure spectrally mapped signals using far fewer measurements than required by Nyquist sampling.

\section{JTh2A.124}

Relative CEP-Locking Laser Source: Narrowband CW Injection Seeded Optical Parametric Amplifier, Jingtao Fan ${ }^{1}$, Chenglin $\mathrm{Gu}^{2}$, Jun Zhao', Bo Liư ${ }^{3}$ Chingyue Wang', Ming-lie $\mathrm{Hu}^{1}$; 'Tianjin Univ., China; ${ }^{2}$ East China Normal Univ., China; ${ }^{3}$ Nankai Univ. China. We demonstrate experimentally a passively relative carrier-envelope phase (CEP)-locking, broadly tunable and robus laser source which is based on narrowband cw injection seeding of a two stage femtosecond optical parametric amplifier (OPA).

JTh2A.125

A real-time ultra-broadband radio frequency spectrum analyzer based on parametric spectro-temporal analyzer, Liao Chen ${ }^{1}$, Yuhua Duan', Chi Zhang', Xinliang Zhang'; 'Wuhan National Lab for Optoelec tronics \& School of Optical and Electronic Information, Huazhong Univ. of Science and Technology, China. We report an all-optica real-time ultra-broadband radio frequency (RF) spectrum analyzer based on parametric spectro-temporal analyzer (PASTA). It not only has the large RF bandwidth (over $800 \mathrm{GHz}$ ), but also achieves over $90-\mathrm{MHz}$ frame rate.

\section{JTh2A.126}

Two-Pulse Photoluminescence Correlation Technique for Studying Ultrafast Carrie Dynamics in Deep-UV Few Monolayer Thick Nitride Quantum Wells, Okan Koksal', SM Islam', Tongbo Wei', Huili (Grace) Xing ${ }^{1}$ Debdeep Jena ${ }^{1}$, Farhan. Rana ${ }^{1} ;{ }^{1}$ Cornell Univ., USA. We a present a new two-pulse photoluminescence correlation technique and use it to study ultrafast dynamics of photoexcited carriers in few monolayer thick deep-UV GaN/AIN quantum wells with picosecond time resolution.

JTh2A.127

Reshaping of Telecom Band Optical Signals Using Programmable Pump Pulses, Paritos Manurkar2,1 Nitin Jain 2,3 Prem Kumar 2,4 Gregory S. Kanter'; ${ }^{2}$ NIST Boulder, USA ${ }^{2}$ EECS, Northwestern Univ., USA; ${ }^{3}$ Physics, Technical Univ. of Denmark, Denmark ${ }^{4}$ Physics and Astronomy, Northwestern Univ. USA. We demonstrate reshaping of optica signals in telecom band without a change in wavelength by designing appropriate pump pulses for interaction in a nonlinear $\$$ $\operatorname{chi}^{\wedge}\{(2)\} \$$ waveguide. 
Exhibit Hall 1, 2 \& 3

\section{0:00-12:00 JTh2A • Poster Session III}

\section{JTh2A.128}

Ultrafast Diode Laser with Self-Adapting Pulse-Shaping in Passive, Active and Hybrid Mode-Locking Operation, Rouven Pilny', Benjamin Döpke', Carsten Brenner ${ }^{1}$, Andreas Klehr², Andrea Knigge², Günther Tränkle ${ }^{2}$, Martin Hofmann ${ }^{1}{ }^{1}$ Ruhr Universitat Bochum, Germany: ${ }^{2}$ Ferdinand-Braun-Institut für Höchstfrequenztechnik im Forschungsverbund Berlin e.V., Germany. We present an ultrafast edge-emitting diode laser system, which is able to self-adapt the resonator internal phase and amplitude. The best operating conditions for passive, active and hybrid mode-locking are analyzed.

\section{JTh2A.129}

Coherently seeded optical parametric amplifier with $500 \mathrm{~nJ}$ short-wave infrared signal at $1 \mathrm{MHz}$, Scott Domingue ${ }^{2}$, David G. Winters ${ }^{2}$, mathew kirchner ${ }^{2}$, Sterling J. Backus 2,1; ${ }^{1}$ Colorado State Univ., USA ${ }^{2}$ Kapteyn-Murnane Labs, USA. We introduce a near-infrared pumped, white-light seeded optical parametric amplifier driven by a 3.5 $\mu \mathrm{J}, 130 \mathrm{fs}$ pulse at $1040 \mathrm{~nm}$ from a KMLabs Y-Fi HP. The signal conversion efficiency is as high $14 \%$
JTh2A.130

Single-walled carbon nanotube modelocked $\mathrm{Yb}^{3+}$-doped $\mathrm{CaF}_{2}$ laser, Naoyuk Yokoshima', SHOTARO KITAJIMA', Akira Shirakawa', Sunyoung $\mathrm{Choi}^{2}$, Fabian Rotermund ${ }^{3} ;{ }^{1}$ Inst. for Laser Science, UEC, Japan; ${ }^{2}$ Institut für Laser-Physik, Universität Hamburg, Germany; ${ }^{3}$ Korea Advanced Inst. of Science and Technology, South Korea. We demonstrated SWCNT-assisted Kerr-lens mode-locked $\mathrm{Yb}: \mathrm{CaF}_{2}$ laser. The pulse duration of $87 \mathrm{fs}$ with a $260 \mathrm{~mW}$ output power and the shortest pulse duration of $77 \mathrm{fs}$ with a 94 $\mathrm{mW}$ output power were obtained.

\section{JTh2A.131}

SHG-FROG characterization of a nove multichannel synchronized AWG-based mode-locked laser, Songtao Liu1,2, Dan Lu $^{1,2}$, Lingjuan Zhao ${ }^{1,2}$, Wei Wang ${ }^{1,2}$, Ronald Broeke ${ }^{3}$, Chen $\mathrm{Ji}^{1,2} ;{ }^{1}$ Key Lab of Semiconductor Materials Science, Inst. of Semiconductors, CAS, China; ${ }^{2}$ Univ. of Chinese Academy of Sciences, China; ${ }^{3}$ Bright Pho tonics, Netherlands. We report the second harmonic generation frequency-resolved optical gating (SHG-FROG) measurements on a monolithically integrated multichannel mode-locked semiconductor laser based on arrayed waveguide grating. Clear phase correlation between synchronized mode-locked channels was demonstrated.
JTh2A.132

Time Range Extension of Ultrafast Waveform Measurement by Using Optical Frequency Comb Synthesizer/Analyzer, Takashi Hasegawa', Takayuki Miyamoto' Tatsutoshi Shioda'; 'Saitama Univ., Japan. Single-shot ultrafast waveform measurement system with time-resolution in femto-second and time-range of 40 pico-second has been developed using $200 \mathrm{GHz}$ optical frequency comb synthesizer and analyzer by means of the proposed time window extension technology.

JTh2A.133

High efficiency Kerr-lens mode-locked Yb:GSO oscillator, Wenlong Tian ${ }^{1,2}$, Yingnan Peng ${ }^{1,2}$, Jiangfeng Zhu', Zhiyi Wei ${ }^{2}$, Jun $\mathrm{Xu}^{3}$. 'Xidian Univ., China; ${ }^{2}$ nst. of Physics, Chinese Academy of, China; ${ }^{3}$ Tongji Univ., School of Physics \& Engineering, Shanghai Engineering Research Center for Sapphire China. A fiber laser pumped Kerr-lens mode-locked $\mathrm{Yb}: G S O$ oscillator delivering 4-W, 249-fs pulses at the repetition rate of $92 \mathrm{MHz}$ is demonstrated for the first time. The corresponding optical-to-optical efficiency is as high as $54 \%$
JTh2A.134

Asynchronous and synchronous dualwavelength pulse generation in a non zero-dispersion fiber laser, Guoging $\mathrm{Hu}^{1}$

Ting Li', Yingling Pan', Xin Zhao ${ }^{1}$, Meng Zhang ${ }^{1}$, Zheng Zheng ${ }^{1,2}$; ${ }^{1}$ School of Electronic and Information Engineering, Beihang Univ. China: ${ }^{2}$ Collaborative Innovation Center of Geospatial Technology, China. We show both asynchronous and synchronous, dualwavelength femtosecond pulse generation from an SWNT-mode-locked fiber laser by tailoring the intracavity anomalous dispersion, suggesting the effect of the mode-locke on pulse synchronization in the presence of dispersion. 
Executive Ballroom

$210 \mathrm{~A}$

\section{Joint}

14:00-16:00

JTh3A - Symposium on

Multimodal Imaging in

Biophotonics I

Presider: Wolfgang Drexler;

Medizinische Universität Wien,

Austria

\section{JTh3A.1 • 14:00 Invited}

Multimodal Label-free Low Fluence Nonlinear Imaging of Living Systems with High-Throughput, Carlos Macias-Romero' Vitalijs Zubkovs ${ }^{1}$, Siyuan Wang ${ }^{1}$, Sylvie Roke ${ }^{1}$; 'Ecole Polytechnique Federale de Lausanne, Switzerland. Photo-damage thresholds in aqueous solutions and living cells are determined for wide field multiphoton imaging. Possible dwell times are $10^{\wedge} 6$ times longe compared to scanning confocal imaging High throughput time-resolved multiphoton neuroimaging is performed.
Executive Ballroom 210B

Executive Ballroom $210 \mathrm{C}$

\section{CLEO: Applications \& Technology}

\section{4:00-16:00}

ATh3B • Optical Devices \& Components

Presider: Jana Jágerská; UiT Norges Arktiske Universitet, Norway

ATh3B.1 • 14:00

Ultrahigh extinction on-chip amplitude modulators with broadband operation Sheng Liu', Hong Cai', Christopher DeRose ${ }^{1}$ Paul Davids ${ }^{1}$, Andrew Pomerene', Andrew Starbuck', Douglas Trotter', Junji Urayama', Ryan M. Camacho', Anthony Lentine ${ }^{1}$; ${ }^{1}$ San dia National Labs, USA. We experimentally demonstrate amplitude modulators (AMs) with $>65 \mathrm{~dB}$ extinction across over a $160 \mathrm{~nm}$ spectral range. The output optical phase response is also characterized when the amplitude is modulated.

\section{ATh3B.2 • 14:15}

Broadside Beam Routing by Dielectric Micro-Prism, Arnab Dewanjee ${ }^{1}$, J. Stewart Aitchison'1, Mo. Mojahedi'1; 'Univ. of Toronto, Canada. We propose and demonstrate the design and fabrication of a broadside beam routing and also a stress induced broadside beam scanning mechanism using high index dielectric micro prism structures compatible to integrated photonics.
14:00-16:00

ATh3C • A\&T Topical Review on Extreme Ultraviolet and Soft X-ray Sources and Application I Presider: Carmen Menoni; Colorado State Univ., USA

\section{ATh3C.1 - 14:00 Invited}

Toward Compact and Ultra-intense Lase Based Soft X-ray Lasers, Stephane Sebban ${ }^{1}$ 'Laboratoire d'Optique Appliquée, France. We report here recent work on an opticalfield ionized (OFI) high-order harmonicseeded $32.8 \mathrm{~nm}$ laser. The gain duration monotonically decreased from $7 \mathrm{ps}$ to an unprecedented shortness of $450 \mathrm{fs}$ FWHM as the amplification peak rose from 150 to 1,200 with an increase of the plasma density from $3 \times 10^{18} \mathrm{~cm}^{-3}$ up to $1.2 \times 10^{20} \mathrm{~cm}^{-3}$. The integrated energy of the EUV laser pulse was also measured, and found to be up to $14 \mu \mathrm{J}$.

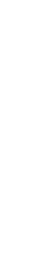

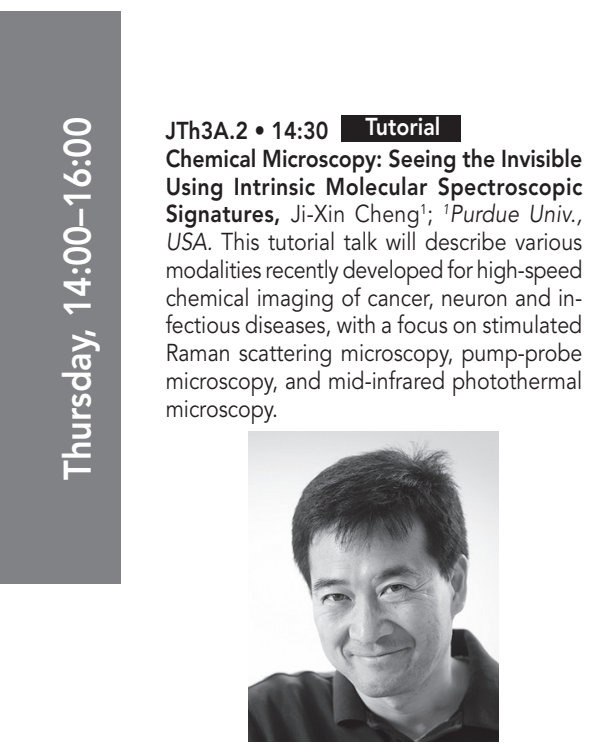

Ji-Xin Cheng was born in China in 1971. He attended University of Science and Technology of China from 1989 to 1994. From 1994 to 1998 , he carried out his PhD study on bond-selective chemistry. After postdoc training at Harvard University, Ji-Xin Cheng joined Purdue University in 2003 as Assistant Professor in Weldon School of Biomedical Engineering and Department of Chemistry, promoted to Associate Professor in 2009 and to Full Professor in 2013. He will join Boston University as the Moustakas Chair Professor in Photonics and Optoelectronics in July 2017.

Executive Ballroom 210D

\section{CLEO: QELS- Fundamental Science}

14:00-16:00

FTh3D • PT Symmetry and

Beyond

Presider: Demetrios

Christodoulides; CREOL, Univ. of

Central Florida, USA

FTh3D.1 • 14:00

Polarization state conversion through exceptional point encirclement, Absar U. Has $\operatorname{san}^{1}$, Bo Zhen ${ }^{2,3}$, Marin Soljacic ${ }^{2}$, Mercedeh Khajavikhan', Demetrios Christodoulides 'Univ. of Central Florida, CREOL, USA ${ }^{2} \mathrm{MIT}$, USA; ${ }^{3}$ Technion, Israel. We present an integrated optical configuration for converting any arbitrary polarization into a desired output state. This topologically robust chiral process relies on encircling the system's exceptional point through parameter variations along the propagation direction.

\section{FTh3D.2 • 14:15}

PT-symmetric Micro-resonators: High Sensitivity at Exceptional Points, Hossein Hodaei ${ }^{1}$, Absar U. Hassan ${ }^{1}$, Demetrios Christodoulides', Mercedeh Khajavikhan'; ${ }^{1}{ }^{1}$ Univ. of Central Florida, USA. Enhanced sensitivity is demonstrated in PT-symmetric coupled micro-resonator arrangements biased at an exceptional point. The spectral response of such a system is shown to follow a square root dependence on externally introduced perturbations.

ATh3B.3 • 14:30

Tunable Enhanced Mid-Infrared Light Absorption in Graphene, alireza safaei ${ }^{1}$, Sayan Chandra', Michael N. Leuenberger ${ }^{1}$, Debashis Chanda'; 'Univ. of Central Florida, USA. We demonstrate extraordinary $\sim 45 \%$ absorption of light by graphene in midinfrared region by exciting graphene surface plasmons. The absorption wavelength is tunable by gate voltage. Further, we show that the absorption bandwidth depends on the carrier mobility of graphene.

\section{ATh3C.2 • 14:30 Invited}

Next Generation High-Order Harmonic Sources and Application, Katsumi Midorikawa'; 'RIKEN Center for Advanced Photonics, Japan. High-order harmonics are established as a high-output coherent light source in the XUV region and the sole source of attosecond pulses. Recent efforts on high harmonic generation and application at RIKEN is reviewed.

\section{FTh3D.3・14:30}

Electrically Pumped Coupled Waveguide Lasers by Parity-Time Symmetry, Ruizhe Yao ${ }^{1}$, Chi-Sen Lee ${ }^{1}$, Viktor A. Podolskiy ${ }^{1}$, Wei Guo'; 'Univ. of Massachusetts Lowell, USA We demonstrate single transverse-mode operation of InAs quantum dot (QD) broadarea coupled waveguide lasers enabled by parity-time symmetry breaking. By tuning the loss, suppression and revival of higher order modes from fundamental mode is obtained. 
Executive Ballroom

210E

\section{Joint}

14:00-16:00

JTh3E • Quantum Photonics II

Presider: Marcelo Davanco; NIST, USA

\section{JTh3E.1 1 14:00 Invited}

Coherent Control and Photonic Interfacing of Color Centers in Diamond, Jonas Becker ${ }^{1}$, Johannes Görlitz', Philipp Fuchs' ${ }^{1}$, Thomas Jung ${ }^{1}$, Elke Neu', Carsten Arend', Christoph Becher'; 'Universitaet des Saarlandes, Germany. We present all-optical, ultrafast coherent control of single silicon vacancy color centers in diamond and single qubit operations both on a direct transition and in a $\Lambda$-system. We further discuss methods for efficient photonic interfacing.

\section{4:00-16:00}

Molecular Dynamics

Presider: Chih-Wei Lai; US Army

Research Lab, USA

\section{JTh3E.2 • 14:30}

High-Q Diamond Microdisks for Coupling to SiV Quantum Emitters, Tamiko Masuda ${ }^{1}$ Matthew Mitchell2,3, Behzad Khanaliloo 2,3, David Lake ${ }^{2,3}$, Thomas Lutz ${ }^{2}$, JP Hadden 1,2, Wolfgang Tittel'2, Paul E. Barclaye,3; ${ }^{2}$ Univ. of Calgary, Canada: ${ }^{2}$ Inst. for Quantum Science and Technology, Univ. of Calgary, Canada; ${ }^{3}$ National Inst. for Nanotechnology, Canada. We present diamond microdisk optical cavities with record quality $(Q)$ factors $\left(Q \sim 1 \times 10^{5}\right.$ at wavelengths near the opitical transitions of silicon vacancy (SiV) quantum emitters. Simulations indicate that $\mathrm{Q} / \mathrm{V}>1.2 \times 10^{4}$ is possible in these structures with optimized dimensions.

\section{Executive Ballroom 210F}

\section{CLEO: QELS- Fundamental Science}

FTh3F • Ultrafast Lattice and

\section{FTh3F.1 • 14:00}

Ferroelectric Phonon-Polariton Dynamics in a Wide Temperature Range Revealed via Single-Shot Spectroscopy, Jun Takeda' Tomonori Kuribayashi', Yusuke Arashida', Iku-

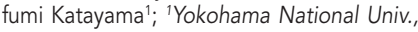
Japan. Using single-shot time-frequency two-dimensional spectroscopy, we could successfully observed the E-mode phononpolariton dynamics of ferroelectric $\mathrm{LiNbO}_{3}$ in a wide temperature range of $10-400 \mathrm{~K}$, while avoiding photorefractive effects persistently appeared at low temperatures.

FTh3F.2 • 14:15

Strong Local-Field Enhancement of the Nonlinear Softmode Response in Aspirin Giulia Folpini', Klaus Reimann', Michae Woerner ${ }^{1}$, Thomas Elsaesser ${ }^{1}$, Johannes $\mathrm{Hoja}^{2}$, Alexandre Tkatchenko2: ${ }^{1} \mathrm{Max}$-BornInstitut, Germany; ${ }^{2}$ Univ. of Luxembourg, Luxembourg. Softmode excitations in aspirin are studied with two-dimensional terahertz spectroscopy. The coupling of $\mathrm{CH}_{3}$ rotational modes and collective excitations of $\pi$ elec trons results in a nonperturbative nonlinear response induced by moderate $\mathrm{THz}$ fields.

\section{Executive Ballroom} 210G

\section{Joint}

14:00-16:00

JTh3G • Symposium on

Optomechanics: Towards the Second Quantum Revolution I

Presider: Pierre Verlot; Ecole

Polytechnique Federale de

Lausanne, Switzerland

\section{JTh3G.1 • 14:00}

Invited

Quantum Optomechanics with Micro- and Nano-Mirrors, Antoine Heidmann'; 'Laboratoire Kastler Brossel, France. We introduce the main concepts of cavity optomechanics in which the quantum behaviour of mechanical systems is governed by radiation pressure of light. We present recent results obtained with micro-mirrors or with photonic-crystal nanomembranes.

FTh3F.3 • 14:30

Coherent Control of Carrier and Phonon Dynamics in Photoexcited Bismuth, YuHsiang Cheng ${ }^{1}, Y_{i} G_{a o}{ }^{1}$, Keith Nelson ${ }^{1}:{ }^{1} M I T$, USA. Using single-shot pump-probe spectroscopy, the carrier relaxation and phonon oscillation in highly photo-excited bismuth are coherently controlled with double-pulse excitation. Beside phonon amplitude and phase, the magnitudes of monotonic decays also oscillate with inter-pump delay.
JTh3G.2 • 14:30

Optomechanically Induced Transparency in Diamond Microdisks, David Lake ${ }^{1,2}$, Matthew Mitchell1,2, JP Hadden', Paul E. Barclay 1,2; 1 Inst. for Quantum Science and Technology, Canada; ${ }^{2}$ National Inst. for Nanotechnology, Canada. Optomechanically induced transparency in a single--crystal diamond microdisk supporting a $2.4 \mathrm{GHz}$ mechanical mode is demonstrated. A cooperativity of $\mathrm{C} \approx 1.2$ for $\mathrm{N} \approx 2 \times 10^{6}$ intracavity photons is reached, sufficient for coherent photon-phonon coupling.
Executive Ballroom $210 \mathrm{H}$

\section{CLEO: QELS- Fundamental Science}

14:00-16:00

FTh3H • Photonics Crystals for Light Manipulation and Concentration

Presider: Qing Gu; Univ. of California San Diego, USA

\section{FTh3H.1 • 14:00}

Observation of edge states at telecom wavelengths in topological photonic crys tal, Sabyasachi Barik',2, Hirokazu Miyake ${ }^{2}$ Wade DeGottardi ${ }^{2}$,Edo Waks ${ }^{1,2}$, Mohammad Hafezi ${ }^{2,1} ;{ }^{1}$ IREAP, USA; ${ }^{2} J Q I$, USA. We report on the experimental observation of edge states of light with wavelength near 1500 $\mathrm{nm}$ in a nanoscale all-dielectric topological photonic crystal waveguide system. Transmission spectra agree with three-dimensional finite-difference time-domain simulations.

\section{FTh3H.2 • 14:15}

Bowtie Photonic Crystal with Deep Subwavelength Mode Confinement in a Dielectric Material, Shuren $\mathrm{Hu}^{1,3}$, Marwan Khater $^{2}$ Rafael Salas-Montiel ${ }^{4}$, Ernst Kratschmer ${ }^{2}$, Sebastian Engelmann², William Green², Sharon M. Weiss'; ${ }^{1}$ Vanderbilt Univ., USA; ${ }^{2}$ IBM, USA ${ }^{3}$ GlobalFoundries, USA; ${ }^{4}$ Univ. de technologie de Troyes, France. We report the design and experimental characterization of deep subwavelength optical modes $\left(\mathrm{V}_{\mathrm{m}} \sim 10^{-3}(\lambda\right.$ $\left.\left.\mathrm{n}_{\mathrm{s}}\right)^{3}\right)$ in high quality factor dielectric photonic crystals $\left(\mathrm{Q} \sim 3 \times 10^{4}\right)$. Our approach offers low-loss alternative to metals for achieving extreme light concentration.

\section{FTh3H.3 • 14:30}

Self-similar Photonic Crystal Cavity with Ultrasmall Mode Volume for Single-photon Nonlinearities, Hyeongrak Choi ${ }^{1}$, Mikke Heuck' ${ }^{1,2}$, Dirk Englund'; 'Research Lab of Electronics, MIT, USA; ${ }^{2}$ Photonics Engineering, Technical Univ. of Denmark, Denmark. We propose a photonic crystal cavity design with self-similar structure to achieve ultrasmal mode volume. We describe the concept with a silicon-air nanobeam cavity at $\lambda \sim 1550 \mathrm{~nm}$ reaching a mode volume of $\sim 7.01 \times 10^{-5} \lambda^{3}$. 


\section{CLEO: Science \& Innovations}

14:00-16:00

STh3I • Quantum Confined

Materials \& Devices

Presider: Roberto Paiella; Boston

Univ., USA

\section{STh3I.1 • 14:00}

Flexible Light Emitting Diodes Based on Nitride Nanowires, Nan Guan' ${ }^{1}$ Xing Dai', Agnès Messanvi ${ }^{1,2}$, Hezhi Zhang ${ }^{1}$, Jianchang Yan $^{1,3}$, Eric Gautier ${ }^{2,4}$, Catherine Bougerol ${ }^{2,5}$, Martin Vallo ${ }^{2,6}$, François H. Julien', Christophe Durand 2,6 , Joël Eymery 2,6, Maria Tchernycheva ${ }^{1} ;{ }^{1}$ Centre de Nanosciences et de Nanotechnologies, Université Paris-Saclay, France; ${ }^{2}$ Université Grenoble Alpes, France; ${ }^{3}$ Inst. of Semiconductors, Chinese Academy of Sciences, China; ${ }^{4}$ INAC-SPINTEC, CEA, France; ${ }^{5} \mathrm{CNRS}$, Institut Néel, France; ${ }^{6}$ INACPHELIOS, CEA, France. Flexible blue/green/ white light emitting diodes based on nitride nanowires embedded in polymer layers are demonstrated. The fabrication and physics of these novel hybrid light sources will be described.

\section{STh3I.2 • 14:15}

Strain-Engineered SiGe Nanomembrane Quantum-Well Infrared Photodetectors, Habibe Durmaz ${ }^{1,2}$, Pornsatit Sookchoo ${ }^{3}$, X. Cui ${ }^{3}$, RB Jacobson ${ }^{3}$, D.E. Savage ${ }^{3}$, M.G. Lagally', Roberto Paiella'; 'Boston Univ., USA; ${ }^{2}$ Recep Tayyip Erdogan Univ., Turkey; ${ }^{3}$ Univ. of Wisconsin, USA. SiGe quantum-well nanomembranes, where stress from lattice mismatch is relaxed via elastic strain sharing rather than defect formation, are used to develop intersubband photodetectors showing improved performance compared to identical devices grown on rigid substrates.

\section{STh31.3 • 14:30}

Study of SiGeSn/GeSn/SiGeSn Quantum Well towards All Group-IV-Optoelectronics, Wei Du 1,2, Seyed Ghetmiri², Sattar H. Al-Kabi², Joe Margetis ${ }^{3}$, Yiyin Zhou ${ }^{2}$, Wei Dou², Aboozar Mosleh" ${ }^{4}$, Jifeng Liu ${ }^{5}$, Gregory Sun ${ }^{6}$, Richard Soref ${ }^{6}$, John Tolle ${ }^{3}$, Baohua $\mathrm{Li}^{7}$, Mansour Mortazavi ${ }^{1}$, Shui-Qing Yu ${ }^{2}$; ${ }^{\prime}$ Univ. of Arkansas at Pine Bluff, USA; ${ }^{2}$ Univ. of Arkansas, USA; ${ }^{3} \mathrm{ASM}$, USA; ${ }^{4}$ Arkansas Tech Univ., USA; ${ }^{5}$ Dartmouth College, USA; ${ }^{6}$ Univ. of Massachusetts Boston, USA; ${ }^{7}$ Arktonics, LLC, USA. SiGeSn/ $\mathrm{GeSn} / \mathrm{SiGeSn}$ quantum well was grown on Ge buffered Si substrate via chemical vapor deposition. Photoluminescence spectra were obtained using three excitation lasers, which could in-depth probe the optical transition characteristics of the quantum well.

\section{4:00-15:45}

STh3J • Ultrafast Laser-Material Interactions

Presider: Richard Haglund;

Vanderbilt Univ., USA

\section{STh3J.1 • 14:00 Invited}

Exploring Energy Deposition of Ultrashort Lasers at the Surface of Dielectrics at the Femtosecond Scale, Olivier P. Uteza ${ }^{1}$, Maxime Lebugle ${ }^{1}$, Nadezda Varkentina ${ }^{1}$, Marc Sentis', Nicolas Sanner'; 'LP3-CNRS-AMU, France. Using high resolution pump-probe experiments, we provide deep understanding of laser energy deposition at the surface of dielectric material irradiated by single femtosecond pulse. This knowledge paves the way to smart engineering of material excitation.

\section{4:00-16:00 \\ STh3K • Nonlinear Fiber \\ Photonics II}

Presider: Camille-Sophie Bres;

Ecole Polytechnique Federale de Lausanne, Switzerland

\section{STh3K.1 - 14:00 Invited}

Advanced Ultrafast Laser Sources Harnessing Fiber-optic Nonlinearities, Guoqing Chang ${ }^{1} ;{ }^{1}$ Center for Free Electron Laser Science, Germany. We demonstrate an energy scalable method of implementing widely tunable femtosecond sources. Based on fiber-optic nonlinearities, 100 -fs pulses tunable in $825-1700 \mathrm{~nm}$ are achieved, well suited for driving multiphoton microscopy.
14:00-16:00 STh3L • Dual Frequency Comb Techniques

Presider: lan Coddington; NIST, USA

\section{STh3L.1 • 14:00}

Electro-optic frequency combs for multiplexed pump-probe spectroscopy, David Long ${ }^{1}$, Adam J. Fleisher ${ }^{1}$, David F. Plusquellic $^{2}$, Joseph T. Hodges ${ }^{1} ;{ }^{1}$ NIST, USA; ${ }^{2}$ NIST USA. Electro-optic frequency combs were generated using a train of frequency chirped waveforms. These combs had up to 10,000 teeth with a spacing as narrow as $200 \mathrm{kHz}$ which enabled Doppler and sub-Doppler spectroscopy of ${ }^{39} \mathrm{~K}$.
STh3J.2 • 14:30

Temperature-dependent Evolution and Properties of Laser-induced Periodic Surface Structures on Fused Silica, Stephan Gräf ${ }^{1}$, Clemens Kunz ${ }^{1}$, Sebastian Engel ${ }^{1}$, Frank A. Müller'; ' ${ }^{1}$ Friedrich-Schiller-Universität Jena, Germany. We report the formation of laser-induced periodic surface structures in fused silica by fs-laser irradiation at different substrate temperatures. Using scanning electron microscopy, we reveal the evolution process and properties of these structures.
STh3K.2 • 14:30

Characterization of Intermodal Group Index Matched Soliton Interactions leading to MW Peak Powers at $1300 \mathrm{~nm}$ Lars Rishoj', Boyin Tai', Poul Kristensen², Siddharth Ramachandran'; ${ }^{1}$ Boston Univ. USA; ${ }^{2}$ OFS-Fitel, Denmark. We demonstrate record 1.1-MW peak power ultrashort pulse source $(80 \mathrm{~nJ}, 74 \mathrm{fs}$ ) directly out of fibers at the biologically significant $~ 1300-n m$ spectral window using a new process of intermodal frequency conversion between ultrafast pulses.
STh3L.2 • 14:15

Dual-comb Spectroscopy using On-chip Mode-locked Frequency Combs, Avik Dutt ${ }^{2,1}$, Chaitanya S. Joshi ${ }^{2,1}$, Xingchen $\mathrm{Ji}^{2,1}$ Jaime Cardenas ${ }^{1,3}$, Yoshitomo Okawachi ${ }^{1}$, Alexander L. Gaeta ${ }^{1}$, Michal Lipson ${ }^{1} ;{ }^{1}$ Columbia Univ., USA; ${ }^{2}$ Cornell Univ., USA; ${ }^{3}$ Inst. of Optics, Univ. of Rochester, USA. We generate broadband soliton mode-locked dual combs on the same chip and demonstrate high SNR (> $40 \mathrm{~dB}$ ) near-infrared dual-comb spectroscopy of dichloromethane over a 170 $\mathrm{nm}$ optical bandwidth with a short acquisition time of $20 \mu \mathrm{s}$.
STh3L.3 • 14:30

Dead-band-free, real-time high-resolution microwave frequency measurement with a multi-comb laser, Cui Li', Xin Zhao', Ruixiao $\mathrm{Li}^{1}$, Ting $\mathrm{Li}^{1}$, Guoqing $\mathrm{Hu}^{1}$, Takeshi Yasui ${ }^{3,4}$ Zheng Zheng, ${ }^{1,2}$; ${ }^{1}$ School of Electronic and Information Engineering, Beihang Univ. China; ${ }^{2}$ Collaborative Innovation Center of Geospatial Technology, China; ${ }^{3}$ Graduate School of Science and Technology, Tokushima Univ., Japan; ${ }^{4}$ JST, ERATO, MINOSHIMA Intelligent Optical Synthesizer Project, Japan. Triple-comb pulses generated from one multi-wavelength mode-locked laser enable real-time microwave frequency measurement without the 'dead-band' limitation in previous dual-comb schemes. $1.5^{\star} 10^{-10}$ measurement accuracy is achieved at $20 \mathrm{GHz}$ using a lowcost, compact fiber-optic setup. 
Marriott

Salon III

Joint

14:00-16:00

JTh3M • Symposium on Optical Microcavities for Ultrasensitive

Detection I

Presider: Yun-Feng Xiao; Peking

Univ., China

\section{JTh3M.1 • 14:00 Invited}

Optical Microresonators as Single-Particle Absorption Spectrometers: Fano Resonances, Attometer Sensitivity, and Working Toward Single-Molecule Spectroscopic Identification, Randall H. Goldsmith'; ' Univ. of Wisconsin Madison, USA. We present a single-particle optical microresonator spectrometer capable of extremely low limit of detection. Spectroscopy of gold nanorods shows signatures of photonic-plasmonic hybridization, including formation of Fano interferences.

Marriott

\section{CLEO: Science \& Innovations}

14:00-16:00

STh3N • Light Emitters and

Lasers

Presider: Zhihong Huang; Hewlett Packard Labs, USA

STh3N.1 • 14:00

Wavelength Tuning in InGaN/GaN Lightemitting Diodes with Strain-induced Through Nanosphere Lithography., SungWen Huang Chen ${ }^{1}$, Hao-chung Kuo ${ }^{1}$, ShengWen Wang', Kuo-Bin Hong', An-Jye Tzuo', You-Chen Chu', Po-Tsung Lee' ${ }^{1}$ Chien Chung Lin'; 'Dept. of Photonics \& Inst. of ElectroOptical Engineering, National Chiao Tung Univ., Taiwan; ${ }^{2}$ Inst. of Photonic System, National Chiao Tung Univ., Taiwan. Nanoring light emitting diodes with different wall width shows that the effective bandgap can be tuned by reducing the strain. This research successful to make the devices with four colors emission on the same wafer.

STh3N.2 • 14:15

Fully-Integrated CMOS-Compatible Q-

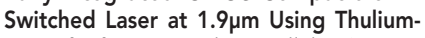
Doped $\mathrm{Al}_{2} \mathrm{O}_{3}$, Patrick T. Callahan', Katia Shtyrkova', Nanxi Li ${ }^{1}{ }^{12}$, Emir S. Magden', Purnawirman Purnawirman', Christopher Baiocco $^{3}$, Douglas Coolbaugh ${ }^{3}$, Erich P. Ippen', Michael Watts', Franz Kaertner 1,4. 'MIT, USA; ${ }^{2}$ Harvard Univ., USA; ${ }^{3}$ College of $\mathrm{Na}$ noscale Science and Engineering, State Univ. of New York, USA; ${ }^{4}$ Center for Free-Electron Laser Science, Germany. A fully-integrated $\mathrm{Q}$-switched laser is demonstrated at $1.9 \mu \mathrm{m}$ using thulium-doped aluminum oxide waveguides, with the potential for achieving an on-chip passively mode-locked laser. All components of the laser are fabricated in a CMOS-compatible silicon photonics process.

STh3N.3 $14: 30$

Lasing of Site-Controlled InGaAs/InP Quantum Well Nanopillars Grown on Silicon, Fabian Schuster ${ }^{1}$, Jonas Kapraun', Gilliard N. Malheiros-Silveira', Saniya Deshpande ${ }^{1}$, Connie J. Chang-Hasnain'; 'UC Berkeley, USA. Site-controlled InP nanopillars MOCVD grown on Silicon show $0.87 \mu \mathrm{m}$ lasing at room temperature. Integrated InGaAs quantum wells enable the first realization of a silicon transparent III-V nanolaser $(1.21 \mu \mathrm{m})$ monolithically integrated on Silicon.
14:00-16:00

STh30 • Free-Space Optical

Communications

Presider: David Geisler; MIT

Lincoln Lab, USA

\section{STh30.1 • 14:00 Tutorial}

Introduction to Free Space Laser Communications, Hamid Hemmati'. ' ${ }^{\text {Facebook }}$ Inc., USA. This tutorial describes the status lasercom technology at a very high level, starting with the state-of-theart, followed by the effects of atmosphere, laser beam acquisition, tracking, and pointing, optics assemblies, transmitters and receivers specific to lasercom.

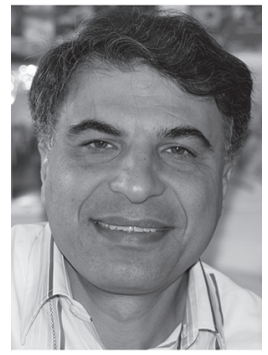

Hamid Hemmati, Ph.D., is a Director of Engineering at Facebook Inc. Prior to that, he was with the JPL/Caltech for 28 years working on the lasercom technology. He is the editor and author of two books: "Deep Space Optical Communications" and "Near-Earth Laser Communications" and author of five other book chapters. 
Executive Ballroom

$210 \mathrm{~A}$

Joint

JTh3A • Symposium on Multimodal Imaging in Biophotonics I-Continued

Executive Ballroom

210B

Executive Ballroom

$210 \mathrm{C}$

\section{CLEO: Applications \\ \& Technology}

ATh3B • Optical Devices \& Components-Continued

ATh3B.4 • 14:45

Demonstration of Compact Silicon Nitride Grating Coupler Arrays for Fan-out of Multicore Fibers, Sarvagya Dwivedi', Bowen Song', yuan liu', Renan Moreira², Leif Johansson $^{2}$, Jonathan Klamkin': ${ }^{1}$ Univ. of California Santa Barbara, USA; ${ }^{2}$ Freedom Photonics, USA. We propose a compact on-chip fan-out for multicore fiber using a focusing grating coupler array realized on a single-etch silicon nitride platform. Coupling loss of $11 \mathrm{~dB}$ with a 3-dB bandwidth of $30 \mathrm{~nm}$ and channe crosstalk lower than $28 \mathrm{~dB}$ are demonstrated.

ATh3B.5 • 15:00

Metal Assisted Focused-Ion Beam Etching for High-Fidelity Fabrication of Nanophotonic Devices, Akash Kannegulla', Li-Jing Cheng ${ }^{1}$; ${ }^{1}$ Oregon State Univ., USA. We report an MAFIB technique that significantly improves the fidelity of nanofabrication that allows direct milling of nanostructures on various materials with fine edges and smooth surfaces. The technique supports precise fabrication of nanophotonic devices.

ATh3B.6 • 15:15

Nanolithography Toolbox: Device design at the nanoscale, K. C. Balram,2, Daron Westly ${ }^{1}$, Marcelo I. Davanco', Karen Grutter, ${ }^{1,2}$, Qing Li $^{1,2}$, Thomas Michels ${ }^{1}$, Christopher Ray ${ }^{1}$, Liya $\mathrm{Yu}^{1}$, Richard Kaseca', Christopher Wallin ${ }^{1,2}$ Ian Gilbert ${ }^{1,2}$, Brian Bryce ${ }^{3}$, Gregory Simelgor ${ }^{4}$, Juraj Topolancik ${ }^{5}$, Nicolae Lobontiu ${ }^{6}$, Yuxiang Liư ${ }^{7}$, Pavel Neuzil ${ }^{8,9}$, Vojtech Svatos ${ }^{8}$, Kristen Dill ${ }^{1}$, Neal Bertrand ${ }^{1}$, Meredith Metzler $^{10}$, Gerald Lopez ${ }^{10}$, David Czaplewski ${ }^{11}$ Leonidas Ocola ${ }^{11}$, Kartik Srinivasan", Samuel Stavis ${ }^{1}$, Vladimir Aksyuk ${ }^{1}$, J. A. Liddle', Slava Krylov ${ }^{12}$, B. R. Ilic $;{ }^{1}$ NIST, USA; ${ }^{2}$ Univ. of Maryland, USA; ${ }^{3}$ Harvey Mudd College, USA; ${ }^{4}$ Edico Genome, USA; ${ }^{5}$ Roche Sequenc ing Solutions, USA; 'Univ. of Alaska, USA ${ }^{7}$ Worcester Polytechnic Inst., USA; ${ }^{8} \mathrm{Brno}$ Univ. of Technology, Czech Republic; ${ }^{9}$ Northwestern Polytechnical Univ., China; ${ }^{10}$ Univ. of Pennsylvania, USA; "Argonne National Lab, USA; ${ }^{12} \mathrm{Tel}$ Aviv Univ., Israel. We have developed a platform-independent software package for designing nanometer scaled device architectures. The Nanolithography Toolbox is applicable to a broad range of design tasks in the fabrication of microscale and nanoscale devices.
ATh3C • A\&T Topical Review on Extreme Ultraviolet and Soft X-ray Sources and Application l-Continued
ATh3C.3 • 15:00

Phase Matching of Noncollinear Sum and Difference Frequency High Harmonic Generation, Jennifer L. Ellis ${ }^{1}$, Kevin M. Dorney ${ }^{1}$ Charles Durfee², Carlos Hernandez-Garcia ${ }^{3}$, Franklin Dollar ${ }^{1}$, Christopher Mancuso' Tingting Fan ${ }^{1}$, Dmitriy Zusin ${ }^{1}$, Christian Gentry ${ }^{1}$, Patrik Grychtol', Henry Kapteyn ${ }^{1}$ Margaret Murnane ${ }^{1}$, Daniel Hickstein ${ }^{1}$ 1 JILA - NIST and Dept. of Physics, Univ. of Colorado Boulder, USA; ${ }^{2}$ Dept. of Physics, Colorado School of Mines, USA; ${ }^{3}$ Grupo de Investigacion en Aplicaciones del Laser y Fotonica, Univ. of Salamanca, Spain. We experimentally investigate phase matching of high harmonic generation in a noncollinear geometry and demonstrate phase matching above critical ionization using noncollinear high-order-difference-frequency generation, which provides a route to maximize the generated photon energies.

\section{ATh3C.4 1 15:15}

Single shot nano-holography with compact soft X-Ray laser, Alex P. Rockwood ${ }^{2}$, Yong Wang ${ }^{3}$, Shoujun Wang ${ }^{3}$, Chan Kyaw ${ }^{3}$, Carmen Menoni ${ }^{1,3}$, Mario Marconi ${ }^{3}$, Weilun $\mathrm{Chao}^{4}$, Patrick Naulleau ${ }^{4}$, Jorge Rocca ${ }^{1,3}$. ${ }^{1}$ XUV Lasers, USA; ${ }^{2}$ Physics, Colorado State Univ. , USA; ${ }^{3}$ Electrical and Computer Engineering, Colorado State Univ., USA; ${ }^{4}$ Center for X-Ray Optics, Lawrence Berkeley Nationa Lab, USA. High resolution Fourier transform holograms with $5 p$ s time resolution were obtained using a $\lambda=18.9 \mathrm{~nm}$ plasma based compact soft X-Ray laser.
Executive Ballroom 210D

CLEO: QELSFundamental Science

FTh3D • PT Symmetry and Beyond-Continued

FTh3D.4 • 14:45

Observation of the Linear Response of a Laser to an Externally Incident Probe, Ali Kazemi Jahromi ${ }^{1}$, Alexander Cerjan ${ }^{2}$, Alfred D. Stone ${ }^{3}$, Ayman F. Abouraddy'; 'Univ. of Central Florida, CREOL, USA; ${ }^{2}$ Stanford Univ. USA; ${ }^{3}$ Applied Physics, Yale Univ., USA. Investigating the optical response of a lasing cavity to a probe signal not coinciding with the lasing wavelength can give rise to paradoxes. We address this question experimentally along with steady-state ab-initio laser theory.

FTh3D.5 • 15:00

Laser self-termination in complex photonic molecules, Mohammad Hosain Teimourpour', Ramy El Ganainy'; 'Michigan Technological Univ., USA. We demonstrate numerically and analytically that laser selftermination can occur in complex photonic molecules made of more than two cavities. We also confirm our results in the presence of gain saturation nonlinearities.
FTh3D.6 • 15:15

Spontaneous Symmetry Breaking of Counterpropagating Light in Microresonators Leonardo Del Bino ${ }^{1,2}$, Jonathan Silver ${ }^{1}$, Sarah L. Stebbings' ${ }^{1}$, Pascal Del'Haye'; ${ }^{1}$ National Physical Lab (NPL), UK; ${ }^{2}$ Inst. of Photonics and Quantum Sciences, Heriot-Watt Univ. UK. We demonstrate spontaneous symmetry breaking of counter-propagating states of light in optical microresonators. The symmetry breaking is induced by nonlinear interac tion of counterpropagating light and leads to a splitting of clockwise and counterclockwise resonance frequencies. 
Executive Ballroom

210E

\section{Joint}

JTh3E • Quantum Photonics II-Continued
Executive Ballroom

210F

CLEO: QELSFundamental Science

FTh3F • Ultrafast Lattice and Molecular DynamicsContinued

FTh3F.4 • 14:45

Phonon Dephasing in Bulk and Monolayer MoS $_{2}$, Liuyang Sun ${ }^{1}$, Kha Tran ${ }^{1}$, Sebastian Roesch" ${ }^{2}$ Junho Choi ${ }^{1}$, Eduardo Priego ${ }^{1}$, Galan Moody ${ }^{3}$, Yu-Ming Chang ${ }^{4}$, Kevin Silverman ${ }^{3}$, Richard Mirin ${ }^{3}$, Xiaogin Li'; ${ }^{1}$ Univ. of Texas at Austin, USA; ${ }^{2}$ Dept. of Physics, Univ. of Tübingen, Germany; ${ }^{3}$ National Inst. of Standards and Technology, USA; ${ }^{4}$ National Taiwan Univ., Taiwan. We investigate phonon dephasing times in $\mathrm{MoS}_{2}$ using a two-pulse coherent Raman spectroscopy method. The phonon dephasing times are 3.9 and $4.9 \mathrm{ps}$ in monolayer and bulk $\mathrm{MoS}_{2}$ at room temperature.

FTh3F.5 • 15:00

JTh3E.4 • 15:00

Integrated Silicon Photonics for HighSpeed Quantum Key Distribution, Jake E. Kennard ${ }^{1}$, Philip Sibson ${ }^{1}$, Stasja Stanisic', Chris Erven', Jeremy L. O'Brien', Mark Thompson'; ' Univ. of Bristol, UK. Integrated silicon photonics offers great potential for quantum communication devices in terms of robustness and scalability. Here we demonstrate high-speed low-error QKD using silicon photonic devices combining slow thermo-optic DC biases and fast carrierdepletion modulation. meter size excited by optical transient gratings, Mathias Sander ${ }^{1}$, Marc Herzog ${ }^{1}$ Jan-Etienne Pudell ${ }^{1}$, Matias Bargheer ${ }^{1}$, Peter Gaal'; ${ }^{1}$ Inst. of Physics and Astronomie, Univ. of Potsdam, Germany; ${ }^{2}$ Inst. of Solid-State and Nanostructure Physics, Univ. of Hamburg Germany. The amplitude of surface distortions induced by transient grating excitation has been measured via time-resolved $\mathrm{x}$-ray diffraction (XRD). Results show 4 times higher amplitude of the thermal surface deformation compared to the propagating surface acoustic waves with a total amplitude of 4 Angström.
Tailored surface distortions of sub nano-
Executive Ballroom 210G

\section{Joint}

JTh3G • Symposium on Optomechanics: Towards the Second Quantum Revolution IContinued

\section{JTh3G.3 • 14:45 Invited}

Force Metrology Using Quantum Correlations of Light Generated Due to a Roomtemperature Mechanical Oscillator, Tobias J. Kippenberg ${ }^{1}$, Vivishek Sudhir ${ }^{1}$, Ryan Schil ling', Sergey Fedorov', Hendrik Schütz' ${ }^{1}$, Dalziel Wilson'; 'Ecole Polytechnique Federale de Lausanne, Switzerland. We report on the observation of quantum correlations developed in a light beam that has interacted with a room-temperature nanomechanical oscillator. Broadband homodyne measurements of the light reveal correlations at the level of $5 \%$. We use these correlations for quantumenhanced force metrology.
JTh3E.5 • 15:15

Scalable Quantum Tomography in a Photonic Chip, James G. Titchener ${ }^{1,2}$, Markus Gräfe ${ }^{2}$, Rene Heilmann², Alexander S. Solntsev ${ }^{1}$, Alexander Szameit ${ }^{2}$, Andrey Sukhorukov'; ${ }^{1}$ Australian National Univ., Australia; ${ }^{2}$ Inst. of Applied Physics, Friedrich-SchillerUniversität Jena, Germany. We formulate a method of quantum tomography that scales linearly with the number of photons and involves only one optical transformation. We demonstrate it experimentally for two-photon entangled states using a special photonic chip.
FTh3F.6 • 15:15

Chirality Dependent Coherent Phonon Dynamics in Carbon Nanotube Solutions, Ikufumi Katayama', Renjie Xu', Yasuo Minami ${ }^{1}$, Kazuhiro Yanagi ${ }^{2}$, Masahiro Kitajima ${ }^{1,3}$, Jun Takeda'; ' Yokohama National Univ., Japan ${ }^{2}$ Tokyo Metropolitan Univ., Japan; ${ }^{3}$ LxRay Co. Ltd., Japan. Using probe-wavelengthresolved coherent phonon spectroscopy, we investigated coherent phonon dynamics in chirality-mixed carbon nanotube solutions. The chirality dependent electronphonon coupling is clearly visualized in phonon-frequency vs. probe-wavelength two-dimensional mapping.
JTh3G.4 • 15:15

Optomechanical Quantum Correlations Thomas Purdy', Karen Grutter', Kartik Srinivasan', Nikolai Klimov'1,2, Zeeshan Ahmed', Jacob Taylor ${ }^{1,2} ;{ }^{1}$ NIST, USA; ${ }^{2}$ Joint Quantum Inst., USA. We present methods to measure optical quantum correlations arising from an optomechanical interaction even when large classical noise sources are present. We demonstrate quantum-backaction-noisecalibrated Brownian motion thermometry as a metrological application of quantum optomechanics.
Executive Ballroom $210 \mathrm{H}$

\section{CLEO: OELS- Fundamental Science}

FTh3H • Photonics Crystals for Light Manipulation and Concentration-Continued

\section{FTh3H.4 • 14:45 Invited}

An Integrated Diamond Nanophotonics Platform for Quantum Optical Networks Alp Sipahigil'1; ${ }^{1}$ Harvard Univ., USA. We integrate silicon-vacancy color centers into diamond nanophotonic devices. Using this platform, we demonstrate a quantum-optical switch controlled by a single color center and entanglement generation between two emitters in a single nanophotonic device.
FTh3H.5 • 15:15

The Origin and Limit of Asymmetric Transmission in Chiral Resonators, P. Nikhil ${ }^{1}$, F. Alpeggiani ${ }^{1,2}$, L. Kuipers ${ }^{1,2}$, E. Verhagen ${ }^{1}$ ${ }^{1} \mathrm{FOM}$ Inst. AMOLF, Netherlands; ${ }^{2}$ Kavli Inst. of Nanoscience, Netherlands. We develop a theoretical formalism which explains asymmetric transmission (AT) in chiral resonators from their eigenmodes. We derive a fundamental limit for AT and propose the design of a chiral photonic crystal offering $84 \%$ AT. 


\section{CLEO: Science \& Innovations}

\section{STh3l • Quantum Confined Materials \& Devices-Continued}

\section{STh31.4 $14: 45$}

Growth of InGaP Alloy Nanowires with Widely Tunable Bandgaps on Silicon Substrates, Seyed Ebrahim Hashemi Amiri ${ }^{1,2}$ Praneeth Ranga ${ }^{1}$, Dongying $\mathrm{Li}^{1}$, fan fan ${ }^{1}$ Cun-Zheng Ning 1,2; ${ }^{1}$ School of Electrical, Computer, and Energy Engineering, Arizona State Univ., USA; ${ }^{2}$ School of Molecular Sciences, Arizona State Univ., USA. InGaP alloy nanowires with In-composition continuously varying from $12 \%$ to $73 \%$ are demonstrated using chemical vapor deposition on a single silicon substrate, corresponding peak of photoluminescence changing from $580 \mathrm{~nm}$ to $780 \mathrm{~nm}$.

\section{STh3I.5 • 15:00}

Analysis of Position and Thickness Dependence of $\mathrm{ZnGeN}_{2}$ Layer in Type-II InGaN$\mathrm{ZnGeN}_{2}$ Quantum Wells Light-Emitting Diodes, Jonathon Grgat', Lu Han'1, Hongping Zhao'; 'Case Western Reserve Univ., USA. Closely lattice-matched Type-II InGaN$\mathrm{ZnGeN}_{2}$ quantum well is analyzed as active region for high performance light emitters with the focus on studying the position and thickness dependence of the $\mathrm{ZnGeN}_{2}$ layer within the In GaN quantum well.
STh31.6 $15: 15$

Temperature Dependent Diffusion Characterization of $\ln (\mathrm{Ga}) \mathrm{As} / \mathrm{InAsSb}$ Type-II Superlattice Infrared Detectors, Narae Yoon', Charles J. Reyner², Gamini Ariyawansa², John E. Scheihing ${ }^{2}$ Jim Mabon ${ }^{3}$, Daniel Wasserman'; 'The Univ. of Texas at Austin, USA; ${ }^{2}$ Air Force Research Lab, USA; ${ }^{3}$ Frederick Seitz Materials Research Lab, USA. We present the temperature dependent minority carrier mobility of Ga-content varying $\operatorname{lnGaAs} / \ln A s S b$ superlattice infrared detectors by combining time-resolved photoluminescence measurements and electron beam induced current technique with a new numerical approach to minimize the uncertainty.

\section{STh3J • Ultrafast Laser-Material Interactions-Continued}

STh3J.3 • 14:45

Ablation rate reduction of silicon interacted with double pulse beam of femtosecond lasers, Masaki Hashida', Shinichiro Masuno' Yuki Furukawa', Mitsuhiro Kusaba², Shunsuke Inoue ${ }^{1}$, Shuji Sakabe ${ }^{1}$, Hitoshi Sakagmi ${ }^{3}$ Masahiro Tsukamoto4; ${ }^{1}$ Kyoto Univ., Japan, ${ }^{2}$ Osaka Sangyo Univ., Japan; ${ }^{3}$ National Inst. for Fusion Science, Japan; ${ }^{4}$ JWRI Osaka Univ. Japan. The ablation rate is investigated on a silicon surface irradiated by a double-pulse beam cross-polarized in time delays of -1000 - 1000 ps. The reduced ablation rate is found clearly at delay times of $600 \mathrm{ps}$

\section{STh3J.4 • 15:00}

Direct inscription of waveguides in bulk glass for the photoexcitation of on-surface nanoparticles, Jean-Philippe Bérubé ${ }^{\text {, Al }}$ exandre Grégoire', Denis Boudreau1, Réa Vallée'; 'Universite Laval, Canada. A novel method allowing for the inscription of optical waveguides up to the surface of almost any transparent material is introduced. The resulting waveguides are employed for the photoexcitation of fluorescence-emitting nanostructures.

\section{STh3K • Nonlinear Fiber Photonics II-Continued}

\section{STh3K.3 • 14:45}

Self-Organized Instability in Disordered Multimode Fiber, Logan Wright' ${ }^{1}$, Zhanwei Liu', Daniel Nolan², Ming-Jun Li², Demetrios Christodoulides ${ }^{3}$, Frank W. Wise ${ }^{1} ;{ }^{1}$ Cornell Univ., USA; ${ }^{2}$ Sullivan Park, Corning Incorporated, USA; ${ }^{3} \mathrm{CREOL/College} \mathrm{of} \mathrm{Optics}$ and Photonics, Univ. of Central Florida, USA. We observe the self-organization of light into its most spatiotemporally-unstable state through propagation in graded-index multimode fiber. We understand this effect in terms of mode-coupling caused by dissipation, disorder, and nonlinearity.

\section{STh3K.4 • 15:00}

Towards 2-4 $\mu \mathrm{m}$ supercontinuum with $\mathrm{mW}$ / nm-level spectral density from large-core tellurite glass fiber, Hongxing Shi ${ }^{1}$, Xian Feng ${ }^{1}$, Fangzhou Tan ${ }^{1}$, Peng Wang ${ }^{1}$, Pu Wang'; ' 'Beijing Univ. of Technology, China. We report 2-3.8 $\mu \mathrm{m}$ supercontinuum with $5.1 \mathrm{~W}$ output from a dehydrated large-core tellurite fiber. The $3 \mathrm{~dB}$ bandwidth of the supercontinuum in $2-3 \mu \mathrm{m}$ is $0.985 \mu \mathrm{m}$, with the power spectral density above $2.0 \mathrm{~mW} / \mathrm{nm}$.

STh3J.5 • 15:15

Self-consistent modeling of laser matter interactions in laser-based 3D printing of metals \& alloys, Raj K. Vinnakota ${ }^{1} ;{ }^{1}$ Louisiana Tech Univ, USA. We present self-consistent model of laser mater interactions in powder beds of 3D printing of metals. Results are in exceptional agreement with developed analytical model, providing guidelines to ward improved control over the processes scan rates.
STh3K.5 • 15:15

Fiber-Based SBS Pulse Compression Using Bragg Grating Reflection Feedback of Stokes Seed, Masayuki Matsumoto', Genya Miyashita', Hitoshi Kiso'; 'Wakayama Univ. Japan. Efficient pulse compression using SBS in fiber is reported where a Stokes seed copropagating with a pump is reflected back by a fiber Bragg grating located at the end of the fiber. $1.1 \mu \mathrm{s}$ pulse is compressed to $12 \mathrm{~ns}$ with peak power $\sim 30 \mathrm{~W}$.

STh3L • Dual Frequency Comb Techniques-Continued

\section{STh3L.4 • 14:45}

Dual Comb Generation in a Single Microresonator, Xin Zhao ${ }^{2,1}$, Jonathan M. Silver ${ }^{2}$ Leonardo Del Bino 2,3 , Pascal Del'Haye ${ }^{2} ;{ }^{1} B e i$ hang Univ., China; ${ }^{2}$ National Physical Lab, UK ${ }^{3}$ Heriot-Watt Univ., UK. We present a nove scheme for dual comb generation in a single microresonator using orthogonal polarization states. The mode spacing difference of the dual combs has the potential for dual comb spectroscopy applications.

\section{STh3L.5 • 15:00}

Dual-comb single-pixel imaging for scanless hyperspectral imaging, Kyuki Shibuya ${ }^{1,2}$ Takeo Minamikawa ${ }^{1,2}$, Yasuhiro Mizutani, ${ }^{3,2}$ Takeshi Yasuil',2, Tetsuo Iwata, 2, ' ${ }^{1}$ Tokushima Univ., Japan; ${ }^{2}$ JST, ERATO MINOSHIMA Intelligent Optical Synthesizer (IOS), Japan; ${ }^{3}$ Osaka Univ., Japan. Combination of the dual comb spectroscopy with the single-pixe imaging is effectively applied for a scan-less hyperspectral imaging of an object having spectral-dependent absorption.

\section{STh3L.6 • 15:15}

Passively Stable Astrocomb from 550-890nm for High Resolution On-Sky Spectroscopy, Richard A. McCracken', Eric Depagne², Rudolph B. Kuhn², Nicolas Erasmus², Lisa A. Crause 2 , Derryck T. Reid'1 'Heriot-Watt Univ., UK; ${ }^{2}$ South African Astronomical Observatory, South Africa. A broadband visible astrocomb was implemented on the 10-m Southern African Large Telescope, delivering complete calibration of one channel of its high-resolution spectrograph. The passively stable master comb removed the need for overnight CEO-frequency locking. 
Marriott

Salon III

Joint

JTh3M • Symposium on Optical Microcavities for Ultrasensitive Detection I-Continued

Marriott

Marriott

Salon V \& VI

\section{CLEO: Science \& Innovations}

STh3N • Light Emitters and Lasers-Continued
STh3O • Free-Space Optical Communications-Continued
STh3N.4 • 14:45

Coherence and Dynamics of a Metallo-dielectric Nanolaser, Si Hui Pan ${ }^{1,2}$, Oing Gu ${ }^{3,2}$, Abdelkrim El Amili2', Felipe Vallini ${ }^{2}$, Yeshaiahu' Fainman'; 'Physics, Univeristy of California, San Diego, USA; ${ }^{2}$ Electrical and Computer Engineering, Univeristy of California, San Diego, USA; ${ }^{3}$ Electrical Engineering, Univ. of Texas at Dallas, USA. We conducted the second-order intensity-correlation measurement on a high- $\beta$ metallo-dielectric nanolaser under nanosecond pulse pumping. Our results confirm fully coherent emission can be achieved and dynamical hysteresis can be observed via the width of the emission pulse.

JTh3M.3 • 15:00

Breaking the Limitation of Evanescent Wave Sensing with Subwavelength Grating Waveguides, Hai Yan ${ }^{1}$, Lijun Huang ${ }^{2,1}$ Xiaochuan $\mathrm{Xu}^{3}$, Swapnajit Chakravarty ${ }^{3}$ Naimei Tang ${ }^{3}$, Huiping Tian², Ray T. Chen ${ }^{1,3}$; ${ }^{1}$ The Univ. of Texas at Austin, USA; ${ }^{2}$ Beijing Univ. of Posts and Telecommunications, China; ${ }^{3}$ Omega Optics Inc., USA. Microring resonator based on subwavelength grating waveguides was studied and demonstrated in biosensing experiment to show its thicknessindependent high sensitivity, which breaks the limitation in conventional evanescent wave sensors.

\section{JTh3M.4 • 15:15}

Multiplexed Subcellular Lasing in Cancer Tissues for Molecular Diagnostics, YuCheng Chen', Xiaotian Tan', Qiushu Chen ${ }^{1}$ Xudong Fan'; ${ }^{1}$ Biomedical Engineering, Univ. of Michigan, Ann Arbor, USA. The first demonstration of multiplexed lasing in human cancerous tissues with subcellular resolution was achieved by using antibodies and nucleic acid probes. Our work represents a critical milestone to implement optofluidic lasers in clinical applications for cancer diagnosis and prognosis.

\section{STh3N.5 • 15:00}

A Yellow Emitting InGaN/GaN Nanowiresbased Light Emitting Diode Grown on Scalable Quartz Substrate, Aditya Prabaswara' Tien Khee $\mathrm{Ng}^{1}$, Chao Zhao ${ }^{1}$, Bilal Janjua', Ahmed Alyamani², Munir El-desouki², Boon S. Ooi ${ }^{1}$; ${ }^{1}$ King Abdullah Univ. of Science and Technology, Saudi Arabia; ${ }^{2}$ King Abdulaziz bia. The first InGaN/GaN nanowires-based yellow $(\lambda=590 \mathrm{~nm}$ ) light-emitting diodes on scalable quartz substrates are demonstrated, by utilizing a thin Ti/TiN interlayer to achieve simultaneous substrate conductivity and transparency.

\section{STh3N.6 • 15:15}

Demonstration of Athermally Synchronized Distributed Feedback Laser with Microring Filter, Nanxi Li ${ }^{1,2}$, Zhan Su ${ }^{1,3}$, Purnawirman Purnawirman', Emir S. Magden', Alfonso Ruocco', Neetesh K. Singh"1, Matthew. Byrd', Christopher V. Poulton 1,3, Jonathan Bradley, Gerald Leake ${ }^{5}$, Douglas Coolbaugh ${ }^{5}$, Michael Watts'. 'Research Lab of Electronics, MIT, USA; ${ }^{2}$ John A. Paulson School of Engineering ${ }^{3}$ Analog Photonics, USA; ${ }^{4}$ Dept. of Engineering Physics, McMaster Univ., Canada; ${ }^{5} \mathrm{Col}-$ lege of Nanoscale Science and Engineering, Univ. at Albany, USA. We demonstrate an athermally synchronized distributed feedback laser cascaded with microring filters on a silicon photonic platform, with $>10 \mathrm{~dB}$ extinction ratio and a synchronized wavelength shift of $0.02 \mathrm{~nm} /{ }^{\circ} \mathrm{C}$ from 20 to $50^{\circ} \mathrm{C}$ City for Science and Technology, Saudi Araand Applied Science, Harvard Univ., USA;
STh30.2 • 15:00 Invited

Next-generation Free-space Optical Transceivers for High-capacity Space-based Communications, David O Caplan ${ }^{1}$, J.P. Wang', Mark L. Stevens', C Burton', J.C. Carney', Barry Romkey', N.W. Spellmeyer', Hemonth G. Rao', David J. Geisler', A Horvath', M Scheinbart', Garvin Lund'1, O Mikulina', J.D. Moores', Scott A. Hamilton"; 'MIT Lincoln Lab, USA. Building upon multirate optical transceivers developed for both near-Earth and deep-space communications, we present scalable next-generation designs for future systems requiring more compact implementation and both power- and photon-efficient performance. 
Executive Ballroom

$210 \mathrm{~A}$

Joint

JTh3A • Symposium on

Multimodal Imaging in

Biophotonics I-Continued

\section{JTh3A.3 • 15:30 Invited}

Looking at Tissue with a New Light: Clinical Advances of Multispectral Optoacoustic Tomography, Vasilis Ntziachristos ${ }^{2,1}$ ${ }^{1}$ Inst. of Biological and Medical Imaging, Helmholtz Zentrum Munchen, Germany; ${ }^{2}$ Chair of Biological Imaging, Technical Univ. of Munich, Germany. The talk discusses progress in multi-spectral optoacoustic tomography (MSOT) that brings unprecedented imaging performance in visualizing anatomical, physiological and molecular imaging biomarkers. Clinical applications and complementarity with other imaging modalities will also be addressed.

Executive Ballroom

210B

Executive Ballroom

$210 \mathrm{C}$

\section{CLEO: Applications \\ \& Technology}

\section{ATh3B • Optical Devices \& Components-Continued}

\section{ATh3B.7 • 15:30}

The self-calibrating dual-mode $\mathrm{Si}$ detector - Improved design based on Comsol Multiphysics simulations, Marit Ulset Nordsveen ${ }^{1,2}$, Chi K. Tang', Jarle Gran'; ; 'Justervesenet, Norway; ${ }^{2}$ Dept. of Physics, Univ. of Oslo, Norway. Our dual-mode optical power detector combines the principles of electrical substitution with measuring photocurrent. We present a new detector design, which shows a considerable reduction in nonequivalence between optical and electrical heating during electrical substitution.
ATh3B.8 • 15:45

Microcavity-ECDL for super-cavity frequency stabilization, Jinkang Lim ${ }^{1}$, Anatoliy Savchenkov², Andrey Matsko², Shu-Wei Huang ${ }^{1}$, Lute Maleki ${ }^{2}$, Cheewei Wong'; ' ${ }^{1}$ Univ. of California Los Angeles, USA; ${ }^{2}$ OEwaves Inc., USA. We report on the development of a tunable microresonator-based sub-kHz extended cavity diode laser (ECDL) for frequency stabilization to an ultrastable high finesse super-cavity. The relative spectral linewidth after the stabilization is $\sim 1 \mathrm{~Hz}$ limited by the super-cavity.
ATh3C • A\&T Topical Review on Extreme Ultraviolet and Soft X-ray Sources and Application I-Continued

ATh3C.5 • 15:30

Valley-dependent Carrier and Lattice Dynamics in Silicon measured by Transient XUV Spectroscopy, Scott Cushing ${ }^{1}$, Lucas Carneiro', Michael Zurch', Peter Kraus ${ }^{1}$ Chris Kaplan', Hung-Tzu Chang', Stephen R. Leone ${ }^{2,3}$; 'Chemistry, UC Berkeley, USA; ${ }^{2}$ Chemistry and Physics, UC Berkeley, USA; ${ }^{3}$ Chemical Sciences Division, Lawrence Berkeley National Lab, USA. Transient XUV core level spectroscopy is used to resolve photoexcited electron and hole distributions, as well as carrier-phonon and phononphonon scattering times, in the $\Gamma, L$, and $X$ valleys of silicon.

\section{ATh3C.6 $\bullet$ 15:45}

Extreme ultraviolet laser ablation mass spectrometry for sensitive materials studies and nanoscale chemical imaging, llya Kuznetsov', Tyler Green ${ }^{1}$, Andrew Duffin ${ }^{2}$ Tomas Burian ${ }^{3}$, Libor Juha ${ }^{3}$, Weilun Chao ${ }^{4}$ Jorge Rocca' ${ }^{1}$ Carmen S. Menoni' ${ }^{1}{ }^{1}$ Colorado State Univ., USA; ${ }^{2}$ Pacific Northwest National Lab, USA; ${ }^{3}$ Inst. of Physics of the ASCR, Czech Republic; ${ }^{4}$ Center for X-Ray Optics, Lawrence Berkeley Lab, USA. We demonstrate three dimensional nanoscale molecular composition imaging of inorganic samples by extreme ultraviolet laser ablation mass spectrometry. The method has applications in studies of surface and interface chemistry, diffusion and contamination at nanoscale dimensions.
Executive Ballroom 210D

CLEO: QELSFundamental Science

FTh3D • PT Symmetry and Beyond-Continued

FTh3D.7 • 15:30

Spontaneous chiral symmetry breaking in a nonlinear microresonator, Qi-Tao Cao ${ }^{1,2}$ Heming Wang ${ }^{1,2}$, Chun-Hua Dong ${ }^{3}$, Hui Jing $^{4}$, Rui-Shan Liu ${ }^{1,2}, X_{i}$ Chen ${ }^{1,2}$, Li Ge ${ }^{5,6}$ Qihuang Gong1,2, Yun-Feng Xiao, 2, ' ${ }^{1}$ State Key Lab for Mesoscopic Physics and School of Physics, Peking Univ.; China; ${ }^{2}$ Collaborative Innovation Center of Quantum Matter China; ${ }^{3}$ Univ. of Science and Technology of China, China; ${ }^{4}$ Hunan Normal Univ., China; ${ }^{5}$ College of Staten Island, CUNY, USA; ${ }^{6}$ The Graduate Center, CUNY, USA. We demonstrate experimentally spontaneous chirality in a whispering-gallery microresonator. Above an input threshold, the intensities of clockwise and counterclockwise propagating waves grow unbalanced, due to the Kerrnonlinearity-modulated coupling between the counter-propagating waves.

\section{FTh3D.8 • 15:45}

Shaping Polaritons to Reshape Selection Rules, Francisco Leal Machado 1,2, Nicholas Rivera ${ }^{2}$, Hrvoje Buljan ${ }^{3}$, Marin Soljacic ${ }^{2}$, Ido Kaminer2; 'Physics, Univ. of California Berkeley, USA: ${ }^{2}$ Physics, MIT, USA: ${ }^{3}$ Physics, Univ. of Zagreb, Croatia. In this work we show that by imbuing extremely highly confined polaritons with orbital angular momentum it is possible to induce new electronic selection rules in a controllable fashion. 
Executive Ballroom

210E

\section{Joint}

JTh3E • Quantum Photonics II-Continued

JTh3E.6 • 15:30

An On-chip Homodyne Detector for Measuring Quantum States, Dylan H. Mahler ${ }^{1}$, Francesco Raffaelli', Giacomo Ferranti', Philip Sibson', Jake E. Kennard', Alberto Santamato ${ }^{1}$, Gary Sinclair', Damien Bonneau', Mark Thompson', Jonathan Matthews'; ' 'Univ. of Bristol, UK. Here we present the first quantum homodyne detector on a silicon chip. We demonstrate all of the characteristics required for detection of quantum states: high speed, signal-to-noise ratio, and common-mode rejection ratio.

JTh3E.7 • 15:45

Large-Area 64-pixel Array of WSi Superconducting Nanowire Single Photon Detectors, Jason P. Allmaras ${ }^{1,2}$, Andrew Beyer ${ }^{1}$, Ryan Briggs ${ }^{1}$, Francesco Marsili ${ }^{1}$, Matthew Shaw', Giovanni Resta', Jeffrey Stern' Varun Verma ${ }^{3}$, Richard Mirin ${ }^{3}$, Sae Woo Nam ${ }^{3}$, William Farr'; ' Jet Propulsion Lab, USA ${ }^{2}$ California Inst. of Technology, USA; ${ }^{3}$ National Inst. of Standards and Technology, USA. We developed a 64-pixel $320 \mu \mathrm{m}$ diameter array of WSi SNSPDs for the ground receiver of a deep-space optical communication link. The SNSPD array exhibits a free-space system detection efficiency of $40 \%$ at $1550 \mathrm{~nm}$.
Executive Ballroom $210 \mathrm{~F}$

CLEO: QELSFundamental Science

FTh3F • Ultrafast Lattice and Molecular DynamicsContinued

FTh3F.7 • 15:30

Unraveling Phonon Couplings in a Cdse/ ZnS Colloidal Quantum Dot Ensemble Using Two-Dimensional Coherent Spectroscopy, Diogo Almeida', Albert Liu', Wan K. Bae ${ }^{2}$, Lázaro A. Padilha ${ }^{3}$, Steven T. Cundiff' ${ }^{1}$ Univ. of Michigan, USA; ${ }^{2}$ Korea Inst. of Science and Technology, Korea (the Republic of): ${ }^{3}$ Universidade Estadual de Campinas, Brazil. We resolve the phonon structure from from lowest exciton transition of $\mathrm{CdSe} /$ $\mathrm{ZnS}$ quantum dots ensemble by separating the homogeneous and inhomogeneous linewidths through optical multidimensiona coherent spectroscopy at low temperatures.

FTh3F.8 • 15:45

Graphene-induced Strong Quenching of Optical Phonons in III-V Semiconductor Heterostructures, Peter Q. Liü,2, John Reno', Igal Brener'; 'Sandia National Labs, USA: ${ }^{2}$ Electrical Engineering, The State Univ. of New York at Buffalo, USA. We demonstrate that monolayer graphene transferred onto III-V semiconductor heterostructures induces strong quenching of the optical phonon in III-V semiconductor epilayers. Such graphene-induced optical phonon quenching is even stronger than that of thin metal films.
Executive Ballroom

210G

Joint

JTh3G • Symposium on Optomechanics: Towards the Second Quantum Revolution IContinued

\section{JTh3G.5 • 15.30 Invited}

Non-classical Sources of Light and Their Applications to Gravitational Wave Detection, David E. McClelland ${ }^{1}$ L LIGO S. Collaboration': 'Q ${ }^{1}$ uantum Science, Australian National Univ., Australia; ${ }^{2}$ LIGO Scientific Collaboration, USA. Audio-band laser interferometer gravitational-wave detectors are massive opto-mechanical devices. Quantum enhancement requires sources at the operating wavelength with more than a factor of 10 squeezing from $1 \mathrm{~Hz}$ to $10 \mathrm{kHz}$, in a frequency-tailored quadrature.
Executive Ballroom $210 \mathrm{H}$

\section{CLEO: QELS- Fundamental Science}

FTh3H • Photonics Crystals for Light Manipulation and Concentration-Continued

FTh3H.6 • 15:30

Butterflies Regulate Wing Temperatures Using Radiative Cooling, Nanfang $Y_{u^{1}}{ }^{1}$ Cheng-Chia Tsai ${ }^{1}$, Norman N. Shi ${ }^{1}$, Julianne Pelaez ${ }^{2}$, Naomi Pierce ${ }^{3}$; ${ }^{1}$ Columbia Univ. USA; ${ }^{2}$ UC Berkeley, USA; ${ }^{3}$ Harvard Univ., USA We discovered that the spatial distribution of coloration and emissivity of butterfly wings help regulate wing temperatures, and that the spatial variation of thermal emissivity is mainly controlled by nano-structures of the wing scales.
FTh3H.7 • 15:45

Biomimetic Radiative-Cooling Metasurfaces Inspired by Saharan Silver Ants, Norman N. Shi', Cheng-Chia Tsai ${ }^{1}$, Rüdige Wehner ${ }^{2}$, Nanfang Yu'; ${ }^{1}$ Columbia Univ. USA; ${ }^{2}$ Brain Research Inst., Univ. of Zürich, Switzerland. Biomimetic radiative-cooling metasurfaces inspired by the Saharan silver ants are fabricated using 3D printing 3D-printed structures are used as proof of concept to demonstrate various reflectivity and emissivity enhancement mechanisms discovered in the ants. 

Meeting Room
$211 \mathrm{~B} / \mathrm{D}$
Meeting Room
$212 \mathrm{~A} / \mathrm{C}$
Meeting Room

$212 \mathrm{~B} / \mathrm{D}$
Marriott

Salon I \& II

\section{CLEO: Science \& Innovations}

\section{STh3l • Quantum Confined} Materials \& Devices-Continued

STh3I.7 • 15:30

246 nm AIN-delta-GaN Quantum Well UItraviolet Light-Emitting Diode, Cheng Liu ${ }^{1}$, Yu Kee Ooi ${ }^{1}$, SM Islam², Huili (Grace) Xing ${ }^{2}$, Debdeep Jena ${ }^{2}$, Jing Zhang ${ }^{1} ;{ }^{1}$ Rochester Inst. of Technology, USA; ${ }^{2}$ Cornell Univ., USA. The $246 \mathrm{~nm}$ AlN-delta-GaN quantum well ultraviolet light-emitting diode was proposed and realized experimentally, with the dominant transverse electric-polarized emission been verified by both the $k \cdot p$ simulation and the room-temperature polarization-dependent electroluminescence measurements.

\section{STh3I.8 • 15:45}

High-quality GaAs Grown on Aluminum Film, Chia-Chu Cheng', Yen-Ting Fan"; ${ }^{1} N C T U$, Taiwan. We have grown GaAs layers on an aluminum nanofilm by using molecular beam epitaxy. Defect-free GaAs and InAs quantum dots are investigated with $\mathrm{X}$-ray diffraction, transmission electron microscopy, and room-temperature photoluminescence.

\author{
STh3J • Ultrafast Laser-Material \\ Interactions-Continued
}

STh3J.6 • 15:30

Comparison of Filament-Generated $\mathrm{Pe}$ riodic Surface Features using Different Laser Wavelengths, Anthony Valenzuela ${ }^{1}$, Kristopher Behler², Zachary Brunson ${ }^{3}$, Al Rastegari ${ }^{4}$, Chengyong Feng ${ }^{4}$, Christopher Wolfe ${ }^{1}$, Laura Vanderhoef ${ }^{1}$, Brian Kramer ${ }^{4}$, Ladan Arissian ${ }^{4}$, Aaron Schweinsberg ${ }^{5}$, Jean-Claude Diels ${ }^{4}$, Aaron Stebner ${ }^{3} ;{ }^{1}$ US Army Research Lab, USA; ${ }^{2}$ TKC Global, USA; ${ }^{3}$ Mechanical Engineering, Colorado School of Mines, USA; ${ }^{4}$ The Center for High Technology Materials, Univ. of New Mexico, USA; ${ }^{5} \mathrm{Oak}$ Ridge Inst. for Science and Education, USA. Filament-induced periodic surface structures are generated on a wide variety of materials with near-IR and UV lasers. The surface structure features demonstrate the relation to laser wavelength and polarization and energy distribution in a filament.

\section{STh3K • Nonlinear Fiber Photonics II-Continued}

STh3K.6 • 15:30

Efficient Polarization-Insensitive Four-Wave Mixing Assisted by Raman Amplification, Xiaojie Guo ${ }^{1}$, Chester Shu ${ }^{2}{ }^{1}{ }^{1}$ Inst. of Photonics Technology, Jinan Univ., China; ${ }^{2}$ Dept. of Electronic Engineering, The Chinese Univ. of Hong Kong, Hong Kong. We report efficient polarization-insensitive four-wave mixing in a two-orthogonal-pump configuration with the assistance of backward Raman amplification. Due to Raman enhancement, conversion efficiency of $\sim 0 \mathrm{~dB}$ is obtained in a nonlinear fiber without stimulated-Brillouin-scattering suppression.

\section{STh3K.7 • 15:45}

Data transmission through polarization domain walls in normally dispersive optical fibers, Marin Gilles ${ }^{1}$, Pierre-Yves Bony' Josselin Garnier², Antonio Picozzi', Massimiliano Guasoni ${ }^{1,3}$, Julien Fatome ${ }^{1} ;{ }^{1}$ CNRS - Universite de Bourgogne Franche-Comté, France; ${ }^{2}$ Univ. of Paris VII, France; ${ }^{3}$ Optoelectronics Research Centre, UK. We report the experimental observation of polarization domain-walls in conventional optical fibers. Moreover, we exploit their topological properties for data transmission beyond the Ker limits imposed in normally dispersive fibers.
STh3L • Dual Frequency Comb Techniques-Continued

STh3L.7 • 15:30

Hybrid Dual-comb Interferometer Using Electro-optic Comb and Free-running Femtosecond Laser, Shuai Wang ${ }^{1}$, Xinyu Fan', Qingwen Liu', Zuyuan $\mathrm{He}^{1}$; ${ }^{1}$ Shanghai Jiao Tong Univ., China. We demonstrate rapid-scan-rate dual-comb interferomete employing an electro-optic comb generated from Fabry-Perot modulator and a freerunning femtosecond laser. High dynamic range has been achieved without the necessity of complicated phase-locking and laser stabilization.

\section{STh3L.8 • 15:45}

Gas Spectroscopy with a Dual-Comb Semiconductor Disk Laser, Sandro M. Link', Deran J. Maas ${ }^{2}$, Dominik Waldburger ${ }^{1}$, Cesare G. Alfieri', Matthias Golling', Florian Emaury ${ }^{1}$ Ursula Keller'; ${ }^{1}$ ETH Zurich, Switzerland; ${ }^{2}$ ABB, Corporate Research, Switzerland. For the first time, we use a stabilized dual-comb modelocked semiconductor disk laser to perform dual-comb spectroscopy. A water vapor absorption spectrum around $968 \mathrm{~nm}$ is measured with our very compact, simple and cost-efficient system. 
Marriott

Salon III

Joint

JTh3M • Symposium on Optical Microcavities for Ultrasensitive Detection I-Continued

JTh3M.5 • 15:30

Ultrahigh-Q/V single point-defect photonic crystal nanocavity with embedded subwavelength air-slot, Eiichi Kuramochi', Jun K. Kim ${ }^{1}$, Hideaki Taniyama ${ }^{1}$, Akihiko Shinya ${ }^{1}$ Shota Kita', Masaya Notomi'; ; ${ }^{1}$ TT Corporation, Japan. $\mathrm{H} 1$ photonic crystal nanocavitie with a short sub-wavelength air-slot that can concentrate an electric field to one antinode with $V<0.025\left(\mathrm{~N} / \mathrm{n}^{3}\right.$ experimentally exhibited a $Q$ factor over $2 \times 10^{5}$ and a $Q / V$ reaching $10^{7}$

JTh3M.6 • 15:45

Size spectrometry of environmental particulate matter using optical evanescent fielda nanofiber array, Xiao-Chong $\mathrm{Yu}^{1}$ Yanyan Zhi' ${ }^{1,2}$, Bei-Bei Li', Qihuang Gong ${ }^{1,2}$ Yun-Feng Xiao ${ }^{1,2}$; ${ }^{1}$ Peking Univ., China; ${ }^{2} \mathrm{Col}$ laborative Innovation Center of Quantum Matter, China. We propose and build an optical evanescent field based size spectrometer using a nanofiber array by measuring the distinction. This method is practically applied to monitor the particulate matters in atmosphere in Beijing.

Marriott

\section{CLEO: Science \& Innovations}

STh3N • Light Emitters and Lasers-Continued

STh3N.7 • 15:30

Resonant Light Emission from Highly $\mathrm{N}$ doped Germanium-on-Insulator Microdisks with Circular Bragg Grating, Xuejun Xu', Hideaki Hashimoto ${ }^{1}$, Kentarou Sawano', Takuya Maruizumi'; 'Tokyo City Univ., Japan. Resonant light emission with high Q-factor and fringe contrast, corresponding to FabryPerot modes, have been observed over 250 $\mathrm{nm}$ wavelength range from highly $\mathrm{n}$-doped Ge-on-Insulator microdisks by combining with highly reflective circular Bragg grating.

STh3N.8 • 15:45

Individually Addressable Micron-Sized LED Color Pixels with Integrated Condenser Lenses, Brandon Demory ${ }^{1}$, Kunook Chung ${ }^{1}$, Jingyang Sui', Pei-Cheng Ku'; ' ${ }^{1}$ Univ. of Michigan, USA. A multi-color LED chip with integrated parabolic lenses is shown. The emission collimates within a 0.5NA zone with percentages of $75 \%$ for red, $83 \%$ for green, and $95 \%$ for blue, of the total emission, respectively.

\section{STh30 • Free-Space Optical} Communications-Continued
STh30.3 • 15:30

First Demonstration of $400 \mathrm{Mb} / \mathrm{s}$ PAM4 Signal Transmission Over 10-meter Underwater Channel Using a Blue LED and a Digital Linear Pre-Equalizer, Boyuan Zhuang ${ }^{1}$, Chao

$\mathrm{Li}^{1}$, Nan Wu${ }^{1}$, Zhengyuan $\mathrm{Xu}^{1}$; ${ }^{1}$ USTC, China. A $400 \mathrm{Mb} / \mathrm{s}$ PAM4 signal is experimentally generated and transmitted over 10-meter underwater channel using a single blue LED and a simple digital linear pre-equalizer for the first time.
STh30.4 $15: 45$

Near-Infrared Wireless Optical Communication with Particulates In-Suspension over the Underwater Channel, It E. Lee ${ }^{2,1}$, Yujian Guo ${ }^{2}$, Tien Khee $\mathrm{Ng}^{2}$, Ki-Hong Park ${ }^{2}$ Mohamed-Slim Alouini ${ }^{2}$, Boon S. Ooi ${ }^{2}$. ${ }^{1}$ Faculty of Engineering (FOE), Multimedia Univ. (MMU), Malaysia; ${ }^{2}$ Computer, Electri$\mathrm{cal}$ and Mathematical Sciences and Engineering (CEMSE) Division, King Abdullah Univ. of Science and Technology (KAUST), Saudi Arabia. We demonstrate a gigabit near-infrared-based underwater wireless optical communication link using an 808-nm laser diode to mitigate the particle scattering effect in turbid medium. An improvement in the error performance is observed with increasing concentrations.

16:00-16:30 Coffee Break, Concourse Level
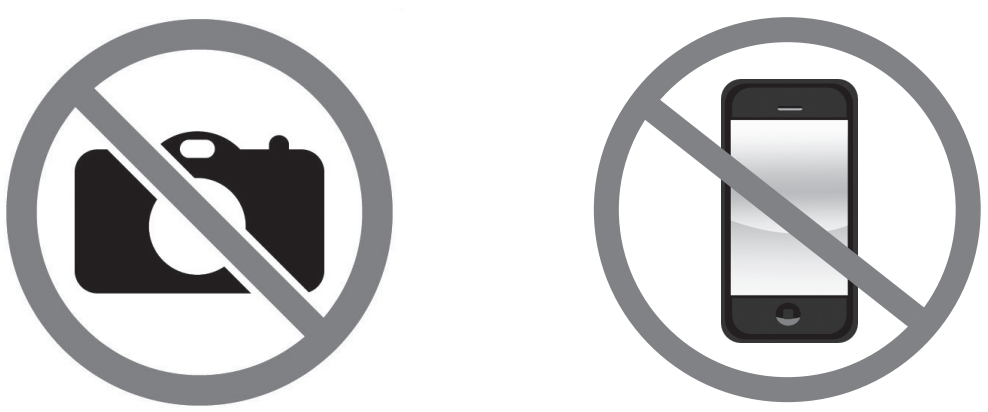
Executive Ballroom

$210 \mathrm{~A}$

\section{Joint}

16:30-18:30

JTh4A - Symposium on

Multimodal Imaging in

Biophotonics II

Presider: Ji-Xin Cheng; Purdue

Univ., USA

\section{JTh4A.1 • 16:30 Invited}

Assessing Airway Smooth Muscle Microstructure and Contractile Force in Vivo Using Birefringence Microscopy, Melissa Suter'; 'Massachusetts General Hospital, Harvard Medical School, USA. Excessive contraction of airway smooth muscle (ASM) is responsible for the majority of the symptoms of asthma. We have developed a birefringence microscopy platform to volumetric assess ASM in patients and to quantify and predict ASM contractile force as a function of optical retardation.
Executive Ballroom 210B

Executive Ballroom $210 \mathrm{C}$

\section{CLEO: Applications \\ \& Technology}

16:30-18:30

ATh4B - Process Evaluation \&

Microscopy

Presider: Brian Simonds; NIST USA

ATh4B.1 - 16:30 Invited

Chemical Segregation and Microstructural Evolution of Fiber Laser Beam Welded Low Carbon Sheet Steel, Ann Chiaramont Debay', Paul T. Blanchard', Stephanie M. Kaster $^{1}$, Jeffrey W. Sowards', James R. Fekete': 'NIST, USA. This study provides fundamental data for phase transformation prediction during the rapid solidification of laser beam welded low carbon sheet steel. Findings can strengthen modeling efforts by providing kinetic and thermodynamic data based on real-world observations.
16:30-18:30

ATh4C • A\&T Topical Review on Extreme Ultraviolet and Soft X-ray Sources and Application II Presider: Alex Ershov; Cymer Inc, USA

ATh4C.1 - 16:30 Invited

Nuclear Photonics Enabled by MeV LaserCompton Sources, Christopher P. Barty'; 'Lawrence Livermore National Lab, USA. This paper reviews the design and optimization of $\mathrm{MeV}$ laser-Compton sources and the development of the unique nuclear science and applications enabled by them, i.e. nuclear photonics.
Executive Ballroom 210D

\section{CLEO: QELS- Fundamental Science}

16:30-18:30

FTh4D - Solitons and Temporal Wave Guiding

Presider: J. Stewart Aitchison; Univ. of Toronto, Canada

FTh4D.1 • 16:30

Stokes Solitons in Optical Microcavities Qifan Yang ${ }^{1}$, Xu Yi ${ }^{1}$, Ki Y. Yang ${ }^{1}$, Kerry Vahala', ${ }^{\prime}$ California Inst. of Technology, USA. A soliton is reported that regenerates by optimizing its Raman interaction with another soliton within a shared optical potential well. The soliton is observed in a high-Q silica optical microcavity.

\section{JTh4A.2 • 17:00 Invited}

Complete Cutaneous Vasculature Imaging and Its Clinical Translation Using Multimodal Photoacoustic and Optical Coherence Tomography Angiography, Mengyang Liu', Zhe Chen', Behrooz Zabihian', Christoph Sinz ${ }^{1}$, Edward Zhang ${ }^{2}$, Paul Beard ${ }^{2}$, Eric Hoover $^{3}$, Micheal Minneman ${ }^{3}$, Jason Ensher ${ }^{3}$, Rainer A. Leitgeb ${ }^{1}$, Harald Kittler ${ }^{1}$, Wolfgang Drexler'; ${ }^{1}$ Medical Univ. of Vienna, Austria; 2Univ. College London, UK; ${ }^{3}$ Insight Photonic Solutions, USA. A multimodal optical imaging system combining all optical photoacoustic tomography and optical coherence tomography angiography is developed for human skin imaging. Various different types of skin disorders can be imaged by this system.
ATh4B.2 • 17:00

High Speed Hyperspectral Thermal Imaging of the Melt Pool Dynamics During Metal Additive Manufacturing, Nicholas P. Calta ${ }^{1}$, Gabe Guss', Sheldon S. Wu', Sonny Ly', Dave Deane', Michael F. Crumb', Manyalibo Matthews'; 'Lawrence Livermore National Lab, USA. We use high speed multiwavelength thermal imaging to quantify cooling rates and temperature gradients generated by the laser - powder interaction during a metal powder bed fusion process to understand rapidly solidified material properties.

\section{ATh4B.3 • 17:15}

Dependence of $\mathrm{THz}$ Signals on Carbon Black Compounding Amount in Vulcanized Rubber, Yasuyuki Hirakawa', Tatsuhiro Yamauchi', Takuya Kamino', Toyohiko Gondo' Seiichi Hirano2 ${ }^{2}$ Tsuyoshi Noguchi'; ${ }^{1} N I T$ Kurume College, Japan; ${ }^{2}$ Technical Service Dept., DAIKIN INDUSTRIES, LTD., Japan. Dependence of the $\mathrm{THz}$ absorbance and reflectance on carbon black(CB) compounding amount in vulcanized rubber was investigated. It was found that the $\mathrm{THz}$ absorbance was not proportional to the $\mathrm{CB}$ concentration at higher amount.
ATh4C.2 • 17:00

Coherent extreme ultraviolet pulse generation using metal-sapphire nanostructures, Seunghwoi Han', Hyunwoong Kim', Yong Woo Kim', Seung-Woo Kim'; ; Korea Advanced Inst of Science \& Tech, Korea (the Republic off. Coherent extreme ultraviolet (EUV) light pulses are produced from metalsapphire nanostructures by irradiation of infrared femtosecond pulses. Single-crysta sapphire emitters subject to plasmonic field enhancement enable high-order harmonics generation up to the 13 th order.

\section{ATh4C.3 • 17:15 Invited}

Elliptically Polarized Attosecond Pulse Trains Produced via Circularly Polarized High Harmonic Generation, Kevin Dorney' Jennifer L. Ellis ${ }^{1}$, Carlos Hernandez-Garcia² Daniel Hickstein ${ }^{1}$, Christopher Mancuso ${ }^{1}$ Tingting Fan', Guangyu Fan³, Patrik Grychtol', Dmitriy Zusin', Christian Gentry', Henry Kapteyn', Margaret Murnane 1; '1 JILA - Univ. of Colorado Boulder, USA; ${ }^{2}$ Grupo de Investigacion en Aplicaciones del Laser y Fotonica - Univ. of Salamanca, Spain; ${ }^{3}$ Photonics Inst. - Vienna Univ. of Technology, Austria. We present a straightforward method to produce ellipticity polarized attosecond pulse trains via bicircular-driven high harmonic generation. The intensity ratio of the bicircular field can be tuned to generate attosecond pulses of nearly arbitrary polarization.
FTh4D.2 • 16:45

Counter-Propagating Solitons in Microresonators, Chaitanya S. Joshi ${ }^{1,2}$, Yoshitomo Okawachi ${ }^{1}$, Mengjie Yu ${ }^{1,3}$, Alexander Klenner ${ }^{1}$, Xingchen $\mathrm{Ji}^{3,4}$, Kevin Luke ${ }^{3}$, Micha Lipson ${ }^{4}$, Alexander Gaeta'; ${ }^{1}$ Dept. of Applied Physics and Applied Mathematics, Columbia Univ., USA: ${ }^{2}$ School of Applied and Engineering Physics, Cornell Univ., USA; ${ }^{3}$ School of Electrical and Computer Engineering, Cornell Univ., USA; ${ }^{4}$ Dept. of Electrical Engineering, Columbia Univ., USA. Using a single pump laser, we demonstrate simultaneous soliton modelocked frequency combs in a siliconnitride microresonator in the clockwise and counter-clockwise directions with slightly different repetition comb spacings.

\section{FTh4D.3 17:00 Invited}

Breathing Dissipative Solitons in Microresonators, Erwan Lucas ${ }^{1}$, Maxim Karpov ${ }^{1}$, Hairun Guo', Michael L. Gorodetsky², Tobias J. Kippenberg ${ }^{1}{ }^{1}$ Ecole Polytechnique Fédérale de Lausanne, Switzerland; ${ }^{2}$ Faculty of Physics, Lomonosov Moscow State Univ., Russia. We present a comprehensive analysis of breathing dissipative solitons in two microresonator platforms. Numerical simulations and theoretical analyses are in good agreement with experimental observations, providing insights into the dynamical instabilities in these systems. 
Executive Ballroom $210 \mathrm{E}$

Executive Ballroom $210 \mathrm{~F}$

\section{CLEO: QELS-Fundamental Science}

16:30-18:30

FTh4E • Single-Photon Sources and Quantum Communications Presider: Alexander Sergienko; Boston Univ., USA

FTh4E.1 • 16:30

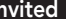

Quantum Communication with Temporal Modes of Pulsed Light, Christine Silberhorn ${ }^{1}$, Vahid Ansari ${ }^{1}$, Markus Allgaier ${ }^{1}$ Benjamin Brecht'2, Christof Eigner', Viktor Quiring', Raimund Ricken', Georg Harder' Linda Sansoni'1. 'Dept. of Physics, Paderborn Univ., Germany; ${ }^{2}$ Clarendon Lab, Oxford Univ., UK. We present a framework for quantum communication using tempora modes of quantum light with orthogonal spectral-temporal shapes. These span a high-dimensional Hilbert space and are ideally suited for efficient quantum information coding for network applications.

\section{6:30-18:15}

FTh4F • Imaging Electron

Dynamics on the Nano-, FemtoScale

Presider: Ilias Perakis; Univ. of Alabama at Birmingham, USA

\section{FTh4F.1 • 16:30 Tutorial}

Ultrafast Microscopy of Electronic Excitations in Nanostructured Materials, Hrvoje Petek'; 'Univ. of Pittsburgh, USA. I describe ultrafast microscopy on the femto-nano scale of plasmonic resonances in Ag nanocrystals grown on Si substrates by broadly tunable (IRUV) femtosecond NOPA multi-photon excitation and aberration corrected photoemission electron microscopy imaging.

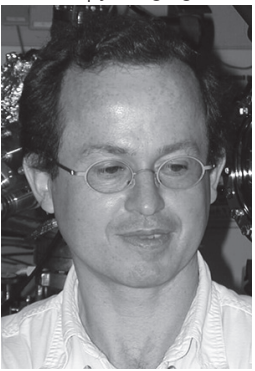

Hrvoje Petek is the Mellon Chair of Physics. He graduated from MIT (BS-1980) and U.C. Berkeley (PhD-1985). After a 15 yr career in Japan at IMS and Hitachi ARL, he joined the University of Pittsburgh. $\mathrm{He}$ is the Editorin-Chief of Progress in Surface Science and inventor of ultrafast coherent photoemission electron microscopy.
FTh4E.2 • 17:00

Temporal Multiplexing of Heralded Single Photons with a Resource-Efficient Fiber Loop, Rowan A. Hoggarth'1, Robert J. FrancisJones ${ }^{1}$, Peter Mosley ${ }^{1} ;{ }^{1}$ Physics, Univ. of Bath, UK. We have implemented resource-efficient active multiplexing that synchronises single photons generated by several consecutive pump pulses with a single optical switch. We demonstrate an increase in delivery probability per mode of heralded single photons.

Th4E.3・17:15

Relative time multiplexing of heralded single photons for efficient quantum communication, Fumihiro Kaneda', Alexander Hill1' Paul G. Kwiat ${ }^{1 \cdot}{ }^{1}$ Univ of Illinois at UrbanaChampaign, USA. We demonstrate relative time multiplexing of heralded single-photon sources, using a low-loss photon storage technique. Our scheme can realize efficient two-photon quantum communication protocols, e.g., measurement-device-independent quantum key distribution.
Executive Ballroom

210G

\section{Joint}

16:30-18:15

JTh4G • Symposium on

Optomechanics: Towards the Second Quantum Revolution II

Presider: Pierre-François

Cohadon; Laboratoire Kastler

Brossel, France

JTh4G.1 • 16:30

High-fidelity ground state cooling of a mechanical resonator via squeezed light driving, David Vitali1,2, Muhammad Asjad ${ }^{1}$ Stefano Zippilli1,2; ' 'Universita di Camerino, Italy; ${ }^{2}$ Sezione di Perugia, INFN, Italy. We show that preparation of nonclassical states of a mechanical resonator with close-to-one fidelity is possible by driving a cavity optomechanical system with squeezed vacuum light.

\section{JTh4G.2 • 16:45 Invited}

Topology of Light and Sound, Florian Marquardt1,2. ${ }^{1}$ Max Planck Inst. Science of Light, Germany; ${ }^{2}$ Dept. of Physics, Univ. of Erlangen-Nuremberg, Germany. I will show how the interaction of light and sound can be used to engineer topologically robust chiral transport of phonons and photons on the nanoscale. This connects the fields of optomechanics and topological transport.

JTh4G.3 • 17:15

Exceptional Points in an Optomechanical System, David Mason', Haitan Xu1, Luyao Jiang', Jack Harris',2. 'Physics, Yale Univ. USA; ${ }^{2}$ Applied Physics, Yale, USA. Here, we investigate exceptional points in a cavity optomechanical system. We demonstrate the predicted topology of the eigenvalues, as well as non-reciprocal energy transfer via closed adiabatic cycles.
Executive Ballroom $210 \mathrm{H}$

\section{CLEO: QELS- Fundamental Science}

16:30-18:30

FTh4H • Optical and Thermal Superresolution Imaging and Nanofocusing

Presider: Esther Wertz; Rensselaer

Polytechnic Inst., USA

FTh4H.1 • 16:30

Interscale Mixing Microscopy: Far-field Imaging Beyond the Diffraction Limit, Bo Fan' ${ }^{1}$ Christopher Roberts ${ }^{1}$, Nicolas Olivier ${ }^{2}$ William Wardley ${ }^{2}$, Sandeep Inampudi ${ }^{3,1}$ Wayne Dickson², Anatoly. Zayats², Viktor A. Podolskiy'; 'Univeristy of Massachusetts at Lowell, USA; ${ }^{2}$ Physics, King's College London, UK: ${ }^{3}$ Northeastern Univ., USA. We present analytical and experimental demonstration of interscale mixing microscopy, imaging technique that allows imaging and spectroscopy of deep subwavelength objects with far field measurements.

\section{FTh4H.2 • 16:45}

Near-field Imaging With Pseudo-thermal Sources, Roxana Rezvani Naraghi ${ }^{1,2}$, Luiz Gustavo Cancado ${ }^{3}$, Aristide Dogariu'. ${ }^{1}$ Univ. of Central Florida, CREOL, USA: ${ }^{2}$ Physics, Univ. of Central Florida, USA; ${ }^{3}$ Physics, Universidade Federal de Minas Gerais (UFMG) Brazil. We provide a simple solution for significant deficiency of near-field microscopy. We demonstrate experimentally that spurious effects caused by interference can be eliminated in passive near-field imaging by implementing a random illumination.

\section{FTh4H.3 17:00 Invited}

Near-field Studies of Thermal Radiation and Local Density of States, Yannick De Wilde'; 'Langevin Inst., France. The thermal radiation, governed by the local density of states, is probed with a thermal radiation scanning tunneling microscope (TRSTM). We investigate plasmonic modes on doped/ undoped semiconductor heterostructures. We demonstrate that TRSTM produces undistorted near-field images at large scale. 


\section{CLEO: Science \& Innovations}

\section{6:30-18:30 \\ STh4l • Emerging Optical Materials \\ Presider: Frank Wang; Nanjing Univ., China}

\section{STh4l.1 • 16:30}

Broadband planar multilayer absorber tuned by $\mathrm{VO}_{2}$ phase transition, $\mathrm{Hao}$ Peng ${ }^{1}$, Yi Luo', Xiangxiao Ying ${ }^{1}$, Yang $\mathrm{Pu}^{1}{ }^{1}$, Zhe $\mathrm{Li}^{1}$, Yadong Jiang', Zhijun Liu'; 'Univ of Electronic Sci \& Tech of China, China. A dynamically tunable planar multiplayer absorber is demonstrated using the $\mathrm{VO}_{2}$ phase-change material. As the $\mathrm{VO}_{2}$ phase transition is thermally triggered, a modulation depth of $72.6 \%$ is achieved over a broad wavelength range from $4-8.2 \mu \mathrm{m}$.

\section{STh4l.2 • 16:45}

Nano-structured Wild Moth Cocoon Fibers as Radiative Cooling and Waveguiding Optical Materials, Norman N. Shi', ChengChia Tsai', Catherine Craig ${ }^{2}$, Nanfang Yu'; 'Columbia Univ., USA; ${ }^{1}$ Harvard Univ., USA. The study shows that comet moth cocoon fibers exhibit radiative cooling properties with enhanced solar reflectivity and thermal emissivity. Optical waveguiding due to transverse Anderson localization of light is also observed in these natural fibers.

\section{STh4l.3 • 17:00}

Control over Emissivity of Zero-StaticPower Thermal Emitters Based on Phase Changing Material GST, Kaikai Du1, Qiang $\mathrm{Li}^{1}$, Yanbiao Lyu ${ }^{2}$, Jichao Ding ${ }^{1}$, Yue Lu1, Zhiyuan Cheng', Min Qiu'; 'State Key Lab of Modern Optical Instrumentation, College of Optical Science and Engineering, Zhejiang Univ., China; ${ }^{2}$ Inst. of Microelectronics and Nanoelectronics, College of Information Science \& Electronic Engineering, Zhejiang Univ., China. A switchable, tunable and wavelength-selective thermal emitter is experimentally demonstrated with simple layered structures by controlling the phases of $\mathrm{Ge}_{2} \mathrm{Sb}_{2} \mathrm{Te}_{5}$, achieving a high emission extinction ratio of $11 \mathrm{~dB}$.

\section{STh4l.4 • 17:15}

All-solid-state tunable Bragg filters based on a phase transition material, Xi Wang ${ }^{1}$, Zilun Gong ${ }^{1}$, Kaichen Dong ${ }^{1}$, Shuai Lou', Jonathan Slack ${ }^{2}$, Andre Anders ${ }^{2}$, Jie Yao'. 'Univ. of California, Berkeley, USA; ${ }^{2}$ Accelerator Technology and Applied Physics Division, Lawrence Berkeley National Lab, USA. We demonstrate an all-solid-state tunable Bragg filter with a phase transition material as the defect layer. Dynamic tunability and hysteresis properties of the Bragg filter promise more applications by combining phase transition materials and optical cavities.

\section{6:30-18:30 \\ STh4J • Ultrafast Laser \\ Processing}

Presider: Pere Serra; Universitat de Barcelona, Spain

STh4J.1 • 16:30 Invited

First-Principles Description for Initial Stage of Femtosecond Laser Processing, Kazuhiro Yabana'; 'Univ. of Tsukuba, Japan. Interactions of intense laser pulse and dielectrics in femtosecond time scale are described using time-dependent density functional theory coupled with Maxwell's equations. Threshold and depth of the laser damage are estimated from the calculation.

\section{6:30-18:30 \\ STh4K • Imaging and Nonlinear Fiber Effects}

Presider: Ming-lie Hu; Tianjin

Univ., China

\section{STh4K.1 - 16:30 Invited}

Broadband Coherent Raman Imaging Method Development and Application to Tissue Imaging, Marcus T. Cicerone ${ }^{1}$, Charles H. Camp'; ${ }^{1}$ NIST, USA. I will discuss efforts to render spectroscopic coherent Raman imaging sufficiently simple and robust for general users. I will discuss progress in signal generation, data reduction, and extraction of information from rich spectroscopic images.
STh4J.2 • 17:00

Rapid fabrication of depressed cladding with shaped femtosecond laser pulses, Ya Cheng ${ }^{1}$, Peng Wang ${ }^{1}$, Jia $\mathrm{Qi}^{1}$, Yang Liao ${ }^{1}$, Zhengming Liu', Wei Chu'; 'Shanghai Inst of Optics and Fine Mech, China. We report on rapid fabrication of square-shaped depressed cladding optical waveguides deeply buried in lithium niobate using shaped femtosecond laser pulses. Low loss single-mode waveguid ing of both s- and p- polarized beams has been demonstrated. optical waveguides in lithium niobate

STh4K.2 • 17:00

Real-Time Observation of Microsecond Order Periodic Velocity Change of Fiber Fuse using Heterodyne Detection, Shoulin Jiang ${ }^{1}$, Lin $\mathrm{Ma}^{1}$, Shuai Wang ${ }^{1}$, Zuyuan $\mathrm{He}^{1}$. 'Shanghai Jiao Tong Univ., China. We studied the propagation speed of fiber fuse using heterodyne detection method by analyzing the Doppler shift based on short-time Fourier transform. We observed periodic velocity changes about $160 \mu$ s with a constant launched power.

\section{STh4J.3 • 17:15}

Fabrication of Novel Biomimetic Structures on Steel Via Femtosecond Laser OverScans, Camilo Florian Baron'1, Daniel Puerto ${ }^{1}$ Yasser I. Fuentes Edfuf', Evangelos Skoulas², Emmanuel Stratakis ${ }^{2}$, Javier Solis' ${ }^{1}$, Jan Siegell'; 'Instituto de Óptica, Spanish National Research Council, Spain; ${ }^{2}$ Inst. of Electronic Structure and Laser, Foundation for Research and Technology, Greece. We present different biomimetic structures on steel fabricated with a high repetition rate femtosecond laser. We show that their wetting properties can be defined by the irradiation conditions and the overall number of over-scans.
STh4K.3 • 17:15

Spectral dynamics of polarization-rotating vector solitons, Bowen $\mathrm{Li}^{1}$, Xiaoming Wei ${ }^{1}$, Ying YU', Kenneth Kin-Yip Wong'; ' Univ. of Hong Kong, China. Vector soliton is obtained by using a fiber stretcher inside a dispersionengineered nonlinear-polarization-rotation (NPR) mode-locked fiber laser. Fascinating real-time spectral dynamics of vector soliton is observed for the first time using dispersive Fourier transform (DFT).
16:30-18:30

STh4L • Precision Timing and Ranging

Presider: Josue Davila-Rodriguez; NIST, USA

\section{STh4L.1 • 16:30}

Jitter Analysis of Timing Distribution Systems, Kemal Shafak ${ }^{1,2}$, Ming Xin ${ }^{1,3}$, Qing Zhang ${ }^{1}$, Shih-Hsuan Chia ${ }^{1}$, Oliver Muecke ${ }^{1,2}$, Franz Kaertner ${ }^{1,3} ;{ }^{1}$ Center for Free-Electron Laser Science, Deutsches Elektronen-Synchrotron, Germany; ${ }^{2}$ Physics Dept. and the Hamburg Center for Ultrafast Imaging, Univ. of Hamburg, Germany; ${ }^{3}$ Research Lab of Electronics, MIT, USA. We present a powerful jitter analysis method for timing distribution systems based on feedback flow between setup elements. Our comprehensive feedback model yields excellent agreement with the experimental results and identifies seven uncorrelated noise sources.

\section{STh4L.2 • 16:45}

Timing jitter analysis for mode-locked lasers by asynchronous optical sampling, Haosen Shi', Youjian Song ${ }^{1}$, Jiahe Yu', Runmin $\mathrm{Li}^{1}$, Chingyue Wang', Ming-lie $\mathrm{Hu}^{1}$; ${ }^{1}$ Tianjin Univ., China. We demonstrate a simple subfemtosecond precision timing jitter measurement method for mode-locked lasers based on the time scaling effects of asynchronous optical sampling.

\section{STh4L.3 • 17:00 Invited}

Digital Control and Processing of Optical Frequency Combs for Precision Measurement, Jean-Daniel Deschênes ${ }^{1}$, Hugo Bergeron ${ }^{1}$, Laura Sinclair ${ }^{2}$, lan R. Coddington', Nate Newbury ${ }^{2}$; Universite Laval, Canada; ${ }^{2}$ NIST, USA. We discuss concepts related to digital control specifically applied to frequency combs. Applications targeted are spectroscopy, radio-frequency signal generation and time and frequency transfer. A free and open-source digital platform is described in details. 
Marriott

Salon III

Joint

16:30-18:30

JTh4M • Symposium on Optical Microcavities for Ultrasensitive

Detection II

Presider: Randall Goldsmith; Univ. of Wisconsin Madison, USA

\section{JTh4M.1 • 16:30 Invited}

Monolithically Integrated Ring Resonator Systems On-chip, Hengky Chandrahalim' Xudong Fan'; ' 'Univ. of Michigan, USA. We review the recent development in robust, monolithically integrated optical ring resonator systems fabricated on-chip using photolithographic and femtosecond laser writing technologies, which potentially has broad applications in passive/active photonic devices and bio/chemical sensing.
JTh4M.2 • 17:00

Impact of Varying Vacuum Levels on Self-Heating in Photonic Thermometers, Zeeshan Ahmed', Nikolai Klimov', Hames Hands', James Fedchak'; 'PML, NIST, USA. Here we examine the impact of vacuum levels on self-heating in photonic crystal cavity thermometers. Our results suggest that background gas pressure has a negligible impact on self-heating correction to the temperature-wavelength calibration.
Marriott

Salon IV
Marriott

Salon V \& VI

\section{CLEO: Science \& Innovations}

16:30-18:30

STh4N • Photonic Crystals \&

Their Applications

Presider: Masaya Notomi; NTT

Basic Research Labs, Japan

STh4N.1 $16: 30$

Forward-biased photonic crystal photodetector towards amplifier-free bias-free receiver, Kengo Nozaki ${ }^{2,1}$, Shinji Matsuo ${ }^{2,3}$, Takuro Fujiii,3, Koji Takeda ${ }^{2,3}$, Eiichi Kuramochi $^{2,1}$, Akihiko Shinya ${ }^{2,1}$, Masaya Notomi ${ }^{2,1}$; ${ }^{1}$ NTT Basic Research Labs, Japan; ${ }^{2}$ Nanophotonics Center, Japan; ${ }^{3}$ NTT Device Technology Labs, Japan. We demonstrate a photonic-crystal photodetector under a forward bias voltage maintaining a $0.88-\mathrm{A} / \mathrm{W}$ responsivity and a 40-Gbit/s bitrate. This will allow an ultralow-energy receiver with highimpedance termination that requires neither amplifiers nor a bias circuit.

STh4N.2 • 16:45

High quality $\mathrm{LiNbO}_{3}$ photonic crystal nanobeams, Hanxiao Liang', Rui Luo', Qiang Lin'; 'Univ. of Rochester, USA. We report, for the first time, high-quality LiNbO3 onedimensional photonic crystal nanabeams, with optical $\mathrm{Q}$ up to $1.09 \times 10^{5}$, which allows us to observe strong reversible photorefractive effect.

STh4N.3 • 17:00

Thresholdless lasing with quantum dot gain, Yasutomo Ota', Daisaku Takamiya², Masahiro Kakuda', Katsuyuki Watanabe', Satoshi Iwamoto 1,2, Yasuhiko Arakawa 1,2. ' Nanoquine, The Univ. of Tokyo, Japan; ${ }^{2} I I S$, The Univ. of Tokyo, Japan. We demonstrate thresholdless lasing with quantum dot gain, operated under cavity resonant excitation. The lasing behavior is systematically compared with that under conventional above bandgap excitation, providing a firm verification of the thresholdless operation.
16:30-18:30

STh4O • Quantum Cascade

Lasers

Presider: Amr Helmy; Univ. of

Toronto, Canada

\section{STh40.1 • 16:30 Tutorial}

Broadband, Tunable, and Monolithic Quantum Cascade Lasers, Manijeh Razeghi'; 'Northwestern Univ., USA. This article describes the state of research and recent developments related to broadband quantum cascade lasers. Monolithic tuning and system development is also discussed.

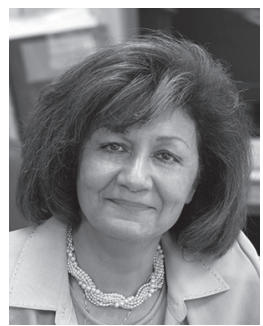

Manijeh Razeghi started at Thomson-CSF as the Head of the Exploratory Materials Laboratory. In 1991, she joined Northwestern University, as a Walter P. Murphy Professor and Director of the Center for Quantum Devices. She is one of the leading scientists in the field of semiconductor science and technology.
JTh4M.3 • 17:15

Ultrasensitive and broadband magnetometry with cavity optomechanics, Beibei Li', , Douglas Bulla ${ }^{3}$, Jan Bilek', Varun Prakash', Stefan Forstner ${ }^{1}$, Eoin Sheridan ${ }^{1}$, Lars Madsen', Halina Rubinsztein-Dunlop', Scott Foster $^{3}$, Clemens Schäfermeier ${ }^{4}$, Tobias Gehring ${ }^{4}$, Ulrik L. Andersen ${ }^{4}$, Warwick Bowen ${ }^{4}$; 'The Univ. of Queensland, Australia; ${ }^{2}$ Qian Xuesen Lab for Space Technology, China; ${ }^{3}$ Defense Science and Technology Group, Australia; ${ }^{4}$ Technical Univ. of Denmark, Denmark. We achieved sensitivity of $30 \mathrm{pT} / \mathrm{Hz}^{1 / 2}$ and working bandwidth larger than $100 \mathrm{MHz}$, using cavity optomechanical magnetometry, and also demonstrated quantum light enhanced sensitivity in such a magnetometer.
STh4N.4 $17: 15$

Guiding of laser light from a nanocavity in a three-dimensional photonic crystal, Takeyoshi Tajiri', Shun Takahashi ${ }^{2}$, Yasutomo Ota ${ }^{2}$, katsuyuki watanabe ${ }^{2}$, Satoshi Iwamoto 1,2, Yasuhiko Arakawa ${ }^{1,2}$; ${ }^{1}$ Inst. of Industrial Science, Univ. of Tokyo, Japan; ${ }^{2}$ Inst. of Nano Quantum Information Electronics, Univ. of Tokyo, Japan. A nanocavity laser and two orthogonal waveguides are integrated in a three-dimensional photonic crystal. Guiding of the laser light from the nanocavity through the waveguides is demonstrated experimentally. 
Executive Ballroom $210 \mathrm{~A}$

Joint

Multimodal Imaging in

Biophotonics II-Continued

JTh4A.3 • 17:30

Invited

New Directions in Multimodal Imaging and Light Sheet Microscopy, Kishan Dholakia'; Univ. of St Andrews, UK. I will describe new work combining Raman imaging with morphological imaging (e.g. digital holographic microscopy and OCT). Additionally, I will describe new directions using light sheet microscopy with studies in neuroscience and developmental biology.

Executive Ballroom

210B

Executive Ballroom

$210 \mathrm{C}$

\section{CLEO: Applications \\ \& Technology}

JTh4A • Symposium on

ATh4B - Process Evaluation \& Microscopy-Continued

\section{ATh4B.4 • 17:30}

Lensfree On-chip Microscopy Achieves Accurate Measurement of Yeast Cell Viability and Concentration Using Machine Learning, Alborz Feizi', Yibo Zhang', Alon Greenbaum 1,2, Alex Guziak', Michelle Luong', Raymond Chan ${ }^{1}$, Brandon Berg', ${ }^{1,}$, Haydar Ozkan', Wei Luo', Michael Wu ${ }^{1}$, Yichen $\mathrm{Wu}^{1}$, Aydogan Ozcan'; ' ${ }^{1}$ univ. of California Los Angeles, USA; ${ }^{2}$ California Institution of Technology, USA; ${ }^{3}$ Univ. of Michigan, USA. Automatic measurement of yeast viability and concentration is achieved by coupling a lensfree on-chip holographic microscope with a machine learning based classification algorithm that counts the number of live/ dead cells stained with methylene blue.

\section{ATh4B.5 • 17:45}

Time-Domain Measurements Reveal Spatial Aberrations in a Sub-Surface Two-Photon Microscope, Marius Rutkauskas ${ }^{1}$, Derryck T. Reid", Jesus Garduño-Mejía², Martha Rosete 2 ; ${ }^{1}$ Heriot-Watt Univ., UK; ${ }^{2}$ Centro de Ciencias Aplicadas y Desarrollo Tecnológico, Universidad Nacional Autónoma de México , Mexico. We experimentally demonstrate that in a sub-surface microscope the effects of chromatic and spherical aberrations are revealed by a difference in the focal positions corresponding to the shortest pulse duration and the maximum autocorrelation amplitude.

\section{ATh4B.6 • 18:00}

JTh4A.4 18:00 Invited structural Properties of Breast Tissue Using Optical Coherence Tomography, Brendan F. Kennedy ${ }^{1,2}$; ${ }^{1}$ The Univ. of Western Australia, Australia; ${ }^{2}$ BRITElab, Harry Perkins Inst. of Medical Research, Australia. New techniques are needed to improve intraoperative assessment of tumor margins in breast-conserving surgery. Here, the potential of optical coherence tomography and optical coherence elastography to provide micro-scale, realtime feedback on margin status is presented.
Enhanced Parallel Bridge Defect Inspection Using a Metalens Assisted Off-Focus Scanning Imaging, Jinlong Zhu', Lynford Goddard'; ${ }^{1}$ Univ. of Illinois at Urbana-Champaign, USA. A near-field metalens is utilized to assist a conventional brightfield microscope for significantly enhancing the signal-to-noise ratio associated with a parallel bridge defect on a $7 \mathrm{~nm}$ node patterned wafer.
ATh4C • A\&T Topical Review on Extreme Ultraviolet and Soft X-ray Sources and Application II-Continued

\section{ATh4C.4 • 17:45}

Quasi-phase-matched high harmonic generation in gas-filled photonic crystal fibers, Patrick Anderson', Florian Wiegandt ${ }^{1}$, Fei Yu ${ }^{2}$ Daniel Treacher', David Lloyd ${ }^{1}$, Peter Mosley ${ }^{2}$ Simon Hooker', Ian A. Walmsley'; ' ${ }^{1}$ Clarendon Lab, Univ. of Oxford, UK; ${ }^{2}$ Centre for Photonics and Photonic Materials, Dept. of Physics, Univ. of Bath, UK. We investigate HHG in gas-filled PCFs with microjoule driving lasers. QPM is implemented for the first time enhancing the flux at $30 \mathrm{eV}$ by a factor of 60 .

\section{ATh4C.5 • 18:00 Invited}

Characterization and Scaling of Laser Produced Plasma EUV Light Source for Lithography, Jayson Stewart'; ${ }^{1}$ AMSL, USA. We review ASML's enabling technologies for producing clean and stable high power EUV LPP sources, including new tools to characterize ions from the Sn plasma, droplet generators, and dose control methods to deliver in-spec stability.
Executive Ballroom 210D

\section{CLEO: QELS-} Fundamental Science

FTh4D - Solitons and Temporal Wave Guiding-Continued

FTh4D.4 • 17:30

Soliton Breathing Induced by Avoided Mode Crossing in Optical Microresonators Hairun Guo', Martin Pfeiffer', Erwan Lucas' Maxim Karpov' ${ }^{1}$ Miles Anderson' ${ }^{1}$, Junqiu Liu' ${ }^{1}$ Michael Geiselmann ${ }^{1}$, John Jost ${ }^{1}$, Tobias J. Kippenberg ${ }^{1}{ }^{1}$ Ecole Polytechnique Fédérale De Lausanne, Switzerland. We observed soliton breathing induced by avoided mode crossing, in soliton-based Kerr frequency combs in two microresonator platforms. We present an understanding of avoided mode crossing as a Lorentzian response that reveals the breathing dynamics.

FTh4D.5 • 17:45

Temporal Dissipative Solitons in a $\mathrm{Mi}$ croresonator Driven by Optical Pulses, Ewelina Obrzud ${ }^{1,2}$, Steve Lecomte ${ }^{1}$, Tobias Herr'; ${ }^{1}$ CSEM, Switzerland; ${ }^{2}$ Observatoire de Geneve, Switzerland. A nonlinear optical microresonator is driven by picosecond laser pulses resulting in formation of temporal dissipative solitons. These femtosecond solitons are stable and generated at a fraction of the power required in continuous-wave driven system.

\section{FTh4D.6 1 18:00 Invited}

Temporal Waveguiding of Optical Pulses, Govind P. Agrawal', Brent W. Plansinis' 'Univ. of Rochester, USA. We discuss the temporal analog of total internal reflection inside a dispersive medium and its use for temporal waveguiding of optical pulses. Spectral changes induced during this process are also described together with potential applications. 
Executive Ballroom $210 \mathrm{E}$

Executive Ballroom 210F

\section{CLEO: QELS-Fundamental Science}

\section{FTh4E • Single-Photon \\ Sources and Quantum Communications-Continued}

FTh4E.4 • 17:30

Temporal-mode tomography of single photons, Vahid Ansari ${ }^{1}$, Markus Allgaier ${ }^{1}$ Linda Sansoni ${ }^{1}$, Benjamin Brecht ${ }^{2}$, Jonathan Roslund ${ }^{3}$, Nicolas Treps ${ }^{3}$, Georg Harder ${ }^{1}$ Christine Silberhorn'. 1/ntegrated Quantum Optics, Paderborn Univ., Germany; ${ }^{2}$ Clarendon Lab, Dept. of Physics, Univ. of Oxford UK: ${ }^{3}$ Laboratoire Kastler Brossel, UPMC-Sorbonne Universites, ENS-PSL Research Univ. France. Employing a quantum pulse gate, we perform temporal mode tomography of pure mixed heralded single-photon states in 7-dimensional Hilbert space with $99 \%$ fidelity.

FTh4E.5 • 17:45

Sub-Megahertz Linewidth Single Photon Source Suitable for Quantum Memories Markus Rambach', Wing Yung S. Lau', Aleksandrina Nikolova ${ }^{1}$ Till Weinhold ${ }^{1}$ Andrew White'; ${ }^{1}$ Univ. of Queensland, Australia. We report $100 \%$ duty cycle generation of sub$\mathrm{MHz}$ linewidth single photon pairs at the Rubidium $\mathrm{D}_{1}$ line. The photons are well-suited for storage in quantum memory schemes with sub-natural linewidths, such as gradient echo memories.

FTh4E.6 • 18:00

Ultrabright Single Photon Source with Subnatural Linewidth, Chih-Hsiang Wu' Chih-Sung Chuu', Tsung-Yao Wu', Yung-Chin Yeh', Po-Hui Liu', Chin-Hsuan Chang', ChiaoKai Liu', Ting Cheng'; 'National Tsing Hua Univ., Taiwan. We demonstrate an ultrabright, subnatural-linewidth single-photon source and the controlled absorption of the single photons in an atomic vapor. The single photons have a spectral brightness one order-ofmagnitude higher than previously reported and a waveform controllable by EOM.
FTh4F • Imaging Electron

Dynamics on the Nano-, FemtoScale-Continued

FTh4F.2 • 17:30

Imaging electron motion in 2D semiconductor heterojunctions, Michael K. Man ${ }^{1}$ Skylar Deckoff-Jones', Takaaki Harada' E Laine Wong ${ }^{1}$, Athanasios Margiolakis ${ }^{1}$ M Bala Murali Krishna ${ }^{1}$ Julien Madéo ${ }^{1}$ Andrew Winchester ${ }^{1}$, Sidong Lei $^{2}$, Robert Vajtai ${ }^{2}$, Pulickel M. Ajayann², Keshav Dani ${ }^{1}$ ${ }^{1}$ Femtosecond Spectroscopy Unit, Okinawa nst. of Science and Technology Graduate Univ., Japan; ${ }^{2}$ Dept. of Materials Science and Nanoengineering, Rice Univ., USA. Transfer of electrons through semiconductor heterojunctions is the key process in all electronics. Here we make movies of electron dynamics in InSe/ GaAs through time and spectrally-resolved photoemission electron microscopy, bringing insights to this fundamental process.

FTh4F.3 • 17:45

Imaging Complex Electron Dynamics Within a Photoexcitation Spot, E Laine Wong ${ }^{1}$, Andrew Winchester ${ }^{1}$, Michae Man', Vivek Pareek', Julien Madéo', Keshav Dani'; 'OIST, Japan. Complex electron flow within a photoexcitation spot is observed on homogeneous $\mathrm{GaAs}$ surface by bringing spatial resolution into traditional ultrafast pump probe technique via time-resolved photoemission electron microscopy.

FTh4F.4 • 18:00

Exploring Ultrafast Electron Dynamics in Space, Time, Momentum and Energy, Andrew Winchester ${ }^{1}$, E Laine Wong ${ }^{1}$, Michae Man', Vivek Pareek', Julien Madéo', Keshav Dani'; ${ }^{1}$ Okinawa Inst. of Science and Tech nology, Japan. We show that time-resolved photoemission spectroscopy enables investigation of electron dynamics in materials with space, time, momentum and energy resolution. With this capability we track the evolution of photoexcited carriers in p-doped gallium arsenide.
Executive Ballroom

210G

Joint

JTh4G • Symposium on Optomechanics: Towards the Second Quantum Revolution II-Continued

\section{JTh4G.4 • 17:30 Invited}

Hybrid Atom-Membrane Optomechanics, Philipp Treutlein'; 'Dept. of Physics, Univ. of Basel, Switzerland. We present a hybrid optomechanical system in which a membran oscillator is coupled to ultracold atoms. We report cooling of the membrane vibrations due to its coupling to atoms and discuss perspectives for quantum control.
Executive Ballroom $210 \mathrm{H}$

\section{CLEO: QELS -} Fundamental Science

FTh4H • Optical and Thermal Superresolution Imaging and Nanofocusing-Continued

FTh4H.4 • 17:30

Nanoscale Control over Optical Dislocations, Evgeny E. Ostrovsky', Kobi Cohen 1 Bergin Gjonaj ${ }^{1}$, Guy Bartal'; 'Technion, Israel. We demonstrate nano-scale tuning of the spatial location of plasmonic vortices on metal-air interface. This is enabled by controling the polarization state of the light coupled to the surface plasmons through a spiral slit.

\section{FTh4H.5 • 17:45}

Metamaterial based compressive spatialspectral transformation microscope, Qia $\mathrm{Ma}^{1}$, Huan Hu${ }^{1}$, Eric Huang ${ }^{2}$, Zhaowei Liu' Electrical and Computer Engineering, Univ. of California San Diego, USA; ${ }^{2}$ Physics, Univ. of California San Diego, USA. We present a new super-resolution imaging approach by using hyperbolic metamaterial to compres sively encode high-resolution spatial information to a spectrum. We show numerical results of resolving sub- $15 \mathrm{~nm}$ resolution by a practically achievable $\mathrm{Ag} / \mathrm{SiO}_{2}$ multilayer metamaterial.

JTh4G.5 • 18:00

Quantum Back Action Evading Measurements in a Spin-Mechanics Hybrid System, Rodrigo A. Thomas ${ }^{1}$, Christoffer B. Møller ${ }^{1}$, Georgios Vasilakis ${ }^{1,3}$, Emil Zeuthen ${ }^{2}$ Yeghishe Tsaturyan', Kasper Jensen', Albert Schliesser ${ }^{1}, K^{\prime}$ lemens Hammerer ${ }^{2}$, Eugene S. Polzik'; 'Niels Bohr Inst., Univ. of Copenhagen, Denmark; ${ }^{2}$ Inst. for Theoretical Physics and Inst. for Gravitational Physics, Leibniz Univ. Hannover, Germany; ${ }^{3}$ Inst. fo Electronic Structure and Laser, Foundation for Research and Technology, Greece. A back action evading joint quantum measurement of a mechanical and a spin oscillator in the negative mass reference frame is reported and its importance towards a quantum link in hybrid systems is discussed.

\section{FTh4H.6 • 18:00}

Imaging with multilayer hyperbolic metamaterials - what are the limits?, Tengfei Li ${ }^{1}$ Vivek Nagal ${ }^{2}$, David Gracias ${ }^{2}$, Jacob Khurgin ${ }^{1}$. 'Electrical and Computer Engineering, Johns Hopkins Univ., USA; ${ }^{2}$ Chemical and Biomolecular Engineering, Johns Hopkins Univ., USA Using the Eigen-mode approach we analyze the imaging performance of multilayer hyperbolic metamaterials and show that resolution decreases with the number of layers and amount of metal loss. 


\section{CLEO: Science \& Innovations}

\section{STh4l • Emerging Optical Materials-Continued}

\section{STh4l.5 $17: 30$}

Chalcogenide Glass-on-Graphene Photonics, Hongtao Lin ${ }^{1}$, Yi Song ${ }^{2}$, Yizhong Huang', Derek Kita', Kaiqi Wang ${ }^{1}$, Lan Li', Junying Li $^{1}$, Hanyu Zheng ${ }^{1}$, Zhengqian Luo', Spence Novak ${ }^{3}$, Chung-Che Huang ${ }^{4}$, Daniel Hewak ${ }^{4}$ Kathleen Richardson ${ }^{3}$, Jing Kong2, Juejun $\mathrm{Hu}^{1} ;{ }^{1}$ Materials Science and Engineering, MIT, USA; ${ }^{2}$ Electrical Engineering and Computer Science, MIT, USA; ${ }^{3}$ The College of Optics \& Photonics, Univ. of Central Florida, USA; ${ }^{4}$ Optoelectronics Research Centre, Univ. of Southampton, UK. Photonic integration with 2-D materials conventionally relied on transfer processes. We developed an approach to monolithically fabricate photonic devices on 2-D materials including graphene and demonstrated its significant potential for high-performance photonic integration.

\section{STh4l.6 • 17:45}

Growth, Spectroscopy and Laser Operation of Tm-doped Monoclinic Magnesium Tungstate (Tm:MgWO ${ }_{4}$ ), Xavier Mateos ${ }^{1,2}$ L Zhang ${ }^{3}, \mathrm{Z} \mathrm{\text {Lin } ^ { 3 } , \mathrm { H } \mathrm { Lin } { } ^ { 3 } , \mathrm { G } \text { Zhang } ^ { 3 } , \text { Pavel }}$ Loiko ${ }^{4}$, Josep M. Serres ${ }^{1}$, Magdalena Aguiló ${ }^{1}$ Francesc Díaz', Yicheng Wang ${ }^{2}$, Uwe Griebner $^{2}$, Valentin Petrov ${ }^{2}$, Elena Vilejshikova ${ }^{5}$, Konstantin Yumashev ${ }^{5}$, Weidong $\mathrm{Chen}^{3}$; 'Universitat Rovira I Virgili, Spain; ${ }^{2}$ Max Born Inst., Germany; ${ }^{3}$ Fujian Inst. of Research on the Structure of Matter, China; ${ }^{4}$ ITMO Univ., Russia; ${ }^{5}$ Belarusian National Technical Univ., Belarus. A novel monoclinic magnesium tungstate crystal, Tm: $\mathrm{MgWO}_{4}$, was grown and characterized in terms of structure, spectroscopy and laser generation. The output power was $772 \mathrm{~mW}$ at 2017-2029 nm with a slope efficiency of $39 \%$.

\section{STh4l.7 • 18:00}

Photocarrier dynamics in Weyl semimetal $\mathrm{WTe}_{2}$ thin films, Chunhui Zhu' ${ }^{1}$, Ming Gao', Yongbing $\mathrm{Xu}^{1}$, Xuefeng Wang ${ }^{1}$, Frank (Fengq iu) Wang'; ${ }^{1}$ Nanjing Univ., China. We report the first photocarrier dynamics investigation on type-II Weyl semimetal WTe $\mathrm{W}_{2}$. A transient feature where an initial photo-bleaching signal is found followed by a persistent photoinduced absorption over the 1.8-2.5 $\mu \mathrm{m}$ range is revealed.

\section{STh4J • Ultrafast Laser Processing-Continued}

STh4J.4 • 17:30

Stealth dicing with ultrafast Bessel beams with engineered transverse profiles, Rém Meyer $^{1}$, Jassem Safioui ${ }^{1}$, Remo Giust ${ }^{1}$, Pierre-Ambroise Lacourt ${ }^{1}$, Luca Furfaro ${ }^{1}$ John Michael Dudley ${ }^{1}$, Francois courvoisier ${ }^{1}$. ${ }^{1}$ FEMTO-ST, France. We investigate highspeed glass cleaving with ultrafast laser beams with engineered transverse intensity profile. We achieve accuracy of $\sim 1 \mu \mathrm{m}$ at 25 $\mathrm{mm} / \mathrm{s}$ and drastically enhance cleavability compared to standard Bessel beams.

STh4J.5 • 17:45

Very Fine Refractive Index Tuning of Silicon by Single Femtosecond Laser Pulses Below Melting Threshold, Daniel Bachman', Zhijiang Chen ${ }^{1}$, Robert Fedosejevs ${ }^{1}$, Ying Tsui ${ }^{1}$ Vien Van': 'Univ. of Alberta, Canada. We report measurements of very small refractive index changes in crystalline silicon by fs laser irradiation below melting threshold. Our measured threshold for permanent optical change is up to five times lower than previously reported.

STh4J.6 • 18:00

Beyond the Drude Approach: a KeldyshVinogradov Model of Dynamics of Ultrafast Laser-Induced Electron Excitation Vitaly Gruzdev', Drake R. Austin², Olga N. Sergaeva', Enam Chowdhury2; 'Univ. of Missouri-Columbia, USA; ${ }^{2}$ Dept. of Physics, The Ohio State Univ., USA. High-power lasersemiconductor interactions are frequently simulated with the Keldysh photoionizationrate formula and Drude model to describe generation and dynamics of conductionband electrons. We report a novel approach utilizing the multi-band Keldysh formula and Vinogradov equation.

\section{STh4K • Imaging and Nonlinear Fiber Effects-Continued}

STh4K.4 • 17:30

Characterization of Chirped Pump FourWave Mixing in Nonlinear Fibers using only Continuous-Wave-Lasers, Mads Lillieholm ${ }^{1}$ Pengyu Guan ${ }^{1}$, Morten S. Møller-Kristensen ${ }^{1}$ Michael Galili', Lars Grüner-Nielsen², Leif K. Oxenløwe'; 'Technical Univ. of Denmark, Denmark; ${ }^{2}$ OFS, Denmark. We propose a novel fiber characterization method that reveals the four-wave mixing bandwidth for chirped pump operation, using two tunable continuous-wave-lasers. The method accurately predicts the bandwidth for optical time lenses with broadband multi-carrier input.
STh4L • Precision Timing and Ranging-Continued

\section{STh4L.4 • 17:30}

Free-Space Terminals for Optical Two-Way Time-Frequency Transfer, William C. Swann ${ }^{1}$ Laura Sinclair ${ }^{1}$, Isaac Khader ${ }^{1}$, Nate Newbury' 'NIST, USA. Optical two-way time-frequency transfer (O-TWTFT) places stringent requirements on the free-space optical terminals used to transmit precise frequency comb pulses. We discuss a compact free-space terminal design that enables O-TWTFT ove long, highly turbulent links.

\section{STh4K.5 • 17:45}

Sensitivity Enhancement of Brillouin Frequency Shift Measurement Based on Multispectral Pump and Probe, Yosuke Tanaka ${ }^{1}$ Yuta Ozaki', Takashi Kurokawa ${ }^{1,2 ;}{ }^{1}$ Tokyo Univ. of Agriculture and Technology, Japan; ${ }^{2} \mathrm{Na}$ tional Astronomical Observatory of Japan Japan. We propose and demonstrate a nove method for measuring the change in Brillouin frequency shift in an optical fiber with high sensitivity using multispectral pump and probe. Experimental result shows 600 times sensitivity enhancement.
STh4K.6 • 18:00

Phase and Combining Efficiency in Divided Pulse Amplification, Koji Iwata ${ }^{1}, \mathrm{Ei} \mathrm{Jo}^{1}$ Henrik Tunnermann ${ }^{1}$, Akira Shirakawa ${ }^{1} ;{ }^{1}$ Inst. for Laser Science, UEC, Japan. We show amplitude and phase retrieval of both pulses in a divided pulse amplification setup. We use this information to calculate the spectra on the combined and the rejected port and compare with our setup.
STh4L.5 • 17:45

Comb-based Optical Frequency Transfer in Free Space, Hyun Jay Kang ${ }^{1}$, Byung Jae Chun'2, Jaewon Yang1, Young-Jin Kim² Seung-Woo Kim "; ' 1 KAIST, Korea (the Republic of); ${ }^{2}$ School of Mechanical and Aerospace Engineering, Nanyang Technology Univ. Singapore. A comb-based scheme of coherent optical frequency transfer in free space is proposed and demonstrated. This method enables concurrent transfer of multiple optical carriers with simultaneous detection and cancellation of the atmospheric phase noise.
STh4L.6 • 18:00

Ultrafast Dual-Comb Distance Metrology Using Dissipative Kerr Solitons, Denis Ganin', Philipp Trocha', Martin Pfeiffer ${ }^{2}$ Maxim Karpov², Arne Kordts², Jonas Krockenberger ${ }^{1}$, Pablo Marin-Palomo', Stefan Wolf' Sebastian Randel ${ }^{1}$, Wolfgang Freude ${ }^{1}$, Tobias J. Kippenberg ${ }^{2}$, Christian Koos ${ }^{1,3}$; ${ }^{1}$ Inst. of Photonics and Quantum Electronics (IPQ) Karlsruhe Inst. of Technology (KIT), Germany ${ }^{2}$ Ecole Polytechnique Fédérale de Lausanne (EPFL), Switzerland: ${ }^{3}$ Inst. of Microstructure Technology (IMT), Karlsruhe Inst. of Technology (KIT), Germany. We demonstrate ultra-fast high-precision distance measurements using a pair of dissipative Kerr-soliton frequency combs. We achieve sub- $\mu \mathrm{m}$ accuracies for static targets and measurement rates of $10 \mathrm{MHz}$ that allows sampling of air-gun bullets on the fly. 
Marriott

Salon III

Joint

JTh4M • Symposium on Optical Microcavities for Ultrasensitive Detection II-Continued

\section{JTh4M.4 • 17:30 Invited}

Cavity Optomechanics for Sensing Applications, Wenyan $\mathrm{Yu}^{1}$, Wei Jiang ${ }^{2}$, Qiang Lin 2,3, Tao Lu'; ${ }^{1}$ Electrical and computer of Optics, Univ. of Rochester, USA; ${ }^{3}$ Electrical and Computer Engineering, Univ. of Rochester, USA. This talk reviews the progresses on cavity optomechanical sensing. A comparison of mass induced and optical spring based sensing approaches are presented. Future improvements that may enable the detection of single atoms are discussed.

Marriott

\section{CLEO: Science \& Innovations} engineering, Univ. of Victoria, Canada; ${ }^{2}$ nst.

STh4N • Photonic Crystals \& Their Applications-Continued

STh4N.5 • 17:30

Cavity-enhanced light emission from an electrically-driven van der Waals heterostructures, Chang-Hua Liu', Genevieve Clark $^{3}$, Taylor Fryett ${ }^{2}$, Sanfeng $\mathrm{Wu}^{1}$, Jiajiu Zheng $^{2}$, Xiaodong $\mathrm{Xu}^{1}$, Arka Majumdar ${ }^{2}$. ${ }^{1}$ Dept. of Physics, Univ. of Washington, USA; ${ }^{2}$ Dept. of Electrical Engineering, Univ. of Washington, USA; ${ }^{3}$ Dept. of Materials Science and Engineering, Univ. of Washington, USA. We demonstrate a novel light source, based on a photonic crystal cavity integrated van der Waals light emitting diode, which can be operated at room temperature with fast modulation speed.

STh4N.6 • 17:45

Enhanced and Preferential Optical Trapping in a Slot-Graphite Photonic Crystal, Aravind Krishnan', Michelle Povinelli', Ningfeng Huang', Luis J. Martinez', Shao-Hua Wu'; ' USC, USA. High-stiffness optical traps are developed for dielectric and metallic particles by exploiting the strong confinement of light in slot-graphite photonic crystal. An optical sieve is realized using the difference in growth kinetics of different nanoparticles.

STh4N.7 • 18:00

JTh4M.5 • 18:00 Invited

Cavity Ring-Up Spectroscopy for Sensing in a Whispering Gallery Mode Resonator, Sho Kasumie ${ }^{1}$, Ramgopal Madugani ${ }^{1}$, Yong Yang ${ }^{1}$, Jonathan M. Ward', Sile Nic Chormaic ${ }^{1}$, ${ }^{1}$ Okinawa Inst. of Science and Technology Graduate Univ., Japan. We investigate cavity ring-up spectroscopy in order to perform distinctive dispersive and dissipative transient sensing in a silica whispering gallery mode resonator coupled to a tapered optical fiber. Experiments support the theoretical model.
Marriott

Salon V \& VI

STh4O • Quantum Cascade

Lasers-Continued

STh40.2 • 17:30

Broadband Continuous Tuning of a $\mathrm{THz}$ Quantum-Cascade VECSEL, Christopher A. Curwen', Luyao $\mathrm{Xu}^{1}$, John Reno ${ }^{2}$, Tatsuo Itoh ${ }^{1}$, Benjamin Williams ${ }^{1}$; ${ }^{1}$ UCLA, USA; ${ }^{2}$ Sandia Labs, USA. We report tuning of a herahertz quantum-cascade vertical-externalcavity surface-emitting-laser (VECSEL) using piezoelectric actuators and an intra-cryostat cavity. Continuous tuning over $260 \mathrm{GHz}$ at a center frequency of $3.3 \mathrm{THz}$ is demonstrated.

STh40.3 • 17:45

Narrow-Beam, 4.7 micron-Emitting NearResonant Leaky-Wave-Coupled Quantum Cascade Laser Phase-Locked Array, Chris Sigler ${ }^{1}$, Colin Boyle ${ }^{1}$, Jeremy Kirch ${ }^{1}$, Don Lindberg- ||$\|^{2}$, Thomas Earles², Joshua Myers $^{3}$, Robert Bedford ${ }^{3}$, Dan Botez ${ }^{1}$, Luke J. Mawst'; ${ }^{1}$ Univ. of Wisconsin-Madison, USA; ${ }^{2}$ Intraband, LLC, USA; ${ }^{3}$ Air Force Research Lab, Sensors Directorate, USA. Narrow beam (3.2xD.L.) is demonstrated up to $\sim 5.85 \mathrm{~W}$ pulsed output power from a five-element phase-locked array of $4.7 \mu \mathrm{m}$-emitting quantum cascade lasers. Devices are fabricated by a MOCVD process and operate predominately in an in-phase array mode, in agreement with design simulation studies.

STh40.4 • 18:00

Full Dispersion Compensation of Terahertz Quantum Cascade Laser Frequency Combs, Yang Yang ${ }^{1}$, David P. Burghoff', John Reno ${ }^{2}$ Qing $\mathrm{Hu}^{1}$; ${ }^{1} \mathrm{MIT}$, USA; ${ }^{2}$ Sandia National Labs, USA. Utilizing a genetic algorithm, we optimized the dispersion compensation of terahertz quantum cascade laser frequency combs up to fourth-order. The fully dispersion-compensated device shows a larger dynamic range and a broader spectral coverage, exhibiting comb formation over $800 \mathrm{GHz}$. 
Executive Ballroom $210 \mathrm{~A}$

\section{Joint}

JTh4A • Symposium on Multimodal Imaging in Biophotonics II-Continued
Executive Ballroom

210B

CLEO: Applications

\& Technology

ATh4B • Process Evaluation \& Microscopy-Continued
ATh4C • A\&T Topical Review on Extreme Ultraviolet and Soft X-ray Sources and Application II-Continued
Executive Ballroom 210D
CLEO: QELSFundamental Science

FTh4D - Solitons and Temporal Wave Guiding-Continued

ATh4B.7 • 18:15

CMOS-Compatible Wavelength-Selective

Infrared Sensors, Tsung Ting $\mathrm{Wu}^{1}$, Chia-

Chien Hsieh1, Ming-Chang Lee1, Yu-Ting

Wang'; 'Photonics Technologies, National

Tsing Hua Univ., Taiwan. We propose

wavelength-selective infrared sensors made

by multiple metallic resonant waveguide gratings integrated on a germanium photodetector array. The detective spectrum can cover from $1.25 \mu \mathrm{m}$ to $1.55 \mu \mathrm{m}$ with the FWHM less than $25 \mathrm{~nm}$. 
Executive Ballroom $210 \mathrm{E}$

Executive Ballroom

210F

\section{CLEO: QELS-Fundamental Science}

FTh4E • Single-Photon

Sources and Quantum

Communications-Continued

FTh4E.7 • 18:15

Distribution of four-dimensional time-bin entangled state over $100 \mathrm{~km}$ of fiber Takuya Ikuta', Hiroki Takesue'; 'NTT Basic Research Labs, Japan. We distributed fourdimensional time-bin entangled photons over $100-\mathrm{km}$ optical fiber. We reconstructed the quantum density operator by utilizing cascaded Mach-Zehnder interferometers, and obtained an average fidelity of $0.935 \pm 0.015$.
FTh4F • Imaging Electron Dynamics on the Nano-, FemtoScale-Continued
Executive Ballroom $210 \mathrm{G}$

Joint

JTh4G • Symposium on Optomechanics: Towards the Second Quantum Revolution II-Continued
Executive Ballroom $210 \mathrm{H}$

\section{CLEO: QELS-} Fundamental Science

FTh4H • Optical and Thermal Superresolution Imaging and Nanofocusing-Continued

FTh4H.7 • 18:15

Efficient Waveguide-to-Plasmon Coupling and Adiabatic Nanofocusing for HAMR Applications, Patrick W. Flanigan', Chuan Zhong ${ }^{1}$, Brian Jennings ${ }^{1}$, Gwenael Atcheson' Frank Bello' David McCloskey ${ }^{1}$ John Donegan"; 'Trinity College Dublin, Ireland. We present several specific and actionable steps to improve the quality of HAMR devices. Recommendations include using a cladding layer to improve coupling efficiency and designing the near-field transducer to achieve adiabatic (low loss) nanofocusing 
Meeting Room
$211 \mathrm{~B} / \mathrm{D}$
Meeting Room

$212 \mathrm{~A} / \mathrm{C}$
Meeting Room

$212 \mathrm{~B} / \mathrm{D}$
Marriott

Salon I \& II

\section{CLEO: Science \& Innovations}

STh4l • Emerging Optical Materials-Continued

STh4l.8 • 18:15

Biologically Inspired Optical Materials and Devices - Harnessing Nature's Light Manipulation Strategies for Dynamic Optical Materials, Mathias Kolle ${ }^{1}$, Sara Nagelberg ${ }^{1}$, Joseph Sandt ${ }^{1}$, Cécile Chazot ${ }^{1}$; 'MIT, USA. Useful concepts for the realization of photonic elements can be identified by taking a careful look at nature. We present a choice of material systems and devices that employ bio-inspired photonic architectures with stimuli-responsive behavior.

\section{STh4J • Ultrafast Laser} Processing-Continued

STh4J.7 • 18:15

Femtosecond Laser Direct Writing with Gating Exposure, Yan-Hao Yu', Jun Jiang', Qi-Dai Chen', Hong-Bo Sun'; 'State Key Lab on Integrated Optoelectronics, College of Electronic Science and Engineering, Jilin Univ., China. We proposed a gating exposure technique for femtosecond laser direct writing. The average laser power could be linearly controlled with the scanning speed while maintaining the exposure dose on the scanning path.
STh4K • Imaging and Nonlinear Fiber Effects-Continued

STh4K.7 • 18:15

Vector Solitons in Harmonically Modelocked Tm/Ho Doped Fiber Laser, Ahmet E. Akosman ${ }^{1}$, Junjie Zeng ${ }^{1}$, Panagis D Samolis ${ }^{1}$, Michelle Y. Sander ${ }^{1,2} ;{ }^{1}$ Electrical and Computer Engineering, Boston Univ., USA ${ }^{2}$ Materials Science and Engineering, Boston Univ., USA. Vector solitons and the evolution of their polarization evolution frequency are studied in harmonically mode-locked $\mathrm{Tm} / \mathrm{Ho}$ co-doped fiber laser states.
STh4L • Precision Timing and Ranging-Continued

STh4L.7 • 18:15

Absolute distance measurement using synthetic wavelength interferometry of optical frequency combs, Guanhao $\mathrm{Wu}^{1}$ Lei Liao'; ' ${ }^{1}$ Tsinghua Univ., China. We present a synthetic-wavelength based heterodyne interferometer of optical frequency combs with wide dynamic measurement range for absolute distance measurement, which can realize an accuracy of $75 \mathrm{~nm}$ in $350 \mathrm{~mm}$ distance measurement.

18:30-20:00 Dinner Break (on your own)

\section{CALL FOR CLEO 2018 SYMPOSIUM PROPOSALS}

The 2018 CLEO CLEO Program Committee is seeking special symposium proposals for consideration from members of the optics and photonics community. Submissions should

consist of timely, cutting-edge topics and/or new material in rapidly advancing areas.

Submissions need to address the following questions.

1. Why is this symposium topic important now and needed in contrast to other years?

2. Which existing topic subcommittees if any, would this topic be most aligned with?

3. Proposed invited speaker list and talk titles.

Submission Deadline: 10 July 2017 at 12:00 EDT (16:00 GMT)

For more information, visit www.cleoconference.org/symposiaproposals 
Marriott

Salon III

Joint

JTh4M • Symposium on Optical Microcavities for Ultrasensitive Detection II-Continued
Marriott

Salon IV
Marriott

Salon V \& VI

\section{CLEO: Science \& Innovations}

STh4N • Photonic Crystals \& Their Applications-Continued

STh4N.8 • 18:15

2D Photonic Crystal Structures in Silicon Rich Nitride Platform, Kapil Debnath'1, Thalia

Dominguez Bucio ${ }^{1}$, Matteo Galli' ${ }^{2}$, Daniele Bajoni², Abdelrahman Al-Attili', Ali Z. Khokhar', Swe Z. Oo', Shinichi Saito', Frederic Gardes'; ${ }^{1}$ Univ. of Southampton, UK; ${ }^{2}$ Univ. of Pavia, Italy. Here we report experimental demonstration of 2D Photonic Crystal waveguide $(\mathrm{PhC})$ and cavity in suspended silicon rich nitride platform. We demonstrate W0.7 PhC waveguide with $70 \mathrm{~nm}$ transmission bandwidth and $\mathrm{PhC}$ cavity ultra-high Q-factor of over 100,000.
STh4O - Quantum Cascade Lasers-Continued

STh40.5 • 18:15

Efficient $\mathrm{THz}$ Generation in Long-Wavelength Infrared Quantum Cascade Lasers, Yifan Jiang', Jae Hyun Kim', Seungyong Jung ${ }^{1}$, Frederic Demmerle'2, Gerhard Boehm²,

M.-C. Amann", Mikhail A. Belkin'; ' ${ }^{1}$ niv. of Texas at Austin, USA; ${ }^{2}$ Walter Schottky Institut, Technische Universität München, Germany. We report more than an order of magnitude improvement in the mid-IR-to$\mathrm{THz}$ conversion efficiency in 1-3 THz sources based on intra-cavity difference-frequency generation in quantum cascade lasers designed to provide mid-infrared gain in $14 \sim 15 \mathrm{~mm}$ range.

18:30-20:00 Dinner Break (on your own)

20:00-22:00 Postdeadline Paper Sessions 
Executive Ballroom 210A

Executive Ballroom 210B

\section{CLEO: Applications \& Technology}

08:00-10:00

AF1A • A\&T Topical Review on Supercontinuum and Applications I

Presider: Robert Alfano; CUNY

City College, USA

\section{AF1A.1 - 08:00 Invited}

Structured Light using OAM and Wavelength Domains for Terabit/sec Communications, Alan E. Willner'; 'Dept. of Electrical Engineering, Univ. of Southern California, USA. Light can be tailored in different domains, including space (e.g., orbital angular momentum) and wavelength. Each domain can also be encoded with information, and multiplexing of multiple beams can increase system capacity. This presentation will discuss recent advances and technical challenges in applying the tailoring of light to communication systems.
08:00-10:00

AF1B - Application \& Advances of Frequency Combs

Presider: Dirk Mueller; Coherent Inc., USA

\section{AF1B.1 08:00 Invited}

Mid-Infrared Spectrometer Featuring $\mu$-second Time Resolution Based on Dualcomb Quantum Cascade Laser Frequency Combs, Andreas Hugi', Anne-Mazarine Lyon', Markus Mangold', Markus Geiser, ${ }^{1,2}$ Wolf Wüster ${ }^{2}$, Filippos Kapsalidis², Jouy Pierre ${ }^{2}$, Jérôme Faist ${ }^{2} ;{ }^{1} I R s w e e p A G$, Switzerland: ${ }^{2} E T H$ Zurich, Switzerland. We present dual-comb spectrometer based on $\mathrm{OCL}$ frequency combs. It features a large optical bandwidth and high-resolution. One key benefit of this instrument is the ability to measure broadband $\mu$ s time-resolved mid-IR spectra.

\section{Executive Ballroom} $210 \mathrm{C}$

\section{CLEO: Science \& Innovations}

08:00-10:00

SF1C • Frequency Comb

Technology

Presider: Guanhao Wu; Tsinghua Univ., China

SF1C.1 $\bullet$ 08:00

Fully-Stabilized Optical Frequency Comb from a Diode-Pumped Solid-State Lase with $\mathrm{GHz}$ Repetition Rate, Sargis Hakobyan', Valentin J. Wittwer', Pierre Brochard ${ }^{1}$ Kutan Guerel ${ }^{1}$, Stephane Schilt ${ }^{1}$, Aline Sophie Mayer $^{2}$, Ursula Keller ${ }^{2}$, Thomas Sudmeyer ${ }^{1}$ 'Université de Neuchâtel, Switzerland; ' ${ }^{2}$ Inst. of Quantum Electronics, ETH, Switzerland. We show full frequency-stabilization of a $\mathrm{GH} z$ diode-pumped solid-state laser frequency comb. The CEO is stabilized via pump-curren modulation. We present a thorough characterization of the comb in terms of noise and frequency stability.
SF1C.2 $\bullet 08: 15$

Optimizing the Power Efficiency of a SESAM Fiber Comb Laser, Shaokang Wang ${ }^{1}$, Curtis R. Menyuk', Stefan Droste ${ }^{2}$ Laura Sinclair ${ }^{2}$, lan R. Coddington ${ }^{2}$, Nate Newbury ${ }^{2}{ }^{1}$ Computer Science and Electrical Engineering, Univ. of Maryland, Baltimore County, USA; ${ }^{2}$ National Inst. of Standard and Technology, USA. The power efficiency of femtosecond fiber lasers with semiconducto saturable absorbers is limited by the wake instability. We computationally optimize the output power and efficiency by increasing the output coupling ratio and gain fiber length.
Executive Ballroom 210D

\section{Joint}

08:00-10:00

JF1D • Symposium on

Thermal Noise in Precision

Interferometry I

Presider: Gregory Harry;

American Univ., USA

JF1D.1 $\bullet$ 08:00

Introduction to the Special Symposium On Thermal Noise: Fostering Collaboration Between the Gravitational Wave and Cavity-stabilized Laser Communities, Garrett D. Cole'; ${ }^{1}$ Crystalline Mirror Solutions ULC. USA. Precision optical interferometers have now enabled the direct detection of gravitational waves as well as the construction of lasers with linewidths below $10 \mathrm{mHz}$. It is becoming increasingly clear that thermal noise now stands as a significant impedimen to continued progress in the development of such advanced optical systems. This symposium will serve to educate interested partie on the fundamental scientific aspects as well as implications for advanced applications in precision metrology, bringing together researchers from the fields of gravitationalwave astronomy and laser-based precision metrology. Our aim is to foster collaboration between materials scientists, physicists, and optical engineers interested in developing components and systems with reduced levels of thermal noise.

JF1D.2 • 08:15 Invited

Thermal Noise in Mirror Coatings for Gravitational Wave Detection, Martin M. Fejer'; ' ${ }^{1}$ E.L. Ginzton Lab, Stanford Univ., USA Mid-band sensitivity of gravitational-wave detectors is limited by Brownian noise in interferometer mirrors. We discuss connection between thermal noise and elastic dissipation in mirrors, and between that dissipation and the structure of amorphous films.
AF1A. 2 • 08:30 Invited

Supercontinuum in Telecom Applications, Juan D. Ania-Castanon ${ }^{2}$, Sergey V. Smirnov ${ }^{1}$ Sergey Kobtsev', Sergei Turitsyn ${ }^{3}$; ${ }^{1}$ Division of Laser Physics and Innovative Technologies, Novosibirsk State Univ., Russia; ${ }^{2}$ nstituto de Optica (IO-CSIC), Consejo Superior de Investigaciones Científicas, Spain: ${ }^{3}$ Aston Inst. of Photonic Technologies, Aston Univ., UK. We provide a general overview of spectral broadening and SC generation application in fiber-optic telecommunications from a historical perspective, with a particular focus on the most recent developments.
AF1B.2 • 08:30

Absorption spectroscopy based on polarization-multiplexed dual-frequency femtosecond fiber laser combs, Rongqing Huil'; ${ }^{1}$ Univ. of Kansas, USA. Coherent duel-frequency optical combs are generated using common-cavity approach based on polarization-multiplexing in an all-PM fibe laser configuration. We demonstrate the application of these optical combs in spectroscopy without the need of active phase synchronization.
SF1C.3・08:30

Coherent Supercontinuum Generation with Picosecond Pulses, Adrea R. Johnson ${ }^{1,2}$ Xingchen $\mathrm{Ji}^{1}{ }^{1,2}$, Michael R. Lamont ${ }^{2}$, Yoshitomo Okawachi" ${ }^{1}$, Michal Lipson ${ }^{1}$, Alexander Gaeta ${ }^{1} ;{ }^{1}$ Columbia, USA; ${ }^{2}$ Cornell, USA. We theoretically show the possibility of generating a coherent, octave-spanning supercontinuum with $>1$-ps pulses. Our proof-of-principle experiments demonstrate the feasibility of utilizing long waveguides and multiple cross sections for supercontinuum generation at ultralow pulse energies. 


\section{CLEO: QELS-Fundamental Science}

08:00-10:00

FF1E - Single-Photon Detectors

Presider: Christine Silberhorn; Universität Paderborn, Germany

FF1E.1 1 08:00

Single-photon detection with near unity efficiency, ultra-high detection-rates, and ultra-high time resolution, Val Zwiller ${ }^{2,1}$, Iman Esmaeil Zadeh', Johannes Los', Ronan Gourgues', Violette Steinmetz', Sergiy Dobrovolskiy', Sander N. Dorenbos': ' ${ }^{1}$ Single Quantum B. V., Netherlands; ${ }^{2}$ KTH Royal Inst. of Technology, Sweden. In this work we demonstrate a broadband single-photon detector with efficiency higher than $92 \%$, over $150 \mathrm{MHz}$ photon detection-rate, dark counts below $130 \mathrm{~Hz}$ and a record low jitter of 14.80 ps.
08:00-10:00

FF1F • Optical \& THZ

Spectroscopy of Quantum

Matter

Presider: Robert Kaindl; Lawrence Berkeley National Lab., USA

FF1F.1 1 08:00

Photoinduced dynamics of terahertz plasmonics response in $\mathrm{Bi}_{2} \mathrm{Se}_{3}$ topological insulator, Flavio Giorgianni', Mostafa Shalaby' ${ }^{1}$ C. Vicario ${ }^{1}$, Christoph P. Hauri', Stefano lupi2; ' ${ }^{2}$ SwissFEL, Paul Scherrer Institut, Switzerland: ${ }^{2}$ Dept. of Physics, Sapienza Univ. Of Rome, Italy. We present time-resolved photo-induced plasmonic response in $\mathrm{Bi}_{2} \mathrm{Se}_{3}$ topological insulator investigated by means of optical-pump/THz-probe spectroscopy. We have found that topological insulators offer a non-trivial relaxation dynamics due to their complex bulk-surface interactions.
08:00-10:00

FF1G • Nanoparticle

Mediated Emission and Field

Enhancement

Presider: Berardi SensaleRodriguez; Univ. of Utah, USA

FF1G.1 $08: 00$

Magnetic vs Electric Second-Harmonic Generation from AlGaAs Nanoantennas, Sergey S. Kruk', Lei Xu' ${ }^{1}$ Rocio CamachoMorales', Mohsen Rahmani', Lei Wang', Daria Smirnova', Guoquan Zhang', Hoe $\operatorname{Tan}^{3}$, Chennupati Jagadish ${ }^{3}$, Yuri Kivshar ${ }^{1}$, Dragomir Neshev'; 'Australian National Univ., Australia; ${ }^{2}$ Nankai Univ., China; ${ }^{3}$ Electronic Materials Engineering, Australian National Univ. Australia. We suggest and demonstrate experimentally AlGaAs nanoantennas for efficient second-harmonic generation (SHG). We show that the SHG directionality and efficiency are defined by either electric or magnetic multipoles and controlled by incident polarization and design.
FF1E.2 • 08:15

Bandwidth-enhanced Superconducting Nanowire Single Photon Detectors for Telecom Wavelengths, Stephan Krapick ${ }^{1,2}$, Marina Hesselberg ${ }^{2}$, Varun Verma ${ }^{2}$, Sae Woo Nam², Richard Mirin'; ' 'Dept. of Physics, Univ. of Paderborn, Germany; ${ }^{2}$ Applied Physics Division, National Inst. of Standards and Technology, USA. We present a singlephoton detector providing system detection efficiencies of at least \$\left(86.7\pm0.9\ right), $1 \% \$$ from $1450 \mathrm{~nm}$ to $1640 \mathrm{~nm}$. It comprises bilayer superconducting WSi nanowires in conjunction with all-dielectric structures for optical impedance matching.
FF1E. 3 • 08:30 Invited

Superconducting Single Photon Detector Science and Engineering, Sae Woo Nam"; ${ }^{1}$ NIST, USA. Single-photon detectors are an essential tool for a wide range of applications. Ideally, a single photon detector generates a measurable signal only when a single photon is absorbed. Since the first reported detection of a single photon using a superconducting nanowire in 2001, rapid progress has been made in the development and application of superconducting nanowire single photon detectors with ideal properties. I will briefly describe recent progress in detector development at NIST.
FF1F.2 • 08:15

Terahertz investigation of Dirac plasmons and phonon interaction in the topological insulator $\mathrm{Bi}_{2} \mathrm{Se}_{3}$ metamaterials, Chihun $\ln ^{1,4}$, Sangwan Sim 1 , Sungjoon Park', Hyemin Bae ${ }^{1}$, Nikesh Koirala ${ }^{2}$, Jisoo Moon², Maryam Salehi², Seongshik Oh², Dohun $\mathrm{Kim}^{3}$, Hyunyong Choi'; ' ${ }^{\text {Yonsei Univ., Ko- }}$ rea (the Republic of); ${ }^{2}$ Rutgers Univ., USA; ${ }^{3}$ Seoul National Univ., Korea (the Republic of); ${ }^{4}$ Korea Atomic Energy Research Inst. Korea (the Republic of). We have measured the terahertz responses of plasmon-phonon interaction in the topological insulator $\mathrm{Bi}_{2} \mathrm{Se}_{3}$ metamaterials. Upon photoexcitation, we observed transient phonon stiffening of $\sim 0.1$ $\mathrm{THz}$ when the plasmon frequency is above the phonon energy.

FF1F.3 • 08:30

Terahertz Pump-Probe Study of the Weyl Semimetal TaAs, Mohammad Mehdi Jadidi', Martin Mittendorff', Stephan Winnerl'2, Bing Shen $^{3}$, Andrei B. Sushkov ${ }^{4}$, Greg S. Jenkins ${ }^{4}$ H. Dennis Drew ${ }^{4}$, Thomas E. Murphy'; ${ }^{1}$ Inst. for Research in Electronics and Applied Physics, Univ. of Maryland, College Park, MD 20742, USA, USA: ${ }^{2} \mathrm{Helmholtz-Zentrum}$ Dresden-Rossendorf, P.O. Box 510119, 01314 Dresden, Germany, Germany; ${ }^{3}$ Dept. of Physics and Astronomy, Univ. of California at Los Angeles, Los Angeles, CA 90095, USA, USA; ${ }^{4}$ Center for Nanophysics and Advanced Materials, Univ. of Maryland, College Park, Maryland 20742, USA, USA. We use terahertz reflection pump-probe measurements to study carrier dynamics in the newly discovered Weyl semimetal tantalum arsenide ( $(\mathrm{Ta} A \mathrm{~s})$. Our measurements reveal the relaxation dynamics of intra- and inter-band excited carriers near the Weyl points.
FF1G.2 • 08:15

Mid-Infrared Third-Harmonic Emission from Heavily-Doped Germanium Plasmonic Nanoantennas, Marco Patrick Fischer ${ }^{1}$ Aaron Riede', Alexander Grupp ${ }^{1}$, Kevin Gallacher², Jacopo Frigerio ${ }^{3}$, Giovanni Pellegrini ${ }^{4}$, Michele Ortolani ${ }^{5}$, Douglas J. Paul2 ${ }^{2}$ Giovanni Isella ${ }^{3}$, Alfred Leitenstorfer ${ }^{1}$, Paolo Biagioni ${ }^{4}$, Daniele Brida'; 'Dept. of Physics and Center for Applied Photonics, Univ. of Konstanz, Germany: ${ }^{2}$ School of Engineering, Univ. of Glasgow, UK; ${ }^{3}$ L-NESS, Dipartimento di Fisica, Politecnico di Milano, Italy; ${ }^{4}$ Dipartimento di Fisica, Politecnico di Milano, Italy; ${ }^{5}$ Dept. of Physics, Sapienza Univ. of Rome, Italy. We investigate the nonlinear optical properties of single resonant plasmonic antennas fabricated from heavily-doped Germanium films. Excitation with intense and ultrashort mid-infrared pulses at $10.8 \mu \mathrm{m}$ wavelength produces emission at $3.7 \mu \mathrm{m}$ via third-harmonic generation.

FF1G.3 • 08:30

Do Low-Loss Doped Semiconductor Nanoparticles Yield Stronger Field Enhancement?, Jacob Khurgin ${ }^{3}$, Pin C. $\mathrm{Wu}^{2}$, Din P. Tsai ${ }^{2}$ Ning Liu ${ }^{4}$, Wen T. Hsieh ${ }^{1}$, Gregory Sun'; 'Univ. of Massachusetts Boston, USA; ${ }^{2}$ Physics, National Taiwan Univ., Taiwan; ${ }^{3} E C E$, Johns Hopkins Univ. USA; ${ }^{4}$ Physics and Energy, Univ. of Limerick, Ireland. We show that using nanoparticles made of low-loss doped semiconductors in place of noble metals with higher losses does not lead to the anticipated superior enhancement of the electric field. 

Executive Ballroom
$210 \mathrm{H}$
Meeting Room
$211 \mathrm{~B} / \mathrm{D}$

Meeting Room

$212 \mathrm{~A} / \mathrm{C}$

\section{CLEO: Science \& Innovations}

08:00-10:00

SF1H $\bullet$ Waveguides and Ring

Resonators

Presider: Jian Wang; Huazhong

Univ of Science and Tech, China

\section{SF1H.1 • 08:00}

Coherent Beam Combining On Silicon Chip Through Hybrid Integration, Yeyu Zhu", Yunsong Zhao', Lin Zhu': ' ${ }^{1}$ lemson Univ., USA. We demonstrate hybrid integration of passive coherent beam combining cavity integrated with a diode laser array on silicon photonics platform. Silicon nitride adiabatic coupler is used to obtain a broadband power splitting ratio of 50:50.

SF1H.2 $\bullet 08: 15$

A Compact Silicon Photonic Add-Drop Multiplexer with Misaligned Sidewall Bragg Gratings in a MZI, Md. Ghulam Saber Zhenping Xing ', David Patel', Eslam El-Fiky', Nicolás Abadía', Yun Wang', David V. Plant'; ${ }^{1}$ Dept. of Electrical and Computer Engineering, McGill Univ., Canada. We experimentally demonstrate a compact optical add-drop multiplexer based on misaligned sidewall Bragg gratings in a MZI. We achieved $51 \mathrm{~dB}$ transmission isolation and $5 \mathrm{~nm} 3-\mathrm{dB}$ bandwidth with a footprint of $400 \mu \mathrm{m} \times 125 \mu \mathrm{m}$.

\section{SF1H.3 $\bullet$ 08:30}

Automatic Monitor-Based Tuning of Reconfigurable Silicon Photonic $2^{\text {nd }}$ Order APF-Based Pole/Zero Filters, Gihoon Choo', Shengchang Cai', Binhao Wang', Christi Madsen', Kamran Entesari', Samuel Palermo'; 'Electrical and Computer Engineering, Texas A\&M Univ., USA. We demonstrate automatic monitor-based filter tuning with a Si-photonic $2^{\text {nd }}$-order APF-based filter. The proposed tuning approach calibrates the initial response by controlling each pole and zero individually to reconfigure to different bandwidths and center wavelengths.
08:00-10:00

SF1I - Integrated Photonic Devices

Presider: Jian Wang; Huazhong

Univ of Science and Tech., China

\section{SF1I.1 08:00 Tutorial}

From Concept to a Working Silicon Photonic Chip, Lukas Chrostowski'; 'Univ. of British Columbia, Canada. This presentation describes approaches to designing silicon photonic circuits while taking manufacturing variability into account to ensure a functioning chip. Our method is an enhanced Monte Carlo technique that includes layout-specific correlated manufacturing variations.

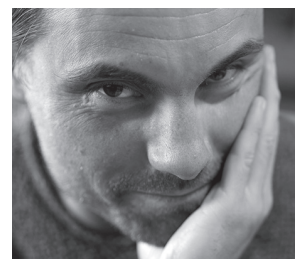

Lukas Chrostowski is a Professor of Electrical and Computer Engineering at the University of British Columbia, Vancouver, BC, Canada. He earned a B.Eng. from McGill University and $\mathrm{PhD}$ from the University of California at Berkeley. His research interests are in silicon photonics, optoelectronics, fabrication and test, for applications in optical communications and biophotonics. He co-authored the book "Silicon Photonics Design". He is the Program Director the Silicon ElectronicPhotonic Integrated Circuits (Si-EPIC) program in Canada.
08:00-10:00

SF1J - Micro- and

Nanophotonic Devices

Presider: Kenneth Crozier; Univ. of Melbourne, Australia

\section{SF1J.1 $\bullet$ 08:00}

A Black Phosphorus Optoelectronic Mixer, Ryan J. Suess', Lei Chen', Joseph D. Hart', Edward Leong', Thomas E. Murphy', Martin Mittendorff'; ${ }^{1}$ Inst. for Research in Electronics and Applied Physics, Univ. of Maryland, USA. An optoelectronic mixer based on the nonlinear photoconductivity of black phosphorus is presented. We demonstrate mixing with a maximum conversion loss of $31 \mathrm{~dB}$ at local oscillator frequencies up to $640 \mathrm{MHz}$.

\section{SF1J.2 • 08:15}

Passive and Active Light Control using Computational Metamaterials, Apratim Majumder', Bing Shen', Randy Polson', Rajesh Menon'; ' ${ }^{1}$ niv. of Utah, USA. We report on our latest developments in computational metamaterials based nanophotonics devices to design and implement ultra-compact onchip polarization rotator, waveguide cloaks and all-optical modulators.

\section{SF1J.3 • 08:30}

Optical trapping using all-dielectric silicon nanoantennas with ultra-low heat generation, Zhe $\mathrm{Xu}^{1}$, Wuzhou Song ${ }^{1,2}$, Kenneth B. Crozier ${ }^{1,3}$; ${ }^{1}$ School of Physics, Univ. of Melbourne, Australia; ${ }^{2}$ School of Materials Science and Engineering, Huazhong Univ. of Science and Technology, China; ${ }^{3}$ Dept. of Electrical and Electronic Engineering, Univ. of Melbourne, Australia. Silicon nanoantennas are used to trap polystyrene nanospheres (20 nm diameter). Fluorescence microscopy is used to monitor trapped particle position as a function of time. The nanoantennas produce subwavelength field enhancement with negligible heat generation. 
CLEO: Science \& Innovations

08:00-10:00

SF1K • Laser Facilities and Applications

Presider: Thomas Spinka; Lawrence Livermore National Lab, USA

\section{SF1K.1 08:00 Invited}

Overview of the 1.15 PW PETAL Lser in the LMJ Fcility, Nathalie Blanchot ${ }^{1}$ Christelle Damiens-Dupont ${ }^{1}$, Herve Coic ${ }^{\prime}$ Claude Rouyer ${ }^{1}$, Fabrice Laniesse ${ }^{1}$, Jerome Neauport ${ }^{1}$, Gilles Behar ${ }^{1}$, Laurent Hilsz' Eric Lavastre ${ }^{1}$, Didier Raffestin ${ }^{1}$, Martin Sozet ${ }^{1}$ Laurent Lamaignere ${ }^{1}$, Steeve Chardavoine ${ }^{1}$ Jean-Paul Goossens ${ }^{1}$, Melanie Mangeant ${ }^{1}$ Jacques Luce ${ }^{1}$, Cyril Present ${ }^{1}$, Nathalie Santacreu', Christian Chappuis' ${ }^{1}$, Stephane Bouillet ${ }^{1}$, Sandrine Freville ${ }^{1}$, Francois Macias ${ }^{1}$ ${ }^{1}$ CEA Cesta, France. PETAL is an additional PW beamline to the LMJ. The kJ shots in the amplifier section, the compressor alignment and the 1.15 PW @ $850 \mathrm{~J}$ operations are detailed. Damage issues encountered are also addressed.

SF1K.2 $\bullet 08: 30$

$10^{22} \mathrm{~W} / \mathrm{cm}^{2}, 0.1 \mathrm{~Hz}$ J-KAREN-P laser facility at QST, Hiromitsu. Kiriyama', Mamiko Nishiuchi', Pirozhkov Alexander ${ }^{1}$, Hironao Sakaki', Nicholas Dover ${ }^{1}$, Akito Sagisaka', Kotaro Kondo', Keita Nishitani', Yuji Fukuda?' Koichi Ogura', Michiaki Mori', James Koga', Yasuhiro Miyasaka', Esirkepov Timur' ${ }^{1}$, Yukio Hayashi ${ }^{1}$, Hideyuki Kotaki ${ }^{1}$, Kai Huang ${ }^{1}$ Nobuhiko Nakanii', Sergei Bulanov', Masaki Kando', Kiminori Kondo'; ${ }^{1}$ National Inst. Quantum \& Rad Sc \& Tech, Japan. Broadband-pulses are amplified to $63 \mathrm{~J}$ and compressed to $30 \mathrm{fs}$. A peak intensity of $10^{22}$ $\mathrm{W} / \mathrm{cm}^{2}$ by focusing a $0.3 \mathrm{PW}$ laser beam with an $\mathrm{f} / 1.4$ off-axis parabolic mirror is achievable on target.

\section{8:00-09:30 \\ SF1L • Data Center \\ Communications \\ Presider: Michael Vasilyev; Univ. \\ of Texas at Arlington, USA}

SF1L. $1 \bullet 08: 00$

An Efficient Hybrid Equalizer for $50 \mathrm{~Gb} / \mathrm{s}$ PAM-4 Signal Transmission Over $50 \mathrm{~km}$ SSMF in a 10-GHz DML-Based IM/DD system, Jing Zhang ${ }^{1}$, Taiping $\mathrm{Ye}^{1}{ }^{1}$ Xingwen $\mathrm{Yi}^{1}$, Changyuan Yu${ }^{2}$, Kun Qiu'; ${ }^{1}$ Uestc, China; ${ }^{2}$ The Hong Kong Polytechnic Univ., China. We experimentally demonstrate an effective hybrid equalizer with FFE and truncated Volterra filter for a 50-Gb/s PAM-4 over 50-km SSMF in a DML-based IM/DD system. The results show significant computational complexity savings without performance degradation.

SF1L.2・08:15

Simplified Demultiplexing Scheme for Two PDM-IM/DD Links Utilizing a Single Stokes Analyzer, Yan Pan ${ }^{1}$, Lianshan Yan ${ }^{1}$, Anlin Yi ${ }^{1}$, Lin Jiang', Wei Pan', Bin Luo', Xihua Zou' ${ }^{1}$. 'Southwest Jiaotong Univ., China. A simplified demultiplexing scheme for two PDM-IM/ DD links utilizing a single Stokes analyzer is experimentally demonstrated. Results of $4 \times 10-$ Gbit/s transmission over 2-km SSMF show $<2-d B$ power penalty compared to the single PDM-IM/DD system.

SF1L. $3 \bullet 08: 30$

Chirp Control in Directly Modulated 25G PAM4 Transmitters for Optical Access Networks, Marco Dalla Santa ${ }^{1}$, Cleitus Antony ${ }^{1}$, Giuseppe Talli', Paul Townsend". ${ }^{1}$ Tyndall National Inst., Ireland. Narrowband filtering chirp control is demonstrated for a $25 \mathrm{~Gb} / \mathrm{s}$ PAM4 signal in directly modulated transmitters for next generation optical access systems, allowing $50 \mathrm{~km}$ transmission without chromatic dispersion compensation with blue-shift filtering offering the best performance.

\section{8:00-10:00 \\ SF1M - Aerosol and Gas Sensing \\ Presider: Michael Wojcik; Space \\ Dynamics Lab, USA}

\section{SF1M.1 • 08:00}

Rapid switching between spectral windows for NO isotope sensing using an external cavity quantum cascade laser Brian Brumfield ${ }^{1}$, Mark C. Phillips' ${ }^{1}{ }^{1}$ Pacific Northwest National Lab, USA. We present high-resolution spectroscopy and sensing of NO isotopes using a new external cavity quantum cascade laser rapidly switched between two spectral windows separated by $13 \mathrm{~cm}-1$.

SF1M.2 • 08:15

Towards the Robust Trace Detection of Radiocarbon via Linear Absorption Spectroscopy, Adam J. Fleisher ${ }^{1}$, David A. Long' Qingnan Liu', Joseph T. Hodges'; ' ${ }^{1}$ NIST, USA. Reported here is the optical detection of radiocarbon below contemporary levels using cavity ring-down spectroscopy in the linear absorption regime. Petrogenic and biogenic samples of $\mathrm{CO}_{2}$ are readily distinguished by repeated optical measurements.

\section{SF1M.3 • 08:30 Invited}

Infrared fingerprint-region aerosol spectroscopy, Luke Maidment ${ }^{1,2}$, Rhea J. Clewes ${ }^{2}$, Martin D. Bowditch², Christopher R. Howle ${ }^{2}$, Derryck T. Reid'; 'Heriot-Watt Univ., UK' ${ }^{2}$ Defence Science and Technology Lab, UK. Mie theory predicts aerosols should exhibit different mid-infrared spectral signatures to bulk materials. We verify this experimentally using broadband pulses from a 7.1-8.85- $\mu \mathrm{m}$ femtosecond optical parametric oscillator, obtaining close agreement with theoretical calculations. 
Executive Ballroom 210A

Executive Ballroom 210B

\section{CLEO: Applications \& Technology}

AF1A • A\&T Topical Review on Supercontinuum and Applications I-Continued
AF1B • Application \& Advances of Frequency CombsContinued

\section{AF1B. $3 \bullet 08: 45$}

Internally phase stabilized Kerr comb Abhinav Vinod', Shu-Wei Huang', Jinghu Yang', Mingbin Yu ${ }^{2}$, Dim-Lee Kwong ${ }^{2}$, Chee Wei Wong'; ${ }^{1}$ Electrical Engineering, UCLA USA; ${ }^{2} I M E$, Singapore. We demonstrate a phase stabilized Kerr comb without the use of external references or non-linear interferometry. Out-of-loop measurements confirm good coherence and stability across the comb, with measured optical frequency fractional instabilities of $5 \times 10^{-11} / \sqrt{T}$.

\section{AF1A.3 • 09:00 Invited}

Supercontinuum Laser Sources Future Await Wide Applications, Adam Devine ${ }^{1}$, Lucy E. Hooper ${ }^{1}$, John R. Clowes ${ }^{1}$, Thomas V. Andersen' ${ }^{1}$, Peter M. Moselund ${ }^{1}$, Christian V. Poulsen ${ }^{1}$, Carsten L. Thomsen ${ }^{2}$, Ole Bang ${ }^{2}$ ${ }^{1}$ Fianium Ltd., UK; ${ }^{2}$ NKT Photonics A/S, Denmark. Recent advances in supercontinuum generation technology have enabled the development of lasers with higher power and broader spectral content, allowing exploitation of these light sources in a range of new and exciting application areas.

\section{AF1B.4 $\bullet$ 09:00}

Generation of Carrier-Envelope Phase Stabilized Laser from Solid Plates and Application in High-Harmonic Generation, Yangyang Liu', Peng $\mathrm{He}^{2}$, Kun Zhao Hangdong Huang ${ }^{2}$, Yujiao Jiang ${ }^{2}$, Pei Huang ${ }^{3}$, Hao Teng ${ }^{1}$, Xinkui He ${ }^{1}$, Shaobo Fang ${ }^{1}$, Xun $\mathrm{Hou}^{3}$, Zhiyi Wei'; 'Inst. of Physics, Chinese Academy of Sciences, China; ${ }^{2}$ Xidian Univ. China; ${ }^{3}{ }^{\prime} i^{\prime} a n$ Inst. of Optics and Precision Mechanics, Chinese Academy of Sciences, China. We demonstrated carrier-envelope phase (CEP) stabilized pulse as short as $5.4 \mathrm{fs}$ by compressing the octave-spanning spectrum from solid-state plates. Continuous and discrete HHG were observed by using the laser pulse as driver with different CEP.

\section{AF1B.5 $\bullet 09: 15$}

Active control of absorption in a hybrid graphene-microfiber modulator, Zhuang Zhao', Daniel Popa', Ugo Sassi', Zongyin Yang ${ }^{1}$, Yingxin $\mathrm{Xu}^{2}$, Limin Tong ${ }^{2}$, Andrea Ferrari'; ' 'Engineering, Univ. of Cambridge, UK ${ }^{2} Z$ hejiang Univ., China. We report a hybrid graphene-microfiber odulator with low insertion loss $\sim 0.4 \mathrm{~dB}$, and active, $\sim 6.7 \%$, control of absorption. This can be used for active control of ultrafast laser working in continuous wave, Q-switching and mode-
Executive Ballroom $210 \mathrm{C}$

\section{CLEO: Science \& Innovations}

\section{SF1C • Frequency Comb} Technology-Continued

\section{SF1C. $4 \cdot 08: 45$}

Octave broadening of a $15 \mathrm{GHz}$ Kerr soliton comb, Erin S. Lamb², Jordan R. Stone ${ }^{2}$ Myoung-Gyun Suh ${ }^{1}$, Kerry Vahala ${ }^{1}$, Scot Diddams ${ }^{2}$, Scott Papp ${ }^{2} ;{ }^{1}$ CalTech, USA; ${ }^{2}$ NIST, USA. We generate single Kerr-cavity solitons from a $15 \mathrm{GHz}$ silica resonator. These pulses are compressed in normal dispersion fiber and used to generate a coherent octavespanning supercontinuum.

SF1C.5 $\bullet$ 09:00

Electro-optic modulator for rapid control of the carrier-envelope offset frequency, Wolfgang Hänsel ${ }^{1}$, Michele Giunta ${ }^{1,2}$, Matthia Lezius ${ }^{1}$, Marc Fischer ${ }^{1}$, Ronald Holzwarth ${ }^{1,2}$ Menlo Systems GmbH, Germany; ${ }^{2}$ MaxPlanck-Inst. for Quantum Optics, Germany. We report on an ultra-low noise optical frequency comb simultaneously stabilized at its carrier-envelope offset frequency and one optical mode, using a novel electrooptic group-velocity shifter with a fix-poin in the optical domain with nanosecond response time.
Executive Ballroom 210D

\section{Joint}

JF1D • Symposium on Thermal Noise in Precision Interferometry I-Continued

\section{JF1D.3 $08: 45$ Invited}

Thermal Noise in Ultrastable Cavity-Referenced Lasers, Uwe Sterr', Dan-Gheorghit Matei ${ }^{1}$, Thomas Legero', Sebastian Häfner ${ }^{1}$ Robin Weyrich ${ }^{1}$, Wei Zhang ${ }^{2}$, John Robinson ${ }^{2}$ Lindsay Sonderhouse ${ }^{2}$, Paula Heu4, David Follman ${ }^{4}$, Christoph Deutsch ${ }^{5}$, Garrett D. Cole ${ }^{4,5}$, Markus Aspelmeyer ${ }^{3}$, Fritz Riehle ${ }^{1}$ Jun $\mathrm{Ye}^{2}$; ${ }^{1}$ Physikalisch Technische Bunde sanstalt, Germany; ${ }^{2}$ JILA, NIST, USA; ${ }^{3}$ Faculty of Physics, Univ. of Vienna, Vienna Center for Quantum Science and Technology (VCO) Austria; ${ }^{4}$ Crystalline Mirror Solutions LLC USA; ${ }^{5}$ Crystalline Mirror Solutions $\mathrm{GmbH}$ Austria. Thermal noise is now limiting the most stable lasers that are employed in optical clocks and for precision measure ments to a few times $10^{-17}$. Laser systems and ways towards further improvements will be presented. locking operations.

SF1C.6・09:15

Coherent Control of Relative Carrier Envelope Phase in Dual-Comb Spectroscopy, Akifumi Asahara 1,2 Ken-ichi Kondo 1,2 Yue Wang ${ }^{1,2}$, Kaoru Minoshima ${ }^{1,2}$. ' Univ. of Electro-Communications, Japan; ${ }^{2} J S T$, ERATO MINOSHIMA Intelligent Optical Synthesizer Japan. Relative carrier envelope phase control was actively exploited in dual-comb spectroscopy, and polarization modulated pulse train generation and its coherent detection were demonstrated. This proof-of-principle experiment promotes advanced coherent spectroscopy using optical frequency combs.

SF1C.7 • 09:30

AF1B.6 • 09:30

\section{AF1A.4 09:30 Invited}

The Early Days of Self-Phase Modulation and Supercontinuuim Generation, Robert A. Fisher'; 'RA Fisher Associates, USA. The early days of SPM and SC are explored with a special attention to the physical principles involved. Here we will find that the human traits of impatience and patience govern different approaches to physical principles.
Fully-Integrated artificial saturable absorber based on Kerr nonlinearity in silicon nitride, Katia Shtyrkova', Patrick T. Callahan ${ }^{1}$ Michael Watts ${ }^{1}$, Erich P. Ippen ${ }^{1}$, Franz Kaertner'; ' ${ }^{1}$ IT, USA. An integrated artificial fast saturable absorber at $1.9 \mu \mathrm{m}$ is demonstrated in a CMOS-compatible process. It is based on the Kerr effect in a nonlinear Mach-Zehnder interferometer using silicon nitride waveguides embedded in $\mathrm{SiO} 2$. zation in time-domain using heterodyne interferometry, Xiaosheng Zhang ${ }^{1}$, Minghao $\mathrm{Hu}^{1}$, Shilin Xiong', Guanhao Wu'; ${ }^{1} T$ singhua Univ., China. We propose a time-domain $f_{\text {ceo }}$ stabilization method that $f$ is stabilized using heterodyne interference phase between two delay-stabilized pulse trains. Allan derivations of stabilized $f_{\text {ceo }}$ are $1.48 \times 10^{-9}$ at $1 \mathrm{~s}$ and $4.90 \times 10^{-10}$ at $100 \mathrm{~s}$.
Carrier-envelope offset frequency stabili-
JF1D.4 • 09:15

A Thermal Noise Limited, Rigidly-held Optical Reference Cavity for Ultra-low Noise Microwave Generation, Josue Davila-Rodriguez², Fred N. Baynes', Andrew Ludlow $^{2}$, Tara M. Fortier ${ }^{2}$, Holly F. Leopardi2,3, Scott Diddams ${ }^{2,3}$, Franklyn Quinlan"2; 'Inst. for Photonics and Advanced Sensing (IPAS) and School of Physical Sciences, The Univ. of Adelaide, Australia; ${ }^{2}$ Time and Frequency Division, National Inst. of Standards and Technology, USA; ${ }^{3}$ Phyiscs, Univ. of Colorado Boulder, USA. A simple, rigidly-held $25 \mathrm{~mm}$ ong reference cavity is presented. A lase stabilized to it supports $10 \mathrm{GHz}$ generation with phase noise near $-100 \mathrm{dBc} / \mathrm{Hz}$ at $1 \mathrm{~Hz}$ offset and $<-173 \mathrm{dBc} / \mathrm{Hz}$ for offsets $>600 \mathrm{~Hz}$.

\section{JF1D.5 • 09:30 Invited}

Thermal Noise in Microfabricated AlGaAs Structures, Thomas Corbitt ${ }^{1}$, Jonathan Cripe', Robinjeet Singh'; ' Louisiana State Univ., USA. Multilayer crystalline AIGaAs stacks have the potential to reduce coating thermal noise in future gravitational-wave interferometers. The results of direct measurements of thermal noise in microfabricated AlGaAs structures will be presented. 
Executive Ballroom $210 \mathrm{E}$
Executive Ballroom 210F
Executive Ballroom 210G

\section{CLEO: QELS-Fundamental Science}

\section{FF1E • Single-Photon}

Detectors-Continued
FF1E.4 0 09:00

Reduced Effect of Single-Photon-Detector Deadtime Using a Switchable Detector Array in an Orbital-Angular-Momentum (OAM) Encoded Quantum System, Cong Liu', Yongxiong Ren'1, Jiapeng Zhao'², Seyed M. Rafsanjani², Guodong Xie', Kai Pang', Haogian Song ${ }^{1}$, Zhe Zhao ${ }^{1}$, Zhe Wang ${ }^{1}$, Long $\mathrm{Li}^{1}$, Joshua Bienfang ${ }^{3}$, Alan Migdall ${ }^{3,4}$, Moshe Tur $^{5}$, Robert Boyd ${ }^{2}$, Alan E. Willner'; ${ }^{1}$ Univ. of Southern California, USA; ${ }^{2}$ Dept. of Physics and Astronomy, Univ. of Rochester, Rochester, USA; ${ }^{3}$ NIST and Univ. of Maryland, USA; ${ }^{4}$ Joint Quantum Inst., Univ. of Maryland, USA; ${ }^{5}$ School of Electrical Engineering, Tel Aviv Univ., Israel. We explore using a switchable detector array to reduce the deadtime effect in an OAM-encoded quantum system. For a 4-OAM-state system, the switchable 16-detector array could provide $>15 \mathrm{X}$ incident photon rate improvement, as compared to a non-switchable 4-detector array.

FF1E.5 • 09:15

Vortex-Crossing-Induced Timing Jitter of Superconducting Nanowire SinglePhoton Detectors, Hao $\mathrm{Wu}^{1,2}$, Chao $\mathrm{Gu}^{1,2}$, Yuhao Cheng ${ }^{1,2}$, Xiaolong Hu${ }^{1,2}$; ${ }^{1}$ School of Precision Instrument and Optoelectronic Engineering, Tianjin Univ., China; ${ }^{2}$ Key Lab of Optoelectronic Information Science and Technology, Ministry of Education, China. We show that single-photon-triggered vortex (or anti-vortex) crossing in a superconducting nanowire single-photon detector induces timing jitter, which fundamentally limits the time-resolving capability of the detector.

\section{FF1F • Optical \& THZ Spectroscopy of Quantum Matter-Continued}

FF1F.4 0 08:45

Polarization-dependent surface-bulk scattering in the Weyl semimetal NbAs, Yaomin Dai $^{1}$, Bing Shen ${ }^{2}$, Lingxiao $\mathrm{Zhao}^{3}$, Bing Xu ${ }^{3}$, Yongkang Luo ${ }^{1}$, Aiping Chen ${ }^{1}$, Run Yang ${ }^{3}$, Xianggang $\mathrm{Qiu}^{3}$, Genfu Chen ${ }^{3}$, $\mathrm{Ni} \mathrm{Ni}^{2}$, Stuart Trugman', Jian-xin Zhu', Antionette Taylor', Dmitry Yarotski', Rohit P. Prasankumar'; ${ }^{1}$ Los Alamos National Lab, USA; ${ }^{2}$ Univ. of California, Los Angeles, USA Minor Outlying Islands; ${ }^{3}$ IOP CAS, China. Ultrafast optical spectroscopy reveals surface-bulk scattering in the Weyl semimetal NbAs within 50 femtoseconds. The direction of this scattering can be controlled by the pump and probe polarizations, suggesting potential ultrafast device applications.

FF1F.5 • 09:00

Pressure-Induced Metallization in $\mathrm{VO}_{2}$ Studied by Optical Pump - THz Probe Spectroscopy, Johannes M. Braun ${ }^{1,2}$, Harald Schneider ${ }^{1}$, Manfred Helm,2, Rafal Mirek ${ }^{3}$, Lynn A. Boatner ${ }^{4}$, Robert E. Marvel ${ }^{5}$, Richard F. Haglund ${ }^{5}$, Alexej Pashkin'; '1 Inst. of Ion Beam Physics and Materials Research, Helmholtz-Zentrum Dresden-Rossendorf, Germany; ${ }^{2}$ Technische Universität Dresden, Germany; ${ }^{3}$ Univ. of Warsaw, Poland; ${ }^{4}$ Materials Science and Technology Division, Oak Ridge National Lab, USA; ${ }^{5}$ Dept. of Physics and Astronomy, Vanderbilt Univ., USA. We have investigated pressurized $\mathrm{VO}_{2}$ using optical pump - $\mathrm{THz}$ probe spectroscopy. Distinct pump-probe signals and an excitation threshold are observed even in the metallic state. Our results are consistent with a pressure-driven Mott-Hubbard transition.

\section{FF1F.6 • 09:15}

Probing the Phase Transition in $\mathrm{VO}_{2}$ Using Few-Cycle, Few-Femtosecond Pulses, Mina Bionta ${ }^{1}$, Vincent Wanie ${ }^{1}$, Philippe Lassonde ${ }^{1}$, Vincent Gruson 1,2, Dominic Lepage', Jeremie Chaillou', Mohamed Chaker', Francois Legare': 'I/NRS-Energie Materiaux et Telecom, Canada; ${ }^{2}$ Dept. of Physics, The Ohio State Univ., USA. We demonstrate in $\mathrm{VO}_{2}$, a nearly instantaneous band gap collapse which initiates a phase transition using few-cycle, few-femtosecond laser pulses to measure the change in the optical transmission of the sample in a pump-probe configuration.

\section{FF1G • Nanoparticle Mediated Emission and Field Enhancement-Continued \\ FF1G.4 08:45 Invited}

Strangely Shaped Plasmonic Nanoparticles and Luminescence, Thomas A. Klar'; ' 'Johannes Kepler Univ. Linz, Austria. The fundamentals how pyramid-, star- and sponge-shaped gold nanoparticles influence extrinsic and intrinsic luminescence, are discussed. Applications comprise random lasers and organic light emitting diodes.
FF1G.5 • 09:15

Multi-Resonant Optical Nanocavities with Continuous Spectral Tunability by Metaldielectric Multilayer Engineering, Wei Zhou'; ' Virginia Tech, USA. We demonstrated and studied a new type of multi-resonant optical nanocavities with ultra-small mode volume. By geometric engineering of metaldielectric multilayers, the total number of resonances and their spectral peaks can be tuned continuously.
FF1E.6 0 09:30

High-Efficiency, Low Noise UV Superconducting Nanowire Single-Photon Detectors Operating Above 4 K, Emma E. Wollman', Varun Verma ${ }^{2}$, Ryan Briggs ${ }^{1}$, Andrew Beyer ${ }^{1}$, Richard Mirin², Sae Woo Nam², Francesco Marsilii, Matthew Shaw'; ${ }^{1}$ Jet Propulsion Lab, USA; ${ }^{2}$ National Inst. of Standards and Technology, USA. We have demonstrated 80\% detection efficiency of $370 \mathrm{~nm}$ photons using superconducting nanowire single-photon detectors (SNSPDs) operating at $4.2 \mathrm{~K}$, with system dark count rates below 1 count/s. Prospects for detecting shorter wavelengths are discussed.
FF1F.7 • 09:30

$\mathrm{THz}$ Nonlinear Response of Landau-Quantized Graphene, Jacob C. König-Otto ${ }^{1,2}$, Yongrui Wang ${ }^{3}$, Alexey Belyanin ${ }^{3}$, Alexej Pashkin ${ }^{1}$, Harald Schneider ${ }^{1}$, Manfred Helm ${ }^{1,2}$, Stephan Winnerl'; 'Helmholtz Zentrum Dresden-Rossendorf, Germany; ${ }^{2}$ Technische Universität Dresden, Germany; ${ }^{3}$ Texas A\&M Univ., USA. The third-order nonlinear susceptibility of Landau-quantized graphene is studied by degenerate time-integrated fourwave mixing in the $\mathrm{THz}$ regime. The revealed resonance behavior and the observed field dependencies are in agreement with our theoretical calculations.
FF1G.6 0 09:30

Magneto-optics enhanced by Mie resonances, Maria G. Barsukova', Alexander S. Shorokhov', Alexander Musorin' ${ }^{1}$ Maxim Shcherbakov', Dragomir Neshev², Yuri Kivshar', Andrey Fedyanin'; 'Lomonosov Moscow State Univ., Russia; ${ }^{2}$ The AustraIian National Univ., Australia. We study experimentally and numerically magnetooptic effects in hybrid Ni/Si metasurfaces and demonstrate a multifold enhancement of the magneto-optical response due to the magnetic dipole Mie resonance of high-index nanoparticles. 


$\begin{array}{ccc}\begin{array}{c}\text { Executive Ballroom } \\ 210 \mathrm{H}\end{array} & \text { Meeting Room } & \text { Meeting Room } \\ 211 \mathrm{~B} / \mathrm{D} & 212 \mathrm{~A} / \mathrm{C}\end{array}$

CLEO: Science \& Innovations

$\mathrm{SF} 1 \mathrm{H} \bullet$ Waveguides and Ring Resonators-Continued

\section{SF1H.4 $08: 45$}

Integrated polarization beam-splitter with $116 \mathrm{THz}$ bandwidth via topographically anisotropic photonics, Jeff Chiles' Tracy Sjaardema', Ashutosh Rao', Sasan Fathpour': ' $U$ niv. of Central Florida, USA. Topographically anisotropic integrated photonics is proposed for extremely broadband polarization-selective devices. Polarization beam-splitting with an unprecedented 116 $\mathrm{THz}$ of bandwidth (0.52 octaves), insertion losses $<1.2 \mathrm{~dB}$ and extinction ratio $>16 \mathrm{~dB}$ is experimentally demonstrated.

\section{SF1H.5 • 09:00}

An integrated high-extinction-ratio lowloss polarization rotator for silicon photonics across $\mathrm{C}+\mathrm{L}$ bands, Andrew P. Li ${ }^{1}$, Xuan Cui ${ }^{1}$, Yongnan Li ${ }^{1}$, Mingbin Yu ${ }^{2}$, Dim-Lee Kwong ${ }^{2}$, Cheewei Wong ${ }^{1} ;{ }^{1}$ Mesoscopic Opitcs and Quantum Electronics Lab, Univ. of California, Los Angeles, USA; ${ }^{2}$ Inst. of Microelectronics, Singapore. We report a integrated silicon polarization rotator, with dual-level aligned fabrication. The rotator has record high 25-dB extinction, low 2-dB loss, with near-perfect rotation of $90.03 \pm 8.85$ degrees across 100 -nm wavelengths for chipscale polarization diversity.

SF1H.6 09:15

Observation of Synchronization in Air-slot Photonic Crystal Optomechanical Oscillator, Yongjun Huang ${ }^{1,2}$, Jiagui $\mathrm{Wu}^{2,3}$, Jaime G. Flores ${ }^{2}$, Mingbin Yu ${ }^{4}$, Dim-Lee Kwong ${ }^{4}$ Guangjun Wen', Chee Wei Wong'; ' ${ }^{1}$ School of Communication and Information Engineering, Univ. of Electronic Science and Technology of China, China; ' $U n i v$. of California, Los Angeles, USA: ${ }^{3}$ College of Electronic and Information Engineering, Southwest Univ. China; ${ }^{4}$ Inst. of Microelectronics, Singapore. Synchronization between two very close mechanical modes in air-slot $\mathrm{PhC}$ optomechanical oscillators is observed with drive powers above threshold. Improvement in phase noise $(-70 \mathrm{dBc} / \mathrm{Hz}$ at $10 \mathrm{kHz}$ offset) for the synchronized $\mathrm{OMO}$ is reported.

SF1H.7 • 09:30

A 2/3-Octave-Spanning Three Spectral Band Splitter on a Si3N4 Photonic Integrated Circuit Platform, Tiehui Su', Siwei $\mathrm{Li}^{1}$, Shaogi Feng ${ }^{1}$, Weicheng Lai ${ }^{1}$, Guangyao Liu', S. J. Ben Yoo'; ${ }^{1}$ Univ. of California Davis, USA. We show a three spectral band splitte that separates the wavelength bands of $1292 \mathrm{~nm}, 1550 \mathrm{~nm}$ and $1937 \mathrm{~nm}$. The device is fabricated using silicon nitride photonic integrated circuit platform. Optical characterization shows $<3 \mathrm{~dB}$ loss for the three channels, and $11 \sim 30 \mathrm{~dB}$ inter-channel crosstalk.

\section{SF1I - Integrated Photonic \\ Devices-Continued}

\section{SF1I.2 • 09:00}

Perfect Vertical Grating Coupler with Directionality of $97 \%$ on a Standard SOI Platform, Tatsuhiko Watanabe ${ }^{1,2}$, Masafumi Ayata ${ }^{1}$, Ueli Koch ${ }^{1}$, Yuriy Fedoryshyn ${ }^{1}$, Juerg Leuthold'; 'Inst. of Electromagnetic Fields, ETH Zurich, Switzerland; ${ }^{2}$ Yokohama National Univ. , Japan. A grating coupler with perfect vertical coupling and a high directionality is demonstrated using a standard $220 \mathrm{~nm}$ silicon-on-insulator wafer. The experimental coupling loss with a single-mode fiber is $-2.6 \mathrm{~dB}$ at the peak wavelength.

\section{SF1I.3 • 09:15}

High Resolution Silicon Arrayed Waveguide Gratings for Photonic Signal Processing Applications, Michael R. Gehl', Douglas Trotter $^{1}$, Andrew Starbuck ${ }^{1}$, Andrew Pomerene ${ }^{1}$, Anthony Lentine ${ }^{1}$, Christopher DeRose"; 'Sandia National Labs, USA. We design, fabricate and demonstrate the operation of a compact, $1 \mathrm{GHz}$ resolution silicon arrayed waveguide grating. Active phase correction allows for low channel cross-talk, enabling the demonstration of spectral shaping and RF signal analysis.

\section{SF11.4 0 09:30}

Silicon Photonic Polarization Insensitive Filter with Low Polarization Dependent Extinction Radio, Tingge Dai ${ }^{1}$, Gencheng Wang', Yuehai Wang', Yubo Li ${ }^{1}$, Hui Yu', Xiaoqing Jiang', Jianyi Yang'; 'Zhejiang Univ., China. We proposed and experimentally demonstrated a novel polarization-insensitive bandstop filter with a low polarization dependent extinction radio $(<1 \mathrm{~dB})$ and low polarization dependent loss $(<1.5 \mathrm{~dB})$ on a $\mathrm{SOI}$ chip. The insertion loss is about $2.6 \mathrm{~dB}$.

\section{SF1J $\bullet$ Micro- and Nanophotonic Devices- Continued}

SF1J.4 $\bullet$ 08:45

Grating-assisted counter-directional resonators for on-chip mode conversion, Jordan Davis', Andrew Grieco', Mário C. Souza ${ }^{2,1}$, Yeshaiahu Fainman'; 'Univ. of California San Diego, USA; ${ }^{2}$ Universidade Estadual de Campinas, Brazil. We demonstrate a multimode device simultaneously resonant at the 1 st and 2 nd order modes of adjacent silicon waveguides. This device introduces design flexibility and represents an interesting alternative to traditional mode conversion devices.

\section{SF1J.5 • 09:00}

Resonance-Free Light Recycling in Waveguides, You-Chia Chang', Samantha P. Roberts ${ }^{1}$, Brian Stern ${ }^{1}$, Ipshita Datta' ${ }^{1}$ Michal Lipson ${ }^{1}{ }^{1}$ Columbia Univ., USA. We demonstrate an approach based on mode conversion to recycle light within a waveguide without relying on resonance. The broadband approach enables a seven fold increase in the optical path of compact integrated interferometers.

\section{SF1J.6 $\bullet$ 09:15}

Cylindrical Polymer Optical Waveguides with Polarization Independent Performance, Aleksandrs Marinins ${ }^{1}$, Oskars Ozolins $^{2}$, Xiaodan Pang ${ }^{2}$, Aleksejs Udalcovs², Jaime Rodrigo Navarro ${ }^{2}$, Aditya Kakkar ${ }^{1}$ Richard Schatz ${ }^{1}$, Gunnar Jacobsen ${ }^{2}$, Sergei Popov'; ' KTH, Sweden; ${ }^{1}$ Netlab, Acreo AB, Sweden. Heating of poly(methyl methacrylate) ridge optical waveguides slightly above glass transition temperature minimizes surface roughness and provides cylindrical shape. We experimentally demonstrate propagation loss decrease and polarization insensitivity as a result of waveguide thermal treatment.

SF1J.7 • 09:30

Large Bandwidth Silicon Nitride Spot-Size Converter for Efficient Supercontinuum Coupling to Chalcogenide Waveguide, Jean-Etienne Tremblay ${ }^{1}$, Yung-Hsiang Lin ${ }^{1}$, Po-Kai Hsu', Marcin Malinowski², Spencer Novak², Pengfei Qiao', Guillermo F. Camacho-Gonzalez ${ }^{2}$, Connie J. Chang-Hasnain ${ }^{1}$, Kathleen Richardson ${ }^{2}$, Sasan Fathpour ${ }^{2}$, Ming C. Wu'; ' Univ. of California, Berkeley, USA; ${ }^{2} \mathrm{CREOL}$, The College of Optics and Photonics, Univ. of Central Florida, USA. Silicon nitride spot-size converters were fabricated in a damascene process to improve the performance of supercontinuum generation in arsenic-free chalcogenide waveguides. Supercontinuum generation covering $950-1750$ $\mathrm{nm}$ with $200 \mathrm{pJ}$ pulses is demonstrated. 
CLEO: Science \& Innovations

Design update and recent results of the Apollon 10 PW facility, Bruno J. Le Garrec $^{1}$, Dimitris N. Papadopoulos ${ }^{1}$, Catherine Le Blanc' ${ }^{1}$, Ji-Ping Zou' ${ }^{1}$, Antoine Freneaux ${ }^{1}$, Luc Martin', Nathalie Lebas' ${ }^{1}$ Audrey Beluze ${ }^{1}$, Francois Mathieu', Patrick Audebert', Gilles Cheriaux', Patrick Georges², Frederic Druon²; 'LULI-Ecole Polytechnique, France; ${ }^{2}$ OOGS, France. The Apollon 10-PW facility, currently under construction in France, is delivering $30 \mathrm{~J}$ before compression at a repetition rate of 1 shot per minute and we are currently increasing to get $100 \mathrm{~J}$

\section{SF1K.4 $\bullet$ 09:00}

Development of High Power Glass Laser systems in NLHPLP, Jianqiang Zhu', Jian Zhu', Xuechun Li', Baogiang Zhu', Weixin $\mathrm{Ma}^{1}$, Dean Liu', Cheng Liu', Guowen Zhang', Zunqi Lin'; 'Shanghai Inst of Optics and Fine Mech, China. A new laser facility with 30kJ/ ns/3w(8 beams) output energy has begun operation with good performance. Another single laser prototype pushes $1 \omega$ output energy to $17.5 \mathrm{~kJ} / 21 \mathrm{~ns}$ in $350 \mathrm{~mm} \times 350 \mathrm{~mm}$ aperture with four-pass main amplifier architecture.

SF1K.5 • 09:15

The CERN/ISOLDE Laser Ion Source, Bruce Marsh ${ }^{1}$, Valentin Fedosseev ${ }^{1}$, Katerina Chrysalidis ${ }^{1,2}$, Thomas Day Goodacre', Pierre B. Larmonier ${ }^{1}$, Ralf E. Rossel', Sebastian Rothe $^{1}$, Christoph Seiffert ${ }^{1}$, Klaus Wendt ${ }^{2}$; ${ }^{1}$ CERN, Switzerland; ${ }^{2}$ Institut für Physik, Johannes Gutenberg-Universität, Germany. Laser resonance photo-ionization is an essential aspect of radioactive ion beam production for fundamental and applied physics research. The CERN/ISOLDE laser ion source, described here, is the most versatile of its type worldwide.

\section{SF1L • Data Center \\ Communications-Continued}

SF1L.4 $\bullet$ 08:45

DSP Equalization-free Data Center Communication with High Dispersion Tolerant Optical Duobinary-PAM4 Signal, Jhih-Heng Yan', Tzu-Yu Yeh', Yen-Hsiang Chang', YiChen Wu', Kai-Ming Feng': 'National Tsing Hua Univ., Taiwan. We propose and experimentally evaluate the dispersion tolerance of a data center communication system with DSP equalization free optical duobinaryPAM4. Without dispersion compensation, its dispersion tolerance is three times higher than DFE equalized PAM4.

\section{SF1L.5 • 09:00}

Wavelength-Controlled Beam Steering for Optical Wireless Transmission Using an In-Fiber Diffraction Grating, Guoging Wang', Usman Habib' ${ }^{1}$ Chao Wang ${ }^{1}$, Nathan J. Gomes ${ }^{1}$, Zhijun Yan ${ }^{3,2}$, Lin Zhang ${ }^{2} ;{ }^{1}$ Univ. of Kent at Canterbury, UK; ${ }^{2}$ Aston Univresity, UK; ${ }^{3}$ Huazhong Univ. of Science and Technology, China. Passive beam steering for optical wireless transmission based on wavelength tuning using a novel in-fiber diffraction grating featuring compactness, high diffraction efficiency and inherent fiber-compatibility, is proposed and experimentally demonstrated for the first time.

SF1L.6 • 09:15

Interplay of Bit Rate, Linewidth, and Reach on DMT vs. PAM Performance, Aminreza Yekani Khoei', Leslie A. Rusch'; 'Université Laval, Canada. We theoretically study the effect of system bandwidth, bit rate, laser linewidth and fiber length on DMT and PAM. DMT is optimized in terms of signal-tocarrier- power ratio and the performance of the optimal DMT configuration is compared to that of PAM.
SF1M - Aerosol and Gas Sensing-Continued

\section{SF1M.4 • 09:00}

Quartz Enhanced Photoacoustic Spectroscopy for Human Breath Analysis, Mikael Lassen', Laurent Lamar ${ }^{2}$, Poul Feng ${ }^{3}$, Andre Peremans ${ }^{2}$, Jan C. Petersen'; 'Danish Fundamental Metrology, Denmark; ${ }^{2}$ Laserspec BVBA, Belgium; ${ }^{3}$ COPAC A/S, Denmark. Simultaneous monitoring of several trace gases requires source tunability and highly sensitive detection. To serve this purpose a MIR-OPO light source pumping a quartzenhanced photoacoustic sensor is demonstrated. The sensor targets exhaled human breath analysis.

SF1M.5 • 09:15

Online Gas Monitoring with Mid-Infrared Optical Parametric Oscillator Based Dual-Comb Spectrometer, Julien Mandon', Simona M. Cristescu', Frans J. Harren'; ' Radboud Universiteit Nijmegen, Netherlands. A dual-comb spectrometer in the 3-5 $\mu \mathrm{m}$ spectral region is used for online gas monitoring. The spectrometer is combined with a 37-m absorption cell to reach a minimal absorption coefficient of $1.1 \times 10^{-7} \mathrm{~cm}^{-1}$.
SF1K.6 • 09:30

Scaling of X-ray Flux from High-Intensity Laser-Solid Interactions as a Function of Energy, Dean R. Rusby ${ }^{1,2}$, Ceri Brenner ${ }^{2}$, Chris Armstrong ${ }^{1,2}$, Lucy Wilson ${ }^{2}$, Rob Clarke ${ }^{2}$, Aaron Alejo ${ }^{3}$, Robert Deas ${ }^{4}$, Paul McKenna', Satya $\mathrm{Kar}^{3}$, David Neely2; 'Strathclyde Univ., UK; ${ }^{2}$ Central Laser Facility, UK; ${ }^{3}$ Queens Univ. Belfast, UK; ${ }^{4}$ Security Sciences Dept., DSTL, UK. The bremsstrahlung $x$-rays from a laser-solid interaction have been investigated for the use of radiography. The scaling of the $\mathrm{x}$-rays as a function of energy has been characterized and modelled and agrees with previous measurements.
SF1M.6 • 09:30

Multiheterodyne Spectroscopy with Interband Cascade Lasers, Jonas Westberg ${ }^{1}$ Lukasz A. Sterczewski ${ }^{1,4}$, Link Patrick ${ }^{1}$, Chul Soo Kim², Mijin Kim³, Chadwick L. Canedy², William W. Bewley ${ }^{2}$, Charles Merritt' ${ }^{2}$, Igor Vurgaftman², Jerry R. Meyer'2, Gerard Wysocki'; 'Princeton Univ., USA; ${ }^{2}$ U.S. Naval Research Lab, USA; ${ }^{3}$ Sotera Defense Solutions, Inc., USA; ${ }^{4}$ Faculty of Electronics, Wroclaw Univ. of Science and Technology, Poland. A multiheterodyne spectroscopy system based on Fabry-Pérot interband cascade lasers is demonstrated for broadband spectroscopic assessments of gaseous methane. The spectrometer is capable of $\sim 240 \mathrm{GHz}$ of spectral coverage around $3.21 \mu \mathrm{m}$. 
Executive Ballroom

$210 \mathrm{~A}$

Executive Ballroom

210B

\section{CLEO: Applications}

\& Technology

AF1A • A\&T Topical Review on Supercontinuum and Applications I-Continued
AF1B - Application \& Advances of Frequency CombsContinued

AF1B.7 • 09:45

Comparative study of the reflectometry and cut-back techniques for the distributed measurement of supercontinuum generation along optical fibers, Régis D. Hontinfinde ${ }^{1}$, Saliya Coulibaly², Patrice Megret ${ }^{1}$ Majid Taki², Marc Wuilpart'; 'Univ. of Mons, Belgium; ${ }^{2}$ PhLAM, Université de Lille, France. We propose a non-destructive measurement technique for the distributed measurement of supercontinuum generation in fibers. For validation purposes, we compare our results with those obtained thanks to the cut-back technique. A good agreement was observed.
Executive Ballroom $210 \mathrm{C}$

\section{CLEO: Science \&} Innovations

SF1C $\bullet$ Frequency Comb Technology-Continued

SF1C.8 • 09:45

Modal Approach Towards Complete Characterization of Frequency Comb Noise, Syamsundar $\mathrm{De}^{1}$, Valérian Thiel ${ }^{1,2}$, Jonathan Roslund', Nicolas Treps'; ; ${ }^{1}$ Laboratoire Kastler Brossel „UPMC-Sorborne Université, France ${ }^{2}$ Claredon Lab, Dept. of Physics, Univ. of Oxford, UK. The fluctuations of the global parameters of an ultrafast frequency comb are analyzed using a novel measurement scheme and a modal representation. The propagation of excess noise added to the pump is also investigated.
Executive Ballroom 210D

\section{Joint}

JF1D • Symposium on Thermal Noise in Precision Interferometry l-Continued
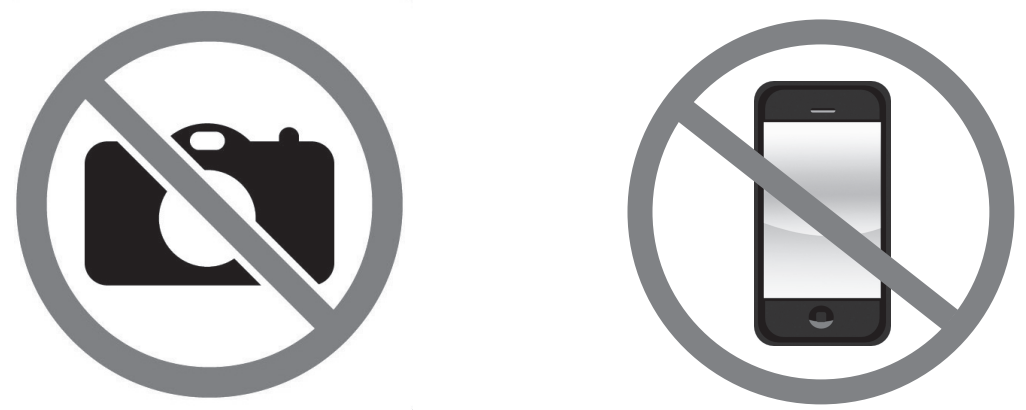


\section{CLEO: QELS-Fundamental Science}

FF1E • Single-Photon

Detectors-Continued

FF1E.7 • 09:45

High-Operating-Temperature Superconducting Nanowire Single Photon Detectors Based on Magnesium Diboride, Ange Velasco ${ }^{1}$, Daniel P. Cunnane ${ }^{1}$, Simone Frasca ${ }^{2}$

Thomas Melbourne ${ }^{3}$, Narendra Acharya ${ }^{3}$ Ryan Briggs ${ }^{1}$, Andrew Beyer ${ }^{1}$, Matthew Shaw ${ }^{1}$, Boris Karasik', Matthaus Wolak ${ }^{3}$, Varun Verma ${ }^{4}$ Adriana Lita ${ }^{4}$ Hiroyuki Shibata ${ }^{5,6}$ Masataka Ohkubo ${ }^{7}$, Nobuyuki Zen ${ }^{7}$, Masahiro Ukibe $^{7}$, Xiaoxing $\mathrm{Xi}^{3}$, Francesco Marsili'i' ${ }^{1}$ Jet Propulsion Lab, USA; ${ }^{2}$ Univ. of Pisa, Italy; ${ }^{3}$ Temple Univ., USA: ${ }^{4}$ National Inst. of Standards and Technology, USA; ${ }^{5}$ Kitami Inst. of Technology, Japan; ${ }^{6}$ NTT Basic Research Lab Japan: ${ }^{7}$ National Inst. of Advanced Industria Science and Technology, Japan. We report on optically sensitive $15 \mathrm{~nm}$ thick, $100 \mathrm{~nm}$ wide $\mathrm{MgB}_{2}$ nanowires in the operating-temperature range $4-11 \mathrm{~K}$.
FF1F • Optical \& THZ Spectroscopy of Quantum Matter-Continued

FF1F.8 • 09:45

Nonlinear polaron dynamics in colossal magnetoresistance manganites driven by intense THz pulses, Pamela R. Bowlan', Mostafa Shalaby², Stuart Trugman², Aiping Chen ${ }^{1}$, Q.X. Jia ${ }^{1}$, C. Vicario ${ }^{2}$, Antionette Taylor', Dmitry Yarotski', Christoph P. Hauri2,3, Rohit P. Prasankumar'; ; Los Alamos National Lab, USA: ${ }^{2}$ Paul Scherrer Inst., Switzerland; ${ }^{3}$ École Polytechnique Fédérale de Lausanne, Switzerland. Using intense, few-cycle $\mathrm{THz}$ pulses we investigate the strong-field interaction with polarons in $\mathrm{La}_{07} \mathrm{Ca}_{3} \mathrm{MnO}_{3}$. By probing the optical reflectivity, we observe a $\mathrm{THz}$-induced detrapping of electrons from polarons, followed by thermalization of phonons with spins.
FF1G • Nanoparticle Mediated Emission and Field Enhancement-Continued

FF1G.7 • 09:45

Low-loss plasmonics via dielectric nanoparticles on metallic films, Yi Yang ${ }^{1}$, Owen Miller $^{2}$, Thomas Christensen', John Joannopoulos ${ }^{1}$, Marin Soljacic ${ }^{1} ;{ }^{1}$ MIT, USA; ${ }^{2}$ Yale Univ., USA. We theoretically propose a pathway to low-loss plasmonics. We show that dielectric-on-metal nanoresonators scatter more strongly than is possible in all-metal or all-dielectric approaches, offer near-unity-efficiency spontaneous-emission enhancements, and are robust to quantum corrections.

10:00-10:30 Coffee Break, Concourse Level 
Executive Ballroom $210 \mathrm{H}$
Meeting Room

$211 \mathrm{~B} / \mathrm{D}$
Meeting Room

$212 \mathrm{~A} / \mathrm{C}$

\section{CLEO: Science \& Innovations}

SF1H $\bullet$ Waveguides and Ring Resonators-Continued

SF1H.8 $\bullet$ 09:45

Monolithic integration of vertical $\mathrm{SiN}_{\mathrm{x}}$ microrings on a ridge waveguide to achieve multi-channel photonic coupling, Xin $\mathrm{Yu}^{1}$, Lynford Goddard ${ }^{1}$, Xiuling Li ${ }^{1}$, Xiaogang Chen': 'Univ of Illinois at Urbana-Champaign, USA. Multi-channel vertical photonic coupling was observed, by integrating two different $\mathrm{SiN}_{\mathrm{x}}$ vertical microring couplers $(\mathrm{V} \mu \mathrm{RC})$ monolithically on a single ridge waveguide. This work represents a critical step to $3 D$ photonic integration using $V \mu R C^{\prime}$ 's.
SF1I • Integrated Photonic Devices-Continued

SF1I.5 • 09:45

Polarization Independent Adiabatic 3-dB Coupler for Silicon-on-Insulator, Luhua $\mathrm{Xu}^{1}$, Yun Wang ${ }^{1}$, David Patel', Eslam El-Fiky', Zhenping Xing ${ }^{1}$, Rui Li ${ }^{1}$, Md. Ghulam Saber ${ }^{1}$ Maxime Jacques', David V. Plant'; 'McGill Univ., Canada. We demonstrate a polarization independent adiabatic 3-dB coupler for the siliconon-on-insulator platform, with a measured bandwidth of $100 \mathrm{~nm}$ and power splitting ratios of $3 \pm 0.7 \mathrm{~dB}$ for both the transverse electric and transverse magnetic modes.
SF1J $\bullet$ Micro- and Nanophotonic DevicesContinued

\section{SF1J.8 • 09:45}

Inverse Design of an Ultra-Compact Mode (De)multiplexer Based on Subwavelength Structure, Weijie Chang', Minming Zhang', Luluzi Lu', Feiya Zhou', Dongyu Li', Zepeng Pan', Deming Liu'; ${ }^{1}$ School of Optical and Electrical Information, Huazhong Univ. of Science and Technology, China. A novel ultra-compact mode (de)multiplexer using inverse design method is proposed and experimentally demonstrated with low crosstalk $<25 \mathrm{~dB}$ and a footprint of only $2.4 \times 2.4 \mu \mathrm{m}^{2}$, fabricated by only one-step etching. 
CLEO: Science \& Innovations

SF1K • Laser Facilities and Applications-Continued

SF1K.7 • 09:45

Velocity Map Imaging for Photocathode Characterization, Hong $\mathrm{Ye}^{1,2}$, Sebastian $\mathrm{H}$.

Trippel ${ }^{1,3}$, Michele Di Fraia', Arya Fallahi',

Oliver D. Mücke ${ }^{1,3}$, Jochen Küpper ${ }^{1,2}$, Franz

X. Kärtner ${ }^{1,2},{ }^{1}$ Center for Free-Electron Laser

Science, Deutsches Elektronen-Synchrotron

Germany; ${ }^{2}$ Dept. of Physics, Univ. of Ham-

burg, Germany: ${ }^{3}$ The Hamburg Center for

Ultrafast Imaging, Univ. of Hamburg, Ger-

many. A velocity map imaging spectrometer

(VMI) to characterize the transverse phase

space of electron emission from solid sur-

faces is described. A first instrument test is

presented using multi-photon emission from

a planar Au surface.
SF1L • Data Center

Communications-Continued
SF1M - Aerosol and Gas Sensing-Continued
SF1M.7 • 09:45

Evanescent-Wave Gas Sensing with DualComb Spectroscopy, Zaijun Chen ${ }^{1,2}$, Ming Yan ${ }^{1,2}$, Theodor W. Hänsch ${ }^{1,2}$, Nathalie Picque $^{1,2} ;{ }^{1}$ Max-Planck Inst. of quantum optics, Germany; ${ }^{2}$ Physics Dept., Ludwig Maximilian Univ. of Munich, Germany. Attenuated total reflection spectroscopy with tapered fibers is combined to coherent multiheterodyne spectroscopy in the near-infrared region. Evanescent sensing is extended to broadband high-resolution spectroscopy in the gas phase at high signal-to-noise ratio. 
Executive Ballroom 210A

Executive Ballroom 210B

\section{CLEO: Applications \& Technology}

10:30-12:30

AF2A - A\&T Topical Review on Supercontinuum and Applications II

Presider: Robert Fisher; RA Fisher Associates LLC, USA

\section{AF2A.1 • 10:30 Invited}

Supercontinuum Sources - Past, Present Any Future?, J. R. Taylor': 'Physics, Imperial College London, UK. For nearly fifty years the supercontinuum source, a result of the understanding and control of the underlying physical processes, has evolved as a scientific and commercial success, providing spectral versatility well beyond the limitations of the transmission window of silica and pumped by diverse temporal formats. The relevant physical processes, characteristics and current status are reviewed with a look to future development and application.

10:30-12:30 Colorado at Boulder, USA

\section{AF2B.1 • 10:30 Invited} quantification of gas plumes.

\section{AF2A.2 • 11:00 Invited}

Ultrahigh Resolution Optical Coherence Tomography Using Supercontinuum and Their Wavelength Dependence, Norihiko Nishizawa1, Hiroyuki Kawagoe1, Masahito Yamanaka'; ${ }^{1}$ Nagoya Univ., Japan. Supercontinuum is useful light source for ultrahigh resolution optical coherence tomography (OCT) imaging. In this talk, the recent investigations about ultrahigh resolution $\mathrm{OCT}$ imaging using supercontinuum and their wavelength dependence are reviewed.
AF2B - Applied Spectroscopy

Presider: Gregory Rieker; Univ. of

Gas Mapping LiDAR for Large-area Leak Detection and Emissions Monitoring Applications, Michael Thorpe'; ${ }^{1}$ Bridger Photonics, Inc, USA. We present gas mapping LiDAR that combines coherent ranging and pathintegrated gas concentration measurements. Spatial scanning of the LiDAR beam produces high-quality, geo-registered imagery of terrestrial scenes for precise localization and

AF2B.2 $\bullet 11: 00$

Multi-species Trace Gas Analysis with Dual-wavelength DFB-QCLs, Morten Hundt ${ }^{1}$, Mehran Shamohammadi², Filippos Kapsalidis², Béla Tuzson? ${ }^{1}$ Chang Liư Philipp Scheidegger ${ }^{1}$, Martin Süess ${ }^{2}$, Herbert Looser ${ }^{1,3}$, Jérôme Faist ${ }^{2}$, Lukas Emmenegger ${ }^{1} ;{ }^{1}$ Empa, Switzerland; ${ }^{2}$ ETH Zürich, Switzerland; ${ }^{3} \mathrm{FHNW}$, Switzerland. We evaluate two designs of dual-wavelength QCLs for high-resolution multi-species laser absorption spectrometers. Several room-temperature dual QCLs were combined to measure concentrations of the most important pollutants and greenhouse gases in a compact instrument with state-of-the-art precision.

AF2B.3 • 11:15

Using a Sagnac Fourier Spectrometer for Laser-Induced Breakdown Spectroscopy, Matthias Lenzner ${ }^{1}$, Ali Rastegari ${ }^{2}$ Jean-Claude Diels ${ }^{2} ;{ }^{1}$ Lenzner Research LLC, USA; ${ }^{2}$ CHTM, Univ. of New Mexico, USA. A new type of interferometric spectrograph is realized by placing a transmission grating into a Sagnac interferometer. Two diffracted orders propagate in opposite directions; the Fizeau interferogram at the output yields a heterodyned spectrum.
Executive Ballroom

$210 \mathrm{C}$

\section{CLEO: Science \& Innovations}

10:30-12:30

$\mathrm{SF} 2 \mathrm{C} \bullet$ Frequency Comb

Applications

Presider: Guanhao Wu; Tsinghua Univ., China

\section{SF2C.1 1 10:30 Tutorial}

Optical Frequency Comb Applications Beyond Frequency Metrology, Kaoru Minoshima1,2: 'Univ. of Electro-Communications, Japan; ${ }^{2}$ JST, ERATO MINOSHIMA Intelligent Optical Synthesizer, Japan. Optical frequency comb provides powerful tools in broad area not only in frequency metrology as "ultraprecise frequency ruler". In this tutorial, various new metrology applications by full use of the properties of combs are presented.

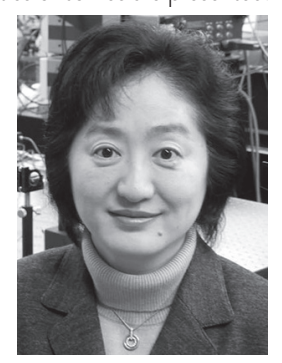

Kaoru Minoshima is a Professor at the University of Electro-Communications and the research director of ERATO MINOSHIMA Intelligent Optical Synthesizer (IOS) Project, JST. She has worked at the National Institute of Advanced Industrial Science and Technology (AIST), the University of Bordeaux and MIT. In 2011 she served as the Genera Co-Chair for CLEO. She is a fellow of The Optical Society. Her areas of research are frequency combs, ultrafast optical science and technology, and their application to optical metrology.
Executive Ballroom 210D

\section{Joint}

10:30-12:30

JF2D • Symposium on

Thermal Noise in Precision

Interferometry II

Presider: Steven Penn; Hobart and William Smith Colleges, USA

\section{JF2D.1 • 10:30 Invited}

Laser Frequency Stabilization for Ion Optical Clocks at NIST, David B. Hume', David R. Leibrandt'; 'NIST, USA. The lon Storage Group at NIST develops stable optical cavities, which enable our trapped-ion optical clocks. I will give an overview of these efforts and describe a new cryogenic cavity with significantly reduced thermal noise.

\section{JF2D.2 • 11:00 Invited}

Thermal Noise Reduction Techniques for High Precision Interferometric Measurements, Harald Lueck',2; 'Leibniz Universität Hannover, Germany; ${ }^{2}$ Laser Interferometry and Gravitational Wave Astronomy, MaxPlanck Inst. for Gravitational Physics, Ger many. An overview of some thermal noise reduction techniques (Khalili cavities and etalons, modified amorphous dielectric coatings, crystalline coatings, monolithic coatings, cooling etc.) will be given including those foreseen for the sub-SQL experiment in the AEl-10m-prototype interferometer facility in Hannover, Germany, where suspension thermal noise is mitigated by using monolithic fused-silica suspensions and coating thermal noise by using crystalline $\mathrm{AlGaAs}$ coatings with large beam spots. 


\section{CLEO: QELS-Fundamental Science}

10:30-12:30

FF2E • Quantum Technologies

Presider: Todd Pittman; Univ. of

Maryland Baltimore County, USA

FF2E.1 • 10:30

Directionally Unbiased Linear-Optical Multiports for Quantum Information Processing, Alexander V. Sergienko', David Simon ${ }^{2}{ }^{1}$, Casey Fitzpatrick'; ${ }^{1}$ Boston Univ., USA; ${ }^{2}$ Stonehill College, USA. The concept of directionally unbiased optical multiport is introduced, in which photons may reflect back out the input direction. It acts as universal qubit and Bell-state processor to implement probabilistic quantum gates.

FF2E.2 • 10:45

Single-Photon-Level Spatial-Mode-Selective Frequency Up-Conversion in a Multimode $\mathbf{X}^{(2)}$ Waveguide, Young Bong Kwon', Mohan Giribabu ${ }^{1,3}$, Carsten Langrock ${ }^{2}$, Martin M. Fejer ${ }^{2}$, Michael Vasilyev' ${ }^{1}{ }^{1}$ Univ. of Texas at Arlington, USA; ${ }^{2}$ Stanford Univ., USA $;{ }^{3}$ Corning Inc., USA. We experimentally demonstrate selective up-conversion of either $\mathrm{TM}_{00}$ or $\mathrm{TM}_{01}$ mode of a $1540 \mathrm{~nm}$ single-photon signal into $\mathrm{TM}_{01}$ mode at $775 \mathrm{~nm}$ with $>70 \%$ efficiency, better than $-10 \mathrm{~dB}$ crosstalk, and $>10^{2}$ signal-to-noise-photon ratio.

FF2E.3 • 11:00

Hong-Ou-Mandel Interference in the Frequency Domain, Chaitali Joshi ${ }^{1,2}$, Alessandro Farsi ${ }^{2}$, Alexander Gaeta2; ${ }^{1}$ Applied and Engineering Physics, Cornell Univ., USA; ${ }^{2}$ Applied Physics and Applied Mathematics, Columbia Univ., USA. We demonstrate the first observation of Hong-Ou-Mandel interference between two energy correlated single photons of different frequencies with a visibility of $0.68 \pm 0.03$, using Bragg-scattering four wave mixing as the active frequency-domain beamsplitter.

FF2E.4 • 11:15

Quantum Frequency Conversion: Into the Strong Coupling Regime, Zachary Vernon', Marco Liscidini ${ }^{2}$, John Sipe ${ }^{1} ;{ }^{1}$ Univ. of Toronto, Canada; ${ }^{2}$ Univ. of Pavia, Italy. We show that Rabi-like coherent oscillations should be observable in integrated microresonators used for quantum frequency conversion, revealing a new regime of strongly coupled photonic modes.
10:30-12:30

FF2F • Valley Coherence and

Polariton Dynamics in 2D

Materials

Presider: Chih-Wei Lai; US Army

Research Lab, USA

\section{FF2F.1 • 10:30 Invited}

Exciton-Polaritons in Atomically Thin Semiconductors and Their Heterostructures, Alexander Tartakovskii'; ' 1 Physics and Astronomy, Univ. of Sheffield, UK. Exciton-polaritons are observed in monolayer $\mathrm{MoSe}_{2}$ and $\mathrm{WSe}_{2}$ in optical microcavities. Relaxation of the valley pseudospin and valley coherence is inhibited by the exciton-photon coupling, the effect controlled by the exciton-cavitymode detuning.

\section{FF2F.2 • 11:00}

Room temperature Tamm-Plasmon ExcitonPolaritons in atomic monolayer, Nils Lundt ${ }^{1}$, Sebastian Klembt ${ }^{1}$, Sebastian Stoll ${ }^{1}$, Evgeniia Cherotchenko², Oliver Iff', Anton V. Nalitov², Martin Klaas ${ }^{1}$, Alexey Kavokin ${ }^{2}$, Sven Höfling ${ }^{1,3}$, Christian Schneider'; ${ }^{1}$ Univ. of Wuerzburg, Germany; ${ }^{2}$ Univ. of Southampton, UK; ${ }^{3}$ Univ. of St. Andrews, UK. We integrated $\mathrm{WSe}_{2}$ and $\mathrm{WS}_{2}$ monolayers into photonic Tamm-structures in order to observe Tammplasmon exciton-polaritons under ambient conditions. The characteristic dispersion observed by in-plane momentum-resolved micro-photoluminescence spectroscopy confirms their existence at room temperature.

FF2F.3 • 11:15

Ultrafast Photo-activation of Surface Polaritons in Black Phosphorus Heterostructures, Markus A. Huber ${ }^{1}$, Fabian Mooshammer ${ }^{1}$, Markus Plankl' ${ }^{1}$ Leonardo Viti², Fabian Sandner ${ }^{1}$, Lukas Z. Kastner ${ }^{1}$, Tobias Frank ${ }^{1}$, Jaroslav Fabian', Miriam S. Vitiello², Tyler L. Cocker'1, Rupert Huber'; ' 'Dept. of Physics, Univ. of Regensburg, Germany; ${ }^{2}$ NEST, CNR - Istituto Nanoscienze and Scuola Normale Superiore, Italy. Photo-activated surface plasmon polaritons in black phosphorus couple with surface phonon polaritons of $\mathrm{SiO}_{2}$ to form switchable hybrid modes. We resolve these modes in time, energy, and space with scattering-type scanning near-field multi-THz microscopy.
10:30-12:30

FF2G • Nanoscale Control of Quantum Emission

Presider: Sergey Kruk; Australian

National Univ, Australia

FF2G.1 • 10:30

Shaping UV Emission through Graphene Plasmons, Jamison M. Sloan', Nicholas Rivera', Ido Kaminer', Marin Soljacic'; 'MIT, USA. We demonstrate that combining Purcell-enhancement engineering, graphene plasmonics, and radiative cascade can result in a new type of UV emitter whose properties can tuned by electrically doping graphene.

\section{FF2G.2 • 10:45}

Enhanced light matter interactions in plasmonic- molecular gas hybrid system, Roy T. Zektzer ${ }^{1}$, Liron Stern ${ }^{1}$, Noa Mazurski ${ }^{1}$ Uriel Levy'; 'The Hebrew Univ. of Jerusalem, Israel. We demonstrate enhanced light matter interactions between surface plasmon and acetylene. Dispersion and absorption are controlled by the interplay between the molecular line and plasmonic resonance. Fano line shapes are observed, and applications are discussed.

\section{FF2G.3 • 11:00 Invited}

Chiral Nanophotonics and Quantum Optics, Arno Rauschenbeutel'; ' Atominstitut, Tu Wien, Austria. Tightly confined light fields exhibit an inherent link between their local polarization and their propagation direction. Their interaction with emitters therefore features chiral, i.e., propagation-directiondependent, effects which are interesting both conceptually and for quantum-photonic applications. 


\section{Executive Ballroom $210 \mathrm{H}$}

Meeting Room
$211 \mathrm{~B} / \mathrm{D}$

Meeting Room

\section{CLEO: Science \& Innovations}

10:30-12:30

SF2I - Detectors and Other

Novel Devices

Presider: Jian Wang; Huazhong

Univ of Science and Tech, China

SF2I.1 • 10:30

10-GHz 32-pixel 2-D photodetector array for advanced optical fiber communications,

Toshimasa Umezawa', Takahide Sakamoto', Kouichi Akahane', Atsushi Matsumoto', Atsushi Kanno ${ }^{1}$, Naokatsu Yamamoto', Tetsuya Kawanishi ${ }^{1}$, ; ${ }^{1}$ National Inst of Information \& Comm Tech, Japan; ${ }^{2}$ Waseda Univ., Japan. We studied a 10-GHz 32-pixel two-dimensional photodetector array for advanced optical fiber communications and LIDAR applications. Electromagnetic field simulation revealed the RF crosstalk problem between pixels. A fabricated high-speed photodetector array will be discussed.

\section{SF2I.2 • 10:45}

Electrically Tunable Photoresponse in a Graphene Heterostructure Photodetector, Dehui Zhang ${ }^{1}$, Gong Cheng ${ }^{2}$, Zhen $\mathrm{Xu}^{1}{ }^{12}$, CheHung Liu', Thomas E. Beechem³ ${ }^{3}$, Michael Goldflam ${ }^{3}$, David Peters ${ }^{3}$, Minmin Zhou ${ }^{1,4}$ Theodore Norris',2, Zhaohui Zhong ${ }^{1}$; 'Electrical Engineering and Computer Science, Univ. of Michigan, USA: ${ }^{2}$ Center for Photonics and Multiscale Nanomaterials, Univ. of Michigan, USA; ${ }^{3}$ Sandia National Labs, USA; ${ }^{4}$ School of Electronic Science and Engineering, Nanjing Univ., China. We report an electrically tunable photodetector design based on photogating effect in graphene heterostructures. We demonstrate electrically tunable photoresponse up to $60 \mathrm{~A} / \mathrm{W}$ with a sub-millisecond speed. The detector's spectra tunability will also be investigated.

\section{SF2I.3 • 11:00 Invited}

Low-voltage three-terminal avalanche photodiodes, Xiaoge Zeng ', Zhihong Huang ${ }^{1}$, Di Liang', Marco Fiorentino', Ray Beausoleil'; ${ }^{1}$ Hewlett Packard Labs, USA. We demonstrate a novel three-terminal avalanche photodiode detector with a measured breakdown voltage of only $-6 \mathrm{~V}$, a $3 \mathrm{~dB}$ bandwidth of $18.6 \mathrm{GHz}$, a $D C$ gain of 15.6 and an open eye diagram at $10 \mathrm{Gbps}$.
$10: 30-12: 30$

SF2J • Surface Emitting Lasers

Presider: Hongping Zhao, Case

Western Reserve Univ., USA

\section{SF2J.1 • 10:30 Invited}

Coherent Vertical Cavity Phased Microlaser Arrays, Kent D. Choquette', Stewart T. Fryslie', Zihe Gao', Bradley J. Thompson', Harshil Dave', Katherine Lakomy', P. S. Carney ${ }^{1}{ }^{1}$ Univ. of Illinois, USA. The characteristics and performance of implanted photonic crystal coherent vertical cavity laser arrays are reported. We discuss control of the coherence, phase, brightness, and beam steering, as well as achieving record small signal bandwidth for digital modulation.

\section{SF2J.2 $\bullet 11: 00$}

Investigation of Air-Hole Shapes for Direct Emission of Circularly-Polarized Beam from Photonic-Crystal Surface-Emitting Lasers, Masaya Nishimoto ${ }^{2,1}$, Kyohei Maekawa2, Susumu Noda ${ }^{2}{ }^{1}$ School of Engineering, The Univ. of Tokyo, Japan; ${ }^{2}$ Dept. of Electronic Science and Engineering, Kyoto Univ., Japan. We propose photonic-crystal surfaceemitting lasers with oblique-triangularprism-shaped air holes for direct emission of circularly polarized beam. High degree of circular polarization $\left(S_{3} / S_{0}>0.9\right)$ can be obtained by appropriate height and tilt angle of air holes.

\section{SF2J.3 • 11:15}

Lateral Size Scaling of Photonic Crystal Surface-Emitting Lasers on Si, Shih-Chia Liu', Deyin Zhao ${ }^{1}$, Hongjun Yang ${ }^{1}$, Carl Reuterskiöld-Hedlund², Mattias Hammar², Shanhui Fan ${ }^{4}$, Zhenqiang $\mathrm{Ma}^{3}$, Weidong Zhou'; 'Univ. of Texas at Arlington, USA; ${ }^{2} \mathrm{KTH}$-Royal Inst. of Technology, Sweden; ${ }^{3}$ Univ. of Wisconsin, USA; ${ }^{4}$ Stanford Univ., USA. We report here the lateral size scaling effect of the photonic crystal surface-emitting lasers (PCSELs) on silicon. Lateral and vertical confinement schemes were also investigated towards low threshold lasing of PCSELs with small lateral cavity sizes. 


\section{CLEO: Science \& Innovations}

10:30-12:30

SF2K • Infrared Laser Sources

Presider: Thomas Metzger;

TRUMPF Scientific Lasers GmbH, Germany

\section{SF2K.1 • 10:30 Tutorial}

Mid-IR Ultrafast Laser Technology for Science and Industry, Irina T. Sorokina ${ }^{1}$; ${ }^{1}$ Norges Teknisk Naturvitenskapelige Univ, Norway. The talk reviews fundamentals as well as recent advances in fiber based ultrafast midIR lasers and frequency combs, providing a flexible and robust fiber based technology platform to address demanding requirements set by the most advanced scientific and industrial applications, such as optogenetics, ultrasensitive molecular detection, nonlinear confocal microscopy, 3D-IR and multiphoton nonlinear spectroscopy, astrocombs, neurosurgery as well as fine material processing.

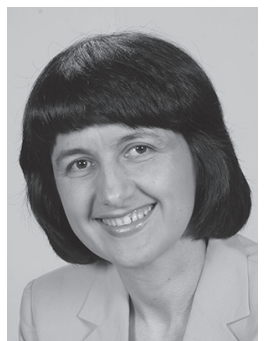

Irina T. Sorokina is a Professor of physics at the Norwegian University of Science and Technology, and a Co-founder and President of ATLA Lasers AS - a company producing industrial grade ultrafast tunable fiber-based lasers operating above $2 \mu \mathrm{m}$. Her over 20 years of research resulted in development and commercialization of the first femtosecond $\mathrm{Cr} 2+: \mathrm{ZnSe}$ laser ("Ti-sapphire of the infrared"). In 2004 she received the IEE Snell Premium award for her contributions to the development of broadly tunable and microchip $\mathrm{Cr}: \mathrm{ZnS}$ lasers and their applications. Sorokina is a Fellow of The Optical Society and an Elected Member of the Norwegian Academy of Science and Letters. She is an author of $>300$ scientific publications, 3 books, 4 patents and several book chapters.
10:30-12:15

SF2L • Advanced Fiber Devices and Concepts

Presider: Sze Set; Univ. of Tokyo, Japan

\section{SF2L. 1 • 10:30}

Programmable passive waveform amplifier based on temporal self-imaging effects,

Jinwoo Jeon' ${ }^{1}$ Reza Maram ${ }^{1}$, James van Howe ${ }^{2}$, Jose Azana'; 'Energie, Matériaux et Télécommunications, Institut National de la Recherche Scientifique, Canada; ${ }^{2}$ Dept. of Physics and Astronomy, Augustana College, USA. We introduce a new design for Talbotbased passive amplifiers, involving temporal phase modulation and dispersion, in which the gain factor can be electrically reconfigurable. In particular, we show gain factors from $m=2$ to 30 using a fixed dispersive line.

SF2L.2 • 10:45

Extended time cloak based on inverse temporal Talbot effect, Bowen $\mathrm{Li}^{1}$, Xie Wang ${ }^{2}$, Jiqiang Kang ${ }^{1}$, Yuan Wei', Kenneth Kin-Yip Wong'; ${ }^{1}$ Univ. of Hong Kong, China: ${ }^{2}$ Huawei Technologies Co., LTD, China. Inverse temporal Talbot effect is used to enhance the performance of time cloak, achieving a continuous cloaking window of $196 \mathrm{ps}$, which is 5.4 time larger than previous record. Pseudo-random temporal event is successfully concealed.

\section{SF2L.3 • 11:00}

Agile photonic generation of arbitrary RF chirped waveforms, Hugues Guillet de Chatellus ${ }^{1,2}$, Luis Romero Cortes $^{2}$, Maurizio Burla ${ }^{3}$, Come Schnébelin', Jose Azana2. 'LIPhy, France: ${ }^{2}$ INRS-EMT, Canada; ${ }^{3} E T H$, Switzerland. We demonstrate a simple platform for reconfigurable generation of arbitrary RF chirped waveforms from a single CW laser. Our scheme is capable of generating $>100$ $\mathrm{GHz}$ bandwidth chirps with a time-bandwidth product up to $\sim 1300$

SF2L.4 $411: 15$

Surface Nanoscale Axial Optomechanics,

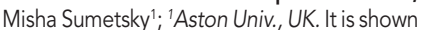
that slow acoustic modes similar to optical modes can be fully controlled by nanoscale variation of the optical fiber radius. Acoustic antibottle microresonators are discovered. Optical frequency combs generated mechanically are investigated.
10:30-12:30

SF2M • Combustion Diagnostics and Imaging

Presider: Scott Howard; Univ. of Notre Dame, USA

SF2M.1 • 10:30

Tracking objects surrounded by scattering media, Milad Akhlaghi Bouzan', Aristide Dogariu': ${ }^{1}$ CREOL, College of Optics and Photonics, Univ. of Central Florida, USA. Statistical properties of integrated scattered intensity from an object, under illumination with dynamic diffused light thorough a scattering wall, are exploited for tracking objects in real time. Experimental demonstrations are presented.

SF2M.2 • 10:45

Underwater Three-Dimensional Imaging Using Single-Photon Detection, Aurora Maccarone ${ }^{1}$, Abderrahim Halimi ${ }^{1}$, Aongus McCarthy', Rachael Tobin', Stephen McLaughlin', Yvan Petillot', Gerald S. Buller ${ }^{1} ;{ }^{1}$ Heriot-Watt Univ., UK. Depth profile measurements are performed in highly scattering underwater environments at stand-off distances equivalent to nine attenuation lengths. The optical system used a scanning transceiver and single-photon detection to produce high-resolution images with milliwatt average powers

SF2M.3 $\bullet 11: 00$

Coherent Noise Reduction Using Heterodyne Detection, Milad Akhlaghi Bouzan ${ }^{1}$ Aristide Dogariu'; ' ${ }^{1}$ CREOL, The College of Optics \& Photonics, Univ. of Central Florida, USA. Coherent stochastic noise affects the information content in imaging systems. We demonstrate the noise reduction using the intrinsic coherence properties of the field with single-shot heterodyne detection and we quantify the efficiency in low-SNR conditions.

SF2M.4 $11: 15$

Mid-Infrared Spectroscopic Imaging with a Low-Cost Microbolometer Array, David Benirschke ${ }^{1}$, Scott S. Howard'; ${ }^{1}$ Electrical Engineering, Univ. of Notre Dame, USA. A low-cost, commercially available vanadium oxide microbolometer array is characterized in terms of its noise equivalent differential temperature and responsivity spectra. The same array is used in an FTIR spectroscopic imaging demonstration. 
Executive Ballroom $210 \mathrm{~A}$

Executive Ballroom 210B

\section{CLEO: Applications \& Technology}

AF2A - A\&T Topical Review on Supercontinuum and Applications II-Continued

\section{AF2A.3 - 11:30 Invited}

Label-free Techniques for the Assessment of Cancer and other Diseases using the Supercontinuum Light Source at the Four NIR Optical Windows, Laura Sordillo1,2, Peter P. Sordillo ${ }^{1,3}$, Lingyan Shi ${ }^{1,4}$, Robert R. Alfano ${ }^{1,2}$ ${ }^{1}$ Inst. for Ultrafast Spectroscopy and Lasers, The City College of New York, USA; ${ }^{2}$ The Grove School of Engineering, The City College of New York, USA; ${ }^{3}$ Dept. of Hematology and Oncology, Lenox Hill Hospital, USA ${ }^{4}$ Chemistry Dept., Columbia Univ., USA. The supercontinuum can generate wavelengths at all four near-infrared windows simultaneously. Additionally, using the supercontinuum can avoid the disadvantages of methods that require contrast agents as it utilizes intrinsic optical properties of the studied material.

\section{AF2B • Applied Spectroscopy-} Continued

\section{AF2B.4 $11: 30$ Invited}

Isotope-Selective Breath Analysis, Albert Manninen', Teemu Kääriäinen'1, Markus Metsälä2, Markku Lehto ${ }^{3,4}$, Eero Hietala ${ }^{1}$ Rami Aikio ${ }^{1}$, Hannu Vasama ${ }^{1}$, Paloma Ruiz Y Kärkkäinen ${ }^{2}$, Craig Richmond ${ }^{1}$, Pekka Suopajärvi'; ${ }^{2}$ TTT Technical Research Centre of Finland, Finland: ${ }^{2}$ Dept. of Chemistry, Univ. of Helsinki, Finland; ${ }^{3}$ Folkhälsan Inst. of Genetics, Folkhälsan Research Center, Finland ${ }^{4}$ Abdominal Center Nephrology, Univ. of Helsinki and Helsinki Univ. Hospital, Finland. Isotope-selective breath analysis offers a non-invasive, fast and reliable alternative to traditional methods in numerous clinical applications. Especially stable isotopologues of carbon dioxide have been applied to tens of different studies.

\section{Executive Ballroom} $210 \mathrm{C}$

\section{CLEO: Science \& Innovations}

\section{SF2C • Frequency Comb Applications-Continued}

SF2C.2 $\bullet 11: 30$

Frequency comb transferred by plasmonic EOT, Young-Jin Kim', Byung Jae Chun' Seungchul Kim²; ${ }^{1}$ Nanyang Technological Univ., Singapore; ${ }^{2}$ Pusan National Univ. Korea (the Republic of). We demonstrate that frequency comb can be transferred by plasmonic nanostructures without noticeable degradation in absolute position and linewidth, which implies frequency comb's potential applications in nanoplasmonic spectroscopy.

SF2C.3 • 11:45

Development of confocal laser scanning microscopy by use of optical frequency comb, Takeo Minamikawa ${ }^{3,1}$. Eiji Hase ${ }^{3,1}$ Shuji Miyamoto ${ }^{3,1}$, Hirotsugu Yamamoto ${ }^{2,1}$, Takeshi. Yasui ${ }^{3,1} ;{ }^{1}$ JST, ERATO MINOSHIMA Intelligent Optical Synthesizer, Japan; ${ }^{2}$ Utsunomiya Univ., Japan; ${ }^{3}$ Tokushima Univ., Japan. We proposed a confocal laser scanning microscopy based on dual-comb spectroscopy, which enables the three-dimensional quantitative amplitude and phase imaging

\section{AF2A.4 12:00 Invited}

Future Supercontinuum Microscope for Medical and Biological Applications Lingyan Shi'2, Robert R. Alfano'; ${ }^{1}$ Physics, City College of New York, USA; ${ }^{2}$ Chemistry, Columbia Univ., USA. A supercontinuum (SC) light microscope is proposed for linear and nonlinear optical resonance and nonresonance processes in medical and biological applications.
AF2B.5 $\bullet 12: 00$

In-line Monitoring of Water Quality by Combined Fluorescence and Raman Spectroscopy, Ivan Maleev², Abdul Khan ${ }^{1}$ Lubna Peerzada', Alexander Khmaladze ${ }^{1}$ Anna Sharikova'; 'Physics, SUNY at Albany, USA; ${ }^{2}$ LiqWiz, USA. Toxic and hazardous contaminants in household water present a significant health risk. We are developing a ow cost, automated, multi-channel integrated system for in-line monitoring of common water contaminants, and are demonstrating preliminary results.

AF2B.6 $\bullet 12: 15$

Spectroscopic Characterization of Si/Mo Thin-film Stack at Extreme Ultraviolet Range, Yen-Yin Li², Yin-Wen Lee', I-Chou $\mathrm{Wu}^{2}$, Sheng-Lung. Huang ${ }^{2,3}$; ${ }^{1}$ Dept. of Electro-Optical Engineering, National Taipei Univ. of Technology, Taiwan; ${ }^{2}$ Inst. of Photonics and Optoelectronics, National Taiwan Univ., Taiwan: ${ }^{3}$ Dept. of Electrical Engineering, National Taiwan Univ., Taiwan. A common-path interferometry based extreme ultraviolet (EUV) spectrometer was used to characterize a Si/Mo thin-film beamsplitter. The complex transfer function of the Si/Mo stack was successfully obtained and verified near the pristine 13.5-nm wavelength range.
Executive Ballroom 210D

\section{Joint}

JF2D • Symposium on Thermal Noise in Precision Interferometry II-Continued

\section{JF2D.3 • 11:30 Invited}

ECR ion beam deposition for the fabrication of ultra-low loss optical coatings, Stuar Reid', Ross Birney ${ }^{1}$, lain Martin ${ }^{2}$, Jessica Steinlechner ${ }^{2 .}{ }^{1}$ Univ of the West of Scotland, UK ${ }^{2}$ Physics and Astronomy, Univ. of Glasgow UK. This presentation will describe a nove ECR (electron cyclotron resonance) technique for fabricating ion beam sputtered optical coatings, developed by the Univ. of the West of Scotland and the Univ. of Glasgow.
SF2C $4 \cdot 12: 00$

Long Depth-of-Focus Imaging by a NonDiffracting Optical Needle under Strong Aberration, Yuichi Kozawa ${ }^{1,2}$, Shunich Sato ${ }^{1} ;{ }^{1}$ Inst. of Multidisciplinary Research for Advanced Materials, Tohoku Univ., Japan 2JST PRESTO Japan. We experimentally investigated the focusing property of an annular-shaped beam. Apparent robustness against optical aberration was revealed when an annular-shaped beam is tightly focused in the presence of strong spherical aberration.

SF2C.5 $\bullet 12: 15$

Non-scanning three-dimensional tomographic imaging using chirped-frequency comb, Takashi Kato ${ }^{1,2}$, Megumi Uchida ${ }^{1,2}$ Yurina Tanaka ${ }^{1}$, Kaoru Minoshima1,2. 'The Univ. of Electro-Communications (UEC) Japan; ${ }^{2} J S T$, ERATO MINOSHIMA Intelligen Optical Synthesizer (IOS), Japan. One-shot 3D tomographic imaging with a chirpedfrequency comb is demonstrated using $2 \mathrm{D}$ pulse-to-pulse spectral interferometry. The profile of the glasses' layered structure is measured, and the thickness of each laye is deduced with 100-nm-level uncertainty.
JF2D $4 \cdot 12: 00$

Time-dependent correlation of cross-polarization mode for microcavity temperature sensing and stabilization, Jinkang Lim ${ }^{1}$ Wei Liang ${ }^{2}$, Andrey Matsko², Lute Maleki², Cheewei Wong'; ${ }^{1}$ Univ. of California Los Angeles, USA: ${ }^{2}$ OEwaves Inc., USA. We report time-dependent negative correlation between the beatnote of cross-polarization resonant modes and one of the polarization resonant modes, which could be utilized for improving the microcavity resonant frequency long-term stability.

JF2D.5 • 12:15

Symposium Wrap Up and Discussion 


\section{CLEO: QELS-Fundamental Science}

FF2E - Quantum TechnologiesContinued

FF2E.5 • 11:30

Practically Noiseless Parametric Frequency Upconverter, Ivan A. Burenkov', Thomas Gerrits $^{2}$, Adriana Lita2 ${ }^{2}$ Sae Woo Nam², Lynden K. Shalm², Sergey V. Polyakov ${ }^{3}$; 'Joint Quantum Inst., NIST and UMD, USA; ${ }^{2}$ National Institure of Standards and Technology, USA; ${ }^{3}$ National Inst. of Standards and Technology, USA. We demonstrate an efficient and inherently ultra-low noise frequency upconverter, resulting in only $\approx 100$ background photons per hour. To measure unprecedentedly low count rate, we introduced a dark count reduction algorithm for a transition edge sensor.

FF2E.6 • 11:45

Quantum Frequency Down-Conversion of $\mathrm{Ca}^{+}$-resonant Polarization-Entangled Photons to the Telecom O-Band, Matthias Bock', Stephan Kucera', Jan Arenskötter ${ }^{1}$, Benjamin Kambs ${ }^{1}$, Sebastian Rühle ${ }^{1}$, An- $^{\prime}$ dreas Lenhard', Jürgen Eschner', Christoph Becher1; 'Universität des Saarlandes, Germany. We demonstrate polarization-preserving quantum frequency conversion of entangled photons from a transition wavelength of trapped $\mathrm{Ca}^{+}-$ions at $854 \mathrm{~nm}$ to the telecom $\mathrm{O}$-band at $1312 \mathrm{~nm}$ and preservation of the entanglement with high fidelity.

FF2E.7 • 12:00

Telecom-Wavelength Quantum Relay Using a Semiconductor Quantum Dot, Jan Huwer ${ }^{1}$, Martin Felle ${ }^{1,2}$, R. Mark Stevenson', Joanna Skiba-Szymanska', Martin B. Ward', lan Farrer², Richard V. Penty², David A. Ritchie$^{2}$, Andrew J. Shields'; ' ${ }^{1}$ Toshiba Research Europe Ltd, UK; ${ }^{2}$ Univ. of Cambridge, UK. A quantum relay is demonstrated using a semiconductor quantum dot emitting at telecom wavelength and weak coherent states from a laser. We achieve low error rates for secure communication protocols and teleportation of arbitrary states.

FF2E.8 • 12:15

A chip-scale single-photon SWAP gate as integrated interface between polarization and spatial-momentum qubits, Zhenda $\mathrm{Xie}^{1}$, Yongnan $\mathrm{Li}^{1}{ }^{3}$, Xinan $\mathrm{Xu}^{2}$, Abhinav $\mathrm{K}$. Vinod ${ }^{1}$, Serdar Kocaman ${ }^{2}$, Tian Zhong ${ }^{4}$, Andrzej Veitia², Mingbin Yu ${ }^{5}$, Dim-Lee Kwong ${ }^{5}$, Franco Wong ${ }^{4}$, Chee Wei Wong ${ }^{1,2}$; ${ }^{1}$ Univ. of California, Los Angeles, USA; ${ }^{2}$ Columbia Univ., USA; ${ }^{3}$ Nankai Univ., China; ${ }^{4}$ MIT, USA; 5 Inst. of Microelectronics, Singapore. We demonstrate a single-photon SWAP gate between polarization and spatial momentum on a SOI chip. $19.8 \%$ error ratio is obtained, and phase coherence of the SWAP operation is measured via single photon interference with up to $58.7 \%$ visibility.

\section{FF2F • Valley Coherence and Polariton Dynamics in 2D Materials-Continued}

FF2F.4 1 11:30

Valley Polarized Exciton Polaritons From Two-dimensional Semiconductor In Microcavity, Zheng Sun 1,2, Jie Gu, , , Areg Ghazaryan 1, Zav Shotan', Christopher R. Considine', Michael Dollar ${ }^{1}$, Pouyan Ghaemi ${ }^{1,2}$, Vinod M. Menon 1,2; 'Physics, City College of New York, USA; ${ }^{2}$ City Univ. of New York, USA. We report the observation of room temperature strongly coupled microcavity polaritons that are valley polarized due to the coupling of the photons with specific helicity to excitons in the distinct valleys of the $2 \mathrm{D}$ material.

\section{FF2F.5 • 11:45}

Valley Polarization Dynamics of Inter- and Intra-valley Trions in Monolayer WSe2, Akshay Singh², Kha Tran², Mirco Kolarczik', Joe Seifert ${ }^{2}$, Yiping Wang ${ }^{2}$, Kai Hao ${ }^{2}$, Dennis Pleskot $^{3}$, Nathaniel Gabor ${ }^{3}$, Sophia Helmrich ${ }^{1}$, Nina Owschimikow', Ulrike Woggon ${ }^{1}$, Xiaoqin Li'; ${ }^{1}$ Technical Univ. Berlin, Germany; ${ }^{2}$ Universtity of Texas at Austin, USA; ${ }^{3}$ Dept. of Physics and Astronomy, Univ. of California, USA. We investigate valley polarization dynamics of trions in WSe\$2\$ in resonant pump-probe experiments. Intra- and inter-valley trions show distinct polarization dynamics, the former exhibits long-lived valley polarization ( $>25 \mathrm{ps}$ ), the latter a fast decay of $\sim 4$ ps.

\section{FF2F.6 • 12:00}

Trion Valley Coherence in Transition Metal Dichalcogenides, Kai Hao', Lixiang $\mathrm{Xu}^{1}$, Fengcheng Wu', Philipp Nagler², Kha Tran', Xin Ma ${ }^{1}$, Christian Schüller ${ }^{2}$, Tobias Korn², Allan MacDonald', Galan Moody ${ }^{3}$, Xiaogin Li': ${ }^{1}$ Dept. of Physics, Univ. of Texas at Austin, USA; ${ }^{2}$ Dept. of Physics, Univ. of Regensburg, Germany; ${ }^{3}$ National Inst. of Standards \& Technology, USA. We use two dimensional Fourier transform spectroscopy to measure coherence between the trions in the opposite valleys in monolayer $\mathrm{MoSe}_{2}$. The trion valley coherence time $\sim 230 \mathrm{fs}$ is limited by pure dephasing of the trion resonance.

\section{FF2F.7 • 12:15}

Generation, transport, and detection of valley-coupled spin-polarized electrons in $\mathrm{WSe}_{2}$-graphene-topological insulator heterostructure devices, Soonyoung Cha', Doeon Lee', Je-Hyun Kim², Minji Noh', Hyemin $\mathrm{Bae}^{1}$, Hoil $\mathrm{Kim}^{3}$, Sungjun $\mathrm{Cho}^{1}$, Wooyoung Shim ${ }^{1}$, Jun Sung $\mathrm{Kim}^{3}$, Dohun Kim², Hyunyong Choi'; ' YYonsei Univ., Korea (the Republic of); ${ }^{2}$ Seoul National Univ., Korea (the Republic of); ${ }^{3}$ Pohang Univsersity of Science and Technology, Korea (the Republic of). We report a novel hybrid heterostructure device that merges the valleytronic and spintronic functionality. We sequentially generate, transport and detect the valley-coupled spin-polarized electrons via non-local photogalvanic effects from WSe ${ }_{2}$-graphene- $\mathrm{Bi}_{1,5} \mathrm{Sb}_{0.5} \mathrm{Te}_{1.7} \mathrm{Se}_{1.3}$.
FF2G • Nanoscale Control of Quantum Emission-Continued

FF2G.4 • 11:30

Ultrafast room-temperature single photon source with plasmonic nanocavities, Maiken H. Mikkelsen"; 'Duke Univ., USA. A single quantum dot coupled to a metallic cavity acts as a directional, efficient, and ultrafast single photon source with a spontaneous emission lifetime of 13 ps, corresponding to a Purcell factor of 540 .

FF2G.5 • 11:45

Multipath Emission Enhancements in Quantum Dot-Plasmon Coupling, Akash Kannegulla', Ye Liu', Bo Wu', Li-Jing Cheng'; 'Oregon State Univ., USA. We report the mechanism of plasmon-enhanced quantum dot (QD) emission as a result of interactions between QDs via plasmon, and interactions between QDs and the plasmon excited by both $\mathrm{QD}$ emission and external illumination.

FF2G.6 • 12:00

Purcell enhanced Spontaneous Emission of Colloidal Perovskite Nanocrystals, Zhili Yang', Maryna Bodnarchuk², Edo Waks'; 'Univ. of Maryland, College Park, USA; ${ }^{2}$ ETH Zürich - Swiss Federal Inst. of Technology Zürich, Switzerland. We demonstrate spontaneous emission Purcell enhancement (by an average factor of 2.9) of $\mathrm{CsPbBr}_{3} \mathrm{col}-$ loidal perovskite nanocrystals coupled to a nanobeam photonic crystal cavity, at room temperature.

\section{FF2G.7 • 12:15}

Probing and Mapping Optical Fields in $\mathrm{Si}$ Disk Arrays with $\mathrm{Eu}^{3+}$, Natalia Noginova', Soheila Mashhadi', Mikhail Noginov', Katie Chong'2, Yuri Kivshar², David Keene', Aleksandr Vaskin ${ }^{3}$, Evgenia Rusak ${ }^{4}$, Carsten Rockstuhl ${ }^{4}$, Thomas Pertsch ${ }^{3}$, Dragomir N.Neshev', Isabelle Staude ${ }^{3}$; ${ }^{1}$ Norfolk State Univ., USA; ${ }^{2}$ The Australian National Univ., Australia; ${ }^{3}$ Friedrich Schiller Univ. Jena," Germany; ${ }^{4}$ Karlsruhe Inst. of Technology, Germany. We experimentally study enhancement of spontaneous emission of $\mathrm{Eu}^{3+}$ at the magnetic dipole transition by Mie-resonances in silicon nanodisks and estimate the spectral branching ratio as the function of the nanodisk radius. 


\section{Executive Ballroom $210 \mathrm{H}$}

Meeting Room
$211 \mathrm{~B} / \mathrm{D}$

Meeting Room

$212 \mathrm{~A} / \mathrm{C}$

\section{CLEO: Science \& Innovations}

SF2I - Detectors and Other Novel Devices-Continued

SF2I.4 • 11:30

Two-dimensional Quantum Walk using 3D Silicon Photonic Fabrication, Libin Yan ${ }^{1}$, Jianguo Huang ', Gong Zhang ', Leong Chuang Kwek ${ }^{1,2}$, Jiangbin Gong ${ }^{2}$, Weibo Gao ${ }^{1}$, Yidong Chong', Wee Ser ${ }^{1}$, Ai Qun Liu'; ${ }^{1}$ Nanyang Technological Univ., Singapore; ${ }^{2}$ National Univ. of Singapore, Singapore. A 2D quantum walk on an integrated quantum photonic circuit is demonstrated by using a multilayer low-loss $\mathrm{Si}_{3} \mathrm{~N}_{4}$ waveguide lattice, which has promising applications in quantum computing and quantum communication, etc.

\section{SF2I.5 • 11:45}

Multivariable Phase Tuning Control and its Application to Wavelength Tracking in High-Order Multi-Ring Filters, Jason Mak', Wesley D. Sacher ${ }^{1,2}$, Jared C. Mikkelsen ${ }^{1}$, Joyce Poon'; 'Univ. of Toronto, Canada; ${ }^{2}$ California Inst. of Technology, USA. We propose a multivariable controller based on a state-space approach and estimation that only requires a single monitor. The procedure is demonstrated on a tunable silicon 5-ring filter to track the passband to a reference wavelength.

\section{SF2I.6 • 12:00}

Power-dependence of high- $\mathrm{Q}$ optomechanical oscillators: from pre-oscillation, to oscillation, to Drude-plasma, Jaime Flor Flores ${ }^{1}$, Yongjun Huang ${ }^{1}$, Lingzhi Li ${ }^{1}$, Vito laia', Chee Wei Wong'; 'UCLA, USA. We demonstrate the power dependence of high$\mathrm{Q} O M O s$, which permits pre-oscillation, oscillation, and chaos like performance. Power dependence on mechanical frequency is also modeled and simulations are compared to the measured data.

\section{SF2I.7 • 12:15}

Organic Membrane Photonic Waveguide with Metal Grating Couplers, Tomo Amemiya ${ }^{1}$, Toru Kanazawa' ${ }^{1}$, Takuo Hiratani' ${ }^{1}$ Daisuke Inoue ${ }^{1}$, Zhichen Gu', Satoshi Yamasaki', Tatsuhiro Urakami2, Shigehisa Arai'; 'Tokyo Inst. of Technology, Japan; ${ }^{2}$ Mitsui Chemicals, Inc., Japan. We made an organic-membrane waveguide with input/output metal grating couplers, a basic element of organic membrane photonic integrated circuits. The propagation loss and coupling loss were $1.4 \mathrm{~dB} / \mathrm{cm}$ and $27 \mathrm{~dB} /$ coupler, respectively.
$\mathrm{SF} 2 \mathrm{~J} \cdot$ Surface Emitting Lasers-Continued

SF2J.4 $11: 30$

Uniform Operation of Coherent Photonic Crystal VCSEL Arrays, Harshil Dave'1, Stewart T. Fryslie', Zihe Gao', Bradley Thompson", Katherine Lakomy ${ }^{1}$, Kent D. Choquette ${ }^{1}$; 'Univ. of Illinois, USA. Coherent 1×2 VCSEL arrays with improved electrical isolation have enhanced output power, visibility, and uniformity for injection locked operation. The current range for locked operation is as large as $0.5 \mathrm{~mA}$, which decreases with increasing bias.

\section{SF2J.5 • 11:45}

Uniform, High Modulation Bandwidth VCSEL Arrays, Stewart Fryslie", Zihe Gao', Harshil Dave', Bradley Thompson', Katherine Lakomy', Kent D. Choquette"; ' ${ }^{1}$ niv. of Illinois Urbana-Champaign, USA. We show improved performance from high-speed 1 2 photonic crystal VCSEL arrays. A modulation bandwidth of $37 \mathrm{GHz}$ is obtained under highly single mode coherently coupled operation in the in-phase coupled mode. Modulation bandwidth $>30 \mathrm{GHz}$ are found for all photonic crystal designs on the sample.

SF2J.6 $12: 00$

GaSb-based Electrically-Pumped Vertical Cavity Surface Emitting Lasers for the 3-4 um Wavelength Range, Ganpath Kumar Veerabathran', Stephan Sprengel', Alexander Andrejew ${ }^{1}$, Markus-Christian Amann ${ }^{1}$; 'Walter Schottky Inst., Technische Universität München, Germany. We present GaSb-based single-mode electrically-pumped VCSELs emitting at 3 and $3.9 \mu \mathrm{m}$. Continuous wave operation at thermo-electrically cooled temperatures and over $19 \mathrm{~nm}$ of electro-thermal tuning range are achieved.

SF2J.7 • 12:15

Projection of freely designed images by integrable phase-modulating surface-emitting lasers, Yoshitaka Kurosaka', Kazuyoshi Hirose', Takahiro Sugiyama', Yu Takiguchi', Yoshiro Nomoto'; 'Hamamatsu Photonics, Japan. We demonstrate semiconductor lasers which emit freely designed images directly without any optical elements or scanners. We introduce holographic modulation in a square lattice photonic crystal. The fabricated devices are on-chip-sized and suitable for integration. 


\section{CLEO: Science \& Innovations}

SF2K • Infrared Laser SourcesContinued

SF2K.2 $111: 30$

High Average Power Tm:YAG Waveguide Lasers, Bert Callicoatt', Glenn Bennett ${ }^{1}$, Michael Hinckley', Eliot Petersen', Andrew Schober', Greg Wagner'; ; ${ }^{1}$ LMCT, USA. Pulsed and $C W$ results are presented for a Tm:YAG self-imaging planar waveguide laser. The laser achieved CW power of $240 \mathrm{~W}$ at 2012 $\mathrm{nm}$ with $23 \%$ electrical-to-optical efficiency.

SF2K.3 • 11:45

Passively Mode-Locked Tm:LuAG Ceramic Laser, Yicheng Wang ${ }^{1}$, Ruijun Lan², Xavier Mateos ${ }^{1,3}$, Jiang Li ${ }^{4}$, Soile Suomalainen ${ }^{5}$, Antti Härkönen ${ }^{5}$, Mircea Guina ${ }^{5}$, Uwe Griebner ${ }^{1}$, Valentin Petrov'; 'Max Born Inst., Germany; ${ }^{2}$ Yantai Univ., China; ${ }^{3}$ Universitat Rovira i Virgili , Spain: ${ }^{4}$ CAS Shanghai Inst. of Ceramics, China; ${ }^{5}$ Tampere Univ. of Technology, Finland. We study a Tm:LuAG ceramic laser operating with $52 \%$ efficiency and $265 \mathrm{~nm}$ tuning range in the continuous-wave regime and delivering 3.8-ps pulses when mode-locked at $2021 \mathrm{~nm}$ by a SESAM

SF2K.4 $\bullet 12: 00$

Continuous-Wave $3.1 \mu \mathrm{m}$ Gas Fiber Laser with 0.47 W Output Power, Mengrong Xu', Fei $\mathrm{Yu}^{1}$, Muhammad R. Hassan ${ }^{1}$, Jonathan Knight ${ }^{1}$; ${ }^{\prime}$ Univ. of Bath, UK. We demonstrate $0.47 \mathrm{~W}$ of continuous-wave $3.1 \mathrm{~mm}$ output from an acetylene-filled hollow-core fiber when pumped by a diode-seeded EDFA at $1.53 \mu \mathrm{m}$. The laser action is dominated by amplified spontaneous emission.

SF2K.5 $\bullet 12: 15$

Mid-IR (4 - $5 \mu \mathrm{m}$ ) Hybrid sub-GW Fe ${ }^{2+}: \mathrm{ZnSe}$ Femtosecond Laser System, Fedor V. Potemkin ${ }^{1}$, Ekaterina Migal', Andrew Pushkin ${ }^{1}$, Anatolij Sirotkin 2,3, Vladimir Kozlovsky ${ }^{4,3}$ Yuri Korostelin', Yuri Podmar'kov', ${ }^{4,5}$, Vladimir Firsov', Mikhail Frolov ${ }^{4,5}$, Vyacheslav Gordienko ${ }^{1} ;{ }^{1}$ M. V. Lomonosov Moscow State Univ., Russia; ${ }^{2}$ A.M. Prokhorov General Physics Inst. of the Russian Academy of Sciences, Russia; ${ }^{3}$ National Research Nuclear Univ. MEPhl, Russia; ${ }^{4}$ P.N. Lebedev Physical Inst. of the Russian Academy of Sciences, Russia; ${ }^{5}$ Moscow Inst. of Physics and Technology, Russia. We demonstrate a first-of-its-kind all-solid-state mid-IR femtosecond laser with tunability from $3.8 \mu \mathrm{m}$ up to $4.8 \mu \mathrm{m}$ based on amplification of AGS-OPA seed in multi-pass $\mathrm{Fe}^{2+}: \mathrm{ZnSe}$ amplifier optically pumped by solid-state 3- $\mu \mathrm{m}$ Cr:Yb:Ho:YSGG laser.
SF2L • Advanced Fiber Devices and Concepts-Continued

SF2L.5 • 11:30

Locking of two widely separated cw lasers through a two-wavelength delayed selfheterodyne interferometer, Naoya Kuse ${ }^{1}$ Martin E. Fermann'; 'IMRA America Inc Boulder Research Labs, USA: ${ }^{2}$ IMRA Americ Inc, USA. Two separate cw lasers are locked through a common delayed self-heterodyne interferometer, based on error signal generation via RF mixing of the self-heterodyn signal at the two separate wavelengths.

SF2L.6 $\bullet 11: 45$

Simultaneous Excitatory and Inhibitory Dynamics in A Graphene Excitable Laser, Philip Y. Ma', Bhavin J. Shastri', Alexander N. Tait ${ }^{1}$, Mitchell A. Nahmias ${ }^{1}$, Thomas Ferreira de Lima', Paul R. Prucnall'; 'Princeton Univ., USA. We demonstrate experimentally for the first time the simultaneous excitatory and inhibitory dynamics in a graphene excitable laser. This technology potentially opens novel spike processing functionality for future neuromorphic photonic systems.

\section{SF2L.7 • 12:00}

Two-dimension Nanomaterial Tungsten Disulfide $\left(\mathrm{WS}_{2}\right)$ Integrated Fiber Device as All Optical Phase Shifter, Switch and Modulator Near 1550 nm, Kan Wu $\mathrm{Wu}^{1}$, Chaoshi Guo ${ }^{1}$, Hao Wang ${ }^{1}$, Xiaoyan Zhang' ${ }^{2}$, Jun Wang ${ }^{2}$, Jianping Chen"; 'Shanghai Jiao Tong Univ., China: ${ }^{2}$ SIOM, CAS, China. An alloptical phase shifter near $1550 \mathrm{~nm}$ is realized by depositing two-dimension nanomaterial tungsten disulfide $\left(\mathrm{WS}_{2}\right)$ to tapered fiber. All-optical switch and modulator are also demonstrated by embedding the phase shifter to a Mach-Zehnder interferometer.

SF2M.7 • 12:15

Nitrogen Dioxide Detection by use of Photoacoustic Spectroscopy with a High Power Violet-Blue Diode Laser, Lei Dong ${ }^{1}$, Xukun Yin', Huandan Zheng', Hongpeng $\mathrm{Wu}^{1}$, Suotang Jia ${ }^{1}$, Frank Tittel2; 'Shanxi Univ., China; ${ }^{2}$ ECE Dept, Rice Univ., USA. A nitrogen dioxide $\left(\mathrm{NO}_{2}\right)$ sensor based on conventional photoacoustic spectroscopy and a $3.5 \mathrm{~W}$ multimode violet-blue diode laser was designed and demonstrated. A minimum detection limit of $~ 54$ pptv was obtained.
SF2M.6 • 12:00

of Krypton Using Femtosecond Pulses, Waruna Kulatilaka', Yejun Wang ${ }^{1}:{ }^{1}$ Texas A\&M Univ., USA. We report a comprehensive spectroscopic study of ultrashort-pulse-based, Krypton gas. Prominent fluorescence emission lines are identified in the near-IR region which are useful for multiple flow diagnostics applications

SF2M • Combustion Diagnostics SF2M 5 11.30 Invited tent Combustion with Tomographic h-speed Imaging, Jonathan $\mathrm{H}$. Sandia National Labs, USA. Laser imaging and $3-D$ velocity field measurements using tomographic PIV at a $10-\mathrm{kHz}$ repetiof transient flows at elevated pressure are demonstrated using a $100-\mathrm{kHz}$ repetition rate 